\title{
Absence of Intermediates in the BINOL-derived Mg(II)/Phosphate-Catalyzed Desymmetrizative Ring Expansion of 1-Vinylcyclobutanols
}

\author{
Estefania Capel, ${ }^{\text {a }}$ Marta Rodríguez-Rodríguez, ${ }^{a}$ Uxue Uria, ${ }^{\text {a }}$ Manuel \\ Pedron, ${ }^{\mathrm{b}}$ Tomas Tejero, ${ }^{\mathrm{c}}$ Jose L. Vicario*a and Pedro Merino*b \\ ${ }^{\mathrm{a}}$ University of the Basque Country (UPV/EHU), P.O. Box 644, 48080 Bilbao (Spain). \\ E-mail:uxue.uria@ehu.eus.joseluis.vicario@ehu.eus \\ ${ }^{\mathrm{b}}$ Instituto de Biocomputación y Física de Sistemas Complejos (BIFI). Universidad de Zaragoza, \\ 50009 Zaragoza, Spain. \\ E-mail:pmerino@unizar.es \\ ${ }^{\mathrm{c}}$ Instituto de Sintesis y Catalisis Homogenea (ISQCH). Universidad de Zaragoza-CSIC, 50009 \\ Zaragoza, Spain.
}

\section{Supporting Information}




\section{Table of Contents}

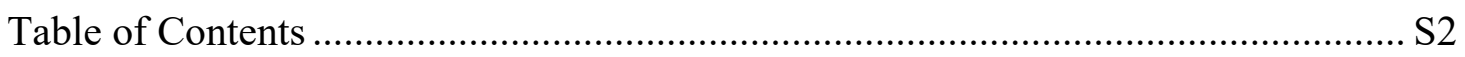

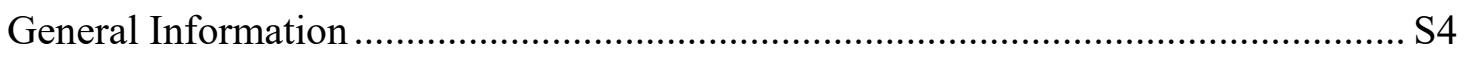

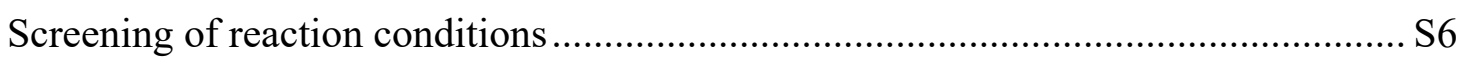

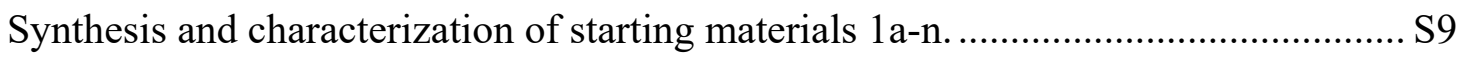

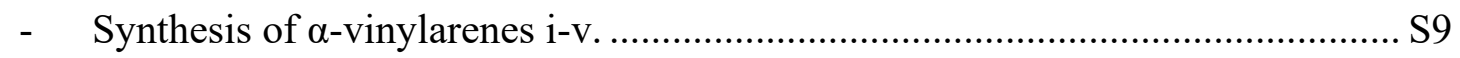

- $\quad$ Synthesis of 3,3-substitutedcyclobutanones I-V ......................................... S10

- Synthesis of 1-bromovinylarenes VI-XIV ….................................................... S12

- $\quad$ Synthesis of 3,3-disubstituted-1-(1-arylvinyl)cyclobutan-1-ols, 1a-1n........... S14

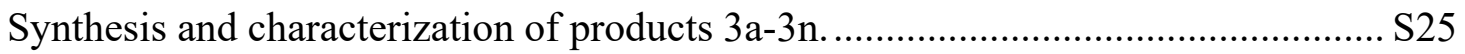

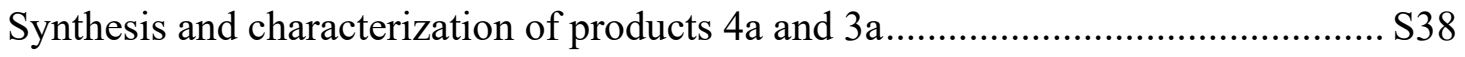

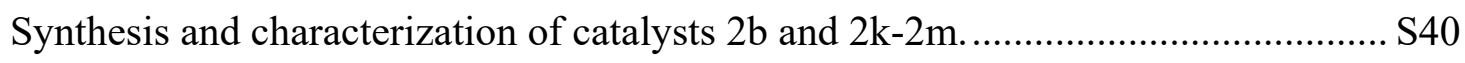

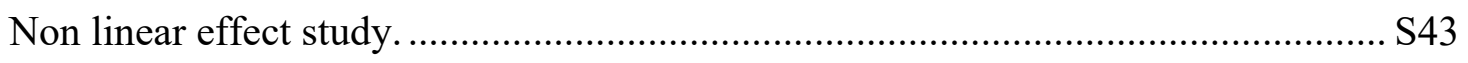

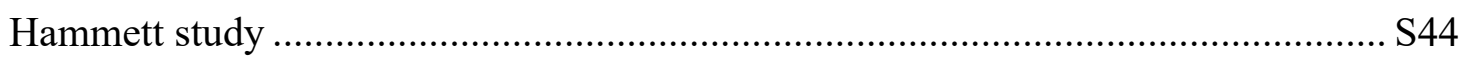

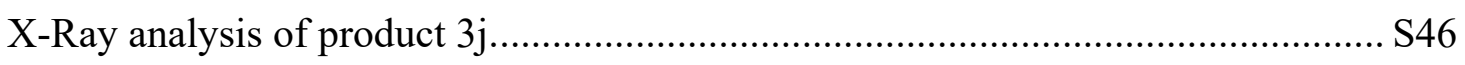

${ }^{1} \mathrm{H},{ }^{13} \mathrm{C}\left\{{ }^{1} \mathrm{H}\right\}(75.5 \mathrm{MHz}),{ }^{19} \mathrm{~F}$ and ${ }^{31} \mathrm{P}$ NMR spectra of cyclobutanones, 1a-n, 3a-n, 4a, 4i

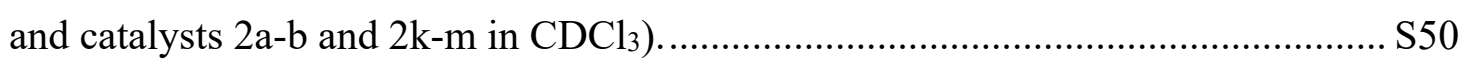

${ }^{31} \mathrm{P}$ NMR $\left(122 \mathrm{MHz}, \mathrm{CDCl}_{3}\right)$ spectra of catalyst mixtures for non linear effect study....

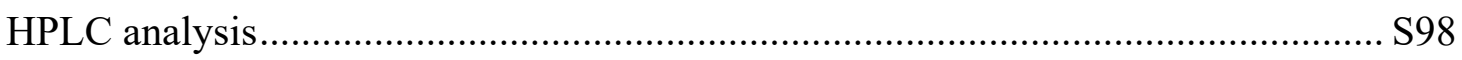

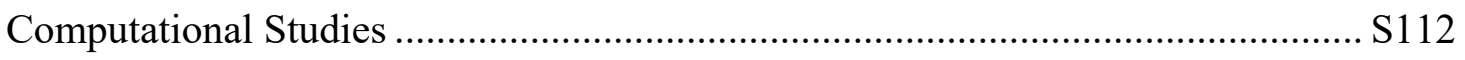

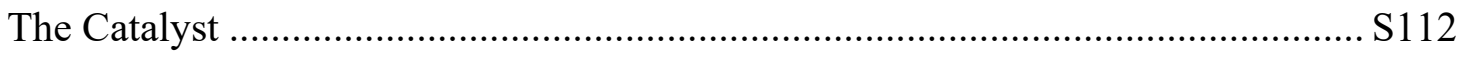

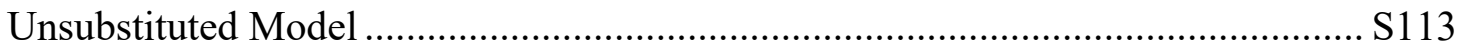

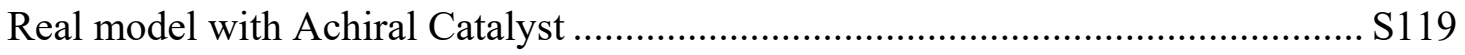

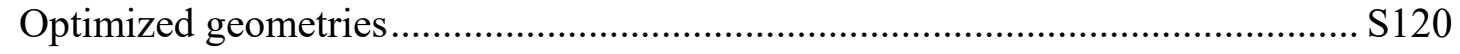

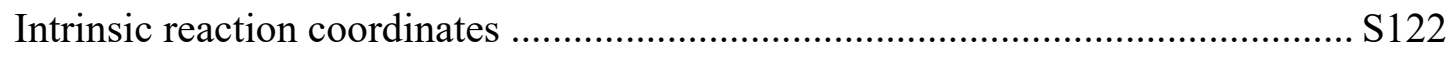

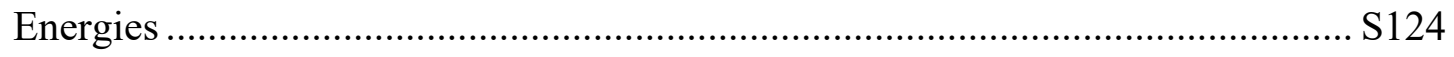

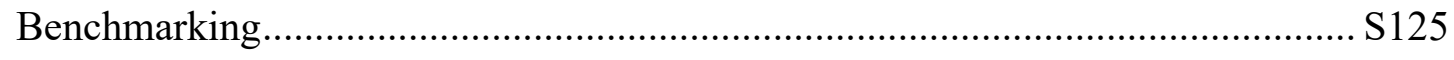


Energy profiles S128

NCI Analysis $\mathrm{S} 130$

ELF Analysis S133

Stabilization of the intermediate. $\mathrm{S} 150$

Real Model with Real Catalyst S153

Molecular Dynamic Simulations. S157

Cartesian Coordinates $\mathrm{S} 162$ 


\section{General Information}

Analytical grade solvents and commercially available reagents were used without further purification. Anhydrous solvents were purified and dried with activated molecular sieves prior to use. ${ }^{1}$ For reactions carried out under inert conditions, the argon was previously dried through a column of $\mathrm{P}_{2} \mathrm{O}_{5}$ and $\mathrm{CaCl}_{2}$. All the glassware was dried for 12 hours prior to use in an oven at $140^{\circ} \mathrm{C}$, and allowed to cool under a dehumidified atmosphere. Reactions were monitored using analytical thin layer chromatography (TLC), in precoated silica-backed plates (Merck Kiesegel 60 F254). Flash column chromatography was performed on standard silica gel (Silicycle 40-63, 230-400 mesh) using standard visualizing agents: UV fluorescence ( 254 and $366 \mathrm{~nm}$ ), potassium permanganate $/ \Delta$ and phosphomolybdic acid stains (PMA)/ $\Delta$. For the removal of the solvents under reduced pressure Büchi R series rotatory evaporators were used. For precision weighting Sartorius Analytical Balance Practum 224-1S was used $( \pm 0.1 \mathrm{mg})$. NMR spectra were recorded at $25^{\circ} \mathrm{C}$ on a Bruker AC-300 spectrometer $\left(300 \mathrm{MHz}\right.$ for ${ }^{1} \mathrm{H}$ and $75.5 \mathrm{MHz}$ for $\left.{ }^{13} \mathrm{C}\right) .{ }^{1} \mathrm{H}$ NMR and ${ }^{13} \mathrm{C}\left\{{ }^{1} \mathrm{H}\right\}$ NMR chemical shifts ( $\delta$ ) are reported in ppm with the solvent (or TMS) resonance as the internal standard $\left(\mathrm{CHCl}_{3}: 7.26 \mathrm{ppm}\left({ }^{1} \mathrm{H}\right)\right)$ and $\left(\mathrm{CDCl}_{3}: 77.16 \mathrm{ppm}\right.$ $\left({ }^{13} \mathrm{C}\right)$. Data are reported as follows: chemical shift, multiplicity $(\mathrm{d}=$ doublet, $\mathrm{t}=$ triplet, $\mathrm{q}$ = quartet, $\mathrm{br}=$ broad, $\mathrm{m}=$ multiplet), coupling constants $(\mathrm{Hz})$ and integration. High resolution mass spectra (HRMS) were recorded using an Aquity UPLC coupled to a QTOF mass spectrometer (SYNAPT G2 HDMS) using electrospray ionization (ESI+). GC-MS analyses were performed on an Agilent 7890A gas chromatograph coupled to an Agilent 5975 quadrupole mass spectrometer under electronic impact ionization (EI) 70 eV. Melting points (M.p.) were measured in a Stuart SMP30 apparatus in open capillary tubes and are uncorrected. The enantiomeric excess (e.e.) of the products was determined by High Performance Liquid Chromatography on a chiral stationary phase in a Waters chromatograph coupled to a Waters photodiode array detector. Daicel Chiralpak AS-H and Chiralcel IA and OD-3 columns $(0.46 \times 25 \mathrm{~cm})$ were used; specific conditions are indicated for each case. Specific optical rotations $\left([\alpha]_{\mathrm{D}^{20}}\right)$ were measured at $20^{\circ} \mathrm{C}$ on a Jasco P-2000 polarimeter with sodium lamp at $589 \mathrm{~nm}$ and a path of length of $1 \mathrm{dm}$. Solvent and concentration are specified in each case. Infrared spectra (IR) were measured

${ }^{1}$ (a) W. L. F. Armarego, C. L. L. Chai. Purification of Laboratory Chemicals, 7th ed.; Elsevier: Oxford, 2012. (b) D. B. G. Williams, M. Lawton, J. Org. Chem. 2010, 75, 8351. 
in a Jasco FT/IR 4100 (ATR) in the interval between 4000 and $600 \mathrm{~cm}^{-1}$ with a $4 \mathrm{~cm}^{-1}$ resolution. X-ray data collections were performed in an Agilent Supernova diffractometer equipped with an Atlas $\mathrm{CCD}$ area detector, and a $\mathrm{CuK} \alpha$ micro-focus source with multilayer optics $(\lambda=1.54184 \AA, 250 \mu \mathrm{m}$ FWHM beam size). The sample was kept at $150 \mathrm{~K}$ with an Oxford Cryosystems Cryostream 700 cooler. The quality of the crystals was checked under a polarizing miscroscope, and a suitable crystal or fragment was mounted on a Mitegen MicromountTM using Paratone N inert oil and transferred to the diffractometer. 


\section{Screening of reaction conditions}

Table S 1. Screening of brominating agents. ${ }^{a}$

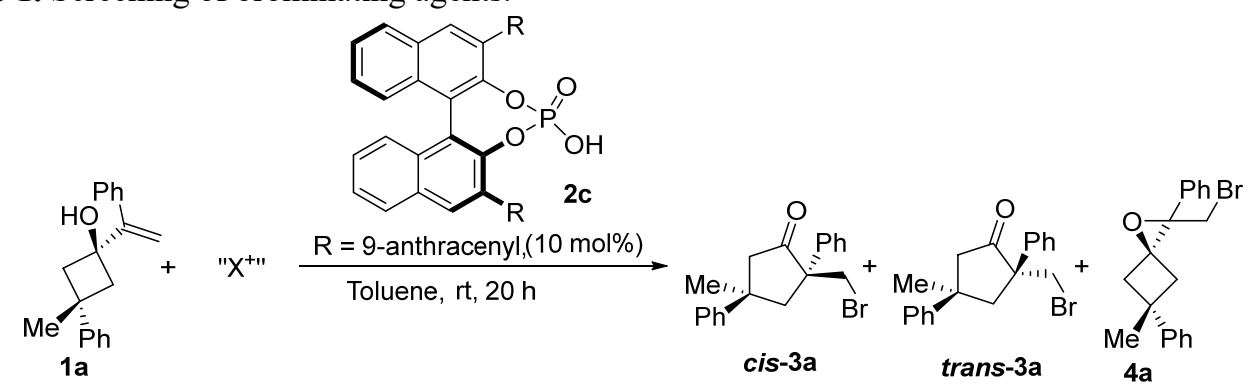<smiles>CC(C)(C)NC(=O)c1cc(C(F)(F)F)cc(C(F)(F)F)c1</smiles>

\begin{tabular}{|c|c|c|c|c|c|c|}
\hline Entry & " $\mathbf{X}^{+} "$ & Conversion & $\begin{array}{c}\text { Global } \\
\text { Yield }(\%)^{b}\end{array}$ & $\begin{array}{c}\text { Ratio } \\
(3 \mathbf{a}: 4 a)^{c}\end{array}$ & $\begin{array}{c}\text { dr 3a (cis: } \\
\text { trans) }\end{array}$ & $\begin{array}{c}\text { ee }(\%)(c i s-3 a / t r a n s \\
3 a / 4 a)^{d}\end{array}$ \\
\hline 1 & I & Complete & 81 & $1.2: 1$ & $6: 1$ & $50 / 5 / 50$ \\
\hline 2 & II & 31 & n.d. & $1: 1.5$ & 1:- & $50 /-/ 50$ \\
\hline 3 & III & 66 & 61 & $6: 1$ & $2: 1$ & $33 / 40 / 25$ \\
\hline 4 & IV & 95 & 70 & $1.3: 1$ & $3: 1:$ & $40 / 74 / 22$ \\
\hline 6 & $\mathbf{V}$ & 83 & 74 & $5: 1$ & $4: 1$ & $30 / 40 / 25$ \\
\hline 7 & VI & 78 & 49 & $1: 1$ & 1:- & $22 /-/ 30$ \\
\hline
\end{tabular}

${ }^{\mathrm{a}}$ Reactions were carried out with $0.10 \mathrm{mmol}$ of ciclobutanol 1a and $0.11 \mathrm{mmol}$ of NBS in toluene $(0.2$ M) at room temperature. ${ }^{b}$ Yields refer to the mixture of isolated products by column chromatography. ${ }^{c}$ Ratio calculated in reaction crude by ${ }^{1} \mathrm{H}-\mathrm{RMN}{ }^{\mathrm{d}}$ Calculated by HPLC on chiral stationary phase. 
Table S 2. Screening of metal phosphates. ${ }^{\mathrm{a}}$

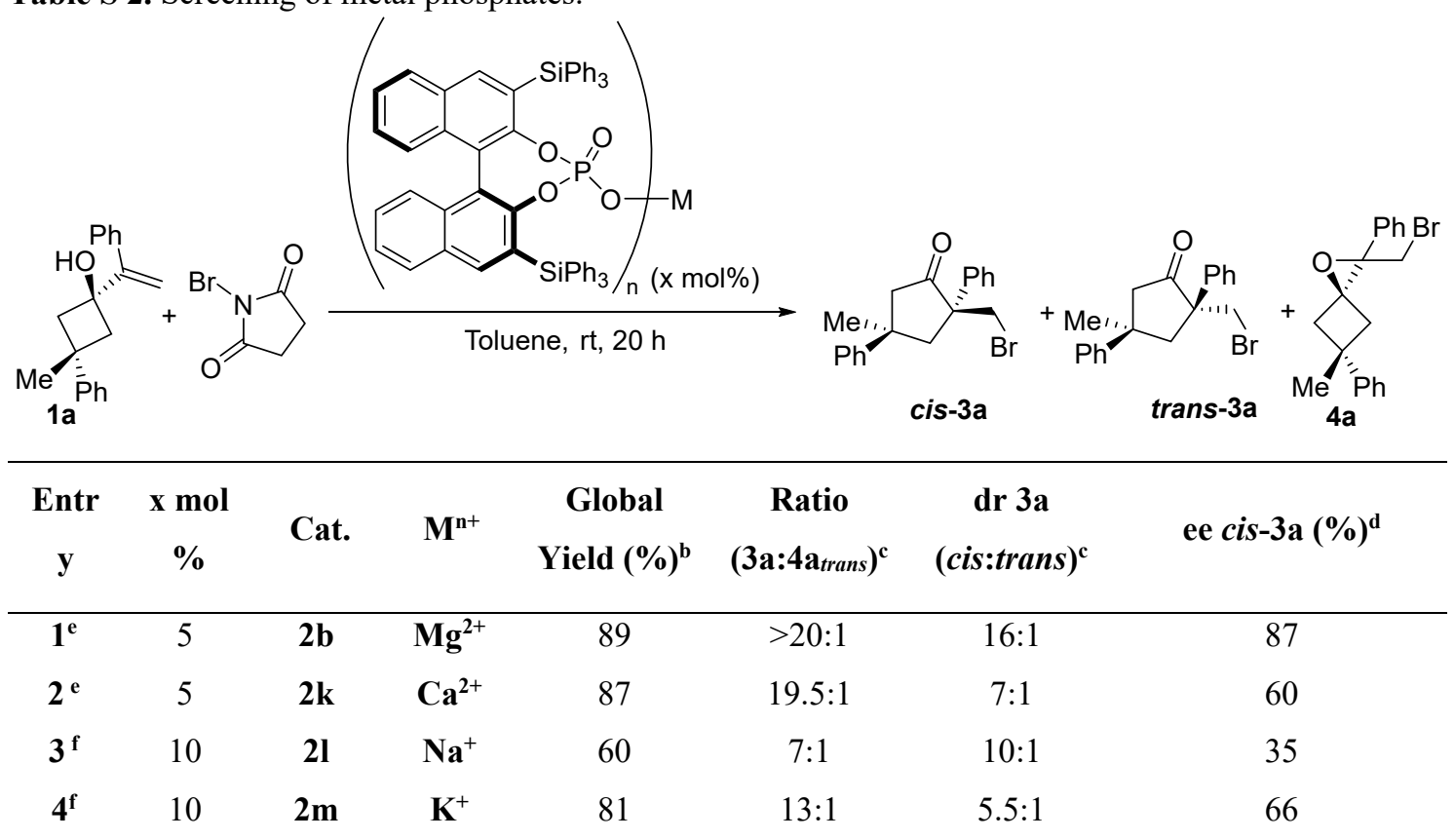

${ }^{\mathrm{a}}$ Reactions were carried out with $0.10 \mathrm{mmol}$ of ciclobutanol 1a and $0.11 \mathrm{mmol}$ of NBS in toluene $(0.2$ M) at room temperature. ${ }^{b}$ Yields refer to the mixture of isolated products by column chromatography. ${ }^{\mathrm{c}}$ Ratio calculated in reaction crude by ${ }^{1} \mathrm{H}-\mathrm{RMN}{ }^{\mathrm{d}}$ Calculated by HPLC on chiral stationary phase. ${ }^{\mathrm{e}}$ Reactions carried out with $5 \mathrm{~mol} \%$ of chiral phosphate. ${ }^{f}$ Reactions carried out with $10 \mathrm{~mol} \%$ of chiral phosphate.

Table S 3. Solvent screening. ${ }^{\text {a }}$

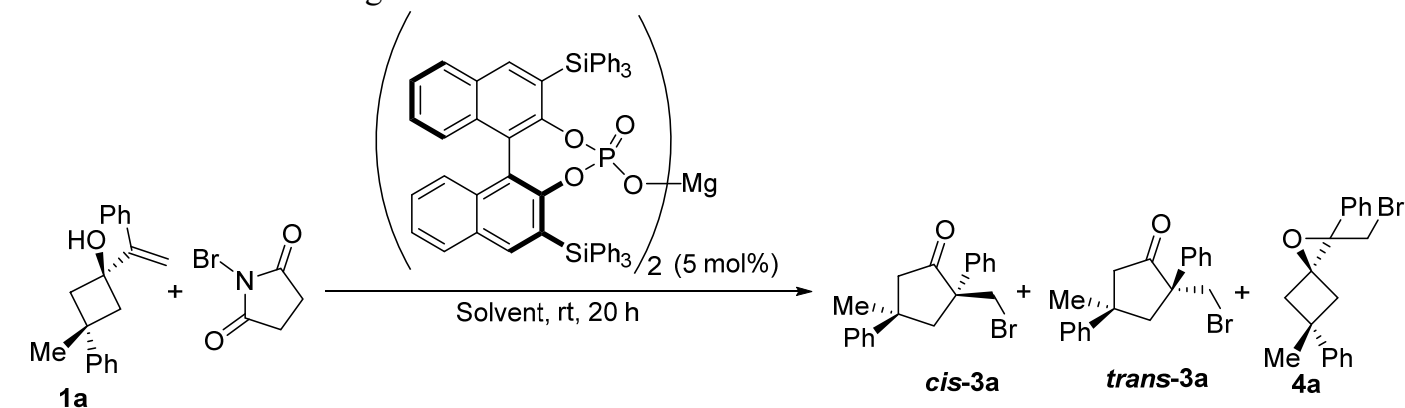

\begin{tabular}{cccccc}
\hline Entry & Solvent & $\begin{array}{c}\text { Global Yield } \\
(\mathbf{\%})^{\mathbf{b}}\end{array}$ & Ratio (3a:4a) $^{\mathbf{c}}$ & dr 3a (cis:trans) $^{\mathbf{c}}$ & ${\text { ee } \text { cis-3a (\%) }^{\mathbf{d}}}^{\text {(3) }}$ \\
\hline $\mathbf{1}$ & Toluene & 89 & $>20: 1$ & $16: 1$ & 87 \\
$\mathbf{2}$ & DCM & 84 & $3.7: 1$ & $10: 1$ & Racemic \\
$\mathbf{3}$ & AcOEt & 73 & $2.7: 1$ & $7: 1$ & 32 \\
$\mathbf{4}$ & THF & 73 & $3.5: 1$ & $6: 1$ & 6 \\
$\mathbf{5}$ & $\boldsymbol{o}$-xylene & 83 & $>20: 1$ & $14: 1$ & 79 \\
$\mathbf{6}$ & $\boldsymbol{m}$-xylene & 84 & $>20: 1$ & $13: 1$ & 87 \\
$\mathbf{7}$ & Mesitylene & 81 & $>20: 1$ & $11: 1$ & 88 \\
\hline
\end{tabular}

${ }^{a}$ Reactions were carried out with $0.10 \mathrm{mmol}$ of ciclobutanol 1a and $0.11 \mathrm{mmol}$ of NBS in toluene $(0.2$ M) at room temperature. ${ }^{b}$ Yields refer to the mixture of isolated products by column chromatography. ${ }^{c}$ Ratio calculated in reaction crude by ${ }^{1} \mathrm{H}-\mathrm{RMN}$. ${ }^{\mathrm{d}}$ Calculated by HPLC on chiral stationary phase. 
Table S 4. Effect of temperature. ${ }^{\mathrm{a}}$

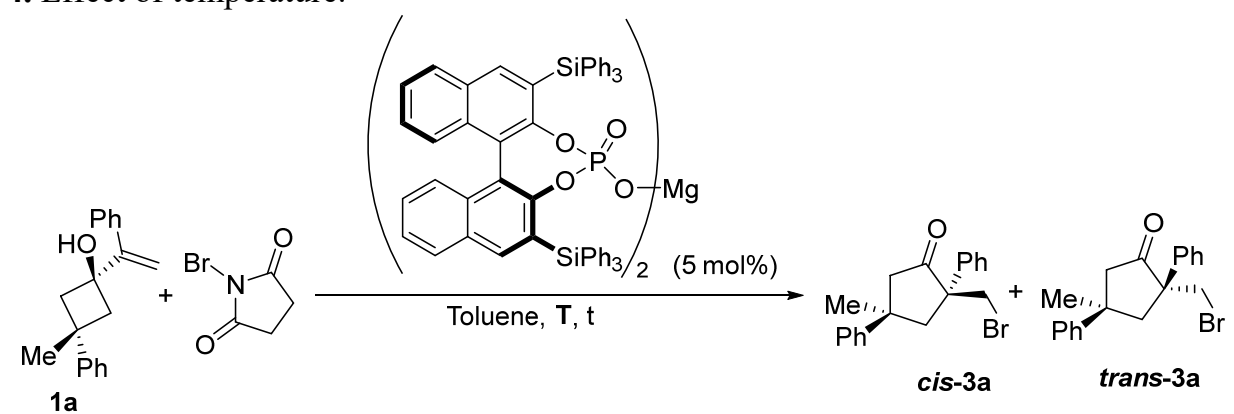

\begin{tabular}{cccccc}
\hline Entry & $\mathbf{T}\left(\mathbf{~}^{\mathbf{0}} \mathbf{C}\right)$ & $\mathbf{t}(\mathbf{h})$ & Yield. 3a (\%) $^{\mathbf{b}}$ & Ratio 3a (cis:trans) $^{\mathbf{c}}$ & ee cis-3a (\%) $^{\mathbf{d}}$ \\
\hline $\mathbf{1}$ & $\mathbf{r t}$ & 20 & 89 & $16: 1$ & 87 \\
$\mathbf{7}$ & $\mathbf{5 0}$ & 8 & 88 & $9.5: 1$ & 87 \\
$\mathbf{8}$ & $\mathbf{0}$ & 20 & 90 & $>20: 1$ & 91 \\
$\mathbf{9}$ & $\mathbf{- 2 0}$ & 48 & 89 & $>20: 1$ & 92 \\
$\mathbf{1 0}^{\mathbf{e}}$ & $\mathbf{0}$ & 72 & 95 & $>20: 1$ & 92 \\
\hline
\end{tabular}

${ }^{\mathrm{a}}$ Reactions were carried out with $0.10 \mathrm{mmol}$ of ciclobutanol 1a and $0.11 \mathrm{mmol}$ of NBS in toluene $(0.2$ M). In all cases the ketone:epoxide ratio was $>20: 1 .{ }^{b}$ Yields refer to the mixture of isolated products by column chromatography. ${ }^{\mathrm{c}}$ Ratio calculated in reaction crude by ${ }^{1} \mathrm{H}-\mathrm{RMN} .{ }^{\mathrm{d}}$ Calculated by HPLC on chiral stationary phase. ${ }^{\text {e }}$ Reaction carried out with $2.5 \mathrm{~mol} \%$ of chiral phosphate $\mathbf{2 b}$. 


\section{Synthesis and characterization of starting materials 1a-n.}

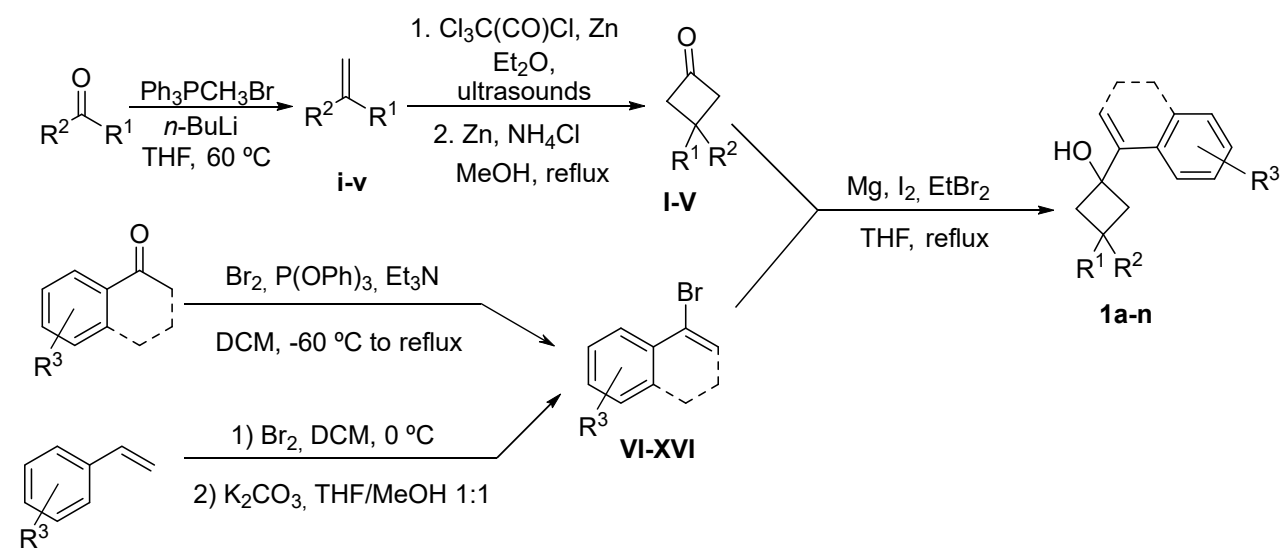

Scheme S 1. General overview of the synthesis of starting materials 1a-n.

- Synthesis of $\alpha$-vinylarenes i-v.

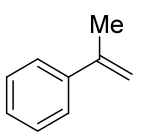

i<smiles>C=C(CC)c1ccccc1</smiles>

ii<smiles>C=C(C)c1ccc2ccccc2c1</smiles>

iii<smiles>C=C(C)c1ccc(C)cc1</smiles>

iv<smiles>C=C(C)c1ccc(Cl)cc1</smiles>

Figure S 1. $\alpha$-vinylarenes employed for the synthesis of cyclobutanones.

Compound $\mathbf{i}$ was purchased from commercial sources and used without further purification. ii- $\mathbf{v}^{2}$ are reported compounds and were prepared by Wittig olefination of the corresponding ketones following the procedures described in the literature ${ }^{3}$.

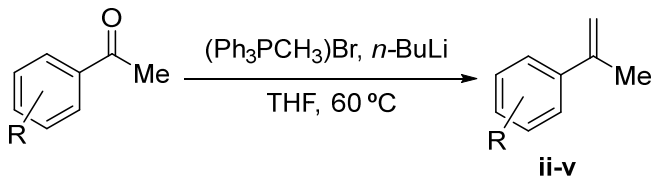

Scheme $\mathbf{S}$ 2. Synthesis of $\alpha$-vinylarenes from carbonyl compounds ii-v.

${ }^{2}$ J. Huang, G. Hu, S. An, D. Chen, M. Li, P. Li, J. Org. Chem. 2019, 84, 9758-9769.

${ }^{3}$ L. Zhang, W. R. Dolbier, B. Sheeller, K. U. Ingold, J. Am. Chem. Soc. 2002, 124, 6362-6366. 


\section{- Synthesis of 3,3-substitutedcyclobutanones I-V.}

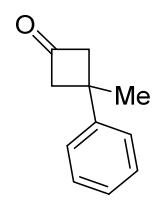

I

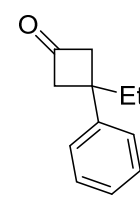

II

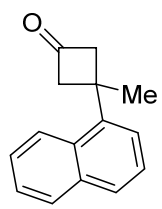

III
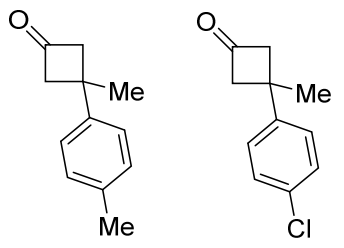

IV

Figure S 2. 3,3-disubstitutedcyclobutanones employed for the synthesis of cyclobutanols.

$\mathbf{I}^{4}, \mathbf{I I}^{5}$ and $\mathbf{I V}-\mathbf{V}^{6}$ are reported compounds. Compounds $\mathbf{I}-\mathbf{V}$ were prepared following the general procedure $\mathrm{A}^{7}$ previously described.

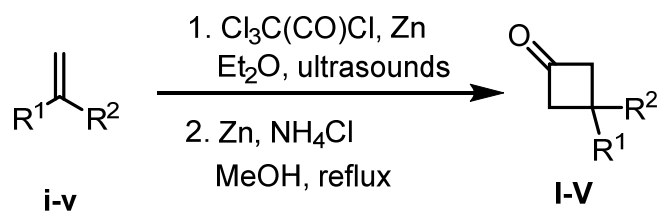

Scheme S 3. General procedure A for the synthesis of cyclobutanones.

General procedure A for the synthesis of cyclobutanones.

In a two necked flask provided with a condenser with dry $\mathrm{Et}_{2} \mathrm{O}(250 \mathrm{~mL})$ and $\mathrm{Zn}$ (4 equiv., $7.8 \mathrm{~g}, 120 \mathrm{mmol})$ under Ar atmosphere, $\alpha$-methylstyrene $(3.90 \mathrm{~mL}, 30 \mathrm{mmol})$ was added followed by the dropwise addition of trichloroacetyl chloride (2 equiv., $6.80 \mathrm{~mL}, 60$ mmol) under sonication. The mixture was kept under sonication for $5 \mathrm{~h}$ until a brown solution was obtained. Then, the solids were filtrated off through a plug of Celita ${ }^{\circledR}$ and washed with $\mathrm{Et}_{2} \mathrm{O}(100 \mathrm{~mL})$. The filtrate was washed with $\mathrm{H}_{2} \mathrm{O}(2 \times 50 \mathrm{~mL})$, saturated aq. $\mathrm{NaHCO}_{3}(4 \times 50 \mathrm{~mL})$ and brine, dried with $\mathrm{Na}_{2} \mathrm{SO}_{4}$ and concentrated under vacuum. The crude was passed through a short pad of silica gel with PE:EtOAc 9:1 to obtain 2,2dichloro-3-methyl-3-phenylcyclobutan-1-one $(6.051 \mathrm{~g}, 26.5 \mathrm{mmol})$ pure enough for the next reaction.

$\mathrm{MeOH}(70 \mathrm{~mL})$ was added into a flask containing the crude of the previous reaction followed by the addition of ammonium chloride ( 2.5 equiv., $3.4 \mathrm{~g}, 63 \mathrm{mmol})$. The mixture

\footnotetext{
${ }^{4}$ K. S. Petersen, B. M. Stoltz, Tetrahedron, 2011, 67, 4352-4357.

5 (a) W.T. Brady, T. C. Cheng, J. Organomet. Chem., 1977, 137, 287-292. (b) T. Seiser, N. Cramer, Angew. Chem. Int. Ed. 2008, 47, 9294.

${ }^{6}$ T. Matsuda, I. Yuihara, Chem. Commun., 2015, 51, 7393-7396.

${ }^{7}$ F. Le Vaillant, M. Garreau, S. Nicolai, G. Gryn'ova, C. Corminboeuf, J. Waser, Chem. Sci., 2018, 9, 58835889.
} 
was cooled to $0{ }^{\circ} \mathrm{C}$ and $\mathrm{Zn}$ (5 equiv., $8.2 \mathrm{~g}, 126 \mathrm{mmol}$ ) was added portion wise under stirring. The flask was provided with a condenser and let stirring at $70{ }^{\circ} \mathrm{C}$ employing a heating mantle overnight. The solids were filtered off trough a plug of Celite ${ }^{\circledR}$. The filtrate was concentrated under vacuum and diluted with $\mathrm{Et}_{2} \mathrm{O}(20 \mathrm{~mL})$ and aq. $1 \mathrm{M} \mathrm{HCl}$ $(20 \mathrm{~mL})$. The layers were separated, and the aqueous layer was extracted with $\mathrm{Et}_{2} \mathrm{O}(3 \mathrm{x}$ $20 \mathrm{~mL}$ ). The crude was purified with PE:EtOAc 9:1 to obtain 3-methyl-3phenylcyclobutan-1-one (2.6 g, $16.25 \mathrm{mmol})$ as a colorless oil.

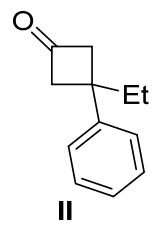

3-ethyl-3-phenylcyclobutan-1-one $\mathbf{I I}^{4}$

Following the general procedure A, II was purified by flash chromatography (PE:EtOAc 9:1) and obtained as an oil (650 $\mathrm{mg} ; 3.73 \mathrm{mmol} ; 40 \%)$ starting from $\alpha$-ethylstyrene $(1.2$ g, $9.14 \mathrm{mmol})$, trichloroacethyl chloride $(2.0 \mathrm{~mL}, 18.3 \mathrm{mmol}), \mathrm{Zn}(2.4 \mathrm{~g} ; 36.6 \mathrm{mmol})$ in $\mathrm{Et}_{2} \mathrm{O}(15.0 \mathrm{~mL}) . \mathrm{Rf}=0.48(\mathrm{PE}: \mathrm{EtOAc}=9: 1) .{ }^{1} \mathbf{H}$ NMR $\left(300 \mathrm{MHz}, \mathrm{CDCl}_{3}\right) \delta 7.41-7.34$ (m, 3H, $\left.\mathrm{C}_{\text {Arom }}-\mathrm{H}\right), 7.31-7.21\left(\mathrm{~m}, 2 \mathrm{H}, \mathrm{C}_{\text {Arom }}-\mathrm{H}\right), 3.46-3.35\left(\mathrm{~m}, 2 \mathrm{H}, \mathrm{C}^{1} \mathrm{H}_{2}\right), 3.19-3.08(\mathrm{~m}$, $\left.1 \mathrm{H}, \mathrm{C}^{2} \mathrm{H}_{2}\right), 1.88\left(\mathrm{q}, J=7.3 \mathrm{~Hz}, 1 \mathrm{H}, \mathbf{C H}_{2} \mathrm{CH}_{3}\right), 0.78\left(\mathrm{t}, J=7.3 \mathrm{~Hz}, 3 \mathrm{H}, \mathrm{CH}_{2} \mathbf{C H}_{3}\right) .{ }^{13} \mathbf{C}\left\{{ }^{1} \mathbf{H}\right\}$ NMR $\left(75.5 \mathrm{MHz}, \mathrm{CDCl}_{3}\right) \delta 206.6$ (CO), 145.9 (C $\left.\mathrm{C}_{\text {Arom-C }}\right), 128.3$ (2xCArom-H), 126.8 (2xCArom-C), $126.2\left(\mathrm{C}_{\text {Arom- }} \mathrm{H}\right), 57.2\left(2 \mathrm{xCH}_{2}\right), 38.4\left(\mathbf{C C H}_{2}\right), 36.0\left(\mathbf{C H}_{2} \mathrm{CH}_{3}\right), 9.8$ $\left(\mathrm{CH}_{2} \mathbf{C H}_{3}\right)$. IR (ATR) $\mathrm{cm}^{-1}: 1716.34(\mathrm{C}=\mathrm{O}) . \mathbf{M S}(\mathrm{EI}) \mathrm{m} / \mathrm{z}(\%): 173.1\left(\mathrm{M}^{+}, 1\right), 132.1(71)$, $117.1(100)$.

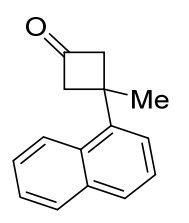

III

3-methyl-3-(naphthalen-1-yl)cyclobutan-1-one III

Following the genera procedure A, III was purified by flash chromatography (PE:EtOAc 9:1) obtained as a white solid (1.0 g; $4.76 \mathrm{mmol} ; 45 \%)$ starting from 2-(prop-1-en-2yl)naphthalene (1.8 g, $10.57 \mathrm{mmol})$, trichloroacethyl chloride $(2.4 \mathrm{~mL}, 21.14 \mathrm{mmol}), \mathrm{Zn}$ $(2.8 \mathrm{~g} ; 42.3 \mathrm{mmol})$ in $\mathrm{Et}_{2} \mathrm{O}(42.0 \mathrm{~mL}) . \mathrm{Rf}=0.52(\mathrm{PE}: \mathrm{EtOAc}=9: 1) . \mathbf{M . p .}=42-43{ }^{\circ} \mathrm{C} .{ }^{1} \mathbf{H}$ 
NMR $\left(300 \mathrm{MHz}, \mathrm{CDCl}_{3}\right) \delta 7.92-7.80\left(\mathrm{~m}, 3 \mathrm{H}, \mathrm{C}_{\text {Arom-H}} \mathrm{H}\right), 7.74\left(\mathrm{~d}, J=1.8 \mathrm{~Hz}, 1 \mathrm{H}, \mathrm{C}_{\text {Arom- }}{ }^{-}\right.$ $\mathrm{H}), 7.57-7.41\left(\mathrm{~m}, 3 \mathrm{H}, \mathrm{C}_{\text {Arom- }} \mathrm{H}\right), 3.67-3.53\left(\mathrm{~m}, 2 \mathrm{H}, \mathrm{C}^{1} \mathrm{H}_{2}\right), 3.29-3.13\left(\mathrm{~m}, 2 \mathrm{H}, \mathrm{C}^{2} \mathrm{H}_{2}\right), 1.71$ $\left(\mathrm{s}, 3 \mathrm{H}, \mathrm{CH}_{3}\right) .{ }^{13} \mathbf{C}\left\{{ }^{1} \mathbf{H}\right\}$ NMR $\left(75.5 \mathrm{MHz}, \mathrm{CDCl}_{3}\right) \delta 206.5(\mathrm{C}=\mathrm{O}), 145.5\left(\mathrm{C}_{\text {Arom- }} \mathrm{C}\right), 133.3$ $\left(\mathrm{C}_{\text {Arom- }} \mathrm{C}\right), 131.9\left(\mathrm{C}_{\text {Arom-C }}\right), 128.6\left(\mathrm{C}_{\text {Arom }}-\mathrm{H}\right), 127.7\left(\mathrm{C}_{\text {Arom }}-\mathrm{H}\right), 127.6\left(\mathrm{C}_{\text {Arom }}-\mathrm{H}\right), 126.4$ $\left(\mathrm{C}_{\text {Arom-H}}-\mathrm{H}\right), 125.8\left(\mathrm{C}_{\text {Arom }}-\mathrm{H}\right), 124.4\left(\mathrm{C}_{\text {Arom }}-\mathrm{H}\right), 123.7\left(\mathrm{C}_{\text {Arom }}-\mathrm{H}\right), 59.3\left(2 \mathrm{xCH}_{2}\right), 34.1$ $\left(\mathbf{C C H}_{2}\right), 30.9\left(\mathrm{CH}_{3}\right)$. IR (ATR) $\mathrm{cm}^{-1}:$ 1781.9. MS (EI) m/z (\%): $210.2\left(\mathrm{M}^{+}, 98\right), 168.2$ (100), 153.1 (99), 128.1 (62). HRMS $\left(\mathrm{ESI}^{+}\right): \mathrm{m} / \mathrm{z}$ calculated for $\left[\mathrm{C}_{15} \mathrm{H}_{14} \mathrm{ONa}\right]^{+} 233.0942$; found $233.0940[\mathrm{M}+\mathrm{Na}]^{+}$

- Synthesis of 1-bromovinylarenes VI-XIV.<smiles>C=C(Br)c1ccccc1</smiles>

VI<smiles>C=C(Br)c1ccc(Cl)cc1</smiles>

IX<smiles>C=C(Br)c1ccc(C(F)(F)F)cc1</smiles><smiles>C=C(Br)c1ccc(C)cc1</smiles><smiles>[X]c1ccc(C(=C)Br)cc1</smiles><smiles>BrC1=CCCc2ccccc21</smiles><smiles>C=C(Br)c1ccc(OC)cc1</smiles><smiles>[X]c1ccc2cc(C(=C)Br)ccc2c1</smiles><smiles>C=C(Br)c1ccccc1C#N</smiles>

Figure S 3. 1-Bromovinylarenes employed for the formation of organomagnesium compounds.

VI was obtained from commercial sources and used without previous purification. Compounds VII-XII ${ }^{8}, \mathbf{X I I I}^{9}$ and $\mathbf{X I V}^{8}$ are reported compounds.

VII-XI and XIII-XIV were prepared following the general procedure $\mathrm{B}^{10}$ and XII following the general procedure $\mathrm{C}^{11}$ described in the literature.

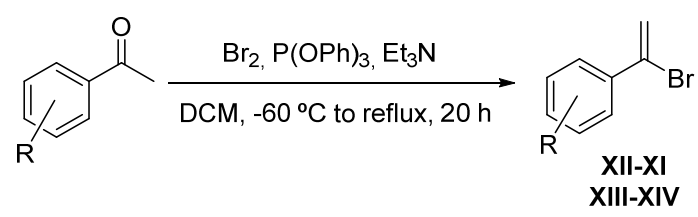

Scheme S 4. General procedure B for the synthesis of 1-bromovinylarenes.

\footnotetext{
${ }^{8}$ S. Alazet, J. Preindl, R. Simonet-Davin, S. Nicolai, A. Nanchen, T. Meyer, J. Waser, J. Org. Chem. 2018, 83, 12334-12356.

${ }^{9}$ E. Napolitano, R. Fiaschi, E. Mastrorilli. Synthesis, 1986, 2, 122-125.

${ }^{10}$ A. Spaggiari, D.Vaccari, P. Davoli, G. Torre, F. Prati, J. Org. Chem. 2007, 72, 2216-2219.

${ }^{11}$ A. V. Chernykh, D. S. Radchenko, A. V. Chernykh, I. S. Kondratov, N. A. Tolmachova, O. P. Datsenko, M. A. Kurkunov, S. X. Zozulya, Y. P. Kheylik, K. Bartels, C. G. Daniliuc, G. Haufe. Eur. J. Org. Chem. 2015, 29, 6466-6471.
} 
General procedure B for the synthesis of 1-bromovinylarenes.

To an oven dry two necked flask provided with a stir bar and a condenser, bromine $(1.25$ equiv., $0.64 \mathrm{~mL}, 12.5 \mathrm{mmol})$ was added to a stirring solution of triphenylphosphite $(1.1$ equiv., $3 \mathrm{~mL}, 11 \mathrm{mmol})$ in anhydrous dichloromethane $(33 \mathrm{~mL})$ at $-60{ }^{\circ} \mathrm{C}$ under $\mathrm{Ar}$ atmosphere. A pail orange solution was formed. Anhydrous triethylamine (1.35 equiv., $1.90 \mathrm{~mL}, 13.5 \mathrm{mmol})$ and 1-(p-tolyl)ethan-1-one $(1.34 \mathrm{~mL}, 10 \mathrm{mmol})$ were also added. The resulting reaction mixture was stirred $18 \mathrm{~h}$ while warming to $\mathrm{rt}$ and then was refluxed employing an aluminium heating block for $2 \mathrm{~h}$. The reaction was cooled to $\mathrm{rt}$, the solvent was removed under vacuum and the crude was purified by silica gel column chromatography with PE:EtOAc 95:5 to obtain 1-(1-bromovinyl)-4-methylbenzene as a pale yellow oil (928 mg, $4.71 \mathrm{mmol}, 47 \%)$.

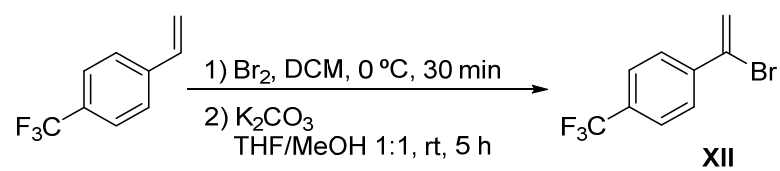

Scheme S 5. General procedure C for the synthesis of 1-bromovinylarene XII.

General procedure $\mathrm{C}$ for the synthesis of 1-bromovinylarenes.

Bromine (1.2 equiv, $14 \mathrm{mmol}, 0.72 \mathrm{~mL}$ ) was slowly added, over a period of 5 minutes, to a solution of 1-(trifluoromethyl)-4-vinylbenzene $(11.7 \mathrm{mmol}, 2.1 \mathrm{~g})$ in anhydrous dichloromethane $(23.4 \mathrm{~mL})$ at $0{ }^{\circ} \mathrm{C}$. The reaction mixture was stirred at this temperature for 30 minutes, controlling by TLC that the starting material was totally consumed. The reaction was quenched with a saturated solution of $\mathrm{Na}_{2} \mathrm{~S}_{2} \mathrm{O}_{3}(15 \mathrm{~mL})$ and the product was extracted with dichloromethane $(3 \times 30 \mathrm{~mL})$. The combined organic layers were washed with brine and dried over $\mathrm{Na}_{2} \mathrm{SO}_{4}$. After filtering the drying agent, the solvent was evaporated and the crude mixture was directly dissolved in THF/MeOH (1:1) (15 mL: 15 $\mathrm{mL}$ ), followed by the addition of $\mathrm{K}_{2} \mathrm{CO}_{3}$ (2 equiv, $23.4 \mathrm{mmol}, 3.2 \mathrm{~g}$ ). The reaction mixture was stirred at room temperature for $5 \mathrm{~h}$. The solvent was evaporated, and water was added $(15 \mathrm{~mL})$. The product was extracted with pentane $(3 \times 20 \mathrm{~mL})$, those extracts were washed with brine and dried over $\mathrm{Na}_{2} \mathrm{SO}_{4}$. The drying agents was removed by filtration and the solvent was evaporated. The desired product was purified by silica gel column chromatography using petroleum ether as eluent to obtain 1-(1-bromovinyl)-4(trifluoromethyl)benzene $(8.3 \mathrm{mmol}, 2.09 \mathrm{~g})$ in a $72 \%$ isolated yield. 
- Synthesis of 3,3-disubstituted-1-(1-arylvinyl)cyclobutan-1-ols, 1a-1n.

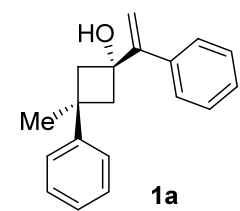<smiles></smiles><smiles>C=C(c1ccccc1)[C@]1(O)C[C@@](C)(c2ccc(C)cc2)C1</smiles><smiles>C=C(c1ccccc1)C1(O)CC1(C)c1ccc(Cl)cc1</smiles><smiles>CC[C@]1(c2ccccc2)C[C@@](O)(c2ccccc2)C1</smiles><smiles>C=C(c1ccc2ccccc2c1)C1CC(c2ccccc2)(c2ccccc2)C1</smiles><smiles>C=C(c1ccc(-c2ccccc2)cc1)C1CC(C)(c2ccccc2)C1</smiles><smiles>C=C(c1ccc(C)cc1)C1CC(C)(c2ccccc2)C[C@@H]1O</smiles><smiles>C=C(c1ccc(OC)cc1)[C@]1(O)C[C@@](C)(c2ccccc2)C1</smiles><smiles>C=C(c1ccc(C(F)(F)F)cc1)C1CC(C)(c2ccccc2)C1</smiles><smiles>Cc1ccccc1C(=O)[C@H]1C[C@@](C)(c2ccccc2)C1</smiles><smiles>C[C@]1(c2ccccc2)C[C@@H](O)[C@]12CCCc1ccccc12</smiles><smiles>C=C(C)C1CC(CC)(c2ccccc2)C1</smiles>

Figure S 4. Synthetized 3,3-disubstituted-1-(1-arylvinyl)cyclobutan-1-ol 1atrans-1ntrans.

Compounds 1a-1m were prepared according to the general procedure D. Compound $1 \mathbf{n}$ was prepared according to the described procedure E. ${ }^{12}$

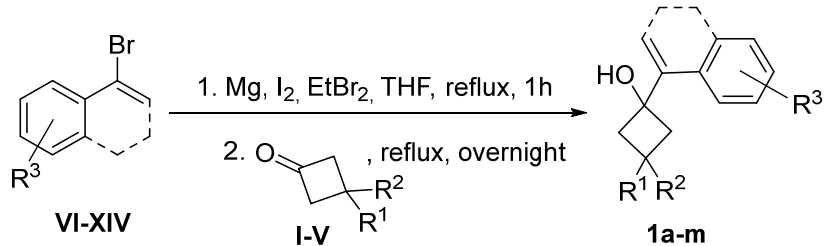

Scheme S 6. General procedure D for the synthesis of 3,3-disubstituted-1-(1-arylvinyl)ciclobutan-1-ol 1a$1 \mathrm{~m}$.

General procedure D for the synthesis of 3,3-disubstituted-1-(1-arylvinyl)ciclobutan-1ols 1a-1m.

To an oven dried two necked flask provided with a condenser and stir bar, one crystal of iodine and 1,2-dibromoethane (0.4 equiv., $0.21 \mathrm{~mL}, 2.50 \mathrm{mmol})$ were added to a suspension of $\mathrm{Mg}$ (3 equiv., $450 \mathrm{mg}, 18.75 \mathrm{mmol}$ ) in THF (20 mL) under Ar atmosphere and was heated to reflux employing an aluminium heating block for at least 10 mins.

12 R. J. Phipps, L. McMurray, S. Ritter, H. A. Duong, M. J. Gaunt, J. Am. Chem. Soc. 2012, 134, 1077310776. 
Thereafter, a drop of the corresponding bromoalkene was added to the refluxing mixture in order to start the formation of organomagnesium compound. Once the bubbling starts, the rest of bromoalkene VI-XIV (1.3 equiv., $8.13 \mathrm{mmol})$ in THF $(5 \mathrm{~mL})$ was added dropwise keeping the bubbling and the reaction was refluxed employing an aluminium heating block for $1 \mathrm{~h}$. Then a solution of ketone $\mathbf{I}-\mathbf{V}(6.25 \mathrm{mmol})$ in THF $(5 \mathrm{~mL})$ was added dropwise and the reaction mixture was refluxed overnight. After being cooled to room temperature it was quenched with saturated aq. $\mathrm{NH}_{4} \mathrm{Cl}$ and extracted with $\mathrm{Et}_{2} \mathrm{O}(3$ x $20 \mathrm{~mL}$ ), the combined organic layers were washed with brine, dried with $\mathrm{Na}_{2} \mathrm{SO}_{4}$, filtered and concentrate under vacuum. The crude was purified by silica gel column chromatography using PE:EtOAc (95:5 to 9:1) as eluent. Further purification with PE:DCM (1:1 to 3:7) was necessary to separate the diastereoisomers.

Procedure E for the synthesis of 3-ethyl-3-phenyl-1-(prop-1-en-2-yl)cyclobutan-1-ol 1n

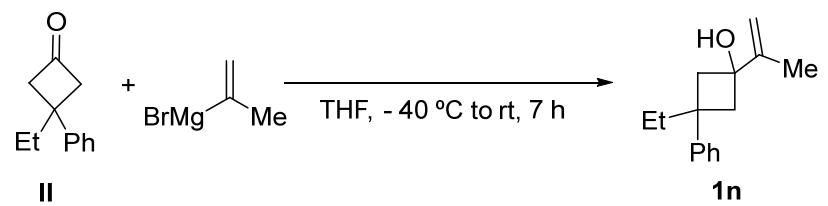

Scheme S 7. Synthesis of 3,3-disubstituted-1-(prop-1-en-2-yl)ciclobutan-1-ol 1n.

3-Ethyl-3-phenylcyclobutanone $(300 \mathrm{mg}, 1.72 \mathrm{mmol}$ ) was dissolved in $1.25 \mathrm{~mL}$ of anhydrous THF under $\mathrm{Ar}$ atmosphere. The solution was cooled to $-40^{\circ} \mathrm{C}$ and isopropenylmagnesium bromide $(5.16 \mathrm{~mL}, 0.5 \mathrm{M}$ in THF) was added to the solution drop by drop over 20 minutes. The reaction was allowed to stir at room temperature for $7 \mathrm{~h}$. After this time, it was quenched with $2 \mathrm{~mL}$ of a saturated solution of $\mathrm{NH}_{4} \mathrm{Cl}$. The product was extracted from the aqueous phase with $\mathrm{Et}_{2} \mathrm{O}(3 \times 5 \mathrm{~mL})$ and the combined organic phases were washed with brine, dried over $\mathrm{Na}_{2} \mathrm{SO}_{4}$ and concentrated in vacuo. The crude was purified by silica gel column chromatography using PE:DCM (70:30) as eluent to obtain trans diastereoisomer as a transparent oil (126 mg; $0.58 \mathrm{mmol} ; 34 \%)$ used in the next reaction and the cis one (78 mg; $0.36 \mathrm{mmol} ; 21 \%$ ). 


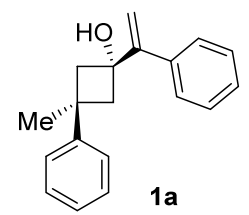

3-methyl-3-phenyl-1-(1-phenylvinyl)cyclobutan-1-ol 1a

The product was prepared following the general procedure $\mathrm{D}$, obtaining a mixture of cis and trans diastereoisomers $(1.510 \mathrm{~g}, 5.69 \mathrm{mmol}, 83 \%)$ with a $\mathrm{dr}_{\text {trans:cis }}=1.4: 1$. The trans isomer 1a was isolated as a transparent oil (780 mg; $2.95 \mathrm{mmol} ; 43 \%)$ by flash chromatography (PE:DCM = 1:1 to 3:7) starting from 1-bromovinylstyrene VI (1.06 mL; $8.13 \mathrm{mmol}), \mathrm{Mg}$ (450 mg; $18.75 \mathrm{mmol})$, THF (30.00 mL) and 3-methyl-3phenylcyclobutanone I (1.000 g; $6.25 \mathrm{mmol}) . \mathrm{Rf}=0.4$ (PE:DCM= 4:6). ${ }^{1} \mathrm{H}$ NMR (500 $\left.\mathrm{MHz}, \mathrm{CDCl}_{3}\right) \delta 7.37\left(\mathrm{~d}, J=7.1 \mathrm{~Hz}, 2 \mathrm{H}, \mathrm{C}_{\text {Arom-H}} \mathrm{H}\right), 7.30-7.15\left(\mathrm{~m}, 5 \mathrm{H}, \mathrm{C}_{\text {Arom-H}}-\mathrm{H}\right), 7.06(\mathrm{t}$, $\left.J=6.3 \mathrm{~Hz}, 3 \mathrm{H}, \mathrm{C}_{\text {Arom }}-\mathrm{H}\right), 5.21-5.17\left(\mathrm{~s}, 1 \mathrm{H}, \mathrm{C}=\mathrm{CH}_{\mathrm{a}} \mathrm{H}_{\mathrm{b}}\right), 5.13\left(\mathrm{~s}, 1 \mathrm{H}, \mathrm{C}=\mathrm{CH}_{\mathrm{a}} \mathbf{H}_{\mathbf{b}}\right), 2.79$ $\left(\mathrm{d}, J=13.3 \mathrm{~Hz}, 2 \mathrm{H}, \mathrm{C}^{1} \mathrm{H}_{2}\right), 2.42\left(\mathrm{~d}, J=13.4 \mathrm{~Hz}, 2 \mathrm{H}, \mathrm{C}^{2} \mathrm{H}_{2}\right), 1.59\left(\mathrm{~s}, 3 \mathrm{H}, \mathrm{CH}_{3}\right) .{ }^{13} \mathbf{C}\left\{{ }^{1} \mathbf{H}\right\}$ NMR $\left(75.5 \mathrm{MHz}, \mathrm{CDCl}_{3}\right) \delta 153.7\left(\mathbf{C}=\mathrm{CH}_{2}\right), 151.9\left(\mathrm{C}_{\text {Arom- }} \mathrm{C}\right), 138.8\left(\mathrm{C}_{\text {Arom- }}-\mathrm{C}\right), 128.2$ $\left(2 \mathrm{xC}_{\text {Arom }}-\mathrm{H}\right), 128.2\left(2 \mathrm{xC}_{\text {Arom }}-\mathrm{H}\right), 127.6\left(\mathrm{C}_{\text {Arom }}-\mathrm{H}\right), 127.5\left(2 \mathrm{xC}_{\text {Arom }}-\mathrm{H}\right), 125.3\left(\mathrm{C}_{\text {Arom }}-\mathrm{H}\right)$, $125.2\left(2 \mathrm{xC}_{\text {Arom }}-\mathrm{H}\right), 113.0\left(\mathrm{C}=\mathbf{C H}_{2}\right), 74.0(\mathrm{C}-\mathrm{OH}), 47.1\left(2 \mathrm{xCH}_{2}\right), 35.8\left(\mathbf{C}-\mathrm{CH}_{2}\right), 32.4$ $\left(\mathrm{CH}_{3}\right)$. IR $(\mathrm{ATR}) \mathrm{cm}^{-1}: 3648(\mathrm{O}-\mathrm{H}), 3024(=\mathrm{C}-\mathrm{H}), 2961(\mathrm{C}-\mathrm{H}), 1625(\mathrm{C}=\mathrm{C}), 1450\left(\mathrm{CH}_{3}\right)$. MS (EI) m/z (\%): $263.2\left(\mathrm{M}^{+}, 34\right)$. HRMS $\left(\mathrm{ESI}^{+}\right): \mathrm{m} / \mathrm{z}$ calculated for $\left[\mathrm{C}_{19} \mathrm{H}_{20} \mathrm{O}-\mathrm{H}_{2} \mathrm{O}+\mathrm{H}\right]^{+}$ 247.1488; found $247.1487\left[\mathrm{M}-\mathrm{H}_{2} \mathrm{O}+\mathrm{H}\right]^{+}$.

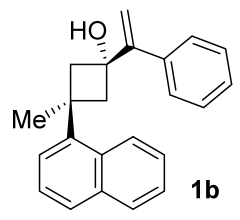

3-methyl-3-(naphthalen-2-yl)-1-(1-phenylvinyl)cyclobutan-1-ol 1b

The product was prepared following the general procedure $\mathrm{D}$, obtaining a mixture of $c$ is and trans diastereoisomers $(396 \mathrm{mg}, 1.26 \mathrm{mmol}, 88 \%)$ with a drans:cis $=1.2: 1$. The trans isomer 1b was isolated as a white solid (198 $\mathrm{mg} ; 0.63 \mathrm{mmol} ; 44 \%$ ) by flash chromatography (PE:DCM = 4:6) starting from 1-bromovinylstyrene VI $(0.24 \mathrm{~mL} ; 1.86$ mmol), Mg (103 mg; $4.29 \mathrm{mmol})$, THF (7 mL), and 3-methyl-3-(naphthalen-1yl)cyclobutan-1-one III (300 mg; 1.43 mmol). Rf = 0.4 (PE:DCM = 4:6). M.p. $=115$ $118^{\circ} \mathrm{C}^{1}{ }^{1} \mathbf{H}$ NMR $\left(300 \mathrm{MHz}, \mathrm{CDCl}_{3}\right) \delta 7.87-7.77\left(\mathrm{~m}, 3 \mathrm{H}, \mathrm{C}_{\text {Arom-H}} \mathrm{H}\right), 7.58(\mathrm{~d}, J=1.7 \mathrm{~Hz}$, 1H, $\left.\mathrm{C}_{\text {Arom }}-\mathrm{H}\right), 7.55-7.44\left(\mathrm{~m}, 4 \mathrm{H}, \mathrm{C}_{\text {Arom }}-\mathrm{H}\right), 7.36\left(\mathrm{~m}, 4 \mathrm{H}, \mathrm{C}_{\text {Arom }}-\mathrm{H}\right), 5.29(\mathrm{~d}, J=0.7 \mathrm{~Hz}$, 
$\left.1 \mathrm{H}, \mathrm{C}=\mathrm{CH}_{\mathrm{a}} \mathrm{H}_{\mathrm{b}}\right), 5.27\left(\mathrm{~d}, J=0.7 \mathrm{~Hz}, 1 \mathrm{H}, \mathrm{C}=\mathrm{CH}_{\mathrm{a}} \mathbf{H}_{\mathbf{b}}\right), 3.03\left(\mathrm{~d}, J=13.4 \mathrm{~Hz}, 2 \mathrm{H}, \mathrm{C}^{1} \mathrm{H}_{2}\right), 2.63$ $\left(\mathrm{d}, J=13.3 \mathrm{~Hz}, 2 \mathrm{H}, \mathrm{C}^{2} \mathrm{H}_{2}\right), 2.10(\mathrm{~s}, 1 \mathrm{H}, \mathrm{OH}), 1.80\left(\mathrm{~s}, 3 \mathrm{H}, \mathrm{CH}_{3}\right) .{ }^{13} \mathbf{C}\left\{{ }^{1} \mathbf{H}\right\}$ NMR $(75.5$ $\left.\mathrm{MHz}, \mathrm{CDCl}_{3}\right) \delta 153.7\left(\mathbf{C}=\mathrm{CH}_{2}\right), 149.0\left(\mathrm{C}_{\text {Arom }}-\mathrm{C}\right), 138.9\left(\mathrm{C}_{\text {Arom- }}-\mathrm{C}\right), 133.3\left(\mathrm{C}_{\text {Arom }}-\mathrm{C}\right), 131.6$ $\left(\mathrm{C}_{\text {Arom-C }} \mathrm{C}\right), 128.3\left(2 \mathrm{xC}_{\text {Arom }}-\mathrm{H}\right), 128.0\left(\mathrm{C}_{\text {Arom }}-\mathrm{H}\right), 127.7\left(2 \mathrm{xC}_{\text {Arom }}-\mathrm{H}\right), 127.5\left(3 \mathrm{xC}_{\text {Arom }}-\mathrm{H}\right)$, $126.0\left(\mathrm{C}_{\text {Arom }}-\mathrm{H}\right), 125.3\left(\mathrm{C}_{\text {Arom }}-\mathrm{H}\right), 124.5\left(\mathrm{C}_{\text {Arom }}-\mathrm{H}\right), 122.9\left(\mathrm{C}_{\text {Arom }}-\mathrm{H}\right), 113.1\left(\mathrm{C}=\mathbf{C H}_{2}\right)$, $73.9(\mathrm{C}-\mathrm{OH}), 47.2\left(2 \mathrm{xCH}_{2}\right), 35.9\left(\mathrm{CCH}_{2}\right), 32.3\left(\mathrm{CH}_{3}\right)$. IR (ATR) $\mathrm{cm}^{-1}: 3547(\mathrm{O}-\mathrm{H}), 3022$ (=C-H), $2961(\mathrm{C}-\mathrm{H}), 1625(\mathrm{C}=\mathrm{C}), 1442\left(\mathrm{CH}_{3}\right) . \mathbf{M S}(\mathrm{EI}) \mathrm{m} / \mathrm{z}(\%): 168.1$ (100), 153.1 (29), 128.1 (26), 103.1 (21). HRMS $\left(\mathrm{ESI}^{+}\right): \mathrm{m} / \mathrm{z}$ calculated for $\left[\mathrm{C}_{23} \mathrm{H}_{22} \mathrm{O}+\mathrm{Na}\right]^{+} 337.1568$; found $337.1563[\mathrm{M}+\mathrm{Na}]^{+}$.

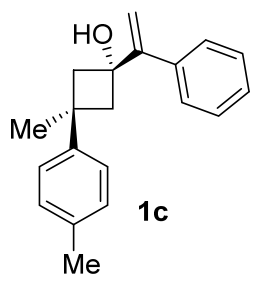

3-methyl-1-(1-phenylvinyl)-3-(p-tolyl)cyclobutan-1-ol 1c.

The product was prepared following the general procedure $\mathrm{D}$, obtaining a mixture of cis and trans diastereoisomers $(416 \mathrm{mg}, 1.49 \mathrm{mmol}, 82 \%)$ with a $\mathrm{dr}_{\text {trans:cis }}=1.3: 1$. The trans isomer 1c was isolated as a transparent oil $(0.67 \mathrm{mmol} ; 188 \mathrm{mg} ; 39 \%)$ by flash chromatography (PE:DCM = 6:4) starting from 1-bromovinylstyrene VI (0.29 mL; 2.24 mmol), Mg (125 mg; $5.16 \mathrm{mmol})$, THF (8.3 mL), and 3-methyl-3-(p-tolyl)cyclobutan-1one IV (300 mg; $1.72 \mathrm{mmol}) . \mathrm{Rf}=0.20$ (PE:DCM =60:40). ${ }^{1} \mathbf{H} \mathbf{~ N M R}\left(300 \mathrm{MHz}, \mathrm{CDCl}_{3}\right)$ $\delta$ 7.49-7.42 (m, 2H, CArom-H), 7.36-7.27 (m, 3H, CArom-H), 7.10 (d, J=8.2 Hz, 2H, CAromH), $7.04\left(\mathrm{~d}, J=8.2 \mathrm{~Hz}, 2 \mathrm{H}, \mathrm{C}_{\text {Arom- }} \mathrm{H}\right), 5.25\left(\mathrm{~s}, 1 \mathrm{H}, \mathrm{C}=\mathrm{CH}_{\mathbf{a}} \mathrm{Hb}_{\mathrm{b}}\right), 5.21\left(\mathrm{~s}, 1 \mathrm{H}, \mathrm{C}=\mathrm{CH}_{\mathrm{a}} \mathbf{H}_{\mathbf{b}}\right)$, $2.85\left(\mathrm{~d}, J=13.5 \mathrm{~Hz}, 2 \mathrm{H}, \mathrm{C}^{1} \mathrm{H}_{2}\right), 2.48\left(\mathrm{~d}, J=13.5 \mathrm{~Hz}, 2 \mathrm{H}, \mathrm{C}^{2} \mathrm{H}_{2}\right), 2.31\left(\mathrm{~s}, 3 \mathrm{H}, \mathrm{C}_{\mathrm{Arom}-\mathrm{CH}_{3}}\right)$, $1.93(\mathrm{~s}, 1 \mathrm{H}, \mathrm{OH}), 1.66\left(\mathrm{~s}, 3 \mathrm{H}, \mathrm{CH}_{3}\right) .{ }^{13} \mathbf{C}\left\{{ }^{1} \mathbf{H}\right\} \mathbf{N M R}\left(75.5 \mathrm{MHz}, \mathrm{CDCl}_{3}\right) \delta 153.7\left(\mathbf{C}=\mathrm{CH}_{2}\right)$, $148.9\left(\mathrm{C}_{\text {Arom }}-\mathrm{C}\right), 138.8\left(\mathrm{C}_{\text {Arom }}-\mathrm{C}\right), 134.7\left(\mathbf{C}_{\text {Arom }}-\mathrm{CH}_{3}\right), 128.8\left(2 \times \mathrm{C}_{\text {Arom }}-\mathrm{H}\right), 128.2$ $\left(2 \mathrm{xC}_{\text {Arom }}-\mathrm{H}\right), 127.6\left(\mathrm{C}_{\text {Arom }}-\mathrm{H}\right), 127.4\left(2 \mathrm{xC}_{\text {Arom }}-\mathrm{H}\right), 125.0\left(2 \mathrm{xC}_{\text {Arom }}-\mathrm{H}\right), 112.9\left(\mathrm{C}=\mathbf{C H}_{\mathbf{2}}\right)$, $73.9(\mathrm{C}-\mathrm{OH}), 47.2\left(2 \mathrm{xCH}_{2}\right), 35.4\left(\mathbf{C}-\mathrm{CH}_{3}\right), 32.4\left(\mathrm{CH}_{3}\right), 20.9\left(\mathrm{C}_{\mathrm{Arom}}-\mathbf{C H}_{3}\right) . \mathbf{I R}(\mathrm{ATR}) \mathrm{cm}^{-}$ 1: $3427(\mathrm{O}-\mathrm{H}), 2974(\mathrm{C}-\mathrm{H}), 1626$ (C=C). MS (EI) m/z (\%): 132.1 (100), 165.0 (15), 245.1 (15), $277.9\left(\mathrm{M}^{+}, 2\right)$. HRMS $\left(\mathrm{ESI}^{+}\right): \mathrm{m} / \mathrm{z}$ calculated for $\left[\mathrm{C}_{20} \mathrm{H}_{22} \mathrm{O}-\mathrm{H}_{2} \mathrm{O}+\mathrm{H}\right]^{+}$261.1643; found $261.1635\left[\mathrm{M}-\mathrm{H}_{2} \mathrm{O}+\mathrm{H}\right]^{+}$. 
1d

3-(4-chlorophenyl)-3-methyl-1-(1-phenylvinyl)cyclobutan-1-ol 1d

The product was prepared following the general procedure $\mathrm{D}$, obtaining a mixture of cis and trans diastereoisomers $(377 \mathrm{mg}, 1.26 \mathrm{mmol}, 35 \%)$ with a drans:cis $=1.7: 1$. The trans isomer 1d was isolated as a transparent oil $(0.16 \mathrm{mmol} ; 47.6 \mathrm{mg} ; 10 \%)$ by flash chromatography (PE:DCM = 7:3) starting from 1-bromovinylstyrene VI $(0.26 \mathrm{~mL} ; 2.03$ mmol), Mg (114 mg; $4.68 \mathrm{mmol})$, THF (7.5 mL), and 3-(4-chlorophenyl)-3methylcyclobutan-1-one V (300 mg; $1.56 \mathrm{mmol}) . \mathrm{Rf}=0.19(\mathrm{PE}: \mathrm{DCM}=6: 4) .{ }^{1} \mathbf{H}$ NMR $\left(300 \mathrm{MHz}, \mathrm{CDCl}_{3}\right) \delta$ 7.39-7.33 (m, 2H, $\mathrm{C}_{\text {Arom-H }}$ ), 7.27-7.19 (m, 3H, $\left.\mathrm{C}_{\text {Arom-H}}-\mathrm{H}\right), 7.18-7.12$ $\left(\mathrm{m}, 2 \mathrm{H}, \mathrm{C}_{\text {Arom }} \mathrm{H}\right), 7.01-6.94\left(\mathrm{~m}, 2 \mathrm{H}, \mathrm{C}_{\text {Arom- }} \mathrm{H}\right), 5.18\left(\mathrm{~s}, 1 \mathrm{H}, \mathrm{C}=\mathrm{CH}_{\mathrm{a}} \mathrm{H}_{\mathrm{b}}\right), 5.13(\mathrm{~s}, 1 \mathrm{H}$, $\left.\mathrm{C}=\mathrm{CH}_{\mathrm{a}} \mathbf{H}_{\mathbf{b}}\right), 2.74\left(\mathrm{dd}, J=13.4,2.6 \mathrm{~Hz}, 2 \mathrm{H}, \mathrm{C}^{1} \mathrm{H}_{2}\right), 2.41\left(\mathrm{dd}, J=13.4,2.6 \mathrm{~Hz}, 2 \mathrm{H}, \mathrm{C}^{2} \mathrm{H}_{2}\right)$, 1.80 (bs, 1H, OH), $1.56\left(\mathrm{~s}, 3 \mathrm{H}, \mathrm{CH}_{3}\right) .{ }^{13} \mathbf{C}\left\{{ }^{1} \mathbf{H}\right\} \mathbf{N M R}\left(75.5 \mathrm{MHz}, \mathrm{CDCl}_{3}\right) \delta 153.5$ $\left(\mathbf{C}=\mathrm{CH}_{2}\right), 150.2\left(\mathrm{C}_{\text {Arom- }} \mathrm{C}\right), 138.7\left(\mathrm{C}_{\text {Arom- }}-\mathrm{C}\right), 131.0\left(\mathbf{C}_{\text {Arom }}-\mathrm{Cl}\right), 128.2(4 \mathrm{xC}$ Arom-H $), 127.7$ $\left(\mathrm{C}_{\text {Arom-H}}\right), 127.4\left(2 \times \mathrm{C}_{\text {Arom- }} \mathrm{H}\right), 126.7(2 \mathrm{xC}$ Arom-H $), 113.1\left(\mathrm{C}=\mathbf{C H}_{2}\right), 73.7(\mathrm{C}-\mathrm{OH}), 47.2$ $\left(2 \mathrm{xCH}_{2}\right), 35.5\left(\mathbf{C}-\mathrm{CH}_{3}\right), 32.2\left(\mathrm{CH}_{3}\right)$. IR (ATR) $\mathrm{cm}^{-1}: 3423(\mathrm{O}-\mathrm{H}), 2972(\mathrm{C}-\mathrm{H}), 2926(\mathrm{C}-$ H), 1626 (C=C). MS (EI) m/z (\%): 264.8 (6), 215 (13), 151.9 (93), 115.1 (96), 77 (100). HRMS $\left(\mathrm{ESI}^{+}\right)$: m/z calculated for $\left[\mathrm{C}_{19} \mathrm{H}_{19} \mathrm{ClO}-\mathrm{H}_{2} \mathrm{O}+\mathrm{H}\right]^{+}$281.1097; found 281.1094 [M$\left.\mathrm{H}_{2} \mathrm{O}+\mathrm{H}\right]^{+}$.

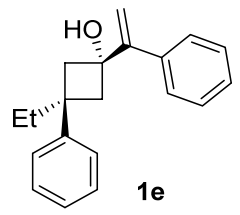

3-ethyl-3-phenyl-1-(1-phenylvinyl)cyclobutan-1-ol 1e

The product was prepared following the general procedure $\mathrm{D}$, obtaining a mixture of $c i s$ and trans diastereoisomers $(288.8 \mathrm{mg}, 1.03 \mathrm{mmol}, 91 \%)$ with a $\mathrm{dr}_{\text {trans:cis }}=1: 1$. The trans isomer 1e was isolated as a white solid (146 mg; $0.52 \mathrm{mmol} ; 46 \%$ ) by flash chromatography (PE:DCM = 4:6) starting from 1-bromovinylstyrene VI $(0.20 \mathrm{~mL} ; 1.49$ mmol), $\mathrm{Mg}$ (83 mg; $3.45 \mathrm{mmol})$, THF (6 mL), and 3-ethyl-3-phenylcyclobutanone II (200 mg; 1.15 mmol). $\mathrm{Rf}=0.4$ (PE:DCM = 4:6). M.p. $=48-50{ }^{\circ} \mathrm{C} .{ }^{1} \mathbf{H}$ NMR $(300 \mathrm{MHz}$, $\left.\mathrm{CDCl}_{3}\right) \delta 7.46\left(\mathrm{~m}, 2 \mathrm{H}, \mathrm{C}_{\text {Arom- }} \mathrm{H}\right), 7.32\left(\mathrm{~m}, 5 \mathrm{H}, \mathrm{C}_{\text {Arom- }} \mathrm{H}\right), 7.18\left(\mathrm{~m}, 1 \mathrm{H}, \mathrm{C}_{\text {Arom }}-\mathrm{H}\right), 7.09$ (m, $\left.1 \mathrm{H}, \mathrm{C}_{\text {Arom-H}} \mathrm{H}\right), 5.25\left(\mathrm{~d}, J=0.7 \mathrm{~Hz}, 1 \mathrm{H}, \mathrm{C}=\mathrm{CH}_{\mathbf{a}} \mathrm{Hb}_{\mathrm{b}}\right), 5.23\left(\mathrm{~d}, \mathrm{~J}=0.7 \mathrm{~Hz}, 1 \mathrm{H}, \mathrm{C}=\mathrm{CH}_{\mathrm{a}} \mathbf{H}_{\mathbf{b}}\right)$, 
$2.85\left(\mathrm{dd}, J=11.0,2.3 \mathrm{~Hz}, 2 \mathrm{H}, \mathrm{C}^{1} \mathrm{H}_{2}\right), 2.54\left(\mathrm{dd}, J=11.0,2.3 \mathrm{~Hz}, 2 \mathrm{H}, \mathrm{C}^{2} \mathrm{H}_{2}\right), 2.07$ (q, $J=$ $\left.7.3 \mathrm{~Hz}, 2 \mathrm{H}, \mathbf{C H}_{2} \mathrm{CH}_{3}\right), 1.96(\mathrm{~s}, 1 \mathrm{H}, \mathrm{OH}), 0.68\left(\mathrm{td}, J=7.3,0.8 \mathrm{~Hz}, 2 \mathrm{H}, \mathrm{CH}_{2} \mathbf{C H}_{3}\right) .{ }^{13} \mathbf{C}\left\{{ }^{1} \mathbf{H}\right\}$ NMR $\left(75.5 \mathrm{MHz}, \mathrm{CDCl}_{3}\right) \delta 153.7\left(\mathbf{C}=\mathrm{CH}_{2}\right), 148.9\left(\mathrm{C}_{\text {Arom-C }} \mathrm{C}\right), 138.9\left(\mathrm{C}_{\text {Arom-C }}\right), 128.2$ $\left(2 \mathrm{xC}_{\text {Arom- }}-\mathrm{H}\right), 127.7\left(2 \mathrm{xC}_{\text {Arom- }} \mathrm{H}\right), 127.6\left(\mathrm{C}_{\text {Arom }}-\mathrm{H}\right), 127.5\left(2 \mathrm{xC}_{\text {Arom }}-\mathrm{H}\right), 126.3\left(2 \mathrm{xC}_{\text {Arom- }}\right.$ $\mathrm{H}), 125.2\left(\mathrm{C}_{\text {Arom }}-\mathrm{H}\right), 113.1\left(\mathrm{C}=\mathbf{C H}_{2}\right), 74.1(\mathrm{C}-\mathrm{OH}), 45.7\left(2 \mathrm{xCH}_{2}\right), 39.6\left(\mathbf{C}-\mathrm{CH}_{2}\right), 36.7$ $\left(\mathbf{C H}_{2} \mathrm{CH}_{3}\right), 8.8\left(\mathrm{CH}_{2} \mathbf{C H}_{3}\right)$. IR (ATR) cm ${ }^{-1}: 3553(\mathrm{O}-\mathrm{H}), 3028$ (=C-H), $2945(\mathrm{C}-\mathrm{H}), 1620$ $(\mathrm{C}=\mathrm{C}), 1442\left(\mathrm{CH}_{3}\right) . \mathbf{M S}(\mathrm{EI}) \mathrm{m} / \mathrm{z}(\%): 146.1(30), 132.1$ (72), 117.1 (100). HRMS $\left(\mathrm{ESI}^{+}\right)$: $\mathrm{m} / \mathrm{z}$ calculated for $\left[\mathrm{C}_{20} \mathrm{H}_{22} \mathrm{O}-\mathrm{H}_{2} \mathrm{O}+\mathrm{H}\right]^{+}$261.1643; found $261.1642\left[\mathrm{M}-\mathrm{H}_{2} \mathrm{O}+\mathrm{H}\right]^{+}$.

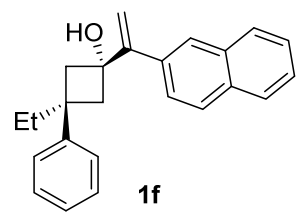

3-ethyl-1-(1-(naphthalen-2-yl)vinyl)-3-phenylcyclobutan-1-ol 1f

The product was prepared following the general procedure $\mathrm{D}$, obtaining a mixture of cis and trans diastereoisomers $(333 \mathrm{mg}, 1.01 \mathrm{mmol}, 59 \%)$ with a $\mathrm{dr}_{\text {trans:cis }}=1.2: 1$. The trans isomer 1f was isolated as a white solid (0.53 mmol; $173 \mathrm{mg} ; 31 \%)$ by flash chromatography (PE:DCM = 7:3 to 1:1) starting from 2-(1-bromovinyl)naphthalene XI (522 mg; $2.24 \mathrm{mmol}), \mathrm{Mg}(125 \mathrm{mg} ; 5.16 \mathrm{mmol})$, THF (11.2 mL), and 3-ethyl-3phenylcyclobutanone II (300 mg; $1.72 \mathrm{mmol}) . \mathrm{Rf}=0.19$ (PE:DCM=6:4). M.p. = 75.3$76.1{ }^{\circ} \mathrm{C} .{ }^{1} \mathbf{H}$ NMR $\left(300 \mathrm{MHz}, \mathrm{CDCl}_{3}\right) \delta$ 7.91-7.88 (m, 1H, CArom-H), 7.84-7.77 (m, 2H, $\left.\mathrm{C}_{\text {Arom- }} \mathrm{H}\right), 7.76\left(\mathrm{~s}, 1 \mathrm{H}, \mathrm{C}_{\text {Arom }}-\mathrm{H}\right), 7.56\left(\mathrm{dd}, J=8.5,1.8 \mathrm{~Hz}, 1 \mathrm{H}, \mathrm{C}_{\text {Arom }}-\mathrm{H}\right), 7.50-7.42(\mathrm{~m}$, 2H, $\left.\mathrm{C}_{\text {Arom }}-\mathrm{H}\right), 7.29-7.20\left(\mathrm{~m}, 3 \mathrm{H}, \mathrm{C}_{\text {Arom }}-\mathrm{H}\right), 7.17-7.10\left(\mathrm{~m}, 1 \mathrm{H}, \mathrm{C}_{\text {Arom }}-\mathrm{H}\right), 7.08-7.02(\mathrm{~m}$, $\left.2 \mathrm{H}, \mathrm{C}_{\text {Arom- }} \mathrm{H}\right), 5.36\left(\mathrm{~s}, 1 \mathrm{H}, \mathrm{C}=\mathrm{CH}_{\mathrm{a}} \mathrm{H}_{\mathrm{b}}\right), 5.30\left(\mathrm{~s}, 1 \mathrm{H}, \mathrm{C}=\mathrm{CH}_{\mathrm{a}} \mathbf{H}_{\mathrm{b}}\right), 2.86(\mathrm{~d}, J=13.5 \mathrm{~Hz}, 2 \mathrm{H}$, $\left.\mathrm{C}^{1} \mathrm{H}_{2}\right), 2.57\left(\mathrm{~d}, J=13.4 \mathrm{~Hz}, 2 \mathrm{H}, \mathrm{C}^{2} \mathrm{H}_{2}\right), 2.06$ (q, $\left.J=7.4 \mathrm{~Hz}, 2 \mathrm{H}, \mathbf{C H}_{2} \mathrm{CH}_{3}\right) 2.01(\mathrm{~s}, 1 \mathrm{H}$, $\mathrm{OH}), 0.66\left(\mathrm{t}, 3 \mathrm{H}, J=7.4 \mathrm{~Hz}, 3 \mathrm{H}, \mathrm{CH}_{3}\right) .{ }^{13} \mathbf{C}\left\{{ }^{1} \mathbf{H}\right\} \mathbf{N M R}\left(75.5 \mathrm{MHz}, \mathrm{CDCl}_{3}\right) \delta 153.9$ $\left(\mathbf{C}=\mathrm{CH}_{2}\right), 149.0\left(\mathrm{C}_{\text {Arom-C}} \mathrm{C}\right), 136.5\left(\mathrm{C}_{\text {Arom- }} \mathrm{C}\right), 133.5$ (C $\left.\mathrm{C}_{\text {Arom- }} \mathrm{C}\right), 133.0$ (C $\left.\mathrm{C}_{\text {Arom- }} \mathrm{C}\right), 128.5$ $\left(\mathrm{C}_{\text {Arom-H}}-\mathrm{H}\right), 128.0\left(2 \mathrm{xC}_{\text {Arom }}-\mathrm{H}\right), 127.9\left(\mathrm{C}_{\text {Arom }}-\mathrm{H}\right), 127.8\left(\mathrm{C}_{\text {Arom }}-\mathrm{H}\right), 126.6\left(2 \mathrm{xC}_{\text {Arom }}-\mathrm{H}\right)$, $126.3\left(\mathrm{C}_{\text {Arom }}-\mathrm{H}\right), 126.2\left(\mathrm{C}_{\text {Arom }}-\mathrm{H}\right), 126.1\left(\mathrm{C}_{\text {Arom }}-\mathrm{H}\right), 125.5\left(\mathrm{C}_{\text {Arom }}-\mathrm{H}\right), 113.9\left(\mathrm{C}=\mathbf{C H}_{2}\right)$, $74.3(\mathrm{C}-\mathrm{OH}), 46.0\left(2 \mathrm{xCH}_{2}\right), 39.9\left(\mathbf{C C H}_{2}\right), 37.0\left(\mathrm{C}_{\mathrm{Arom}}-\mathbf{C H}_{2}-\mathrm{CH}_{3}\right), 9.1\left(\mathrm{C}_{\mathrm{Arom}}-\mathrm{CH}_{2}-\mathbf{C H}_{3}\right)$. IR (ATR) cm : $^{-1} 348$ (O-H), $2964(\mathrm{C}-\mathrm{H}), 2929$ (C-H), 1598 (C=C). MS (EI) m/z (\%): $328.2(\mathrm{M}, 12), 178.0(43), 152$ (100). HRMS $\left(\mathrm{ESI}^{+}\right): \mathrm{m} / \mathrm{z}$ calculated for $\left[\mathrm{C}_{23} \mathrm{H}_{22} \mathrm{O}+\mathrm{Na}\right]^{+}$ 337.1541; found $337.1553[\mathrm{M}+\mathrm{Na}-14]^{+}$. 


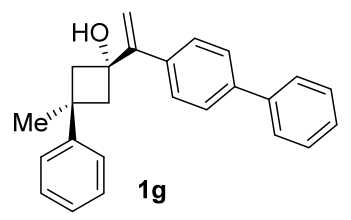

1-(1-([1,1'-biphenyl]-4-yl)vinyl)-3-methyl-3-phenylcyclobutan-1-ol $\mathbf{1 g}$

The product was prepared following the general procedure $\mathrm{D}$, obtaining a mixture of cis and trans diastereoisomers (301 $\mathrm{mg}, 0.88 \mathrm{mmol}, 47 \%)$ with a drtrans:cis $=1.6: 1$. The trans isomer $1 \mathrm{~g}$ was isolated as a white solid $(0.41 \mathrm{mmol} ; 141 \mathrm{mg} ; 22 \%)$ by flash chromatography (PE:DCM = 6:4 to 1:1) starting from 4-(1-bromovinyl)-1,1'-biphenyl $\mathbf{X}$ (630 mg; $2.43 \mathrm{mmol}), \mathrm{Mg}(136 \mathrm{mg} ; 5.61 \mathrm{mmol})$, THF (8.9 mL), and 3-methyl-3phenylcyclobutanone I (300 mg; $1.87 \mathrm{mmol}) . \mathrm{Rf}=0.28(\mathrm{PE}: \mathrm{DCM}=1: 1)$. M.p. $=97.5$ $98.3{ }^{\circ} \mathrm{C} .{ }^{1} \mathbf{H}$ NMR $\left(300 \mathrm{MHz}, \mathrm{CDCl}_{3}\right) \delta 7.55-7.49\left(\mathrm{~m}, 2 \mathrm{H}, \mathrm{C}_{\text {Arom- }} \mathrm{H}\right), 7.46\left(\mathrm{~s}, 4 \mathrm{H}, \mathrm{C}_{\text {Arom- }}\right.$ H), 7.40-7.32 (m, 2H, CArom-H), 7.30-7.16 (m, 3H, CArom-H), 7.11-7.03 (m, 3H, CArom$\mathrm{H}), 5.24\left(\mathrm{~s}, 1 \mathrm{H}, \mathrm{C}=\mathrm{CH}_{\mathrm{a}} \mathrm{H}_{\mathrm{b}}\right), 5.16\left(\mathrm{~s}, 1 \mathrm{H}, \mathrm{C}=\mathrm{CH}_{\mathrm{a}} \mathbf{H}_{\mathbf{b}}\right), 2.82\left(\mathrm{~d}, J=13.4,2.6 \mathrm{~Hz}, 2 \mathrm{H}, \mathrm{C}^{1} \mathrm{H}_{2}\right)$, $2.46\left(\mathrm{~d}, J=13.4,2.6 \mathrm{~Hz}, 2 \mathrm{H}, \mathrm{C}^{2} \mathrm{H}_{2}\right), 1.90(\mathrm{bs}, 1 \mathrm{H}, \mathrm{OH}), 1.61\left(\mathrm{~s}, 3 \mathrm{H}, \mathrm{CH}_{3}\right) .{ }^{13} \mathbf{C}\left\{{ }^{1} \mathbf{H}\right\} \mathbf{~ N M R}$ $\left(75.5 \mathrm{MHz}, \mathrm{CDCl}_{3}\right) \delta 153.2\left(\mathbf{C}=\mathrm{CH}_{2}\right), 151.8\left(\mathrm{C}_{\text {Arom- }} \mathrm{C}\right), 140.7\left(\mathrm{C}_{\text {Arom- }}-\mathrm{C}\right), 140.4\left(\mathrm{C}_{\text {Arom- }}{ }^{-}\right.$ C), $137.7\left(\mathrm{C}_{\text {Arom-C }}\right), 128.8\left(2 \mathrm{xC}_{\text {Arom }}-\mathrm{H}\right), 128.2\left(2 \mathrm{xC}_{\text {Arom }}-\mathrm{H}\right), 127.8\left(2 \mathrm{xC}_{\text {Arom }}-\mathrm{H}\right), 127.3$ $\left(\mathrm{C}_{\text {Arom- }} \mathrm{H}\right), 127.0\left(2 \mathrm{xC}_{\text {Arom }}-\mathrm{H}\right), 126.9\left(2 \mathrm{xC}_{\text {Arom }}-\mathrm{H}\right), 125.3\left(\mathrm{C}_{\text {Arom }}-\mathrm{H}\right), 125.1\left(2 \mathrm{xC}_{\text {Arom }}-\mathrm{H}\right)$, $112.9\left(\mathrm{C}=\mathbf{C H}_{2}\right), 74.0(\mathrm{C}-\mathrm{OH}), 47.2\left(2 \mathrm{xCH}_{2}\right), 35.9\left(\mathbf{C C H}_{3}\right), 32.4\left(\mathrm{C}_{\text {Arom }}-\mathbf{C H}_{3}\right)$. IR (ATR) $\mathrm{cm}^{-1}: 3438(\mathrm{O}-\mathrm{H}), 2971(\mathrm{C}-\mathrm{H}), 2925(\mathrm{C}-\mathrm{H}), 1601$ (C=C). MS (EI) m/z (\%): 340.1 (M, 18), 222.1 (48), 178.0 (100). HRMS $\left(\mathrm{ESI}^{+}\right): \mathrm{m} / \mathrm{z}$ calculated for $\left[\mathrm{C}_{25} \mathrm{H}_{24} \mathrm{O}-\mathrm{H}_{2} \mathrm{O}+\mathrm{H}\right]^{+}$ 323.1800; found $323.1790\left[\mathrm{M}-\mathrm{H}_{2} \mathrm{O}+\mathrm{H}\right]^{+}$.

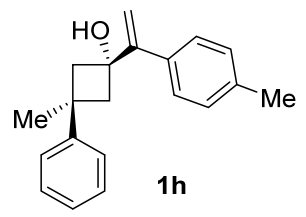

3-methyl-3-phenyl-1-(1-(p-tolyl)vinyl)cyclobutan-1-ol $\mathbf{1 h}$

The product was prepared following the general procedure $\mathrm{D}$, obtaining a mixture of cis and trans diastereoisomers (467.4 $\mathrm{mg}, 1.674 \mathrm{mmol}, 75 \%)$ with a $\mathrm{dr}_{\text {trans:cis }}=1.5: 1$. The trans isomer $1 \mathrm{~h}$ was isolated as a yellow oil $(0.96 \mathrm{mmol} ; 268 \mathrm{mg} ; 43 \%)$ by flash chromatography (PE:DCM = 1:1 to 3:7) starting from 1-(1-bromovinyl)-4methylbenzene VII (424 mg; $2.154 \mathrm{mmol}), \mathrm{Mg}$ (163 mg; $6.81 \mathrm{mmol})$, THF (11.00 mL), and 3-methyl-3-phenylcyclobutanone I (363 mg; $2.27 \mathrm{mmol}) . \mathrm{Rf}=0.4$ (PE:DCM =4:6). ${ }^{1} \mathbf{H}$ NMR $\left(300 \mathrm{MHz}, \mathrm{CDCl}_{3}\right) \delta 7.42\left(\mathrm{~d}, J=8.2 \mathrm{~Hz}, 2 \mathrm{H}, \mathrm{C}_{\text {Arom-H}}\right), 7.32$ (q, $J=7.8 \mathrm{~Hz}, 2 \mathrm{H}$, $\left.\mathrm{C}_{\text {Arom-H}}-\mathrm{H}\right), 7.23-7.15\left(\mathrm{~m}, 5 \mathrm{H}, \mathrm{C}_{\text {Arom- }}-\mathrm{H}\right), 5.29\left(\mathrm{~d}, J=0.5 \mathrm{~Hz}, 1 \mathrm{H}, \mathrm{C}=\mathrm{CH}_{\mathrm{a}} \mathrm{H}_{\mathrm{b}}\right), 5.22(\mathrm{~d}, J=$ 
$\left.0.5 \mathrm{~Hz}, 1 \mathrm{H}, \mathrm{C}=\mathrm{CH}_{\mathrm{a}} \mathbf{H}_{\mathbf{b}}\right), 2.92\left(\mathrm{dd}, J=10.8,2.6 \mathrm{~Hz}, 2 \mathrm{H}, \mathrm{C}^{1} \mathrm{H}_{2}\right), 2.58(\mathrm{dd}, J=10.8,2.6 \mathrm{~Hz}$, $\left.2 \mathrm{H}, \mathrm{C}^{2} \mathrm{H}_{2}\right), 2.40\left(\mathrm{~s}, 3 \mathrm{H}, \mathrm{C}_{\text {Arom }}-\mathrm{CH}_{3}\right), 2.04(\mathrm{~s}, 1 \mathrm{H}, \mathrm{OH}), 1.73\left(\mathrm{~s}, 3 \mathrm{H}, \mathrm{CH}_{3}\right) .{ }^{13} \mathbf{C}\left\{{ }^{1} \mathbf{H}\right\} \mathbf{N M R}$ $\left(75.5 \mathrm{MHz}, \mathrm{CDCl}_{3}\right) \delta 153.5\left(\mathbf{C}=\mathrm{CH}_{2}\right), 151.9\left(\mathrm{C}_{\text {Arom- }}-\mathrm{C}\right), 137.4\left(\mathrm{C}_{\text {Arom }}-\mathrm{C}\right), 135.9\left(\mathrm{C}_{\text {Arom- }}{ }^{-}\right.$ C), $128.9\left(2 \mathrm{xC}_{\text {Arom }}-\mathrm{H}\right), 128.2\left(2 \mathrm{xC}_{\text {Arom }}-\mathrm{H}\right), 127.4\left(2 \mathrm{xC}_{\text {Arom }}-\mathrm{H}\right), 125.3\left(\mathrm{C}_{\text {Arom }}-\mathrm{H}\right), 125.2$ $\left(2 \mathrm{xC}\right.$ Arom-H), $112.3\left(\mathrm{C}=\mathbf{C H}_{2}\right), 74.0(\mathrm{C}-\mathrm{OH}), 47.2\left(2 \mathrm{xCH}_{2}\right), 35.8\left(\mathbf{C}_{-} \mathrm{CH}_{3}\right), 32.4\left(\mathrm{C}_{\text {Arom- }}{ }^{-}\right.$

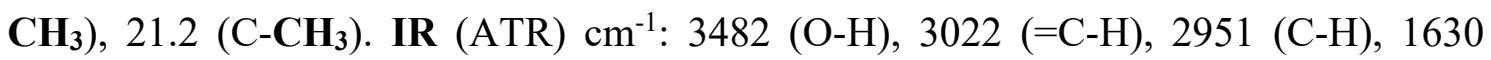
$(\mathrm{C}=\mathrm{C}), 1452\left(\mathrm{CH}_{3}\right) . \mathbf{M S}(\mathrm{EI}) \mathrm{m} / \mathrm{z}(\%): 278.2\left(\mathrm{M}^{+}, 23\right), 160.1$ (57), 145 (100), 132 (44). HRMS $\left(\mathrm{ESI}^{+}\right): \mathrm{m} / \mathrm{z}$ calculated for $\left[\mathrm{C}_{20} \mathrm{H}_{22} \mathrm{O}-\mathrm{H}_{2} \mathrm{O}+\mathrm{H}\right]^{+}$261.1643; found 261.1642 [M$\left.\mathrm{H}_{2} \mathrm{O}+\mathrm{H}\right]^{+}$.

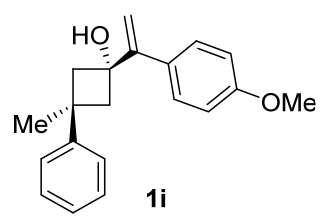

1-(1-(4-methoxyphenyl)vinyl)-3-methyl-3-phenylcyclobutan-1-ol 1i.

The product was prepared following the general procedure $\mathrm{D}$, obtaining a mixture of cis and trans diastereoisomers $(809 \mathrm{mg}, 2.735 \mathrm{mmol}, 88 \%)$ with a $\mathrm{dr}_{\text {trans:cis }}=1.2: 1$. The trans isomer 11 was isolated as a yellow solid (340 $\mathrm{mg} ; 1.15 \mathrm{mmol} ; 37 \%$ ) by flash chromatography (PE:DCM = 4:6) starting from 1-(1-bromovinyl)-4-methoxybenzene VIII (633 mg; $2.97 \mathrm{mmol}), \mathrm{Mg}(225 \mathrm{mg}$; $9.38 \mathrm{mmol})$, THF (15.00 mL), and 3-methyl-3phenylcyclobutanone I (500 mg; $3.13 \mathrm{mmol}) . \mathrm{Rf}=0.4(\mathrm{PE}: \mathrm{DCM}=4: 6)$. M.p. $=44-46$ ${ }^{\circ} \mathrm{C} .{ }^{1} \mathbf{H}$ NMR $\left(300 \mathrm{MHz}, \mathrm{CDCl}_{3}\right) \delta 7.33\left(\mathrm{~d}, J=8.9 \mathrm{~Hz}, 2 \mathrm{H}, 2 \mathrm{xC} \mathrm{Arom}_{\mathrm{H}} \mathrm{H}\right), 7.18(\mathrm{dd}, J=6.4$, $\left.1.0 \mathrm{~Hz}, 2 \mathrm{H}, 2 \mathrm{xC}_{\text {Arom- }} \mathrm{H}\right), 7.10-7.02\left(\mathrm{~m}, 3 \mathrm{H}, 3 \mathrm{xC}_{\text {Arom }}-\mathrm{H}\right), 6.77\left(\mathrm{~d}, J=8.9 \mathrm{~Hz}, 2 \mathrm{H}, 2 \mathrm{xC}_{\text {Arom- }}\right.$ $\mathrm{H}), 5.12\left(\mathrm{~d}, J=0.6 \mathrm{~Hz}, 1 \mathrm{H}, \mathrm{C}=\mathrm{CH}_{\mathbf{a}} \mathrm{H}_{\mathrm{b}}\right), 5.05\left(\mathrm{~d}, J=0.4 \mathrm{~Hz}, 1 \mathrm{H}, \mathrm{C}=\mathrm{CH}_{\mathrm{a}} \mathbf{H}_{\mathbf{b}}\right), 3.73(\mathrm{~s}, 3 \mathrm{H}$, $\left.\mathrm{OCH}_{3}\right), 2.83-2.73\left(\mathrm{~m}, 2 \mathrm{H}, \mathrm{C}^{1} \mathrm{H}_{2}\right), 2.47-2.36\left(\mathrm{~m}, 2 \mathrm{H}, \mathrm{C}^{2} \mathrm{H}_{2}\right), 1.80$ (bs, $\left.1 \mathrm{H}, \mathrm{OH}\right), 1.59$ $\left(\mathrm{s}, 3 \mathrm{H}, \mathrm{CH}_{3}\right) .{ }^{13} \mathbf{C}\left\{{ }^{1} \mathbf{H}\right\} \mathbf{N M R}\left(75.5 \mathrm{MHz}, \mathrm{CDCl}_{3}\right) \delta 159.2\left(\mathbf{C}=\mathrm{CH}_{2}\right), 153.0\left(\mathrm{C}_{\text {Arom- }} \mathrm{C}\right)$, $152.0\left(\mathrm{C}_{\text {Arom- }} \mathrm{C}\right), 131.1\left(\mathrm{C}_{\text {Arom- }} \mathrm{C}\right), 128.6\left(2 \mathrm{xC} \mathrm{C}_{\text {Arom }}-\mathrm{H}\right), 128.2\left(2 \mathrm{x} \mathrm{C}_{\text {Arom }}-\mathrm{H}\right), 125.2\left(\mathrm{C}_{\text {Arom- }}\right.$ $\mathrm{H}), 125.1\left(2 \times \mathrm{C}_{\text {Arom }}-\mathrm{H}\right), 113.6(2 \mathrm{xC}$ Arom- $\mathrm{H}), 111.6\left(\mathrm{C}=\mathbf{C H}_{2}\right), 74.0(\mathrm{C}-\mathrm{OH}), 55.3\left(\mathrm{OCH}_{3}\right)$, $47.2\left(2 \mathrm{xCH}_{2}\right), 35.8\left(\mathbf{C}-\mathrm{CH}_{3}\right), 32.4\left(\mathrm{CH}_{3}\right)$. IR (ATR) cm ${ }^{-1}: 3518(\mathrm{O}-\mathrm{H}), 3021(=\mathrm{C}-\mathrm{H}), 2949$ (C-H), $1640(\mathrm{C}=\mathrm{C}), 1448\left(\mathrm{CH}_{3}\right) . \mathbf{M S}(\mathrm{EI}) \mathrm{m} / \mathrm{z}(\%): 294.2\left[\mathrm{M}^{+}, 11\right], 295.2\left[\mathrm{M}^{+}, 3\right], 176.1$ (98), 161.1 (24), 133.1 (100), 117.1 (63). HRMS $\left(\mathrm{ESI}^{+}\right)$: $\mathrm{m} / \mathrm{z}$ calculated for $\left[\mathrm{C}_{20} \mathrm{H}_{22} \mathrm{O}_{2-}\right.$ $\left.\mathrm{H}_{2} \mathrm{O}+\mathrm{H}\right]^{+}$277.1592; found $277.1594\left[\mathrm{M}-\mathrm{H}_{2} \mathrm{O}+\mathrm{H}\right]^{+}$. 


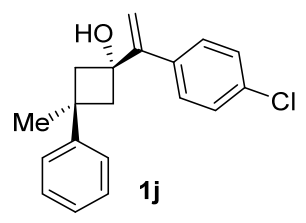

1-(1-(4-chlorophenyl)vinyl)-3-methyl-3-phenylcyclobutan-1-ol 1j

The product was prepared following the general procedure $\mathrm{D}$, obtaining a mixture of $c$ is and trans diastereoisomers (308 mg, $1.034 \mathrm{mmol}, 33 \%$ ) with a $\mathrm{dr}_{\text {trans:cis }}=1.1: 1$. The trans isomer 1j was isolated as a transparent oil (140 $\mathrm{mg} ; 0.47 \mathrm{mmol} ; 15 \%$ ) by flash chromatography (PE:DCM = 4:6) starting from 1-(1-bromovinyl)-4-chlorobenzene IX (646 mg; $2.97 \mathrm{mmol}), \mathrm{Mg}$ (225 mg; $9.38 \mathrm{mmol})$, THF (15.00 mL), and 3-methyl-3phenylcyclobutanone I (570 mg; $3.125 \mathrm{mmol}) . \mathrm{Rf}=0.4(\mathrm{PE}: \mathrm{DCM}=4: 6) .{ }^{1} \mathbf{H}$ NMR $(300$

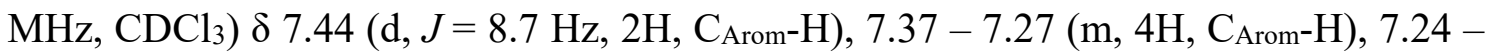
$7.14\left(\mathrm{~m}, 3 \mathrm{H}, \mathrm{C}\right.$ Arom-H), $5.28\left(\mathrm{~s}, 1 \mathrm{H}, \mathrm{C}=\mathrm{CH}_{\mathbf{a}} \mathrm{Hb}_{\mathrm{b}}\right), 5.26\left(\mathrm{~s}, 1 \mathrm{H}, \mathrm{C}=\mathrm{CH}_{\mathrm{a}} \mathbf{H}_{\mathbf{b}}\right), 2.92-2.80(\mathrm{~m}$, $\left.2 \mathrm{H}, \mathrm{C}^{1} \mathrm{H}_{2}\right), 2.60-2.44\left(\mathrm{~m}, 2 \mathrm{H}, \mathrm{C}^{2} \mathrm{H}_{2}\right), 1.99(\mathrm{~s}, 1 \mathrm{H}, \mathrm{OH}), 1.70\left(\mathrm{~s}, 3 \mathrm{H}, \mathrm{CH}_{3}\right) .{ }^{13} \mathbf{C}\left\{{ }^{1} \mathbf{H}\right\} \mathbf{~ N M R}$ $\left(75.5 \mathrm{MHz}, \mathrm{CDCl}_{3}\right) \delta 152.7\left(\mathbf{C}=\mathrm{CH}_{2}\right), 151.6\left(\mathrm{C}_{\text {Arom- }}-\mathrm{C}\right), 137.3\left(\mathrm{C}_{\text {Arom- }}-\mathrm{C}\right), 133.5\left(\mathrm{C}_{\text {Arom- }}{ }^{-}\right.$ C), $128.8\left(2 \mathrm{xC}_{\text {Arom-H}} \mathrm{H}\right), 128.3\left(2 \mathrm{xC}_{\text {Arom }}-\mathrm{H}\right), 128.2\left(2 \mathrm{xC}_{\text {Arom }}-\mathrm{H}\right), 125.4\left(\mathrm{C}_{\text {Arom }}-\mathrm{H}\right), 125.1$ $\left(2 \times C_{\text {Arom- }}-\mathrm{H}\right), 113.5\left(\mathrm{C}=\mathbf{C H}_{2}\right), 73.8(\mathrm{C}-\mathrm{OH}), 47.0\left(2 \mathrm{xCH}_{2}\right), 35.8\left(\mathbf{C C H}_{3}\right), 32.5\left(\mathrm{CH}_{3}\right) . \mathbf{I R}$ (ATR) $\mathrm{cm}^{-1}: 3567(\mathrm{O}-\mathrm{H}), 3030(=\mathrm{C}-\mathrm{H}), 2952(\mathrm{C}-\mathrm{H}), 1643(\mathrm{C}=\mathrm{C}), 1439\left(\mathrm{CH}_{3}\right)$. MS (EI) m/z (\%): 145.1 (65), 137 (14), 118 (100). HRMS (ESI $\left.{ }^{+}\right): \mathrm{m} / \mathrm{z}$ calculated for [ $\mathrm{C}_{19} \mathrm{H}_{19} \mathrm{ClO}-$ $\left.\mathrm{H}_{2} \mathrm{O}+\mathrm{H}\right]^{+}$281.1097; found 281.1089 [M- $\left.\mathrm{H}_{2} \mathrm{O}+\mathrm{H}\right]^{+}$.

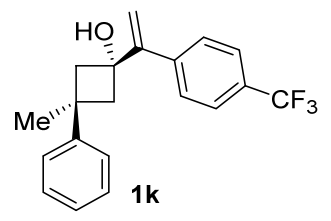

3-methyl-3-phenyl-1-(1-(4-(trifluoromethyl)phenyl)vinyl)cyclobutan-1-ol 1k

The product was prepared following the general procedure $\mathrm{D}$, obtaining a mixture of cis and trans diastereoisomers $(221 \mathrm{mg} ; 0.67 \mathrm{mmol} ; 36 \%)$ with a drans:cis $=3.3: 1$. The trans isomer 1k was isolated as a yellow oil (156 $\mathrm{mg} ; 0.47 \mathrm{mmol} ; 25 \%$ ) by flash chromatography (PE:DCM $=8: 2$ to $7: 3$ to $1: 1$ ) starting from 1-(1-bromovinyl)-4(trifluoromethyl)benzene XII (610 mg; $2.43 \mathrm{mmol}), \mathrm{Mg}$ (136 mg; $5.61 \mathrm{mmol})$, THF (9 $\mathrm{mL}$ ), and 3-methyl-3-phenylcyclobutan-1-one I (300 mg; $1.87 \mathrm{mmol}) . \mathrm{Rf}=0.25$ (PE:DCM = 70:30). ${ }^{1} \mathbf{H}$ NMR (300 MHz, $\left.\mathrm{CDCl}_{3}\right) \delta 7.48\left(\mathrm{~s}, 4 \mathrm{H}, \mathrm{C}_{\text {Arom-H}} \mathrm{H}\right), 7.24-7.15$ (m, 2H, $\left.\mathrm{C}_{\text {Arom- }} \mathrm{H}\right), 7.10-7.00\left(\mathrm{~m}, 3 \mathrm{H}, \mathrm{C}_{\text {Arom- }} \mathrm{H}\right), 5.22\left(\mathrm{~d}, J=1.4 \mathrm{~Hz}, 2 \mathrm{H}, \mathrm{C}=\mathrm{CH}_{2}\right), 2.75(\mathrm{~d}, J=$ $\left.13.4 \mathrm{~Hz}, 2 \mathrm{H}, \mathrm{C}^{1} \mathrm{H}_{2}\right), 2.41$ (d, $\left.J=13.4 \mathrm{~Hz}, 2 \mathrm{H}, \mathrm{C}^{2} \mathrm{H}_{2}\right), 1.87$ (s, $\left.1 \mathrm{H}, \mathrm{OH}\right), 1.58\left(\mathrm{~s}, 3 \mathrm{H}, \mathrm{CH}_{3}\right)$. ${ }^{19} \mathbf{F}$ NMR $\left(282 \mathrm{MHz}, \mathrm{CDCl}_{3}\right) \delta-62.5 .{ }^{13} \mathbf{C}\left\{{ }^{1} \mathbf{H}\right\}$ NMR $\left(75.5 \mathrm{MHz}, \mathrm{CDCl}_{3}\right) \delta 152.7$ 
$\left(\mathbf{C}=\mathrm{CH}_{2}\right), 151.4\left(\mathrm{C}_{\text {Arom }}-\mathrm{C}\right), 142.5\left(\mathrm{C}_{\text {Arom }}-\mathrm{C}\right), 129.5\left(\mathrm{q}, J_{C-F}=32.3 \mathrm{~Hz}, \mathbf{C}_{\text {Arom }}-\mathrm{CF}_{3}\right), 128.2$ (2xC $\left.\mathrm{C}_{\text {Arom- }}-\mathrm{H}\right), 127.7\left(2 \mathrm{xC}_{\text {Arom }}-\mathrm{H}\right), 125.4\left(\mathrm{C}_{\text {Arom }}-\mathrm{H}\right), 125.1\left(2 \mathrm{xC}_{\text {Arom }}-\mathrm{H}\right), 125.0\left(\mathrm{q}, J_{C-F}=3.8\right.$ $\mathrm{Hz}, 2 \mathrm{C}_{\text {Arom-H}}$ ), $124.0\left(\mathrm{q}, J_{C-F}=272.0 \mathrm{~Hz}, \mathrm{CF}_{3}\right), 114.6\left(\mathrm{C}=\mathbf{C H}_{2}\right), 73.7(\mathrm{C}-\mathrm{OH}), 47.0$ $\left(2 \mathrm{xCH}_{2}\right), 35.8\left(\mathrm{CCH}_{3}\right), 32.5\left(\mathrm{CH}_{3}\right)$. IR (ATR) cm $\mathrm{cm}^{-1}: 3416(\mathrm{O}-\mathrm{H}), 2953(\mathrm{C}-\mathrm{H}), 2927(\mathrm{C}-$ H), $1616(\mathrm{C}=\mathrm{C})$. MS (EI) m/z (\%): $314.1\left(\mathrm{M}-\mathrm{H}_{2} \mathrm{O}, 100\right)$. HRMS $\left(\mathrm{ESI}^{+}\right): \mathrm{m} / \mathrm{z}$ calculated for $\left[\mathrm{C}_{20} \mathrm{H}_{19} \mathrm{OF} 3-\mathrm{H}_{2} \mathrm{O}+\mathrm{H}\right]^{+} 315.1361$; found $315.1353\left[\mathrm{M}-\mathrm{H}_{2} \mathrm{O}+\mathrm{H}\right]^{+}$.

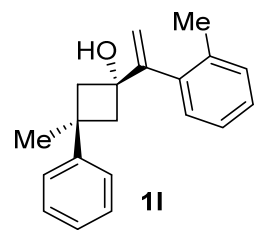

3-methyl-3-phenyl-1-(1-(o-tolyl)vinyl)cyclobutan-1-ol 11

The product was prepared following the general procedure $\mathrm{D}$, obtaining a mixture of cis and trans diastereoisomers $(364 \mathrm{mg} ; 1.31 \mathrm{mmol} ; 70 \%)$ with a drans:cis $=1.7: 1$. The trans isomer 11 was isolated as a transparent oil $(0.46 \mathrm{mmol} ; 129 \mathrm{mg} ; 25 \%)$ by flash chromatography (PE:DCM $=6: 4$ to 1:1) starting from 1-(1-bromovinyl)-2methylbenzene XIV (479 mg; $2.43 \mathrm{mmol}), \mathrm{Mg}$ (136 mg; $5.61 \mathrm{mmol}$ ), THF (10 mL), and 3-methyl-3-phenylcyclobutanone I (300 mg; $1.87 \mathrm{mmol})$. Rf = 0.36 (PE:DCM = 60:40). ${ }^{1} \mathbf{H}$ NMR $\left(300 \mathrm{MHz}, \mathrm{CDCl}_{3}\right) \delta$ 7.32-7.24 (m, 2H, CArom-H), 7.21-7.09 (m, 7H, CArom-H), $5.37\left(\mathrm{~d}, J=1.0 \mathrm{~Hz}, 1 \mathrm{H}, \mathrm{C}=\mathrm{CH}_{\mathrm{a}} \mathrm{Hb}\right), 4.92\left(\mathrm{~d}, J=1.0 \mathrm{~Hz}, 1 \mathrm{H}, \mathrm{C}=\mathrm{CH}_{\mathrm{a}} \mathbf{H}_{\mathrm{b}}\right), 2.87(\mathrm{dd}, J=$ 13.4, $\left.2.6 \mathrm{~Hz}, 2 \mathrm{H}, \mathrm{C}^{1} \mathrm{H}_{2}\right), 2.34\left(\mathrm{dd}, J=13.4,2.6 \mathrm{~Hz}, 2 \mathrm{H}, \mathrm{C}^{2} \mathrm{H}_{2}\right), 2.20$ (s, 3H, $\mathrm{C}_{\text {Arom- }} \mathrm{CH}_{3}$ ), $1.82(\mathrm{~s}, 1 \mathrm{H}, \mathrm{OH}), 1.63\left(\mathrm{~s}, 3 \mathrm{H}, \mathrm{CH}_{3}\right) .{ }^{13} \mathbf{C}\left\{{ }^{1} \mathbf{H}\right\} \mathbf{N M R}\left(75.5 \mathrm{MHz}, \mathrm{CDCl}_{3}\right) \delta 153.5\left(\mathbf{C}=\mathrm{CH}_{2}\right)$, 151.7 ( $\left.\mathrm{C}_{\text {Arom }}-\mathrm{C}\right), 139.4\left(\mathrm{C}_{\text {Arom }}-\mathrm{C}\right), 136.2\left(\mathrm{C}_{\text {Arom }}-\mathrm{C}\right), 130.3\left(\mathrm{C}_{\text {Arom }}-\mathrm{H}\right), 128.8\left(\mathrm{C}_{\text {Arom }}-\mathrm{H}\right)$, $128.2\left(2 \mathrm{xC}_{\text {Arom }}-\mathrm{H}\right), 127.3\left(\mathrm{C}_{\text {Arom }}-\mathrm{H}\right), 125.3\left(\mathrm{C}_{\text {Arom }}-\mathrm{H}\right), 125.2\left(\mathrm{C}_{\text {Arom }}-\mathrm{H}\right), 125.1\left(2 \mathrm{xC}_{\text {Arom- }}\right.$ $\mathrm{H}), 113.8\left(\mathrm{C}=\mathbf{C} \mathbf{H}_{2}\right), 74.2(\mathrm{C}-\mathrm{OH}), 47.2\left(2 \mathrm{xCH}_{2}\right), 35.6\left(\mathbf{C}-\mathrm{CH}_{3}\right), 32.2\left(\mathrm{C}_{\text {Arom }}-\mathbf{C H}_{3}\right), 20.5$ (CH3). IR (ATR) cm cm $^{-1} 416(\mathrm{O}-\mathrm{H}), 2951(\mathrm{C}-\mathrm{H}), 2924(\mathrm{C}-\mathrm{H}), 1600$ (C=C). MS (EI) m/z (\%): $260\left(\mathrm{M}-\mathrm{H}_{2} \mathrm{O}, 6\right), 145$ (23), 117 (100). HRMS (ESI $\left.{ }^{+}\right): \mathrm{m} / \mathrm{z}$ calculated for $\left[\mathrm{C}_{20} \mathrm{H}_{22} \mathrm{O}-\right.$ $\left.\mathrm{H}_{2} \mathrm{O}+\mathrm{H}\right]^{+}$261.1643; found $261.1635\left[\mathrm{M}-\mathrm{H}_{2} \mathrm{O}+\mathrm{H}\right]^{+}$.

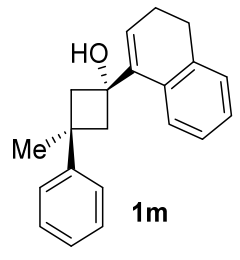

1-(3,4-dihydronaphthalen-1-yl)-3-methyl-3-phenylcyclobutan-1-ol 1 m 
The product was prepared following the general procedure $\mathrm{D}$, obtaining a mixture of cis and trans diastereoisomers (364 mg, $1.245 \mathrm{mmol}, 81 \%$ ) with a $\mathrm{dr}_{\text {trans:cis }}=1.4: 1$. The trans isomer $1 \mathrm{~m}$ was isolated as a transparent oil (184 $\mathrm{mg} ; 0.63 \mathrm{mmol} ; 41 \%)$ by flash chromatography (PE:DCM = 4:6) starting from 4-bromo-1,2-dihydronaphthalene XIII (306 mg; $1.47 \mathrm{mmol}), \mathrm{Mg}$ (111 mg; $4.64 \mathrm{mmol})$, THF (7.70 mL), and 3-methyl-3phenylcyclobutanone I (250 mg; $1.55 \mathrm{mmol}) . \mathrm{Rf}=0.3$ (PE:DCM = 1:1). ${ }^{1} \mathbf{H}$ NMR (300 $\left.\mathrm{MHz}, \mathrm{CDCl}_{3}\right) \delta 7.71\left(\mathrm{~d}, J=7.3 \mathrm{~Hz}, 1 \mathrm{H}, \mathrm{C}_{\text {Arom-H}}\right), 7.48-7.34\left(\mathrm{~m}, 2 \mathrm{H}, \mathrm{C}_{\text {Arom-H}}-\mathrm{H}\right), 7.32-$ $7.19(\mathrm{~m}, 6 \mathrm{H}, \mathrm{C}$ Arom-H), 6.10 (t, $J=4.7 \mathrm{~Hz}, 1 \mathrm{H}, \mathrm{C}=\mathbf{C H}), 3.15-3.04($ app dd, $J=13.3,2.4$ $\left.\mathrm{Hz}, 2 \mathrm{H}, \mathbf{C}^{\mathbf{1}} \mathbf{H}_{2}\right), 2.85-2.66\left(\mathrm{~m}, 4 \mathrm{H}, \mathbf{C}^{2} \mathbf{H}_{2}\right.$ and $\left.\mathrm{C}_{\text {Arom }} \mathrm{CH}_{2}\right), 2.38-2.15\left(\mathrm{~m}, 2 \mathrm{H}, \mathrm{C}_{\text {Arom- }}\right.$ $\mathrm{CH}_{2} \mathbf{C H}_{2}$ and $\left.-\mathrm{OH}\right), 1.83\left(\mathrm{~s}, 3 \mathrm{H}, \mathrm{CH}_{3}\right) .{ }^{13} \mathbf{C}\left\{{ }^{1} \mathbf{H}\right\} \mathbf{N M R}\left(75.5 \mathrm{MHz}, \mathrm{CDCl}_{3}\right) \delta 152.0\left(\mathrm{C}_{\text {Arom- }}\right.$ C), $141.5(\mathbf{C}=\mathrm{CH}), 137.4\left(\mathrm{C}_{\text {Arom- }}-\mathrm{C}\right), 132.3\left(\mathrm{C}_{\text {Arom }}-\mathrm{C}\right), 128.2\left(2 \mathrm{xC}_{\text {Arom }}-\mathrm{H}\right), 127.9\left(\mathrm{C}_{\text {Arom- }}{ }^{-}\right.$ $\mathrm{H}), 126.9\left(\mathrm{C}_{\text {Arom- }}-\mathrm{H}\right), 126.2\left(\mathrm{C}_{\text {Arom }}-\mathrm{H}\right), 125.6\left(\mathrm{C}_{\text {Arom }}-\mathrm{H}\right), 125.3\left(2 \mathrm{xC}_{\text {Arom }}-\mathrm{H}\right), 125.2\left(\mathrm{C}_{\text {Arom- }}\right.$ $\mathrm{H}), 73.4(\mathrm{C}-\mathrm{OH}), 47.6\left(2 \times \mathbf{C H}_{2}\right), 36.4\left(\mathbf{C}-\mathrm{CH}_{2}\right), 32.7\left(\mathrm{CH}_{3}\right), 28.2\left(\mathrm{CArom}-\mathrm{CH}_{2}\right), 23.0$ $\left(\mathrm{C}_{\text {Arom-CH}} \mathbf{C H}_{2}\right)$. IR (ATR) cm ${ }^{-1}: 3562(\mathrm{O}-\mathrm{H}), 3022$ (=C-H), $2954(\mathrm{C}-\mathrm{H}), 1635$ (C=C), $1437\left(\mathrm{CH}_{3}\right) . \mathbf{M S}(\mathrm{EI}) \mathrm{m} / \mathrm{z}$ (\%): 172.1 (90), 154.1 (41) 128 (100). HRMS (ESI $\left.{ }^{+}\right): \mathrm{m} / \mathrm{z}$ calculated for $\left[\mathrm{C}_{21} \mathrm{H}_{22} \mathrm{O}-\mathrm{H}_{2} \mathrm{O}+\mathrm{H}\right]^{+}$273.1643; found $273.1644\left[\mathrm{M}-\mathrm{H}_{2} \mathrm{O}+\mathrm{H}\right]^{+}$.

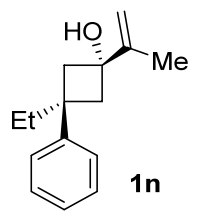

3-ethyl-3-phenyl-1-(prop-1-en-2-yl)cyclobutan-1-ol 1n

The product was prepared following the procedure E, obtaining a mixture of cis and trans diastereoisomers (242 mg; $1.12 \mathrm{mmol} ; 65 \%)$ with a $\mathrm{dr}_{\text {trans:cis }}=1.1: 1$. The trans isomer $\mathbf{1 n}$ was obtained as a transparent oil (126 mg; $0.58 \mathrm{mmol} ; 34 \%)$ by flash chromatography (PE:DCM 7:3) starting from isopropenylmagnesium bromide (5.2 $\mathrm{mL}, 0.5 \mathrm{M}$ in THF), THF (1.25 mL) and 3-ethyl-3-phenylcyclobutanone II (300 mg, $1.72 \mathrm{mmol}) . \mathrm{Rf}=0.29$ (PE:DCM = 6:4). ${ }^{1} \mathbf{H}$ NMR (300 MHz, $\left.\mathrm{CDCl}_{3}\right) \delta$ 7.33-7.24 (m, 2H, CArom-H), 7.19-7.09 $\left(\mathrm{m}, 3 \mathrm{H}, \mathrm{C}_{\text {Arom }}-\mathrm{H}\right), 4.84\left(\mathrm{~s}, 1 \mathrm{H}, \mathrm{C}=\mathrm{CH}_{\mathbf{a}} \mathrm{H}_{\mathrm{b}}\right), 4.73\left(\mathrm{t}, J=1.4 \mathrm{~Hz}, 1 \mathrm{H}, \mathrm{C}=\mathrm{CH}_{\mathrm{a}} \mathbf{H}_{\mathrm{b}}\right), 2.74(\mathrm{~d}, J$ $\left.=13.5 \mathrm{~Hz}, 2 \mathrm{H}, \mathrm{C}^{1} \mathrm{H}_{2}\right), 2.32\left(\mathrm{~d}, J=13.5 \mathrm{~Hz}, 2 \mathrm{H}, \mathrm{C}^{2} \mathrm{H}_{2}\right), 2.01\left(\mathrm{q}, J=7.3 \mathrm{~Hz}, 2 \mathrm{H}, \mathbf{C H}_{2^{-}}\right.$ $\left.\mathrm{CH}_{3}\right), 1.70\left(\mathrm{~s}, 3 \mathrm{H}, \mathrm{CH}_{3}\right), 0.65\left(\mathrm{t}, J=7.3 \mathrm{~Hz}, 3 \mathrm{H}, \mathrm{CH}_{2}-\mathrm{CH}_{3}\right) .{ }^{13} \mathbf{C}\left\{{ }^{1} \mathbf{H}\right\} \mathbf{N M R}(75.5 \mathrm{MHz}$, $\left.\mathrm{CDCl}_{3}\right) \delta 149.2\left(\mathrm{C}_{\text {Arom }}-\mathbf{C}\right), 148.9\left(\mathbf{C}=\mathrm{CH}_{2}\right), 127.7\left(2 \times \mathrm{C}_{\text {Arom- }}-\mathrm{H}\right), 126.3\left(2 \times \mathrm{C}_{\text {Arom }}-\mathrm{H}\right), 125.2$ $\left(\mathrm{C}_{\text {Arom-H}}-\mathrm{H}\right), 109.7\left(\mathrm{C}=\mathbf{C H}_{2}\right), 74.1(\mathrm{C}-\mathrm{OH}), 44.2\left(2 \times \mathrm{CH}_{2}\right), 39.2\left(\mathbf{C C H}_{2} \mathrm{CH}_{3}\right), 36.9\left(\mathbf{C H}_{2}-\right.$ $\left.\mathrm{CH}_{3}\right), 17.4\left(\mathrm{CH}_{3}\right), 8.7\left(\mathrm{CH}_{2}-\mathbf{C H}_{3}\right)$. IR (ATR) cm ${ }^{-1}: 3364(\mathrm{O}-\mathrm{H}), 2967(\mathrm{C}-\mathrm{H}), 2929(\mathrm{C}-\mathrm{H})$, 
$1600(\mathrm{C}=\mathrm{C}) . \mathbf{M S}(\mathrm{EI}) \mathrm{m} / \mathrm{z}(\%): 198\left(\mathrm{M}-\mathrm{H}_{2} \mathrm{O}, 10\right), 169$ (30), $117(100) . \quad$ HRMS $\left(\mathrm{ESI}^{+}\right)$: $\mathrm{m} / \mathrm{z}$ calculated for $\left[\mathrm{C}_{15} \mathrm{H}_{20} \mathrm{O}-\mathrm{H}_{2} \mathrm{O}+\mathrm{H}\right]^{+}$199.1487; found $199.1485\left[\mathrm{M}-\mathrm{H}_{2} \mathrm{O}+\mathrm{H}\right]^{+}$.

\section{Synthesis and characterization of products 3a-3n.}
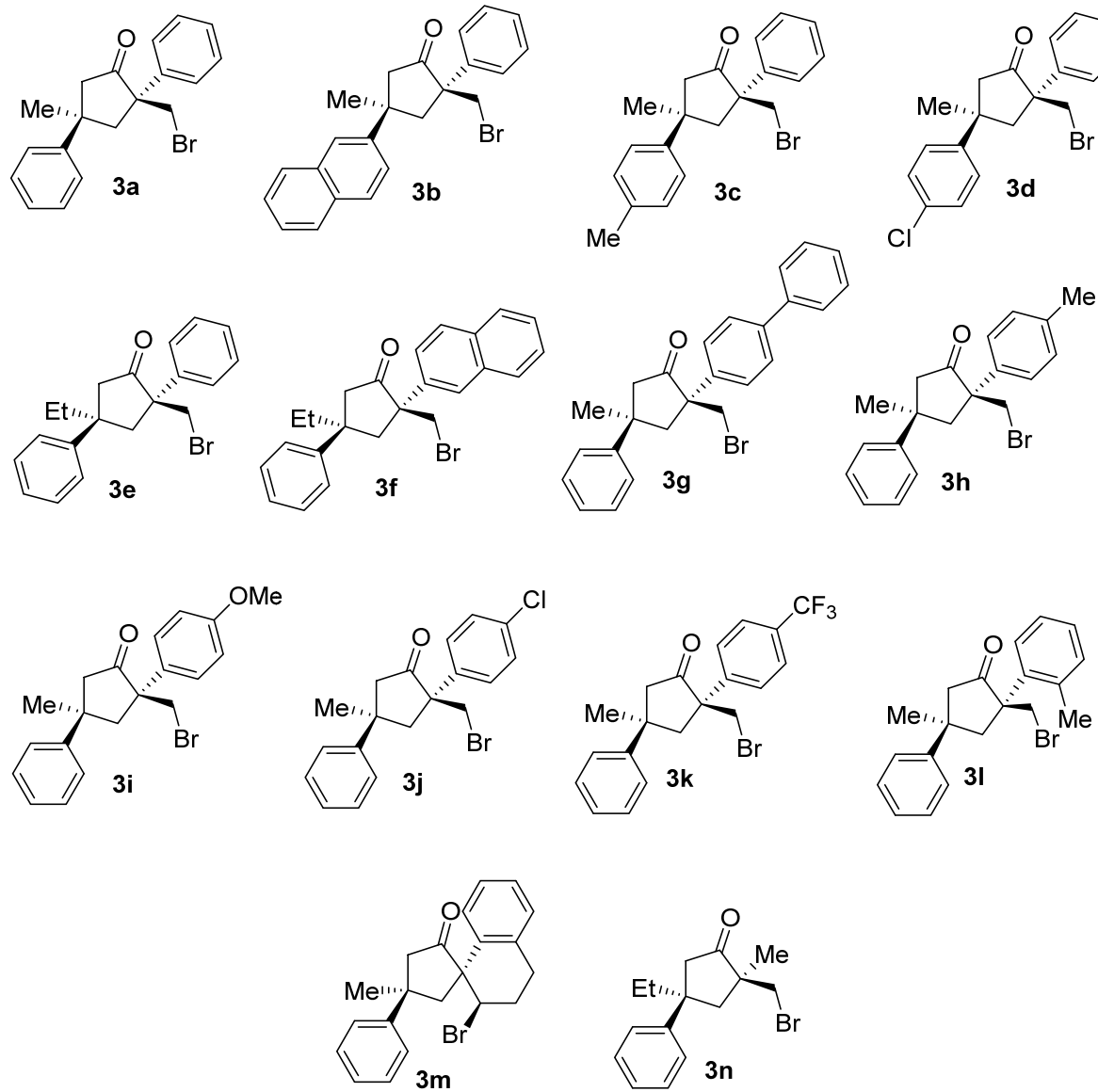

Figure S 5. Synthetized d 2,2,3,3-tetrasubstetutedcyclopentanones 3a-3n.

All compounds were prepared according to the general procedure F.

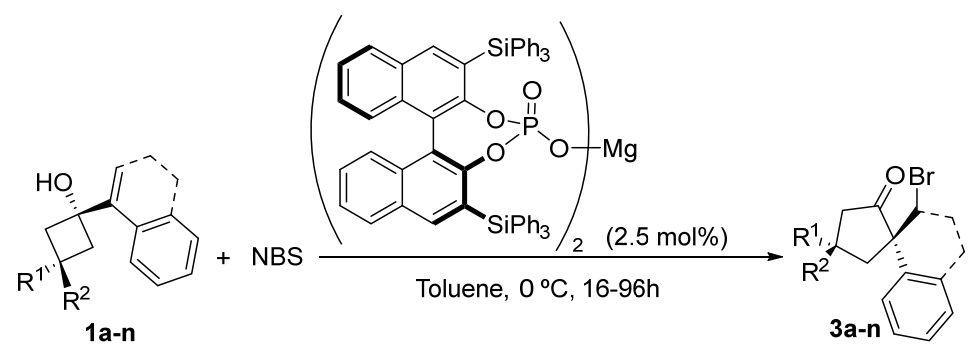

Scheme S 8. General procedure for the synthesis of 2,2,3,3-tetrasubstitutedcyclopentanones 3a-3n.

General procedure F for the synthesis of 2,2,3,3-tetrasubstitutedcyclopentanones.

To a cold solution, $0^{\circ} \mathrm{C}$, of $(1 r, 3 r)$-3-methyl-3-phenyl-1-(1-phenylvinyl)cyclobutan-1-ol $1 \mathrm{a}(26.4 \mathrm{mg}, 0.1 \mathrm{mmol})$ and magnesium 2,6-bis(triphenylsilyl)dinaphtho[2,1-d:1',2'f][1,3,2]dioxaphosphepin-4-olate 4-oxide $\mathbf{2 b}$ (4.3 $\mathrm{mg} ; 0.0025 \mathrm{mmol})$ in toluene $(0.50 \mathrm{~mL})$ 
under Ar atmosphere, $N$-bromosuccinimide (1.1 equiv., $19.6 \mathrm{mg}, 0.11 \mathrm{mmol}$ ) was added in one portion. The mixture was let stirring at $0{ }^{\circ} \mathrm{C}$ for $16-96 \mathrm{~h}$ until full conversion by TLC. Once the reaction was finished, aq. std. $\mathrm{Na}_{2} \mathrm{~S}_{2} \mathrm{O}_{3}(1 \mathrm{~mL})$ was added, the layers were separated and the aqueous layer was extracted with $\mathrm{Et}_{2} \mathrm{O}(3 \times 2 \mathrm{~mL})$, dried with $\mathrm{Na}_{2} \mathrm{SO}_{4}$, filtered and concentrated under vacuum. The crude was purified by a short plug of silica gel column using PE:EtOAc 9:1.

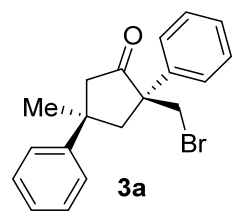

(2R-4R)-2-(bromomethyl)-4-methyl-2,4-diphenylcyclopentan-1-one 3a

Following the general procedure F, 3a was isolated as a colorless oil $(32.7 \mathrm{mg}$; 0.095 mmol; $95 \%$; dr $>20: 1 ; 92 \%$ ee) by flash chromatography (PE:EtOAc 9:1), starting from 3-methyl-3-phenyl-1-(1-phenylvinyl)cyclobutan-1-ol (26.4 mg; $0.10 \mathrm{mmol})$ and $\mathbf{2 b}$ (4.3 $\mathrm{mg} ; 0.0025 \mathrm{mmol})$ in toluene $(0.05 \mathrm{mmol})$ and NBS (19.6 mg; $0.11 \mathrm{mmol})$. The enantiomeric excess of product was determined by HPLC using Chiralpack OD-3 column [Hexane: $i \operatorname{PrOH} 99: 1$; flow $=1 \mathrm{~mL} / \mathrm{min}$; $\operatorname{tr}_{\text {major }}=13.1 \mathrm{~min}$; $\operatorname{tr}_{\text {minor }}=20.4 \mathrm{~min}$. $] .[\boldsymbol{\alpha}]_{\mathrm{D}}{ }^{25}=$ $+21.1\left(\mathrm{c}=0.5, \mathrm{CHCl}_{3}\right) . \mathbf{R f}=0.36(\mathrm{PE}: \mathrm{EtOAc}=95: 5){ }^{\mathbf{1}} \mathbf{H} \mathbf{~ N M R}\left(300 \mathrm{MHz}, \mathrm{CDCl}_{3}\right) \delta$ 7.61-7.55 (m, 2H, CArom-H), 7.46-7.22 (m, 8H, CArom-H), $3.64(\mathrm{~d}, J=10.1 \mathrm{~Hz}, 1 \mathrm{H}$, $\left.\mathrm{CH}_{\mathrm{a}} \mathrm{HbBr}\right), 3.47\left(\mathrm{~d}, J=10.1 \mathrm{~Hz}, 1 \mathrm{H}, \mathrm{CH}_{\mathrm{a}} \mathbf{H}_{\mathrm{b}} \mathrm{Br}\right), 3.09$ (d, $\left.J=13.6 \mathrm{~Hz}, 1 \mathrm{H}, \mathrm{C}^{1} \mathbf{H}_{\mathbf{a}} \mathrm{Hb}_{\mathrm{b}}\right), 3.03$ $\left(\mathrm{dd}, J=13.6,1.7 \mathrm{~Hz}, 1 \mathrm{H}, \mathrm{C}^{1} \mathrm{H}_{\mathrm{a}} \mathbf{H}_{\mathrm{b}}\right), 2.90$ (d, $\left.J=18.3 \mathrm{~Hz}, 1 \mathrm{H}, \mathrm{C}^{2} \mathbf{H}_{\mathrm{a}} \mathrm{H}_{\mathrm{b}}\right), 2.73$ (dd, $J=18.3$, $\left.1.7 \mathrm{~Hz}, 1 \mathrm{H}, \mathrm{C}^{2} \mathrm{H}_{\mathrm{a}} \mathbf{H}_{\mathbf{b}}\right), 1.12\left(\mathrm{~s}, 3 \mathrm{H}, \mathrm{CH}_{3}\right) .{ }^{13} \mathbf{C}\left\{{ }^{1} \mathbf{H}\right\}$ NMR $\left(75.5 \mathrm{MHz}, \mathrm{CDCl}_{3}\right) \delta 216.2$

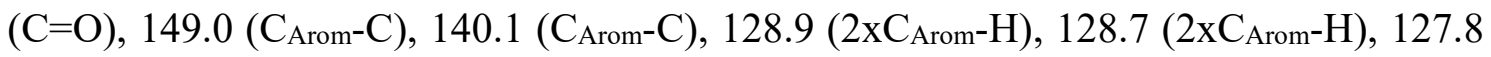
$\left(\mathrm{C}_{\text {Arom }}-\mathrm{H}\right), 126.6\left(2 \times \mathrm{C}_{\text {Arom }}-\mathrm{H}\right), 126.4\left(\mathrm{C}_{\text {Arom }}-\mathrm{H}\right), 125.3\left(2 \mathrm{x}_{\text {Arom }}-\mathrm{H}\right), 57.9\left(\mathbf{C}-\mathrm{CH}_{2} \mathrm{Br}\right), 52.9$ $\left(\mathrm{C}^{1} \mathrm{H}_{2}\right), 45.6\left(\mathrm{C}^{2} \mathrm{H}_{2}\right), 41.1\left(\mathrm{CCH}_{2}\right), 40.9\left(\mathrm{CH}_{2} \mathrm{Br}\right), 31.2\left(\mathrm{CH}_{3}\right)$. IR (ATR) $\mathrm{cm}^{-1}: 3020(=\mathrm{C}-$ H), $2958(\mathrm{C}-\mathrm{H}), 1739$ (C=O), 1643 (C=C). MS (EI) e/z (\%): 263.2 (34), 205.1 (25), 145.1 (39), 117.1 (100). HRMS (ESI $\left.{ }^{+}\right): \mathrm{m} / \mathrm{z}$ calculated for $\left[\mathrm{C}_{19} \mathrm{H}_{19} \mathrm{OBr}+\mathrm{Na}\right]^{+} 365.0517$; found $365.0507[\mathrm{M}+\mathrm{Na}]^{+}$.

Bigger scale: Following the general procedure F, 3a was isolated as a colorless oil (336.2 $\mathrm{mg} ; 0.98 \mathrm{mmol} ; 98 \%$; dr>20:1; $92 \%$ ee) starting from 3-methyl-3-phenyl-1-(1phenylvinyl)cyclobutan-1-ol (264 mg; $1.00 \mathrm{mmol})$ and $\mathbf{2 b}(43 \mathrm{mg} ; 0.025 \mathrm{mmol})$ in toluene (0.5 mmol) and NBS (196 mg; $1.10 \mathrm{mmol})$. 


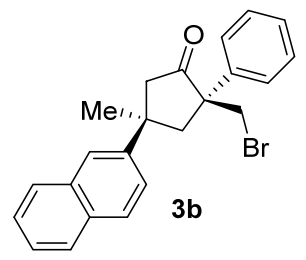

(2R,4R)-2-(bromomethyl)-4-methyl-4-(naphthalen-1-yl)-2-phenylcyclopentan-1-one 3b

Following the general procedure F, $\mathbf{3 b}$ was isolated as a white solid (35.4 mg; 0.090 mmol; $90 \%$; dr>20:1; $92 \%$ ee) by flash chromatography (PE:EtOAc 95:5), starting from 3-methyl-3-(naphthalen-1-yl)-1-(1-phenylvinyl)cyclobutan-1-ol $1 \mathbf{1 b} \quad(31.4 \mathrm{mg} ; 0.10$ $\mathrm{mmol})$ and $\mathbf{2 b}(4.3 \mathrm{mg} ; 0.0025 \mathrm{mmol})$ in toluene $(0.50 \mathrm{~mL})$ and NBS $(19.6 \mathrm{mg} ; .0 .11$ mmol). The enantiomeric excess of product was determined by HPLC using Chiralpack OD-3 column [Hexane: $i \operatorname{PrOH} 99: 1$; flow $=1 \mathrm{~mL} / \mathrm{min}$.; $\operatorname{tr}_{\text {major }}=12.1 \mathrm{~min}$.; $\operatorname{tr}_{\text {minor }}=14.4$ $\min .] .[\alpha]_{\mathrm{D}}{ }^{24}=+27.6\left(\mathrm{c}=0.4, \mathrm{CHCl}_{3}\right) . \mathbf{R f}=0.43(\mathrm{PE}: \mathrm{EtOAc}=95: 5) . \mathbf{M . p .}=115-118$ ${ }^{\circ} \mathrm{C}^{1}{ }^{1} \mathrm{H}$ NMR $\left(300 \mathrm{MHz}, \mathrm{CDCl}_{3}\right) \delta 7.92-7.81\left(\mathrm{~m}, 3 \mathrm{H}, \mathrm{C}_{\text {Arom- }} \mathrm{H}\right), 7.76(\mathrm{~d}, J=1.8 \mathrm{~Hz}, 1 \mathrm{H}$, $\left.\mathrm{C}_{\text {Arom-H}} \mathrm{H}\right), 7.65-7.58\left(\mathrm{~m}, 2 \mathrm{H}, \mathrm{C}_{\text {Arom }}-\mathrm{H}\right), 7.58$ - 7.47 (m, 3H, $\left.\mathrm{C}_{\text {Arom }}-\mathrm{H}\right), 7.46$ - 7.37 (m, $\left.2 \mathrm{H}, \mathrm{C}_{\text {Arom- }} \mathrm{H}\right), 7.34\left(\mathrm{dt}, J=5.0,2.0 \mathrm{~Hz}, 1 \mathrm{H}, \mathrm{C}_{\text {Arom }}-\mathrm{H}\right), 3.65\left(\mathrm{~d}, J=10.1 \mathrm{~Hz}, 1 \mathrm{H}, \mathrm{CH}_{\mathrm{a}} \mathrm{Hb}_{\mathrm{b}} \mathrm{Br}\right)$, $3.49\left(\mathrm{~d}, J=10.1 \mathrm{~Hz}, 1 \mathrm{H}, \mathrm{CH}_{\mathbf{a}} \mathbf{H}_{\mathbf{b}} \mathrm{Br}\right), 3.21\left(\mathrm{~d}, J=13.7 \mathrm{~Hz}, 1 \mathrm{H}, \mathrm{C}^{1} \mathbf{H}_{\mathbf{a}} \mathrm{H}_{\mathrm{b}}\right), 3.12$ (dd, $J=$ 13.7, $\left.1.9 \mathrm{~Hz}, 1 \mathrm{H}, \mathrm{C}^{1} \mathrm{H}_{\mathrm{a}} \mathbf{H}_{\mathbf{b}}\right), 3.02\left(\mathrm{~d}, J=18.3 \mathrm{~Hz}, 1 \mathrm{H}, \mathrm{C}^{2} \mathbf{H}_{\mathrm{a}} \mathrm{H}_{\mathrm{b}}\right), 2.83$ (dd, $J=18.3,1.9 \mathrm{~Hz}$, $\left.1 \mathrm{H}, \mathrm{C}^{2} \mathrm{H}_{\mathrm{a}} \mathbf{H}_{\mathbf{b}}\right), 1.22\left(\mathrm{~s}, 3 \mathrm{H}, \mathrm{CH}_{3}\right) .{ }^{13} \mathbf{C}\left\{{ }^{1} \mathbf{H}\right\} \mathbf{N M R}\left(75.5 \mathrm{MHz}, \mathrm{CDCl}_{3}\right) \delta 216.07(\mathrm{C}=\mathrm{O})$, 146.18 (C $\left.\mathrm{C}_{\text {Arom-C }}\right), 140.13$ ( $\mathrm{C}_{\text {Arom-C }}$ ), 133.30 (C $\left.\mathrm{C}_{\text {Arom-C }}\right), 132.05$ ( $\left.\mathrm{C}_{\text {Arom- }}-\mathrm{C}\right), 128.94$ $\left(2 \times C_{\text {Arom }}-\mathrm{H}\right), 128.56\left(\mathrm{C}_{\text {Arom }}-\mathrm{H}\right), 127.92\left(\mathrm{C}_{\text {Arom }}-\mathrm{H}\right), 127.78\left(\mathrm{C}_{\text {Arom }}-\mathrm{H}\right), 127.51\left(\mathrm{C}_{\text {Arom }}-\mathrm{H}\right)$, 126.66 (2x $\left.\mathrm{C}_{\text {Arom }}-\mathrm{H}\right), 126.33\left(\mathrm{C}_{\text {Arom }}-\mathrm{H}\right), 125.84\left(\mathrm{C}_{\text {Arom }}-\mathrm{H}\right), 124.31\left(\mathrm{C}_{\text {Arom }}-\mathrm{H}\right), 123.30$ $\left(\mathrm{C}_{\text {Arom-H}} \mathrm{H}\right), 58.03\left(\mathrm{CCH}_{2} \mathrm{Br}\right), 52.96\left(\mathrm{C}^{1} \mathrm{H}_{2}\right), 45.52\left(\mathrm{C}^{2} \mathrm{H}_{2}\right), 41.20\left(\mathrm{CH}_{2}\right), 40.90\left(\mathrm{CH}_{2} \mathrm{Br}\right)$, $31.07\left(\mathrm{CH}_{3}\right)$. IR (ATR) cm ${ }^{-1}: 3022(=\mathrm{C}-\mathrm{H}), 2956(\mathrm{C}-\mathrm{H}), 1739(\mathrm{C}=\mathrm{O}), 1456\left(\mathrm{CH}_{2}\right), 1446$ $\left(\mathrm{CH}_{3}\right)$. MS (EI) m/z (\%): $392.1\left(\mathrm{M}^{+}, 4\right), 195.1$ (55), 168.1 (88), 152.1 (75), 128.1 (59), 117.1 (100). HRMS $\left(\mathrm{ESI}^{+}\right): \mathrm{m} / \mathrm{z}$ calculated for $\left[\mathrm{C}_{23} \mathrm{H}_{21} \mathrm{OBr}+\mathrm{Na}\right]^{+} 415.0673$; found $415.0669[\mathrm{M}+\mathrm{Na}]^{+}$.

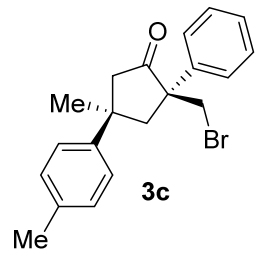

$(2 R, 4 R)$-2-(bromomethyl)-4-methyl-2-phenyl-4-(p-tolyl)cyclopentan-1-one 3c

Following the general procedure F, 3c was isolated as a yellowish oil (31 mg; $0.09 \mathrm{mmol}$; $87 \%$; dr $>20: 1 ; 92 \%$ ee) by flash chromatography $(\mathrm{PE}:$ EtOAc $=95: 5)$, starting from 3methyl-1-(1-phenylvinyl)-3-(p-tolyl)cyclobutan-1-ol 1c $(27.8 \mathrm{mg} ; 0.10 \mathrm{mmol})$ and $\mathbf{2 b}$ 
(4.3 $\mathrm{mg} ; 0.0025 \mathrm{mmol})$ in toluene $(0.5 \mathrm{~mL})$ and NBS $(19.6 \mathrm{mg} ; 0.11 \mathrm{mmol})$. The enantiomeric excess of product was determined by HPLC using Chiralpack OD3 column [Hexane: $i \operatorname{PrOH} 99: 1$; flow $=1 \mathrm{~mL} / \mathrm{min}$; $\operatorname{tr}_{\text {minor }}=9.9$ min.; $\operatorname{tr}_{\text {major }}=11.6 \mathrm{~min}$.]. $[\boldsymbol{\alpha}]_{\mathrm{D}}{ }^{20}=$ $+30.2\left(c=1, \mathrm{CHCl}_{3}\right) . \mathbf{R f}=0.59(\mathrm{PE}: \mathrm{EtAcO}=95: 5) .{ }^{1} \mathbf{H} \mathbf{~ N M R}\left(300 \mathrm{MHz}, \mathrm{CDCl}_{3}\right) \delta 7.52-$ 7.46 (m, 2H, C $\left.\mathrm{C}_{\text {Arom- }} \mathrm{H}\right), 7.34-7.26$ (m, 2H, $\left.\mathrm{C}_{\text {Arom- }} \mathrm{H}\right), 7.26-7.21$ (m, 1H, $\mathrm{C}_{\text {Arom-H) }}$, 7.217.14 (m, 2H, C $\left.\mathrm{C}_{\text {Arom }}-\mathrm{H}\right), 7.14-7.07$ (m, 2H, $\left.\mathrm{C}_{\text {Arom- }} \mathrm{H}\right), 3.54$ (d, J=10.1 Hz, 1H, $\mathrm{CH}_{\mathrm{a}} \mathrm{Hb}_{\mathrm{b}} \mathrm{Br}$ ), $3.37\left(\mathrm{~d}, J=10.1 \mathrm{~Hz}, 1 \mathrm{H}, \mathrm{CH}_{\mathbf{a}} \mathbf{H}_{\mathbf{b}} \mathrm{Br}\right), 2.98\left(\mathrm{~d}, J=13.6 \mathrm{~Hz}, 1 \mathrm{H}, \mathrm{C}^{1} \mathbf{H}_{\mathbf{a}} \mathrm{H}_{\mathrm{b}}\right), 2.91(\mathrm{dd}, J=$ 13.6, 1.7 Hz, 1H, $\left.\mathrm{C}^{1} \mathrm{H}_{\mathrm{a}} \mathbf{H}_{\mathbf{b}}\right), 2.79$ (d, $\left.J=18.2 \mathrm{~Hz}, 1 \mathrm{H}, \mathrm{C}^{2} \mathbf{H}_{\mathrm{a}} \mathrm{H}_{\mathrm{b}}\right), 2.62(\mathrm{dd}, J=18.2 \mathrm{~Hz}, 1 \mathrm{H}$, $\left.\mathrm{C}^{2} \mathrm{H}_{\mathrm{a}} \mathbf{H}_{\mathbf{b}}\right), 2.27\left(\mathrm{~s}, 3 \mathrm{H}, \mathrm{CH}_{3}-\mathrm{C}_{\mathrm{Ar}}\right), 1.03\left(\mathrm{~s}, 3 \mathrm{H}, \mathrm{CH}_{3}\right) .{ }^{13} \mathbf{C}\left\{{ }^{1} \mathbf{H}\right\} \mathbf{N M R}\left(75.5 \mathrm{MHz}, \mathrm{CDCl}_{3}\right) \delta$

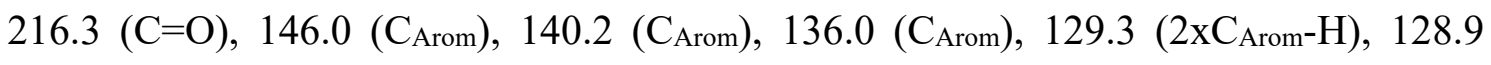
$\left(2 \mathrm{xC}_{\text {Arom }}-\mathrm{H}\right), 127.7\left(\mathrm{C}_{\text {Arom }}-\mathrm{H}\right), 126.6\left(2 \mathrm{xC}_{\text {Arom }}-\mathrm{H}\right), 125.2\left(2 \mathrm{xC}_{\text {Arom }}-\mathrm{H}\right), 58.0\left(\mathbf{C}-\mathrm{CH}_{2} \mathrm{Br}\right)$, $53.1\left(\mathrm{C}^{1} \mathrm{H}_{2}\right), 45.6\left(\mathrm{C}^{2} \mathrm{H}_{2}\right), 40.9\left(\mathrm{CH}_{2} \mathrm{Br}\right), 40.7\left(\mathbf{C C H}_{3}\right), 31.3\left(\mathrm{CCH}_{3}\right), 20.9\left(\mathrm{CH}_{3}\right) . \mathbf{I R}$ (ATR) $\mathrm{cm}^{-1}: 1739(\mathrm{C}=\mathrm{O})$. MS (EI) e/z (\%): 277.1 (12), 132 (43), 115 (100). HRMS $\left(\mathrm{ESI}^{+}\right): \mathrm{m} / \mathrm{z}$ calculated for $\left[\mathrm{C}_{20} \mathrm{H}_{21} \mathrm{OBr}+\mathrm{Na}\right]^{+} 379.0673$; found $379.0672[\mathrm{M}+\mathrm{Na}]^{+}$.

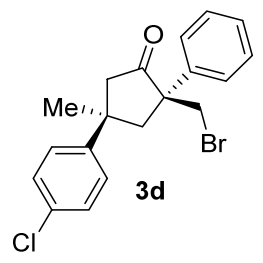

$(2 R, 4 R)$-2-(bromomethyl)-4-(4-chlorophenyl)-4-methyl-2-phenylcyclopentan-1-one 3d Following the general procedure F, 3d was isolated as a yellowish solid (35 mg; 0.09 mmol; $92 \%$; dr $>20: 1 ; 94 \%$ ee) by FC (PE:EtOAc = 95:5), starting from $(1 r, 3 r)-3-(4-$ chlorophenyl)-3-methyl-1-(1-phenylvinyl)cyclobutan-1-ol 3d (29.9 mg; $0.10 \mathrm{mmol})$ and 2b (4.3 mg; $0.0025 \mathrm{mmol})$ in toluene $(0.5 \mathrm{~mL})$ and NBS (19.6 mg; $0.11 \mathrm{mmol})$. The enantiomeric excess of product was determined by HPLC using Chiralpack OD3 column [Hexane: $i \operatorname{PrOH} 99: 1$; flow $=1 \mathrm{~mL} / \mathrm{min}$; $\operatorname{tr}_{\text {minor }}=14.9 \mathrm{~min}$.; $\operatorname{tr}_{\text {major }}=15.9 \mathrm{~min}$.]. $[\boldsymbol{\alpha}]_{\mathrm{D}}{ }^{20}=$ $+23.2\left(c=1, \mathrm{CHCl}_{3}\right) . \mathbf{R f}=0.56(\mathrm{PE}: \mathrm{EtAcO}=95: 5)$. M.p. $=97.3-98.6 .{ }^{\mathbf{1}} \mathbf{H}$ NMR $(300$ $\left.\mathrm{MHz} \mathrm{CDCl}_{3}\right) \delta 7.59-7.52\left(\mathrm{~m}, 2 \mathrm{H}, \mathrm{C}_{\text {Arom- }} \mathrm{H}\right), 7.43-7.25\left(\mathrm{~m}, 7 \mathrm{H}, \mathrm{C}_{\text {Arom }}-\mathrm{H}\right), 3.65(\mathrm{~d}, J=$ $\left.10.1 \mathrm{~Hz}, 1 \mathrm{H}, \mathrm{CH}_{\mathrm{a}} \mathrm{H}_{\mathrm{b} B r}\right), 3.45$ (d, $\left.J=10.1 \mathrm{~Hz}, 1 \mathrm{H}, \mathrm{CH}_{\mathrm{a}} \mathbf{H}_{\mathrm{b}} \mathrm{Br}\right), 3.05$ (d, $J=13.6 \mathrm{~Hz}, 1 \mathrm{H}$, $\left.\mathrm{C}^{1} \mathbf{H}_{\mathrm{a}} \mathrm{H}_{\mathrm{b}}\right), 2.99\left(\mathrm{~d}, J=13.6 \mathrm{~Hz}, 1 \mathrm{H}, \mathrm{C}^{1} \mathrm{H}_{\mathrm{a}} \mathbf{H}_{\mathrm{b}}\right), 2.83\left(\mathrm{~d}, J=18.2 \mathrm{~Hz}, 1 \mathrm{H}, \mathrm{C}^{2} \mathbf{H}_{\mathbf{a}} \mathrm{H}_{\mathrm{b}}\right), 2.70$ (dd, $\left.J=18.2,1.4 \mathrm{~Hz}, 1 \mathrm{H}, \mathrm{C}^{2} \mathrm{H}_{\mathrm{a}} \mathbf{H}_{\mathbf{b}}\right), 1.09\left(\mathrm{~s}, 3 \mathrm{H}, \mathrm{CH}_{3}\right) .{ }^{13} \mathbf{C}\left\{{ }^{1} \mathbf{H}\right\} \mathbf{N M R}\left(75.5 \mathrm{MHz}, \mathrm{CDCl}_{3}\right) \delta$ $215.7(\mathrm{C}=\mathrm{O}), 147.6\left(\mathrm{C}_{\text {Arom-C }} \mathrm{C}\right), 139.9$ (CArom-C), 132.2 (C $\left.\mathrm{C}_{\text {Arom-C }}\right), 129.0(2 \mathrm{xC}$ Arom-H), 128.8 (2xCArom-H), $127.9\left(\mathrm{C}_{\text {Arom }}-\mathrm{H}\right), 126.8$ (2xC Arom-H), $126.6\left(2 \times \mathrm{C}_{\text {Arom }}-\mathrm{H}\right), 58.0(\mathbf{C}$ $\left.\mathrm{CH}_{2} \mathrm{Br}\right), 52.9\left(\mathrm{C}^{1} \mathrm{H}_{2}\right), 45.5\left(\mathrm{C}^{2} \mathrm{H}_{2}\right), 40.8\left(\mathbf{C}-\mathrm{CH}_{3}\right), 40.7\left(\mathrm{CH}_{2} \mathrm{Br}\right), 31.1\left(\mathrm{CH}_{3}\right)$. IR (ATR) 
$\mathrm{cm}^{-1}: 1741(\mathrm{C}=\mathrm{O}), 1096\left(\mathrm{C}_{\mathrm{Ar}}-\mathrm{Cl}\right) . \mathbf{M S}(\mathrm{EI}) \mathrm{e} / \mathrm{z}(\%): 297.1$ (6), 152 (41), 115 (100). HRMS $\left(\mathrm{ESI}^{+}\right): \mathrm{m} / \mathrm{z}$ calculated for $\left[\mathrm{C}_{19} \mathrm{H}_{18} \mathrm{OClBr}+\mathrm{Na}\right]^{+}$399.0127; found $399.0116[\mathrm{M}+\mathrm{Na}]^{+}$.

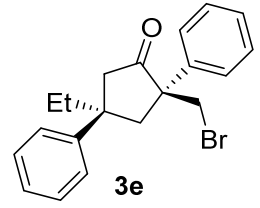

(2R,4R)-2-(bromomethyl)-4-ethyl-2,4-diphenylcyclopentan-1-one 3e

Following the general procedure F, 3e was isolated as a colorless oil ( $30 \mathrm{mg}$; $0.084 \mathrm{mmol}$; $84 \%$; dr>20:1; $93 \%$ ee) starting from 3-ethyl-3-phenyl-1-(1-phenylvinyl)cyclobutan-1ol $1 \mathbf{e}(27.8 \mathrm{mg} ; 0.10 \mathrm{mmol})$ and $\mathbf{2 b}(4.3 \mathrm{mg} ; 0.0025 \mathrm{mmol})$ in toluene $(0.50 \mathrm{~mL})$ and NBS $(19.6 \mathrm{mg} ; 0.11 \mathrm{mmol})$. The enantiomeric excess of product was determined by HPLC using Chiralpack OD-3 column [Hexane: $i \mathrm{PrOH} 99: 1$; flow $=1 \mathrm{~mL} / \mathrm{min}$; $\operatorname{tr}_{\text {minor }}=11.7$ $\min$; $\left.\operatorname{tr}_{\text {major }}=18.0 \mathrm{~min}.\right] .[\alpha]_{\mathrm{D}}{ }^{24}=+5.2\left(\mathrm{c}=0.5, \mathrm{CHCl}_{3}\right) . \mathbf{R f}=0.48(\mathrm{PE}: \operatorname{EtOAc}=95: 5)$. ${ }^{1}$ H NMR (300 MHz, CDCl 3 ) $\delta 7.61-7.54$ (m, 2H, CArom-H), 7.37 (m, 5H, CArom-H), 7.27 (m, 3H, CArom-H), $3.50\left(\mathrm{~d}, J=10.2 \mathrm{~Hz}, 1 \mathrm{H}, \mathrm{CH}_{\mathrm{a}} \mathrm{HbBr}\right), 3.44(\mathrm{~d}, J=10.2 \mathrm{~Hz}, 1 \mathrm{H}$, $\left.\mathrm{CH}_{\mathrm{a}} \mathbf{H}_{\mathbf{b}} \mathrm{Br}\right) 3.10\left(\mathrm{dd}, J=13.7,1.7 \mathrm{~Hz}, 1 \mathrm{H}, \mathrm{C}^{1} \mathbf{H}_{\mathbf{a}} \mathrm{Hb}\right), 3.00\left(\mathrm{~d}, J=13.7 \mathrm{~Hz}, 1 \mathrm{H}, \mathrm{C}^{1} \mathrm{H}_{\mathrm{a}} \mathbf{H}_{\mathbf{b}}\right)$, $2.90\left(\mathrm{~d}, J=18.1 \mathrm{~Hz}, 1 \mathrm{H}, \mathrm{C}^{2} \mathbf{H}_{\mathrm{a}} \mathrm{H}_{\mathrm{b}}\right), 2.78\left(\mathrm{dd}, J=18.0,1.7 \mathrm{~Hz}, 1 \mathrm{H}, \mathrm{C}^{2} \mathrm{H}_{\mathrm{a}} \mathbf{H}_{\mathbf{b}}\right), 1.54-1.35$ $\left(\mathrm{m}, 2 \mathrm{H}, \mathbf{C H}_{2} \mathrm{CH}_{3}\right), 0.46\left(\mathrm{t}, J=7.3 \mathrm{~Hz}, 3 \mathrm{H}, \mathrm{CH}_{2} \mathbf{C H}_{3}\right) .{ }^{13} \mathbf{C}\left\{{ }^{1} \mathbf{H}\right\} \mathbf{N M R}\left(75.5 \mathrm{MHz}, \mathrm{CDCl}_{3}\right)$ $\delta 215.9(\mathrm{C}=\mathrm{O}), 146.1\left(\mathrm{C}_{\text {Arom }} \mathrm{C}\right), 140.0\left(\mathrm{C}_{\text {Arom }}-\mathrm{C}\right), 128.8\left(2 \mathrm{xC}_{\text {Arom }}-\mathrm{H}\right), 128.4\left(2 \mathrm{xC}_{\text {Arom }}-\mathrm{H}\right)$, 127.7 ( $\left.\mathrm{C}_{\text {Arom }}-\mathrm{H}\right), 126.6\left(2 \mathrm{xC}_{\text {Arom }}-\mathrm{H}\right), 126.4\left(\mathrm{C}_{\text {Arom }}-\mathrm{H}\right), 126.4\left(2 \mathrm{xC}_{\text {Arom }}\right), 57.3\left(\mathbf{C C H}_{2} \mathrm{Br}\right)$, $51.1\left(\mathrm{C}^{1} \mathrm{H}_{2}\right), 45.1\left(\mathbf{C C H}_{3}\right), 42.9\left(\mathrm{C}^{2} \mathrm{H}_{2}\right), 41.1\left(\mathrm{CH}_{2} \mathrm{Br}\right), 35.4\left(\mathbf{C H}_{2} \mathrm{CH}_{3}\right), 8.9\left(\mathrm{CH}_{2} \mathbf{C H}_{3}\right)$. IR (ATR) cm ${ }^{-1}: 3022$ (=C-H), $2962(\mathrm{C}-\mathrm{H}), 1738(\mathrm{C}=\mathrm{O}), 1636(\mathrm{C}=\mathrm{C}), 1465\left(\mathrm{CH}_{2}\right), 1446$ $\left(\mathrm{CH}_{3}\right) . \mathbf{M S}(\mathrm{EI}) \mathrm{m} / \mathrm{z}$ (\%): 277.2 (22), 235.2 (16), 159.1 (20), 131.1 (42), 117.1 (100). HRMS $\left(\mathrm{ESI}^{+}\right)$: $\mathrm{m} / \mathrm{z}$ calculated for $\left[\mathrm{C}_{20} \mathrm{H}_{21} \mathrm{OBrNa}\right]^{+} 379.0673$; found $379.0670[\mathrm{M}+\mathrm{Na}]^{+}$.

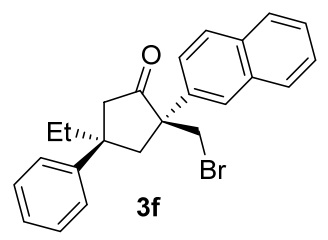

$(2 R, 4 R)$-2-(bromomethyl)-4-ethyl-2-(naphthalen-2-yl)-4-phenylcyclopentan-1-one $\mathbf{3 f}$ Following the general procedure F, $3 \mathbf{f}$ was isolated as a yellowish oil $(31 \mathrm{mg}$; $0.08 \mathrm{mmol}$; $76 \%$; dr $>20: 1 ; 92 \%$ ee) by FC (PE:EtOAc = 95:5), starting from 3-ethyl-1-(1(naphthalen-2-yl)vinyl)-3-phenylcyclobutan-1-ol $\mathbf{1 f}(32.8 \mathrm{mg} ; 0.10 \mathrm{mmol})$ and $\mathbf{2 b}(4.3$ $\mathrm{mg} ; 0.0025 \mathrm{mmol})$ in toluene $(0.5 \mathrm{~mL})$ and NBS $(19.6 \mathrm{mg} ; 0.11 \mathrm{mmol})$. The enantiomeric 
excess of product was determined by HPLC using Chiralpack IA column [Hexane: $i \mathrm{PrOH}$ 99:1; flow $=1 \mathrm{~mL} / \mathrm{min} ; \operatorname{tr}_{\text {minor }}=11.4 \mathrm{~min}$; $\left.\operatorname{tr}_{\text {major }}=14.4 \mathrm{~min}.\right] .[\alpha]_{\mathrm{D}}{ }^{20}=+35.1(c=1$, $\left.\mathrm{CHCl}_{3}\right) . \mathbf{R f}=0.47(\mathrm{PE}:$ EtOAc $=95: 5) .{ }^{1} \mathbf{H} \mathbf{N M R}\left(300 \mathrm{MHz}, \mathrm{CDCl}_{3}\right) \delta 7.94(\mathrm{~d}, J=1.8$ $\left.\mathrm{Hz}, 1 \mathrm{H}, \mathrm{C}_{\text {Arom- }} \mathrm{H}\right), 7.83-7.72\left(\mathrm{~m}, 3 \mathrm{H}, \mathrm{C}_{\text {Arom }}-\mathrm{H}\right), 7.61$ (dd, $\left.J=8.7,2.0 \mathrm{~Hz}, 1 \mathrm{H}, \mathrm{C}_{\text {Arom }}-\mathrm{H}\right)$, 7.46-7.38 (m, 2H, C Arom-H) $_{\text {A }}$ 7.34-7.26 (m, 2H, C $\left.\mathrm{C}_{\text {Arom }}-\mathrm{H}\right), 7.24-7.14$ (m, 3H, $\left.\mathrm{C}_{\text {Arom- }}-\mathrm{H}\right)$, $3.50\left(\mathrm{~d}, J=10.2 \mathrm{~Hz}, 1 \mathrm{H}, \mathrm{CH}_{\mathbf{a}} \mathrm{H}_{\mathrm{b}} \mathrm{Br}\right), 3.45\left(\mathrm{~d}, J=10.2 \mathrm{~Hz}, 1 \mathrm{H}, \mathrm{CH}_{\mathrm{a}} \mathbf{H}_{\mathbf{b}} \mathrm{Br}\right), 3.16$ (dd, $J=$ 13.7, 1.7 Hz, 1H, C $\left.{ }^{1} \mathbf{H}_{\mathbf{a}} \mathrm{H}_{\mathrm{b}}\right), 2.99$ (d, $\left.J=13.7 \mathrm{~Hz}, 1 \mathrm{H}, \mathrm{C}^{1} \mathrm{H}_{\mathbf{a}} \mathbf{H}_{\mathbf{b}}\right), 2.85$ (d, J=18.1 Hz, 1H, $\left.\mathrm{C}^{2} \mathbf{H}_{\mathrm{a}} \mathrm{Hb}_{\mathrm{b}}\right), 2.75\left(\mathrm{dd}, J=18.1,1.7 \mathrm{~Hz}, 1 \mathrm{H}, \mathrm{C}^{2} \mathrm{H}_{\mathbf{a}} \mathbf{H}_{\mathbf{b}}\right), 1.40(\mathrm{dq}, J=13.9,7.3 \mathrm{~Hz}, 1 \mathrm{H}$, $\left.\mathrm{CH}_{\mathbf{a}} \mathrm{H}_{\mathrm{b}} \mathrm{CH}_{3}\right), 1.30$ (dq, $\left.J=13.9,7.3 \mathrm{~Hz}, 1 \mathrm{H}, \mathrm{CH}_{\mathrm{a}} \mathbf{H}_{\mathbf{b}} \mathrm{CH}_{3}\right), 0.35$ (t, $\left.J=7.3 \mathrm{~Hz}, 3 \mathrm{H}, \mathrm{CH}_{3}\right)$. ${ }^{13} \mathbf{C}\left\{{ }^{1} \mathbf{H}\right\}$ NMR $\left(75.5 \mathrm{MHz}, \mathrm{CDCl}_{3}\right) \delta 216.0(\mathrm{C}=\mathrm{O}), 146.2\left(\mathrm{C}_{\text {Arom- }} \mathrm{C}\right), 137.3\left(\mathrm{C}_{\text {Arom- }} \mathrm{C}\right)$, $133.2\left(\mathrm{C}_{\text {Arom }}-\mathrm{C}\right), 132.5\left(\mathrm{C}_{\text {Arom }}-\mathrm{C}\right), 128.6\left(\mathrm{C}_{\text {Arom }}-\mathrm{H}\right), 128.4\left(2 \mathrm{xC}_{\text {Arom }}-\mathrm{H}\right), 128.3\left(\mathrm{C}_{\text {Arom }}-\mathrm{H}\right)$, $127.5\left(\mathrm{C}_{\text {Arom }}-\mathrm{H}\right), 126.5\left(\mathrm{C}_{\text {Arom }}-\mathrm{H}\right), 126.4\left(2 \mathrm{xC}_{\text {Arom }}-\mathrm{H}\right), 126.4\left(2 \mathrm{xC}_{\text {Arom }}-\mathrm{H}\right), 125.8\left(\mathrm{C}_{\text {Arom- }}\right.$ $\mathrm{H}), 124.3\left(\mathrm{C}_{\text {Arom-H}}-\mathrm{H}\right), 57.4\left(\mathbf{C}-\mathrm{CH}_{2} \mathrm{Br}\right), 51.1\left(\mathrm{C}^{1} \mathrm{H}_{2}\right), 45.2\left(\mathbf{C}-\mathrm{CH}_{2} \mathrm{CH}_{3}\right), 43.0\left(\mathrm{C}^{2} \mathrm{H}_{2}\right), 40.9$ $\left(\mathrm{CH}_{2} \mathrm{Br}\right), 35.4\left(\mathbf{C H}_{2} \mathrm{CH}_{3}\right), 8.9\left(\mathrm{CH}_{2} \mathbf{C H}_{3}\right)$. IR $(\mathrm{ATR}) \mathrm{cm}^{-1}: 1736(\mathrm{C}=\mathrm{O})$. MS (EI) e/z (\%): $408.1(\mathrm{M}+\mathrm{H}, \quad 7), 195.1$ (45), 165 (100). HRMS $\left(\mathrm{ESI}^{+}\right): \mathrm{m} / \mathrm{z}$ calculated for $\left[\mathrm{C}_{24} \mathrm{H}_{23} \mathrm{OBr}+\mathrm{Na}\right]^{+}$429.0830; found $429.0825[\mathrm{M}+\mathrm{Na}]^{+}$.

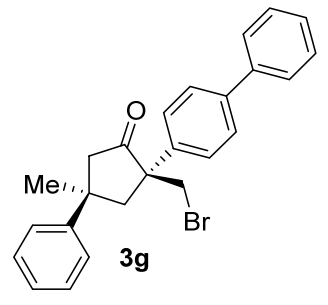

$(2 R, 4 R)-2-([1,1$ '-biphenyl]-4-yl)-2-(bromomethyl)-4-methyl-4-phenylcyclopentan-1-one $3 g$

Following the general procedure F, $\mathbf{3 g}$ was isolated as a amorphous white solid (41 $\mathrm{mg}$; $0.10 \mathrm{mmol} ; 98 \%$; dr>20:1; 92\% ee) by FC (PE:EtOAc = 95:5), starting from 1-(1-([1,1'biphenyl]-4-yl)vinyl)-3-methyl-3-phenylcyclobutan-1-ol 1 g (34 mg; $0.10 \mathrm{mmol}$ ) and 2b (4.3 $\mathrm{mg} ; 0.0025 \mathrm{mmol})$ in toluene $(0.5 \mathrm{~mL})$ and NBS (19.6 mg; $0.11 \mathrm{mmol})$. The enantiomeric excess of product was determined by HPLC using Chiralpack IA column [Hexane: $i \operatorname{PrOH} 99: 1$; flow $=1 \mathrm{~mL} / \mathrm{min}$; $\operatorname{tr}_{\text {minor }}=17.7 \mathrm{~min}$; $\operatorname{tr}_{\text {major }}=35.4 \mathrm{~min}$.]. $[\boldsymbol{\alpha}]_{\mathrm{D}}{ }^{20}=$ $+44.9\left(c=1, \mathrm{CHCl}_{3}\right) . \mathbf{R f}=0.37(\mathrm{PE}: \mathrm{EtAcO}=95: 5) .{ }^{1} \mathbf{H} \mathbf{~ N M R}\left(300 \mathrm{MHz}, \mathrm{CDCl}_{3}\right) \delta 7.71-$ $7.58\left(\mathrm{~m}, 6 \mathrm{H}, \mathrm{C}_{\text {Arom }}-\mathrm{H}\right), 7.52-7.30\left(\mathrm{~m}, 8 \mathrm{H}, \mathrm{C}_{\text {Arom }}-\mathrm{H}\right), 3.68\left(\mathrm{~d}, J=10.2 \mathrm{~Hz}, 1 \mathrm{H}, \mathrm{CH}_{\mathrm{a}} \mathrm{Hb}_{\mathrm{b}} \mathrm{Br}\right)$, $3.51\left(\mathrm{~d}, J=10.2 \mathrm{~Hz}, 1 \mathrm{H}, \mathrm{CH}_{\mathrm{a}} \mathbf{H}_{\mathbf{b}} \mathrm{Br}\right), 3.13\left(\mathrm{~d}, J=13.8 \mathrm{~Hz}, 1 \mathrm{H}, \mathrm{C}^{1} \mathbf{H}_{\mathbf{a}} \mathrm{H}_{\mathrm{b}}\right), 3.07$ (dd, $J=$ $\left.13.8 \mathrm{~Hz}, 1 \mathrm{H}, \mathrm{C}^{1} \mathrm{H}_{\mathbf{a}} \mathbf{H}_{\mathbf{b}}\right), 2.93$ (d, $\left.J=18.2 \mathrm{~Hz}, 1 \mathrm{H}, \mathrm{C}^{2} \mathbf{H}_{\mathbf{a}} \mathrm{H}_{\mathrm{b}}\right), 2.76(\mathrm{dd}, J=18.2 \mathrm{~Hz}, 1 \mathrm{H}$, $\left.\mathrm{C}^{2} \mathrm{H}_{\mathrm{a}} \mathbf{H}_{\mathbf{b}}\right), 1.18\left(\mathrm{~s}, 3 \mathrm{H}, \mathrm{CH}_{3}\right) .{ }^{13} \mathbf{C}\left\{{ }^{1} \mathbf{H}\right\}$ NMR (75.5 MHz, $\left.\mathrm{CDCl}_{3}\right) \delta 216.2(\mathrm{C}=\mathrm{O}), 149.0$ 


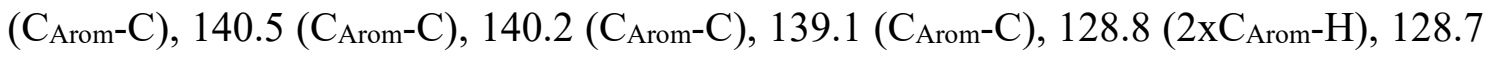
$\left(2 \mathrm{xC}_{\text {Arom- }}-\mathrm{H}\right), 127.6\left(\mathrm{C}_{\text {Arom- }} \mathrm{H}\right), 127.5\left(2 \mathrm{xC}_{\text {Arom }}-\mathrm{H}\right), 127.1\left(2 \mathrm{xC}_{\text {Arom }}-\mathrm{H}\right), 127.0\left(2 \mathrm{xC}_{\text {Arom- }}\right.$ $\mathrm{H}), 126.5\left(\mathrm{C}_{\text {Arom- }} \mathrm{H}\right), 125.4\left(2 \times \mathrm{C}_{\text {Arom }}-\mathrm{H}\right), 57.7\left(\mathbf{C}-\mathrm{CH}_{2} \mathrm{Br}\right), 53.0\left(\mathrm{C}^{1} \mathrm{H}_{2}\right), 45.5\left(\mathrm{C}^{2} \mathrm{H}_{2}\right), 41.1$ $\left(\mathrm{CCH}_{3}\right), 40.9\left(\mathrm{CH}_{2} \mathrm{Br}\right), 31.4\left(\mathrm{CH}_{3}\right)$ IR (ATR) cm ${ }^{-1}: 1738(\mathrm{C}=\mathrm{O})$. MS (EI) e/z (\%): 418.1 (M, 3), 338.1 (5), 221 (11), 178 (100). HRMS (ESI $\left.{ }^{+}\right): \mathrm{m} / \mathrm{z}$ calculated for $\left[\mathrm{C}_{25} \mathrm{H}_{23} \mathrm{OBr}+\mathrm{Na}\right]^{+}$441.0830; found $441.0830[\mathrm{M}+\mathrm{Na}]^{+}$.

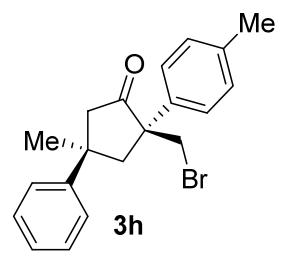

(2R,4R)-2-(bromomethyl)-4-methyl-4-phenyl-2-( $p$-tolyl)cyclopentan-1-one $\mathbf{3 h}$

Following the general procedure F, $\mathbf{3 h}$ was isolated as a colorless oil $(28.2 \mathrm{mg} ; 0.079$ mmol; $79 \%$;r $>20: 1 ; 93 \%$ ee) starting from 3-methyl-3-phenyl-1-(1-(ptolyl)vinyl)cyclobutan-1-ol $\mathbf{1 h}(27.8 \mathrm{mg} ; 0.10 \mathrm{mmol})$ and $\mathbf{2 b}(4.3 \mathrm{mg} ; 0.0025 \mathrm{mmol})$ in Toluene $(0.50 \mathrm{~mL})$ and NBS $(19.6 \mathrm{mg} ; 0.11 \mathrm{mmol})$. The enantiomeric excess of product was determined by HPLC using Chiralpack OD-3 column [Hexane: $i \mathrm{PrOH}$ 99:1; flow $=$ $1 \mathrm{~mL} / \mathrm{min}$; $\operatorname{tr}_{\text {major }}=12.0 \mathrm{~min}$; $\operatorname{tr}_{\text {minor }}=16.8 \mathrm{~min}$. $]$. $[\boldsymbol{\alpha}]_{\mathrm{D}}{ }^{25}=+11.9\left(\mathrm{c}=0.7, \mathrm{CHCl}_{3}\right) . \mathbf{R f}=$ 0.39 (PE:EtOAc = 95:5). ${ }^{1} \mathbf{H}$ NMR $\left(300 \mathrm{MHz} \mathrm{CDCl}_{3}\right) \delta 7.45\left(\mathrm{~d}, J=8.3 \mathrm{~Hz}, 2 \mathrm{H}, \mathrm{C}_{\text {Arom- }}{ }^{-}\right.$ $\mathrm{H}), 7.43-7.33\left(\mathrm{~m}, 4 \mathrm{H}, \mathrm{C}_{\text {Arom- }} \mathrm{H}\right), 7.31-7.23\left(\mathrm{~m}, 1 \mathrm{H}, \mathrm{C}_{\text {Arom }}-\mathrm{H}\right), 7.20(\mathrm{~d}, J=8.0 \mathrm{~Hz}, 2 \mathrm{H}$, $\left.\mathrm{C}_{\text {Arom-H}} \mathrm{H}\right), 3.63\left(\mathrm{~d}, J=10.1 \mathrm{~Hz}, 1 \mathrm{H}, \mathrm{CH}_{\mathbf{a}} \mathrm{H}_{\mathrm{b}} \mathrm{Br}\right), 3.45\left(\mathrm{~d}, J=10.1 \mathrm{~Hz}, 1 \mathrm{H}, \mathrm{CH}_{\mathrm{a}} \mathbf{H}_{\mathrm{b}} \mathrm{Br}\right), 3.07$ $\left(\mathrm{d}, J=13.6 \mathrm{~Hz}, 1 \mathrm{H}, \mathrm{C}^{1} \mathbf{H}_{\mathrm{a}} \mathrm{H}_{\mathrm{b}}\right), 3.01\left(\mathrm{dd}, J=13.6,1.7 \mathrm{~Hz}, 1 \mathrm{H}, \mathrm{C}^{1} \mathrm{H}_{\mathrm{a}} \mathbf{H}_{\mathbf{b}}\right), 2.88(\mathrm{~d}, J=18.3$ $\left.\mathrm{Hz}, 1 \mathrm{H}, \mathrm{C}^{2} \mathbf{H}_{\mathbf{a}} \mathrm{Hb}_{\mathrm{b}}\right), 2.72\left(\mathrm{dd}, J=18.3,1.7 \mathrm{~Hz}, 1 \mathrm{H}, \mathrm{C}^{2} \mathrm{H}_{\mathrm{a}} \mathbf{H}_{\mathbf{b}}\right), 2.36\left(\mathrm{~s}, 3 \mathrm{H}, \mathrm{C}_{\text {Arom- }}-\mathbf{C H}_{3}\right), 1.13$ $\left(\mathrm{s}, 3 \mathrm{H}, \mathrm{CH}_{3}\right) .{ }^{13} \mathbf{C}\left\{{ }^{1} \mathbf{H}\right\}$ NMR $\left(75.5 \mathrm{MHz}, \mathrm{CDCl}_{3}\right) \delta 215.4(\mathrm{C}=\mathrm{O}), 148.1\left(\mathrm{C}_{\mathrm{Arom}}-\mathrm{CH}_{3}\right)$, 136.5 (CArom-C), $136.0\left(\mathrm{C}_{\text {Arom-C }} \mathrm{C}\right), 128.6$ (2xCArom-H), 127.6 (2xCArom-H), 125.5 (C $\mathrm{C}_{\text {Arom- }}$ $\mathrm{H}), 125.4\left(2 \mathrm{xC}_{\text {Arom- }} \mathrm{H}\right), 124.3\left(2 \mathrm{xC}_{\text {Arom }}-\mathrm{H}\right), 56.7\left(\mathrm{CCH}_{2} \mathrm{Br}\right), 51.9\left(\mathrm{C}^{1} \mathrm{H}_{2}\right), 44.4\left(\mathrm{C}^{2} \mathrm{H}_{2}\right)$, $40.0\left(\mathbf{C C H}_{2}\right), 39.9\left(\mathrm{CH}_{2} \mathrm{Br}\right), 30.2\left(\mathrm{C}_{\mathrm{Arom}}-\mathbf{C H}_{3}\right), 19.9\left(\mathrm{CH}_{3}\right)$. IR (ATR) cm ${ }^{-1}: 3018(=\mathrm{C}-$ H), $2970(\mathrm{C}-\mathrm{H}), 1738(\mathrm{C}=\mathrm{O}), 1642$ (C=C). MS (EI) e/z (\%): 277.2 (26), 159.1 (22), 145.2 (22), 131.1 (100). HRMS $\left(\mathrm{ESI}^{+}\right): \mathrm{m} / \mathrm{z}$ calculated for $\left[\mathrm{C}_{20} \mathrm{H}_{21} \mathrm{OBr}+\mathrm{Na}\right]^{+}$379.0673; found $379.0673[\mathrm{M}+\mathrm{Na}]^{+}$. 


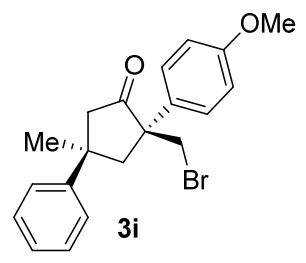

(2R,4R)-2-(bromomethyl)-2-(4-methoxyphenyl)-4-methyl-4-phenylcyclopentan-1-one $3 \mathbf{i}$

Following the general procedure F, 3i was isolated as a white solid (18.3 mg; $0.049 \mathrm{mmol}$; $49 \%$; dr>20:1; $80 \%$ ee) starting from 1-(1-(4-methoxyphenyl)vinyl)-3-methyl-3phenylcyclobutan-1-ol $\mathbf{1 i}(29.4 \mathrm{mg} ; 0.1 \mathrm{mmol})$ and $\mathbf{2 b}$ (4.3 mg; $0.0025 \mathrm{mmol})$ in toluene $(0.50 \mathrm{~mL})$ and NBS $(19.6 \mathrm{mg} ; 0.11 \mathrm{mmol})$. The enantiomeric excess of product was determined by HPLC using Chiralpack OD-3 column [Hexane: $i \mathrm{PrOH} 99: 1$; flow $=1$ $\mathrm{mL} / \mathrm{min}$; $\operatorname{tr}_{\text {major }}=24.5 \mathrm{~min}$; $\operatorname{tr}_{\text {minor }}=30.8 \mathrm{~min}$. $] .[\boldsymbol{\alpha}]_{\mathrm{D}}{ }^{25}=+13.2\left(\mathrm{c}=0.7, \mathrm{CHCl}_{3}\right) . \mathbf{R f}=$ 0.37 (PE:EtOAc = 95:5). M.p. $=86-88^{\circ} \mathrm{C} .{ }^{1} \mathbf{H}$ NMR $\left(300 \mathrm{MHz}, \mathrm{CDCl}_{3}\right) \delta 7.52-7.46$ (m, 2H, $\left.\mathrm{C}_{\text {Arom- }} \mathrm{H}\right), 7.41-7.34\left(\mathrm{~m}, 4 \mathrm{H}, \mathrm{C}_{\text {Arom }}-\mathrm{H}\right), 7.30-7.23\left(\mathrm{~m}, 1 \mathrm{H}, \mathrm{C}_{\text {Arom }}-\mathrm{H}\right), 6.96-$ $6.87\left(\mathrm{~m}, 2 \mathrm{H}, \mathrm{C}_{\text {Arom-H}} \mathrm{H}\right), 3.82\left(\mathrm{~s}, 3 \mathrm{H}, \mathrm{OCH}_{3}\right), 3.62\left(\mathrm{~d}, J=10.1 \mathrm{~Hz}, 1 \mathrm{H}, \mathrm{CH}_{\mathrm{a}} \mathrm{H}_{\mathrm{bBr}}\right), 3.43(\mathrm{~d}$, $\left.J=10.1 \mathrm{~Hz}, 1 \mathrm{H}, \mathrm{CH}_{\mathrm{a}} \mathbf{H}_{\mathrm{b}} \mathrm{Br}\right), 3.06\left(\mathrm{~d}, J=13.6 \mathrm{~Hz}, 1 \mathrm{H}, \mathrm{C}^{1} \mathbf{H}_{\mathbf{a}} \mathrm{H}_{\mathrm{b}}\right), 2.99(\mathrm{dd}, J=13.6,1.9$ $\left.\mathrm{Hz}, 1 \mathrm{H}, \mathrm{C}^{1} \mathrm{H}_{\mathrm{a}} \mathbf{H}_{\mathbf{b}}\right), 2.88\left(\mathrm{~d}, J=18.3 \mathrm{~Hz}, 1 \mathrm{H}, \mathrm{C}^{2} \mathbf{H}_{\mathbf{a}} \mathrm{H}_{\mathrm{b}}\right), 2.72(\mathrm{dd}, J=18.3,1.9 \mathrm{~Hz}, 1 \mathrm{H}$, $\left.\mathrm{C}^{2} \mathrm{H}_{\mathbf{a}} \mathbf{H}_{\mathbf{b}}\right), 1.13\left(\mathrm{~s}, 3 \mathrm{H}, \mathrm{CH}_{3}\right) .{ }^{13} \mathbf{C}\left\{{ }^{1} \mathbf{H}\right\}$ NMR (75.5 MHz, $\left.\mathrm{CDCl}_{3}\right) \delta 216.5(\mathrm{C}=\mathrm{O}), 159.0$ $\left(\mathrm{C}_{\text {Arom-OCH }}\right), 149.1$ ( $\left.\mathrm{C}_{\text {Arom }}-\mathrm{C}\right), 131.9\left(\mathrm{C}_{\text {Arom- }}-\mathrm{C}\right), 128.6\left(2 \mathrm{xC}_{\text {Arom }}-\mathrm{H}\right), 127.8\left(2 \mathrm{xC}_{\text {Arom }}-\mathrm{H}\right)$, $126.4\left(\mathrm{C}_{\text {Arom }}-\mathrm{H}\right), 125.3\left(2 \times \mathrm{C}_{\text {Arom }}-\mathrm{H}\right), 114.2\left(2 \times \mathrm{C}_{\text {Arom }}-\mathrm{H}\right), 57.3\left(\mathrm{CCH}_{2} \mathrm{Br}\right), 55.3\left(\mathrm{OCH}_{3}\right)$, $53.0\left(\mathrm{C}^{1} \mathrm{H}_{2}\right), 45.5\left(\mathrm{C}^{2} \mathrm{H}_{2}\right), 41.0\left(\mathrm{C}-\mathrm{CH}_{3}\right), 40.9\left(\mathrm{CH}_{2} \mathrm{Br}\right), 31.2\left(\mathrm{CH}_{3}\right)$. IR (ATR) $\mathrm{cm}^{-1}: 3020$ $(=\mathrm{C}-\mathrm{H}), 2961(\mathrm{C}-\mathrm{H}), 1737(\mathrm{C}=\mathrm{O}), 1641(\mathrm{C}=\mathrm{C}), 1472\left(\mathrm{CH}_{2}\right), 1456\left(\mathrm{CH}_{3}\right), 1253(\mathrm{C}-\mathrm{O})$. MS (EI) e/z (\%): 147.1 (69), 133.1 (56.0), 121.1 (90), 91.1 (100). HRMS (ESI ${ }^{+}$): m/z calculated for $\left[\mathrm{C}_{20} \mathrm{H}_{21} \mathrm{O}_{2} \mathrm{Br}+\mathrm{Na}\right]^{+}$395.0623; found $395.0616[\mathrm{M}+\mathrm{Na}]^{+}$.

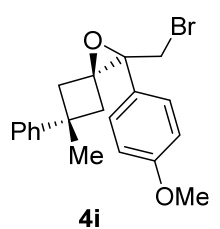

2-(bromomethyl)-2-(4-methoxyphenyl)-5-methyl-5-phenyl-1-oxaspiro[2.3]hexane 4i

Following the general procedure F, 4i was isolated as a colorless oil (17.5 mg; 0.047 mmol; $47 \% ; 81 \%$ ee) starting from 1-(1-(4-methoxyphenyl)vinyl)-3-methyl-3phenylcyclobutan-1-ol 1i (29.4 mg; $0.1 \mathrm{mmol})$ and $\mathbf{2 b}(4.3 \mathrm{mg} ; 0.0025 \mathrm{mmol})$ in toluene $(0.50 \mathrm{~mL})$ and NBS (19.6 mg; $0.11 \mathrm{mmol}) . \mathbf{R f}=0.38(\mathrm{PE}:$ EtOAc $=95: 5) .{ }^{1} \mathbf{H}$ NMR $(300$ 
$\left.\mathrm{MHz}, \mathrm{CDCl}_{3}\right) \delta 7.32\left(\mathrm{dd}, J=16.0,8.6 \mathrm{~Hz}, 3 \mathrm{H}, \mathrm{C}_{\text {Arom }}-\mathrm{H}\right), 7.26-7.13(\mathrm{~m}, 5 \mathrm{H}), 6.88(\mathrm{~d}, J$ $\left.=8.8 \mathrm{~Hz}, 2 \mathrm{H}, \mathrm{C}_{\text {Arom }} \mathrm{H}\right), 3.85-3.78(\mathrm{~m}, 3 \mathrm{H}, \mathrm{OMe}+1 \mathrm{H}, \mathrm{CHaHbBr}), 3.43(\mathrm{~d}, J=10.8 \mathrm{~Hz}$, 1H, CHaHbBr), 3.03 (d, $\left.J=13.1 \mathrm{~Hz}, 1 \mathrm{H}, \mathrm{C}^{1} \mathbf{H a H b}\right), 2.60$ (dt, $J=13.1,1.9 \mathrm{~Hz}, 1 \mathrm{H}$, $\left.\mathrm{C}^{1} \mathrm{HaHb}\right), 2.55-2.46$ (m, 1H, $\left.\mathrm{C}^{2} \mathrm{HaHb}\right), 2.30$ (d, J=13.6 Hz, 1H, $\mathrm{C}^{2} \mathrm{HaHb}$ ), 1.63 (s, 3H, $\left.\mathrm{CH}_{3}\right) .{ }^{13} \mathbf{C}\left\{{ }^{1} \mathbf{H}\right\}$ NMR (75.5 MHz, $\left.\mathrm{CDCl}_{3}\right) \delta 159.21$ (C $\mathrm{C}_{\text {Arom-C }}$ ), $149.04\left(\mathrm{C}_{\text {Arom- }}-\mathrm{C}\right), 128.36$ $\left(2 \times \mathrm{C}_{\text {Arom }}-\mathrm{H}\right), 127.84\left(2 \times \mathrm{C}_{\text {Arom }}-\mathrm{H}\right), 127.80\left(\mathrm{C}_{\text {Arom }}-\mathrm{C}\right), 125.90\left(\mathrm{C}_{\text {Arom }}-\mathrm{H}\right), 125.42\left(2 \mathrm{xC}_{\text {Arom- }}\right.$ $\mathrm{H}), 113.61\left(\mathrm{C}_{\text {Arom- }} \mathrm{H}\right), 69.76\left(\mathbf{C}-\mathrm{CH}_{2} \mathrm{Br}\right), 63.96(\mathbf{C}-\mathrm{O}), 55.23\left(\mathrm{OCH}_{3}\right), 42.70\left(\mathrm{C}^{1} \mathrm{H}_{2}\right), 42.45$ $\left(\mathrm{C}^{2} \mathrm{H}_{2}\right), 36.83\left(\mathbf{C}-\mathrm{CH}_{3}\right), 36.12\left(\mathrm{CH}_{2} \mathrm{Br}\right), 32.14\left(\mathrm{CH}_{3}\right)$. IR (ATR) $\mathrm{cm}^{-1}: 3022(=\mathrm{C}-\mathrm{H}), 2961$ $(\mathrm{C}-\mathrm{H}), 1612(\mathrm{C}=\mathrm{C})$. HRMS $\left(\mathrm{ESI}^{+}\right): \mathrm{m} / \mathrm{z}$ calculated for $\left[\mathrm{C}_{20} \mathrm{H}_{21} \mathrm{O}_{2} \mathrm{Br}+\mathrm{Na}\right]^{+}$395.0623; found $395.0627[\mathrm{M}+\mathrm{Na}]^{+}$.

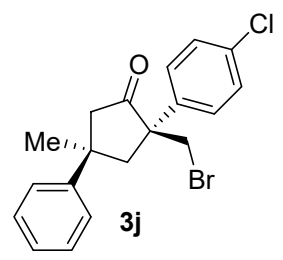

(2R,4R)-2-(bromomethyl)-2-(4-chlorophenyl)-4-methyl-4-phenylcyclopentan-1-one 3j

Following the general procedure F, $3 \mathbf{j}$ was isolated as a white solid $(28.4 \mathrm{mg}$; $0.075 \mathrm{mmol}$; $75 \% ; \quad \mathrm{dr}>20: 1 ; 95 \%$ ee) starting from 1-(1-(4-chlorophenyl)vinyl)-3-methyl-3phenylcyclobutan-1-ol (29.9 mg; $0.10 \mathrm{mmol}) \mathbf{1 j}$ and $\mathbf{2 b}(4.3 \mathrm{mg} ; 0.0025 \mathrm{mmol})$ in Toluene (0.5 L) and NBS (19.6 mg; $0.11 \mathrm{mmol})$. The enantiomeric excess of product was determined by HPLC using Chiralpack OD-3 column [Hexane: $i \mathrm{PrOH}$ 99:1; flow $=1$ $\mathrm{mL} / \mathrm{min}$.; $\operatorname{tr}_{\text {minor }}=19.0 \mathrm{~min}$; $\left.\operatorname{tr}_{\text {major }}=30.7 \mathrm{~min}.\right] .[\boldsymbol{\alpha}]_{\mathrm{D}}{ }^{26}=+391.2\left(\mathrm{c}=0.1, \mathrm{CHCl}_{3}\right) . \mathbf{R f}=$ 0.38 (PE:EtOAc = 95:5). M.p. $=124-125{ }^{\circ} \mathrm{C} .{ }^{1} \mathbf{H}$ NMR $\left(300 \mathrm{MHz}, \mathrm{CDCl}_{3}\right) \delta 7.58-7.51$ (m, 2H, CArom-H), 7.44 - 7.33 (m, 6H, CArom-H), 7.31 - 7.23 (m, 1H, CArom-H), 3.55 (d, $J$ $\left.=10.2 \mathrm{~Hz}, 1 \mathrm{H}, \mathrm{CH}_{\mathrm{a}} \mathrm{HbBr}\right), 3.42\left(\mathrm{~d}, J=10.2 \mathrm{~Hz}, 1 \mathrm{H}, \mathrm{CH}_{\mathrm{aHbBr}}\right), 3.06(\mathrm{~d}, J=13.7 \mathrm{~Hz}, 1 \mathrm{H}$, $\left.\mathrm{C}^{1} \mathbf{H}_{\mathrm{a}} \mathrm{H}_{\mathrm{b}}\right), 3.02-2.86\left(\mathrm{~m}, 2 \mathrm{H}, \mathrm{C}^{1} \mathrm{H}_{\mathrm{a}} \mathbf{H}_{\mathbf{b}}\right.$ and $\left.\mathrm{C}^{2} \mathbf{H}_{\mathbf{a}} \mathrm{H}_{\mathrm{b}}\right), 2.72(\mathrm{dd}, J=18.1,1.9 \mathrm{~Hz}, 1 \mathrm{H}$, $\left.\mathrm{C}^{2} \mathrm{H}_{\mathbf{a}} \mathbf{H}_{\mathbf{b}}\right), 1.14\left(\mathrm{~s}, 3 \mathrm{H}, \mathrm{CH}_{3}\right) .{ }^{13} \mathbf{C}\left\{{ }^{1} \mathbf{H}\right\}$ NMR $\left(75.5 \mathrm{MHz}, \mathrm{CDCl}_{3}\right) \delta 215.8(\mathrm{C}=\mathrm{O}), 148.5$ $\left(\mathrm{C}_{\text {Arom- }} \mathrm{Cl}\right), 138.6\left(\mathrm{C}_{\text {Arom }}-\mathrm{C}\right), 133.4\left(\mathrm{C}_{\text {Arom }}-\mathrm{C}\right), 129.0\left(2 \mathrm{xC}_{\text {Arom }}-\mathrm{H}\right), 128.7\left(2 \mathrm{xC}_{\text {Arom }}-\mathrm{H}\right)$, $128.1\left(2 \times \mathrm{C}_{\text {Arom }}-\mathrm{H}\right), 126.6\left(\mathrm{C}_{\text {Arom- }}-\mathrm{H}\right), 125.3\left(2 \times \mathrm{C}_{\text {Arom }}-\mathrm{H}\right), 57.3\left(\mathrm{CCH}_{2} \mathrm{Br}\right), 52.8\left(\mathrm{C}^{1} \mathrm{H}_{2}\right)$, $45.6\left(\mathrm{C}^{2} \mathrm{H}_{2}\right), 41.1\left(\mathrm{C}_{-} \mathrm{CH}_{3}\right), 40.9\left(\mathrm{CH}_{2} \mathrm{Br}\right), 31.3\left(\mathrm{CH}_{3}\right)$. IR (ATR) $\mathrm{cm}^{-1}: 3029$ (=C-H), 2968 (C-H), 1736 (C=O), 1641 (C=C). MS (EI) m/z (\%): 297.2 (44), 159.1 (30), 153.0 (34), 152.0. (15), 151.0 (100). HRMS $\left(\mathrm{ESI}^{+}\right): \mathrm{m} / \mathrm{z}$ calculated for $\left[\mathrm{C}_{19} \mathrm{H}_{18} \mathrm{OBrCl}+\mathrm{Na}\right]^{+}$ 399.0127; found $399.0136[\mathrm{M}+\mathrm{Na}]^{+}$. 


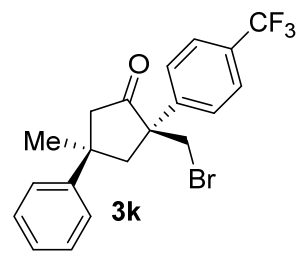

$(2 R, 4 R)$-2-(bromomethyl)-4-methyl-4-phenyl-2-(4-(trifluoromethyl)phenyl)cyclopentan1-one 3k

Following the general procedure F, 3k was isolated as a yellowish oil (11.4 mg; 0.03 mmol; $62 \%$; dr:9:1; $86 \%$ ee) by FC (PE:EtOAc = 95:5), starting from (1r,3r)-3-methyl3-phenyl-1-(1-(4-(trifluoromethyl)phenyl)vinyl)cyclobutan-1-ol $1 \mathbf{k}$ (14.9 mg; 0.045 $\mathrm{mmol})$ and $\mathbf{2 b}(4.0 \mathrm{mg} ; 0.0022 \mathrm{mmol})$ in toluene $(0.22 \mathrm{~mL})$ and NBS $(8.72 \mathrm{mg} ; 0.049$ $\mathrm{mmol})$. The enantiomeric excess of product was determined by HPLC using Chiralpack OD3 column [Hexane: $i \operatorname{PrOH} 99: 1$; flow $=1 \mathrm{~mL} / \mathrm{min}$; $\operatorname{tr}_{\text {minor }}=22.5 \mathrm{~min}$.; $\operatorname{tr}_{\text {major }}=33.7$ min.]. $[\boldsymbol{\alpha}]_{\mathrm{D}}{ }^{20}=+20.8\left(c=1, \mathrm{CHCl}_{3}\right) . \mathbf{R f}=0.51(\mathrm{PE}: \mathrm{EtAcO}=95: 5) .{ }^{1} \mathbf{H} \mathbf{~ N M R}(300 \mathrm{MHz}$, $\left.\mathrm{CDCl}_{3}\right) \delta 7.74\left(\mathrm{~d}, J=8.6 \mathrm{~Hz}, 2 \mathrm{H}, \mathrm{C}_{\text {Arom- }} \mathrm{H}\right), 7.64\left(\mathrm{~d}, J=8.6 \mathrm{~Hz}, 2 \mathrm{H}, \mathrm{C}_{\text {Arom }}-\mathrm{H}\right), 7.43-7.34$ (m, 4H, $\left.\mathrm{C}_{\text {Arom- }} \mathrm{H}\right), 7.34-7.29\left(\mathrm{~m}, 1 \mathrm{H}, \mathrm{C}_{\text {Arom- }} \mathrm{H}\right), 3.53$ (d, J=10.2 Hz, 1H, $\left.\mathrm{CH}_{\mathrm{a}} \mathrm{H}_{\mathrm{b}} \mathrm{Br}\right), 3.44$ $\left(\mathrm{d}, J=10.2 \mathrm{~Hz}, 1 \mathrm{H}, \mathrm{CH}_{\mathrm{a}} \mathbf{H}_{\mathrm{b}} \mathrm{Br}\right), 3.09\left(\mathrm{~d}, J=13.8 \mathrm{~Hz}, 1 \mathrm{H}, \mathrm{C}^{1} \mathbf{H}_{\mathrm{a}} \mathrm{H}_{\mathrm{b}}\right), 3.02(\mathrm{~d}, J=13.8 \mathrm{~Hz}$, $\left.1 \mathrm{H}, \mathrm{C}^{1} \mathrm{H}_{\mathrm{a}} \mathbf{H}_{\mathbf{b}}\right) 2.96\left(\mathrm{~d}, J=18.2 \mathrm{~Hz}, 1 \mathrm{H}, \mathrm{C}^{2} \mathbf{H}_{\mathbf{a}} \mathrm{Hb}_{\mathrm{b}}\right), 2.72\left(\mathrm{dd}, J=18.2,1.7 \mathrm{~Hz}, 1 \mathrm{H}, \mathrm{C}^{2} \mathrm{H}_{\mathrm{a}} \mathbf{H}_{\mathbf{b}}\right)$, $1.14\left(\mathrm{~s}, 3 \mathrm{H}, \mathrm{CH}_{3}\right) .{ }^{19} \mathbf{F}$ NMR $\left(282 \mathrm{MHz}, \mathrm{CDCl}_{3}\right) \delta-62.7 .{ }^{13} \mathbf{C}\left\{{ }^{1} \mathbf{H}\right\} \mathbf{N M R}(75.5 \mathrm{MHz}$, $\left.\mathrm{CDCl}_{3}\right) \delta 215.4(\mathrm{C}=\mathrm{O}), 148.2\left(\mathrm{C}_{\text {Arom- }} \mathrm{C}\right), 144.1\left(\mathrm{C}_{\text {Arom- }} \mathrm{C}\right), 130.0\left(\mathrm{q}, J_{C-F}=32.5 \mathrm{~Hz}, \mathbf{C}_{\text {Arom }^{-}}\right.$ $\left.\mathrm{CF}_{3},\right), 128.8\left(2 \mathrm{xC}_{\text {Arom }}-\mathrm{H}\right), 127.2\left(2 \mathrm{xC}_{\text {Arom }}-\mathrm{H}\right), 126.7\left(\mathrm{C}_{\text {Arom }}-\mathrm{H}\right), 125.7\left(\mathrm{q}, J_{C-F}=3.8 \mathrm{~Hz}\right.$, 2xCArom-H), $125.3\left(2 \times \mathrm{C}_{\text {Arom- }} \mathrm{H}\right), 123.8\left(\mathrm{q}, J_{C-F}=265.5 \mathrm{~Hz}, \mathrm{CF}_{3},\right), 57.6\left(\mathbf{C}-\mathrm{CH}_{2} \mathrm{Br}\right), 52.8$ $\left(\mathrm{C}^{1} \mathrm{H}_{2}\right), 45.7\left(\mathrm{C}^{2} \mathrm{H}_{2}\right), 41.1\left(\mathrm{CCH}_{3}\right), 40.8\left(\mathrm{CH}_{2} \mathrm{Br}\right), 31.3\left(\mathrm{CH}_{3}\right)$. IR (ATR) $\mathrm{cm}^{-1}: 1740$ $(\mathrm{C}=\mathrm{O})$. MS (EI) e/z (\%): 331.1 (5), 289.1 (19), 185 (77), 117. (100). HRMS (ESI $\left.{ }^{+}\right): \mathrm{m} / \mathrm{z}$ calculated for $\left[\mathrm{C}_{20} \mathrm{H}_{18} \mathrm{OF} 3 \mathrm{Br}+\mathrm{Na}\right]^{+} 433.0391$; found $433.0390[\mathrm{M}+\mathrm{Na}]^{+}$.

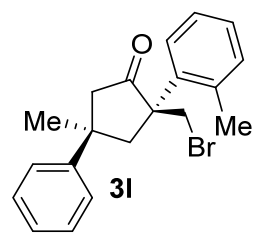

$(2 R, 4 R)$-2-(bromomethyl)-4-methyl-4-phenyl-2-(o-tolyl)cyclopentan-1-one 31

Following the general procedure F, 3l was isolated as a yellowish oil (32 mg; $0.09 \mathrm{mmol}$; $89 \%$; dr>20:1; $45 \%$ ee) by FC (PE:EtOAc = 95:5), starting from 3-methyl-3-phenyl-1(1-(o-tolyl)vinyl)cyclobutan-1-ol $1 \mathbf{l}(27.8 \mathrm{mg} ; 0.10 \mathrm{mmol})$ and $\mathbf{2 b}$ (4.3 mg; $0.0025 \mathrm{mmol})$ in toluene $(0.5 \mathrm{~mL})$ and NBS $(19.6 \mathrm{mg} ; 0.11 \mathrm{mmol})$. The enantiomeric excess of product was determined by HPLC using Chiralpack OD3 column [Hexane: $i$ PrOH 99.5:0.5; flow 
$=0.5 \mathrm{~mL} / \mathrm{min}$; $\operatorname{tr}_{\text {major }}=19.3 \mathrm{~min}$.; $\operatorname{tr}_{\text {minor }}=20.5 \mathrm{~min}$. $] .[\boldsymbol{\alpha}]_{\mathrm{D}}{ }^{20}=+20.3\left(c=1, \mathrm{CHCl}_{3}\right) . \mathbf{R f}=$ 0.56 (PE:EtAcO = 95:5). ${ }^{1} \mathbf{H}$ NMR $\left(300 \mathrm{MHz}, \mathrm{CDCl}_{3}\right) \delta$ 7.14-6.95 (m, 7H, CArom-H), 6.90-6.81 (m, 2H, C Arom-H) $_{\text {- }} 3.85$ (d, $\left.J=10.6 \mathrm{~Hz}, 1 \mathrm{H}, \mathrm{CH}_{\mathrm{a}} \mathrm{H}_{\mathrm{bBr}}\right), 3.66$ (d, $J=10.6 \mathrm{~Hz}$, $\left.1 \mathrm{H}, \mathrm{CH}_{\mathrm{a}} \mathbf{H}_{\mathbf{b}} \mathrm{Br}\right), 3.08$ (d, $\left.J=16.4 \mathrm{~Hz}, 1 \mathrm{H}, \mathrm{C}^{1} \mathbf{H}_{\mathrm{a}} \mathrm{H}_{\mathrm{b}}\right), 2.98\left(\mathrm{~s}, 2 \mathrm{H}, \mathrm{C}^{2} \mathrm{H}_{2}\right), 2.49$ (d, $J=16.4$ $\left.\mathrm{Hz}, 1 \mathrm{H}, \mathrm{C}^{1} \mathrm{H}_{\mathrm{a}} \mathbf{H}_{\mathbf{b}}\right), 2.31$ (s, 3H, $\left.\mathrm{C}_{\text {Arom- }} \mathbf{C H}_{3}\right), 1.43\left(\mathrm{~s}, 3 \mathrm{H}, \mathrm{CH}_{3}\right) .{ }^{13} \mathbf{C}\left\{{ }^{1} \mathbf{H}\right\}$ NMR $(75.5 \mathrm{MHz}$, $\left.\mathrm{CDCl}_{3}\right) \delta 215.3(\mathrm{C}=\mathrm{O}), 149.1\left(\mathrm{C}_{\text {Arom- }} \mathrm{C}\right), 139.5\left(\mathrm{C}_{\text {Arom- }} \mathrm{C}\right), 135.5\left(\mathrm{C}_{\text {Arom- }} \mathrm{C}\right), 133.1\left(\mathrm{C}_{\text {Arom- }}\right.$ $\mathrm{H}), 128.3\left(2 \times \mathrm{C}_{\text {Arom-H}} \mathrm{H}\right), 127.6\left(\mathrm{C}_{\text {Arom-H }} \mathrm{H}\right), 126.9\left(\mathrm{C}_{\text {Arom-H}}\right), 126.1\left(\mathrm{C}_{\text {Arom-H }}\right), 126.0$ (CArom$\mathrm{H}), 125.2\left(2 \times \mathrm{C}_{\text {Arom- }} \mathrm{H}\right), 58.8\left(\mathbf{C}-\mathrm{CH}_{2} \mathrm{Br}\right), 51.5\left(\mathrm{C}^{1} \mathrm{H}_{2}\right), 47.7\left(\mathrm{C}^{2} \mathrm{H}_{2}\right), 40.0\left(\mathbf{C C H}_{3}\right), 36.1$ $\left(\mathrm{CH}_{2} \mathrm{Br}\right), 32.0\left(\mathrm{C}_{\text {Arom}}-\mathbf{C H}_{3}\right), 21.7\left(\mathrm{CH}_{3}\right)$. IR (ATR) cm $\mathrm{cm}^{-1}: 1741(\mathrm{C}=\mathrm{O})$. MS (EI) e/z (\%): 277.1 (2), 159.1 (16), 131.0 (48), 115 (100). HRMS (ESI $\left.{ }^{+}\right): \mathrm{m} / \mathrm{z}$ calculated for $\left[\mathrm{C}_{20} \mathrm{H}_{21} \mathrm{OBr}+\mathrm{Na}\right]^{+}$379.0673; found $379.0671[\mathrm{M}+\mathrm{Na}]^{+}$.

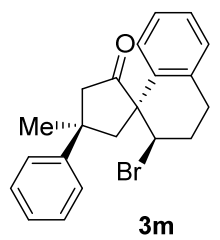

$\left(1 R, 2^{\prime} R, 4 R\right)$-2'-bromo-4-methyl-4-phenyl-3',4'-dihydro-2'H-spiro[cyclopentane-1,1'naphthalen]-2-one $\mathbf{3 m}$

Following the general procedure F, 3m was isolated as a colorless oil (28.0 mg; 0.076 mmol; $76 \%$; dr>20:1; $90 \%$ ee) starting from 1-(3,4-dihydronaphthalen-1-yl)-3-methyl3-phenylcyclobutan-1-ol $\mathbf{1 m}(29.0 \mathrm{mg} ; 0.10 \mathrm{mmol})$ and $\mathbf{2 b}(4.3 \mathrm{mg} ; 0.0025 \mathrm{mmol})$ in toluene $(0.50 \mathrm{~mL})$ and NBS $(19.6 \mathrm{mg} ; 0.11 \mathrm{mmol})$. The enantiomeric excess of product was determined by HPLC using Chiralpack OD-3 column [Hexane: $i \mathrm{PrOH} 99: 1$; flow $=$ $1 \mathrm{~mL} / \mathrm{min}$.; $\operatorname{tr}_{\text {major }}=16.6 \mathrm{~min}$; $\operatorname{tr}_{\text {minor }}=24.2 \mathrm{~min}$.]. $[\boldsymbol{\alpha}]_{\mathrm{D}}{ }^{20}=+20.0\left(\mathrm{c}=0.3, \mathrm{CHCl}_{3}\right) . \mathbf{R f}=$ 0.38 (PE:EtOAc = 95:5). ${ }^{1} \mathbf{H}$ NMR $\left(300 \mathrm{MHz}, \mathrm{CDCl}_{3}\right) \delta 7.47-7.33\left(\mathrm{~m}, 4 \mathrm{H}, \mathrm{C}_{\text {Arom- }}-\mathrm{H}\right)$, $7.33-7.16\left(\mathrm{~m}, 3 \mathrm{H}, \mathrm{C}_{\text {Arom- }} \mathrm{H}\right), 7.16-7.06\left(\mathrm{~m}, 2 \mathrm{H}, \mathrm{C}_{\text {Arom }}-\mathrm{H}\right), 4.22(\mathrm{dd}, J=5.6,3.0 \mathrm{~Hz}$, $1 \mathrm{H}, \mathrm{CHBr}$ ), 3.52 (d, $\left.J=17.0 \mathrm{~Hz}, 1 \mathrm{H} ; \mathrm{C}^{1} \mathbf{H}_{\mathrm{a}} \mathrm{H}_{\mathrm{b}}\right), 3.24$ (ddd, $J=17.1,9.6,6.9 \mathrm{~Hz}, 1 \mathrm{H}$, $\left.\mathrm{C}_{\text {Arom- }}-\mathrm{CH}_{\mathbf{a}} \mathrm{H}_{\mathrm{b}}\right), 3.02\left(\mathrm{~d}, J=14.6 \mathrm{~Hz}, 1 \mathrm{H}, \mathrm{C}^{2} \mathbf{H}_{\mathbf{a}} \mathrm{H}_{\mathrm{b}}\right), 2.82(\mathrm{ddd}, J=17.1,5.7,4.4 \mathrm{~Hz}, 1 \mathrm{H}$, $\left.\mathrm{C}_{\text {Arom- }} \mathrm{CH}_{\mathrm{a}} \mathbf{H}_{\mathbf{b}}\right), 2.71\left(\mathrm{~d}, J=17.1 \mathrm{~Hz}, 1 \mathrm{H}, \mathrm{C}^{1} \mathrm{H}_{\mathrm{a}} \mathbf{H}_{\mathbf{b}}\right), 2.63\left(\mathrm{~d}, J=14.5 \mathrm{~Hz}, 1 \mathrm{H}, \mathrm{C}^{2} \mathrm{H}_{\mathrm{a}} \mathbf{H}_{\mathbf{b}}\right)$, 2.45 - $2.15\left(\mathrm{~m}, 2 \mathrm{H}, \mathrm{C}_{\text {Arom}}-\mathrm{CH}_{2} \mathbf{C H}_{2}\right), 1.53\left(\mathrm{~s}, 3 \mathrm{H}, \mathrm{CH}_{3}\right) .{ }^{13} \mathbf{C}\left\{{ }^{1} \mathbf{H}\right\}$ NMR (75.5 MHz, $\left.\mathrm{CDCl}_{3}\right) \delta 213.5(\mathrm{C}=\mathrm{O}), 149.4\left(\mathrm{C}_{\text {Arom- }} \mathrm{C}\right), 135.6\left(\mathrm{C}_{\text {Arom- }} \mathrm{C}\right), 135.5\left(\mathrm{C}_{\text {Arom }}-\mathrm{C}\right), 129.1\left(\mathrm{C}_{\text {Arom- }}\right.$ $\mathrm{H}), 128.9\left(\mathrm{C}_{\text {Arom- }} \mathrm{H}\right), 128.9\left(2 \mathrm{xC}_{\text {Arom }}-\mathrm{H}\right), 127.0\left(\mathrm{C}_{\text {Arom }}-\mathrm{H}\right), 126.6\left(\mathrm{C}_{\text {Arom }}-\mathrm{H}\right), 126.6\left(\mathrm{C}_{\text {Arom- }}{ }^{-}\right.$ $\mathrm{H}), 125.2\left(2 \times \mathrm{C}_{\text {Arom }} \mathrm{H}\right), 59.1(\mathbf{C}-\mathrm{CHBr}), 55.3\left(\mathrm{C}^{1} \mathrm{H}_{2}\right), 54.9(\mathrm{CHBr}), 51.2\left(\mathrm{C}^{2} \mathrm{H}_{2}\right), 39.2(\mathbf{C}-$ $\left.\mathrm{CH}_{3}\right), 34.2\left(\mathrm{CH}_{3}\right), 27.8\left(\mathrm{C}_{\text {Arom- }}-\mathbf{C H}_{2}\right), 26.1\left(\mathrm{C}_{\text {Arom }}-\mathrm{CH}_{2} \mathbf{C H}_{2}\right)$. IR (ATR) $\mathrm{cm}^{-1}: 3022$ (=C- 
H), $2957(\mathrm{C}-\mathrm{H}), 1744(\mathrm{C}=\mathrm{O}), 1636(\mathrm{C}=\mathrm{C}) . \mathbf{M S}(\mathrm{EI}) \mathrm{m} / \mathrm{z}(\%): 288.2$ (44), 170.1 (44), 141.1 (100). HRMS $\left(\mathrm{ESI}^{+}\right): \mathrm{m} / \mathrm{z}$ calculated for $\left[\mathrm{C}_{21} \mathrm{H}_{22} \mathrm{OBr}\right]^{+}$369.0854; found 369.0848 $[\mathrm{M}+\mathrm{H}]^{+}$.

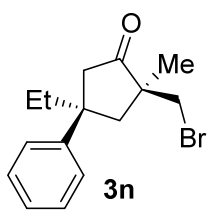

(2S,4R)-2-(bromomethyl)-4-ethyl-2-methyl-4-phenylcyclopentan-1-one 3n

Following the general procedure F, 3n was isolated as a yellowish oil (25.2 mg; 0.08 mmol; $85 \%$; dr: 1.2:1; $1^{\text {st }}$ diastereomer $70 \%$ ee and $2^{\text {nd }}$ diastereomer $72 \%$ ee) by FC $(\mathrm{PE}:$ EtOAc $=95: 5)$, starting from 3-ethyl-3-phenyl-1-(prop-1-en-2-yl)cyclobutan-1-ol (21.6 mg; $0.10 \mathrm{mmol}) \mathbf{1 n}$ and $\mathbf{2 b}(4.3 \mathrm{mg} ; 0.0025 \mathrm{mmol})$ in toluene $(0.5 \mathrm{~mL})$ and NBS (19.6 $\mathrm{mg} ; 0.11 \mathrm{mmol})$. The enantiomeric excess of product was determined by HPLC using Chiralpack OD3 column [Hexane: $i \operatorname{PrOH} 99: 1$; flow $=0.5 \mathrm{~mL} / \mathrm{min} .1^{\text {st }}$ diastereomer: $\operatorname{tr}_{\text {major }}=14.8 \mathrm{~min}$.; $\operatorname{tr}_{\text {major }}=18.8 \mathrm{~min} .2^{\text {nd }}$ diastereomer: $\operatorname{tr}_{\text {minor }}=18.2 \mathrm{~min}$; $\operatorname{tr}_{\text {major }}=25.7$ min.]. $[\boldsymbol{\alpha}]_{\mathrm{D}}{ }^{20}=-36.7\left(c=1, \mathrm{CHCl}_{3}\right) . \mathbf{R f}=0.48(\mathrm{PE}: \mathrm{EtAcO}=95: 5)$. The spectral data is given for a mixture 1.2:1 of both diastereomers: ${ }^{1} \mathbf{H}$ NMR (300 MHz, $\left.\mathrm{CDCl}_{3}\right) \delta 7.32-7.12\left(\mathrm{~m}, 5 \mathrm{H}, \mathrm{C}_{\text {Arom-H }} \mathrm{H}\right.$ of each diastereomers), 3.39 (d, $J=10.0 \mathrm{~Hz}, 1 \mathrm{H}$, $\mathrm{CH}_{\mathrm{a}} \mathrm{Hb} \mathrm{Br}$ minor), 3.22 (d, $J=10.0 \mathrm{~Hz}, 1 \mathrm{H}, \mathrm{CH}_{\mathrm{a}} \mathbf{H}_{\mathbf{b}} \mathrm{Br}$ minor), 3.11 (d, $J=10.0 \mathrm{~Hz}, 1 \mathrm{H}$, $\mathrm{CH}_{\mathrm{a}} \mathrm{HbBr}$ major), $3.05\left(\mathrm{dd}, J=17.5,1.8 \mathrm{~Hz}, 1 \mathrm{H}, \mathrm{C}^{1} \mathbf{H}_{\mathrm{a}} \mathrm{H}_{\mathrm{b}}\right.$ minor), $2.96(\mathrm{~d}, J=10.0 \mathrm{~Hz}$, $1 \mathrm{H}, \mathrm{CH}_{\mathrm{a}} \mathbf{H}_{\mathrm{b}} \mathrm{Br}$ major), $2.89\left(\mathrm{~d}, J=17.4 \mathrm{~Hz}, 1 \mathrm{H}, \mathrm{C}^{1} \mathbf{H}_{\mathrm{a}} \mathrm{H}_{\mathrm{b}}\right.$ major), $2.64(\mathrm{~d}, J=13.8 \mathrm{~Hz}, 1 \mathrm{H}$, $\mathrm{C}^{2} \mathbf{H}_{\mathrm{a}} \mathrm{H}_{\mathrm{b}}$ major), $2.59\left(\mathrm{~d}, J=17.4 \mathrm{~Hz}, 1 \mathrm{H}, \mathrm{C}^{1} \mathrm{H}_{\mathrm{a}} \mathbf{H}_{\mathbf{b}}\right.$ major), $2.50(\mathrm{~d}, J=13.4 \mathrm{~Hz}, 1 \mathrm{H}$, $\mathrm{C}^{2} \mathbf{H}_{\mathrm{a}} \mathrm{Hb}_{\mathrm{b}}$ minor), 2.40 (d, $J=17.5 \mathrm{~Hz}, 1 \mathrm{H}, \mathrm{C}^{1} \mathrm{H}_{\mathrm{a}} \mathbf{H}_{\mathbf{b}}$ minor), 2.22 (dd, $J=13.4,1.8 \mathrm{~Hz}, 1 \mathrm{H}$, $\mathrm{C}^{2} \mathrm{H}_{\mathbf{a}} \mathbf{H}_{\mathbf{b}}$ minor), 2.11 (d, $J=13.8 \mathrm{~Hz}, 1 \mathrm{H}, \mathrm{C}^{2} \mathrm{H}_{\mathbf{a}} \mathbf{H}_{\mathbf{b}}$ major), 1.89 (dq, $J=13.8,7.4 \mathrm{~Hz}, 1 \mathrm{H}$, $\mathrm{CH}_{\mathrm{a}} \mathrm{H}_{\mathrm{b}} \mathrm{CH}_{3}$ minor), $1.81-1.53\left(\mathrm{~m}, 3 \mathrm{H}, \mathrm{CH}_{\mathrm{a}} \mathbf{H}_{\mathrm{b}} \mathrm{CH}_{3}\right.$ minor, $\mathrm{CH}_{\mathbf{a}} \mathrm{H}_{b} \mathrm{CH}_{3}$ major, $\mathrm{CH}_{\mathrm{a}} \mathrm{H}_{\mathrm{b}} \mathrm{CH}_{3}$ major), 1.24 (s, 3H, $\mathrm{CH}_{3}$ major), 0.72 (s, 3H, $\mathrm{CH}_{3}$ minor), 0.58 (t, $J=7.4 \mathrm{~Hz}$, $3 \mathrm{H}, \mathrm{CH}_{2} \mathrm{CH}_{3}$ minor), 0.57 (t, $J=7.4 \mathrm{~Hz}, 3 \mathrm{H}, \mathrm{CH}_{2} \mathrm{CH}_{3}$ major). ${ }^{13} \mathbf{C}\left\{{ }^{1} \mathbf{H}\right\} \mathbf{N M R}(75.5 \mathrm{MHz}$, $\left.\mathrm{CDCl}_{3}\right) \delta 218.3(\mathrm{C}=\mathrm{O}$, minor $), 218.2(\mathrm{C}=\mathrm{O}$, major $), 145.8\left(\mathrm{C}_{\text {Arom}}-\mathrm{C}\right.$, major $), 145.6\left(\mathrm{C}_{\text {Arom- }}\right.$ $\mathrm{C}$, minor), 128.5 (2xC $\mathrm{x}_{\text {Arom- }} \mathrm{H}$ of each diastereomer), 126.5 ( $\mathrm{C}_{\text {Arom-H }}$ major), 126.5 ( $\mathrm{C}_{\text {Arom- }}$ $\mathrm{H}$ minor), 126.4 (2xCArom-H of each diastereomer), $50.0\left(\mathrm{CH}_{2} \mathrm{Br}\right.$, major), $49.6\left(\mathbf{C}-\mathrm{CH}_{2} \mathrm{Br}\right.$, major), 49.5 (C- $\mathrm{CH}_{2} \mathrm{Br}$, minor), 49.4 ( $\mathrm{CH}_{2} \mathrm{Br}$, minor), $47.8\left(\mathrm{C}^{1} \mathrm{H}_{2}\right.$ minor), $46.1\left(\mathrm{C}^{1} \mathrm{H}_{2}\right.$ major), 45.0 (C- $\mathrm{CH}_{2}$ minor), 44.9 ( $\left(\mathrm{C}-\mathrm{CH}_{2}\right.$ major), $41.4\left(\mathrm{C}^{2} \mathrm{H}_{2}\right.$ major), $40.9\left(\mathrm{C}^{2} \mathrm{H}_{2}\right.$ minor), 37.5 ( $\mathbf{C H}_{2} \mathrm{CH}_{3}$ minor), 36.5 ( $\mathbf{C H}_{2} \mathrm{CH}_{3}$ major), 24.6 ( $\mathrm{CH}_{3}$ major), $24.2\left(\mathrm{CH}_{3}\right.$ minor $), 9.5$ 
( $\mathrm{CH}_{2} \mathbf{C H}_{3}$, minor), $9.1\left(\mathrm{CH}_{2} \mathbf{C H}_{3}\right.$, major). IR (ATR) $\mathrm{cm}^{-1}: 1738(\mathrm{C}=\mathrm{O})$. MS (EI) e/z (\%): 296.9 (M+H, 2), 264.9 (20), 157 (95), 55 (100). HRMS (ESI ${ }^{+}$): decomposition. 


\section{Synthesis and characterization of products $4 a$ and $3 a$}

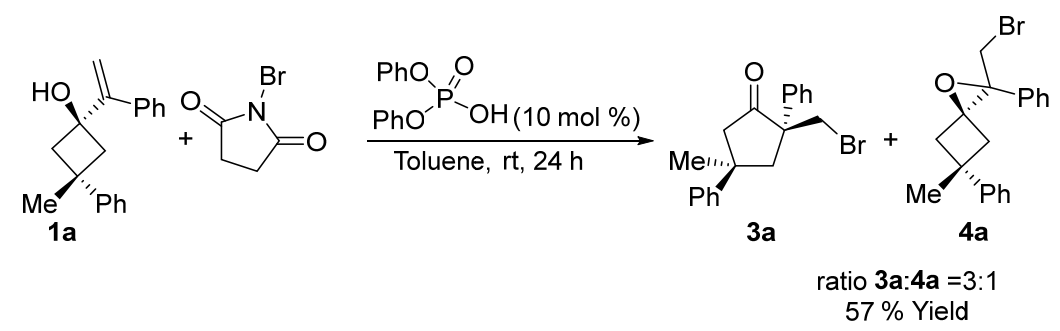

Scheme S 9. Synthesis of epoxide 4a.

Compound 4a was prepared by treating 3-methyl-3-phenyl-1-(1-phenylvinyl)cyclobutan1-ol 1a (79.2 mg; $0.3 \mathrm{mmol})$ and diphenylphosphoric acid (7.5 mg; $0.01 \mathrm{mmol})$ in toluene $(1.5 \mathrm{~mL})$ with NBS $(64.2 \mathrm{mg} ; 0.36 \mathrm{mmol})$ at room temperature. The reaction was let stirring for 24 hours, then it was concentrated under vacuum and directly purified by column chromatography (PE:EtOAc $=95: 5)$ to obtain $58.8 \mathrm{mg}$ as a mixture of 2(bromomethyl)-4-methyl-2,4-diphenylcyclopentan-1-one $\mathbf{3 a}$ and 2-(bromomethyl)-5methyl-2,5-diphenyl-1-oxaspiro[2.3]hexane 4a in a 3:1 ratio. The epoxide 4a was separated by preparative TLC employing PE:EtOAc 95:1. Rf =0.36 (PE:EtOAc = 95:5). ${ }^{1}$ H NMR $\left(300 \mathrm{MHz}, \mathrm{CDCl}_{3}\right) \delta 7.41-7.30$ (m, 5H, CArom-H), 7.29 - 7.17 (m, 5H, CArom$\mathrm{H}), 3.88\left(\mathrm{~d}, J=10.9 \mathrm{~Hz}, 1 \mathrm{H}, \mathrm{CH}_{\mathbf{a}} \mathrm{H}_{\mathrm{b}} \mathrm{Br}\right), 3.44\left(\mathrm{~d}, J=10.9 \mathrm{~Hz}, 1 \mathrm{H}, \mathrm{CH}_{\mathrm{a}} \mathbf{H}_{\mathbf{b}} \mathrm{Br}\right), 3.13-2.94$ (dt, $\left.J=13.1,1.6 \mathrm{~Hz}, 1 \mathrm{H}, \mathrm{C}^{1} \mathbf{H}_{\mathbf{a}} \mathrm{H}_{\mathrm{b}}\right), 2.62\left(\mathrm{ddd}, J=13.1,2.5,1.3 \mathrm{~Hz}, 1 \mathrm{H}, \mathrm{C}^{1} \mathrm{H}_{\mathrm{a}} \mathbf{H}_{\mathbf{b}}\right), 2.49$ (app dt, $\left.J=13.4,1.6 \mathrm{~Hz}, 1 \mathrm{H}, \mathrm{C}^{2} \mathbf{H}_{\mathbf{a}} \mathrm{H}_{\mathrm{b}}\right), 2.31$ (ddd, $J=13.4,2.5,1.3 \mathrm{~Hz}, 1 \mathrm{H}, \mathrm{C}^{2} \mathrm{H}_{\mathrm{a}} \mathbf{H}_{\mathbf{b}}$ ), $1.63\left(\mathrm{~s}, 1 \mathrm{H}, \mathrm{CH}_{3}\right) .{ }^{13} \mathbf{C}\left\{{ }^{1} \mathbf{H}\right\}$ NMR $\left(75.5 \mathrm{MHz}, \mathrm{CDCl}_{3}\right) \delta 148.98\left(\mathrm{C}_{\text {Arom- }} \mathrm{C}\right), 135.80\left(\mathrm{C}_{\text {Arom- }}\right.$ C), $128.36\left(2 \times \mathrm{C}_{\text {Arom }}-\mathrm{H}\right), 128.18(2 \mathrm{xC}$ Arom-H $), 127.89\left(\mathrm{C}_{\text {Arom }}-\mathrm{H}\right), 126.57$ (2xCArom-H), $125.92\left(\mathrm{C}_{\text {Arom-H}}-\mathrm{H}\right), 125.39\left(2 \mathrm{xC}\right.$ Arom-H), $69.88\left(\mathbf{C}-\mathrm{CH}_{2} \mathrm{Br}\right), 64.20(\mathbf{C}-\mathrm{O}), 42.67\left(\mathrm{C}^{1} \mathrm{H}_{2}\right)$, $42.44\left(\mathrm{C}^{2} \mathrm{H}_{2}\right), 36.90\left(\mathbf{C}-\mathrm{CH}_{3}\right), 35.77\left(\mathrm{CH}_{2} \mathrm{Br}\right), 32.13\left(\mathrm{CH}_{3}\right)$. IR (ATR) $\mathrm{cm}^{-1}: 3020$ (=CH), $2958(\mathrm{C}-\mathrm{H})$. HRMS $\left(\mathrm{ESI}^{+}\right): \mathrm{m} / \mathrm{z}$ calculated for $\left[\mathrm{C}_{19} \mathrm{H}_{19} \mathrm{OBr}+\mathrm{Na}\right]^{+}$365.0517; found $365.0507[\mathrm{M}+\mathrm{Na}]^{+}$.
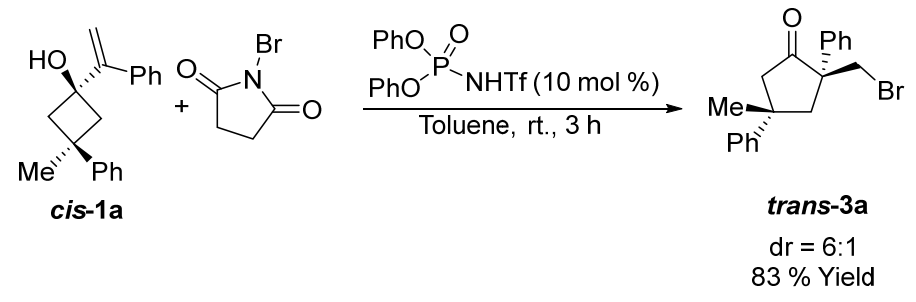

Scheme S 10. Synthesis of ketone 3atrans.

Compound trans-3a was prepared by treating 3-methyl-3-phenyl-1-(1phenylvinyl)cyclobutan-1-ol cis-1a $\quad(26.4 \quad \mathrm{mg} ; 0.1 \quad \mathrm{mmol})$ and diphenyl 
((trifluoromethyl)sulfonyl)phosphoramidate $(3.8 \mathrm{mg} ; 0.01 \mathrm{mmol})$ in toluene $(0.5 \mathrm{~mL})$ with NBS (21.4 mg; $0.12 \mathrm{mmol})$ at room temperature. The reaction was let stirring for 3 $\mathrm{h}$ and then it was concentrated under vacuum and directly purified by column chromatography (PE:EtOAc $=95: 5)$ to obtain 2-(bromomethyl)-4-methyl-2,4diphenylcyclopentan-1-one trans-3a (28.5 mg; $0.083 \mathrm{mmol} ; 83 \%$ yield). $\mathbf{R f}=0.36$ $(\mathrm{PE}: \mathrm{EtOAc}=95: 5)$. IR $($ ATR $) \mathrm{cm}^{-1}: 3020(=\mathrm{C}-\mathrm{H}), 2958(\mathrm{C}-\mathrm{H}), 1739(\mathrm{C}=\mathrm{O}), 1643(\mathrm{C}=\mathrm{C})$, $1465\left(\mathrm{CH}_{2}\right), 1439\left(\mathrm{CH}_{3}\right)$. MS (EI) e/z (\%): 263.2 (34), 205.1 (25), 145.1 (39), 117.1 (100). HRMS $\left(\mathrm{ESI}^{+}\right): \mathrm{m} / \mathrm{z}$ calculated for $\left[\mathrm{C}_{19} \mathrm{H}_{19} \mathrm{OBr}+\mathrm{Na}\right]^{+} 365.0517$; found 365.0507 $[\mathrm{M}+\mathrm{Na}]^{+} .{ }^{1} \mathbf{H}$ NMR $\left(300 \mathrm{MHz}, \mathrm{CDCl}_{3}\right) \delta 7.35-7.27\left(\mathrm{~m}, 1 \mathrm{H}, \mathrm{C}_{\text {Arom }}-\mathrm{H}\right), 7.15-6.95(\mathrm{~m}$, 9H, C Arom-H), 3.68 (d, $\left.J=10.3 \mathrm{~Hz}, 1 \mathrm{H}, \mathrm{CH}_{\mathbf{a}} \mathrm{H}_{\mathrm{b}} \mathrm{Br}\right), 3.53$ (d, $J=10.3 \mathrm{~Hz}, 1 \mathrm{H}, \mathrm{CH}_{\mathrm{a}} \mathbf{H}_{\mathbf{b}} \mathrm{Br}$ ), $3.02\left(\mathrm{dd}, J=13.9,12.1 \mathrm{~Hz}, 2 \mathrm{H}, \mathrm{C}^{1} \mathbf{H}_{\mathrm{a}} \mathrm{H}_{\mathrm{b}}+\mathrm{C}^{2} \mathbf{H}_{\mathrm{a}} \mathrm{H}_{\mathrm{b}}\right), 2.81\left(\mathrm{~d}, J=13.8 \mathrm{~Hz}, 1 \mathrm{H}, \mathrm{C}^{1} \mathrm{H}_{\mathrm{a}} \mathbf{H}_{\mathbf{b}}\right)$, $2.47\left(\mathrm{~d}, J=17.3 \mathrm{~Hz}, 1 \mathrm{H}, \mathrm{C}^{2} \mathrm{H}_{\mathrm{a}} \mathbf{H}_{\mathbf{b}}\right), 1.47\left(\mathrm{~s}, 3 \mathrm{H}, \mathrm{CH}_{3}\right) .{ }^{13} \mathbf{C}\left\{{ }^{1} \mathbf{H}\right\} \mathbf{N M R}\left(75.5 \mathrm{MHz}, \mathrm{CDCl}_{3}\right)$ $\delta 215.01(\mathrm{C}=\mathrm{O}), 147.73\left(\mathrm{C}_{\text {Arom- }} \mathrm{C}\right), 139.13\left(\mathrm{C}_{\text {Arom- }} \mathrm{C}\right), 128.38(2 \mathrm{xC}$ Arom-H $), 128.27$ (2xC Arom-H), $127.28\left(\mathrm{C}_{\text {Arom }}-\mathrm{H}\right), 126.53(2 \mathrm{xC}$ Arom-H $), 126.15\left(\mathrm{C}_{\text {Arom }}-\mathrm{H}\right), 125.53\left(2 \times \mathrm{C}_{\text {Arom- }}\right.$ H), $57.87\left(\mathbf{C}-\mathrm{CH}_{2} \mathrm{Br}\right), 51.74\left(\mathrm{C}^{1} \mathrm{H}_{2}\right), 46.79\left(\mathbf{C H}_{2} \mathrm{Br}\right), 40.99\left(\mathrm{C}^{2} \mathrm{H}_{2}\right), 40.32\left(\mathbf{C}-\mathrm{CH}_{3}\right), 31.74$ $\left(\mathrm{CH}_{3}\right)$. 


\section{Synthesis and characterization of catalysts $2 b$ and $2 k-2 m$.}

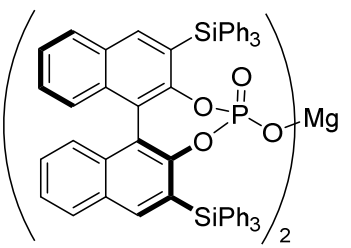

$\mathbf{2 b}$<smiles></smiles>

2)<smiles>O=P(OCc1ccccc1)(OCc1ccccc1)Oc1c([SnH2])cc2ccccc2c1-c1ccccc1</smiles>

$2 \mathrm{k}$<smiles>O=P(O)(Oc1cc2ccccc2cc1[SnH2])Oc1c([SnH2])cc2ccccc2c1-c1ccccc1</smiles>

$2 m$

Figure S 6. Chiral phosphates salts 2b, 2k-2m.

All compounds were prepared according to the reported general procedure $\mathrm{G}^{13}$

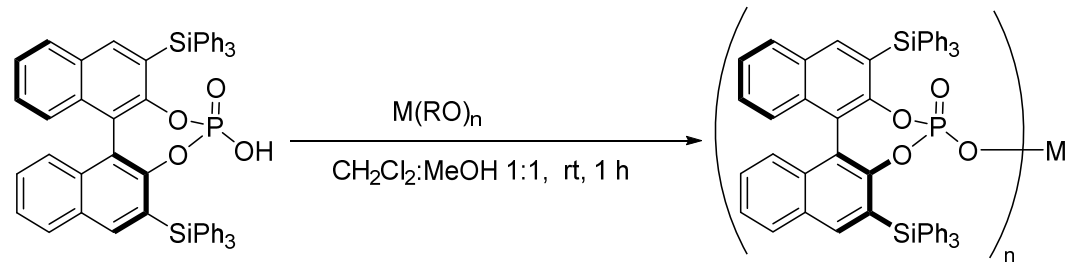

Scheme S 11. Synthesis of catalyst 2d.

General procedure $\mathrm{G}$ for the synthesis of catalysts $\mathbf{2 b}, \mathbf{2} \mathbf{k}-\mathbf{2} \mathbf{m}$.

To a flame dry test tube $\mathbf{2 a}$ and $\mathrm{Mg}(\mathrm{O} t \mathrm{Bu})_{2}, \mathrm{Ca}(\mathrm{OMe})_{2}, \mathrm{NaOMe}$ or $\mathrm{KO} t \mathrm{Bu}$ were added followed by the addition of $0.3 \mathrm{~mL}$ each of dry $\mathrm{CH}_{2} \mathrm{Cl}_{2}$ and dry $\mathrm{MeOH}$ and reaction mixture was stirred for $1 \mathrm{~h}$. Solvents were removed under reduced pressure to obtain catalysts $\mathbf{2 b}, \mathbf{2 k - 2} \mathbf{m}$ quantitatively as a white solid.

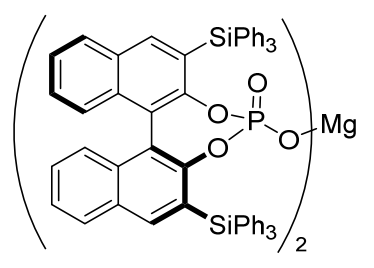

2b

Catalyst 2a (500 mg; $0.579 \mathrm{mmol})$ was dissolved in dry mixture of $\mathrm{CH}_{2} \mathrm{Cl}_{2}: \mathrm{MeOH} \mathrm{1:1}$ $(11.6 \mathrm{~mL})$ in a dry reaction tube and $\mathrm{Mg}(\mathrm{O} t \mathrm{Bu})_{2}(53 \mathrm{mg}, 0.289 \mathrm{mmol})$ was added. The reaction mixture was let stir at room temperature for $1 \mathrm{~h}$, then solvents were evaporated in

${ }^{13}$ G K. Ingle, Y. Liang, M. G. Mormino, G. Li, F. R. Fronczek, J. C. Antilla, Org. Lett. 2011, 13, 2054 2057. M. Klussmann, L. Ratjen, S. Hoffmann, V. Wakchaure, R. Goddard, B. List, Synlett, 2010, 14, 2189 2192. 
vacuum to obtain the catalyst $\mathbf{2 b}(507 \mathrm{mg}, 0.289 \mathrm{mmol}$; quant.) without purification. $[\alpha]_{D^{20}}^{20}-167\left(c=0.31, \mathrm{CHCl}_{3}\right) .{ }^{1} \mathbf{H}$ NMR $\left(300 \mathrm{MHz}, \mathrm{CDCl}_{3}\right) \delta 7.84(\mathrm{~s}, 4 \mathrm{H}), 7.62(\mathrm{~d}, J=$ $8.0 \mathrm{~Hz}, 4 \mathrm{H}), 7.58-7.48(\mathrm{~m}, 24 \mathrm{H}), 7.31-7.10(\mathrm{~m}, 44 \mathrm{H}), 7.03(\mathrm{~d}, J=8.4 \mathrm{~Hz}, 4 \mathrm{H}) .{ }^{13} \mathbf{C}\left\{{ }^{1} \mathbf{H}\right\}$ NMR (75.5 MHz, $\left.\mathrm{CDCl}_{3}\right) \delta 152.85\left(2 \mathrm{xC}_{\text {Arom-C }}\right), 152.73\left(2 \mathrm{xC}_{\text {Arom- }}\right.$ ) $), 141.99$ (4xCArom-

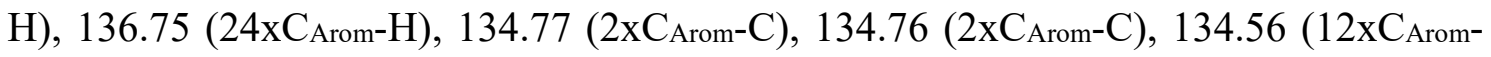

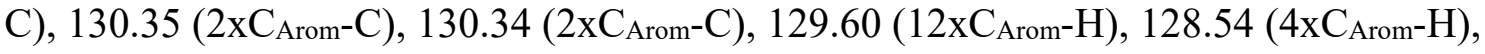
127.85 (24xCArom-H), 127.23 (4xCArom-H), 126.76 (4xCArom-H), 125.71 (2xCArom-C),

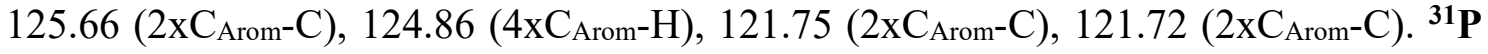
NMR $\left(122 \mathrm{MHz}, \mathrm{CDCl}_{3}\right) \delta 1.27 \mathrm{ppm}$. MALDI-LTQ-ORBITRAP: $\mathrm{m} / \mathrm{z}$ calculated for $\mathrm{C}_{112} \mathrm{H}_{80} \mathrm{O}_{8} \mathrm{P}_{2} \mathrm{Si}_{4} \mathrm{MgNa}: 1774.4182 ; \mathrm{m} / \mathrm{z}$ found for $\mathrm{C}_{112} \mathrm{H}_{80} \mathrm{O}_{8} \mathrm{P}_{2} \mathrm{Si}_{4} \mathrm{MgNa}: 1774.4166$.

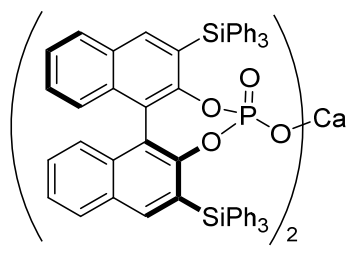

$2 \mathbf{k}$

Catalyst 2a (30 mg; $0.0347 \mathrm{mmol})$ was dissolved in dry mixture of $\mathrm{CH}_{2} \mathrm{Cl}_{2}: \mathrm{MeOH}$ 1:1 $(0.6 \mathrm{~mL})$ in a dry reaction tube and $\mathrm{Ca}(\mathrm{OMe})_{2}(1.8 \mathrm{mg} ; 0.0174 \mathrm{mmol})$ was added. The reaction mixture was let stir at room temperature for $1 \mathrm{~h}$, then solvents were evaporated in vacuum to obtain catalyst $\mathbf{2 k}(30.8 \mathrm{mg}, 0.0174 \mathrm{mmol}$; quant.) without purification.

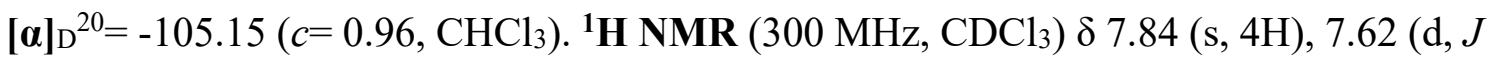
$=8.0 \mathrm{~Hz}, 4 \mathrm{H}), 7.58-7.48(\mathrm{~m}, 24 \mathrm{H}), 7.31-7.10(\mathrm{~m}, 44 \mathrm{H}), 7.03(\mathrm{~d}, J=8.4 \mathrm{~Hz}, 4 \mathrm{H})$. ${ }^{13} \mathbf{C}\left\{{ }^{1} \mathbf{H}\right\}$ NMR $\left(75.5 \mathrm{MHz}, \mathrm{CDCl}_{3}\right) \delta 152.85$ (2x Arom-C), $152.73\left(2 \times \mathrm{C}_{\text {Arom- }}-\mathrm{C}\right), 142.13$

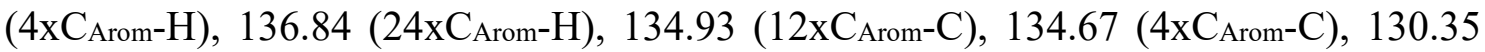
(4xC Arom-C), $129.42\left(12 \mathrm{xC}_{\text {Arom }}-\mathrm{H}\right), 128.60\left(4 \mathrm{xC}_{\text {Arom }}-\mathrm{H}\right), 127.73\left(24 \times \mathrm{C}_{\text {Arom }}-\mathrm{H}\right), 127.22$

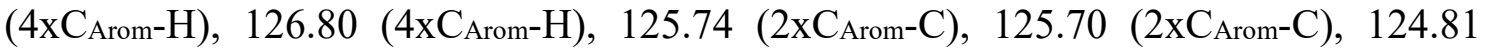
(4xCArom-H), 121.93 (2xCArom-C), 121.91 (2xCArom-C). ${ }^{31} \mathbf{P}$ NMR $\left(122 \mathrm{MHz}, \mathrm{CDCl}_{3}\right) \delta$ $1.83 \mathrm{ppm}$.

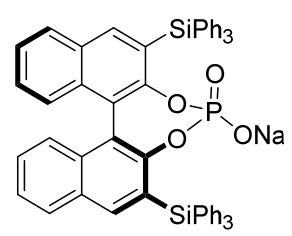

21

Catalyst 2a (30 mg; $0.0347 \mathrm{mmol})$ was dissolved in dry mixture of $\mathrm{CH}_{2} \mathrm{Cl}_{2}: \mathrm{MeOH} 1: 1$ $(0.6 \mathrm{~mL})$ in a dry reaction tube and $\mathrm{NaOMe}(1.9 \mathrm{mg}, 0.0347 \mathrm{mmol})$ was added. The 
reaction mixture was let stir at room temperature for $1 \mathrm{~h}$, then solvents were evaporated in vacuum to obtain catalyst 21 (30.8 $\mathrm{mg}, 0.0347 \mathrm{mmol}$; quant.) without purification. $[\boldsymbol{\alpha}]_{\mathrm{D}}{ }^{20}=$ -159.23 $\left(c=0.07, \mathrm{CHCl}_{3}\right) .{ }^{1} \mathbf{H}$ NMR $\left(300 \mathrm{MHz} \mathrm{CDCl}_{3}\right) \delta 7.84(\mathrm{~s}, 4 \mathrm{H}), 7.62(\mathrm{~d}, J=8.0$ $\mathrm{Hz}, 4 \mathrm{H}), 7.58-7.48(\mathrm{~m}, 24 \mathrm{H}), 7.31-7.10(\mathrm{~m}, 44 \mathrm{H}), 7.03(\mathrm{~d}, J=8.4 \mathrm{~Hz}, 4 \mathrm{H}) .{ }^{13} \mathbf{C}\left\{{ }^{1} \mathbf{H}\right\}$ NMR $\left(75.5 \mathrm{MHz}, \mathrm{CDCl}_{3}\right) \delta 153.20\left(1 \mathrm{xC}_{\text {Arom- }}\right.$ - $), 153.08\left(1 \mathrm{xC}_{\text {Arom- }}\right), 141.83\left(2 \mathrm{xC}_{\text {Arom- }}\right.$

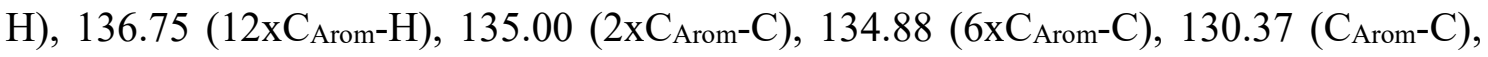
$129.14\left(6 \times \mathrm{C}_{\text {Arom }}-\mathrm{H}\right), 128.59\left(2 \mathrm{xC}_{\text {Arom }}-\mathrm{H}\right), 127.58\left(12 \mathrm{xC}_{\text {Arom }}-\mathrm{H}\right), 127.13\left(2 \times \mathrm{C}_{\text {Arom }}-\mathrm{H}\right)$, 127.03 (2xCArom-H), 126.17 (2x- $\left.\mathrm{C}_{\text {Arom- }} \mathrm{C}\right), 126.13$ (C $\left.\mathrm{C}_{\text {Arom-C }}\right), 124.66$ (2xC $\left.\mathrm{C}_{\text {Arom }}-\mathrm{H}\right), 121.78$ $\left(\mathrm{C}_{\text {Arom-C }}\right), 121.76\left(\mathrm{C}_{\text {Arom-C }}\right) .{ }^{31} \mathbf{P}$ NMR $\left(122 \mathrm{MHz}, \mathrm{CDCl}_{3}\right) \delta 1.49 \mathrm{ppm}$.

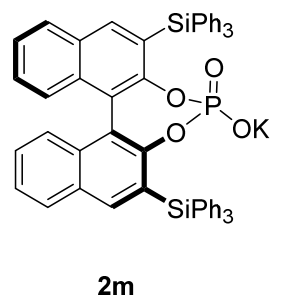

Catalyst 2a (30 mg; $0.0347 \mathrm{mmol}$ ) was dissolved in dry mixture of $\mathrm{CH}_{2} \mathrm{Cl}_{2}: \mathrm{MeOH} \mathrm{1:1}$ $(0.6 \mathrm{~mL})$ in a dry reaction tube and $\mathrm{KO} t \mathrm{Bu}(3.9 \mathrm{mg}, 0.0347 \mathrm{mmol})$ was added. The reaction mixture was let stir at room temperature for $1 \mathrm{~h}$, then solvents were evaporated in vacuumto obtain catalyst $\mathbf{2 m}\left(31 \mathrm{mg}, 0.035 \mathrm{mmol}\right.$; quant.) without purification. $[\boldsymbol{\alpha}]_{\mathrm{D}}{ }^{20}=$ $146.86\left(c=0.17, \mathrm{CHCl}_{3}\right) .{ }^{1} \mathbf{H}$ NMR $\left(300 \mathrm{MHz}, \mathrm{CDCl}_{3}\right) \delta 7.84(\mathrm{~s}, 4 \mathrm{H}), 7.62(\mathrm{~d}, J=8.0 \mathrm{~Hz}$, 4H), $7.58-7.48(\mathrm{~m}, 24 \mathrm{H}), 7.31-7.10(\mathrm{~m}, 44 \mathrm{H}), 7.03(\mathrm{~d}, J=8.4 \mathrm{~Hz}, 4 \mathrm{H}) .{ }^{13} \mathbf{C}\left\{{ }^{1} \mathbf{H}\right\} \mathbf{N M R}$ $\left(75.5 \mathrm{MHz}, \mathrm{CDCl}_{3}\right) \delta 152.28$ (C $\left.\mathrm{C}_{\text {Arom-C }} \mathrm{C}\right), 152.16$ (CArom-C), 140.78 (2xCArom-H), 135.76 (12xCArom-H), 133.79 (2xC Arom-C), 133.57 (6x- Arom-C $_{\text {Arom }}, 129.20$ (2xCArom-C), 128.27 $\left(6 x C_{\text {Arom }}-\mathrm{H}\right), 127.61\left(2 \mathrm{xC}_{\text {Arom }}-\mathrm{H}\right), 126.61\left(12 \mathrm{xC}_{\text {Arom }}-\mathrm{H}\right), 126.23\left(2 \mathrm{xC}_{\text {Arom }}-\mathrm{H}\right), 125.53$ (2xC $\left.\mathrm{Arom}_{\text {Aro }} \mathrm{H}\right), 125.12$ ( $\left.\mathrm{C}_{\text {Arom-C }}\right), 125.08$ ( $\left.\mathrm{C}_{\text {Arom-C }}\right), 123.75\left(2 \mathrm{xC}_{\text {Arom }}-\mathrm{H}\right), 120.99\left(\mathrm{C}_{\text {Arom- }}-\mathrm{C}\right)$, 120.96. (C $\mathrm{C}_{\text {Arom-C }}$ ). ${ }^{31} \mathbf{P}$ NMR $\left(122 \mathrm{MHz}, \mathrm{CDCl}_{3}\right) \delta 1.81 \mathrm{ppm}$. 


\section{Non linear effect study.}

Following the general procedure G, catalysts with different enantiomeric ratios were prepared using different ratios of $R$ and $S$ enantiomers of the corresponding chiral phosphoric acids (Scheme S 12).

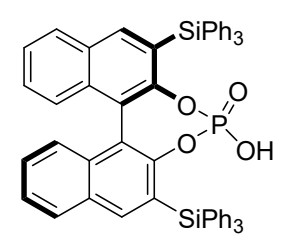

(S)-2a<smiles>O=P(O)(O)Oc1c(S)cc2ccccc2c1OP(=O)(O)O</smiles>

(R)-2a

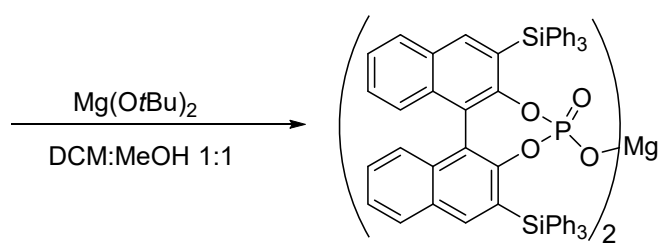

Scheme S 12. Synthesis of catalyst using different ratios of chiral phosphoric acid enantiomers.

In order to verify the formation of catalysts, ${ }^{31} \mathrm{P}-\mathrm{NMR}$ spectra in $\mathrm{CDCl}_{3}$ were recorded, observing two signals that have a similar proportion to the ratio of phosphoric acid enantiomers used in each case.

With these catalysts in hand, the model reaction was carried out under the conditions established in general procedure $\mathrm{F}$, the final product was purified, and the enantiomeric excess was measured by HPLC (Table S 6).

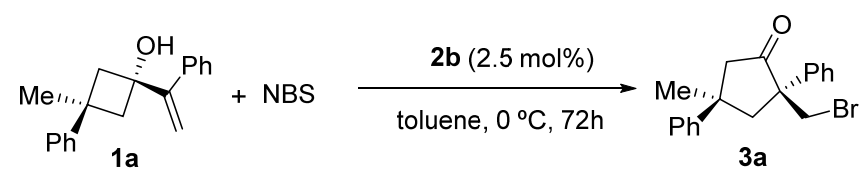

Table S 5. Non linear effect results. ${ }^{\mathrm{a}}$

\begin{tabular}{cccc}
\hline Entry & $\begin{array}{c}\text { er catalyst } \\
(\boldsymbol{R}):(\boldsymbol{S})\end{array}$ & ${ }^{31} \mathbf{P}-\mathbf{N M R}$ signals $(\mathbf{p p m})$ & ${\text { ee product } \mathbf{\%}^{\mathbf{b}}}^{\mathbf{3}}$ \\
\hline 1 & $50: 50(0)$ & $1.84,1.28$ & 6 \\
2 & $60: 40(20)$ & $1.76,1.26$ & 30 \\
3 & $70: 30(40)$ & $1.94,1.46$ & 46 \\
4 & $80: 20(60)$ & $1.66,1.09$ & 56 \\
5 & $90: 10(80)$ & $1.65,1.21$ & 72 \\
6 & $100: 0(100)$ & 1.27 & 92 \\
\hline
\end{tabular}

${ }^{a}$ Reactions carried out with $0.10 \mathrm{mmol}$ of $\mathbf{1 a}, 0.11 \mathrm{mmol}$ of NBS and $2.5 \mathrm{~mol} \%$ of $\mathbf{2 b}$ with different ee ratios in toluene $(0.2 \mathrm{M})$ at $0{ }^{\circ} \mathrm{C}$ for $72 \mathrm{~h}$. The numbers between parenthesis indicate the enaniomeric excess of catalyst $\mathbf{2} \mathbf{b}^{\mathrm{b}}$ Product $\mathbf{3 a}$ was isolated and ee was determined by HPLC on a chiral stationary phase. 


\section{Hammett study}

Hammett study was carried out to analyze the effect of the substitution in para-position of the aromatic ring. The analysis was performed based on competition experiments between the unsubstituted cyclobutanol 1a and its para-substituted equivalent $\mathbf{1 h}-\mathbf{k}$. These competition experiments were carried out with $0.05 \mathrm{mmol}$ of $\mathbf{1 a}, 0.05 \mathrm{mmol}$ of $\mathbf{1 h}$ $1 \mathbf{k}, 0.055 \mathrm{mmol}$ of NBS and $2.5 \mathrm{~mol} \%(0.00125 \mathrm{mmol})$ of catalyst $\mathbf{2 b}$ in toluene $(0.25$ $\mathrm{mL}$ ) at $0{ }^{\circ} \mathrm{C}$ for $45 \mathrm{~h}$. The experiments were quenched with aq. std. $\mathrm{Na}_{2} \mathrm{~S}_{2} \mathrm{O}_{3}(1 \mathrm{~mL})$ and the products were extracted with $\mathrm{Et}_{2} \mathrm{O}(3 \times 2 \mathrm{~mL})$. At this point, $0.05 \mathrm{mmol}$ of 1,3,5trimethoxybenzene was added as standard for subsequent NMR analysis. The mixture was dried with $\mathrm{Na}_{2} \mathrm{SO}_{4}$, filtered, and concentrated under vacuum.

These experiments were stopped at $45 \mathrm{~h}$ and the product ratio $\left[\mathbf{p}_{\mathbf{X}}\right] /\left[\mathbf{p}_{\mathbf{H}}\right]$ was calculated by ${ }^{1} \mathrm{H}-\mathrm{NMR}$ using 1,3,5-trimethoxybenzene as standard. This experimental ratio was approximated to rate constants ratio $\mathbf{k}_{\mathbf{X}} / \mathbf{k}_{\mathbf{H}} \cdot{ }^{14}$

Table S 6. Data obtained from competition experiments in Hammett study.
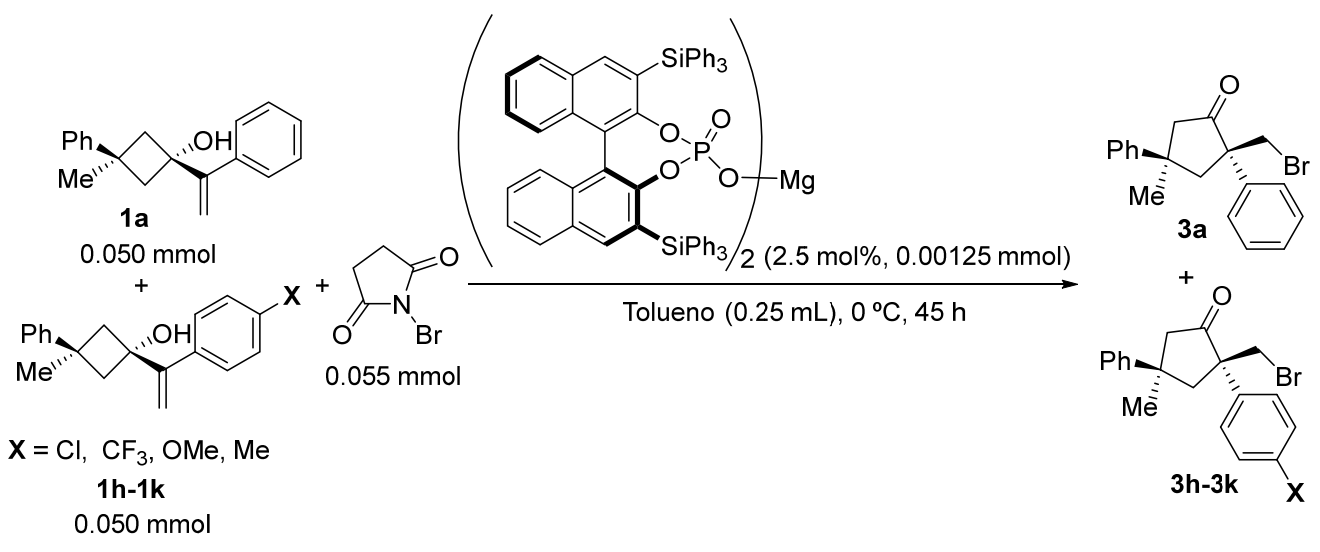

\begin{tabular}{ccccccc}
\hline Entry & $\mathbf{1}$ & Substituent $(\mathbf{X})$ & $\boldsymbol{\sigma}_{\mathbf{p}}{ }^{[\mathbf{a}]}$ & ${\boldsymbol{\boldsymbol { \sigma } _ { \mathbf { p } }}}^{+[\mathbf{b}]}$ & $\mathbf{k}_{[\mathbf{X}]} / \mathbf{k}_{[\mathbf{H}]}$ & $\log [\mathbf{k x}] /[\mathbf{k H}]$ \\
\hline $\mathbf{1}$ & $\mathbf{1 h}$ & $\mathbf{M e}$ & $-0,17$ & $-0,31$ & 5,25 & 0,72 \\
$\mathbf{2}^{[\mathbf{c}]}$ & $\mathbf{1 i}$ & $\mathbf{O M e}$ & $-0,27$ & $-0,78$ & 9,71 & 0,99 \\
$\mathbf{3}$ & $\mathbf{1 j}$ & $\mathbf{C l}$ & 0,23 & 0,11 & 0,6 & $-0,22$ \\
$\mathbf{4}$ & $\mathbf{1 k}$ & $\mathbf{C F}_{3}$ & 0,54 & 0,61 & 0,08 & $-1,08$
\end{tabular}

${ }^{\mathrm{a}}$ Hammett constants for para substituents with inductive effect. ${ }^{15 \mathrm{~b}}$ Hammett constants for para substituents with resonance effect. ${ }^{15} .{ }^{\mathrm{c}}$ Epoxide $4 \mathbf{i}$ was also considered in $\left[\mathbf{p}_{\mathbf{x}}\right]$ in this case.

${ }^{14}$ F. Romanov-Michailidis, M. Romanova-Michaelides, M. Pupier and A. Alexakis, Chem. Eur. J. 2015, 21, 5561-5583.

${ }^{15}$ Hammett, L. P. J. Am. Chem. Soc. 1937, 59, 96-103. 
The logarithms of the rate constants ratios were plotted out with the Hammett substituent constants $\left(\sigma\right.$ and $\left.\sigma^{+}\right)$for each substituent, ${ }^{16}$ according to the known Hammett relationship [Eq. (1)].

$\log \left(\frac{k_{X}}{k_{H}}\right)=\rho \sigma$

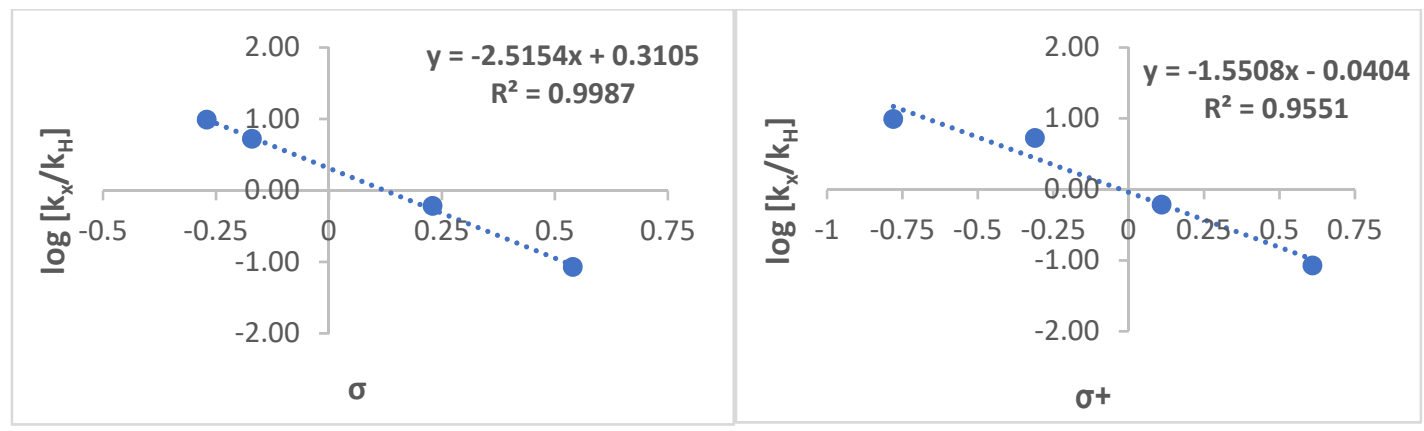

Figure S 7. Hammett plots for $\sigma$ and $\sigma^{+}$.

${ }^{16}$ C. Hansch, A. Leo, R. W. Taft, Chem. Rev. 1991, 91, 165-195. 


\section{X-Ray analysis of product $3 \mathbf{j}$.}
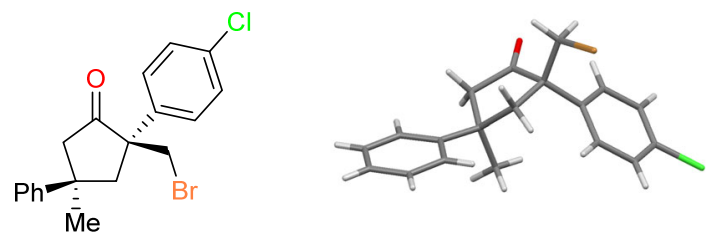

3j

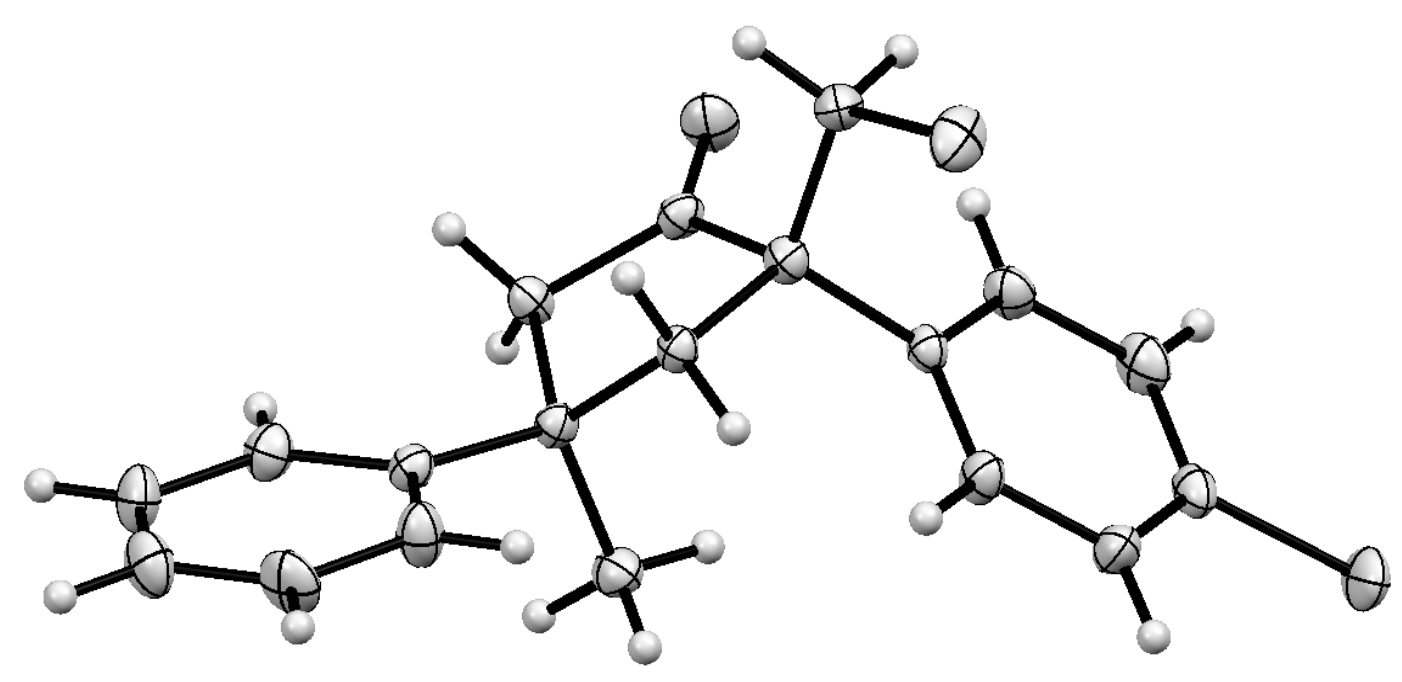

Figure S 8. X-Ray structure and ORTEP diagram (50\% probability) for $\mathbf{3 j}$.

The enantiopure sample of compound 3j was obtained following General Procedure F. Crystals of the sample were obtained after slow evaporation of a mixture of Hexane/Ethyl acetate $1: 1$ in a screw-top vial.

Crystal Data for $\mathrm{C}_{19} \mathrm{H}_{18} \mathrm{BrClO}(M=377.69 \mathrm{~g} / \mathrm{mol})$ (3j, CCDC number 2091677): orthorhombic, space group P212121 (no. 19), $a=6.83880$ (10) $\AA, b=13.6467$ (2) $\AA, c=$ 17.6849(2) $\AA, V=1650.48(4) \AA^{3}, Z=4, T=150.01(10) \mathrm{K}, \mu(\mathrm{CuK} \alpha)=4.863 \mathrm{~mm}^{-1}$, Dcalc $=1.520 \mathrm{~g} / \mathrm{cm}^{3}, 15576$ reflections measured $\left(8.18^{\circ} \leq 2 \Theta \leq 137.86^{\circ}\right), 3075$ unique $\left(R_{\text {int }}=\right.$ $\left.0.0537, \mathrm{R}_{\text {sigma }}=0.0373\right)$ which were used in all calculations. The final $R_{1}$ was 0.0294 (>2sigma(I)) and $w R_{2}$ was 0.0713 (all data). 
Table S 7. Crystal data and structure refinement for $\mathbf{3 j}$.

Identification code

Empirical formula

$\mathrm{C}_{19} \mathrm{H}_{18} \mathrm{BrClO}$

Formula weight

377.69

Temperature/K

$150.01(10)$

Crystal system

orthorhombic

Space group

$\mathrm{P} 2{ }_{1} 2_{1} 2_{1}$

$\mathrm{a} / \AA$

$6.83880(10)$

$\mathrm{b} / \AA$

$13.6467(2)$

$\mathrm{c} / \AA$

$17.6849(2)$

$\alpha /{ }^{\circ}$

90.00

$\beta /{ }^{\circ}$

90.00

$\gamma /{ }^{\circ}$

90.00

Volume $/ \AA^{3}$

$1650.48(4)$

Z

$\rho_{\text {calc } g / \mathrm{cm}^{3}}$

$\mu / \mathrm{mm}^{-1}$

4.863

$\mathrm{F}(000)$

768.0

Crystal size $/ \mathrm{mm}^{3}$

$0.223 \times 0.051 \times 0.043$

Radiation

$\mathrm{CuK} \alpha(\lambda=1.54184)$

$2 \Theta$ range for data collection/ ${ }^{\circ}$

8.18 to 137.86

Index ranges

$-8 \leq \mathrm{h} \leq 8,-16 \leq \mathrm{k} \leq 16,-21 \leq 1 \leq 18$

Reflections collected

15576

Independent reflections

$3075\left[\mathrm{R}_{\text {int }}=0.0537, \mathrm{R}_{\text {sigma }}=0.0373\right]$

Data/restraints/parameters

$3075 / 0 / 200$

Goodness-of-fit on $\mathrm{F}^{2}$

1.058

Final R indexes $[\mathrm{I}>=2 \sigma(\mathrm{I})]$

$\mathrm{R}_{1}=0.0294, \mathrm{wR}_{2}=0.0691$

Final $\mathrm{R}$ indexes [all data] $\mathrm{R}_{1}=0.0323, \mathrm{wR}_{2}=0.0713$

Largest diff. peak/hole / e $\AA^{-3}$

$0.33 /-0.36$

Flack parameter

$-0.031(19)$ 
Table S 8. Fractional Atomic Coordinates $\left(\times 10^{4}\right)$ and Equivalent Isotropic Displacement Parameters $\left(\AA^{2} \times 10^{3}\right)$ for $3 \mathbf{j} . U_{\text {eq }}$ is defined as $1 / 3$ of of the trace of the orthogonalised $U_{\text {IJ }}$ tensor.

\begin{tabular}{|c|c|c|c|c|}
\hline Atom & $x$ & $y$ & $z$ & $\mathrm{U}(\mathrm{eq})$ \\
\hline $\mathrm{Br} 01$ & $4630.2(5)$ & $3194.5(2)$ & $5220.14(18)$ & $28.87(10)$ \\
\hline $\mathrm{Cl} 100$ & $5089.4(13)$ & $6229.1(6)$ & $8115.2(3)$ & $31.33(19)$ \\
\hline $\mathrm{O} 003$ & $-562(3)$ & $5503.4(16)$ & $4859.1(12)$ & $25.9(5)$ \\
\hline C004 & $4661(5)$ & $6448.8(19)$ & $3131.4(14)$ & $16.6(5)$ \\
\hline $\mathrm{C} 005$ & $1071(4)$ & $5617(2)$ & $4613.1(15)$ & $17.4(6)$ \\
\hline $\mathrm{C} 006$ & $4411(4)$ & $5305.4(19)$ & $4276.7(13)$ & $15.4(5)$ \\
\hline $\mathrm{C} 007$ & $5389(5)$ & $5650(2)$ & $5907.8(15)$ & $20.2(6)$ \\
\hline $\mathrm{C} 008$ & $3829(4)$ & $6307(2)$ & $3926.8(15)$ & $15.9(5)$ \\
\hline C009 & $3476(4)$ & $5447(2)$ & $5708.3(15)$ & $16.0(6)$ \\
\hline C00A & $3591(5)$ & $6916(2)$ & $2569.6(16)$ & $22.4(6)$ \\
\hline C00B & $2930(4)$ & $5087(2)$ & $4908.3(15)$ & $16.2(6)$ \\
\hline $\mathrm{C} 00 \mathrm{C}$ & $4514(5)$ & $7168(2)$ & $4418.2(15)$ & $22.1(6)$ \\
\hline C00D & $4397(5)$ & $7090(2)$ & $1860.9(17)$ & $27.5(7)$ \\
\hline C00E & $2504(5)$ & $5769(2)$ & $7005.0(17)$ & $25.9(7)$ \\
\hline $\mathrm{COOF}$ & $4431(5)$ & $5953(2)$ & $7184.1(15)$ & $22.1(6)$ \\
\hline $\mathrm{C} 00 \mathrm{G}$ & $5893(5)$ & $5895(2)$ & $6649.2(16)$ & $23.5(7)$ \\
\hline $\mathrm{COOH}$ & $2379(5)$ & $4004(2)$ & $4955.3(15)$ & $20.6(6)$ \\
\hline $\mathrm{COOI}$ & $2040(5)$ & $5511(2)$ & $6264.3(17)$ & $21.9(6)$ \\
\hline $\mathrm{COOJ}$ & $1590(4)$ & $6248(2)$ & $3939.9(15)$ & $17.5(6)$ \\
\hline $\mathrm{COOK}$ & $6581(5)$ & $6176(3)$ & $2974.3(17)$ & $25.3(7)$ \\
\hline C00L & $7400(5)$ & $6357(3)$ & $2267.1(18)$ & $29.9(7)$ \\
\hline C00M & $6308(5)$ & $6812(3)$ & $1712.9(16)$ & $28.1(7)$ \\
\hline
\end{tabular}


Table S 9. Anisotropic Displacement Parameters $\left(\AA^{2} \times 10^{3}\right)$ for 3j. The Anisotropic displacement factor exponent takes the form: $-2 \pi^{2}\left[\mathrm{~h}^{2} \mathrm{a}^{* 2} \mathrm{U}_{11}+2 \mathrm{hka} \mathrm{b}^{*} \mathrm{U}_{12}+\ldots\right]$.

\begin{tabular}{lrrrrrr} 
Atom & \multicolumn{1}{c}{$\mathrm{U}_{11}$} & \multicolumn{1}{c}{$\mathrm{U}_{22}$} & \multicolumn{1}{l}{$\mathrm{U}_{33}$} & \multicolumn{1}{l}{$\mathrm{U}_{23}$} & \multicolumn{1}{c}{$\mathrm{U}_{13}$} & \multicolumn{1}{c}{$\mathrm{U}_{12}$} \\
Br01 & $34.31(17)$ & $21.47(15)$ & $30.83(15)$ & $-1.30(13)$ & $-7.76(14)$ & $9.04(14)$ \\
C100 & $43.7(5)$ & $37.5(4)$ & $12.8(3)$ & $-3.2(3)$ & $2.7(3)$ & $-7.9(4)$ \\
O003 & $16.2(11)$ & $33.3(11)$ & $28.0(10)$ & $3.8(9)$ & $2.8(9)$ & $1.2(9)$ \\
C004 & $16.2(12)$ & $18.3(12)$ & $15.4(11)$ & $-2.1(9)$ & $0.6(12)$ & $0.5(12)$ \\
C005 & $14.0(13)$ & $20.8(14)$ & $17.3(13)$ & $-1.4(10)$ & $-0.4(10)$ & $0.1(11)$ \\
C006 & $14.2(14)$ & $18.6(13)$ & $13.3(11)$ & $-0.8(10)$ & $1.2(10)$ & $1.0(12)$ \\
C007 & $17.7(13)$ & $28.8(14)$ & $14.2(11)$ & $-2.3(10)$ & $2.9(11)$ & $-1.1(13)$ \\
C008 & $14.0(13)$ & $19.1(14)$ & $14.8(12)$ & $-0.6(10)$ & $0.1(10)$ & $0.1(12)$ \\
C009 & $18.2(15)$ & $16.6(13)$ & $13.2(12)$ & $-0.1(10)$ & $1.8(11)$ & $0.6(11)$ \\
C00A & $22.6(15)$ & $25.3(16)$ & $19.3(13)$ & $-1.8(12)$ & $0.5(11)$ & $3.0(14)$ \\
C00B & $14.7(14)$ & $18.3(14)$ & $15.5(13)$ & $-1.6(10)$ & $2.5(11)$ & $1.1(10)$ \\
C00C & $25.4(15)$ & $21.7(14)$ & $19.2(12)$ & $-2.7(10)$ & $1.4(12)$ & $-3.4(13)$ \\
C00D & $32.4(18)$ & $34.5(17)$ & $15.4(12)$ & $2.3(11)$ & $0.3(13)$ & $2.8(14)$ \\
C00E & $27.6(17)$ & $30.7(17)$ & $19.4(14)$ & $-1.6(12)$ & $10.0(13)$ & $0.3(14)$ \\
C00F & $32.6(18)$ & $19.4(13)$ & $14.1(12)$ & $-0.6(10)$ & $2.8(12)$ & $-2.6(14)$ \\
C00G & $23.1(17)$ & $29.9(16)$ & $17.6(14)$ & $-0.8(12)$ & $-1.4(11)$ & $-6.2(13)$ \\
C00H & $22.3(15)$ & $19.9(14)$ & $19.6(13)$ & $0.6(10)$ & $-2.3(11)$ & $-3.8(12)$ \\
C00I & $17.1(15)$ & $27.0(16)$ & $21.7(14)$ & $2.9(12)$ & $3.2(12)$ & $-0.9(13)$ \\
C00J & $14.3(14)$ & $17.5(14)$ & $20.6(13)$ & $3.5(11)$ & $1.0(10)$ & $2.1(11)$ \\
C00K & $21.0(16)$ & $35.1(18)$ & $19.8(14)$ & $4.8(12)$ & $2.3(12)$ & $4.7(14)$ \\
C00L & $23.4(17)$ & $39.6(19)$ & $26.8(15)$ & $3.2(14)$ & $12.3(14)$ & $-1.6(15)$ \\
C00M & $34.0(18)$ & $32.2(16)$ & $18.1(13)$ & $1.3(14)$ & $7.6(12)$ & $2.1(17)$
\end{tabular}


${ }^{1} \mathrm{H},{ }^{13} \mathrm{C}\left\{{ }^{1} \mathrm{H}\right\}(75.5 \mathrm{MHz}),{ }^{19} \mathrm{~F}$ and ${ }^{31} \mathrm{P}$ NMR spectra of cyclobutanones, $1 \mathrm{a}-$ n, 3a-n, 4a, $4 \mathrm{i}$ and catalysts $2 \mathrm{a}-\mathrm{b}$ and $2 \mathrm{k}-\mathrm{m}$ in $\mathrm{CDCl}_{3}$ ).

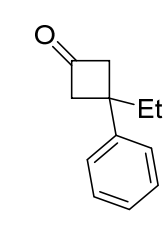

II
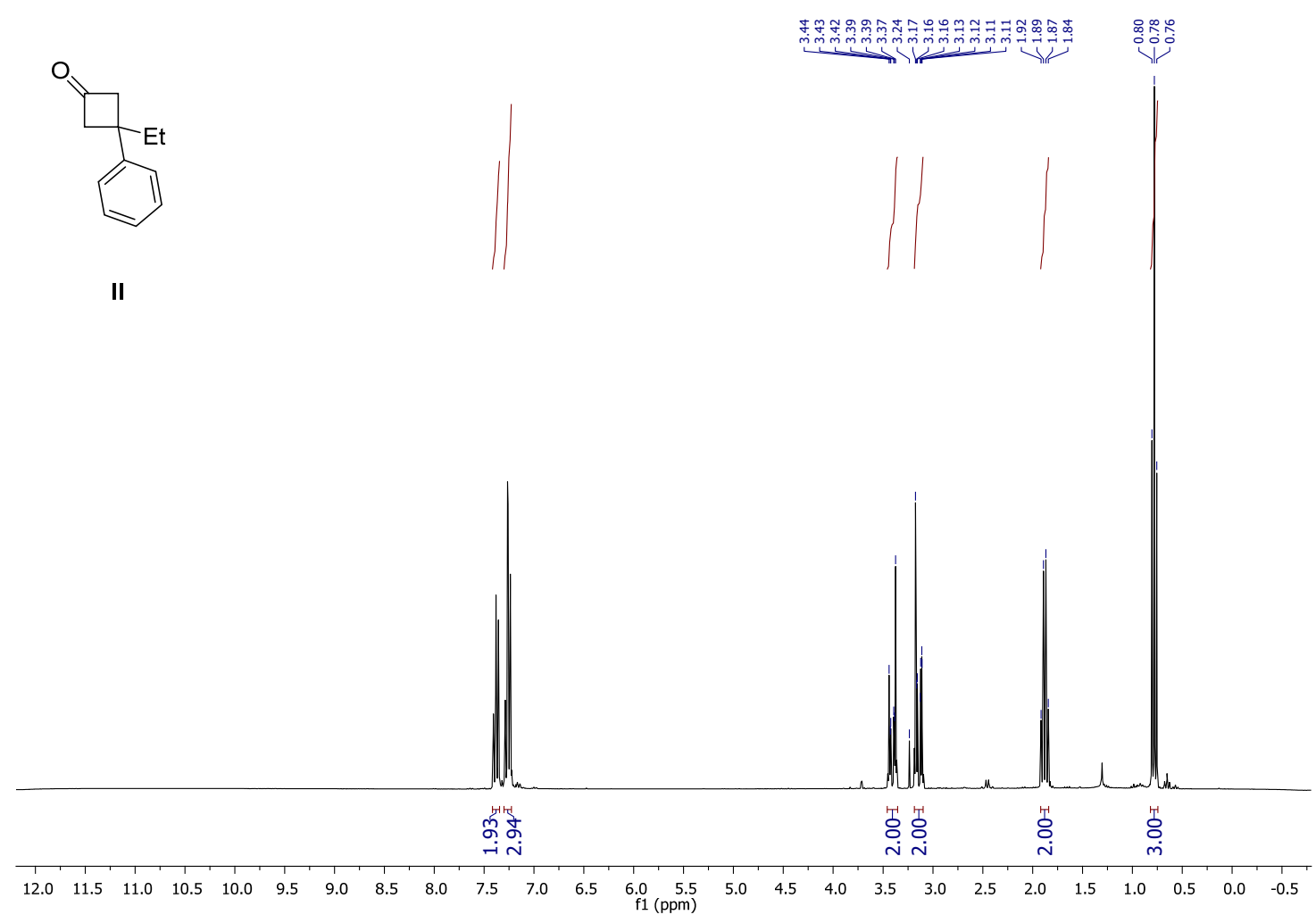

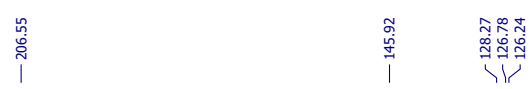
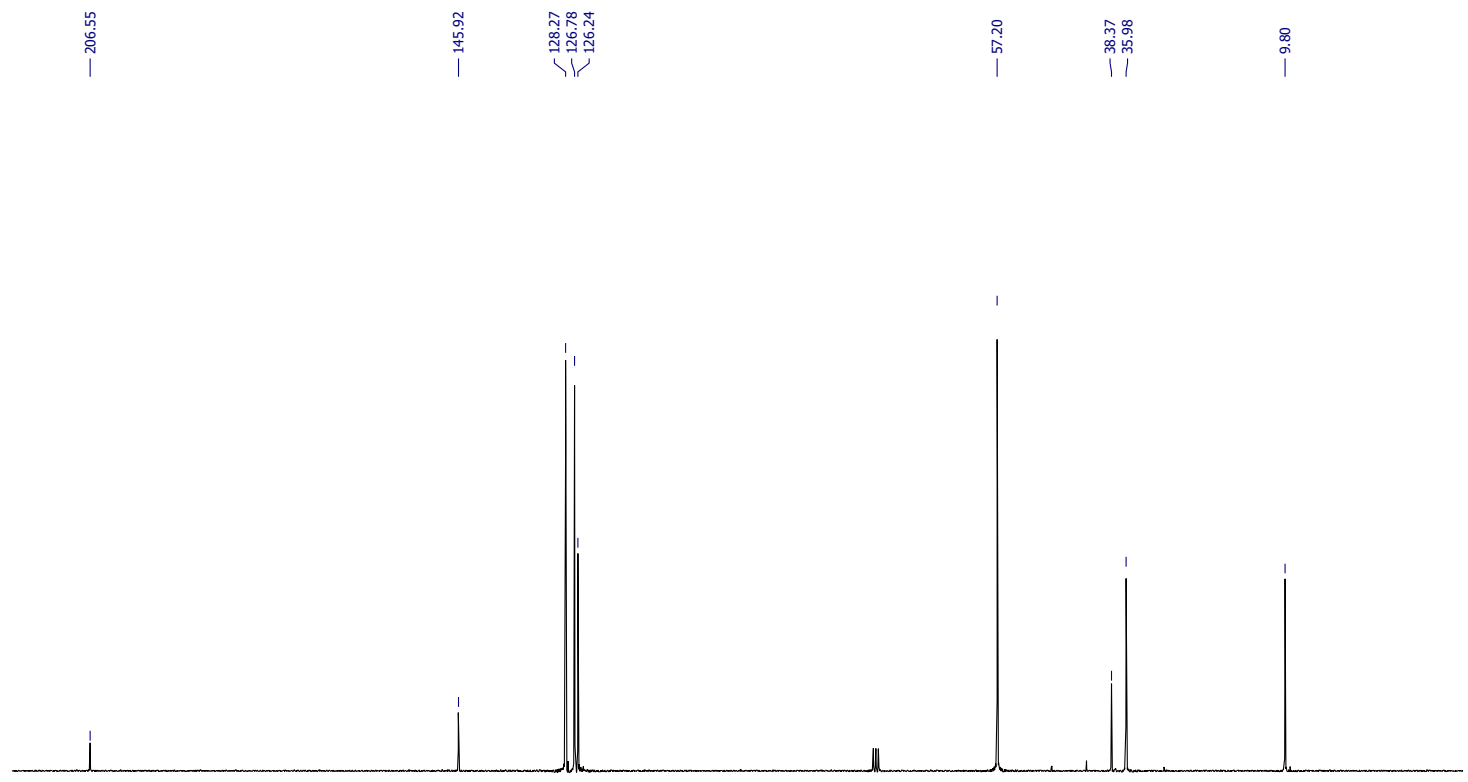

$\begin{array}{llllllllllllllllllllllllll}210 & 200 & 190 & 180 & 170 & 160 & 150 & 140 & 130 & 120 & 110 & \begin{array}{c}100 \\ \mathrm{f} 1(\mathrm{ppm})\end{array} & 90 & 80 & 70 & 60 & 50 & 40 & 30 & 20 & 10 & 0 & -10\end{array}$

Figure S9. ${ }^{1} \mathrm{H}-\mathrm{NMR}\left(300 \mathrm{MHz}, \mathrm{CDCl}_{3}\right)$ and ${ }^{13} \mathrm{C}\left\{{ }^{1} \mathrm{H}\right\}-\mathrm{NMR}\left(75.5 \mathrm{MHz}, \mathrm{CDCl}_{3}\right)$ of compound II 


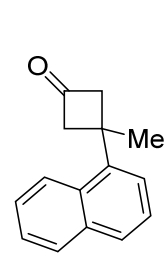

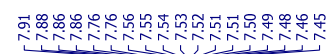

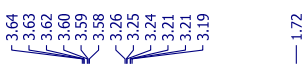

III
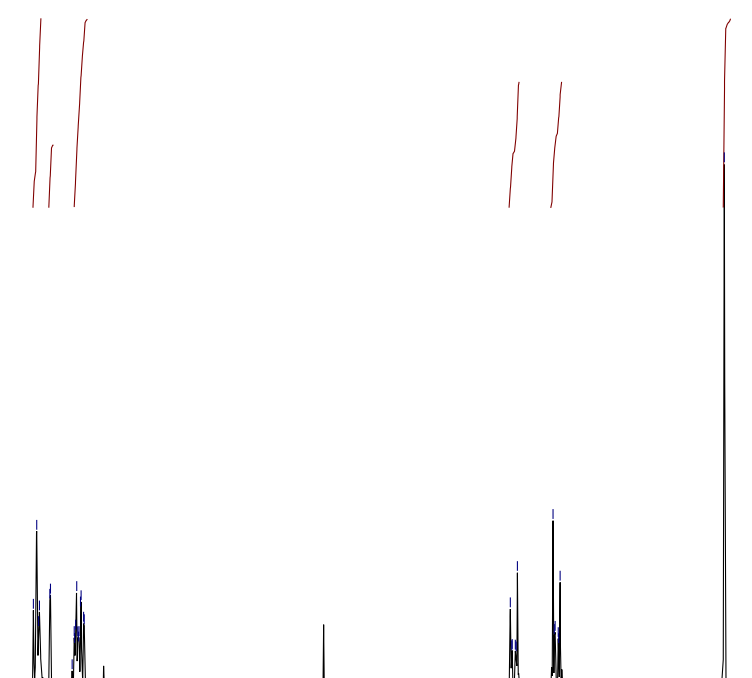

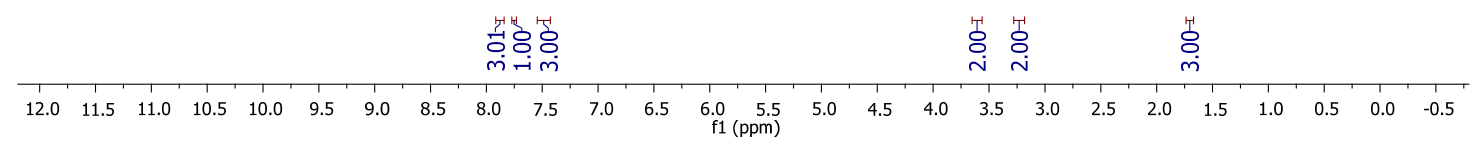

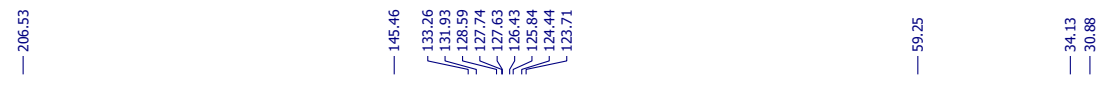

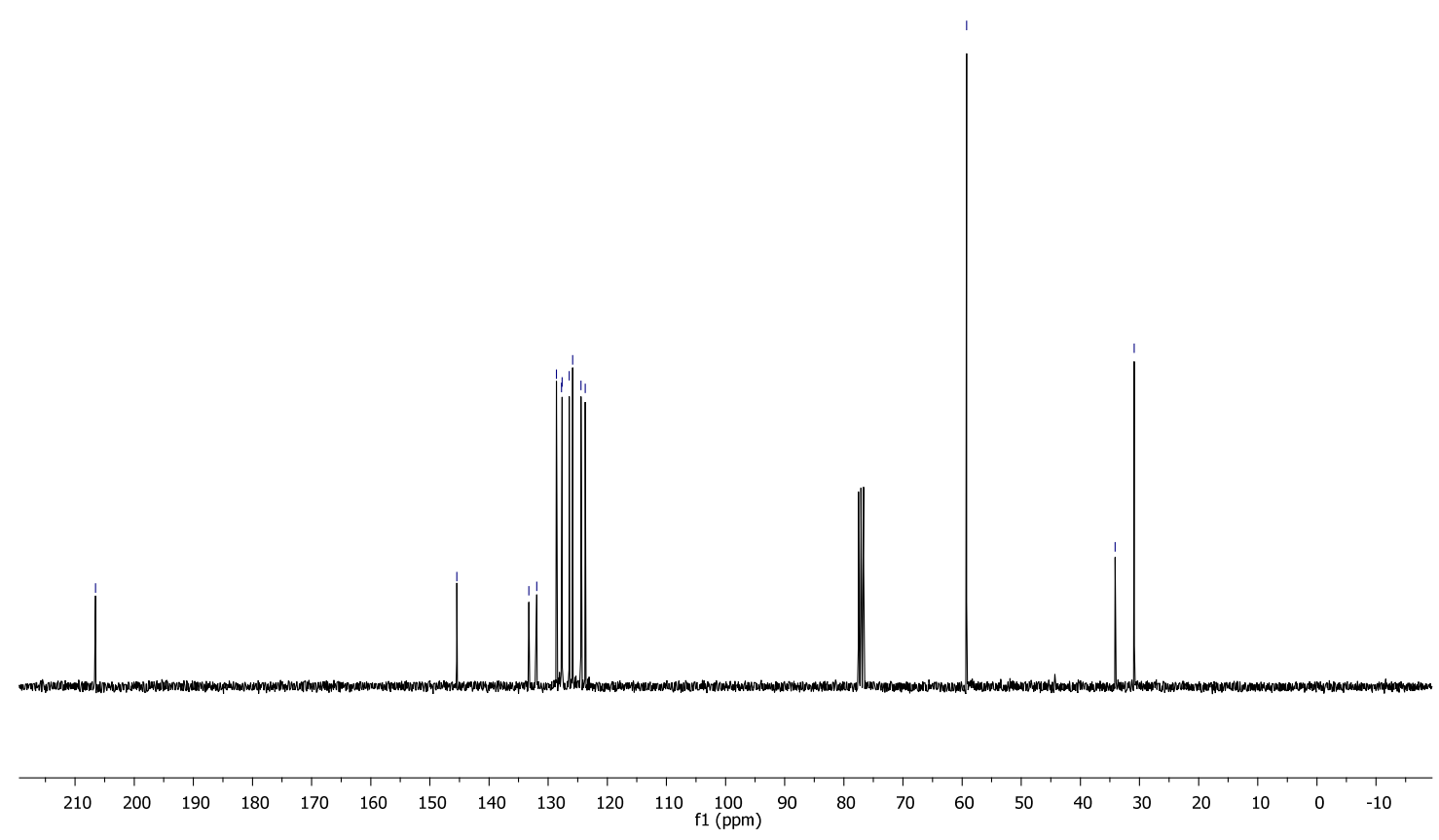

Figure S10. ${ }^{1} \mathrm{H}-\mathrm{NMR}\left(300 \mathrm{MHz}, \mathrm{CDCl}_{3}\right)$ and ${ }^{13} \mathrm{C}\left\{{ }^{1} \mathrm{H}\right\}-\mathrm{NMR}\left(75.5 \mathrm{MHz}, \mathrm{CDCl}_{3}\right)$ of compound III 

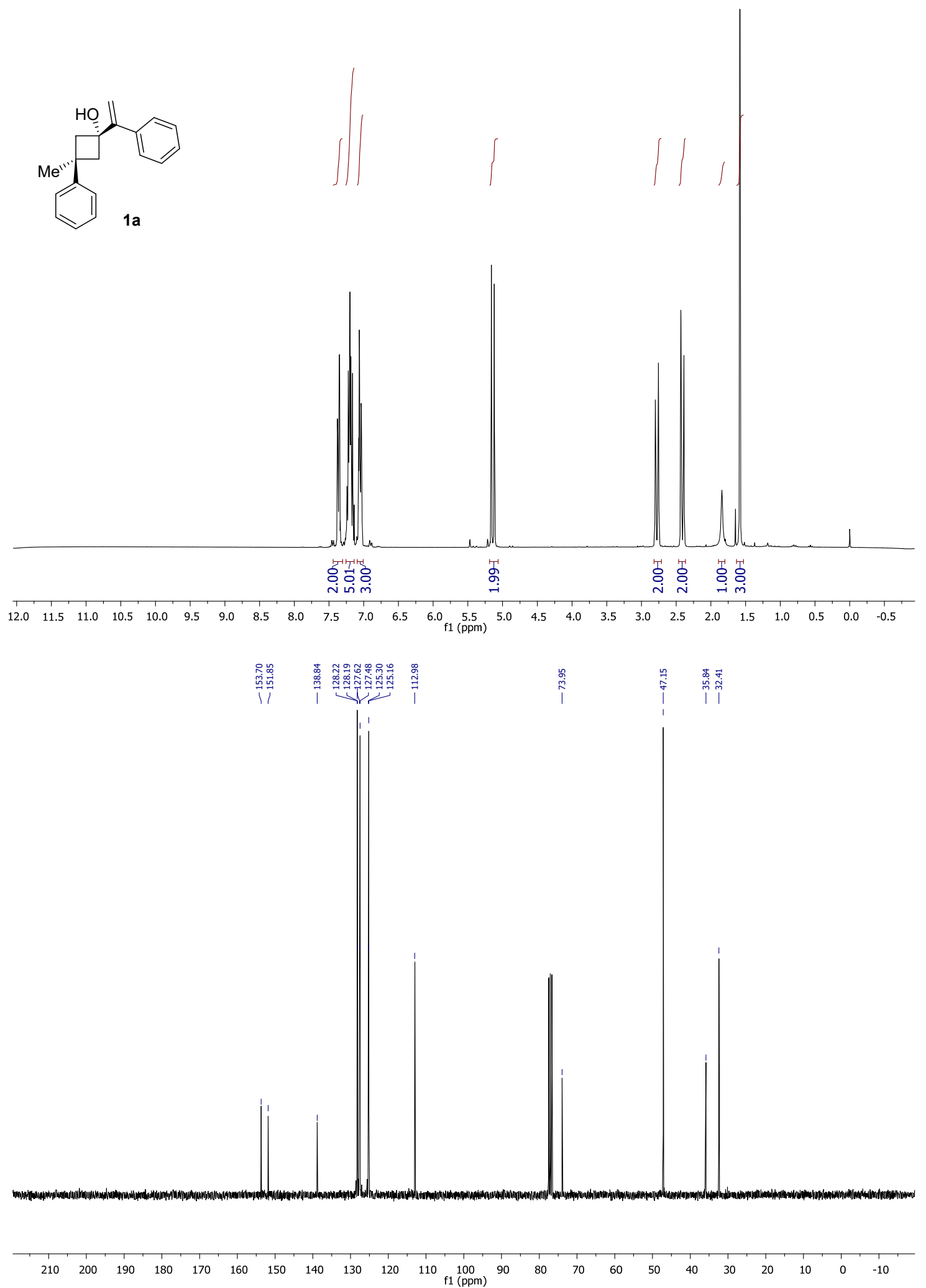

Figure S11. ${ }^{1} \mathrm{H}-\mathrm{NMR}\left(300 \mathrm{MHz}, \mathrm{CDCl}_{3}\right)$ and ${ }^{13} \mathrm{C}\left\{{ }^{1} \mathrm{H}\right\}$-NMR $\left(75.5 \mathrm{MHz}, \mathrm{CDCl}_{3}\right)$ of compound 1a 

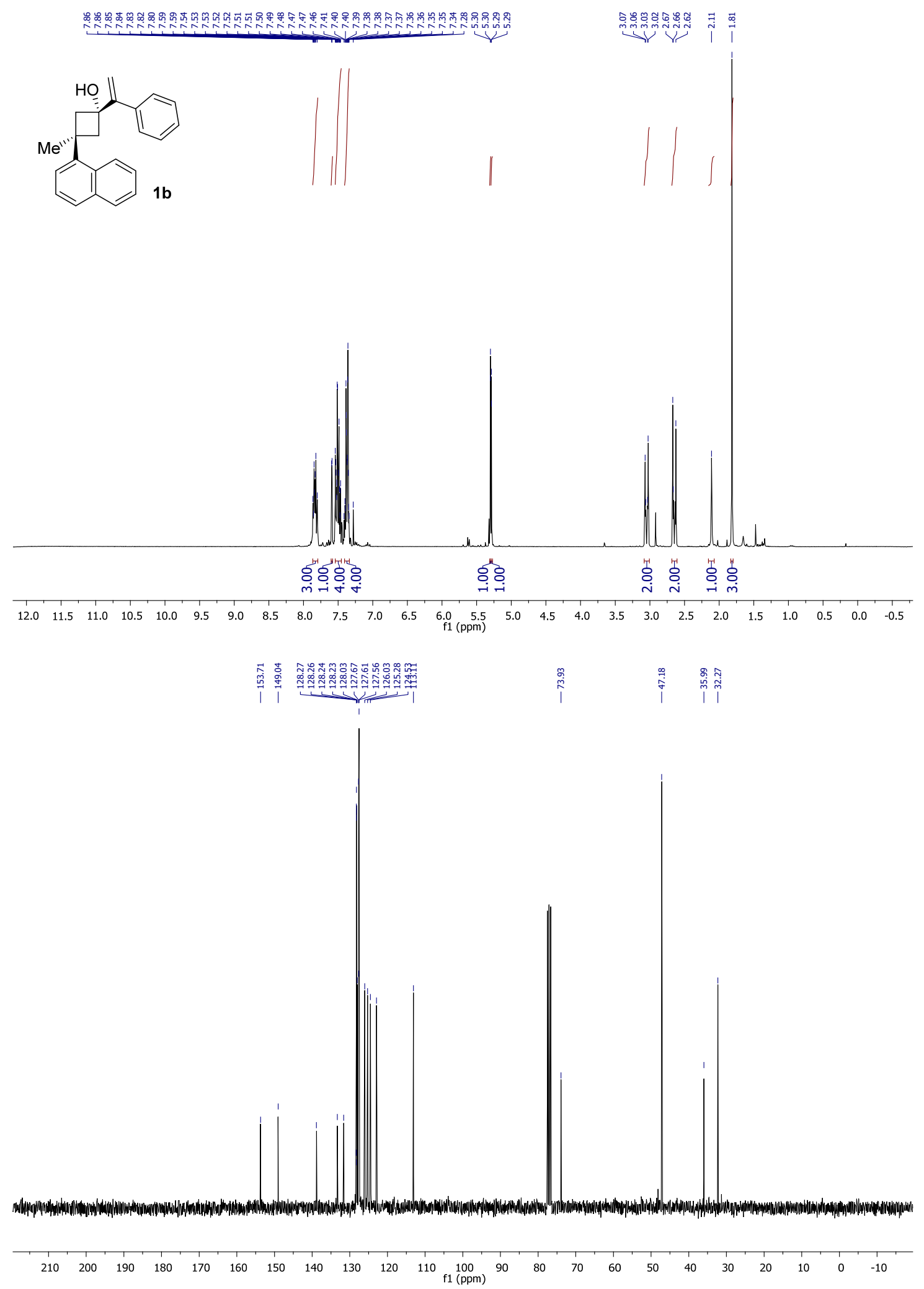

Figure S12. ${ }^{1} \mathrm{H}-\mathrm{NMR}\left(300 \mathrm{MHz}, \mathrm{CDCl}_{3}\right)$ and ${ }^{13} \mathrm{C}\left\{{ }^{1} \mathrm{H}\right\}-\mathrm{NMR}\left(75.5 \mathrm{MHz}, \mathrm{CDCl}_{3}\right)$ of compound $\mathbf{1 b}$ 

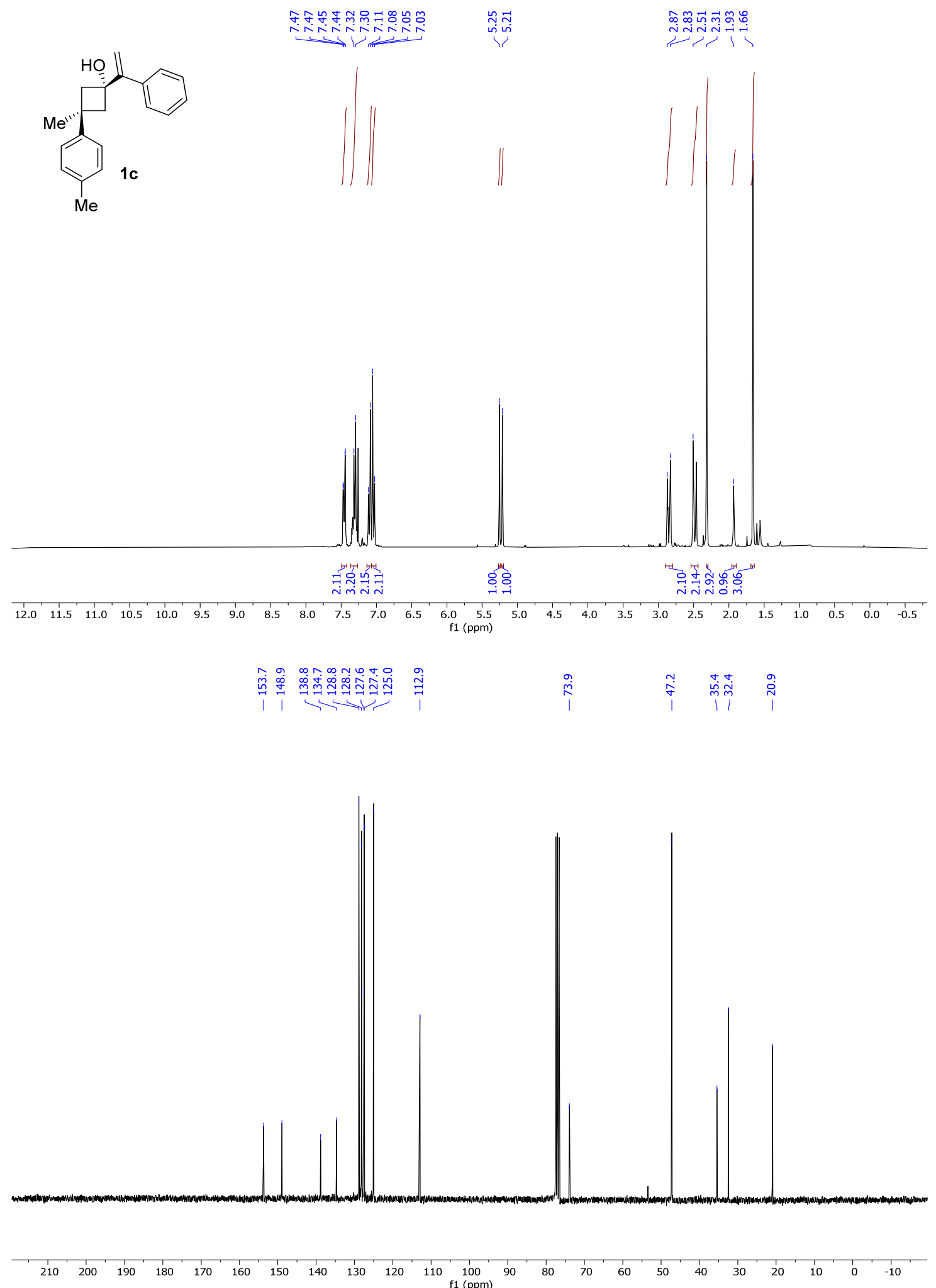

Figure S13. ${ }^{1} \mathrm{H}-\mathrm{NMR}\left(300 \mathrm{MHz}, \mathrm{CDCl}_{3}\right)$ and ${ }^{13} \mathrm{C}\left\{{ }^{1} \mathrm{H}\right\}-\mathrm{NMR}\left(75.5 \mathrm{MHz}, \mathrm{CDCl}_{3}\right)$ of compound 1c 

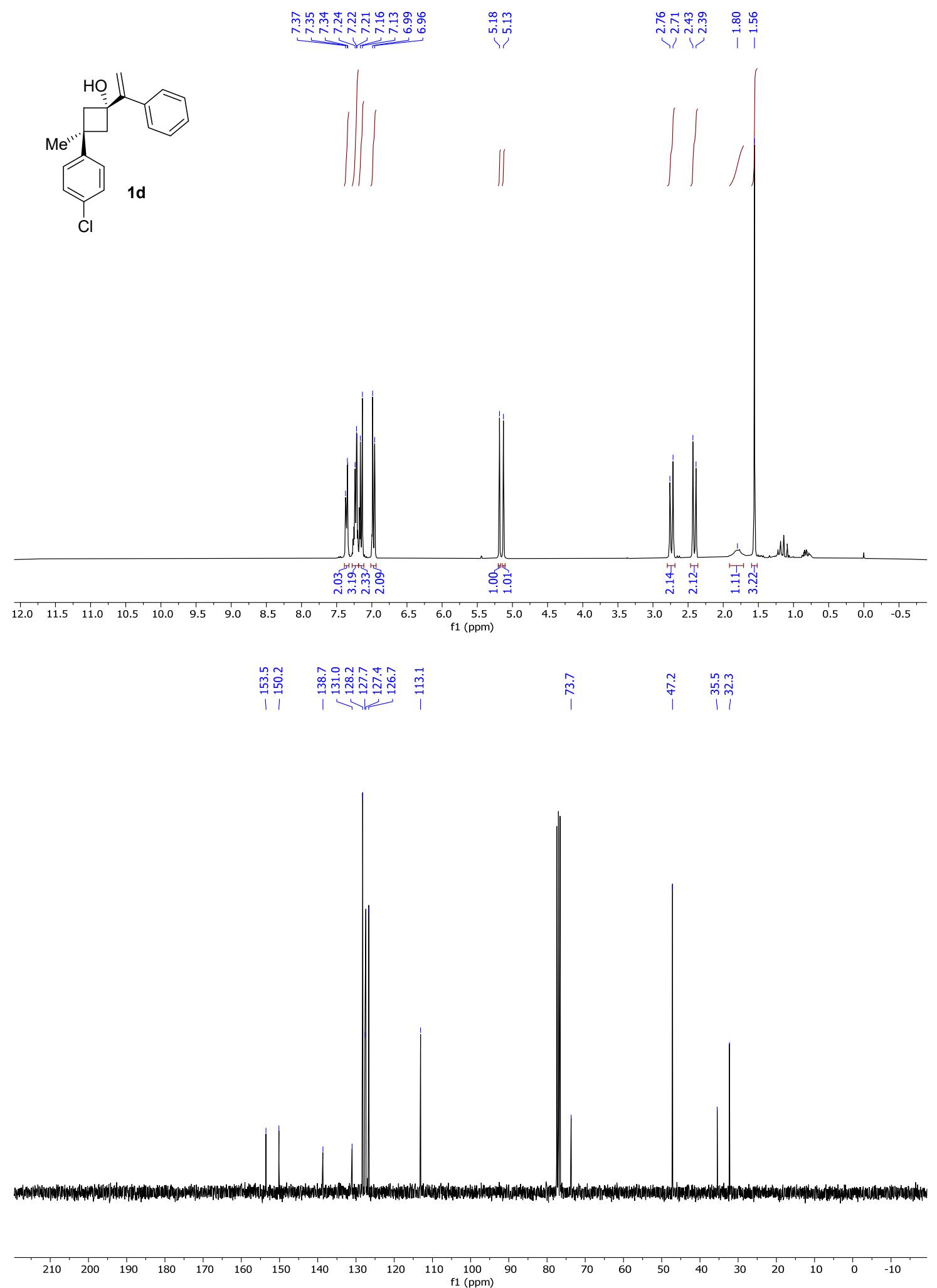

Figure S14. ${ }^{1} \mathrm{H}-\mathrm{NMR}\left(300 \mathrm{MHz}, \mathrm{CDCl}_{3}\right)$ and ${ }^{13} \mathrm{C}\left\{{ }^{1} \mathrm{H}\right\}-\mathrm{NMR}\left(75.5 \mathrm{MHz}, \mathrm{CDCl}_{3}\right)$ of compound 1d 

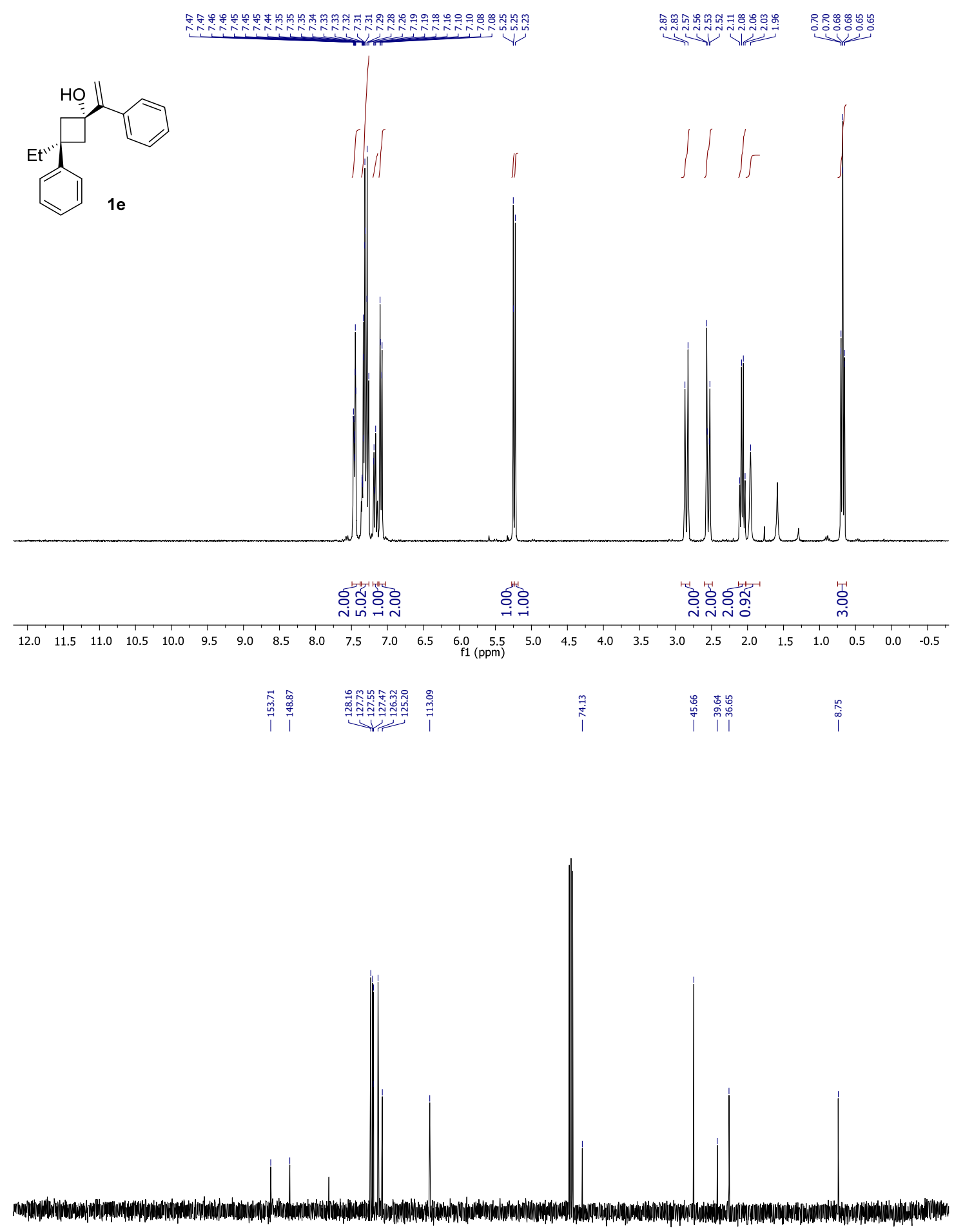

$\begin{array}{lllllllllllllllllllllll}210 & 200 & 190 & 180 & 170 & 160 & 150 & 140 & 130 & 120 & 110 & 100 & 100 & 80 & 70 & 60 & 50 & 40 & 30 & 20 & 10 & 0 & -10\end{array}$

Figure S15. ${ }^{1} \mathrm{H}-\mathrm{NMR}\left(300 \mathrm{MHz}, \mathrm{CDCl}_{3}\right)$ and ${ }^{13} \mathrm{C}\left\{{ }^{1} \mathrm{H}\right\}-\mathrm{NMR}\left(75.5 \mathrm{MHz}, \mathrm{CDCl}_{3}\right)$ of compound 1e 

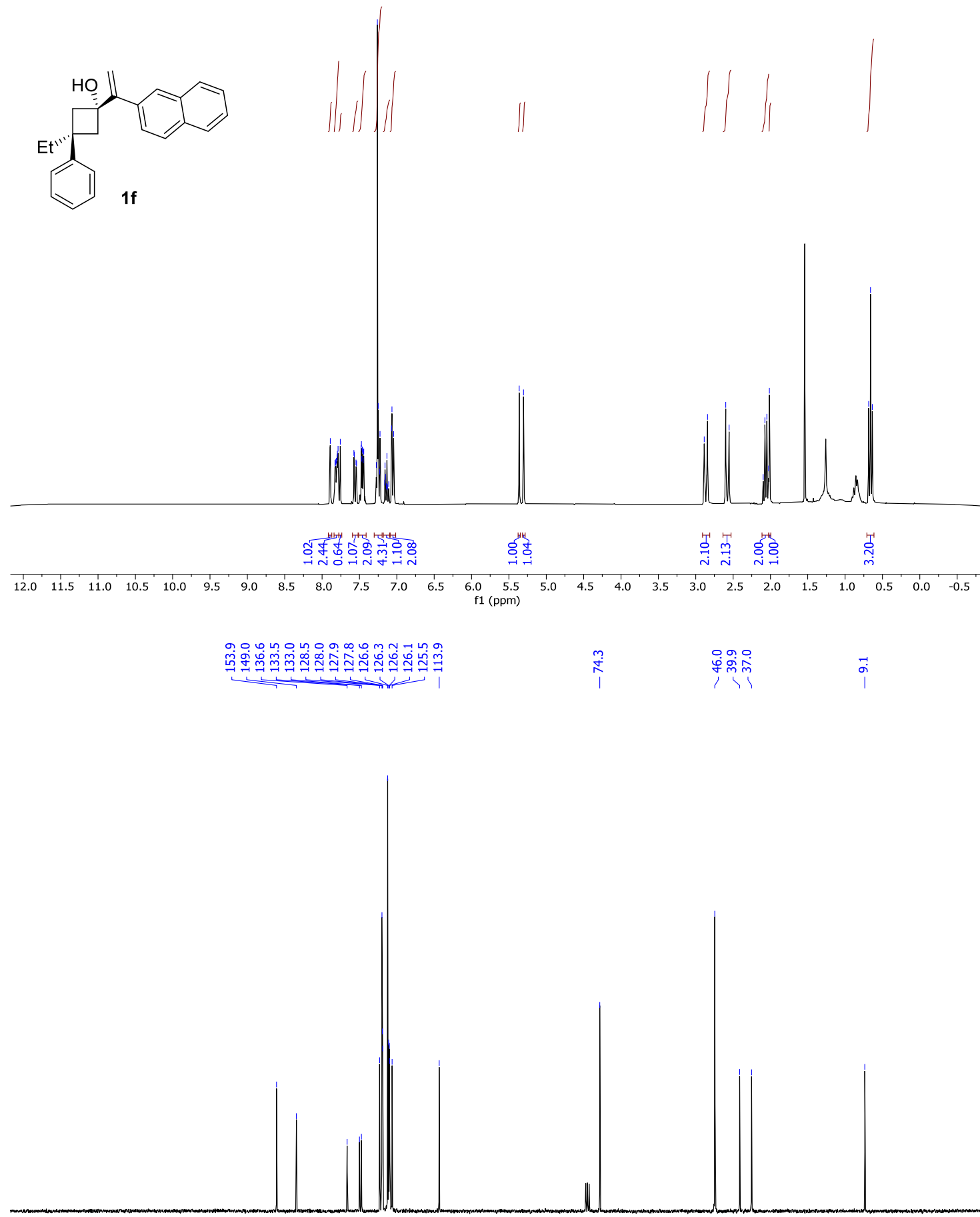

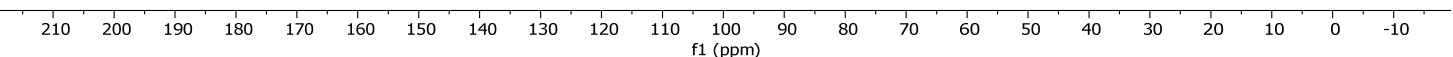

Figure S16. ${ }^{1} \mathrm{H}-\mathrm{NMR}\left(300 \mathrm{MHz}, \mathrm{CDCl}_{3}\right)$ and ${ }^{13} \mathrm{C}\left\{{ }^{1} \mathrm{H}\right\}-\mathrm{NMR}\left(75.5 \mathrm{MHz}, \mathrm{CDCl}_{3}\right)$ of compound $\mathbf{1 f}$ 

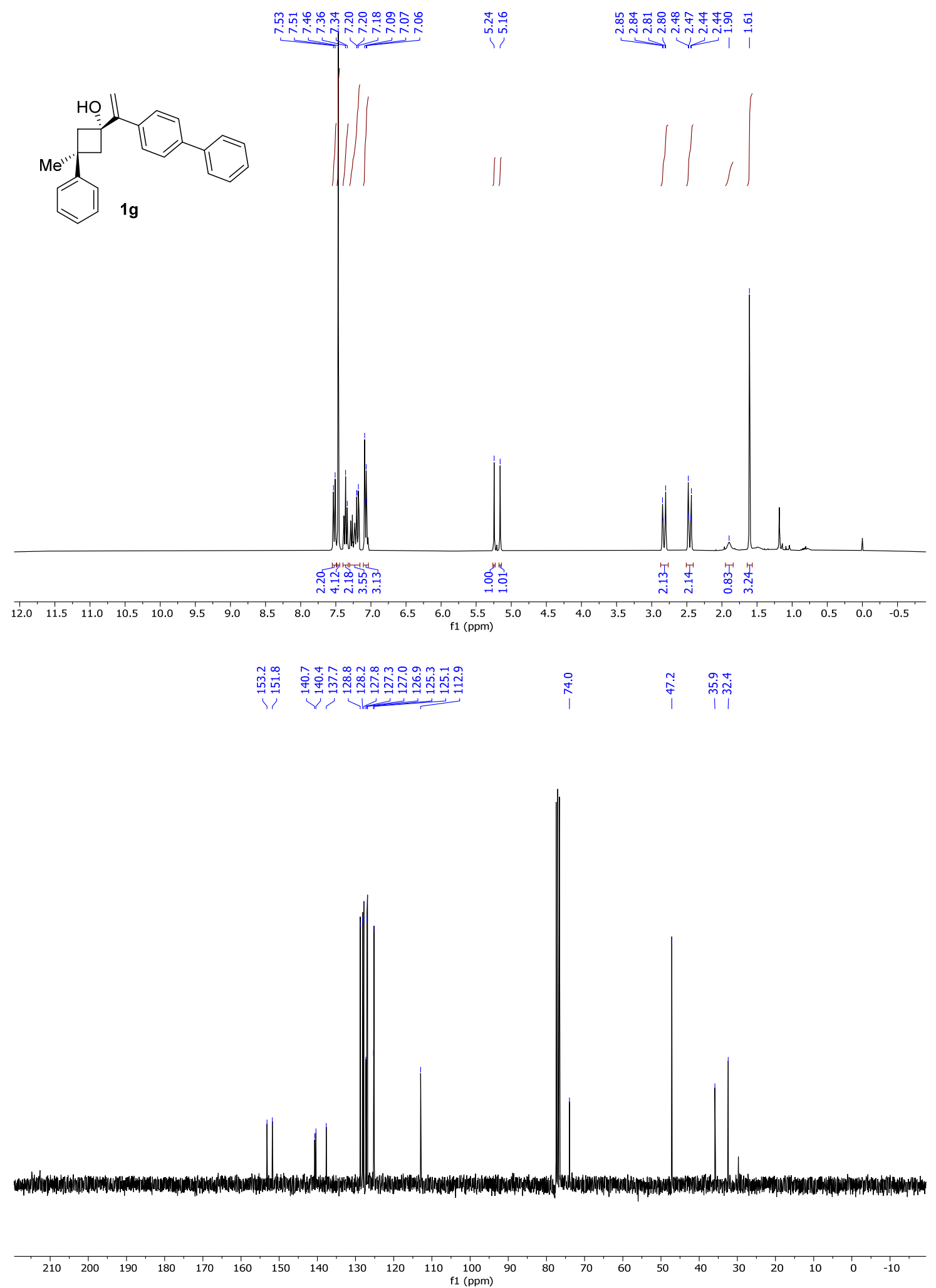

Figure S17. ${ }^{1} \mathrm{H}-\mathrm{NMR}\left(300 \mathrm{MHz}, \mathrm{CDCl}_{3}\right)$ and ${ }^{13} \mathrm{C}\left\{{ }^{1} \mathrm{H}\right\}-\mathrm{NMR}\left(75.5 \mathrm{MHz}, \mathrm{CDCl}_{3}\right)$ of compound $1 \mathrm{~g}$ 

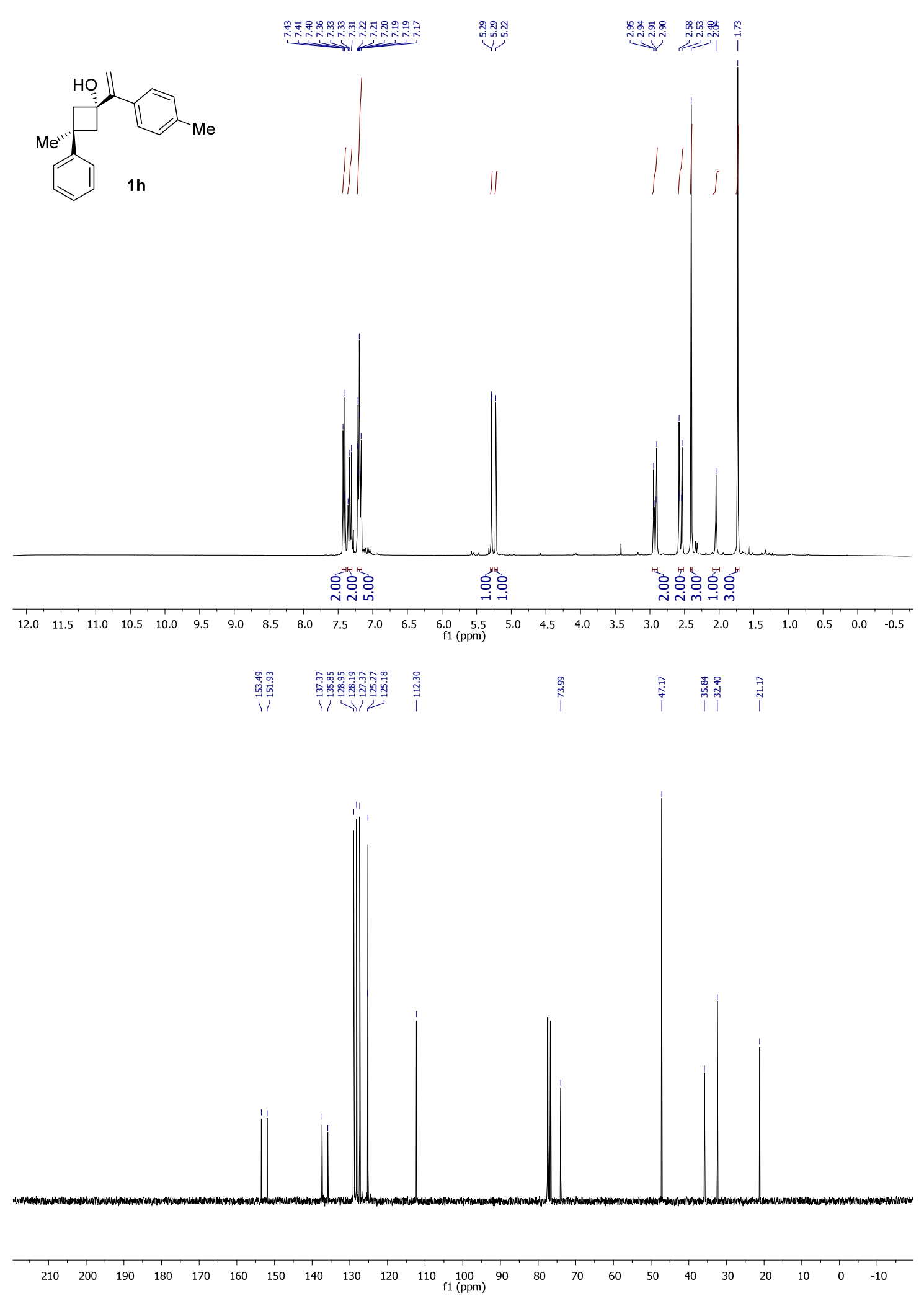

Figure S18. ${ }^{1} \mathrm{H}-\mathrm{NMR}\left(300 \mathrm{MHz}, \mathrm{CDCl}_{3}\right)$ and ${ }^{13} \mathrm{C}\left\{{ }^{1} \mathrm{H}\right\}-\mathrm{NMR}\left(75.5 \mathrm{MHz}, \mathrm{CDCl}_{3}\right)$ of compound $\mathbf{1 h}$ 

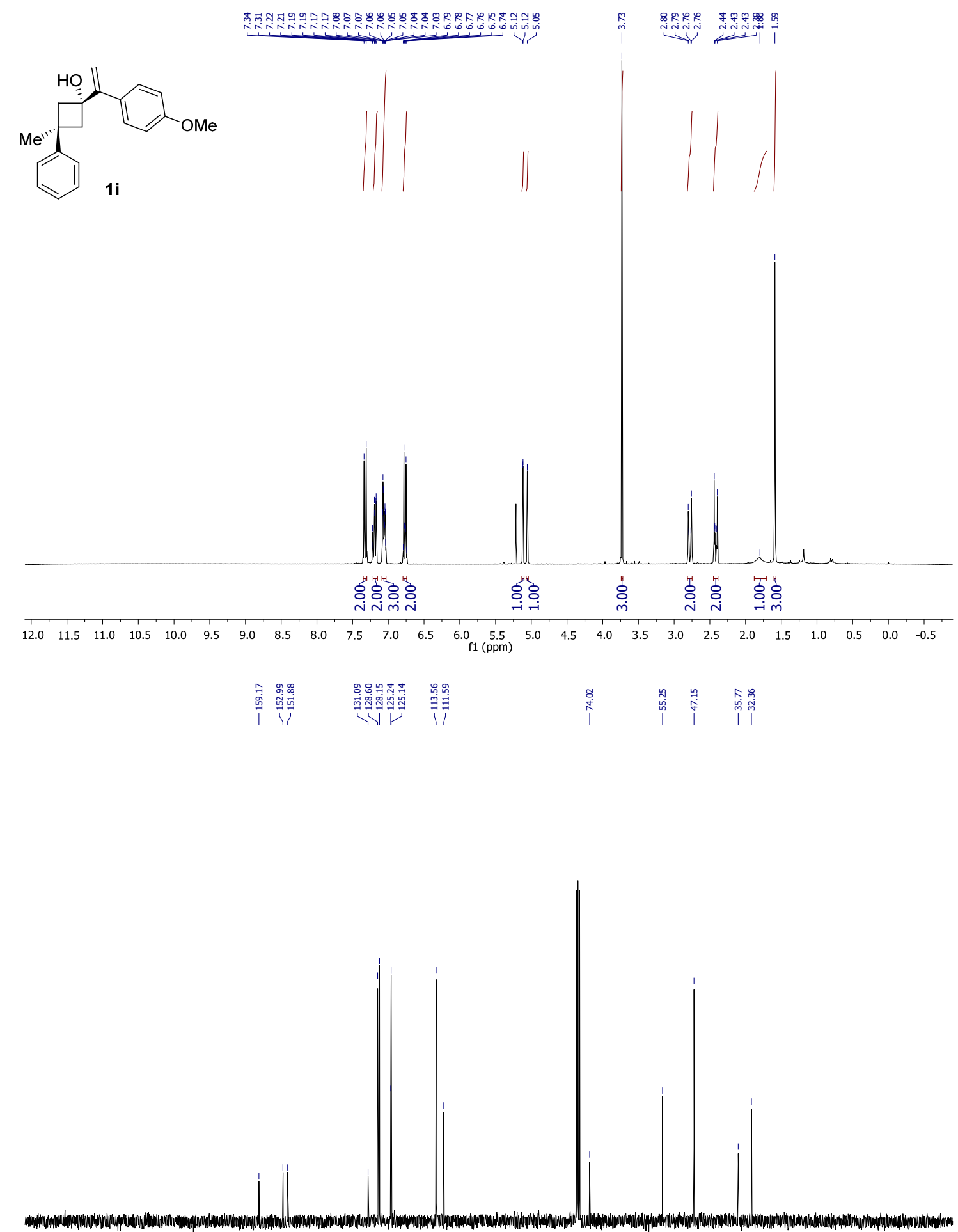

$\begin{array}{lllllllllllllllllllllllll}210 & 200 & 190 & 180 & 170 & 160 & 150 & 140 & 130 & 120 & 110 & 100 & 100 & 80 & 70 & 60 & 50 & 40 & 30 & 20 & 10 & 0 & -10\end{array}$

Figure S19. ${ }^{1} \mathrm{H}-\mathrm{NMR}\left(300 \mathrm{MHz}, \mathrm{CDCl}_{3}\right)$ and ${ }^{13} \mathrm{C}\left\{{ }^{1} \mathrm{H}\right\}-\mathrm{NMR}\left(75.5 \mathrm{MHz}, \mathrm{CDCl}_{3}\right)$ of compound 1i 


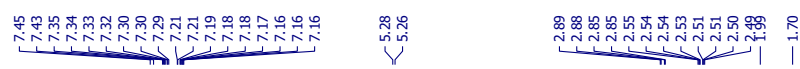
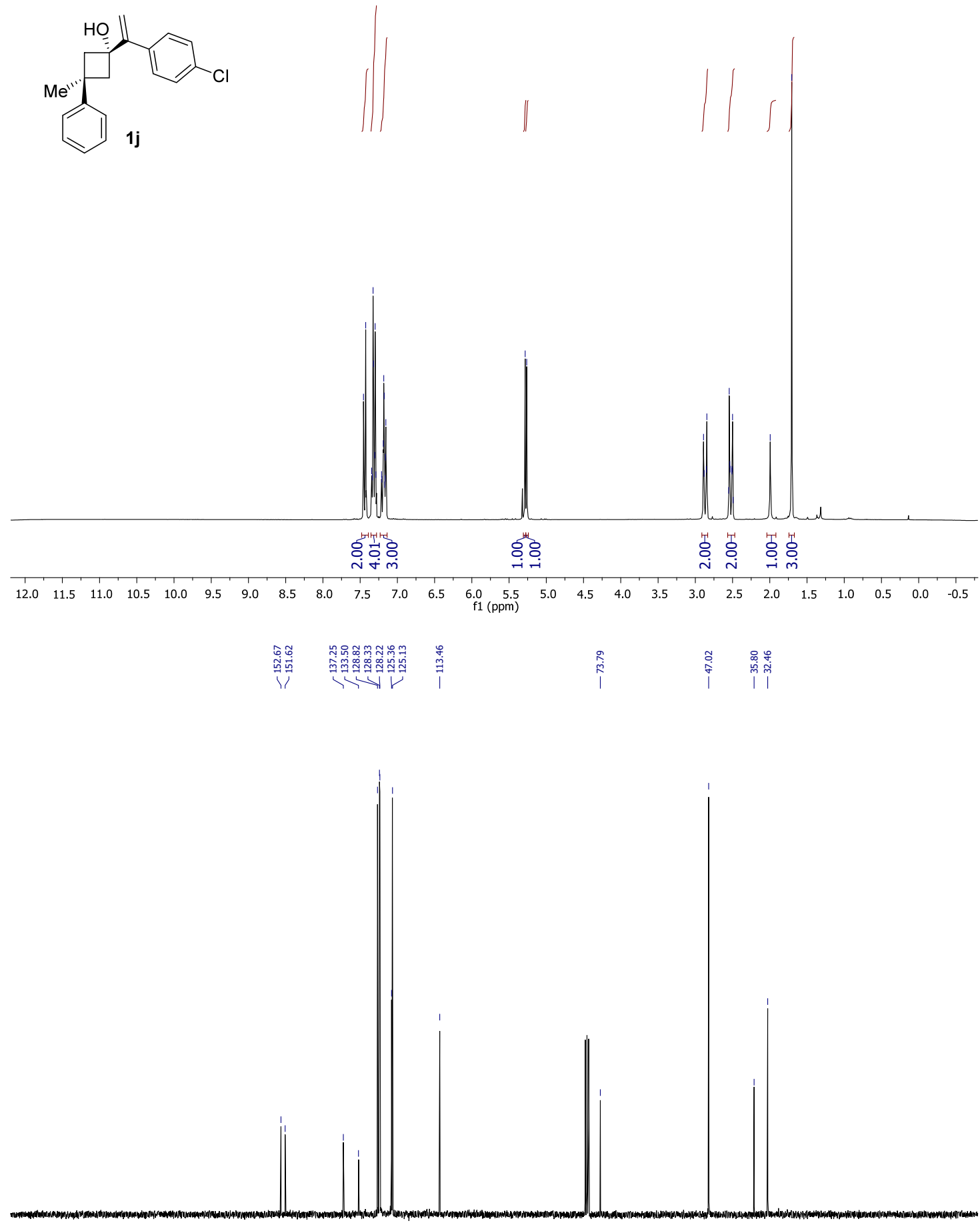

$\begin{array}{lllllllllllllllllllllll}210 & 200 & 190 & 180 & 170 & 160 & 150 & 140 & 130 & 120 & 110 & \begin{array}{c}100 \\ \mathrm{f} 1(\mathrm{ppm})\end{array} & 90 & 80 & 70 & 60 & 50 & 40 & 30 & 20 & 10 & 0 & -10\end{array}$

Figure S20. ${ }^{1} \mathrm{H}-\mathrm{NMR}\left(300 \mathrm{MHz}, \mathrm{CDCl}_{3}\right)$ and ${ }^{13} \mathrm{C}\left\{{ }^{1} \mathrm{H}\right\}-\mathrm{NMR}\left(75.5 \mathrm{MHz}, \mathrm{CDCl}_{3}\right)$ of compound $\mathbf{1 j}$ 

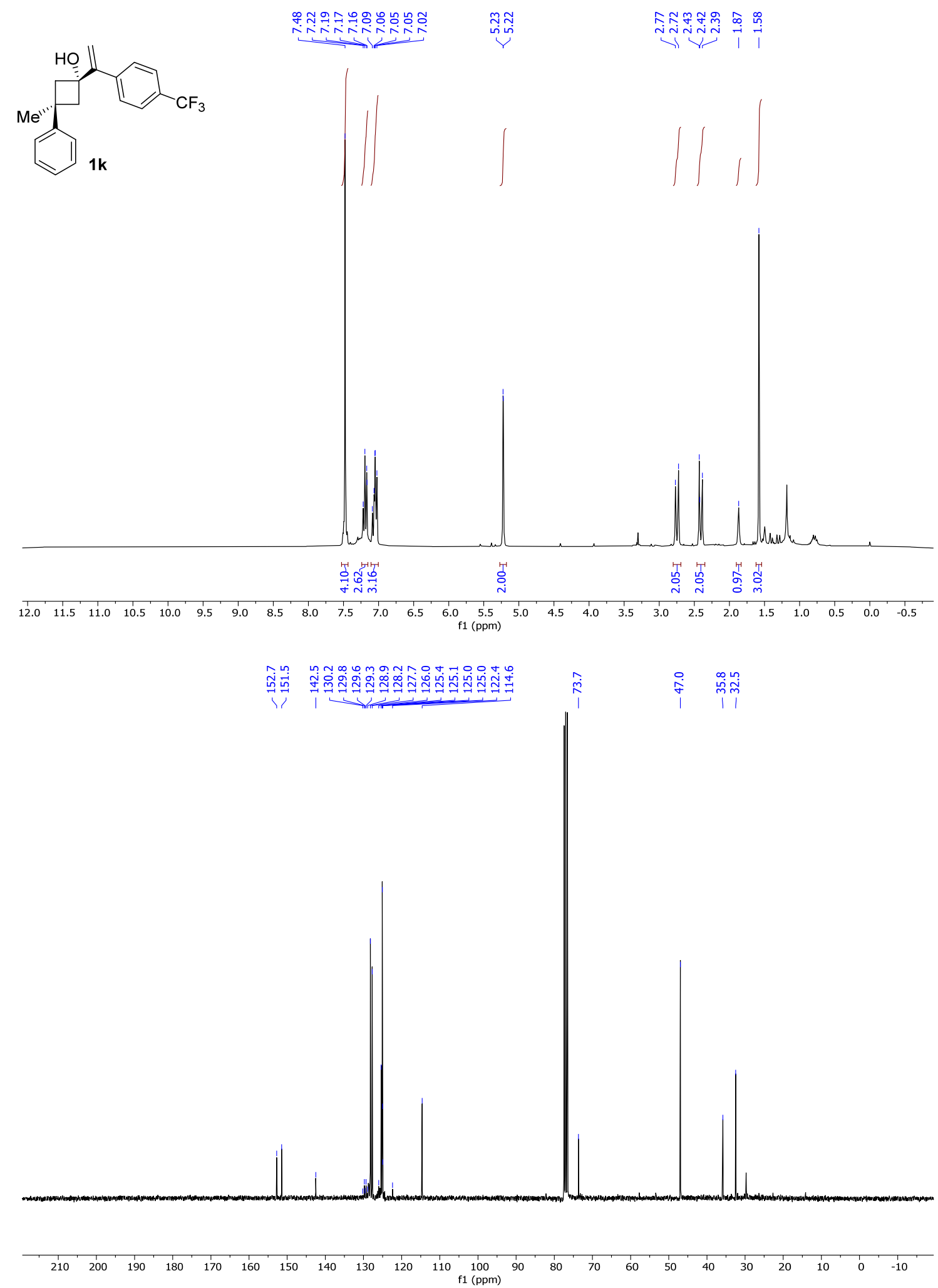

Figure S21. ${ }^{1} \mathrm{H}-\mathrm{NMR}\left(300 \mathrm{MHz}, \mathrm{CDCl}_{3}\right)$ and ${ }^{13} \mathrm{C}\left\{{ }^{1} \mathrm{H}\right\}-\mathrm{NMR}\left(75.5 \mathrm{MHz}, \mathrm{CDCl}_{3}\right)$ of compound $1 \mathbf{k}$ 


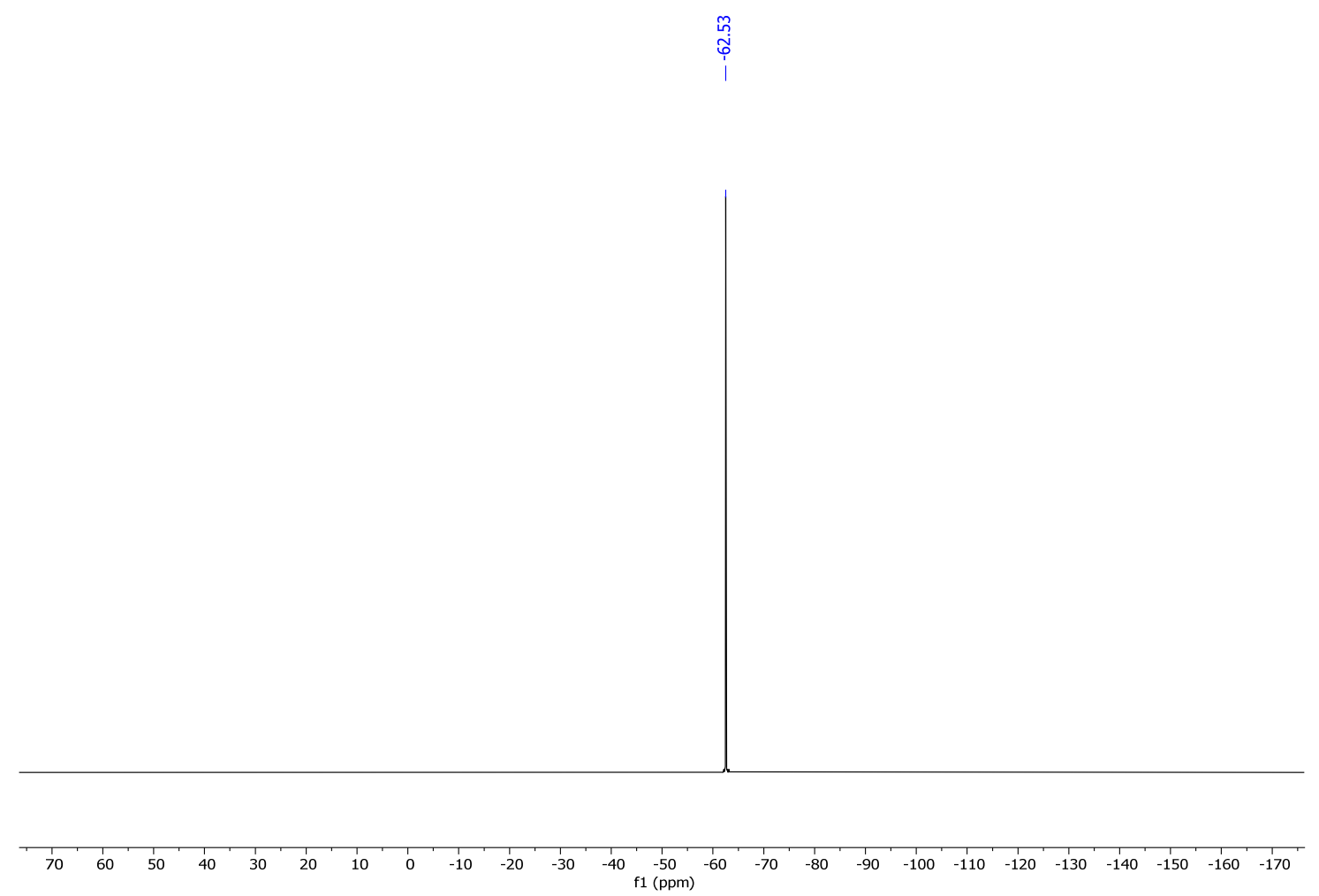

Figure S22. ${ }^{31} \mathrm{P}-\mathrm{NMR}\left(122 \mathrm{MHz}, \mathrm{CDCl}_{3}\right)$ of compound $\mathbf{1 k}$ 


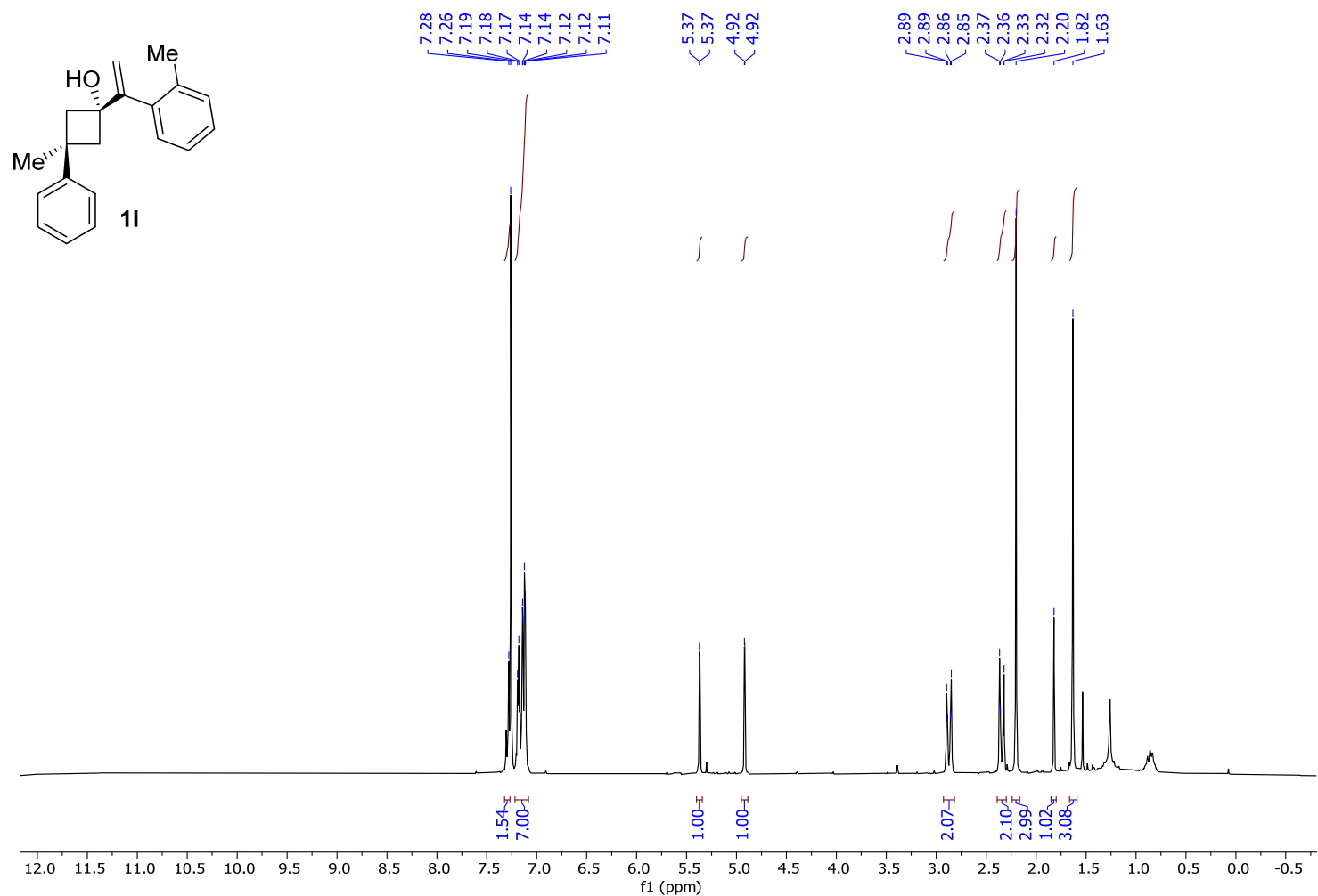

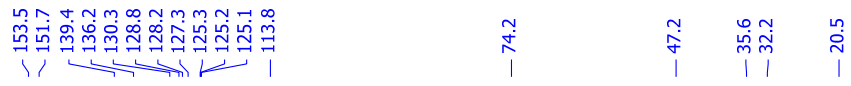

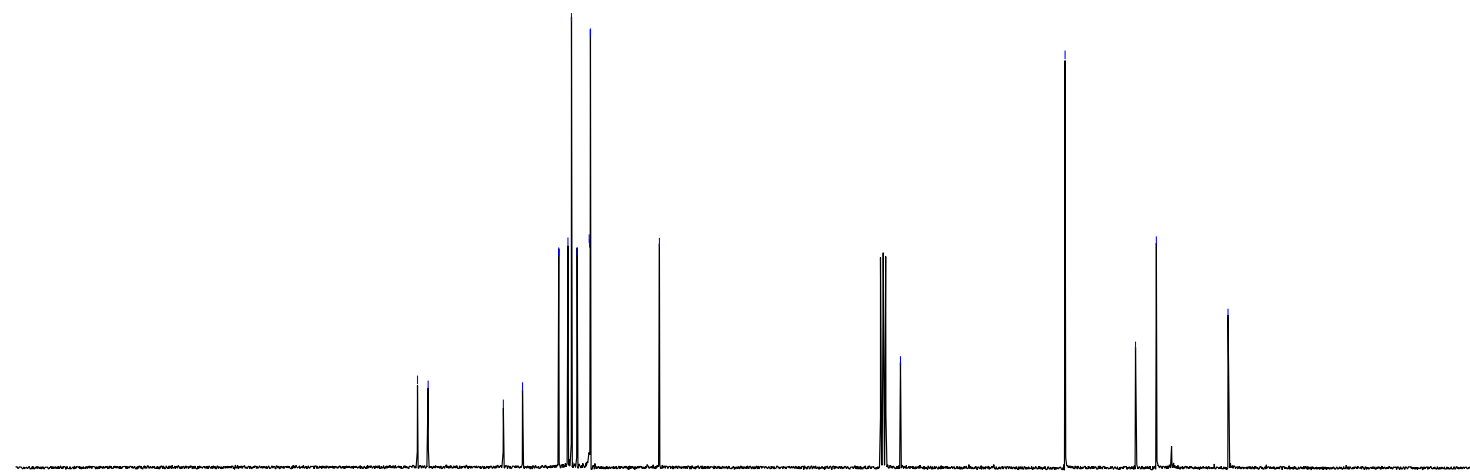

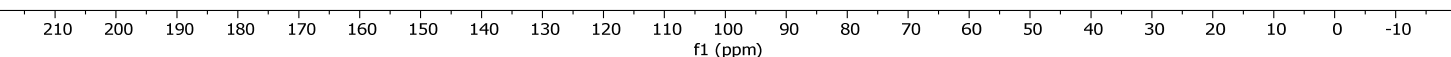

Figure S23. ${ }^{1} \mathrm{H}-\mathrm{NMR}\left(300 \mathrm{MHz}, \mathrm{CDCl}_{3}\right)$ and ${ }^{13} \mathrm{C}\left\{{ }^{1} \mathrm{H}\right\}-\mathrm{NMR}\left(75.5 \mathrm{MHz}, \mathrm{CDCl}_{3}\right)$ of compound 11 

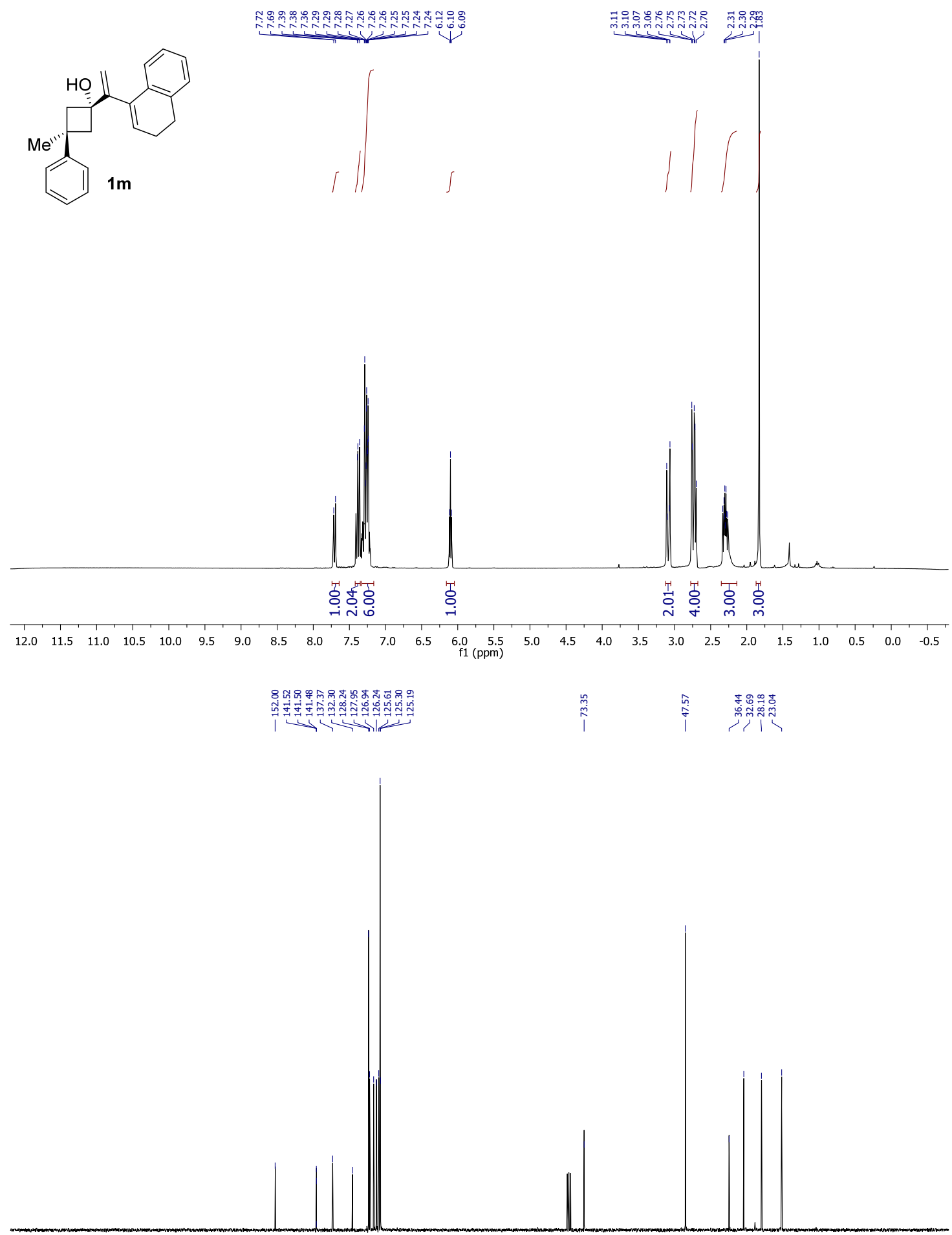

$\begin{array}{lllllllllllllllllllllll}210 & 200 & 190 & 180 & 170 & 160 & 150 & 140 & 130 & 120 & 110 & 100 & 90 & 80 & 70 & 60 & 50 & 40 & 30 & 20 & 10 & 0 & -10\end{array}$

Figure S24. ${ }^{1} \mathrm{H}-\mathrm{NMR}\left(300 \mathrm{MHz}, \mathrm{CDCl}_{3}\right)$ and ${ }^{13} \mathrm{C}\left\{{ }^{1} \mathrm{H}\right\}-\mathrm{NMR}\left(75.5 \mathrm{MHz}, \mathrm{CDCl}_{3}\right)$ of compound $\mathbf{1 m}$ 


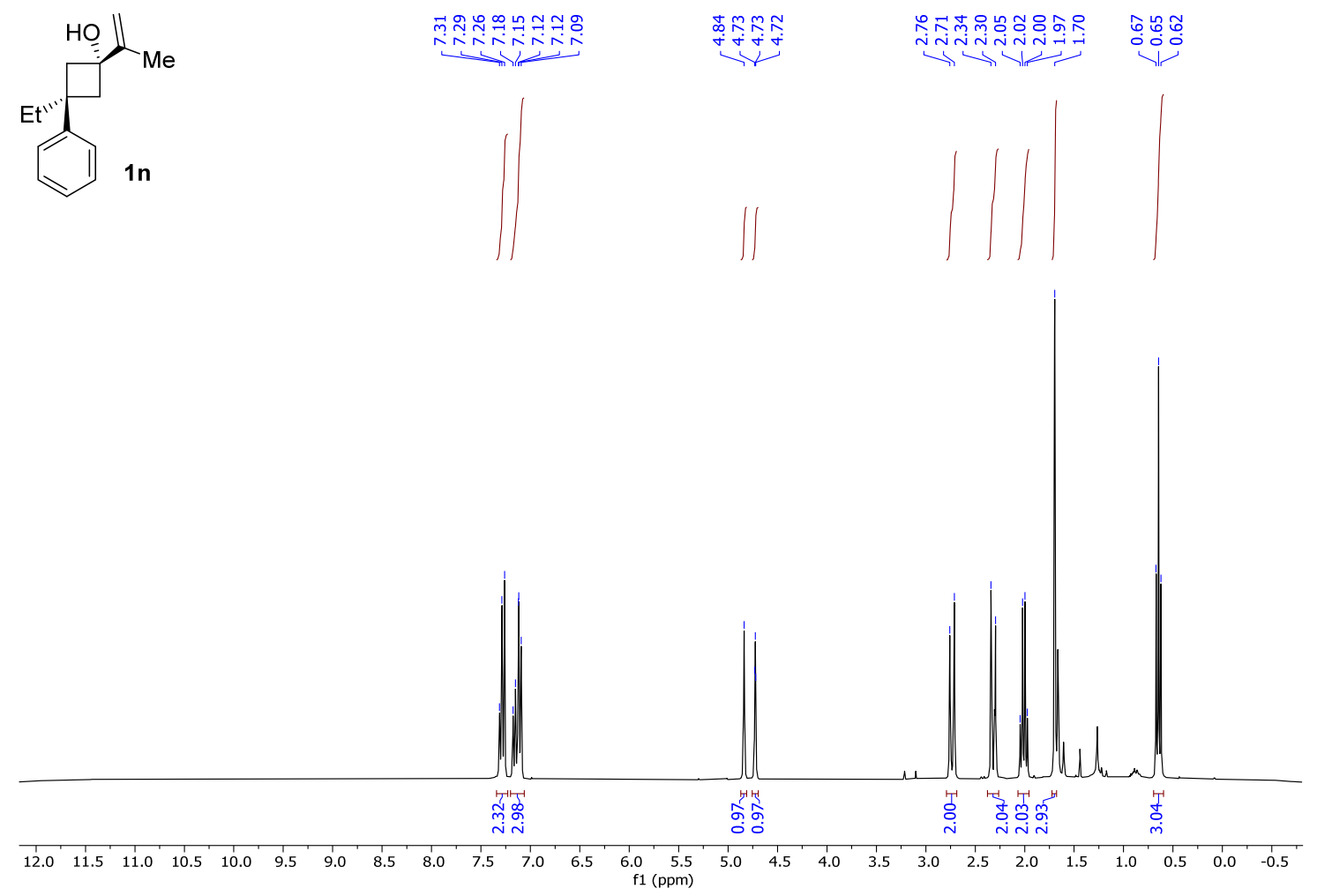

\begin{tabular}{|c|c|c|c|c|c|}
\hline 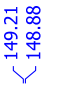 & 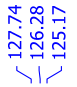 & $\begin{array}{l}\overrightarrow{1} \\
0 \\
0 \\
1 \\
1\end{array}$ & 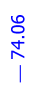 & 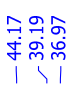 & 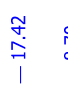 \\
\hline
\end{tabular}

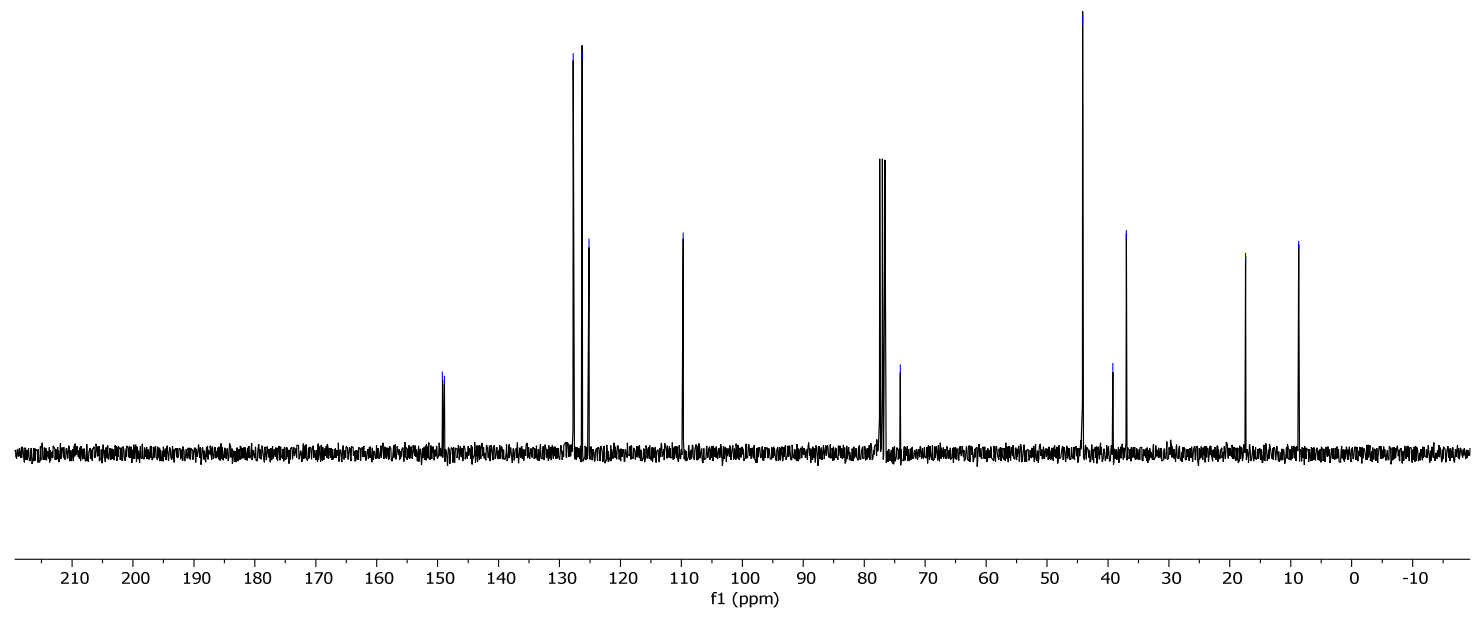

Figure S25. ${ }^{1} \mathrm{H}-\mathrm{NMR}\left(300 \mathrm{MHz}, \mathrm{CDCl}_{3}\right)$ and ${ }^{13} \mathrm{C}\left\{{ }^{1} \mathrm{H}\right\}-\mathrm{NMR}\left(75.5 \mathrm{MHz}, \mathrm{CDCl}_{3}\right)$ of compound 1n 

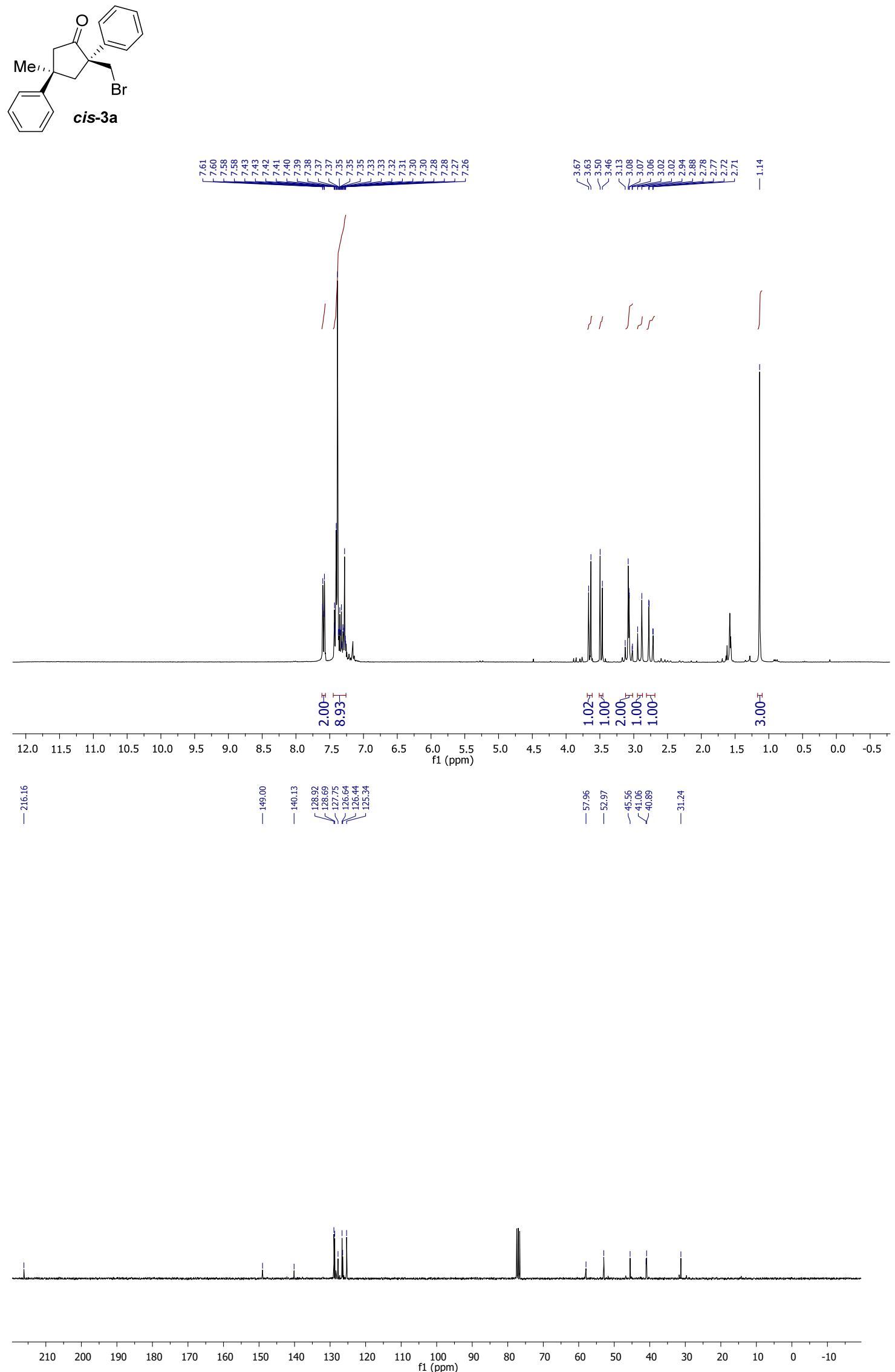

Figure S26. ${ }^{1} \mathrm{H}-\mathrm{NMR}\left(300 \mathrm{MHz}, \mathrm{CDCl}_{3}\right)$ and ${ }^{13} \mathrm{C}\left\{{ }^{1} \mathrm{H}\right\}-\mathrm{NMR}\left(75.5 \mathrm{MHz}, \mathrm{CDCl}_{3}\right)$ of compound cis-3a 

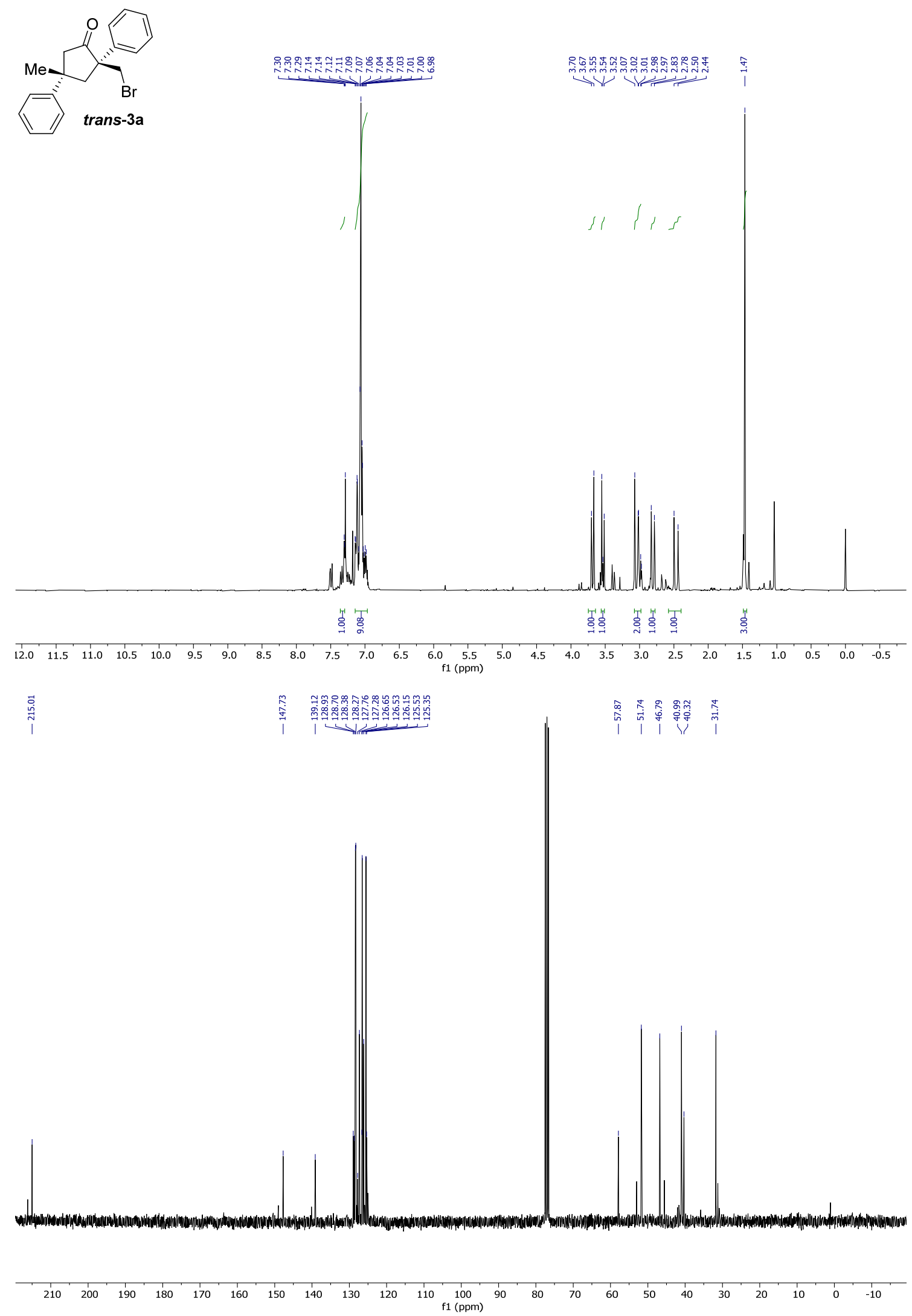

Figure S27. ${ }^{1} \mathrm{H}-\mathrm{NMR}\left(300 \mathrm{MHz}, \mathrm{CDCl}_{3}\right)$ and ${ }^{13} \mathrm{C}\left\{{ }^{1} \mathrm{H}\right\}-\mathrm{NMR}\left(75.5 \mathrm{MHz}, \mathrm{CDCl}_{3}\right)$ of compound trans-3a 
$\underbrace{\mathrm{Br}}_{\mathrm{Me}}$

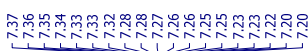

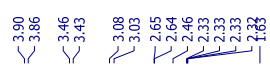

$\|$

$1 / 1 / 1 /$

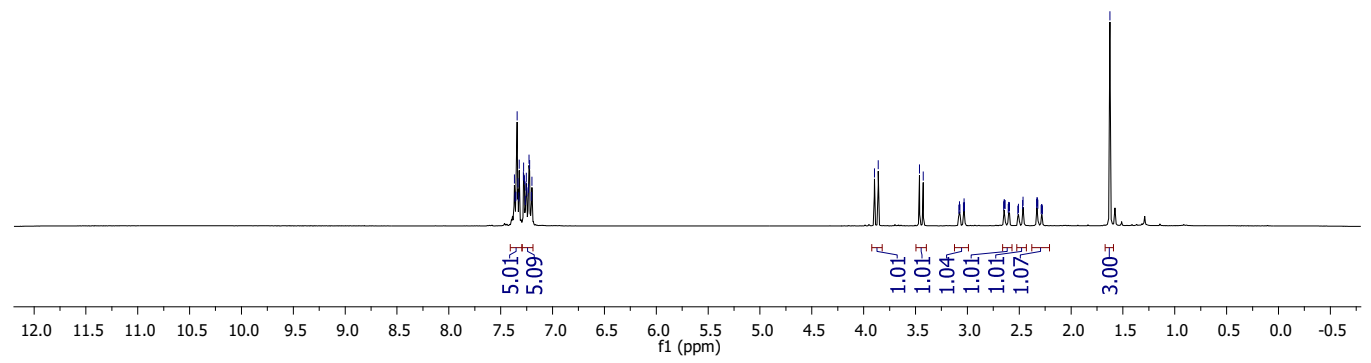

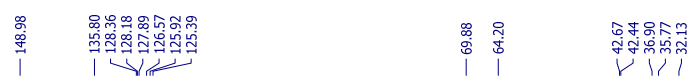

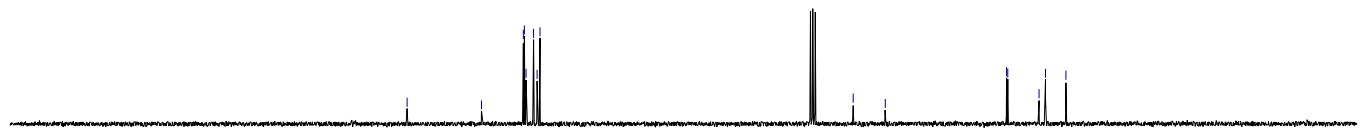

$\begin{array}{llllllllllllllllllllllll}210 & 200 & 190 & 180 & 170 & 160 & 150 & 140 & 130 & 120 & 110 & 100 & 90 & 80 & 70 & 60 & 50 & 40 & 30 & 20 & 10 & 0 & -10\end{array}$

Figure S28. ${ }^{1} \mathrm{H}-\mathrm{NMR}\left(300 \mathrm{MHz}, \mathrm{CDCl}_{3}\right)$ and ${ }^{13} \mathrm{C}\left\{{ }^{1} \mathrm{H}\right\}-\mathrm{NMR}\left(75.5 \mathrm{MHz}, \mathrm{CDCl}_{3}\right)$ of compound 4a 

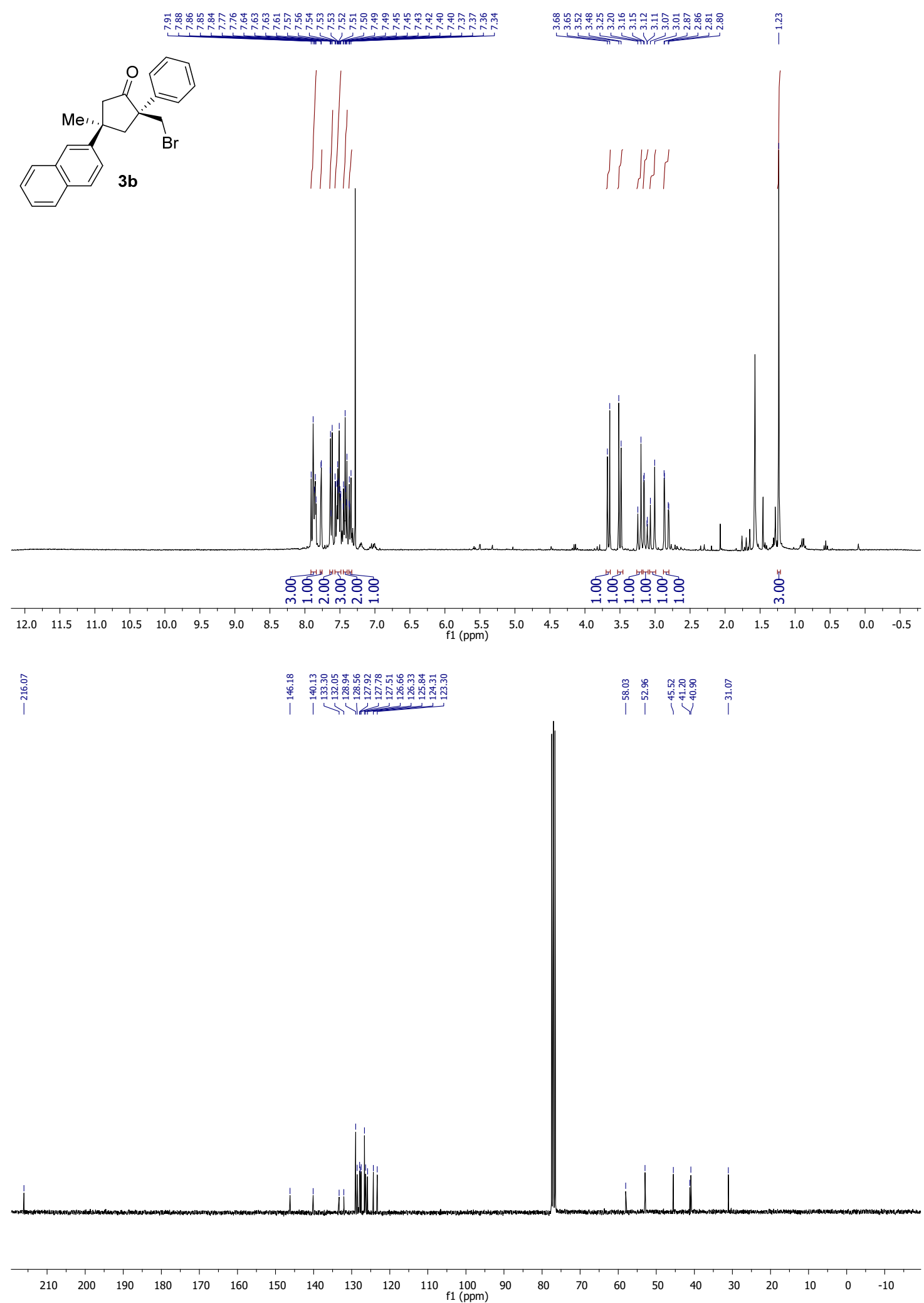

Figure S29. ${ }^{1} \mathrm{H}-\mathrm{NMR}\left(300 \mathrm{MHz}, \mathrm{CDCl}_{3}\right)$ and ${ }^{13} \mathrm{C}\left\{{ }^{1} \mathrm{H}\right\}-\mathrm{NMR}\left(75.5 \mathrm{MHz}, \mathrm{CDCl}_{3}\right)$ of compound $\mathbf{3 b}$ 

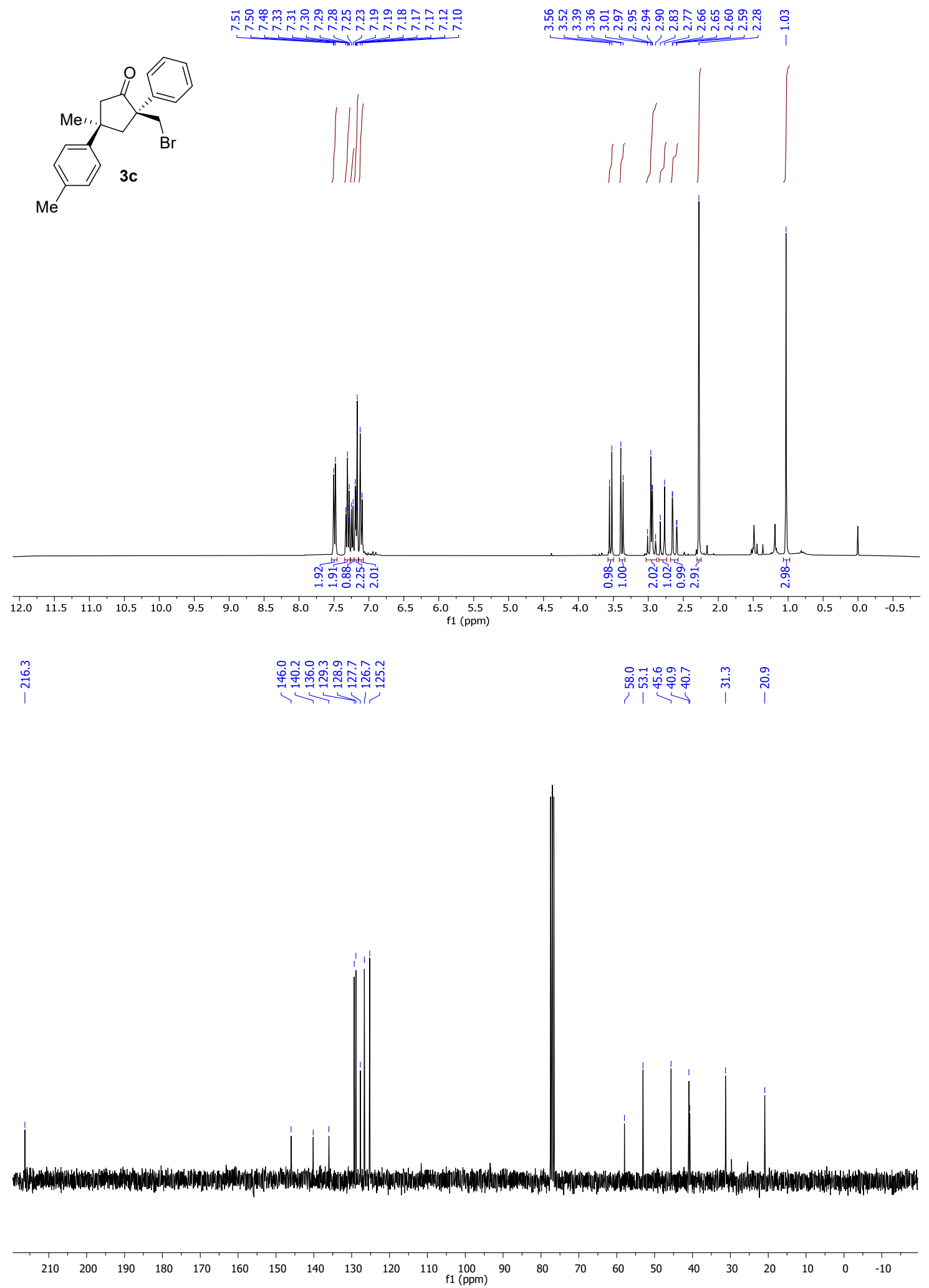

Figure S30. ${ }^{1} \mathrm{H}-\mathrm{NMR}\left(300 \mathrm{MHz}, \mathrm{CDCl}_{3}\right)$ and ${ }^{13} \mathrm{C}\left\{{ }^{1} \mathrm{H}\right\}-\mathrm{NMR}\left(75.5 \mathrm{MHz}, \mathrm{CDCl}_{3}\right)$ of compound 3c 

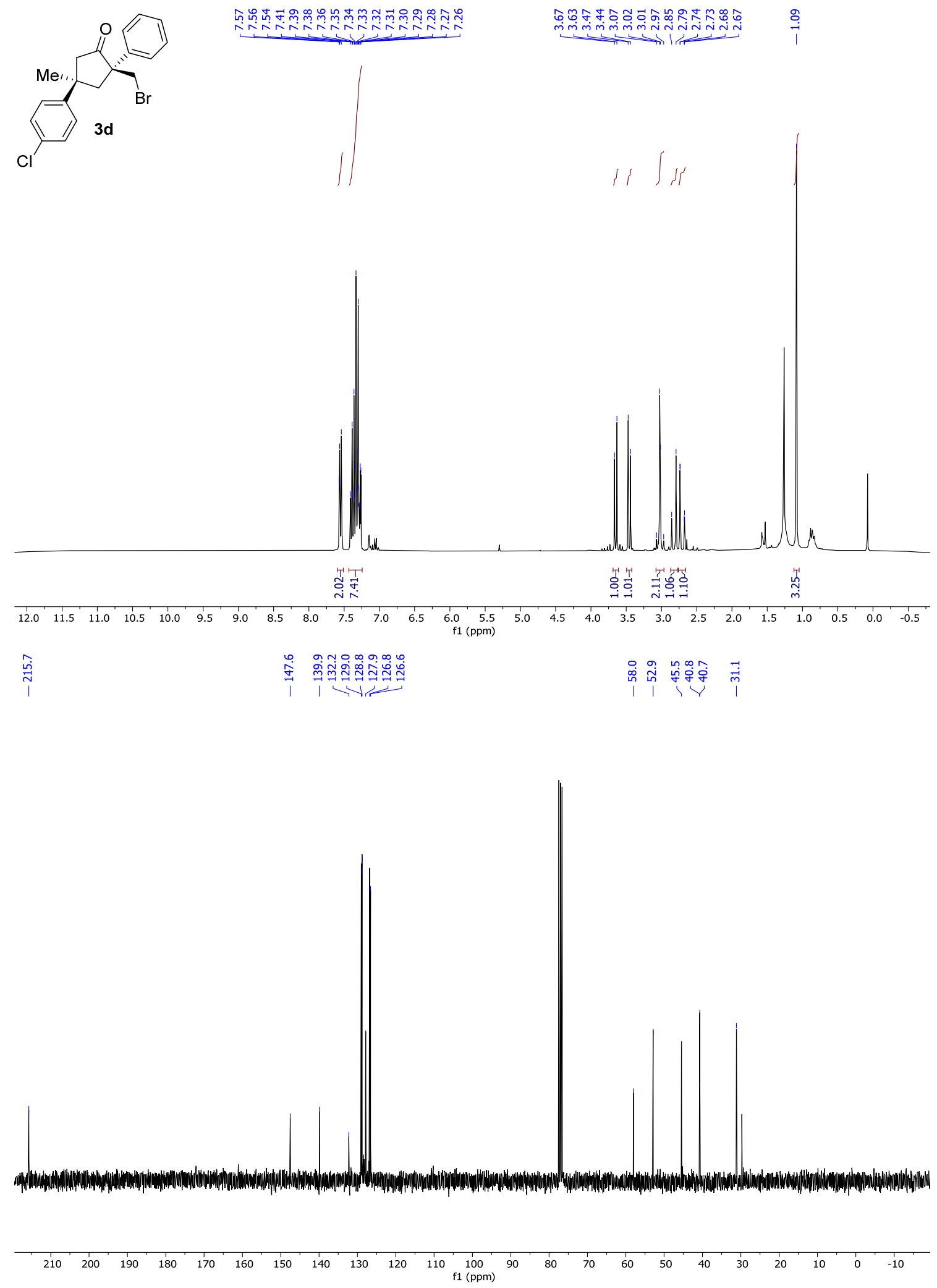

Figure S31. ${ }^{1} \mathrm{H}-\mathrm{NMR}\left(300 \mathrm{MHz}, \mathrm{CDCl}_{3}\right)$ and ${ }^{13} \mathrm{C}\left\{{ }^{1} \mathrm{H}\right\}-\mathrm{NMR}\left(75.5 \mathrm{MHz}, \mathrm{CDCl}_{3}\right)$ of compound 3d 

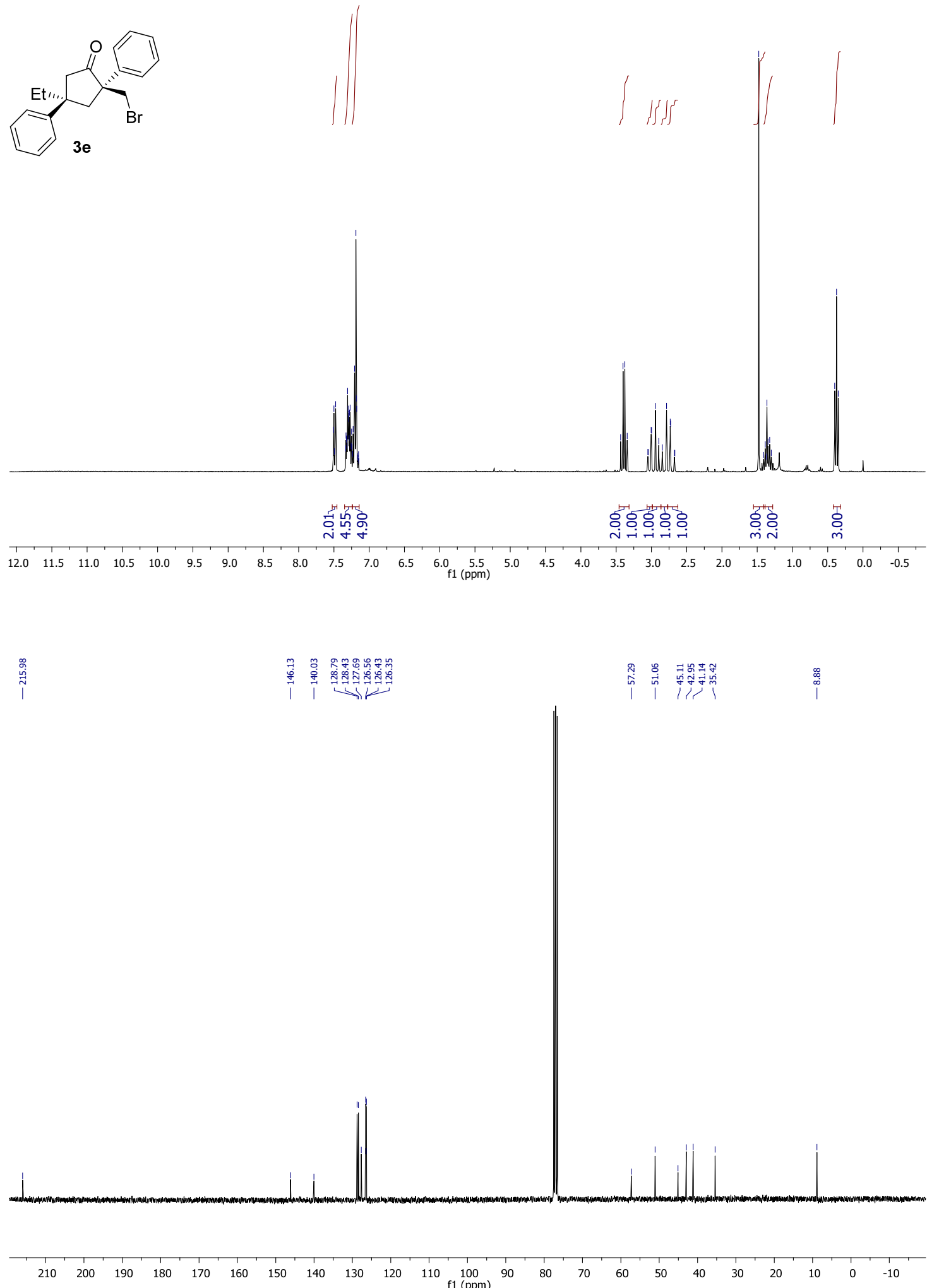

Figure S32. ${ }^{1} \mathrm{H}-\mathrm{NMR}\left(300 \mathrm{MHz}, \mathrm{CDCl}_{3}\right)$ and ${ }^{13} \mathrm{C}\left\{{ }^{1} \mathrm{H}\right\}-\mathrm{NMR}\left(75.5 \mathrm{MHz}, \mathrm{CDCl}_{3}\right)$ of compound $3 \mathbf{e}$ 


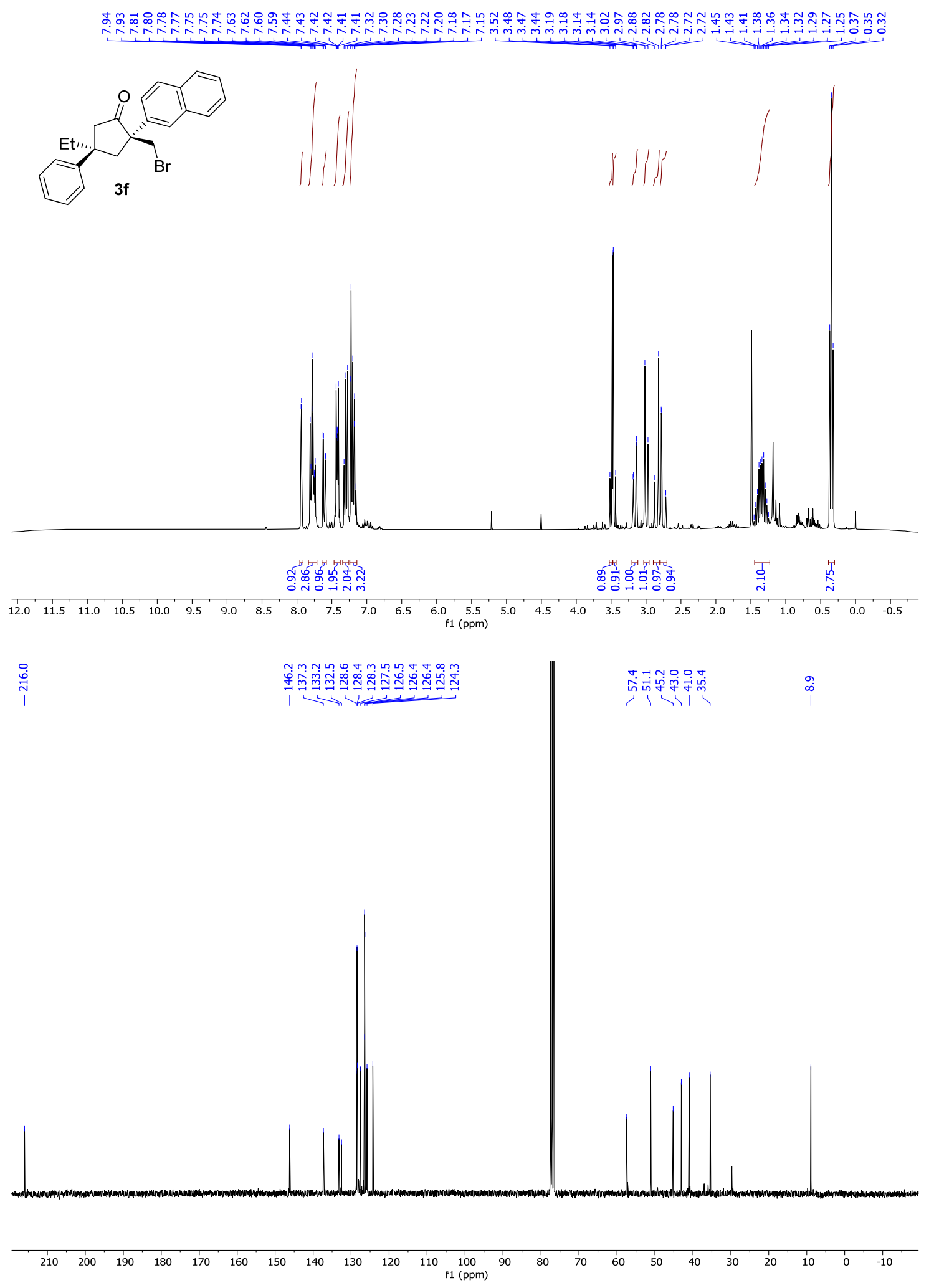

Figure S33. ${ }^{1} \mathrm{H}-\mathrm{NMR}\left(300 \mathrm{MHz}, \mathrm{CDCl}_{3}\right)$ and ${ }^{13} \mathrm{C}\left\{{ }^{1} \mathrm{H}\right\}-\mathrm{NMR}\left(75.5 \mathrm{MHz}, \mathrm{CDCl}_{3}\right)$ of compound $3 f$ 

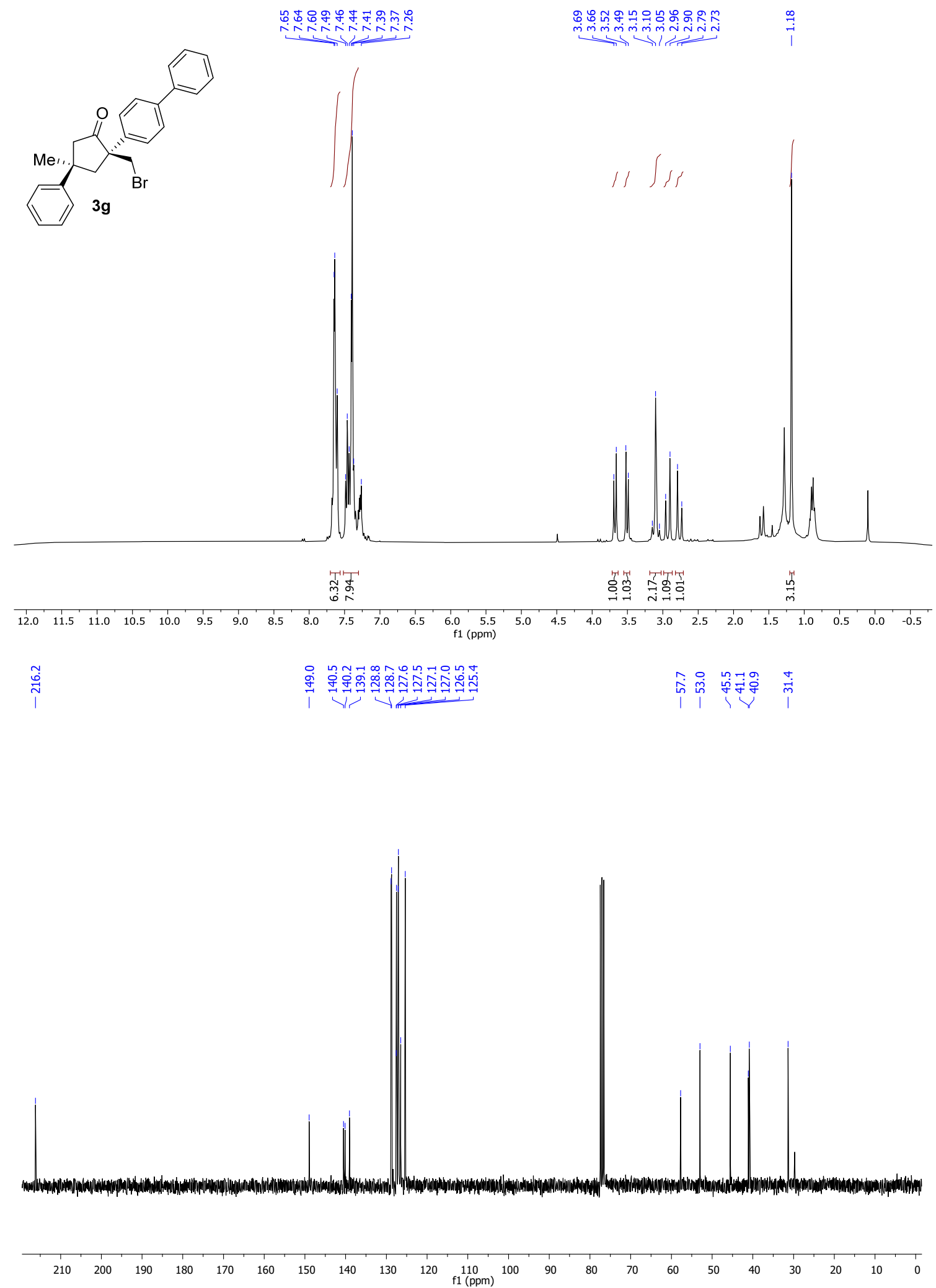

Figure S34. ${ }^{1} \mathrm{H}-\mathrm{NMR}\left(300 \mathrm{MHz}, \mathrm{CDCl}_{3}\right)$ and ${ }^{13} \mathrm{C}\left\{{ }^{1} \mathrm{H}\right\}-\mathrm{NMR}\left(75.5 \mathrm{MHz}, \mathrm{CDCl}_{3}\right)$ of compound $3 g$ 

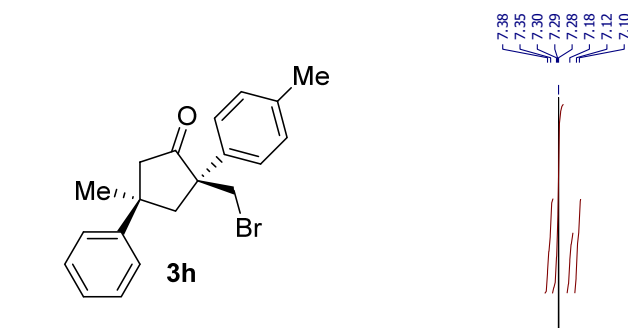

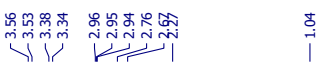

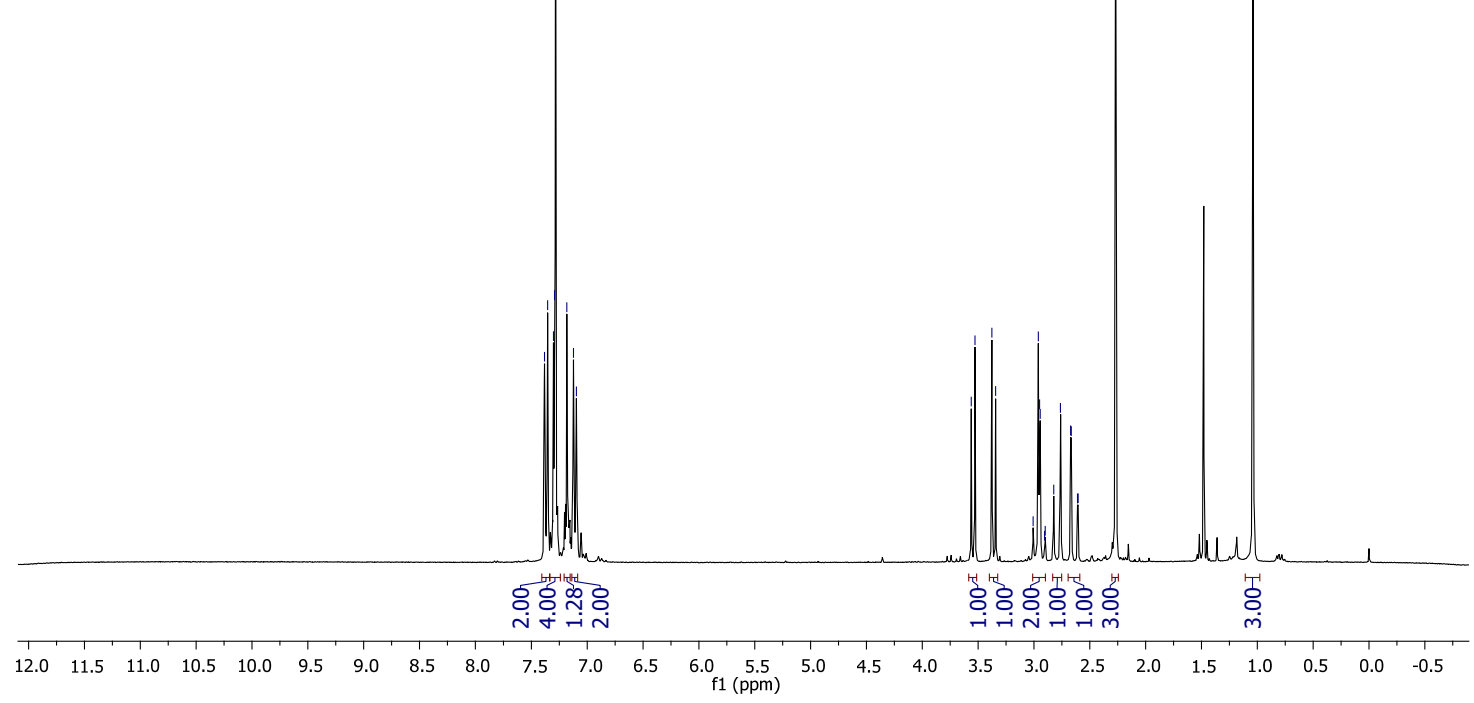

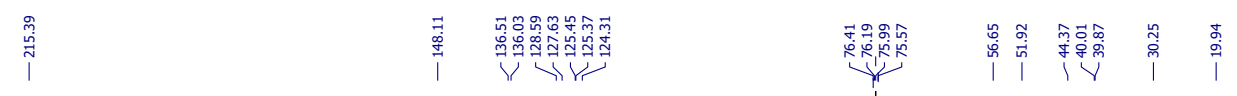

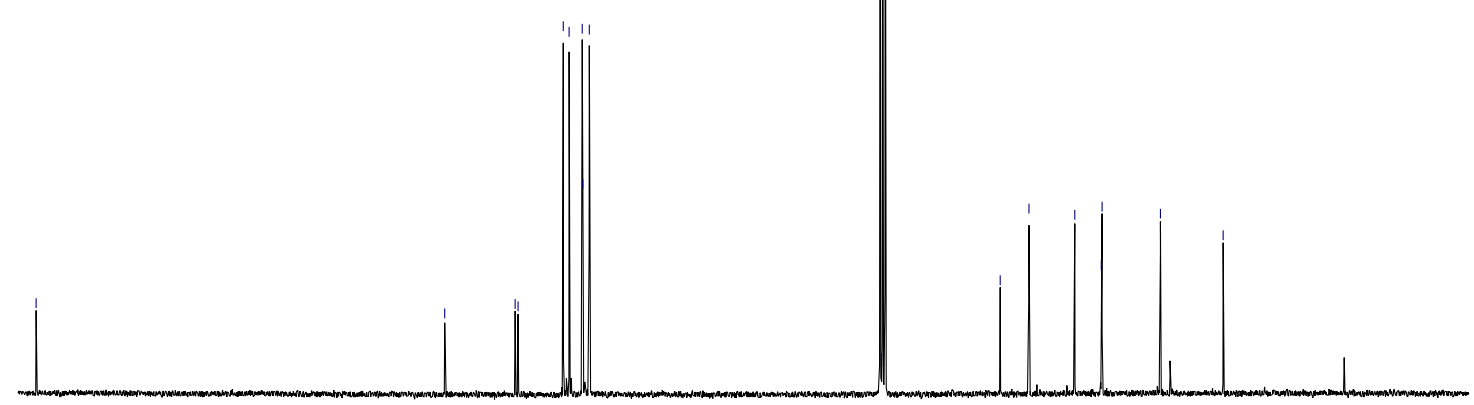

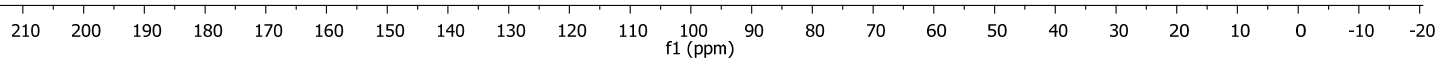

Figure S35. ${ }^{1} \mathrm{H}-\mathrm{NMR}\left(300 \mathrm{MHz}, \mathrm{CDCl}_{3}\right)$ and ${ }^{13} \mathrm{C}\left\{{ }^{1} \mathrm{H}\right\}-\mathrm{NMR}\left(75.5 \mathrm{MHz}, \mathrm{CDCl}_{3}\right)$ of compound $\mathbf{3 h}$ 


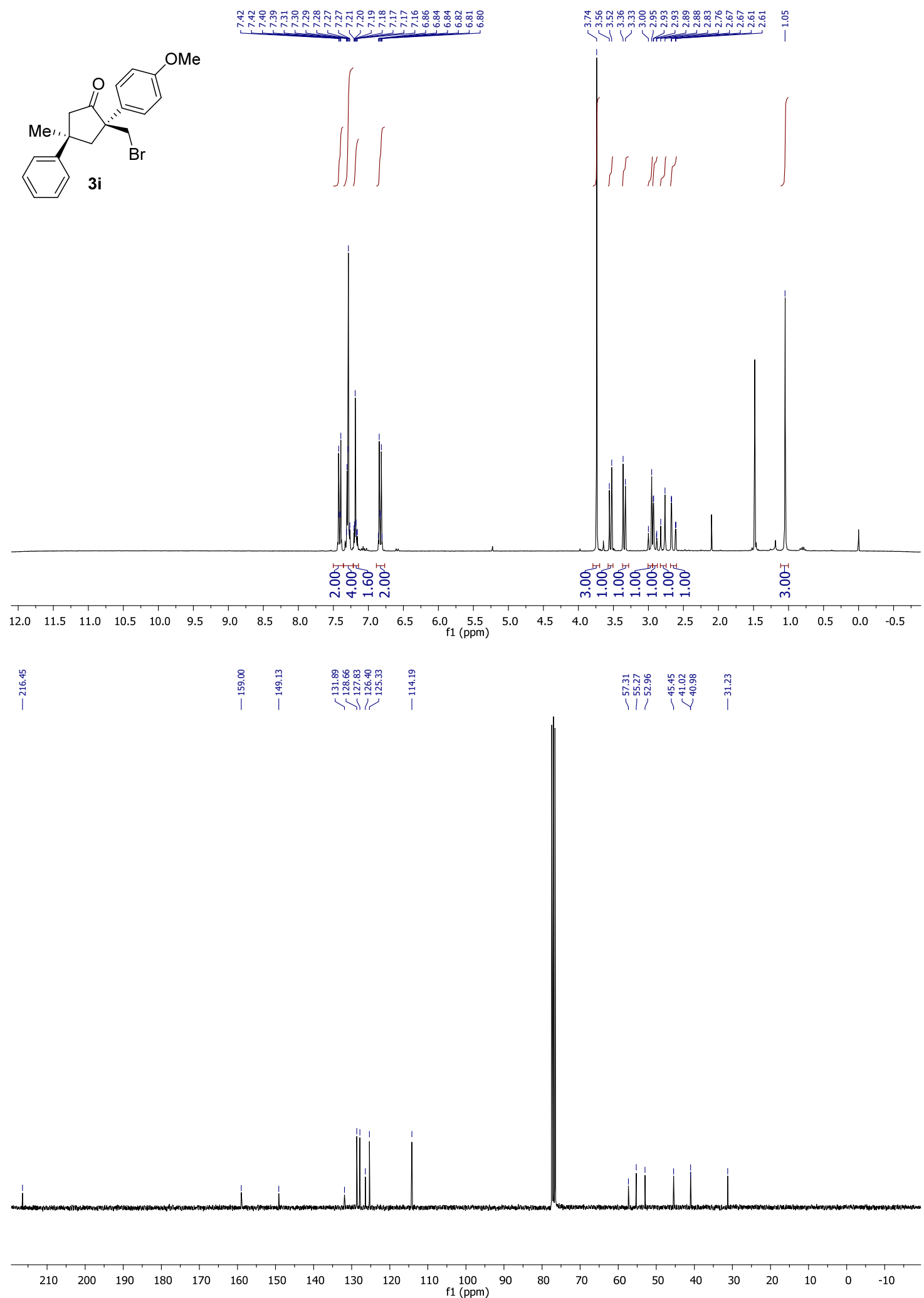

Figure S36. ${ }^{1} \mathrm{H}-\mathrm{NMR}\left(300 \mathrm{MHz}, \mathrm{CDCl}_{3}\right)$ and ${ }^{13} \mathrm{C}\left\{{ }^{1} \mathrm{H}\right\}-\mathrm{NMR}\left(75.5 \mathrm{MHz}, \mathrm{CDCl}_{3}\right)$ of compound $\mathbf{3 i}$ 


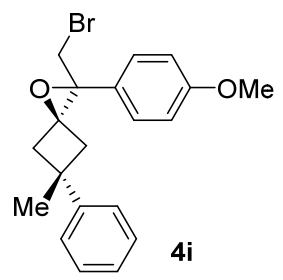

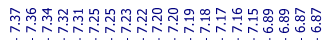

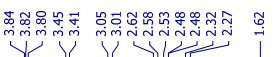

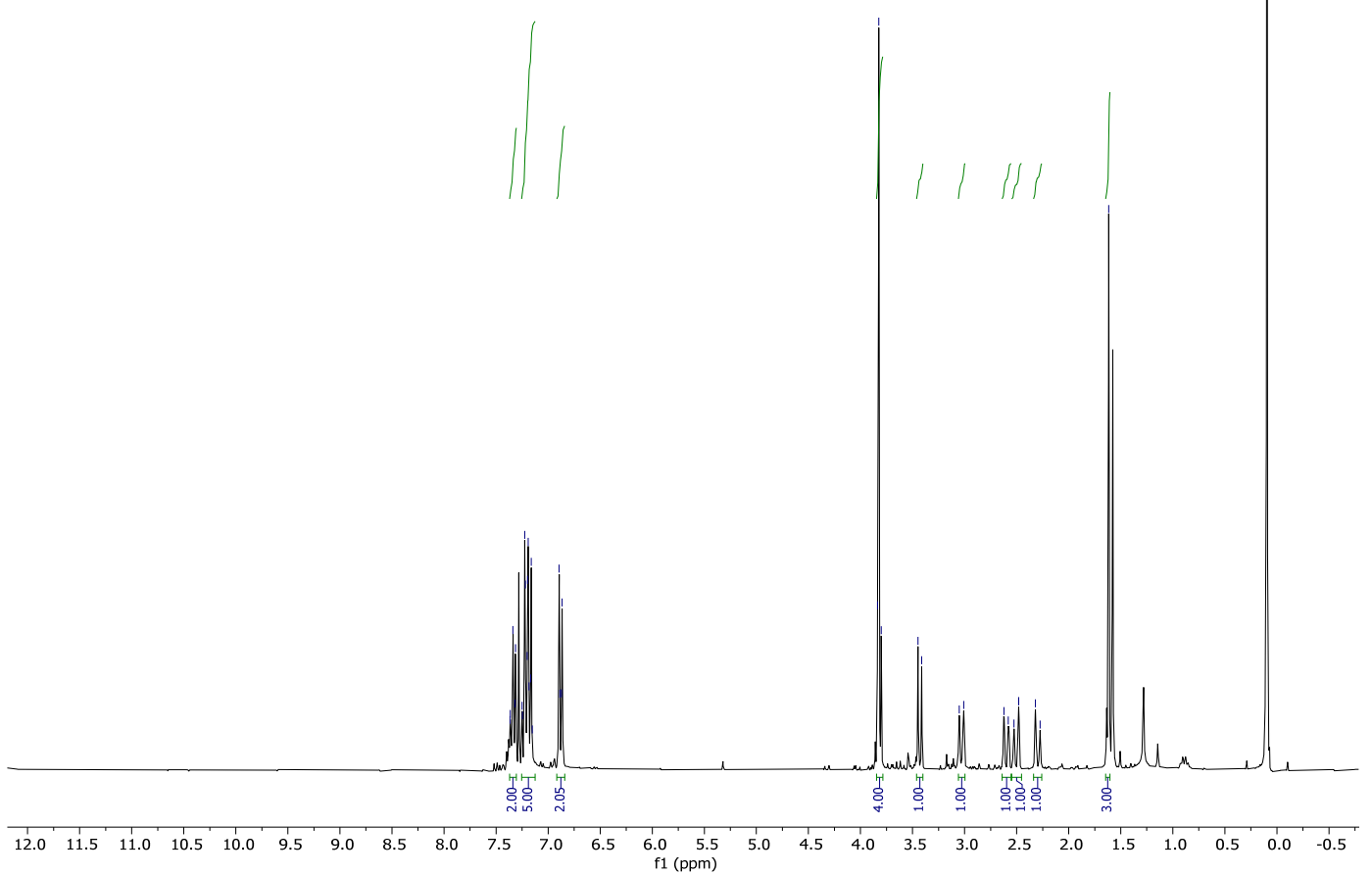

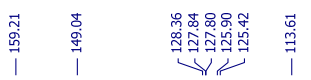

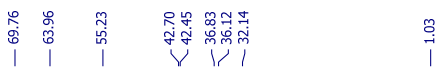

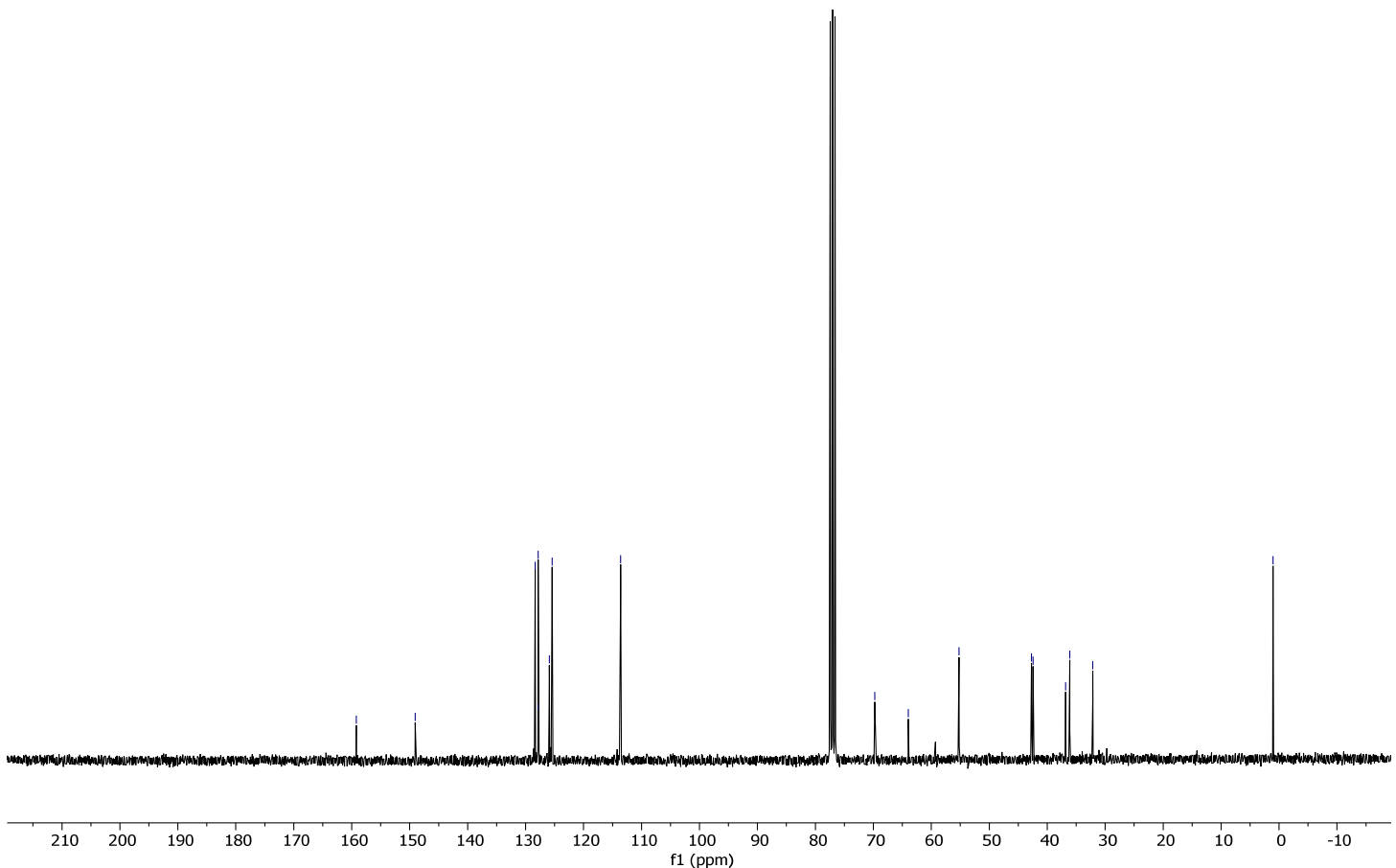

Figure S37. ${ }^{1} \mathrm{H}-\mathrm{NMR}\left(300 \mathrm{MHz}, \mathrm{CDCl}_{3}\right)$ and ${ }^{13} \mathrm{C}\left\{{ }^{1} \mathrm{H}\right\}-\mathrm{NMR}\left(75.5 \mathrm{MHz}, \mathrm{CDCl}_{3}\right)$ of compound $\mathbf{4 i}$ 

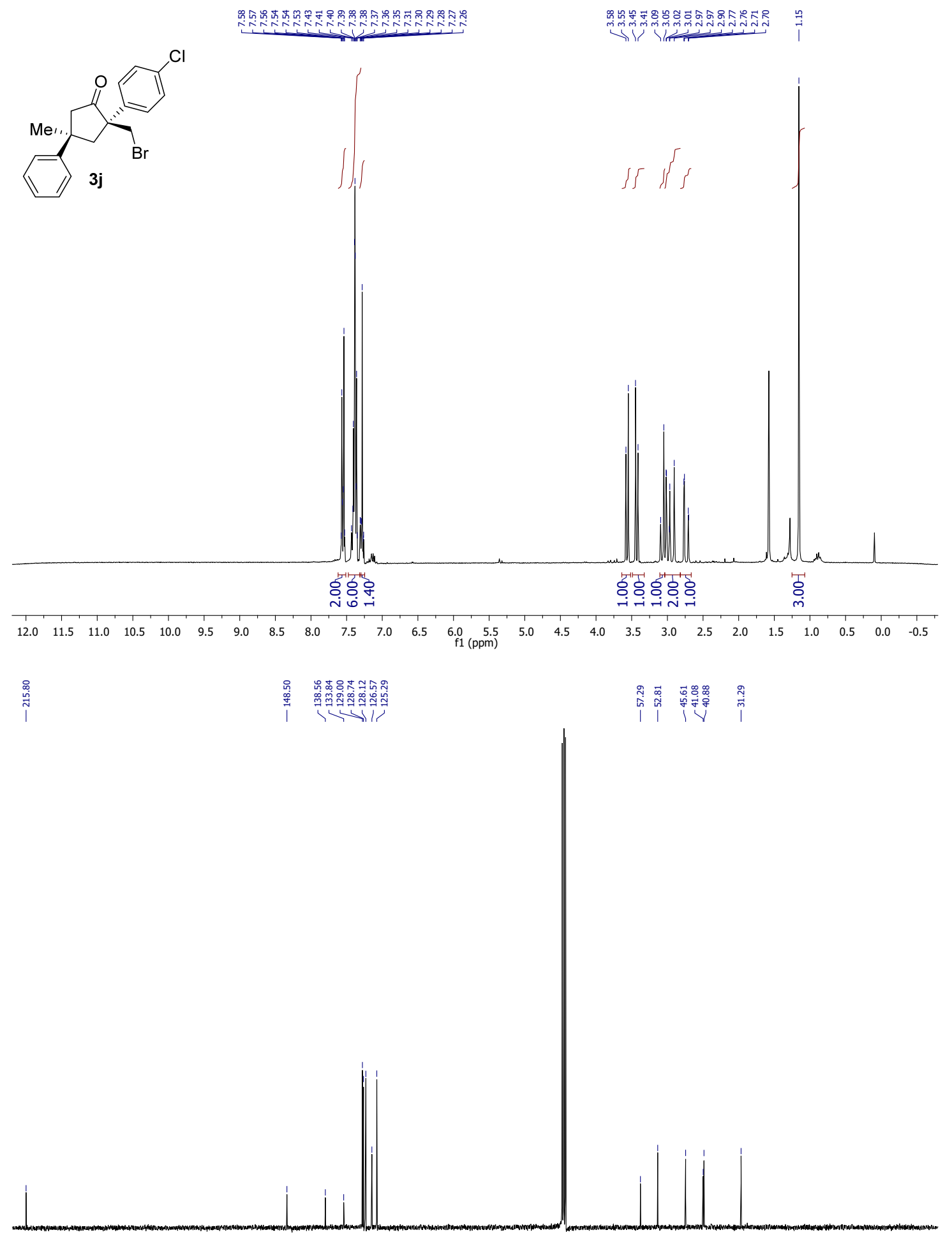

$\begin{array}{llllllllllllllllllllllllll}1 & 210 & 200 & 190 & 180 & 170 & 160 & 150 & 140 & 130 & 120 & 110 & 100 & 90 & 80 & 70 & 60 & 50 & 40 & 30 & 20 & 10 & 0 & -10\end{array}$

Figure S38. ${ }^{1} \mathrm{H}-\mathrm{NMR}\left(300 \mathrm{MHz}, \mathrm{CDCl}_{3}\right)$ and ${ }^{13} \mathrm{C}\left\{{ }^{1} \mathrm{H}\right\}-\mathrm{NMR}\left(75.5 \mathrm{MHz}, \mathrm{CDCl}_{3}\right)$ of compound $\mathbf{3 j}$ 


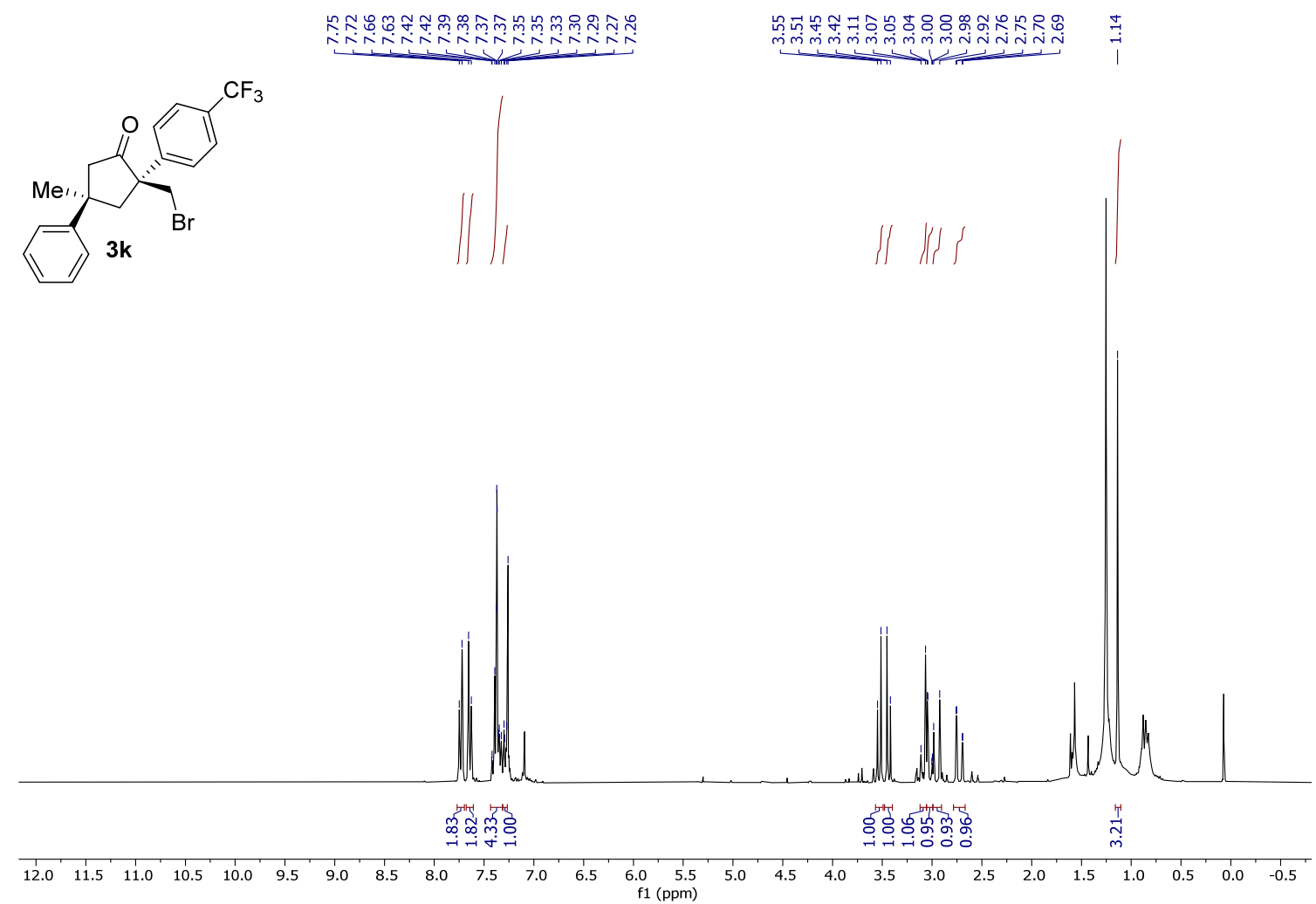

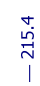

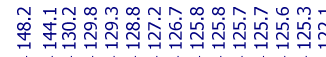

||

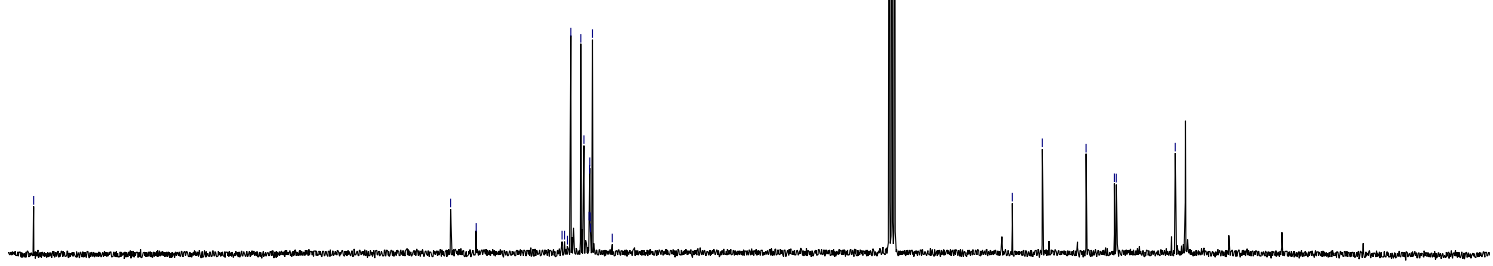

$\begin{array}{lllllllllllllllllllllllll}1 & 210 & 200 & 190 & 180 & 170 & 160 & 150 & 140 & 130 & 120 & 110 & 100 & 90 & 80 & 70 & 60 & 50 & 40 & 30 & 20 & 10 & 0 & -10\end{array}$

Figure S39. ${ }^{1} \mathrm{H}-\mathrm{NMR}\left(300 \mathrm{MHz}, \mathrm{CDCl}_{3}\right)$ and ${ }^{13} \mathrm{C}\left\{{ }^{1} \mathrm{H}\right\}-\mathrm{NMR}\left(75.5 \mathrm{MHz}, \mathrm{CDCl}_{3}\right)$ of compound 3k 


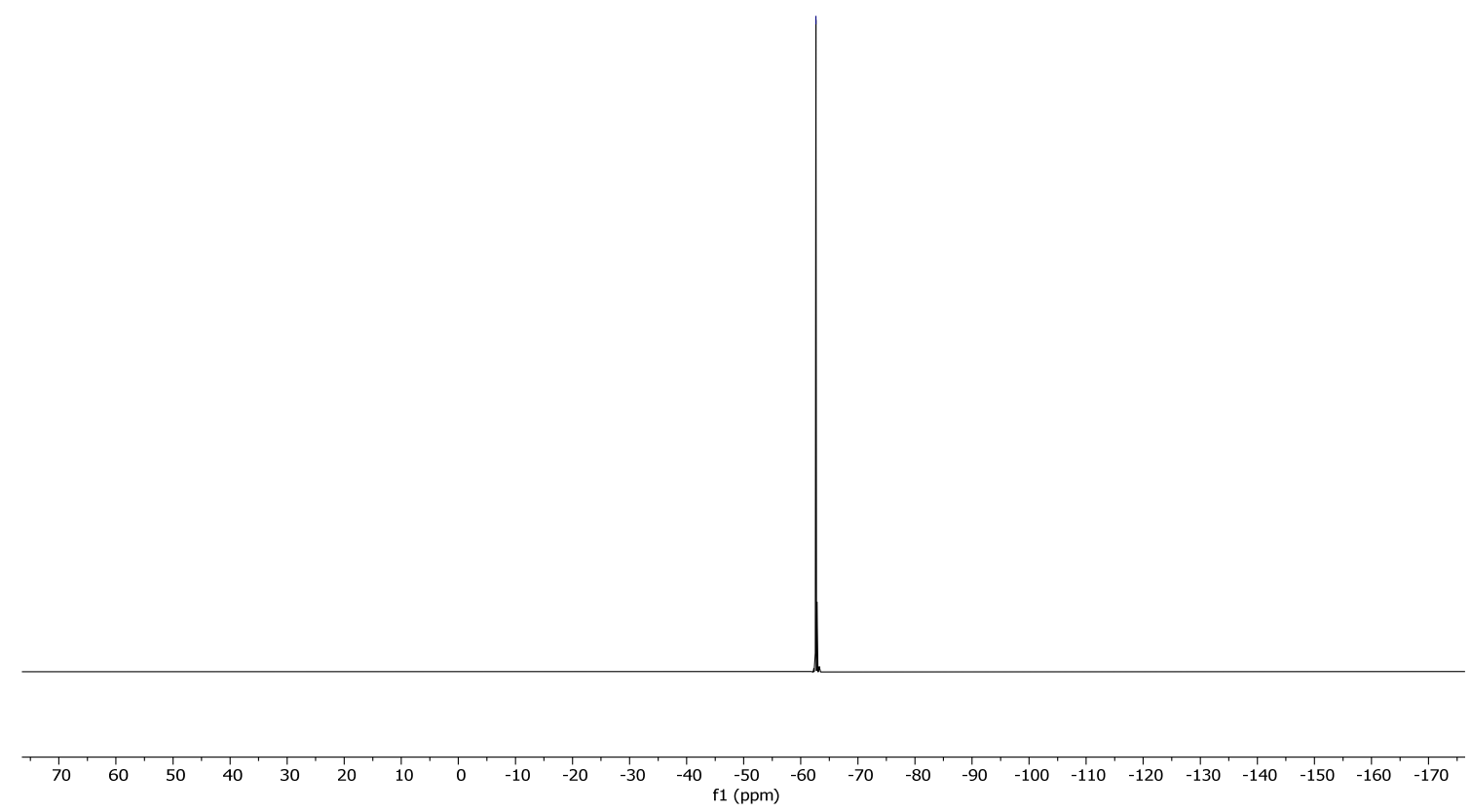

Figure S40. ${ }^{31} \mathrm{P}-\mathrm{NMR}\left(122 \mathrm{MHz}, \mathrm{CDCl}_{3}\right)$ of compound $\mathbf{3 k}$ 

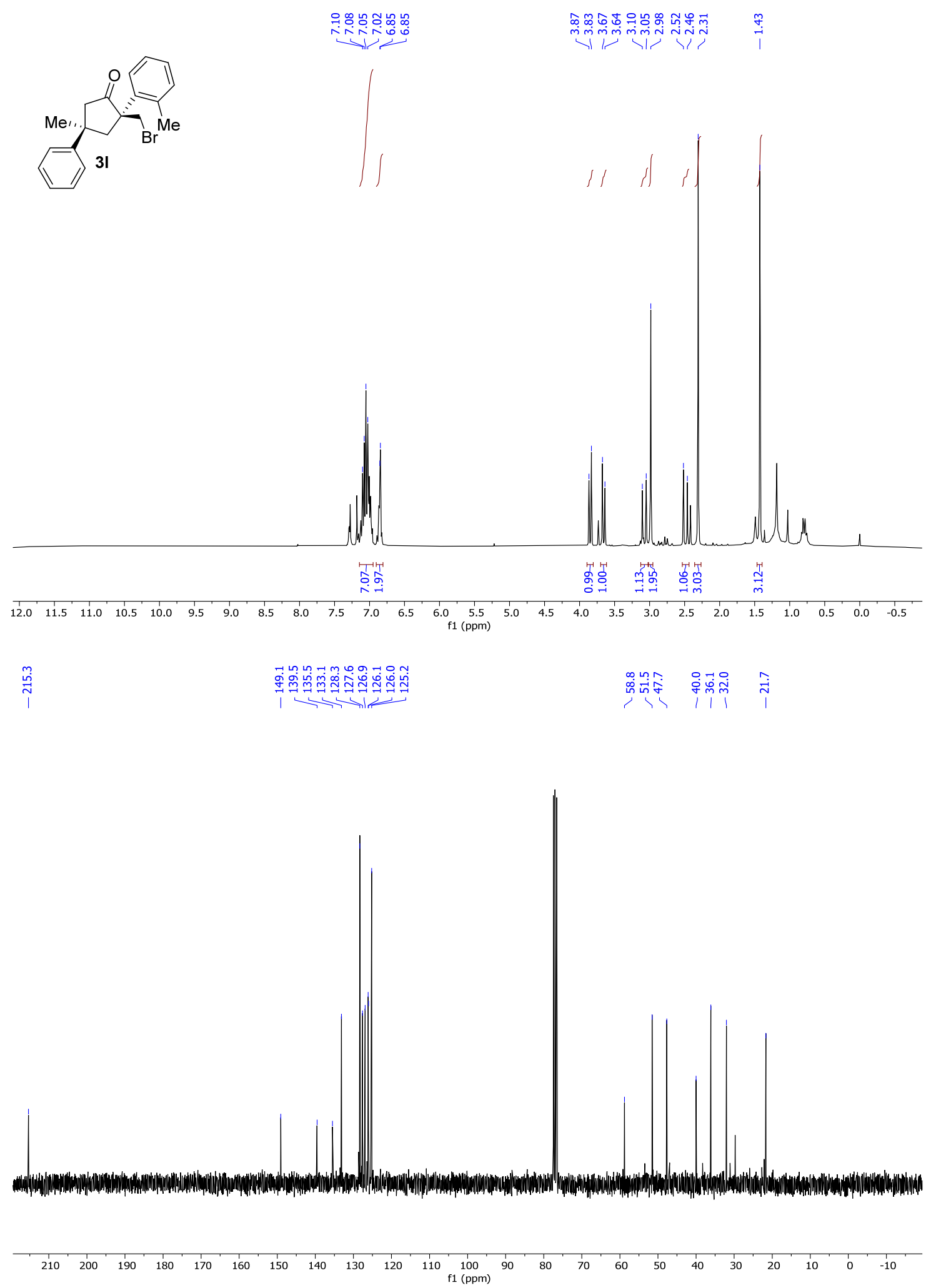

Figure S41. ${ }^{1} \mathrm{H}-\mathrm{NMR}\left(300 \mathrm{MHz}, \mathrm{CDCl}_{3}\right)$ and ${ }^{13} \mathrm{C}\left\{{ }^{1} \mathrm{H}\right\}-\mathrm{NMR}\left(75.5 \mathrm{MHz}, \mathrm{CDCl}_{3}\right)$ of compound $\mathbf{3 l}$ 
<smiles>C[C@]1(c2ccccc2)CC(=O)C[C@@]12c1ccccc1CCC2Br</smiles>
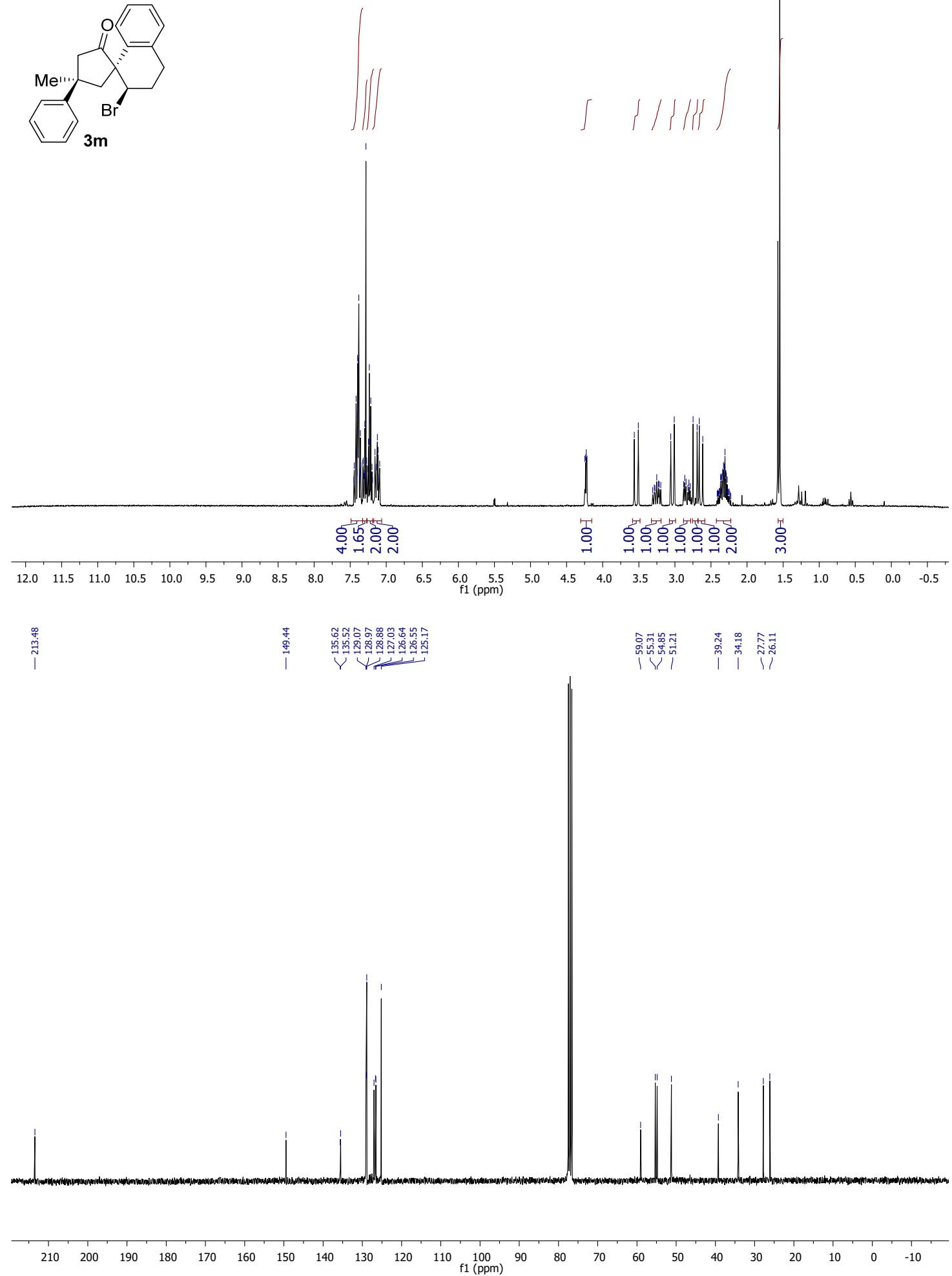

Figure S42. ${ }^{1} \mathrm{H}-\mathrm{NMR}\left(300 \mathrm{MHz}, \mathrm{CDCl}_{3}\right)$ and ${ }^{13} \mathrm{C}\left\{{ }^{1} \mathrm{H}\right\}-\mathrm{NMR}\left(75.5 \mathrm{MHz}, \mathrm{CDCl}_{3}\right)$ of compound $\mathbf{3 m}$ 

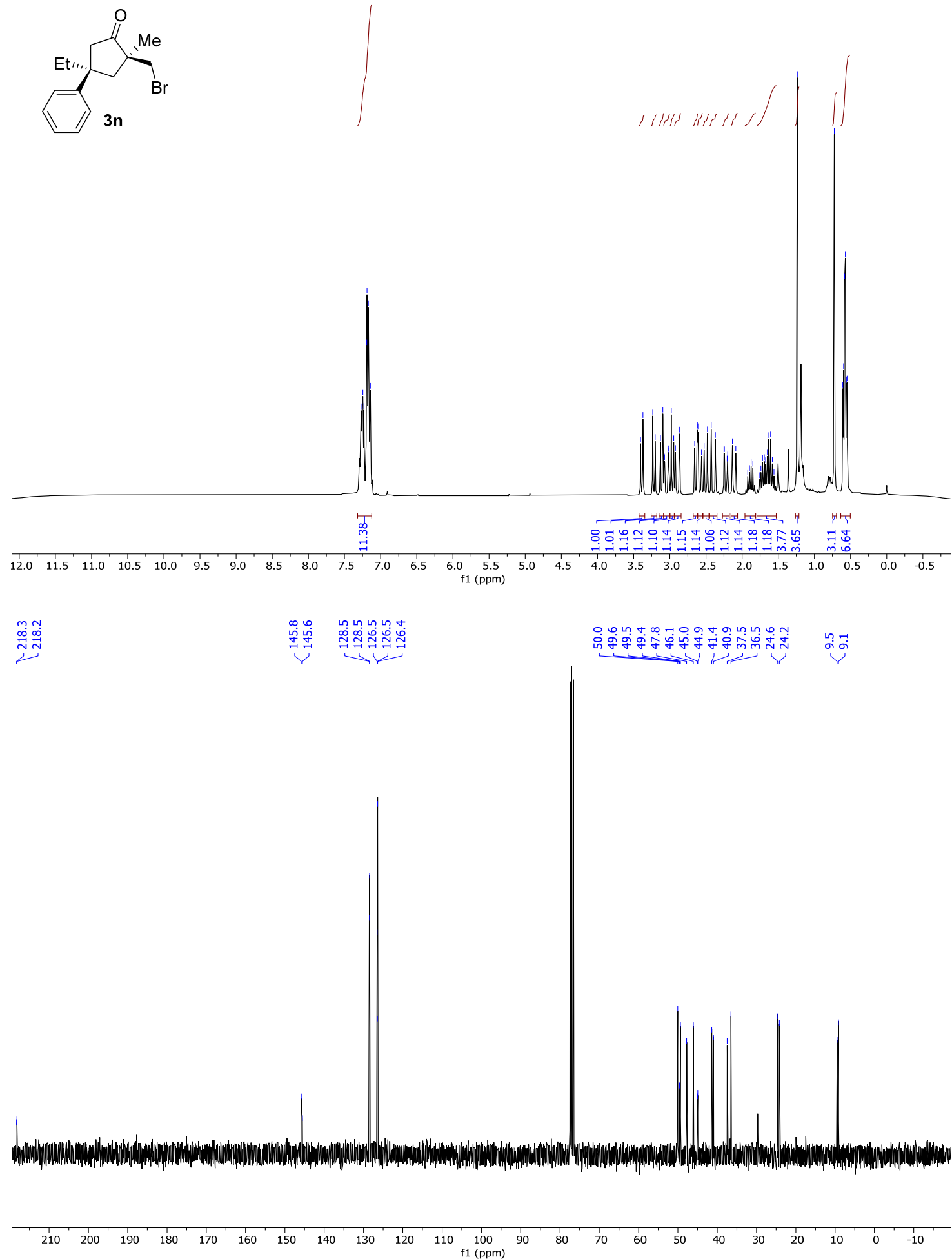

Figure S43. ${ }^{1} \mathrm{H}-\mathrm{NMR}\left(300 \mathrm{MHz}, \mathrm{CDCl}_{3}\right)$ and ${ }^{13} \mathrm{C}\left\{{ }^{1} \mathrm{H}\right\}-\mathrm{NMR}\left(75.5 \mathrm{MHz}, \mathrm{CDCl}_{3}\right)$ of compound 3n 


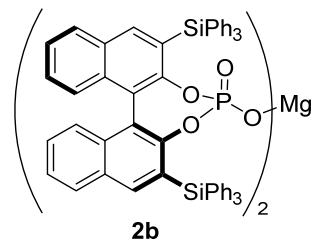

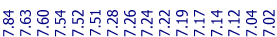

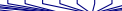
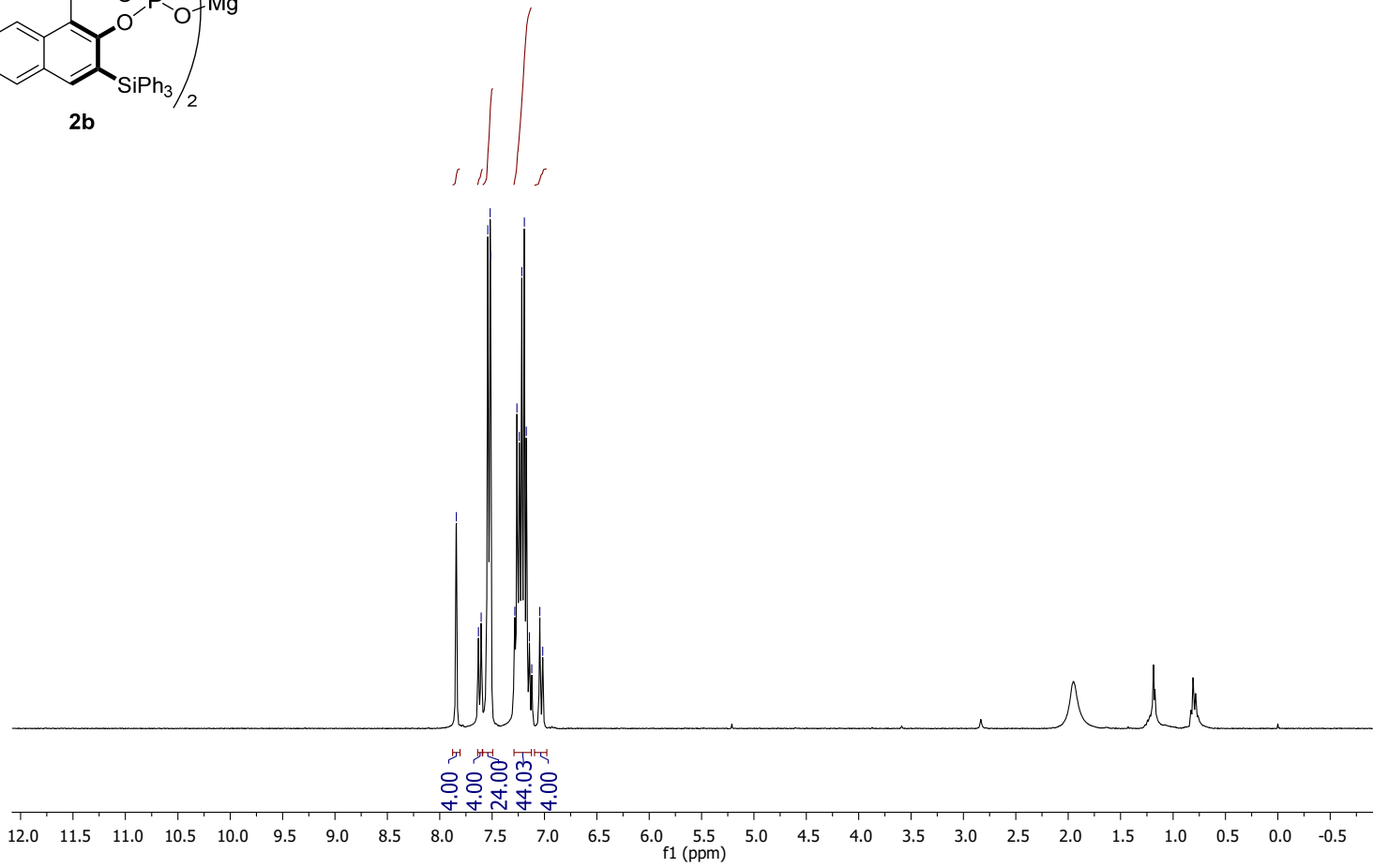

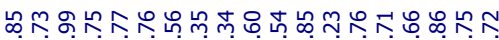

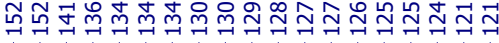

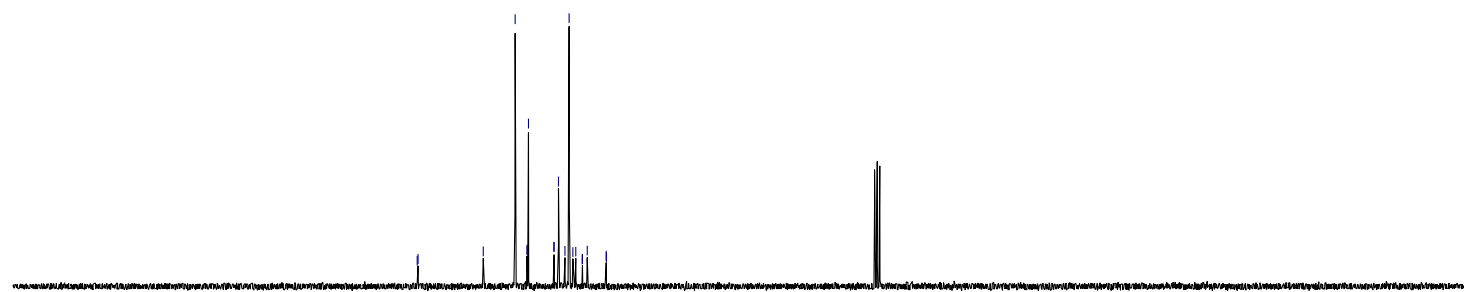

$\begin{array}{llllllllllllllllllllllll}210 & 200 & 190 & 180 & 170 & 160 & 150 & 140 & 130 & 120 & 110 & 100 & 90 & 80 & 70 & 60 & 50 & 40 & 30 & 20 & 10 & 0 & -10\end{array}$

Figure S44. ${ }^{1} \mathrm{H}-\mathrm{NMR}\left(300 \mathrm{MHz}, \mathrm{CDCl}_{3}\right)$ and ${ }^{13} \mathrm{C}\left\{{ }^{1} \mathrm{H}\right\}-\mathrm{NMR}\left(75.5 \mathrm{MHz}, \mathrm{CDCl}_{3}\right)$ of compound $\mathbf{2 b}$ 


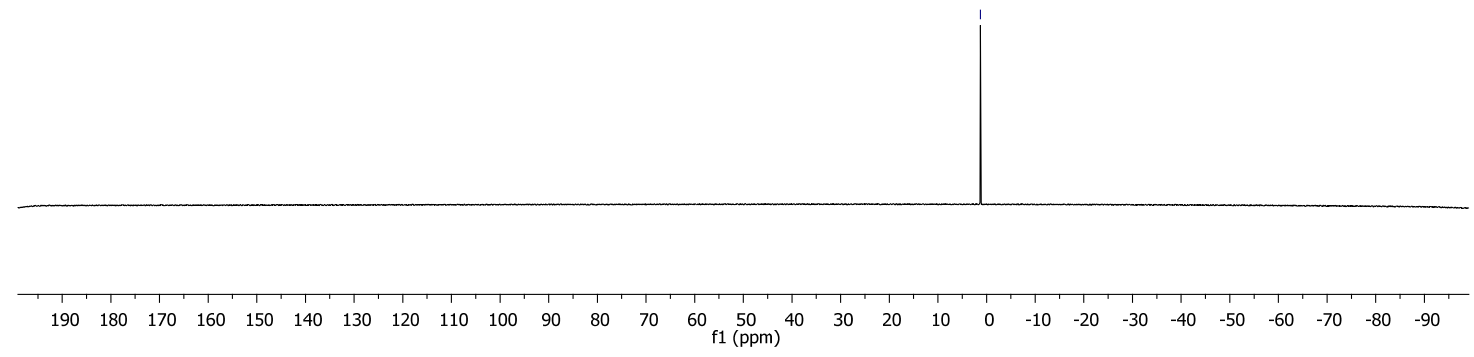

Figure $\mathbf{S 4 5} .{ }^{31} \mathrm{P}-\mathrm{NMR}\left(122 \mathrm{MHz}, \mathrm{CDCl}_{3}\right)$ of compound $\mathbf{2 b}$ 

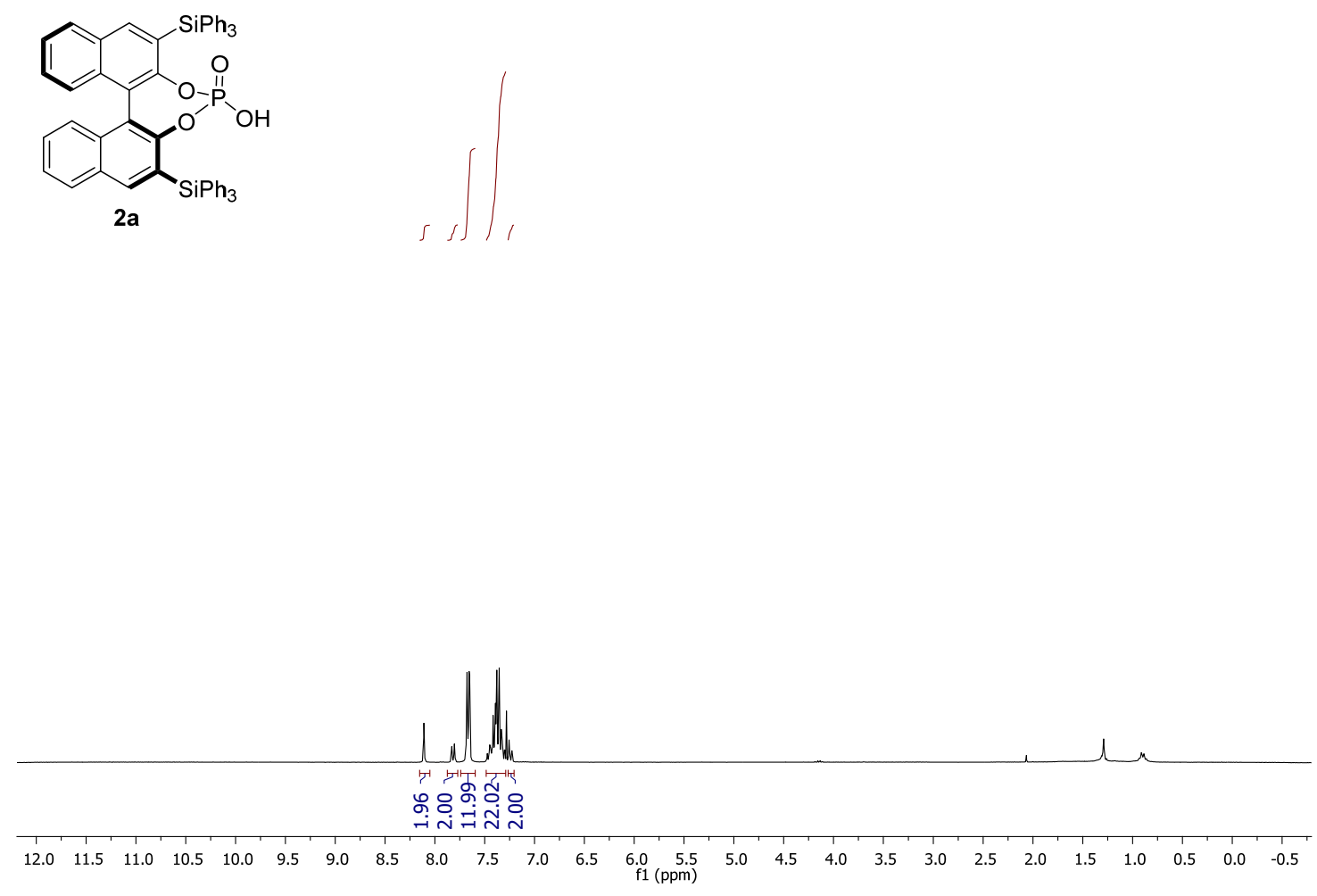

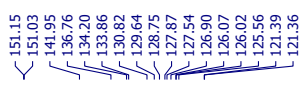

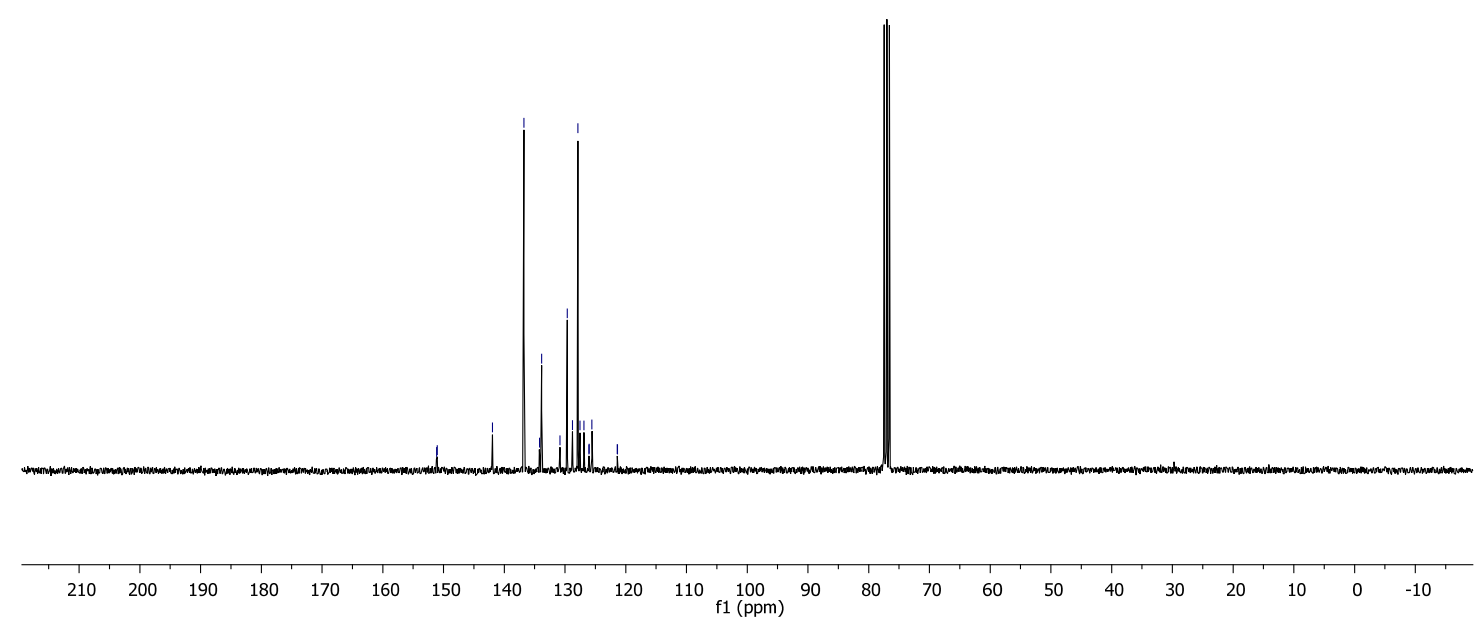

Figure S46. ${ }^{1} \mathrm{H}-\mathrm{NMR}\left(300 \mathrm{MHz}, \mathrm{CDCl}_{3}\right)$ and ${ }^{13} \mathrm{C}\left\{{ }^{1} \mathrm{H}\right\}-\mathrm{NMR}\left(75.5 \mathrm{MHz}, \mathrm{CDCl}_{3}\right)$ of compound 2a 


$\mid$

$\begin{array}{lllllllllllllllllllllllllllll}190 & 180 & 170 & 160 & 150 & 140 & 130 & 120 & 110 & 100 & 90 & 80 & 70 & 60 & 50 & 50 & 30 & 20 & 10 & 0 & -10 & -20 & -30 & -40 & -50 & -60 & -70 & -80 & -90\end{array}$

Figure S47. ${ }^{31} \mathrm{P}-\mathrm{NMR}\left(122 \mathrm{MHz}, \mathrm{CDCl}_{3}\right)$ of compound 2a 


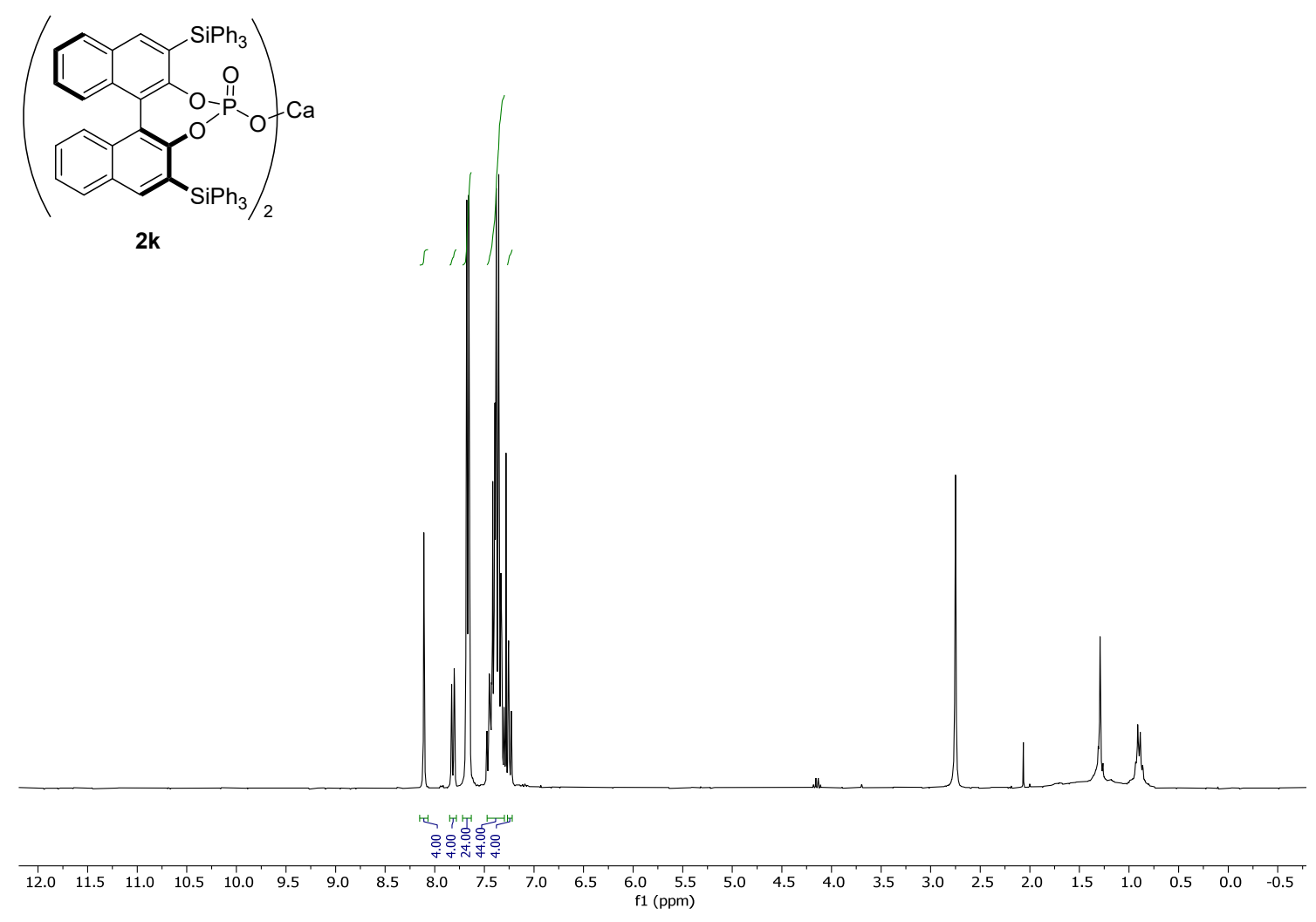

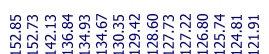

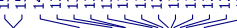

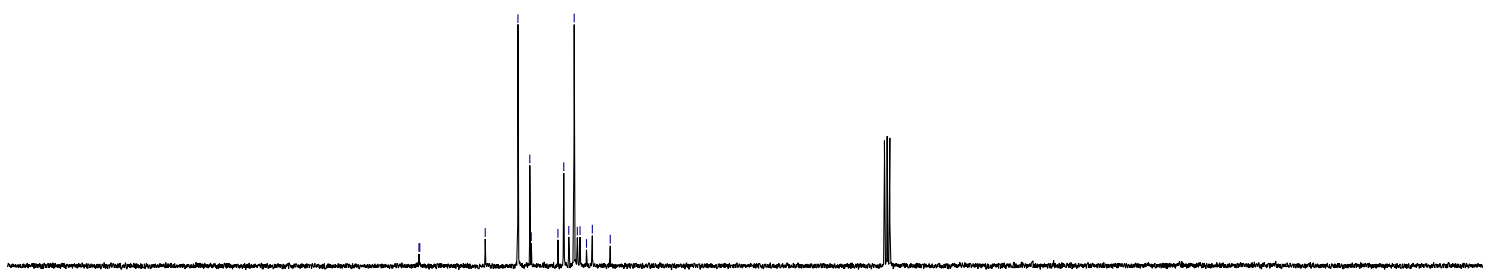

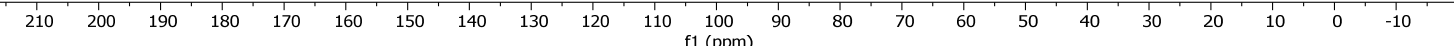

Figure S48. ${ }^{1} \mathrm{H}-\mathrm{NMR}\left(300 \mathrm{MHz}, \mathrm{CDCl}_{3}\right)$ and ${ }^{13} \mathrm{C}\left\{{ }^{1} \mathrm{H}\right\}$-NMR $\left(75.5 \mathrm{MHz}, \mathrm{CDCl}_{3}\right)$ of compound $2 \mathrm{k}$ 


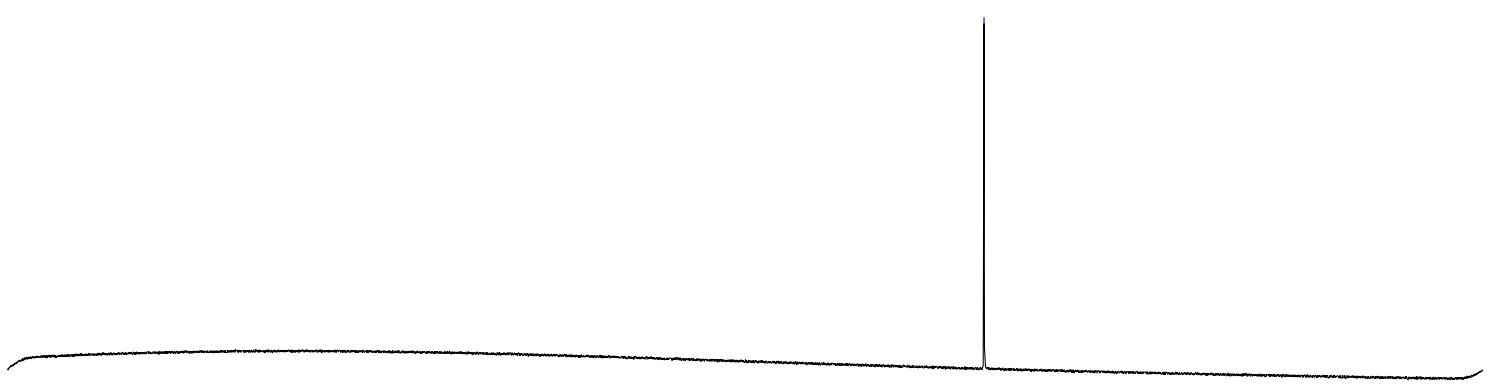

$\begin{array}{lllllllllllllllllllllllllllll}190 & 180 & 170 & 160 & 150 & 140 & 130 & 120 & 110 & 100 & 90 & 80 & 70 & 60 & 50 & 40 & 30 & 20 & 10 & 0 & -10 & -20 & -30 & -40 & -50 & -60 & -70 & -80 & -90\end{array}$ Figure S49. ${ }^{31} \mathrm{P}-\mathrm{NMR}\left(122 \mathrm{MHz}, \mathrm{CDCl}_{3}\right)$ of compound $\mathbf{2 k}$ 


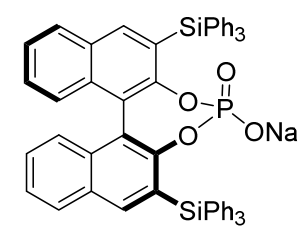

2)
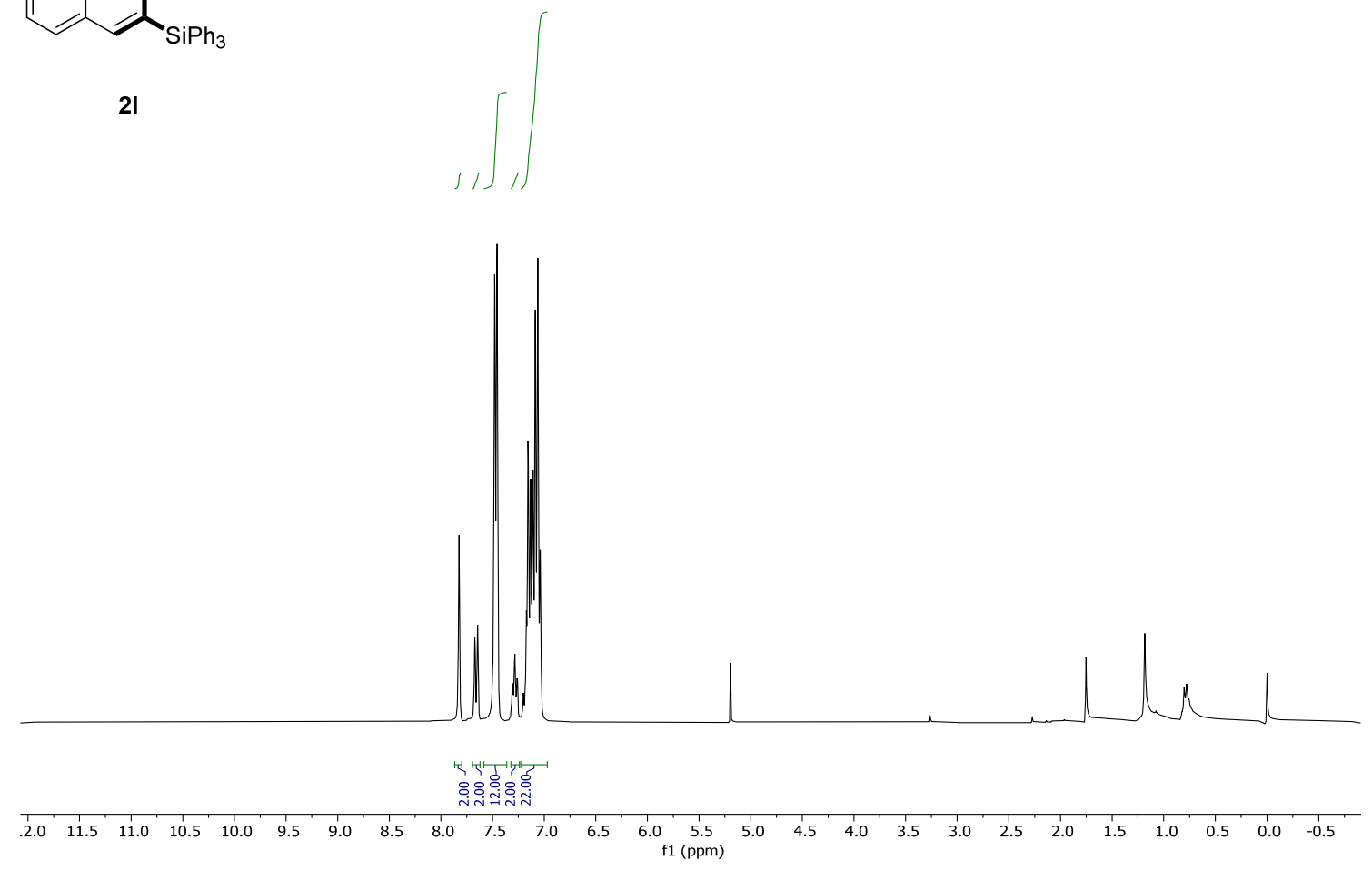

Y.

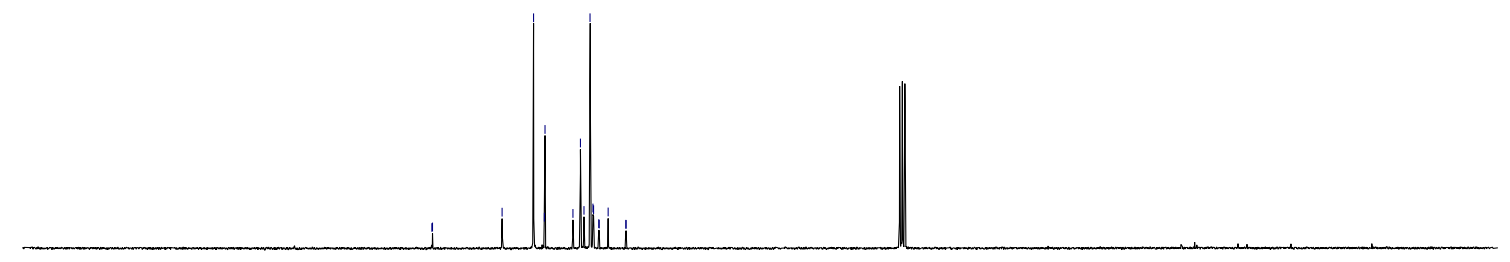

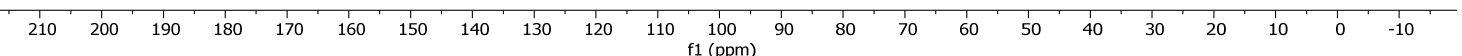

Figure S50. ${ }^{1} \mathrm{H}-\mathrm{NMR}\left(300 \mathrm{MHz}, \mathrm{CDCl}_{3}\right)$ and ${ }^{13} \mathrm{C}\left\{{ }^{1} \mathrm{H}\right\}-\mathrm{NMR}\left(75.5 \mathrm{MHz}, \mathrm{CDCl}_{3}\right)$ of compound 2l 


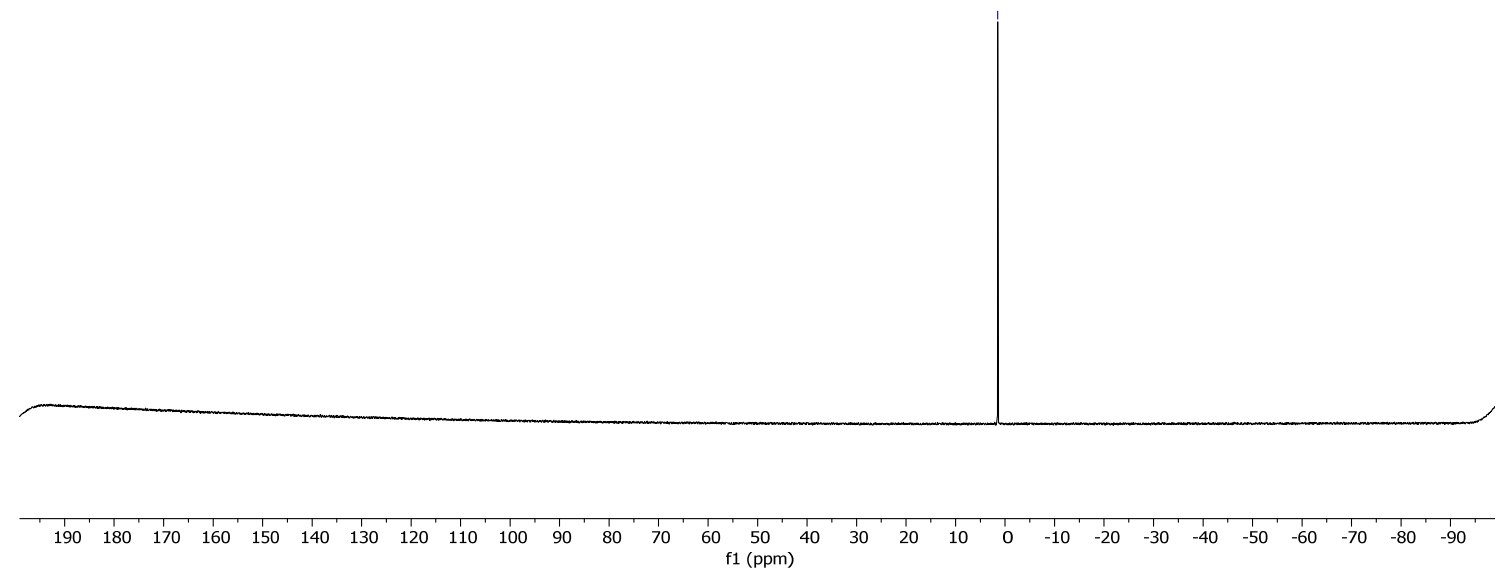

Figure S51. ${ }^{31} \mathrm{P}-\mathrm{NMR}\left(122 \mathrm{MHz}, \mathrm{CDCl}_{3}\right)$ of compound $2 \mathbf{I}$ 

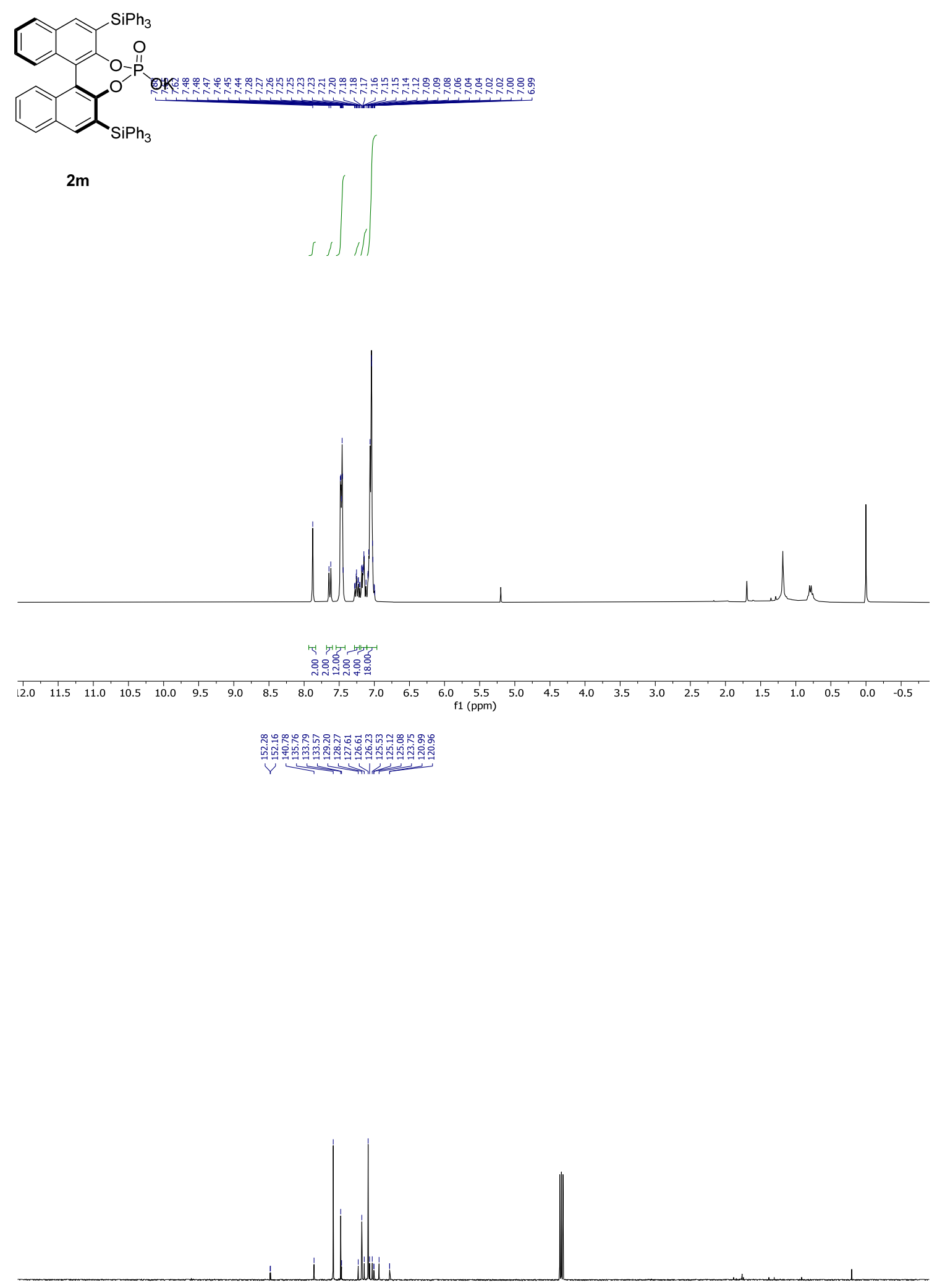

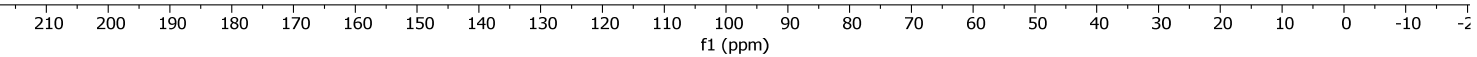

Figure S52. ${ }^{1} \mathrm{H}-\mathrm{NMR}\left(300 \mathrm{MHz}, \mathrm{CDCl}_{3}\right)$ and ${ }^{13} \mathrm{C}\left\{{ }^{1} \mathrm{H}\right\}-\mathrm{NMR}\left(75.5 \mathrm{MHz}, \mathrm{CDCl}_{3}\right)$ of compound $\mathbf{2 m}$ 


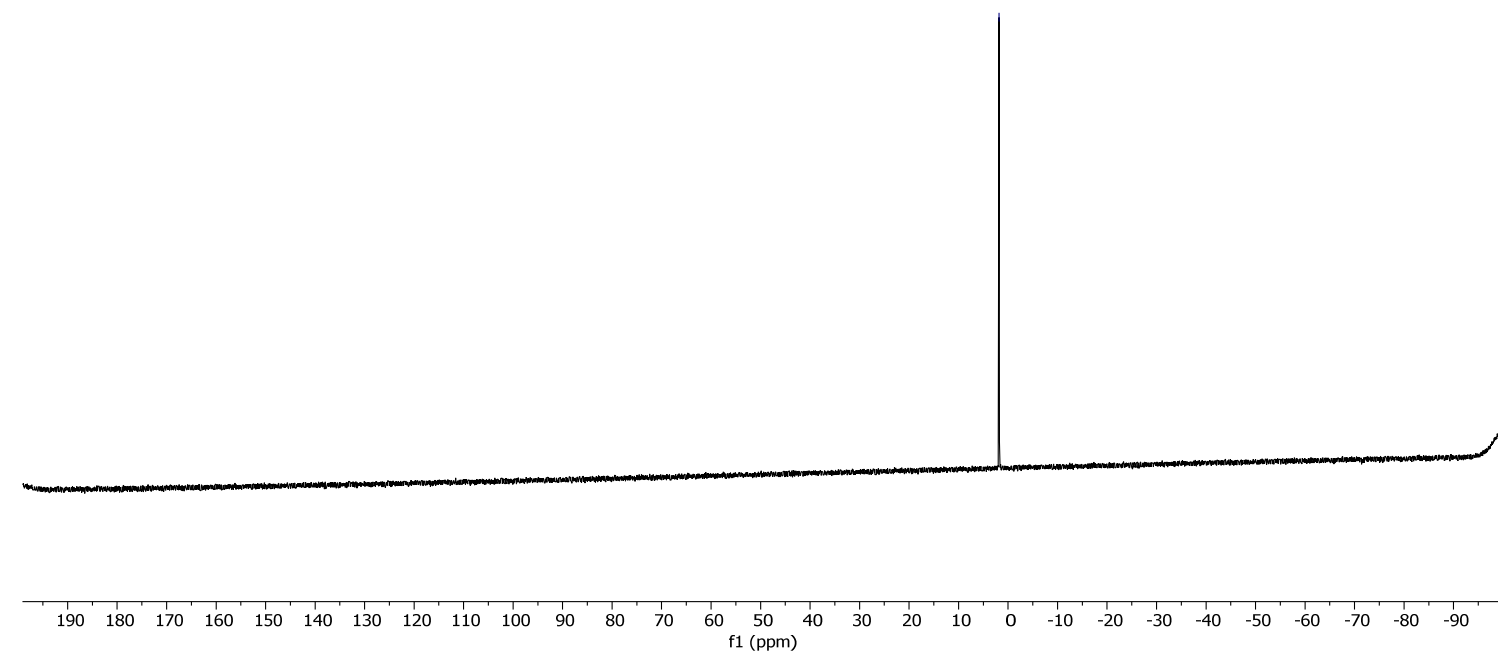

Figure S53. ${ }^{31} \mathrm{P}-\mathrm{NMR}\left(300 \mathrm{MHz}, \mathrm{CDCl}_{3}\right)$ of compound $\mathbf{2 m}$ 
${ }^{31}$ P NMR (122 MHz, $\mathrm{CDCl}_{3}$ ) spectra of catalyst mixtures for non linear effect study

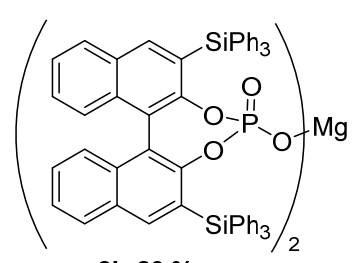

$2 b-80 \%$ ee
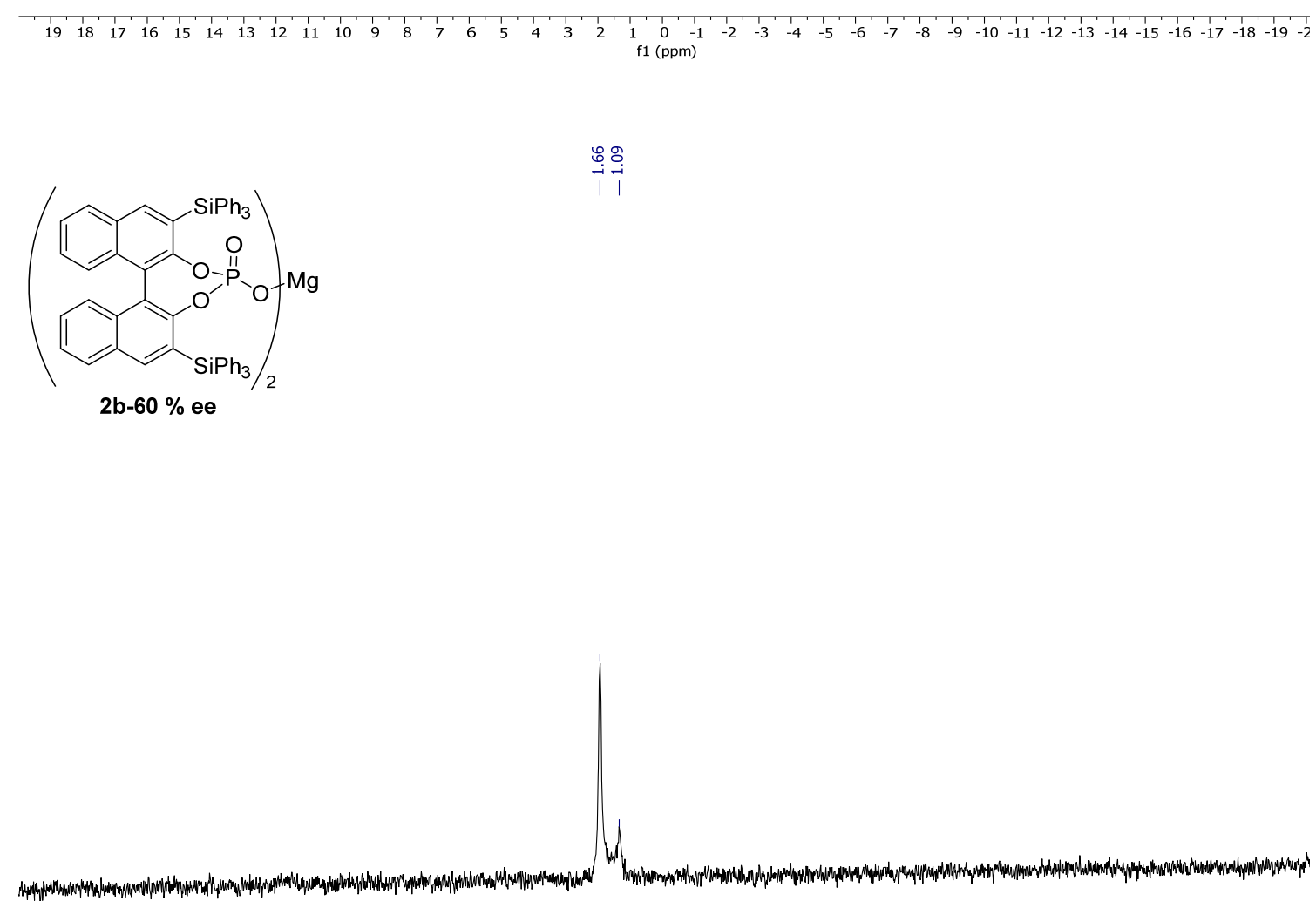

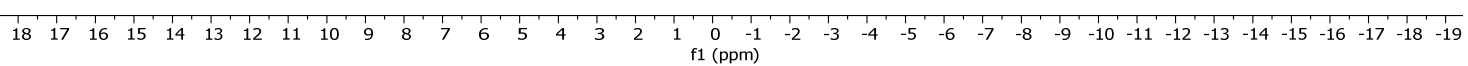




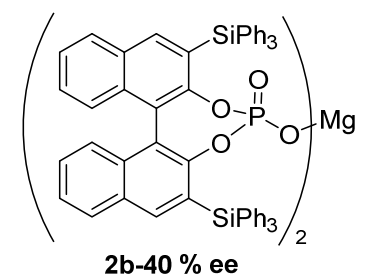

2b-40\% ee

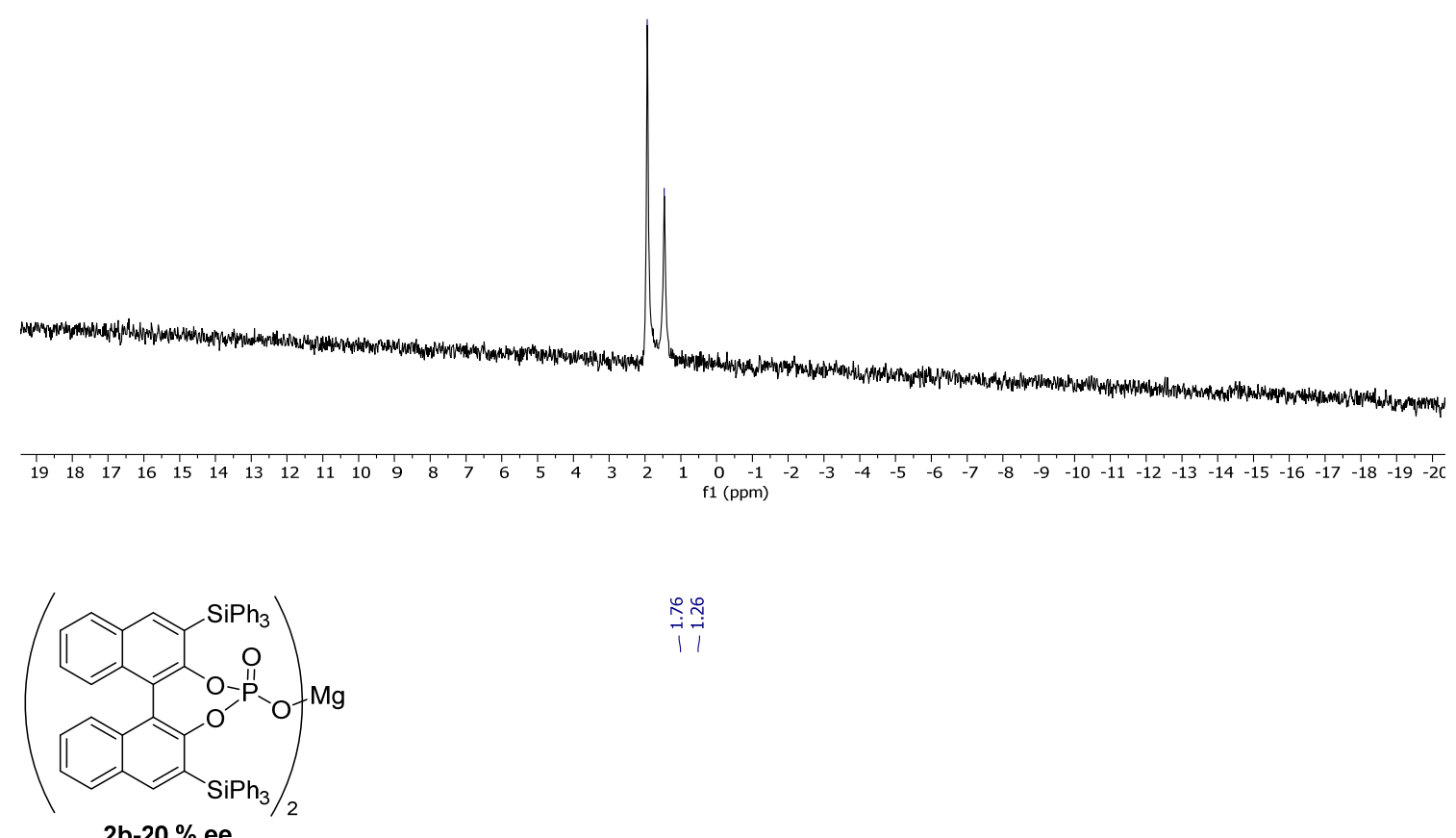

2b-20\% ee

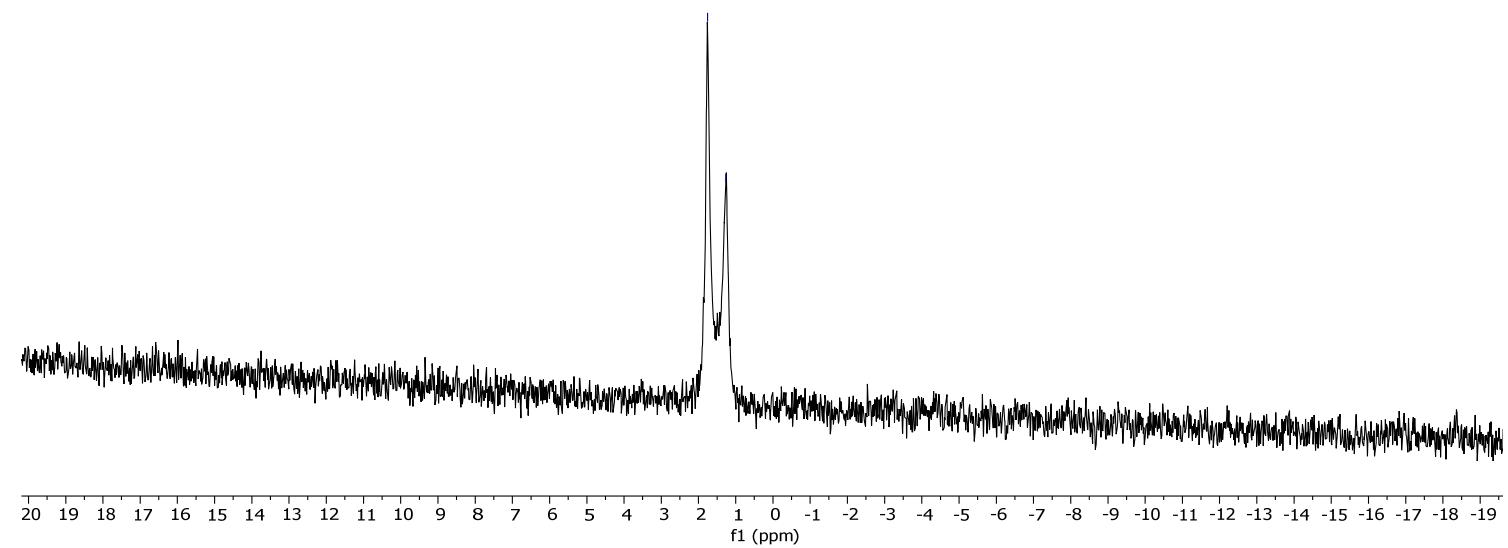




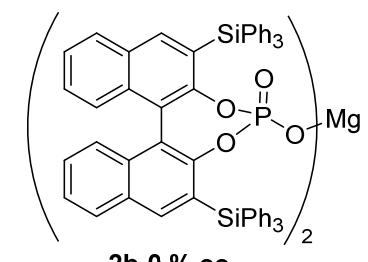

$2 b-0 \%$ ee

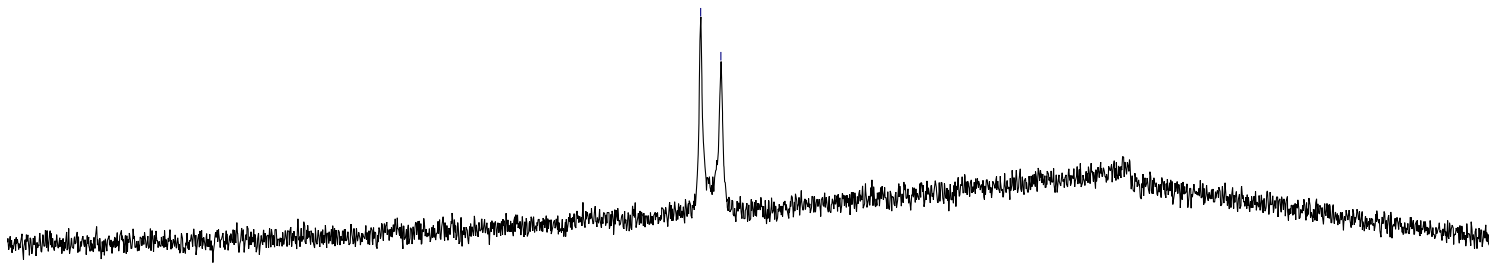

$\begin{array}{lllllllllllllllllllllllllllllllllllllllllllllllllll}20 & 19 & 18 & 17 & 16 & 15 & 14 & 13 & 12 & 11 & 10 & 9 & 8 & 7 & 6 & 5 & 4 & 3 & 2 & 1 & 0 & -1 & -2 & -3 & -4 & -5 & -6 & -7 & -8 & -9 & -10 & -11 & -12 & -13 & -14 & -15 & -16 & -17 & -18 & -19\end{array}$ 


\section{HPLC analysis}
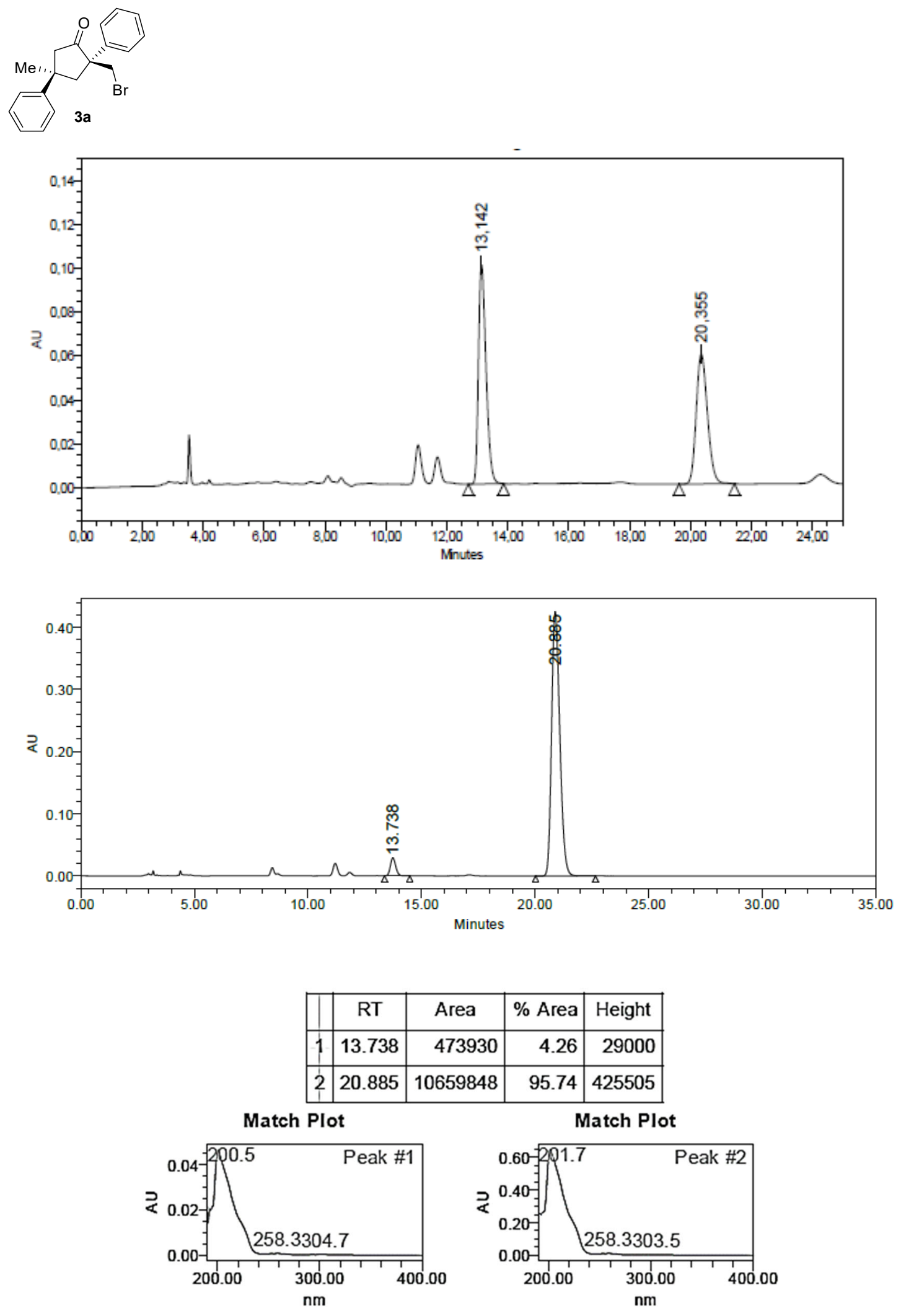

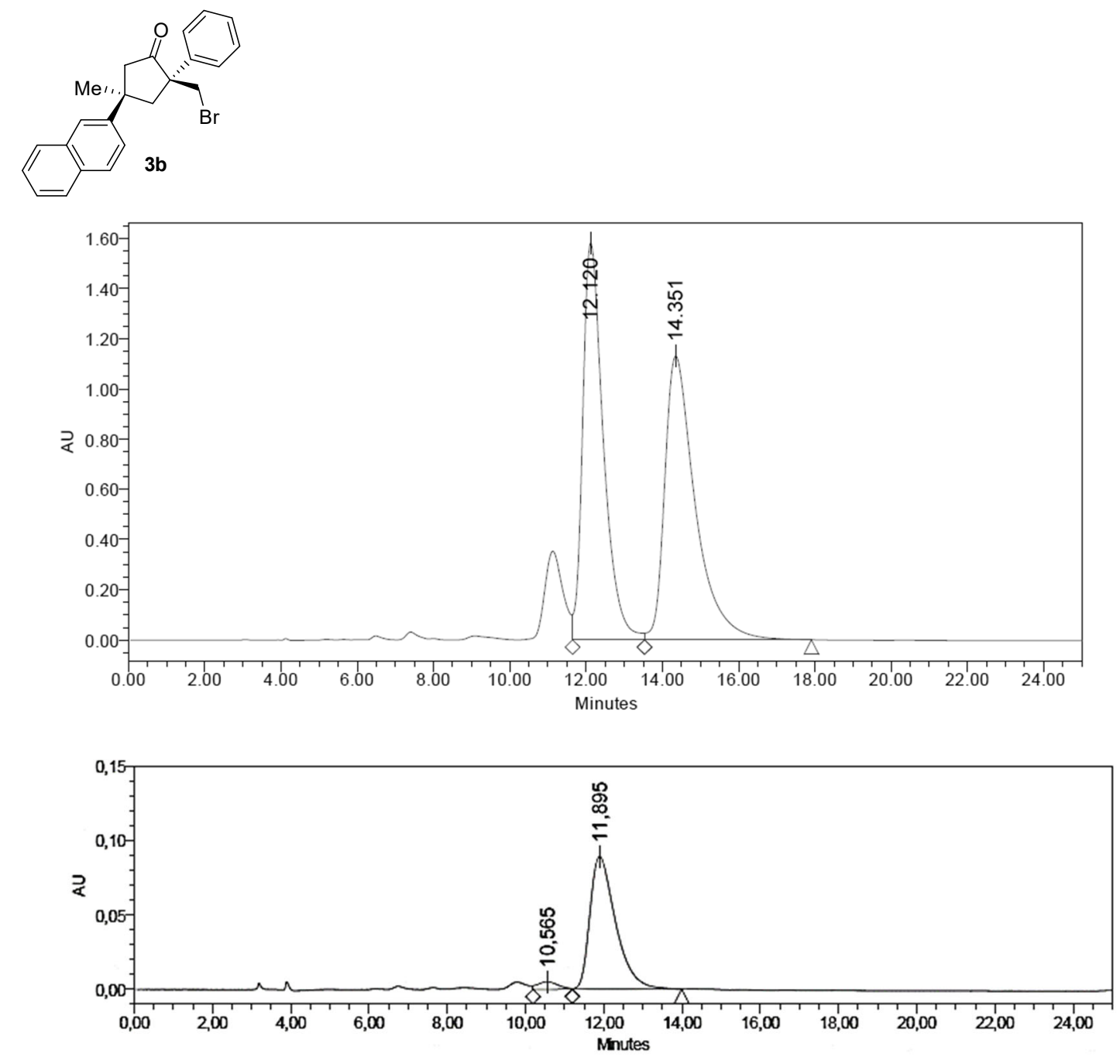

\begin{tabular}{|c|c|c|r|r|}
\hline & RT & Area & Height & \% Area \\
\hline 1 & 10,565 & 189142 & 5256 & 4,43 \\
\hline 2 & 11,895 & 4075833 & 89391 & 95,57 \\
\hline
\end{tabular}
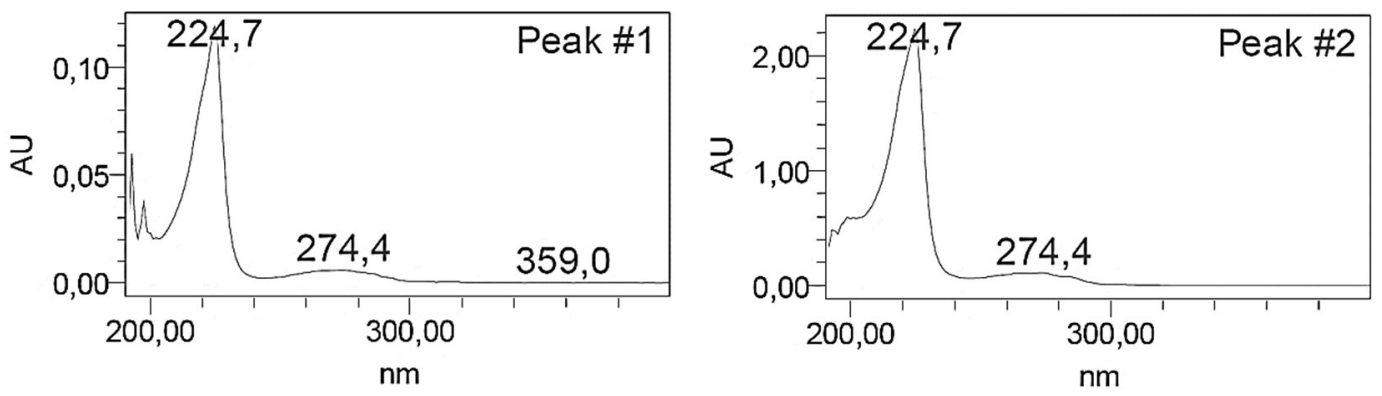

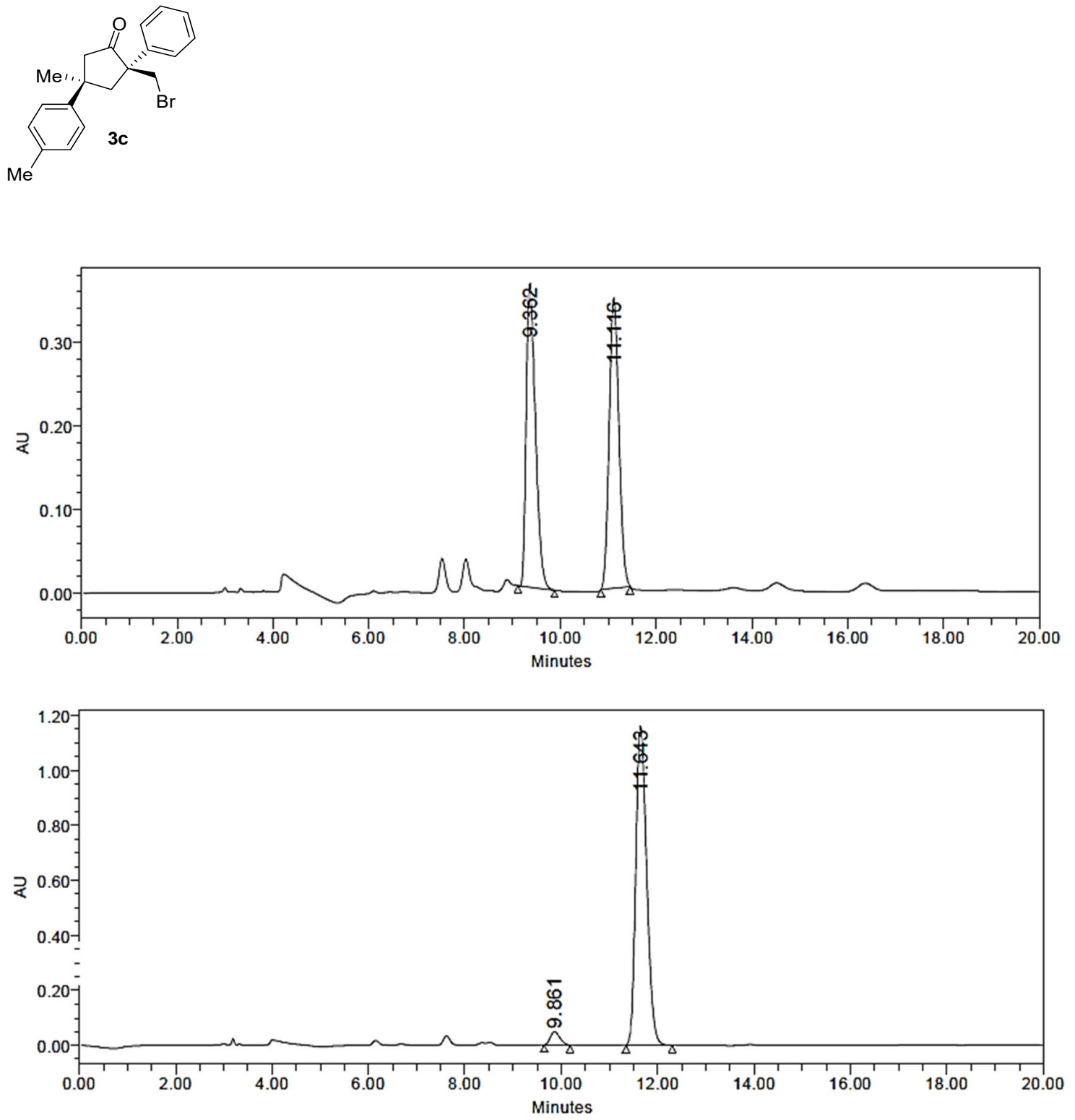

\begin{tabular}{|l|r|r|r|r|}
\hline & \multicolumn{1}{|c|}{ RT } & \multicolumn{1}{c|}{ Area } & \% Area & Height \\
\hline 1 & 9.861 & 677340 & 3.58 & 49539 \\
\hline 2 & 11.643 & 18259198 & 96.42 & 1159915 \\
\hline
\end{tabular}

Match Plot

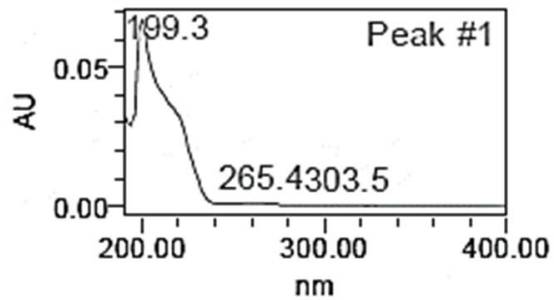

Match Plot

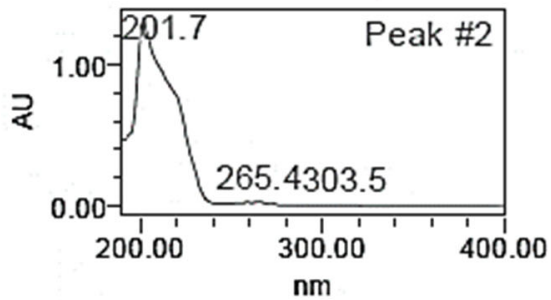



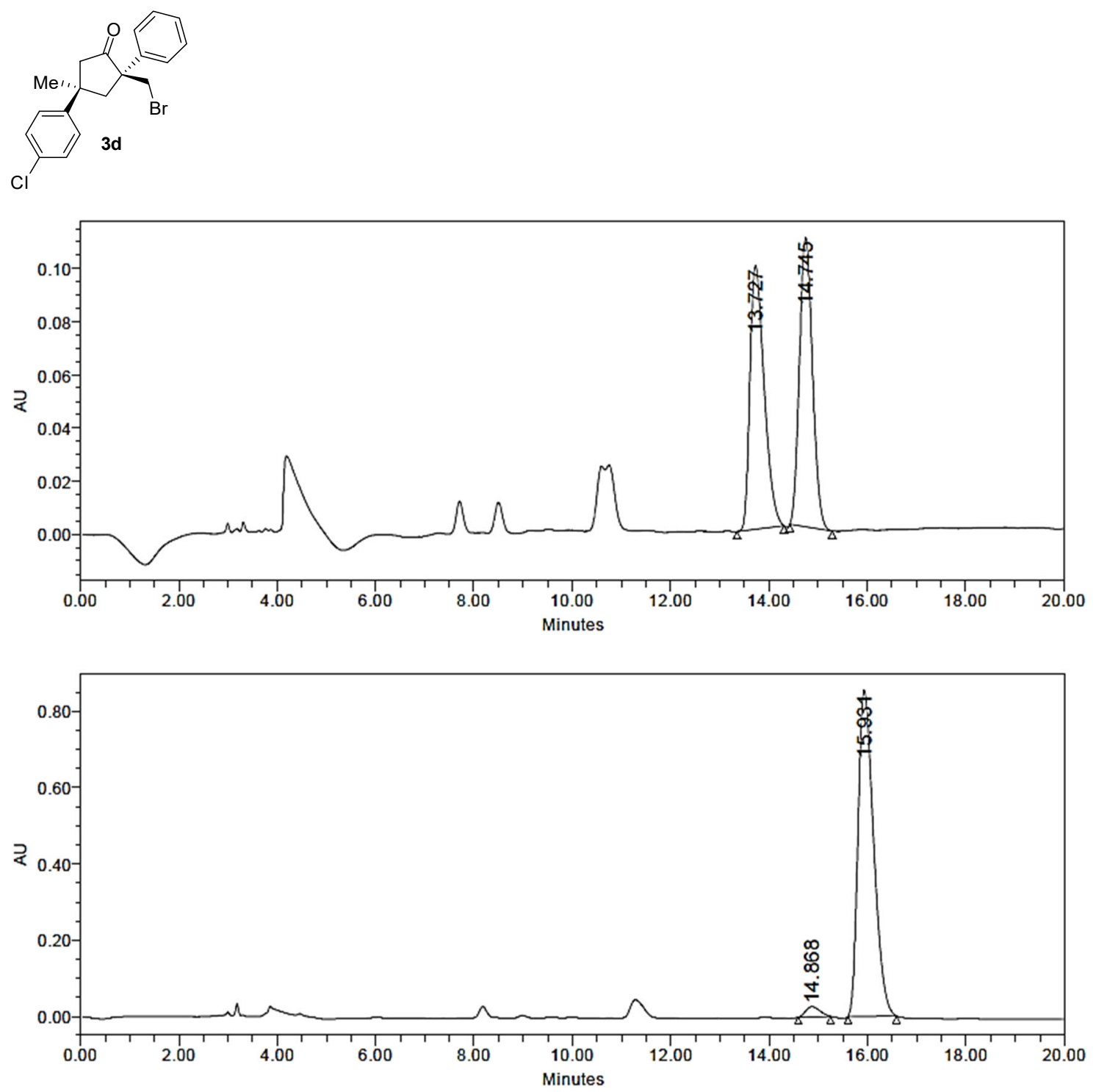

\begin{tabular}{|c|c|r|r|r|}
\hline & RT & Area & \% Area & Height \\
\hline 1 & 14.868 & 537321 & 2.79 & 26877 \\
\hline 2 & 15.931 & 18752930 & 97.21 & 853895 \\
\hline
\end{tabular}

Match Plot

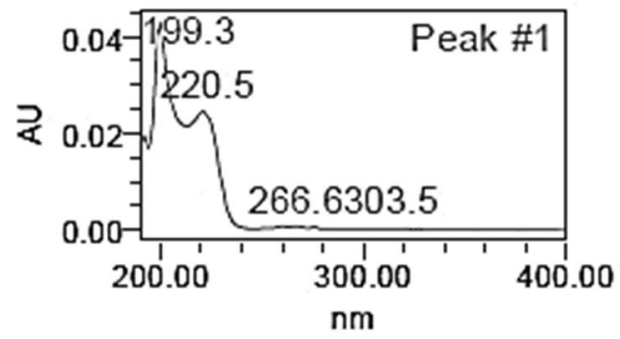

Match Plot

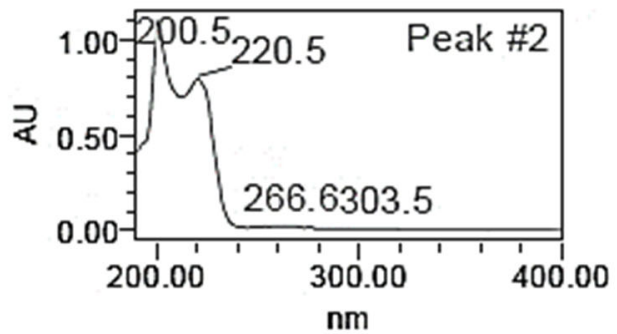



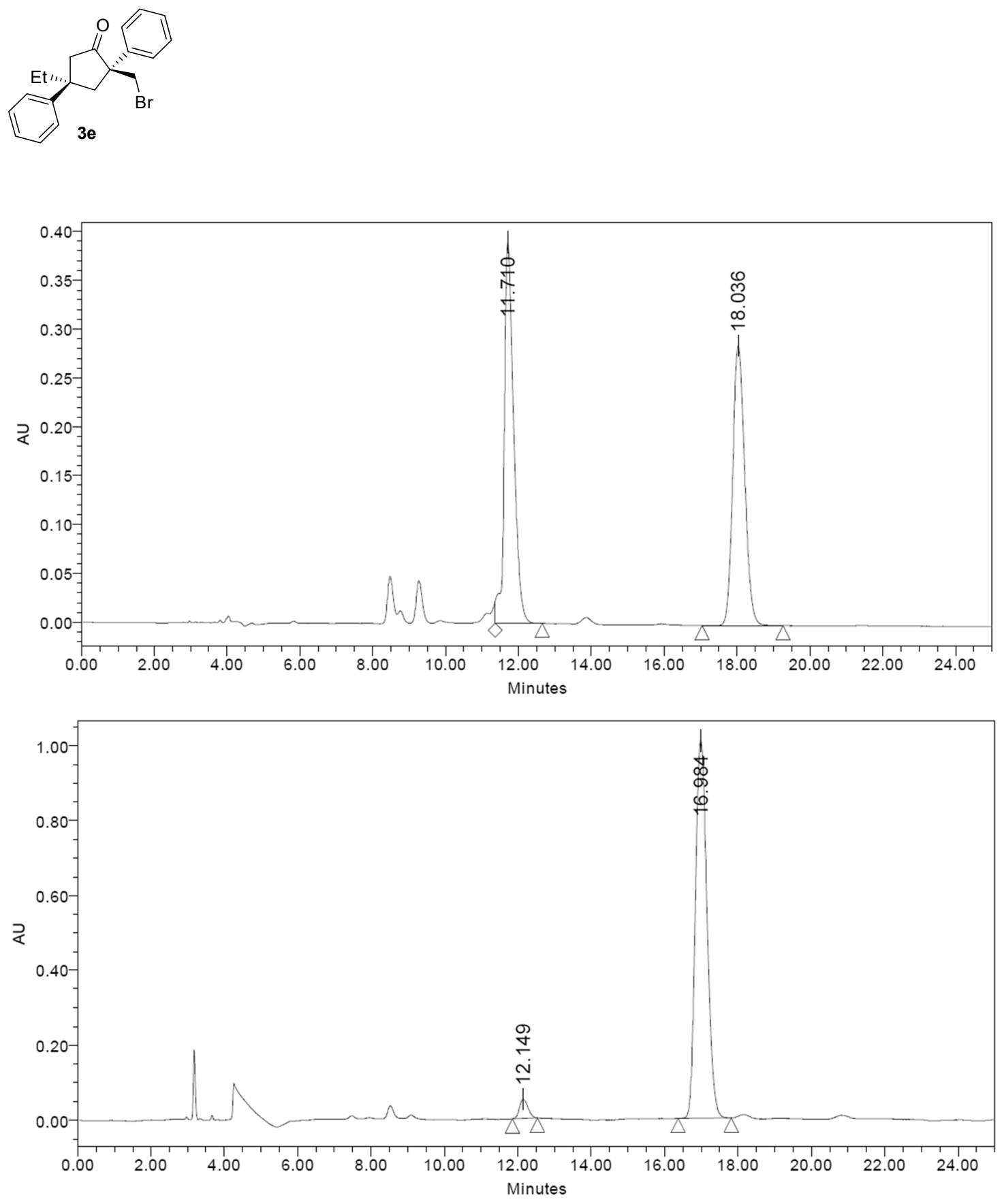

\begin{tabular}{|c|r|c|c|r|}
\hline & Injection & RT & Area & $\%$ Area \\
\hline 1 & 1 & 12.149 & 814443 & 3.55 \\
\hline 2 & 1 & 16.984 & 22135034 & 96.45 \\
\hline
\end{tabular}

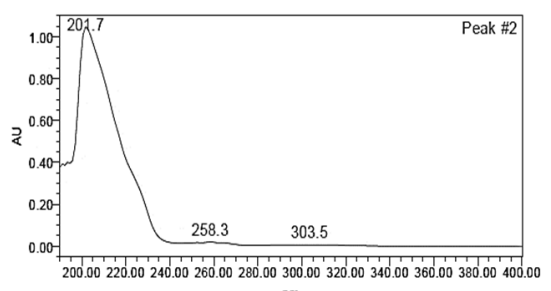

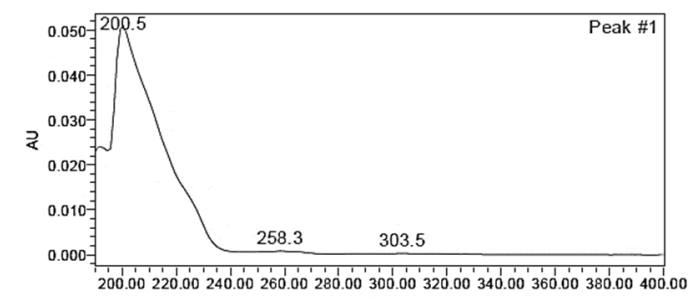



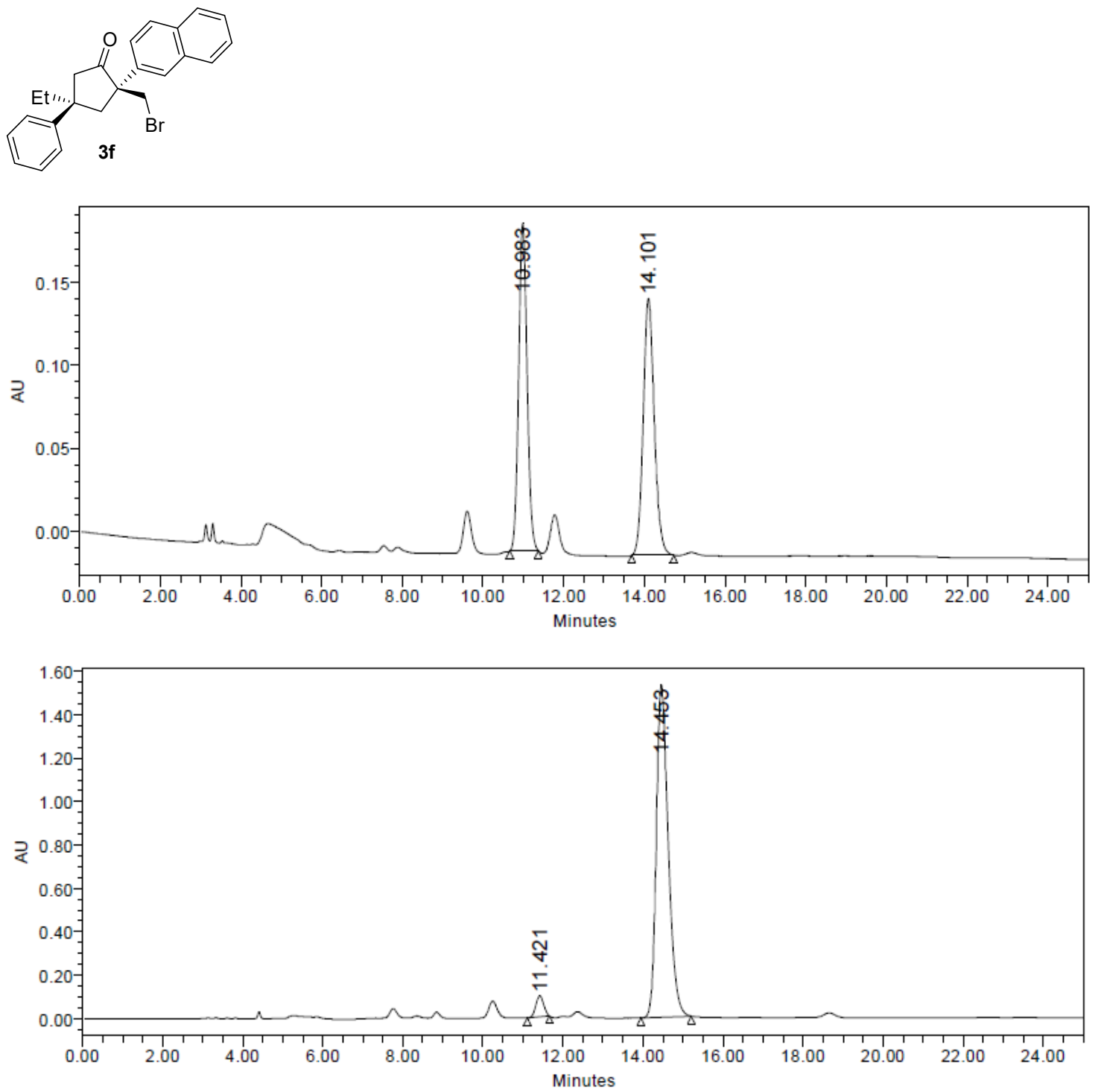

\begin{tabular}{|l|c|c|r|r|}
\hline & RT & Area & \% Area & Height \\
\hline 1 & 11.421 & 1315431 & 4.03 & 96398 \\
\hline 2 & 14.453 & 31290341 & 95.97 & 1528391 \\
\hline
\end{tabular}

Match Plot

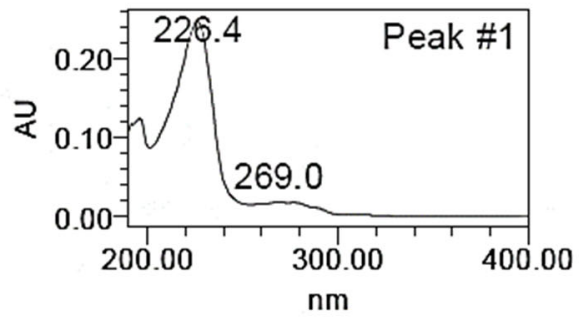

Match Plot

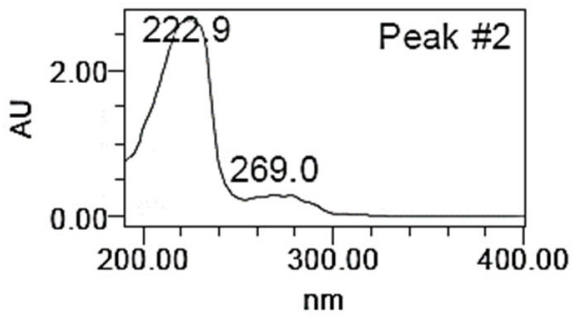



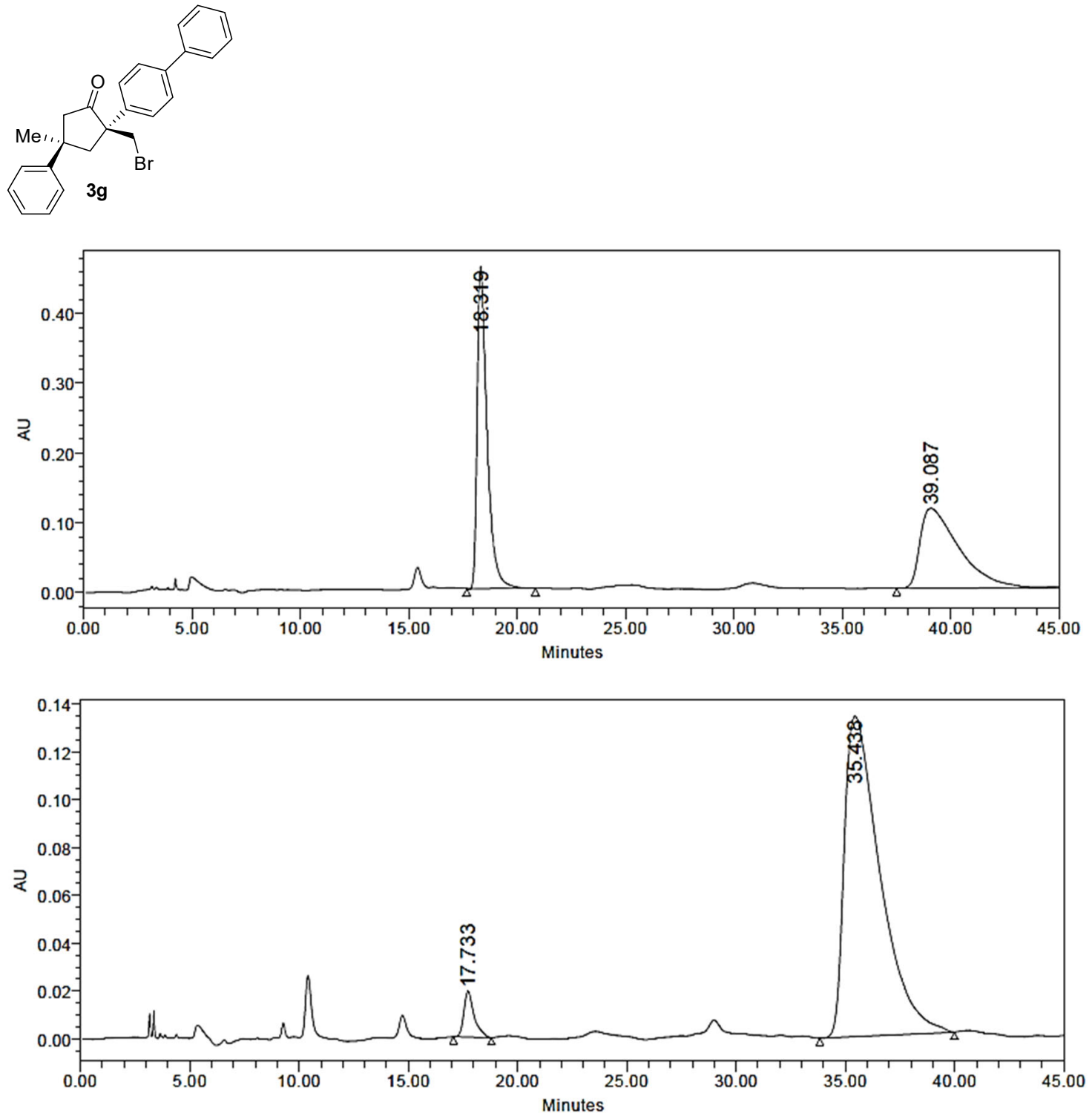

\begin{tabular}{|r|c|r|r|r|}
\hline & RT & Area & \% Area & Height \\
\hline 1 & 17.733 & 628218 & 3.97 & 19161 \\
\hline 2 & 35.438 & 15204624 & 96.03 & 134084 \\
\hline
\end{tabular}

Match Plot

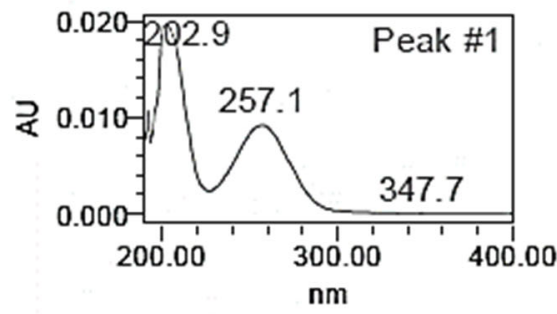

Match Plot

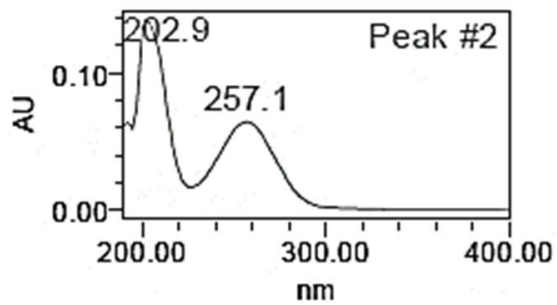



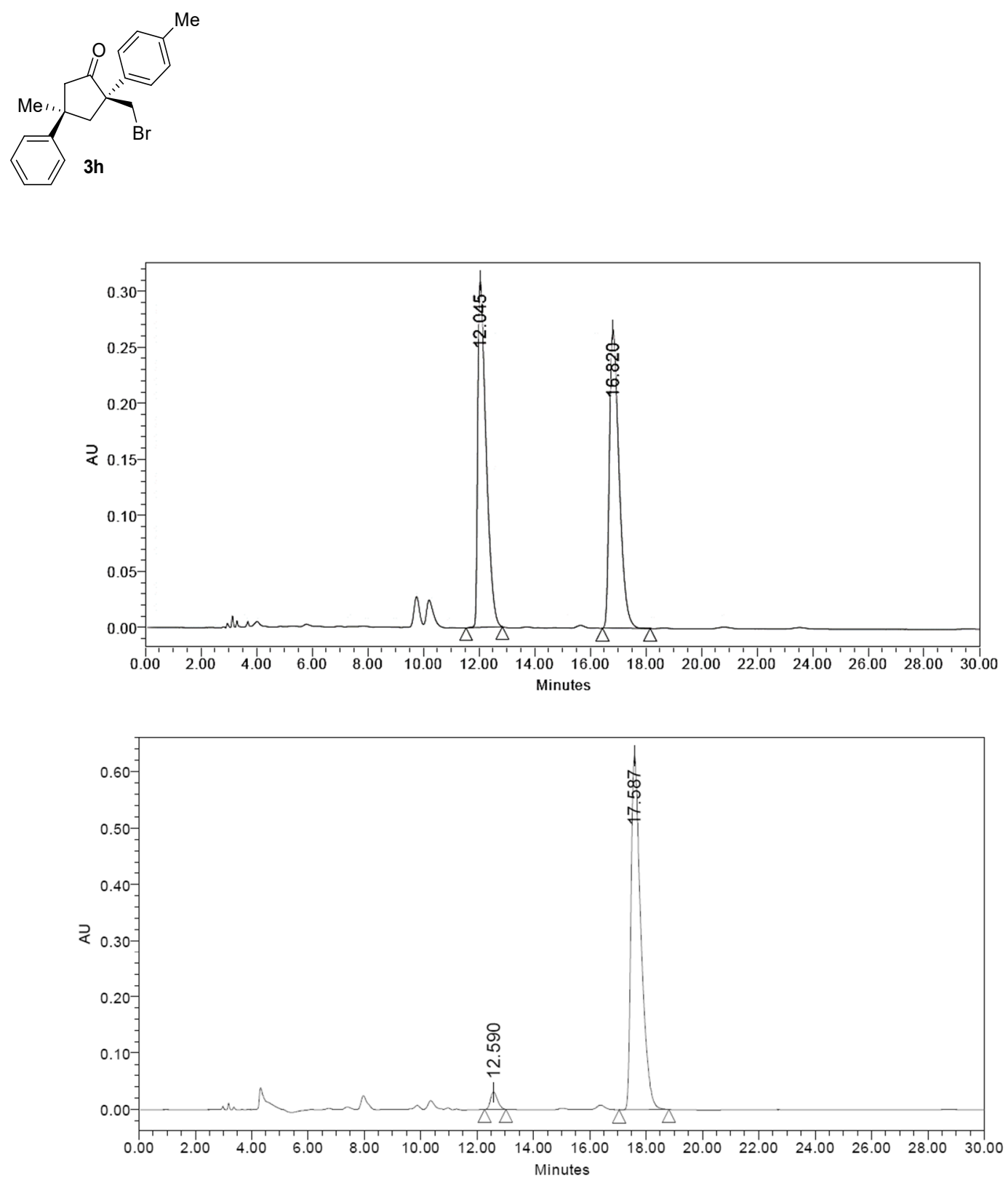

\begin{tabular}{|c|r|c|c|r|}
\hline & Injection & RT & Area & \% Area \\
\hline 1 & 1 & 12.590 & 528568 & 3.34 \\
\hline 2 & 1 & 17.587 & 15307050 & 96.66 \\
\hline
\end{tabular}
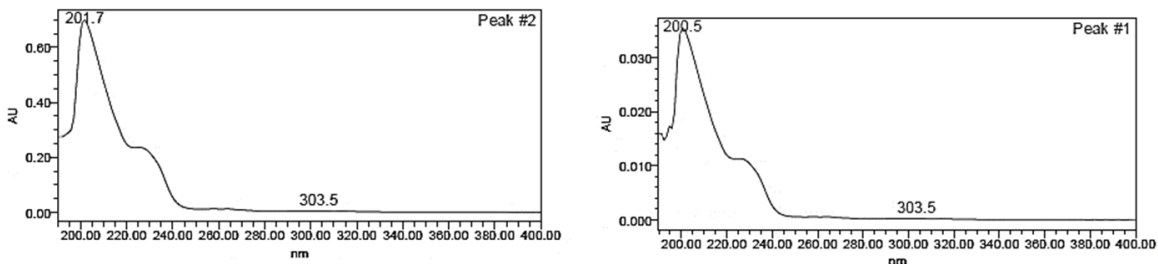

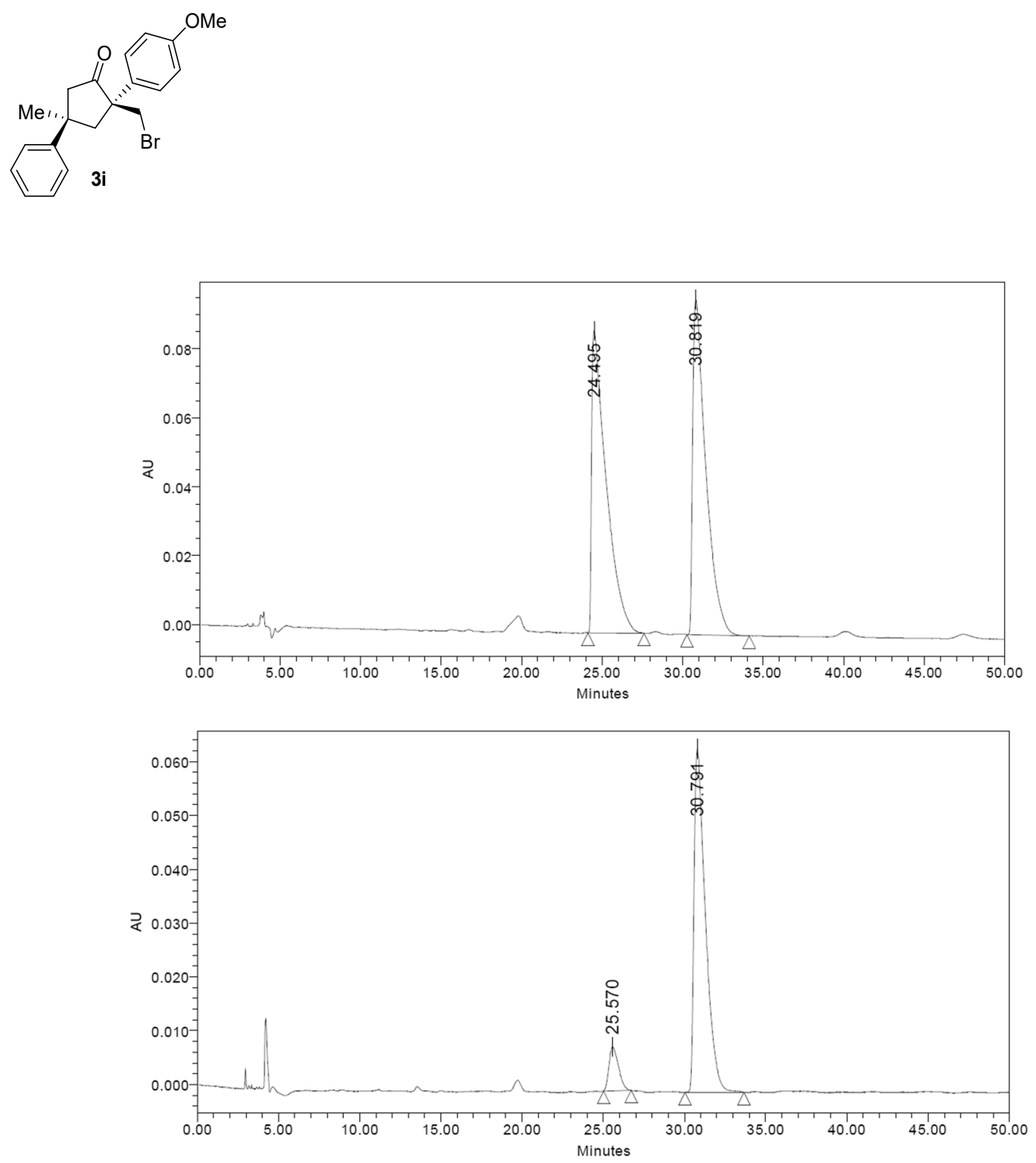

\begin{tabular}{|c|r|c|c|r|}
\hline & Injection & RT & Area & $\%$ Area \\
\hline 1 & 1 & 30.792 & 1685638 & 90.31 \\
\hline 2 & 1 & 25.566 & 180798 & 9.69 \\
\hline
\end{tabular}
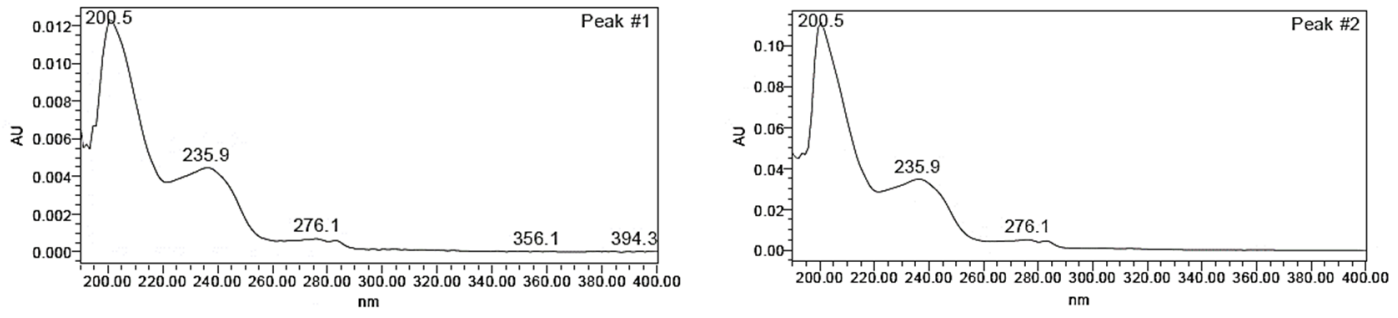

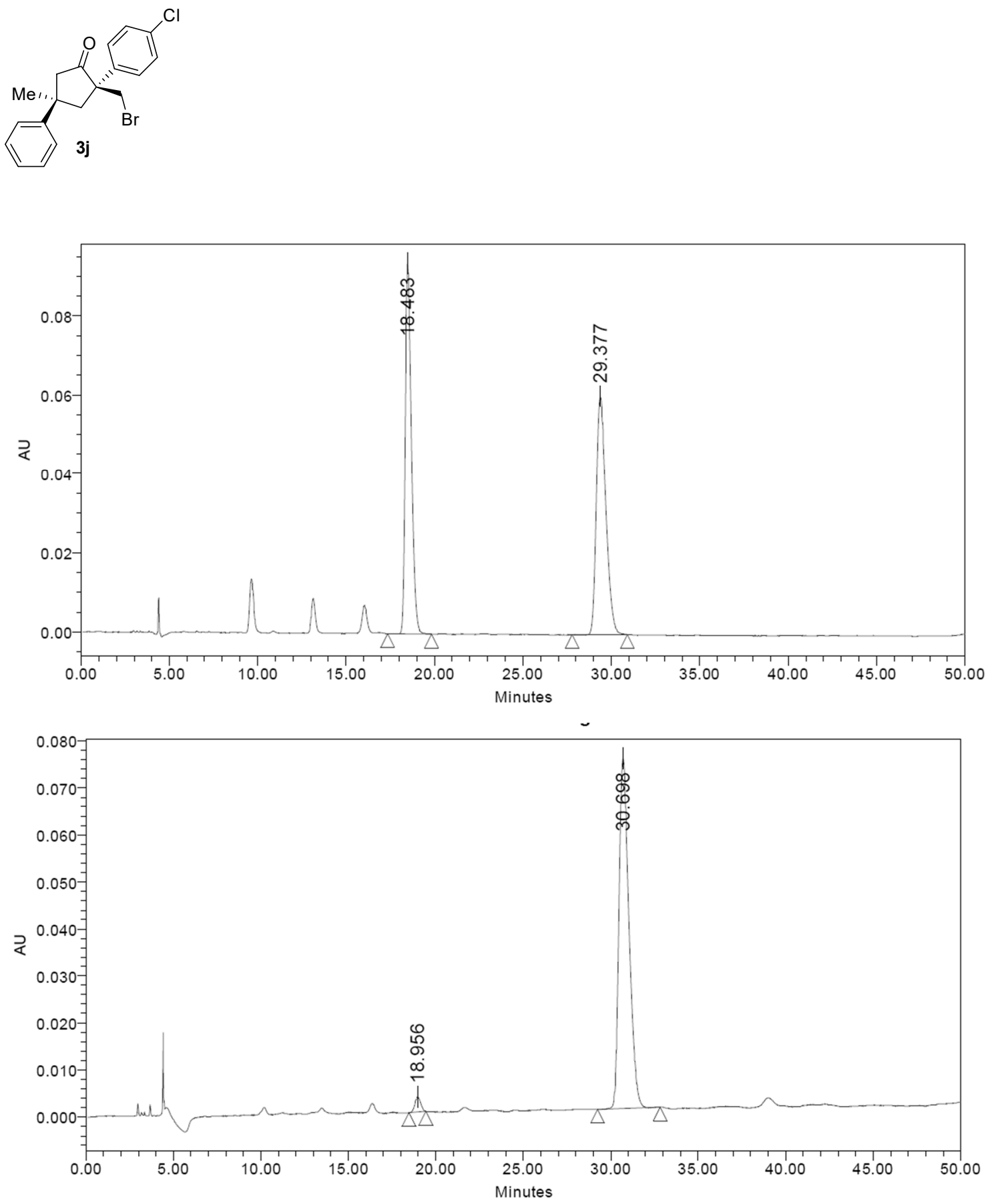

\begin{tabular}{|r|r|c|c|r|}
\hline & Injection & RT & Area & $\%$ Area \\
\hline 1 & 1 & 18.960 & 46809 & 2.42 \\
\hline 2 & 1 & 30.699 & 1887357 & 97.58 \\
\hline
\end{tabular}
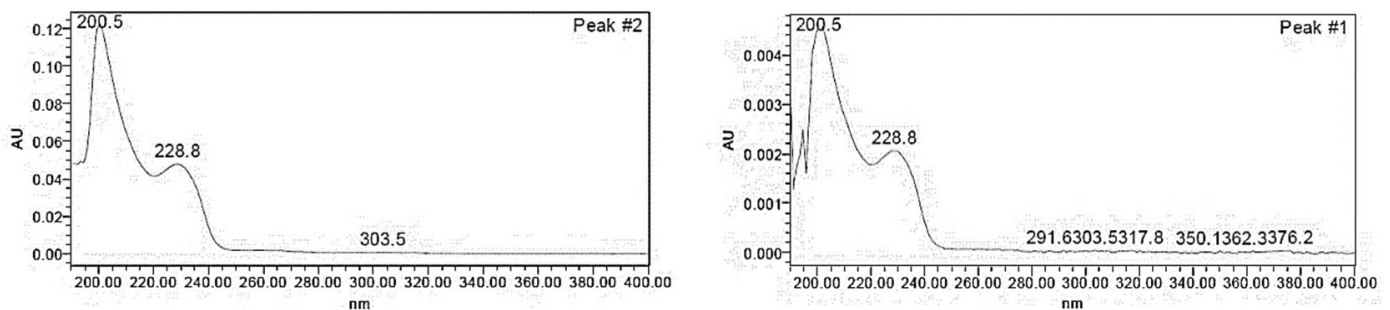

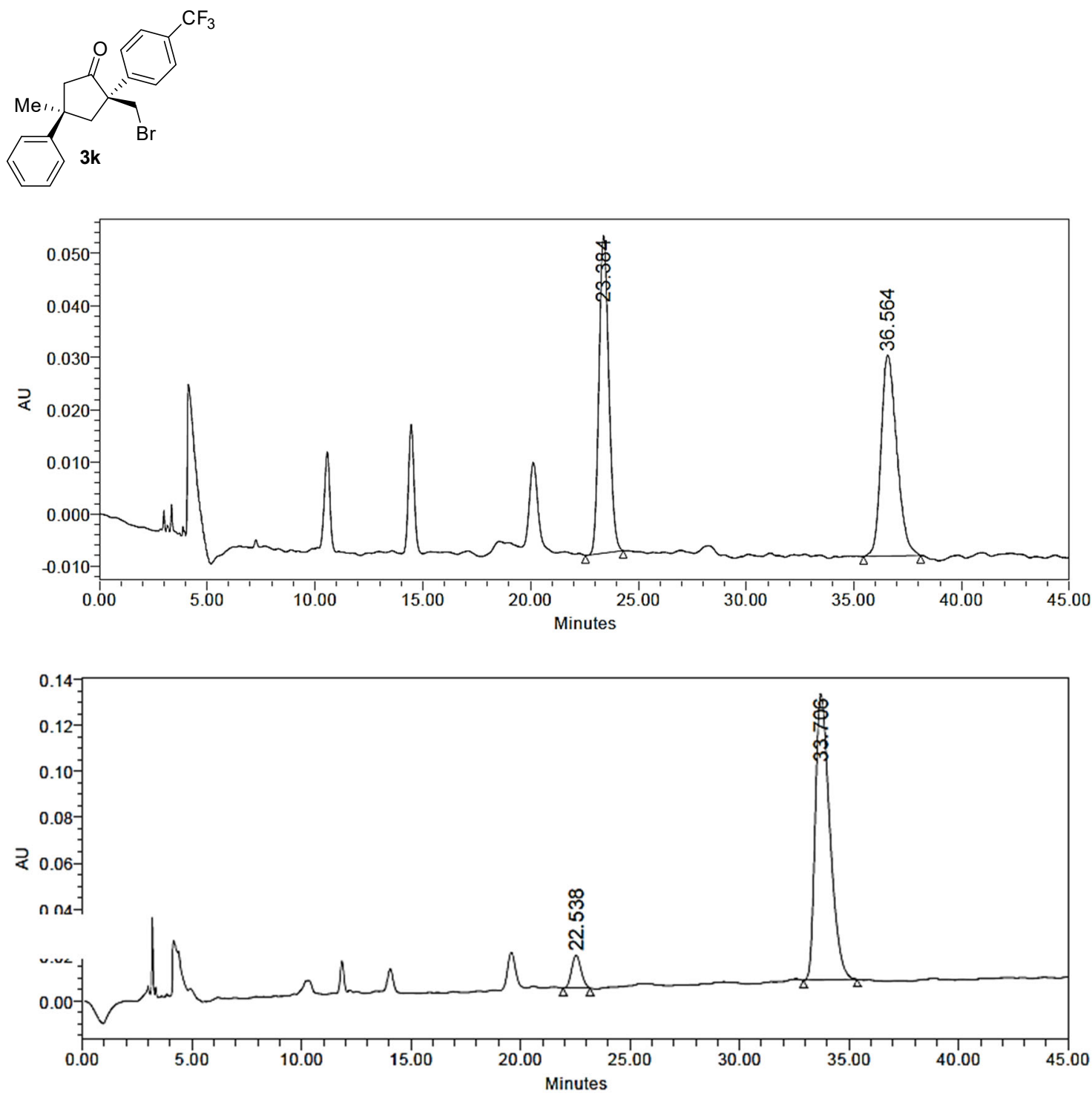

\begin{tabular}{|c|c|c|r|r|}
\hline & RT & Area & \% Area & Height \\
\hline $\mathbf{1}$ & 22.538 & 430251 & 6.92 & 14329 \\
\hline 2 & 33.706 & 5790716 & 93.08 & 124214 \\
\hline
\end{tabular}

Match Plot

Match Plot
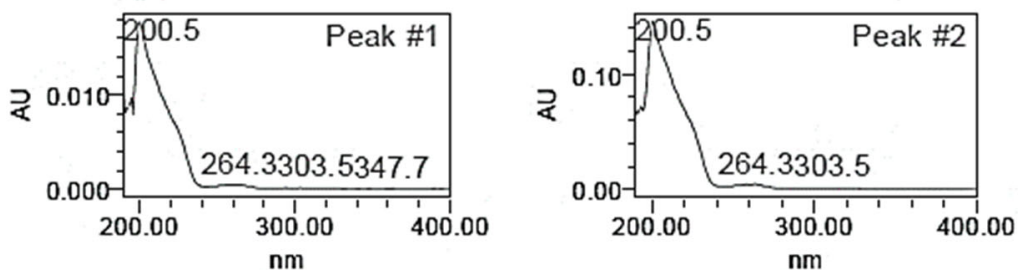

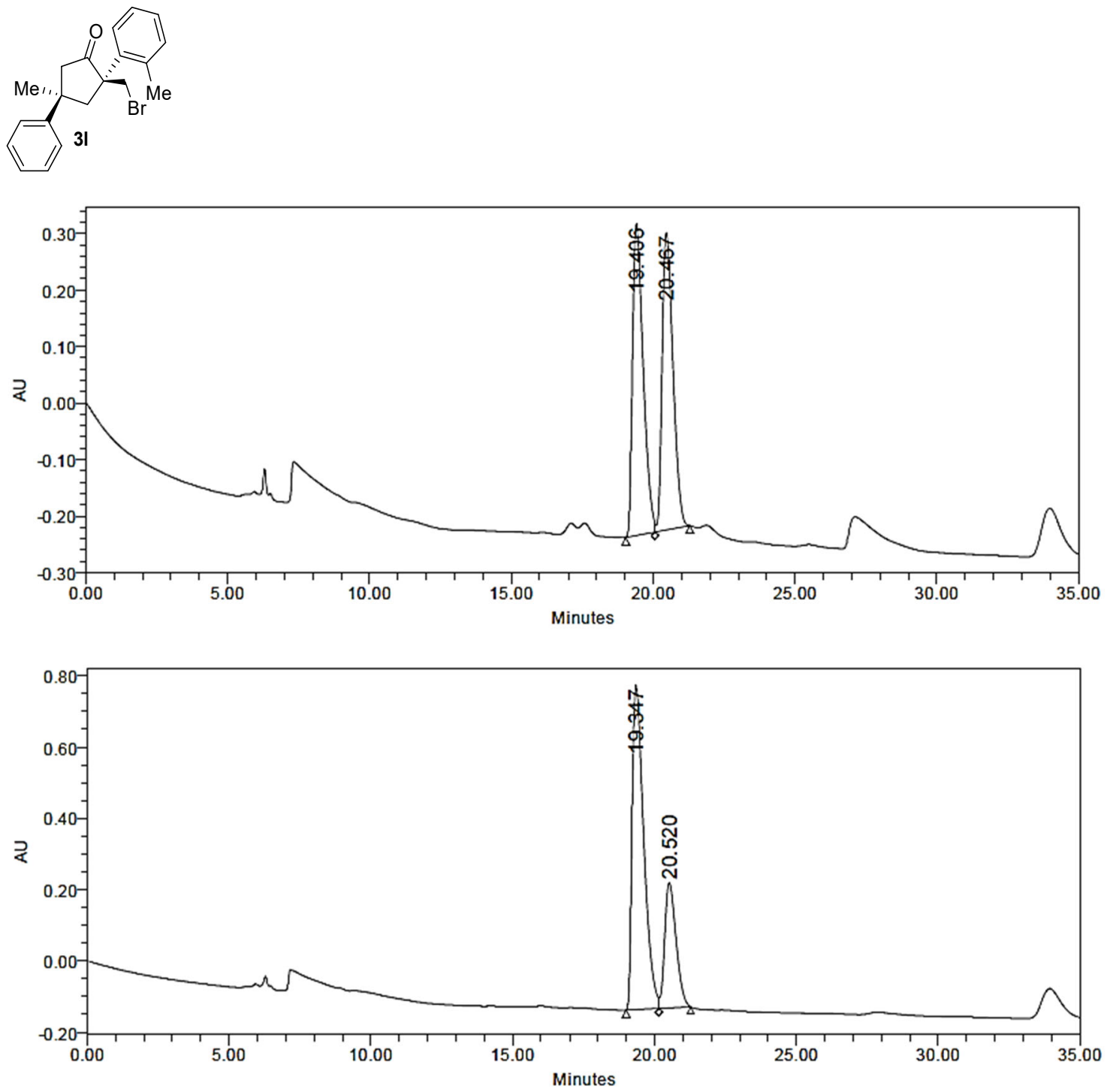

\begin{tabular}{|l|c|r|r|c|}
\hline & RT & Area & \% Area & Height \\
\hline 1 & 19.347 & 25694210 & 72.36 & 908764 \\
\hline 2 & 20.520 & 9816905 & 27.64 & 351098 \\
\hline
\end{tabular}

Match Plot

Match Plot
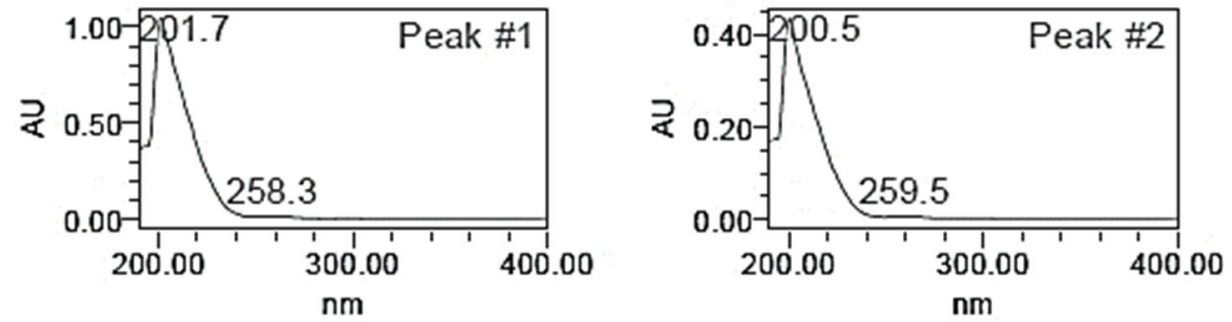

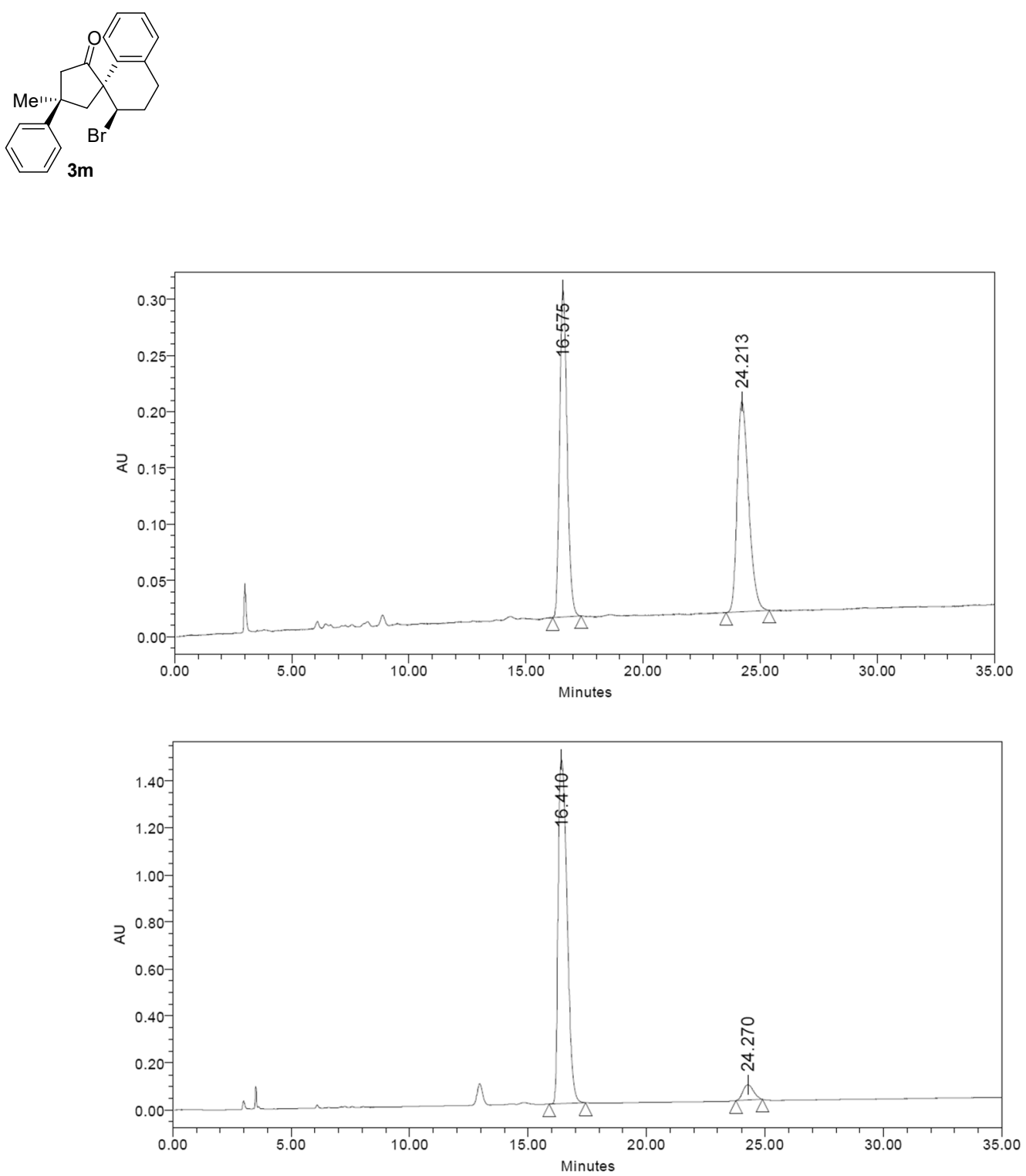

\begin{tabular}{|c|r|c|c|r|}
\hline & Injection & RT & Area & $\%$ Area \\
\hline 1 & 1 & 24.270 & 2038597 & 5.09 \\
\hline 2 & 1 & 16.410 & 38036398 & 94.91 \\
\hline
\end{tabular}
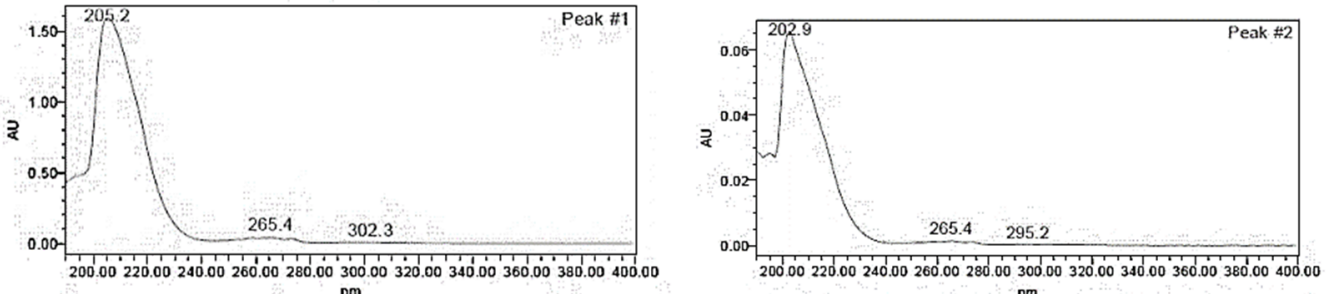
$\mathrm{nm}$. 

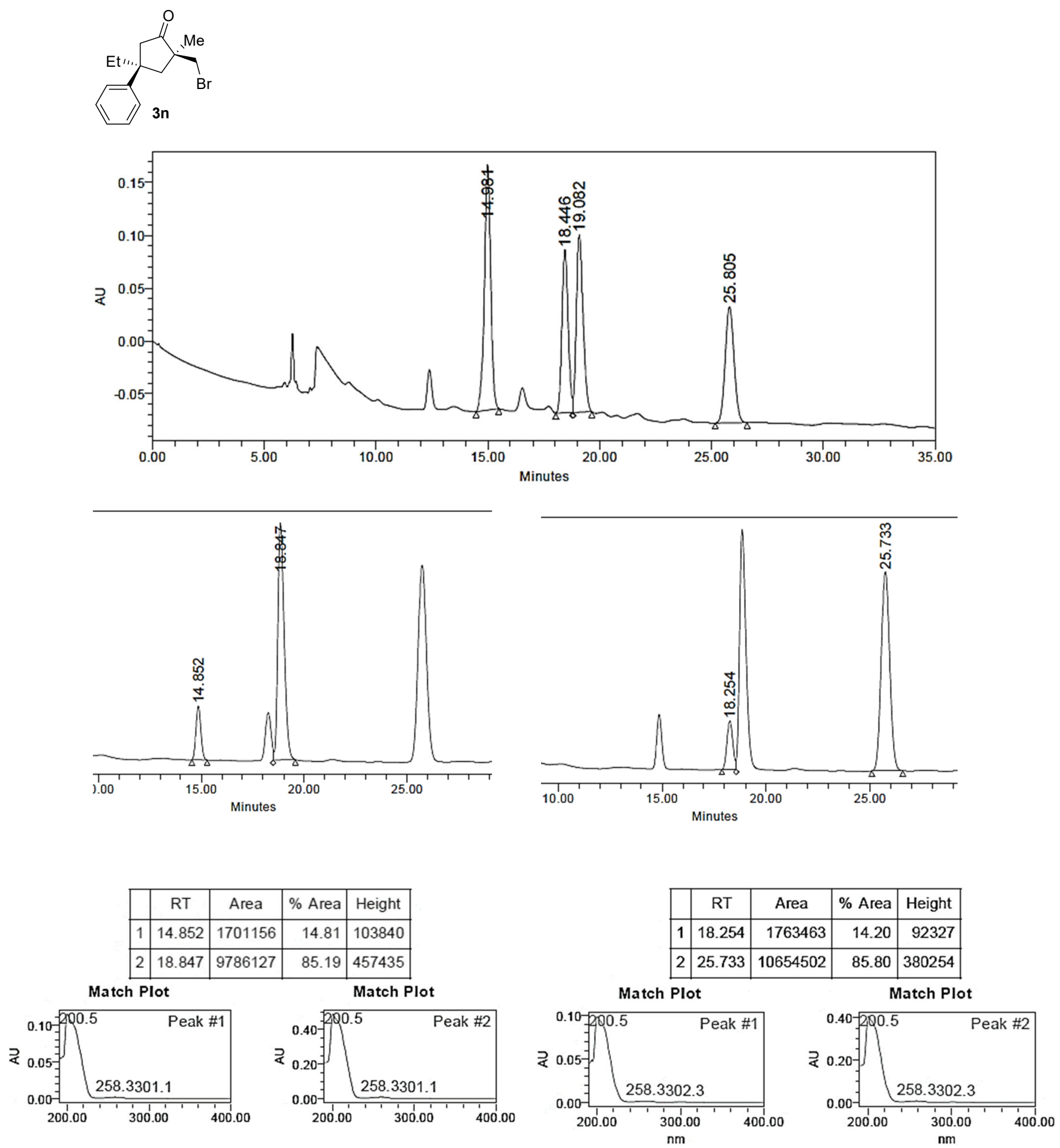


\section{Computational Studies}

\section{The Catalyst}

A close inspection of the minimized structure of the catalyst revealed a linear disposition for the coordination of the magnesium atom due to the large size of the triphenylsilyl moieties (Figure S9), in a similar way to that reported for a chiral Pd-bis-phosphate catalyst. ${ }^{17}$ In our case, two aromatic rings from one phosphate and one from the other phosphate compose a cage in which the metal atom can interact favorably with the electron density of the aromatic rings as evidenced in the $\mathrm{NCI}$ analysis (Figure S10) in a similar way to that reported for cationic magnesium $\pi$-arene complexes. ${ }^{18}$

The structure of the catalyst does not allow the simultaneous coordination of the substrate and the NBS as required in model $\mathbf{A}$ (see main text) without any previous decoordination. Moreover, the arene stabilization might allow the formation of an ionic pair presenting $\eta^{3}$-arene-Mg interactions leading to model $\mathbf{B}$ (see main text) after interaction with the substrate.

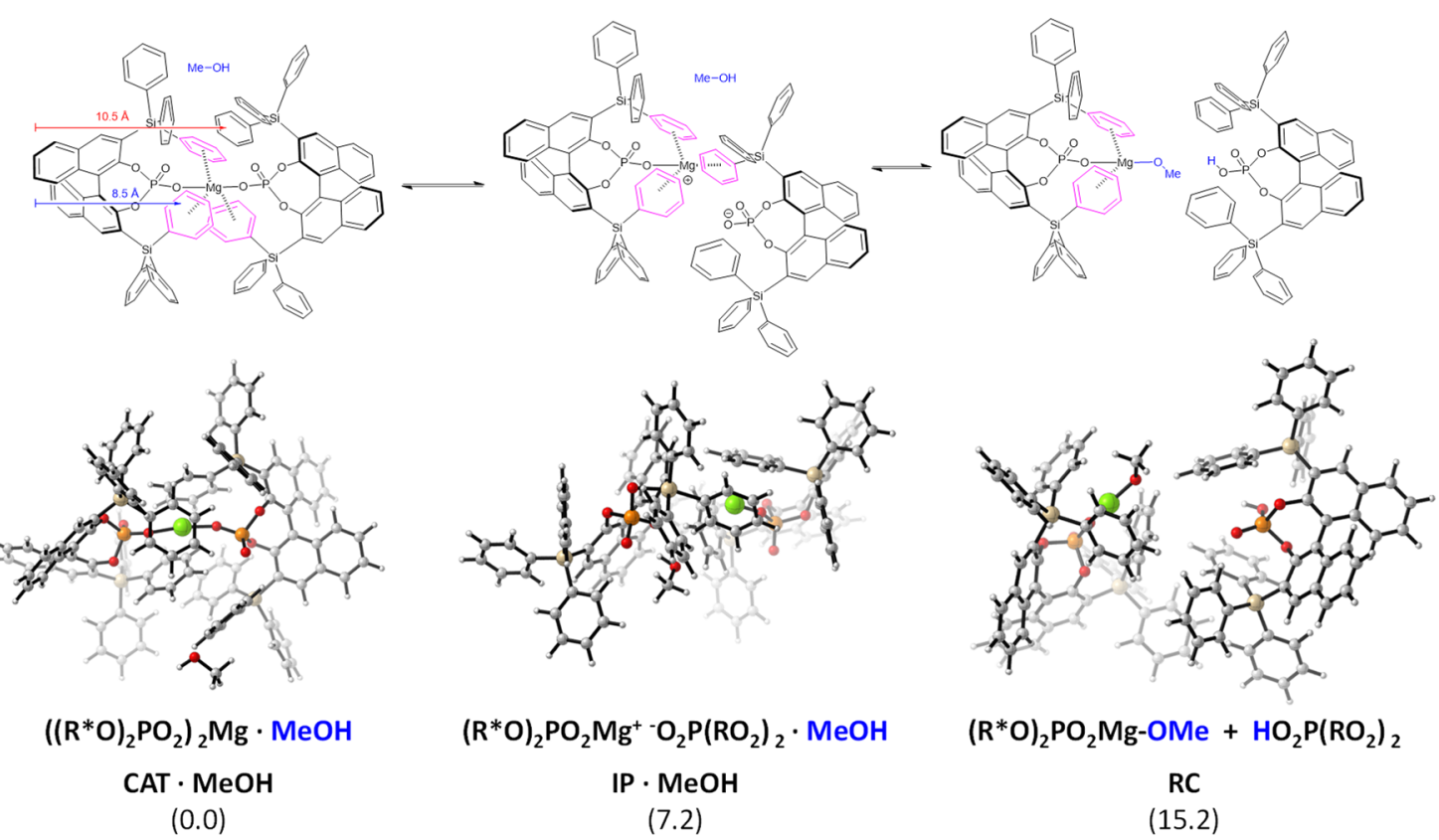

Figure S 54. Optimized structure (PM6) of the catalyst $\mathbf{2 b}$ as a complex (CAT) and as an ionic air (IP) (in the presence of a molecule of $\mathrm{MeOH}$ ), and once the alcohol is deprotonated and coordinated to form a reactive complex RC with an only unit of phosphate (the other is protonated in the form of phosphoric acid). Relative energies are given in $\mathrm{kcal} / \mathrm{mol}$.

\footnotetext{
${ }^{17}$ Jindal, G.; Sunoj, R. B. Deciphering the Origin of Stereoinduction in Cooperative Asymmetric Catalysis Involving Pd(II) and a Chiral Brønsted Acid. Org. Lett. 2015, 17, 2874-2877.

${ }^{18}$ Pahl, J.; Friedrich, A.; Elsen, H.; Harder, S. Cationic Magnesium $\pi$-Arene Complexes. Organometallics 2018, 37, 2901-2909.
} 


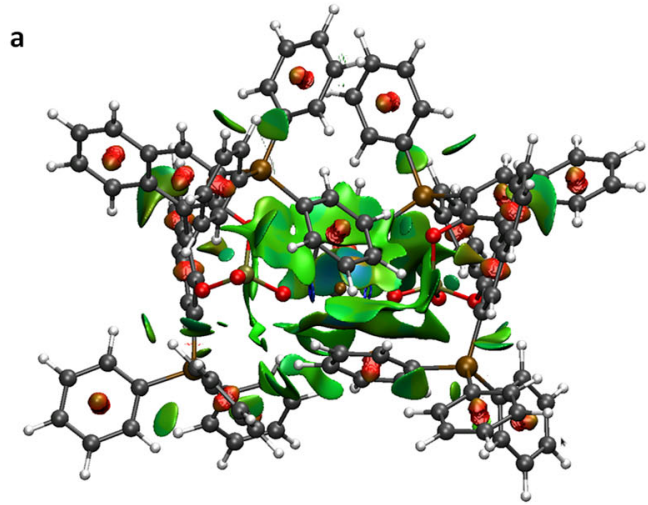

CAT

b

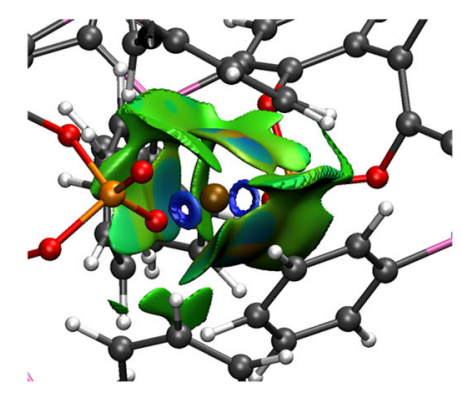

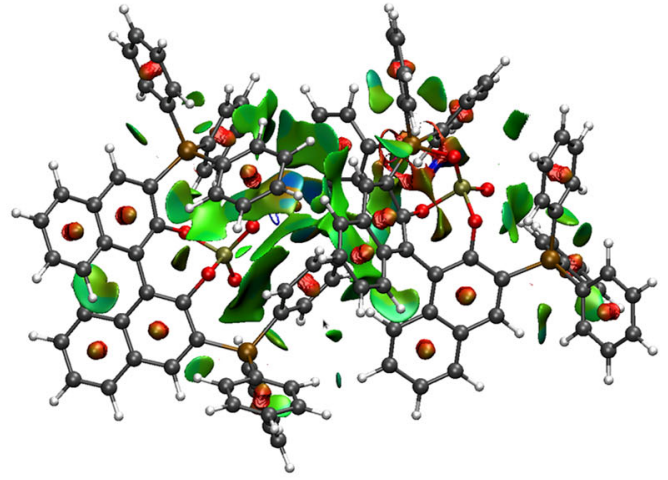

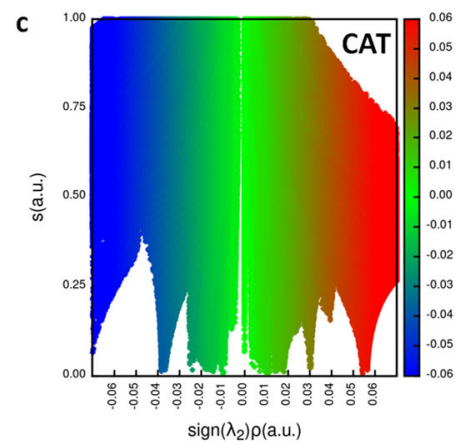

IP

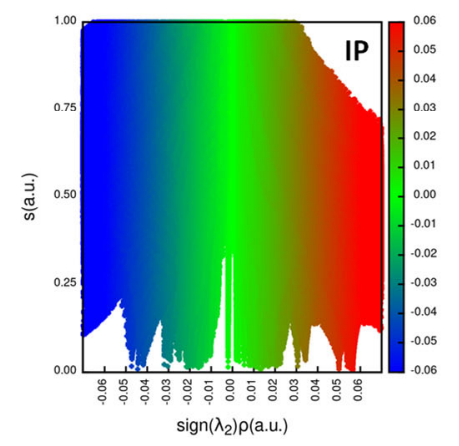

Figure S 55. a: NCI analyses for the catalyst as a complex (CAT) and as an ionic air (IP). b: Details of NCI interactions between the metal and the ligand. Thin, delocalized green surface indicates van der Waals interactions. Small, lenticular, bluish surfaces indicate strong interactions such as hydrogen bonding. Steric clashes are shown as red isosurfaces. c: $s(\mathbf{r})$ against $\operatorname{sign}(\lambda 2) \rho(\mathbf{r})$ plots for CAT (left) and IP (right). Green area corresponds to weak van der Waals interactions. Blue area corresponds to strong interactions and red area corresponds to repulsive forces.

It is plausible to hypothesize the formation of an ionic pair and further coordination of the alcohol to the magnesium atom thus leading to model $\mathbf{B}$ and liberating one unit of phosphoric acid, with a cost (in the case of $\mathrm{MeOH}$ ) of ca. $15 \mathrm{kcal} / \mathrm{mol}$.

\section{Unsubstituted Model}

Preliminary studies were carried out with an unsubstituted model EC1 (Scheme S 14). These studies suggested the non-existence of an intermediate IN but a reaction in one kinetic step presenting a transient carbocation. Considering the whole reaction (bromination followed by rearrangement) there are two possible approaches for the formation of the epoxide and four ones for the formation of cyclopentanone. Taking into account that coordination with magnesium should be maintained, two approaches should be ruled out (Scheme S 14). In consequence, there are one approach (TSa) for obtaining the epoxide and four approaches (TSb-d) leading to the enantiomers of the cyclopentanone. The absence of cyclobutane susbituents in this model makes that 
rearrangement of bonds $\mathrm{C} 2-\mathrm{C} 3$ and $\mathrm{C} 2-\mathrm{C} 5$ are equivalent leading to enantiomeric compounds.

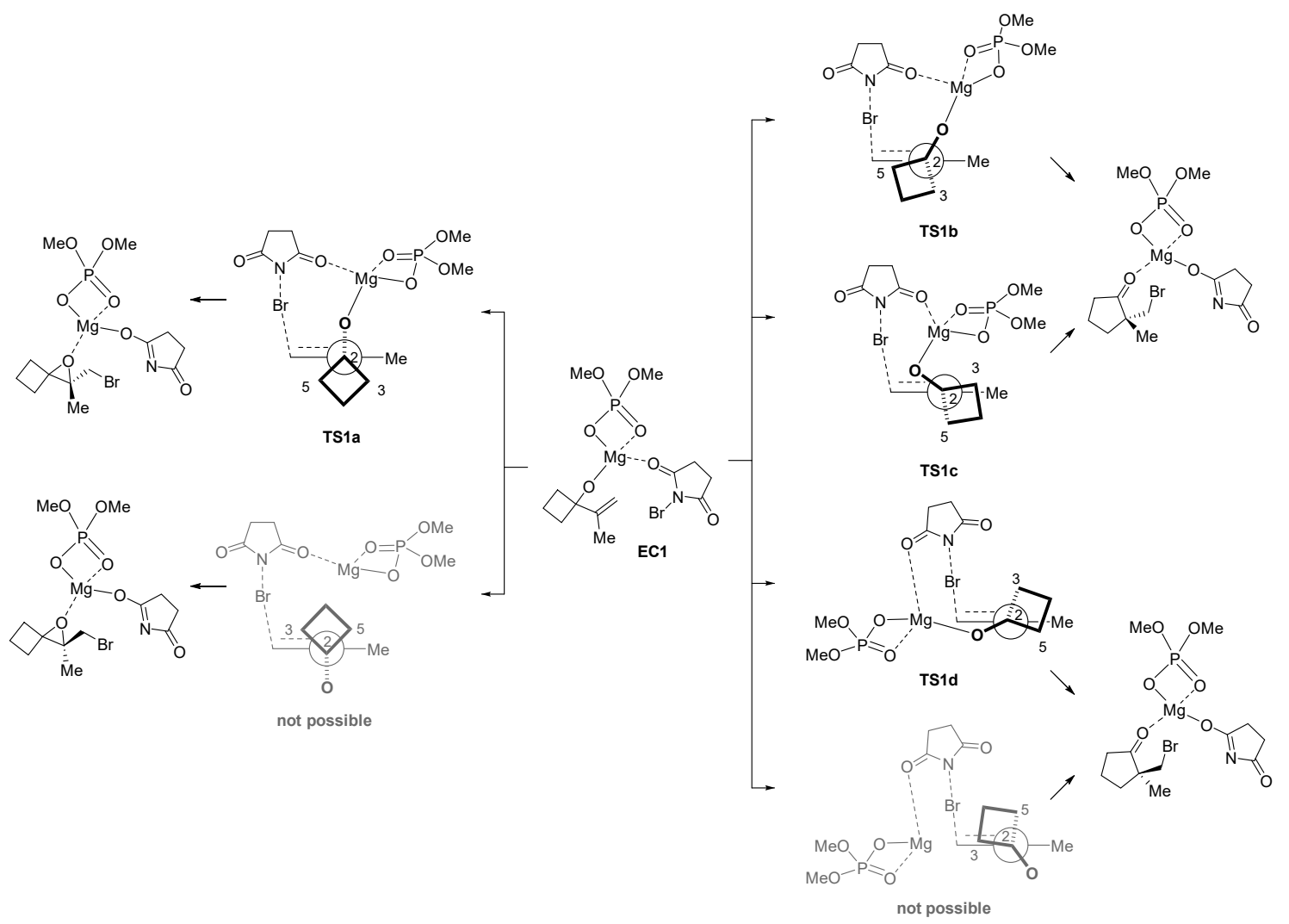

Scheme S 13. Approaches (PES) for the unsubstituted model EC1

The four transition structures as well as the starting minimum (EC1) and final products (EP1 and CY1) were located. The optimized geometries are given in Figure S 56 and Figure S 57, respectively.

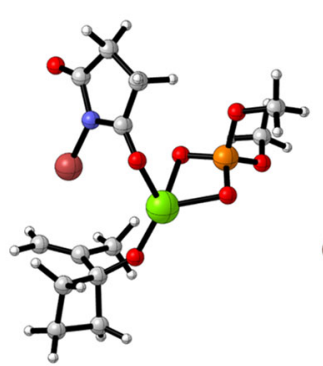

EC1

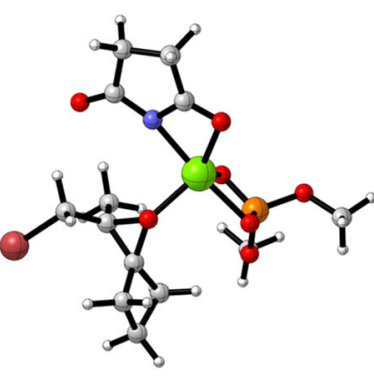

EP1

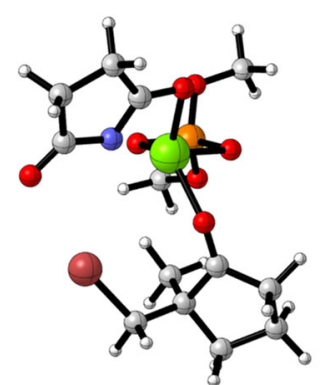

CY1

Figure S 56. Optimized (wb97xd/def2SVP) geometries of encounter complex EC1, epoxide EP1 and cyclopentanone CY1. 


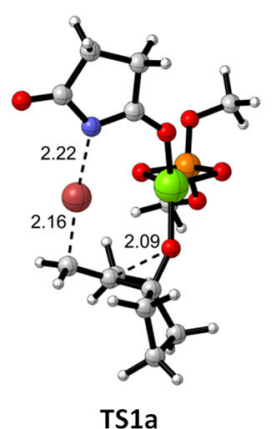

TS1a
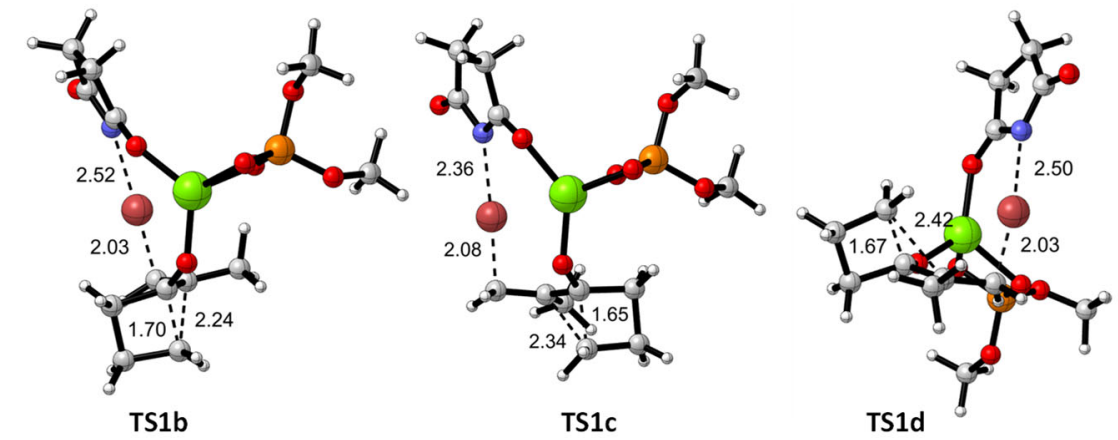

Figure S 57. Optimized (wb97xd/def2SVP) geometries of transition structures TS1a, TS1b, TS1c and TS1d.

The IRC analyses confirming that transition structures connect reagent and products are given in Figure S 58. Interestingly, the IRCs corresponding to TS1b and TS1d showed a clear shoulder typical of a "hidden intermediate".
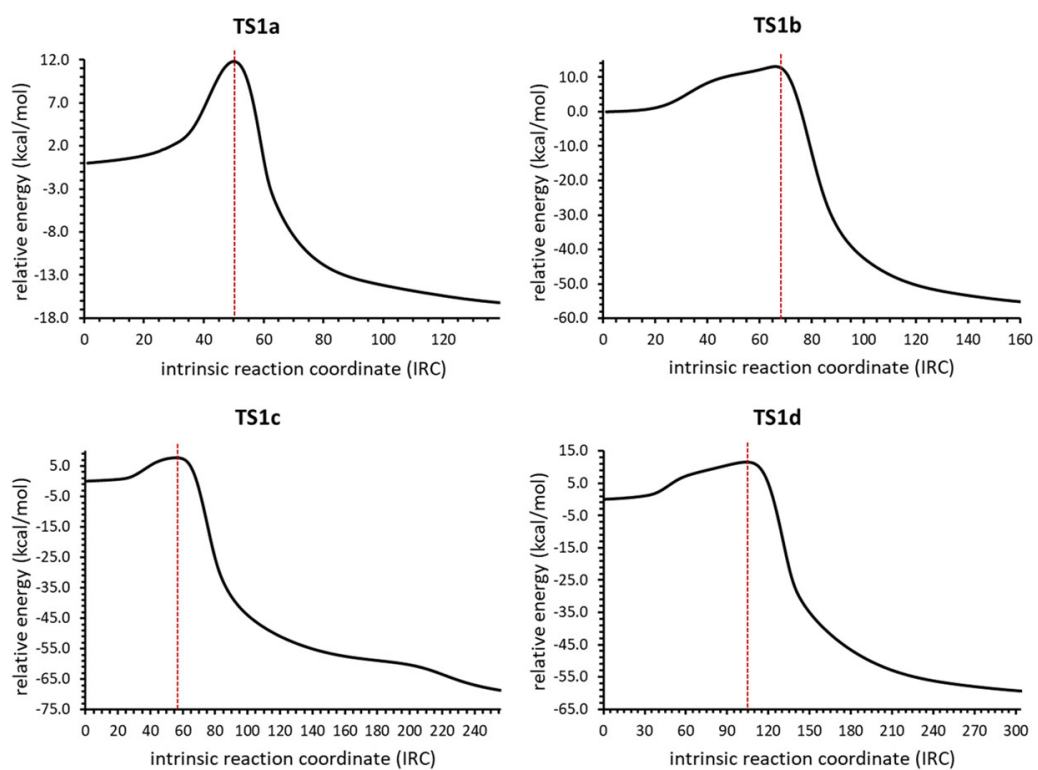

Figure S 58. IRCs (wb97xd/def2svp) corresponding to transition structures TS1a, TS1b, TS1c and TS1d. The red dotted line indicates the transition state.

Energy profiles are given in Figure S 59. Absolute and relative energies are listed in Table S 100. 


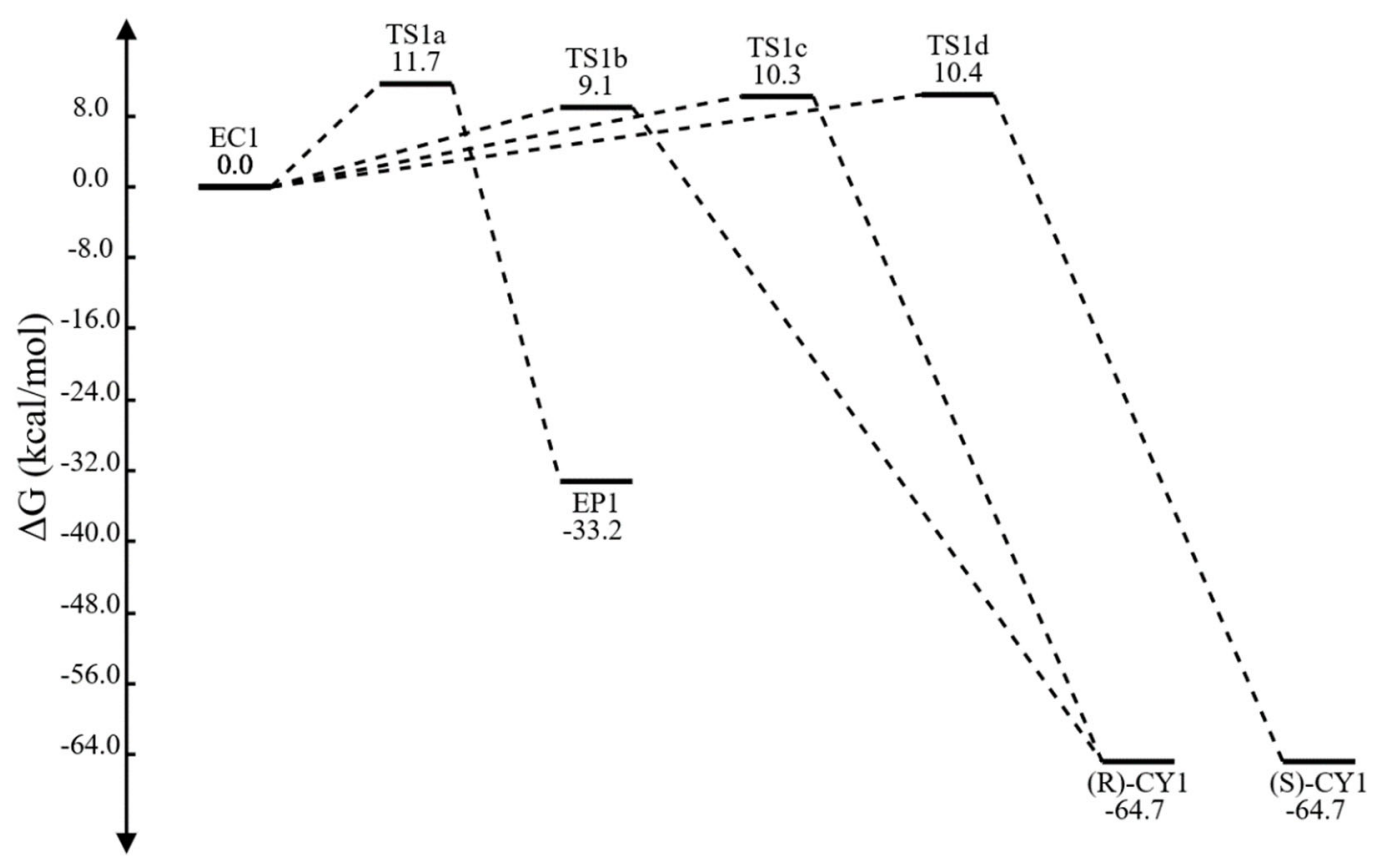

Figure S 59. Energy profiles (wb97xd/def2tzvp/pcm=toluene)//wb97xd/def2svp) for the different pathways from EC1 to EP1 and CY1. Relative free energies are given in $\mathrm{kcal} / \mathrm{mol}$.

Table S 10. Calculated (wb97xd/def2tzvp/pcm=toluene//wb97xd/def2svp) absolute (hartree) and relative (kcal/mol) energies for the transformation of EC1 into EP1 and CY1.

\begin{tabular}{lccccc}
\hline & $\mathrm{E}_{0}$ & $\Delta \mathrm{E}_{0}$ & $\mathrm{G}$ & $\Delta \mathrm{G}$ & im. freq \\
\hline EC1 & -4204.993364 & 0.0 & -4205.074060 & 0.0 & \\
TS1a & -4204.974328 & 11.9 & -4205.055441 & 11.7 & -227.6 \\
TS1b & -4204.978509 & 9.3 & -4205.059523 & 9.1 & -285.9 \\
TS1c & -4204.977618 & 9.9 & -4205.057617 & 10.3 & -132.3 \\
TS1d & -4204.976291 & 10.7 & -4205.057505 & 10.4 & -125.8 \\
CY1 & -4205.094731 & -63.6 & -4205.177200 & -64.7 & \\
EP1 & -4205.044295 & -32.0 & -4205.127047 & -33.2 & \\
\hline
\end{tabular}

An exploration of the potential energy surface (PES) corresponding to the second part of the reaction, i.e. formation of either the O1-C1 bond (epoxide) or C2-C3 bond (cyclopentanone) was made by carrying out relaxed scans at the different $\mathrm{O} 1-\mathrm{C} 1$ and $\mathrm{C} 2-$ C3 distances. The exploration covered sampling of conformations and in all cases the most stable conformers have been considered. The resulting PES (Figure S 61) confirmed two different transition structures for the epoxide and cyclopentanone formation and the absence of a reasonable connection between the two products, in agreement with the experimental observations. 


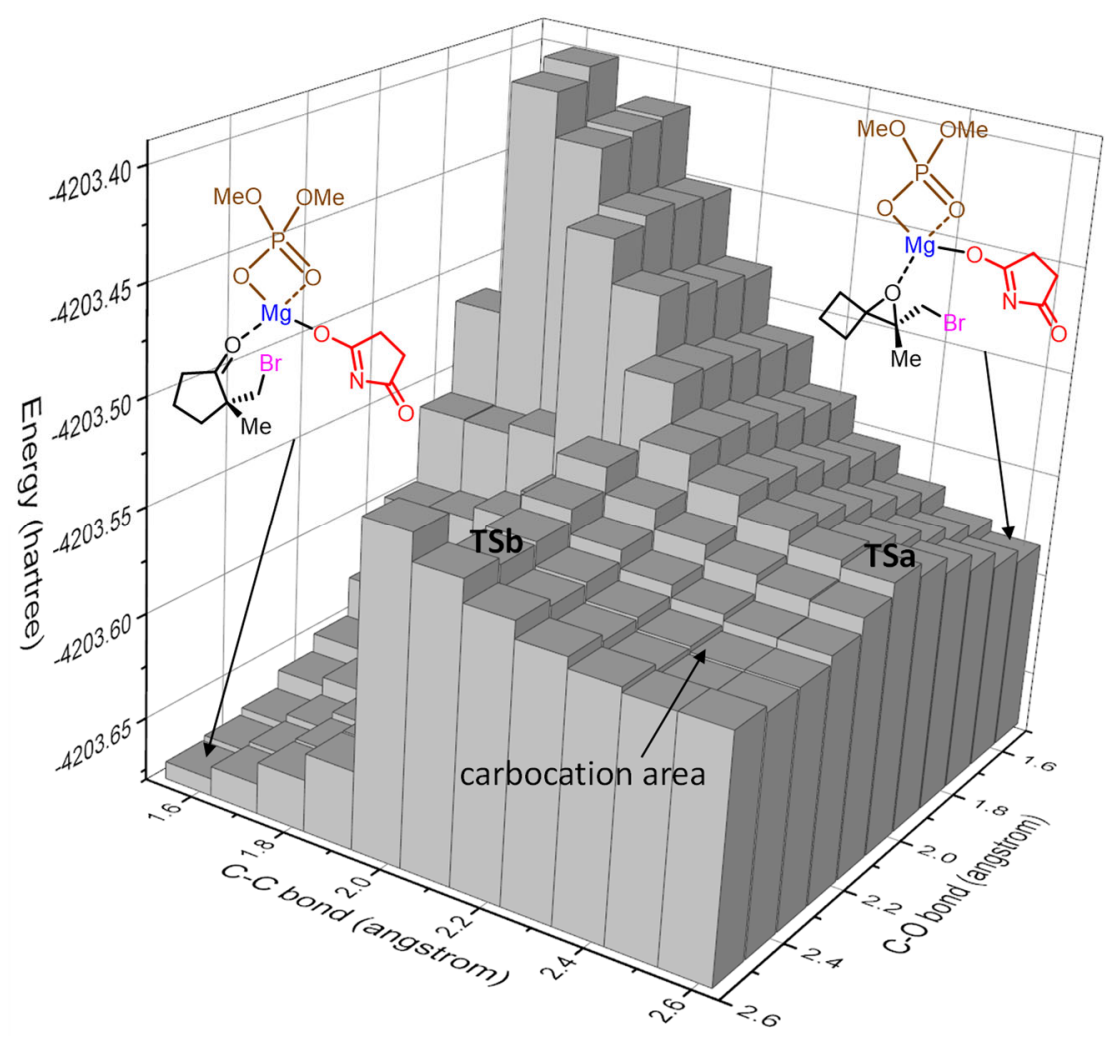

Figure S 60. Potential energy surface (wb97xd/def2svp) related to the formation of O1-C1 and C2-C3 bonds corresponding to the epoxide and the cyclopentanone, respectively.

\section{Benchmarking}

Three additional levels of theory were calculated confirming that the employed level is the closest to experimental results even though all the levels showed the same trend.

Table S 11. Calculated ( $\mathrm{m} 062 \mathrm{x} / \mathrm{cc}-\mathrm{pvtz} / \mathrm{pcm}=$ toluene $/ / \mathrm{m} 062 \mathrm{x} / \mathrm{cc}-\mathrm{pvdz}$ ) absolute (hartree) and relative (kcal/mol) energies for the transformation of EC1 into EP1 and CY1.

\begin{tabular}{lccccc}
\hline & $\mathrm{E}_{0}$ & $\Delta \mathrm{E}_{0}$ & $\mathrm{G}$ & $\Delta \mathrm{G}$ & im. freq \\
\hline EC1 & -4204.873013 & 0.0 & -4204.934009 & 0.0 & \\
TS1a & -4204.849887 & 14.5 & -4204.911619 & 14.1 & -291.8 \\
TS1b & -4204.852338 & 13.0 & -4204.914097 & 12.5 & -317.3 \\
TS1c & -4204.853413 & 12.3 & -4204.913827 & 12.7 & -174.4 \\
TS1d & -4204.850983 & 13.8 & -4204.912320 & 13.6 & -144.3 \\
CY1 & -4204.969752 & -60.7 & -4205.031266 & -61.0 & \\
EP1 & -4204.924108 & -32.1 & -4204.986001 & -32.6 & \\
\hline
\end{tabular}

Table S 12. Calculated (b3lyp-gd3bj/6-311G(d,p)//b3lyp-gd3bj/6-31G(d,p)) absolute (hartree) and relative (kcal/mol) energies for the transformation of EC1 into EP1 and CY1.

\begin{tabular}{lccccc}
\hline & $\mathrm{E}_{0}$ & $\Delta \mathrm{E}_{0}$ & $\mathrm{G}$ & $\Delta \mathrm{G}$ & im. freq \\
\hline EC1 & -4205.215671 & 0.0 & -4205.276833 & 0.0 & \\
TS1a & -4205.203256 & 7.8 & -4205.264809 & 7.5 & -153.1 \\
TS1b & -4205.200089 & 9.8 & -4205.262251 & 9.2 & -397.3 \\
TS1c & -4205.203085 & 7.9 & -4205.265291 & 7.2 & -238.8 \\
TS1d & -4205.199187 & 10.3 & -4205.261305 & 9.7 & -195.9 \\
CY1 & -4205.316731 & -63.4 & -4205.379780 & -64.6 & \\
EP1 & -4205.257793 & -26.4 & -4205.320834 & -27.6 & \\
\hline
\end{tabular}


Table S 13. Calculated (m062x/6-311G(d,p)/pcm=toluene $/ / \mathrm{m} 062 \mathrm{x} / 6-31 \mathrm{G}(\mathrm{d}, \mathrm{p})$ ) absolute (hartree) and relative ( $\mathrm{kcal} / \mathrm{mol})$ energies for the transformation of EC1 into EP1 and CY1.

\begin{tabular}{lccccc}
\hline & $\mathrm{E}_{0}$ & $\Delta \mathrm{E}_{0}$ & $\mathrm{G}$ & $\Delta \mathrm{G}$ & im. freq \\
\hline EC1 & -4204.631744 & 0.0 & -4204.692064 & 0.0 & \\
TS1a & -4204.608624 & 14.5 & -4204.668985 & 14.5 & -290.9 \\
TS1b & -4204.610684 & 13.2 & -4204.671587 & 12.8 & -397.8 \\
TS1c & -4204.613714 & 11.3 & -4204.673755 & 11.5 & -212.2 \\
TS1d & -4204.609753 & 13.8 & -4204.670650 & 13.4 & -164.1 \\
CY1 & -4204.740881 & -68.5 & -4204.802151 & -69.1 & \\
EP1 & -4204.686138 & -34.1 & -4204.747661 & -34.9 & \\
\hline
\end{tabular}




\section{Real model with Achiral Catalyst}

We studied the real model leading to desymmetrization with an achiral catalyst to evaluate the reactivity. Full studies were carried out with $\mathrm{R}=\mathrm{Ph}$ and predictive studies with $\mathrm{R}=$ 4- $\mathrm{MeOC}_{6} \mathrm{H}_{4}$ and $\mathrm{R}=4-\mathrm{F}_{3} \mathrm{CC}_{6} \mathrm{H}_{4}$. In a similar way to the unsubstituted model, four approaches are possible. In this case, due to the presence of substituents in the cyclobutane ring, different isomeric cyclopentanones in addition to the epoxide can be obtained (Scheme S 14).

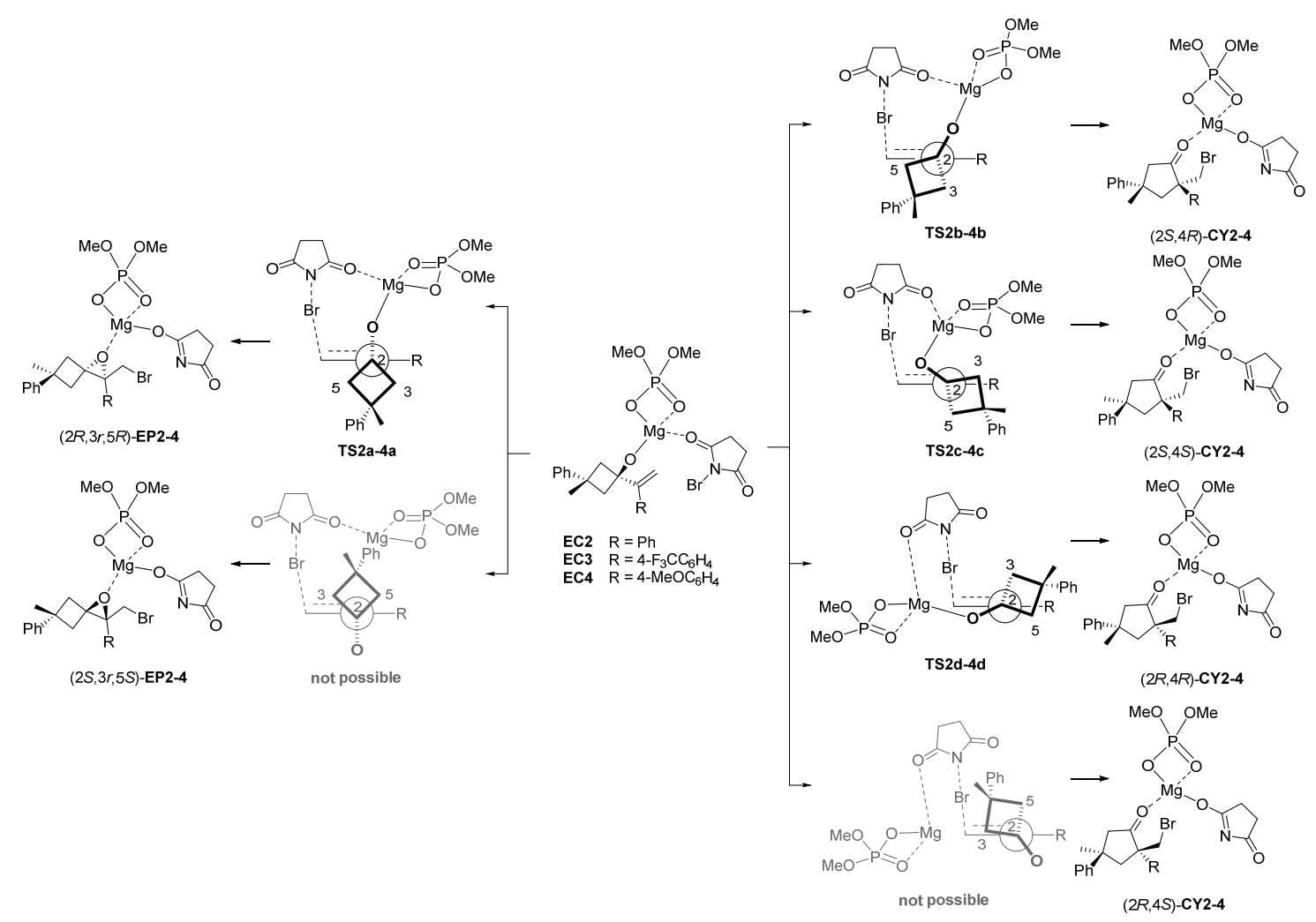

Scheme S 14. Approaches (PES) for the real models EC2-4

The preliminary analysis of the transition structures illustrated in Scheme S15 showed a clear competition between TS2a and TS2b, the only transition structures that show the aromatic ring planar with respect to $\mathrm{C} 6-\mathrm{C} 7$ bond, thus being able of contributing to the stabilization of a transient carbocation. Indeed, in both TS2c and TS2d, due to steric hindrance caused by the cyclobutane ring, the aromatic ring linked to C-6 is placed perpendicular to the double bond circumventing any conjugative effect. ${ }^{19}$ Such a disposition facilitates $\pi, \pi$-interactions between the two aromatic rings linked to $\mathrm{C} 4$ and C6 (confirmed by NCI analysis, see below).

\footnotetext{
${ }^{19}$ This effect is also reflected in the IRCs of TS1c and TS1d that do not show the area corresponding to the transient carbocation, being more similar to that corresponding to TS1a.
} 
Optimized geometries

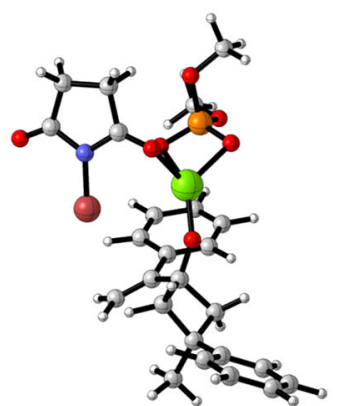

EC2

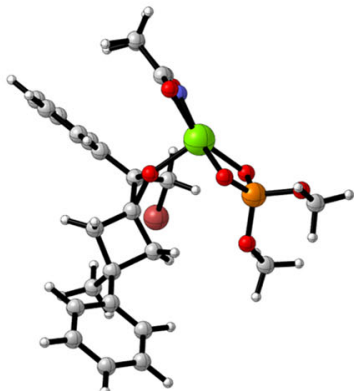

$(2 R, 3 r, 5 R)-\mathrm{EP} 2$

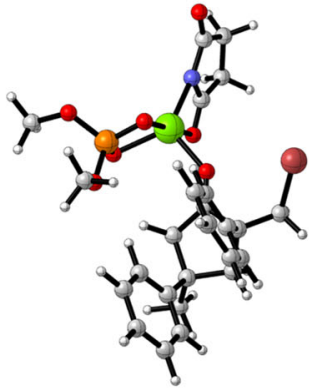

$(2 S, 4 R)-\mathrm{CY} 2$

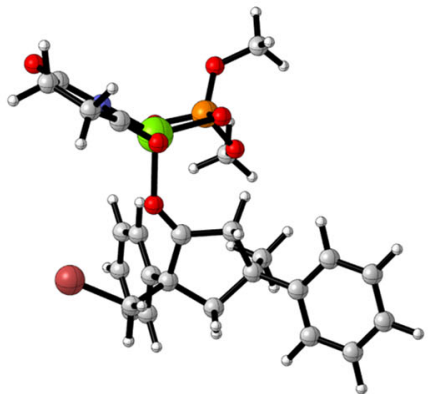

$(2 S, 4 S)-\mathrm{CY} 2$

Figure S 61. Optimized (wb97xd/def2SVP) geometries of encounter complex EC2, epoxide $(2 R, 3 r, 5 R)$ EP2 and cyclopentanones $(2 S, 4 R)-\mathbf{C Y} 2,(2 S, 4 S)-\mathbf{C Y} 2$ and $(2 R, 4 R)-\mathbf{C Y} 2$

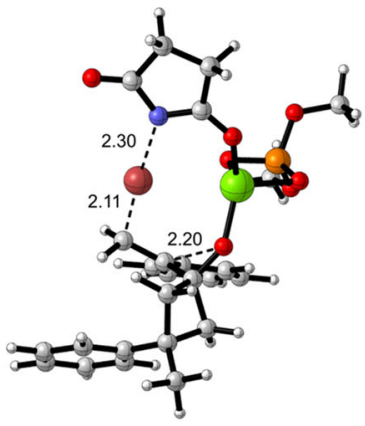

TS2a

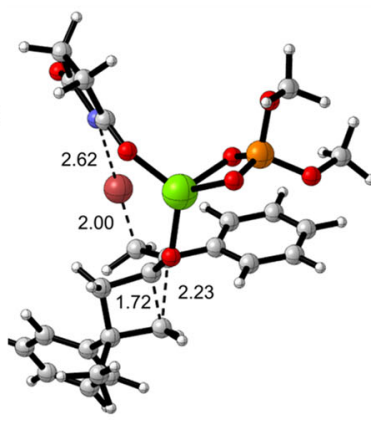

TS2b

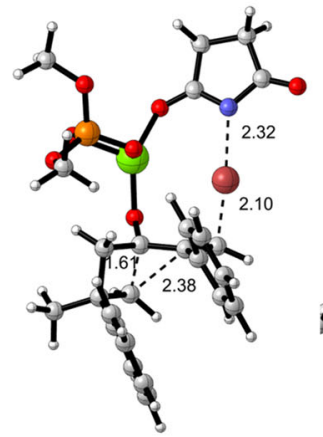

TS2c

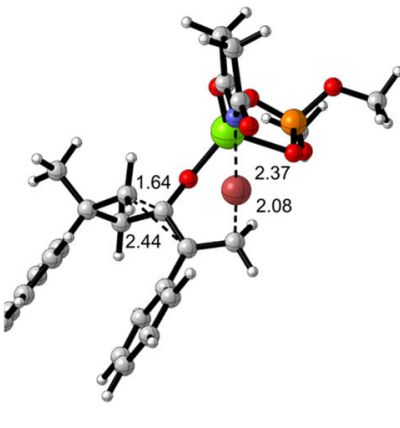

TS2d

Figure S 62. Optimized (wb97xd/def2SVP) geometries of transition structures TS2a, TS2b, TS2c and TS2d.

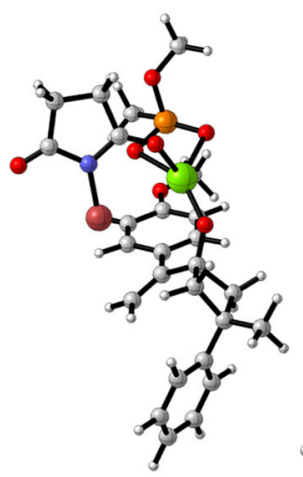

EC3

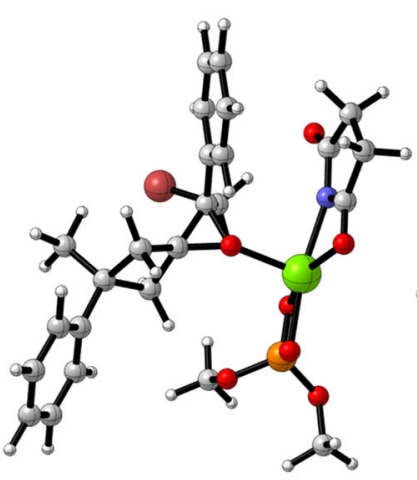

$(2 R, 3 r, 5 R)$-EP3

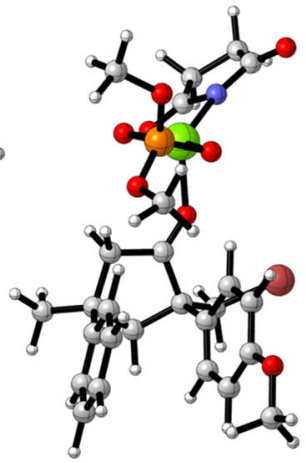

$(2 S, 4 R)-\mathrm{CY} 3$

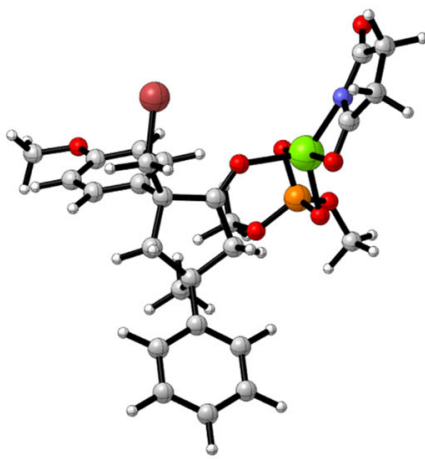

$(2 S, 4 S)-\mathrm{CY} 3$

Figure S 63. Optimized (wb97xd/def2SVP) geometries of encounter complex EC3, epoxide $(2 R, 3 r, 5 R)$ EP3 and cyclopentanones $(2 S, 4 R)$-CY3, $(2 S, 4 S)$-CY3 and $(2 R, 4 R)$-CY3 


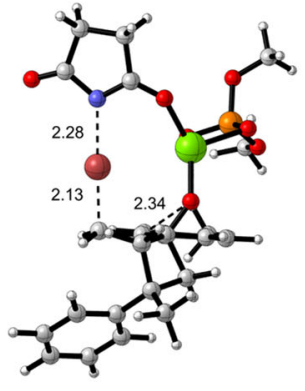

TS3a

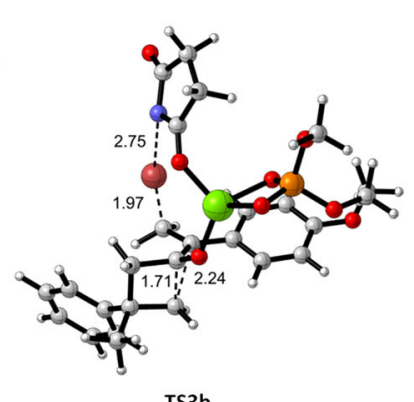

TS3b
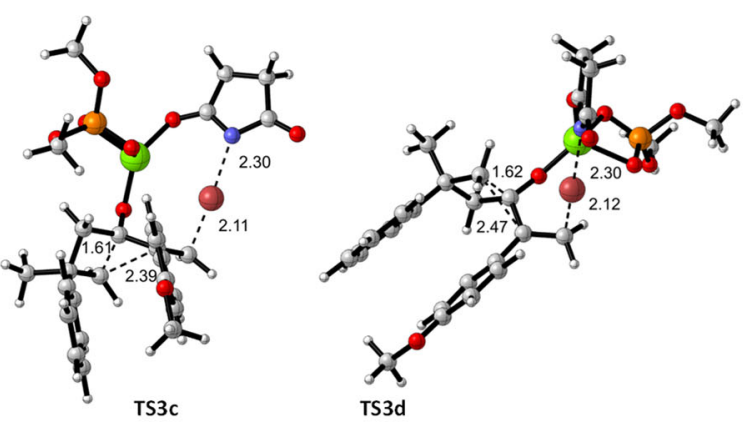

Figure S 64. Optimized (wb97xd/def2SVP) geometries of transition structures TS3a, TS3b, TS3c and TS3d.

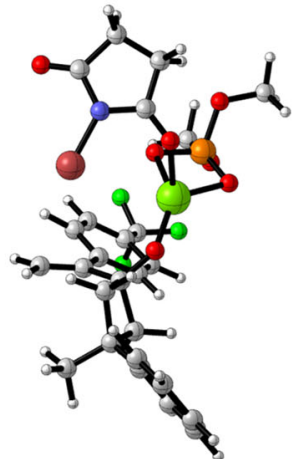

EC4

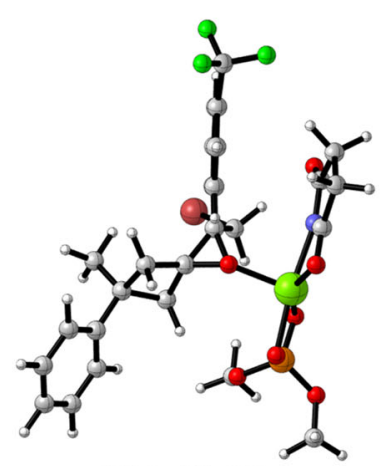

$(2 R, 3 r, 5 R)$-EP4

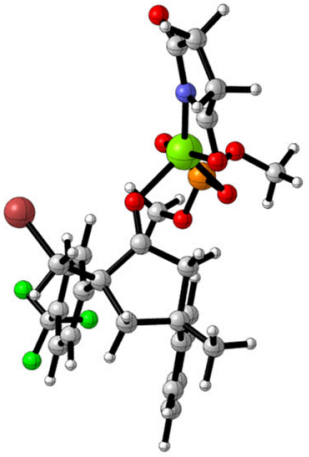

$(2 S, 4 R)-\mathrm{CY} 4$

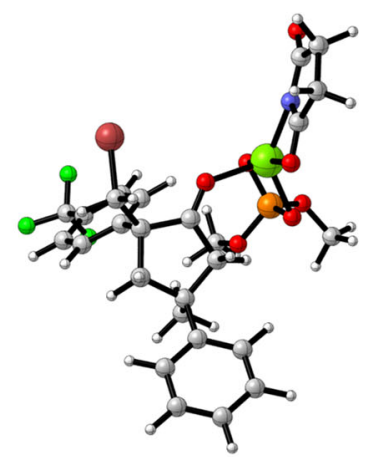

$(2 S, 4 S)-C Y 4$

Figure S 65. Optimized (wb97xd/def2SVP) geometries of encounter complex EC4, epoxide $(2 R, 3 r, 5 R)$ EP4 and cyclopentanones $(2 S, 4 R)$-CY4, $(2 S, 4 S)$-CY4 and $(2 R, 4 R)$-CY4

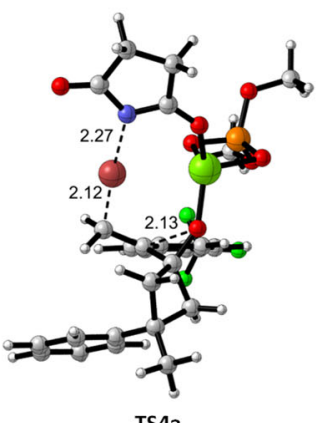

TS4a
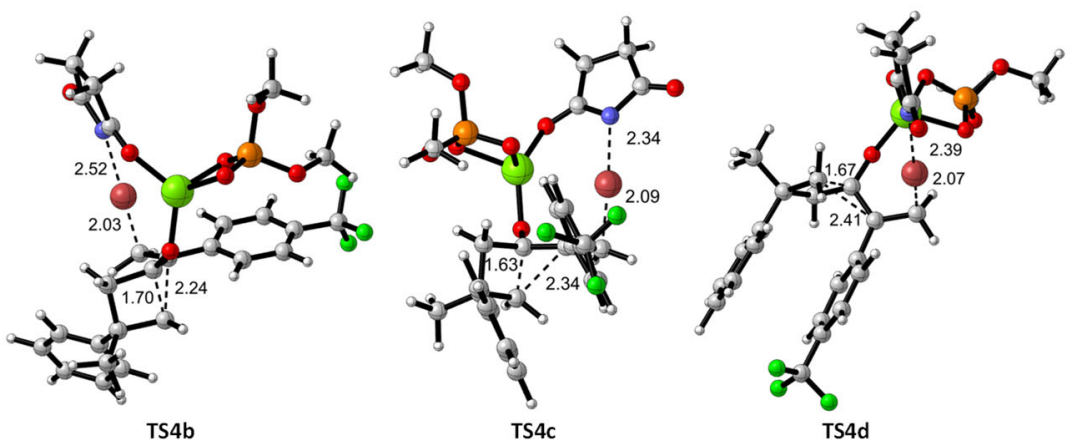

Figure S 66. Optimized (wb97xd/def2SVP) geometries of transition structures TS4a, TS4b, TS4c and TS4d. 

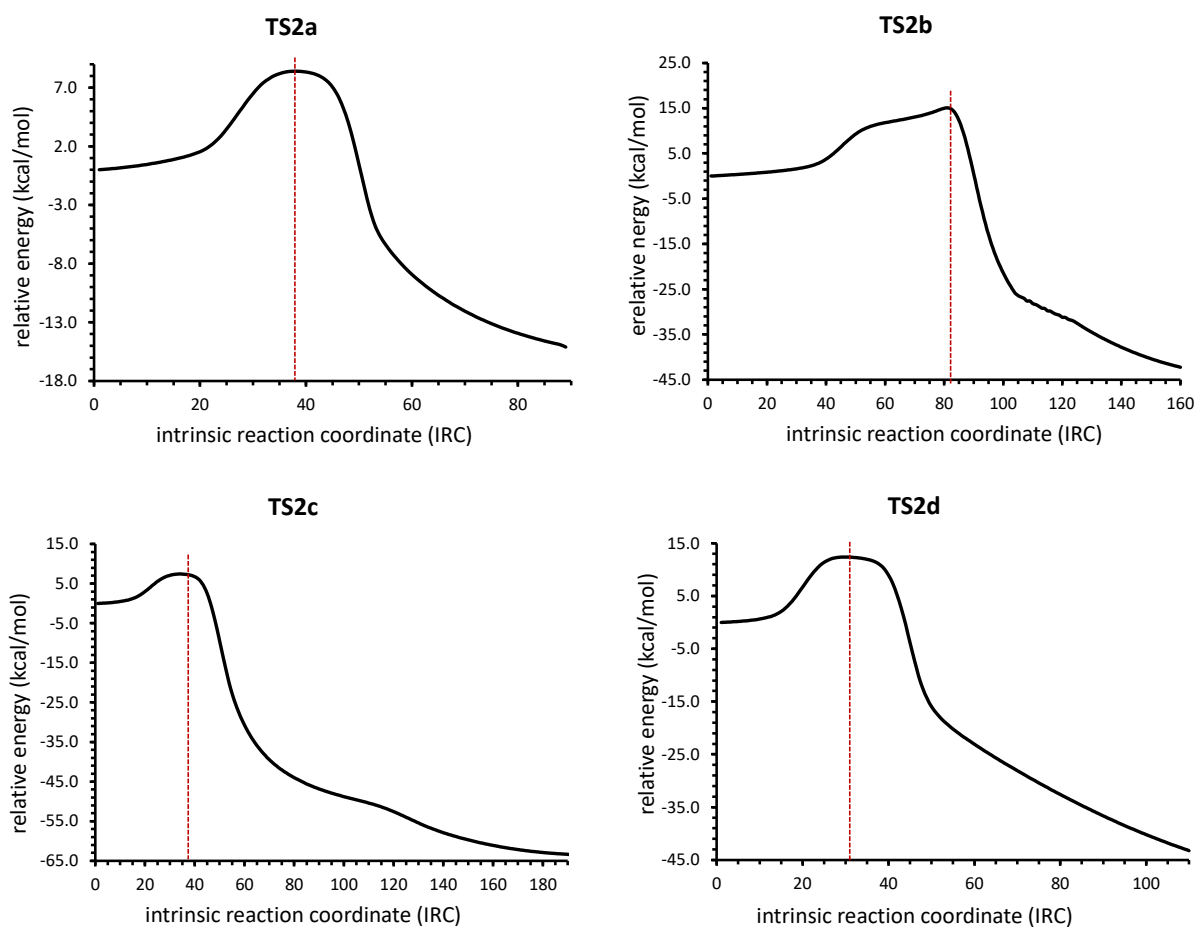

Figure S 67. IRCs (wb97xd/def2svp) corresponding to transition structures TS2a, TS2b, TS2c and TS2d. The red dashed line indicates the transition state.

TS3a

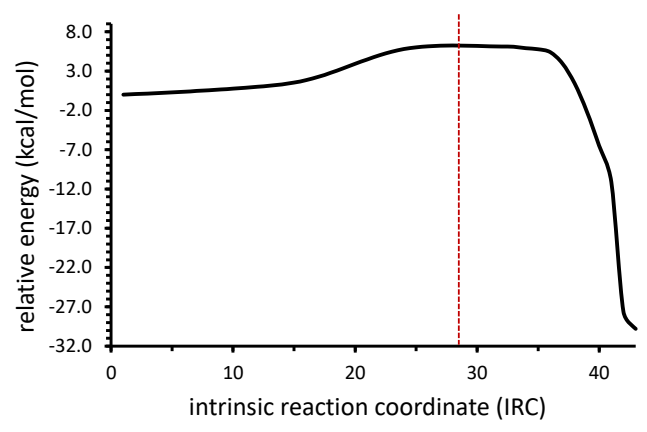

TS3c

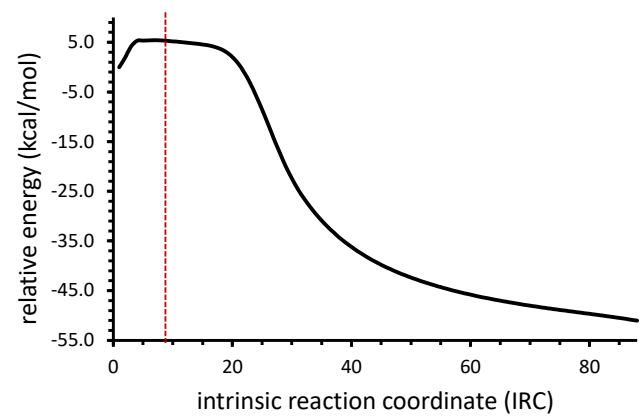

TS3b

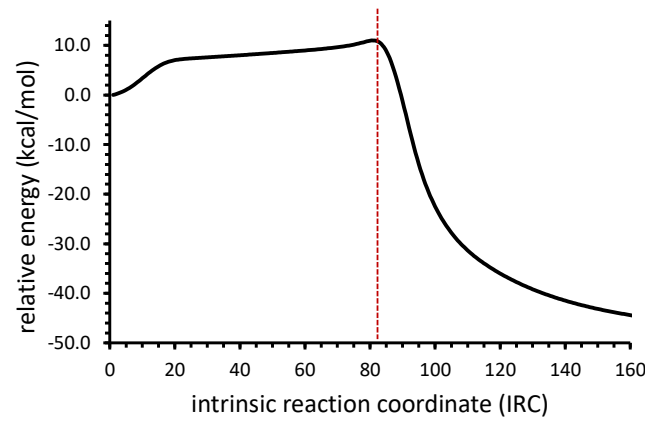

TS3d

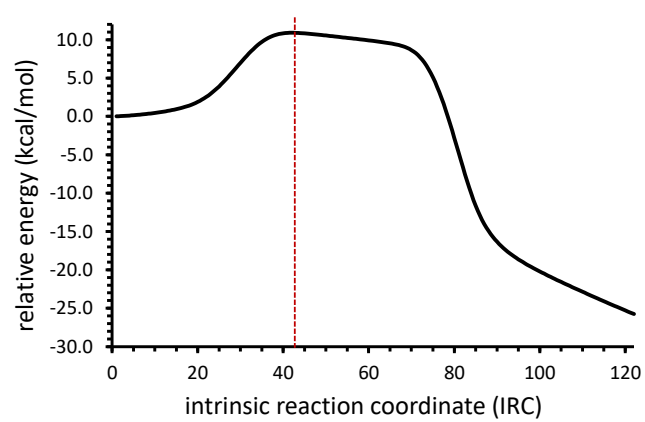

Figure S 68. IRCs (wb97xd/def2svp) corresponding to transition structures TS3a, TS3b, TS3c and TS3d.TS3a and TS3c were completed with relaxed scans after 10 points and before 8 points from the corresponding transition structure. The red dashed line indicates the transition state. 

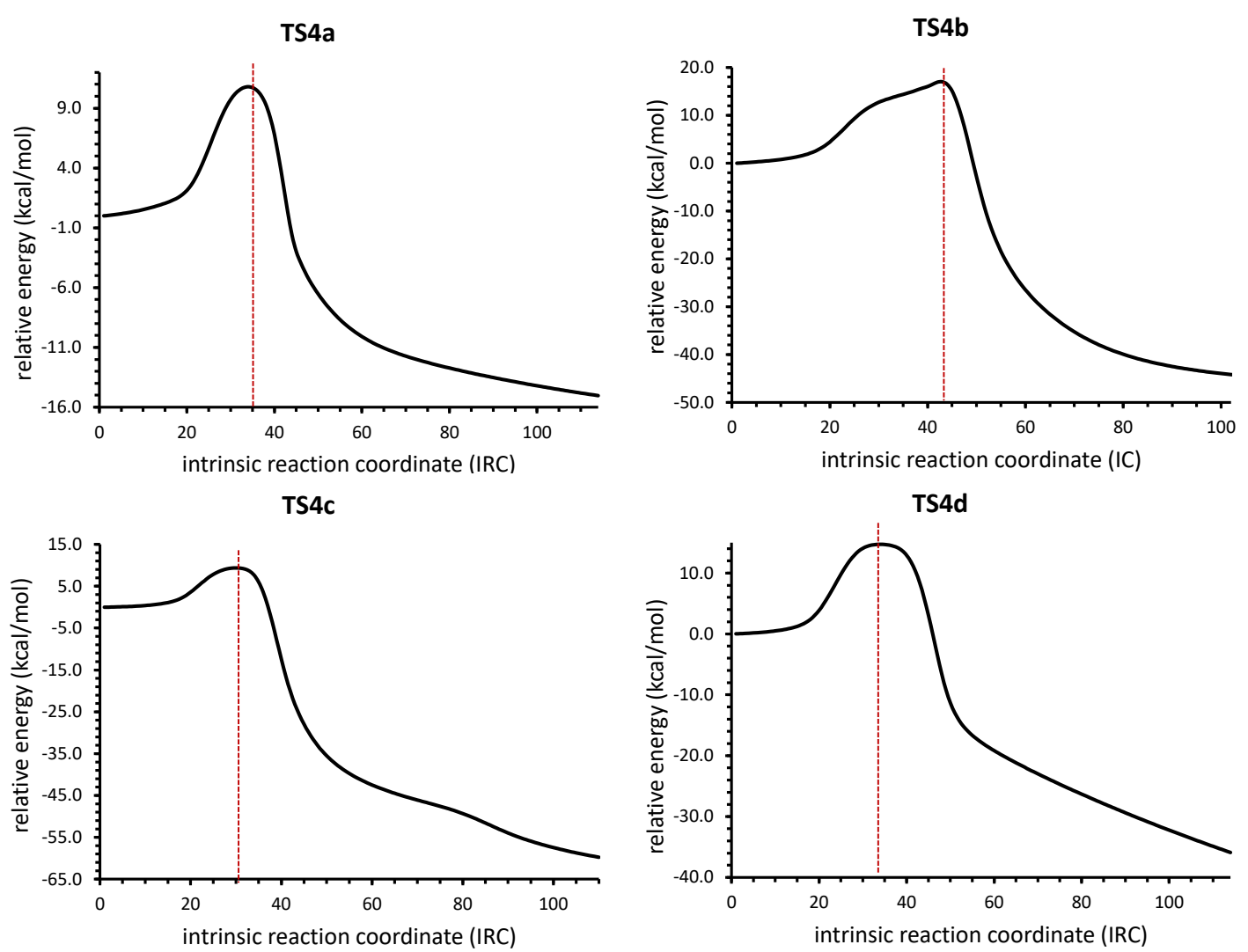

Figure S 69. IRCs (wb97xd/def2svp) corresponding to transition structures TS4a, TS4b, TS4c and TS4d. The red dashed line indicates the transition state. 
Energies

Table S 14. Calculated (wb97xd/def2tzvp/pcm=toluene//wb97xd/def2svp) absolute (hartree) and relative (kcal/mol) energies for the transformation of EC2 into EP2 and CY2 .

\begin{tabular}{lccccc}
\hline & $\mathrm{E}_{0}$ & $\Delta \mathrm{E}_{0}$ & $\mathrm{G}$ & $\Delta \mathrm{G}$ & im. freq \\
\hline EC2 & -4666.936063 & 0.0 & -4667.033127 & 0.0 & \\
TS2a & -4666.921472 & 9.2 & -4667.016934 & 10.2 & -102.3 \\
TS2b & -4666.920881 & 9.5 & -4667.018248 & 9.3 & -283.1 \\
TS2c & -4666.922829 & 8.3 & -4667.016694 & 10.3 & -100.1 \\
TS2d & -4666.914449 & 13.6 & -4667.011436 & 13.6 & -75.2 \\
$(2 R, 4 S)-$ CY2 & -4667.035617 & -62.5 & -4667.136666 & -65.0 & \\
$(2 S, 4 S)-$ CY2 & -4667.034869 & -62.0 & -4667.134881 & -63.9 & \\
$(2 R, 3 r, 5 R)-\mathbf{E P 2}$ & -4666.987781 & -32.5 & -4667.084815 & -32.4 & \\
\hline
\end{tabular}

Table S 15. Calculated (wb97xd/def2tzvp/pcm=toluene//wb97xd/def2svp) absolute (hartree) and relative (kcal/mol) energies for the transformation of EC3 into EP3 and CY3 .

\begin{tabular}{lccccc}
\hline & $\mathrm{E}_{0}$ & $\Delta \mathrm{E}_{0}$ & $\mathrm{G}$ & $\Delta \mathrm{G}$ & im. freq \\
\hline EC3 & -4781.441079 & 0.0 & -4781.540049 & 0.0 & \\
TS3a & -4781.431976 & 5.7 & -4781.532648 & 4.6 & -83.9 \\
TS3b & -4781.430028 & 6.9 & -4781.532229 & 4.9 & -242.3 \\
TS3c & -4781.428921 & 7.6 & -4781.526756 & 8.3 & -87.1 \\
TS3d & -4781.420430 & 13.0 & -4781.521707 & 11.5 & -105.4 \\
$(2 R, 4 S)-\mathbf{C Y 3}$ & -4781.538483 & -61.1 & -4781.642939 & -64.6 & \\
$(2 S, 4 S)-\mathbf{C Y 3}$ & -4781.537674 & -60.6 & -4781.642778 & -64.5 & \\
$(2 R, 3 r, 5 R)-\mathbf{E P 3}$ & -4781.488421 & -29.7 & -4781.589374 & -31.0 & \\
\hline
\end{tabular}

Table S 16. Calculated (wb97xd/def2tzvp/pcm=toluene//wb97xd/def2svp) absolute (hartree) and relative (kcal/mol) energies for the transformation of EC4 into EP4 and CY4 .

\begin{tabular}{lccccc}
\hline & $\mathrm{E}_{0}$ & $\Delta \mathrm{E}_{0}$ & $\mathrm{G}$ & $\Delta \mathrm{G}$ & im. freq \\
\hline EC4 & -5004.038543 & 0.0 & -5004.142061 & 0.0 & \\
TS4a & -5004.019872 & 11.7 & -5004.122592 & 12.2 & -169.8 \\
TS4b & -5004.023355 & 9.5 & -5004.125136 & 10.6 & -248.5 \\
TS4c & -5004.018319 & 12.7 & -5004.122248 & 12.4 & -120.7 \\
TS4d & -5004.014970 & 14.8 & -5004.119556 & 14.1 & -95.3 \\
$(2 R, 4 S)-C Y 4$ & -5004.137163 & -61.9 & -5004.241073 & -62.1 & \\
$(2 S, 4 S)-$ CY4 & -5004.135087 & -60.6 & -5004.243853 & -63.9 & \\
$(2 R, 3 r, 5 R)-$-EP4 & -5004.088621 & -31.4 & -5004.195912 & -33.8 & \\
\hline
\end{tabular}




\section{Benchmarking}

Three additional levels of theory were calculated confirming that the employed level is the closest to experimental results even though all the levels showed the same trend.

Level: $\mathrm{m} 062 \mathrm{x} / \mathrm{cc}-\mathrm{pvtz} / \mathrm{pcm}=$ toluene $/ / \mathrm{m} 062 \mathrm{x} / \mathrm{cc}-\mathrm{pvdz}$

Table S17. Calculated $(\mathrm{m} 062 \mathrm{x} / \mathrm{cc}-\mathrm{pvtz} / \mathrm{pcm}=$ toluene $/ / \mathrm{m} 062 \mathrm{x} / \mathrm{cc}-\mathrm{pvdz})$ absolute (hartree) and relative ( kcal/mol) energies for the transformation of EC2 into EP2 and CY2 .

\begin{tabular}{lccccc}
\hline & $\mathrm{E}_{0}$ & $\Delta \mathrm{E}_{0}$ & $\mathrm{G}$ & $\Delta \mathrm{G}$ & im. freq \\
\hline EC2 & -4666.784591 & 0.0 & -4666.856119 & 0.0 & \\
TS2a & -4666.764352 & 12.7 & -4666.835733 & 12.8 & -134.0 \\
TS2b & -4666.761792 & 14.3 & -4666.834845 & 13.3 & -275.1 \\
TS2c & -4666.766677 & 11.2 & -4666.836856 & 12.1 & -139.9 \\
TS2d & -4666.758944 & 16.1 & -4666.830760 & 15.9 & -122.8 \\
$(2 R, 4 S)-$ CY2 & -4666.877483 & -58.3 & -4666.949556 & -58.6 & \\
$(2 S, 4 S)-$ CY2 & -4666.876534 & -57.7 & -4666.952043 & -60.2 & \\
$(2 R, 3 r, 5 R)$-EP2 & -4666.834672 & -31.4 & -4666.907688 & -32.4 & \\
\hline
\end{tabular}

Table S18. Calculated (m062x/cc-pvtz/pcm=toluene//m062x/cc-pvdz) absolute (hartree) and relative $(\mathrm{kcal} / \mathrm{mol})$ energies for the transformation of EC3 into EP3 and CY3 .

\begin{tabular}{lccccc}
\hline & $\mathrm{E}_{0}$ & $\Delta \mathrm{E}_{0}$ & $\mathrm{G}$ & $\Delta \mathrm{G}$ & im. freq \\
\hline EC3 & -4781.280628 & 0.0 & -4781.353412 & 0.0 & \\
TS3a & -4781.262342 & 11.5 & -4781.336933 & 10.3 & -80.4 \\
TS3b & -4781.259134 & 13.5 & -4781.334920 & 11.6 & -201.3 \\
TS3c & -4781.261625 & 11.9 & -4781.334345 & 12.0 & -97.4 \\
TS3d & -4781.254139 & 16.6 & -4781.328562 & 15.6 & -110.2 \\
$(2 R, 4 S)-$ CY3 & -4781.369477 & -55.8 & -4781.444827 & -57.4 & \\
$(2 S, 4 S)-$ CY3 & -4781.367670 & -54.6 & -4781.446764 & -58.6 & \\
$(2 R, 3 r, 5 R)-\mathbf{E P 3}$ & -4781.325350 & -28.1 & -4781.399192 & -28.7 & \\
\hline
\end{tabular}

Table S19. Calculated ( $\mathrm{m} 062 \mathrm{x} / \mathrm{cc}-\mathrm{pvtz} / \mathrm{pcm}=$ toluene $/ / \mathrm{m} 062 \mathrm{x} / \mathrm{cc}-\mathrm{pvdz})$ absolute (hartree) and relative ( $\mathrm{kcal} / \mathrm{mol}$ ) energies for the transformation of EC4 into EP4 and CY4 .

\begin{tabular}{lccccc}
\hline & $\mathrm{E}_{0}$ & $\Delta \mathrm{E}_{0}$ & $\mathrm{G}$ & $\Delta \mathrm{G}$ & im. freq \\
\hline EC4 & -5003.865918 & 0.0 & -5003.941723 & 0.0 & \\
TS4a & -5003.841662 & 15.2 & -5003.919115 & 14.2 & -239.9 \\
TS4b & -5003.839519 & 16.6 & -5003.916449 & 15.9 & -305.2 \\
TS4c & -5003.845453 & 12.8 & -5003.920524 & 13.3 & -182.4 \\
TS4d & -5003.837628 & 17.8 & -5003.914686 & 17.0 & -165.8 \\
$(2 R, 4 S)-C Y 4$ & -5003.957344 & -57.4 & -5004.034098 & -58.0 & \\
$(2 S, 4 S)-C Y 4$ & -5003.954124 & -55.4 & -5004.034015 & -57.9 & \\
$(2 R, 3 r, 5 R)-E P 4$ & -5003.913108 & -29.6 & -5003.991683 & -31.3 & \\
\hline
\end{tabular}


Level: b3lyp-gd3bj/6-311G(d,p)/pcm=toluene//b3lyp-gd3bj/6-31G(d,p)

Table S20. Calculated (b3lyp-gd3bj/6-311G(d,p)/pcm=toluene//b3lyp-gd3bj/6-31G(d,p)) absolute (hartree) and relative ( $\mathrm{kcal} / \mathrm{mol})$ energies for the transformation of EC2 into EP2 and CY2 .

\begin{tabular}{lccccc}
\hline & $\mathrm{E}_{0}$ & $\Delta \mathrm{E}_{0}$ & $\mathrm{G}$ & $\Delta \mathrm{G}$ & im. freq \\
\hline EC2 & -4667.321862 & 0.0 & -4667.395918 & 0.0 & \\
TS2a & -4667.314886 & 4.4 & -4667.386725 & 5.8 & -69.0 \\
TS2b & -4667.304656 & 10.8 & -4667.378087 & 11.2 & -360.5 \\
TS2c & -4667.314552 & 4.6 & -4667.386559 & 5.9 & -176.6 \\
TS2d & -4667.304663 & 10.8 & -4667.378478 & 10.9 & -86.5 \\
$(2 R, 4 S)-$ CY2 & -4667.410997 & -55.9 & -4667.481286 & -53.6 & \\
$(2 S, 4 S)-$ CY2 & -4667.410058 & -55.3 & -4667.483185 & -54.8 & \\
$(2 R, 3 r, 5 R)-\mathbf{E P 2}$ & -4667.362652 & -25.6 & -4667.433919 & -23.8 & \\
\hline
\end{tabular}

Table S21. Calculated (b3lyp-gd3bj/6-311G(d,p)/pcm=toluene//b3lyp-gd3bj/6-31G(d,p)) absolute (hartree) and relative (kcal/mol) energies for the transformation of EC3 into EP3 and CY3 .

\begin{tabular}{lccccc}
\hline & $\mathrm{E}_{0}$ & $\Delta \mathrm{E}_{0}$ & $\mathrm{G}$ & $\Delta \mathrm{G}$ & im. freq \\
\hline EC3 & -4781.856839 & 0.0 & -4781.932046 & 0.0 & \\
TS3a & -4781.848255 & 5.4 & -4781.923722 & 5.2 & -61.5 \\
TS3b & -4781.838406 & 11.6 & -4781.914980 & 10.7 & -361.4 \\
TS3c & -4781.846994 & 6.2 & -4781.922013 & 6.3 & -145.7 \\
TS3d & -4781.837596 & 12.1 & -4781.913495 & 11.6 & -32.0 \\
$(2 R, 4 S)-$ CY3 & -4781.939974 & -52.2 & -4782.017185 & -53.4 & \\
$(2 S, 4 S)-$ CY3 & -4781.937686 & -50.7 & -4782.017172 & -53.4 & \\
$(2 R, 3 r, 5 R)-$-EP3 & -4781.892203 & -22.2 & -4781.969625 & -23.6 & \\
\hline
\end{tabular}

Table S22. Calculated (b3lyp-gd3bj/6-311G(d,p)/pcm=toluene//b3lyp-gd3bj/6-31G(d,p)) absolute (hartree) and relative (kcal/mol) energies for the transformation of EC4 into EP4 and CY4 .

\begin{tabular}{lccccc}
\hline & $\mathrm{E}_{0}$ & $\Delta \mathrm{E}_{0}$ & $\mathrm{G}$ & $\Delta \mathrm{G}$ & im. freq \\
\hline EC4 & -5004.466015 & 0.0 & -5004.541690 & 0.0 & \\
TS4a & -5004.455464 & 6.6 & -5004.533307 & 5.3 & -96.8 \\
TS4b & -5004.446336 & 12.3 & -5004.524423 & 10.8 & -371.6 \\
TS4c & -5004.456418 & 6.0 & -5004.534177 & 4.7 & -210.1 \\
TS4d & -5004.446274 & 12.4 & -5004.524916 & 10.5 & -132.6 \\
$(2 R, 4 S)-C Y 4$ & -5004.558047 & -57.8 & -5004.633597 & -57.7 & \\
$(2 S, 4 S)-C Y 4$ & -5004.551154 & -53.4 & -5004.636098 & -59.2 & \\
$(2 R, 3 r, 5 R)-$-EP4 & -5004.504154 & -23.9 & -5004.581095 & -24.7 & \\
\hline
\end{tabular}


Level: m062x/6-311G(d,p)/pcm=toluene//m062x/6-31G(d,p)

Table S23. Calculated (b3lyp-gd3bj/6-311G(d,p)/pcm=toluene//b3lyp-gd3bj/6-31G(d,p)) absolute (hartree) and relative ( $\mathrm{kcal} / \mathrm{mol})$ energies for the transformation of EC2 into EP2 and CY2 .

\begin{tabular}{lccccc}
\hline & $\mathrm{E}_{0}$ & $\Delta \mathrm{E}_{0}$ & $\mathrm{G}$ & $\Delta \mathrm{G}$ & im. freq \\
\hline EC2 & -4666.497339 & 0.0 & -4666.568052 & 0.0 & \\
TS2a & -4666.477716 & 12.3 & -4666.547818 & 12.7 & -138.9 \\
TS2b & -4666.472620 & 15.5 & -4666.544921 & 14.5 & -350.1 \\
TS2c & -4666.481340 & 10.0 & -4666.551009 & 10.7 & -167.1 \\
TS2d & -4666.471062 & 16.5 & -4666.541926 & 16.4 & -114.0 \\
$(2 R, 4 S)-$ CY2 & -4666.590419 & -58.4 & -4666.661658 & -58.7 & \\
$(2 S, 4 S)-$ CY2 & -4666.591922 & -59.4 & -4666.668223 & -62.9 & \\
$(2 R, 3 r, 5 R)-$-EP2 & -4666.550061 & -33.1 & -4666.621864 & -33.8 & \\
\hline
\end{tabular}

Table S24. Calculated (b3lyp-gd3bj/6-311G(d,p)/pcm=toluene//b3lyp-gd3bj/6-31G(d,p)) absolute (hartree) and relative (kcal/mol) energies for the transformation of EC3 into EP3 and CY3 .

\begin{tabular}{lccccc}
\hline & $\mathrm{E}_{0}$ & $\Delta \mathrm{E}_{0}$ & $\mathrm{G}$ & $\Delta \mathrm{G}$ & im. freq \\
\hline EC3 & -4780.981389 & 0.0 & -4781.053642 & 0.0 & \\
TS3a & -4780.961835 & 12.3 & -4781.035558 & 11.3 & -36.2 \\
TS3b & -4780.956007 & 15.9 & -4781.031087 & 14.2 & -294.6 \\
TS3c & -4780.962852 & 11.6 & -4781.035513 & 11.4 & -109.5 \\
TS3d & -4780.952437 & 18.2 & -4781.025796 & 17.5 & -90.9 \\
$(2 R, 4 S)-$ CY3 & -4781.069651 & -55.4 & -4781.147045 & -58.6 & \\
$(2 S, 4 S)-$ CY3 & -4781.069510 & -55.3 & -4781.149609 & -60.2 & \\
$(2 R, 3 r, 5 R)-\mathbf{E P 3}$ & -4781.028720 & -29.7 & -4781.102358 & -30.6 & \\
\hline
\end{tabular}

Table S25. Calculated (b3lyp-gd3bj/6-311G(d,p)/pcm=toluene//b3lyp-gd3bj/6-31G(d,p)) absolute (hartree) and relative (kcal/mol) energies for the transformation of EC4 into EP4 and CY4 .

\begin{tabular}{lccccc}
\hline & $\mathrm{E}_{0}$ & $\Delta \mathrm{E}_{0}$ & $\mathrm{G}$ & $\Delta \mathrm{G}$ & im. freq \\
\hline EC4 & -5003.539595 & 0.0 & -5003.614225 & 0.0 & \\
TS4a & -5003.516186 & 14.7 & -5003.591986 & 14.0 & -227.6 \\
TS4b & -5003.511986 & 17.3 & -5003.588616 & 16.1 & -373.4 \\
TS4c & -5003.521491 & 11.4 & -5003.596650 & 11.0 & -217.0 \\
TS4d & -5003.511104 & 17.9 & -5003.586946 & 17.1 & -156.3 \\
$(2 R, 4 S)-$ CY4 & -5003.636309 & -60.7 & -5003.712570 & -61.7 & \\
$(2 S, 4 S)-$ CY4 & -5003.630685 & -57.2 & -5003.711377 & -61.0 & \\
$(2 R, 3 r, 5 R)$-EP4 & -5003.589377 & -31.2 & -5003.666989 & -33.1 & \\
\hline
\end{tabular}


Energy profiles

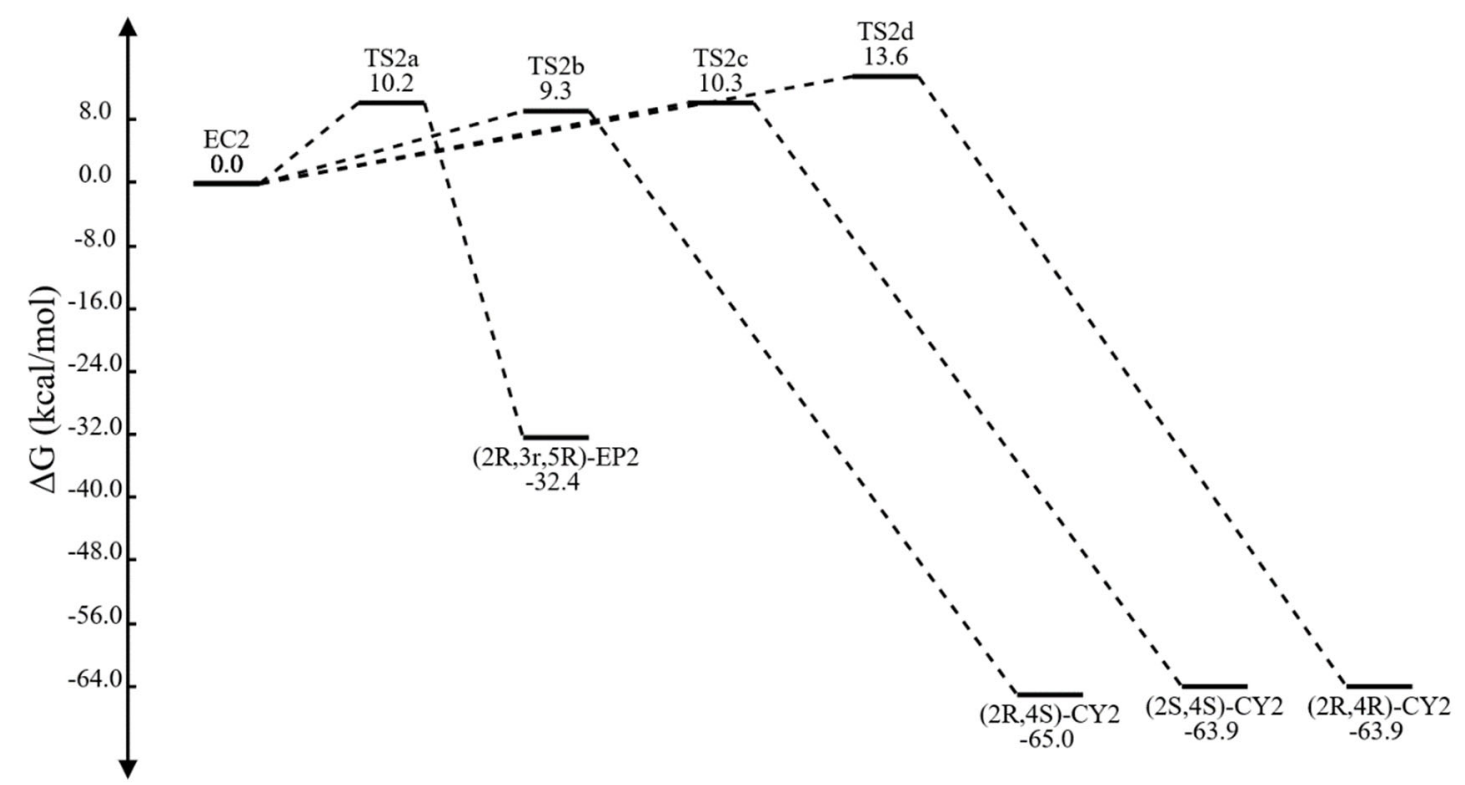

Figure $\mathbf{S}$ 70. Energy profiles (wb97xd/def2tzvp/pcm=toluene)//wb97xd/def2svp) for the different pathways from EC2 to EP2 and CY2. Relative free energies are given in $\mathrm{kcal} / \mathrm{mol}$.

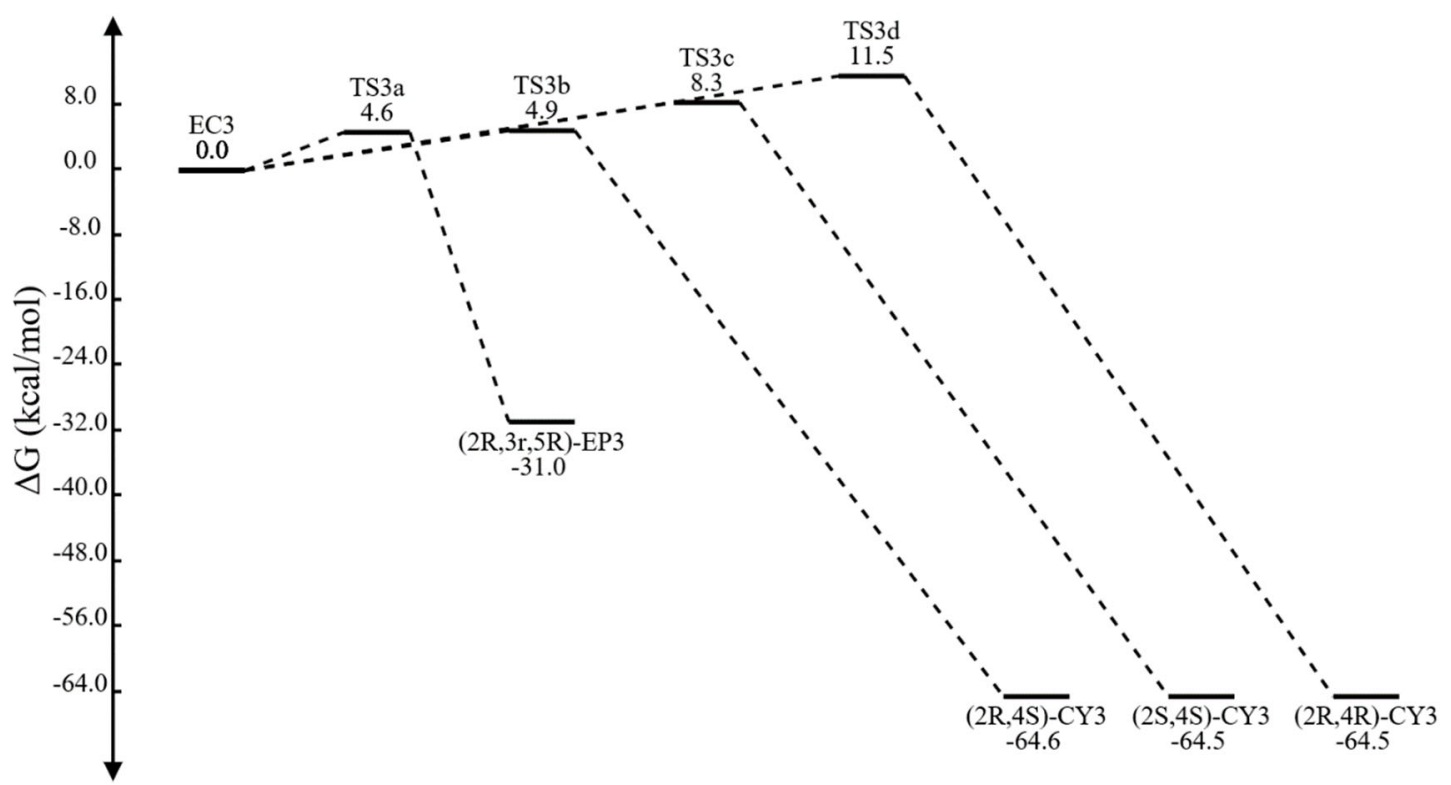

Figure S 71. Energy profiles (wb97xd/def2tzvp/pcm=toluene)//wb97xd/def2svp) for the different pathways from EC3 to EP3 and CY3. Relative free energies are given in $\mathrm{kcal} / \mathrm{mol}$. 


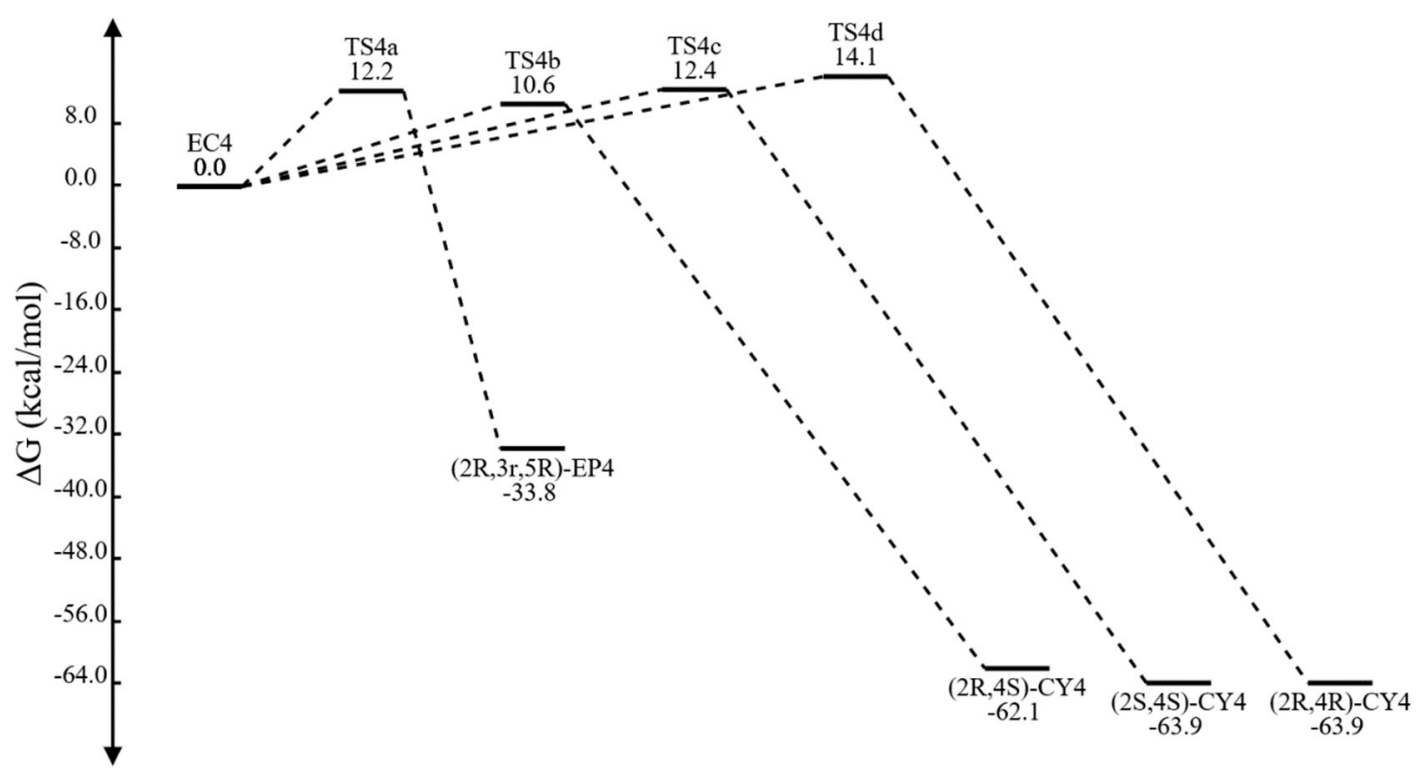

Figure S 72. Energy profiles (wb97xd/def2tzvp/pcm=toluene)//wb97xd/def2svp) for the different pathways from EC4 to EP4 and CY4. Relative free energies are given in $\mathrm{kcal} / \mathrm{mol}$.

A further exploration of the potential energy surface (PES) for the reaction of EP3 $(\mathrm{R}=$ $\left.\mathrm{MeOC}_{6} \mathrm{H}_{4}\right)$ corresponding to the first part of the reaction, i.e. variation of the dihedral angle with respect to the IRC (Figure S 73, left), evidenced the planarity of the surface as it corresponds to the small difference between the two dihedral angles leading to epoxide and cyclopentanone.

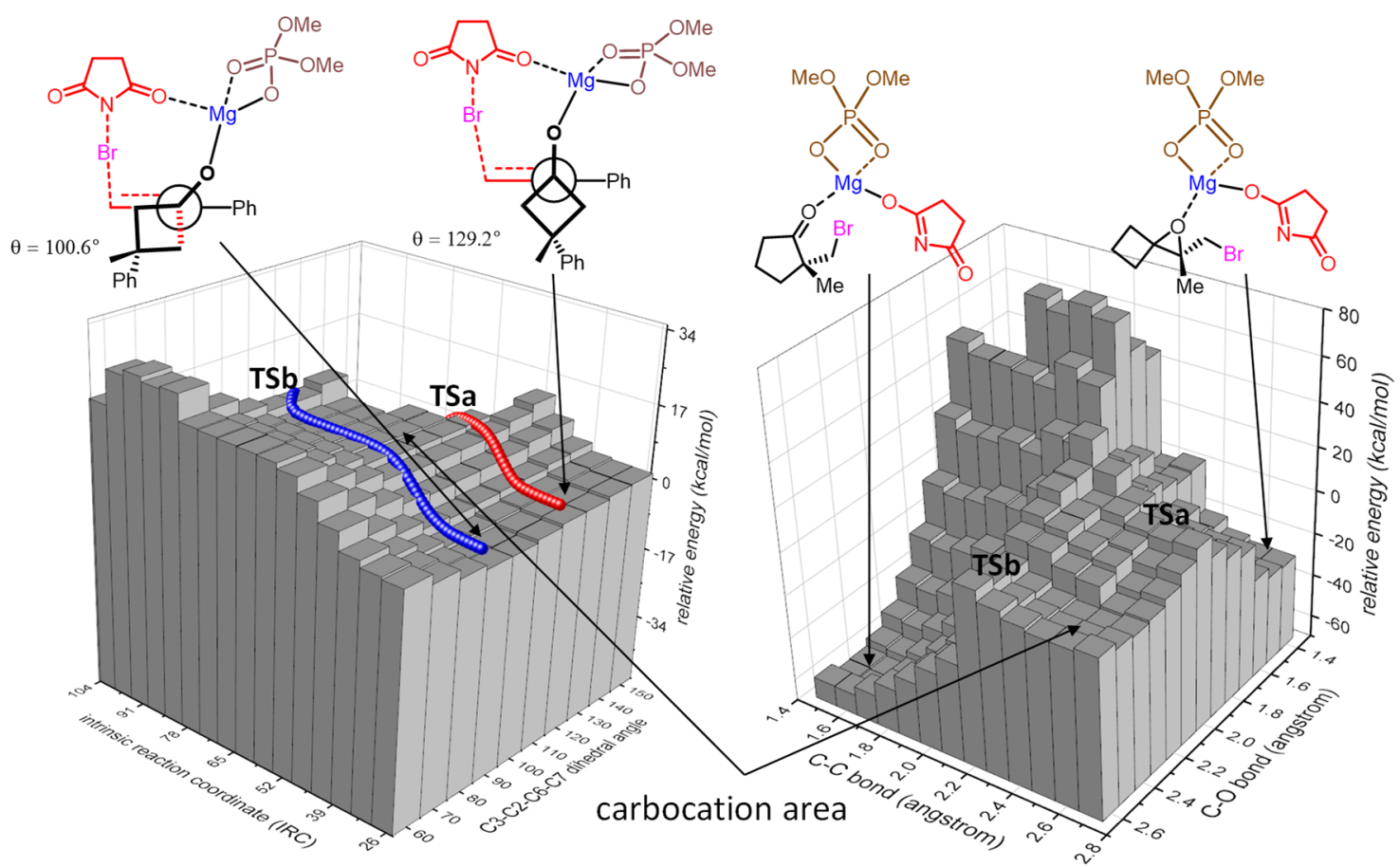

Figure S 73. Left: Potential energy surface (wb97xd/def2svp) related to the variation of the dihedral angle with respect to to the IRC (red: IRC for the formation of the epoxide; blue: IRC for the formation of the cyclopentanone). Right: Potential energy surface (wb97xd/def2svp) corresponding to the formation of O1$\mathrm{C} 1$ and $\mathrm{C} 2-\mathrm{C} 3$ bonds leading to the epoxide and the cyclopentanone, respectively. 
The exploration of the second part of the reaction, i.e. formation of either the $\mathrm{O} 1-\mathrm{C} 1$ bond (epoxide) or C2-C3 bond (cyclopentanone) (Figure S 73, right), was made in a similar way to the unsubstituted model; similar results were obtained confirming the absence of a direct connection between the epoxide and the cyclopentanone. For the PES exploration the most stable conformers were considered.

\section{NCI Analysis}

Non-covalent interactions are shown as isosurfaces in Figure S 74, Figure S 75 and Figure S 76. Thin, delocalized green surfaces indicates van der Waals interactions such as extended surfaces between aromatic rings illustrating $\pi$ - $\pi$ interactions. Small, lenticular, bluish surfaces indicate strong interactions such as hydrogen bonding. Steric clashes are shown as red isosurfaces. Forming bonds are indicated by a blue disc surrounded by a red area. $s(\mathbf{r})$ against $\operatorname{sign}\left(\lambda_{2}\right) \rho(\mathbf{r})$ plots are also given in the bottom part. Quantitative analysis is given in Table S 26, Table S 27 and Table S 28. Blue area corresponds to strong NCI (H-bond, halogen, etc.); green area corresponds to van der Waals interactions and red area corresponds to repulsive interactions (steric clashes, etc.)

In all cases, the $\mathrm{C}-\mathrm{Br}$ bond is essentially done at the transition structure but, interestingly, a strong interaction (disc blue) exists between the nitrogen and the bromine atoms indicating that the process, as a whole, has not finished, yet. This interaction demonstrates that, independently of the advance in the forming bonds like $\mathrm{C}-\mathrm{Br}$, all the process consist of a single kinetic step.

Transition structures $\mathbf{a}$ and $\mathbf{b}$, place the aromatic ring planar with respect to the $\mathrm{sp}^{2}$ carbon developing a positive charge. In those cases, in which the aromatic ring cannot be planar to the carbocationic $\mathrm{sp}^{2}$ carbon for steric reasons (c and $\mathbf{d}$ ), London interactions between the two aromatic rings are visible, compensating in part the destabilization caused by such a lack of planarity. 

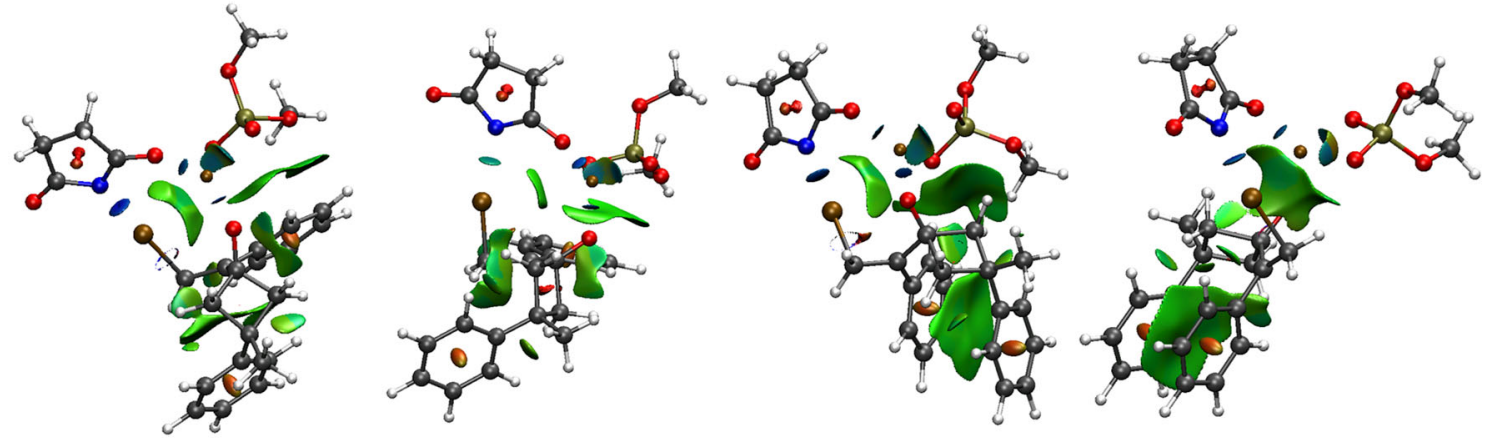

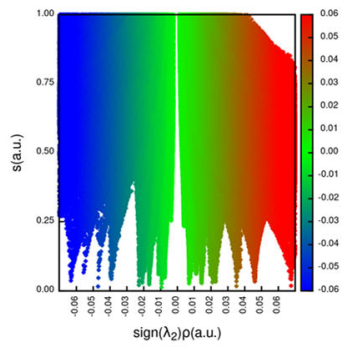

TS2a

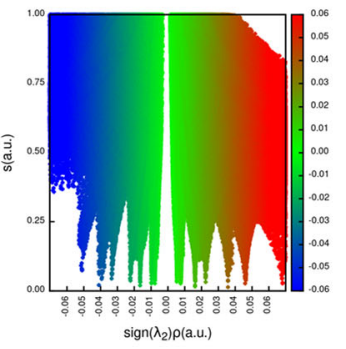

TS2b

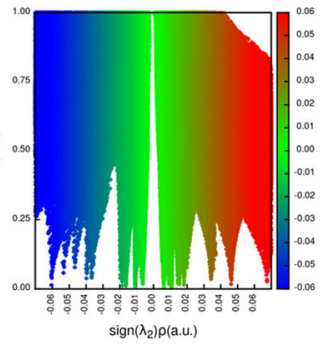

TS2c

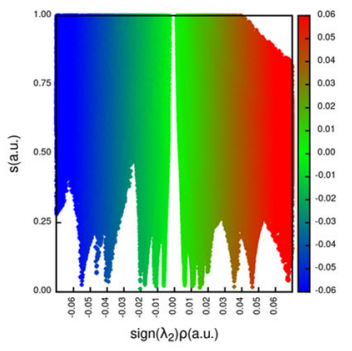

TS2d

Figure S 74. NCI analysis for transition structures TS2a, TS2b, TS4c and TS2d. Top: Isosurfaces. Bottom: $\mathrm{s}(\mathbf{r})$ against $\operatorname{sign}\left(\lambda_{2}\right) \rho(\mathbf{r})$ plots (see text).

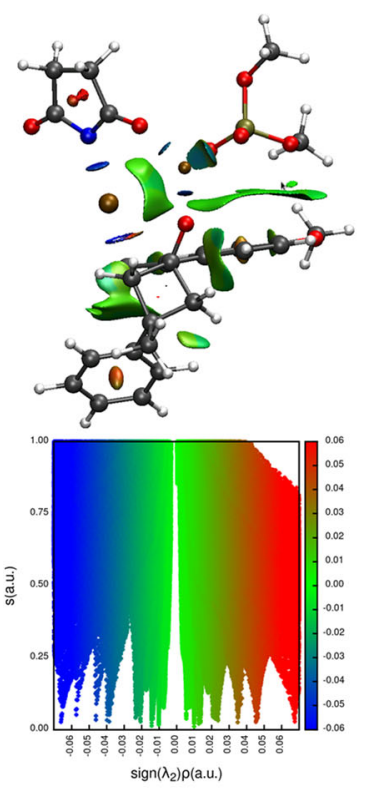

TS3a
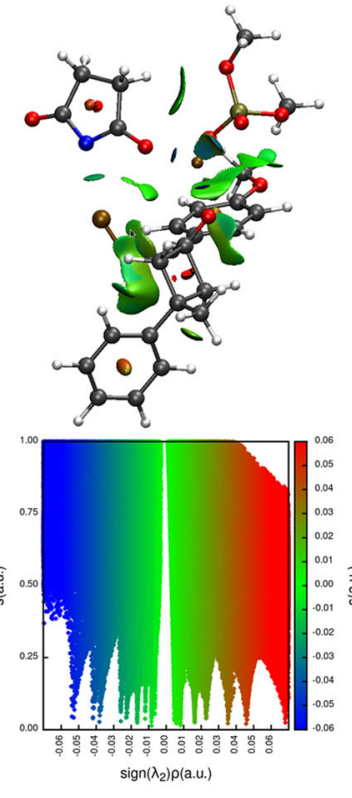

TS3b
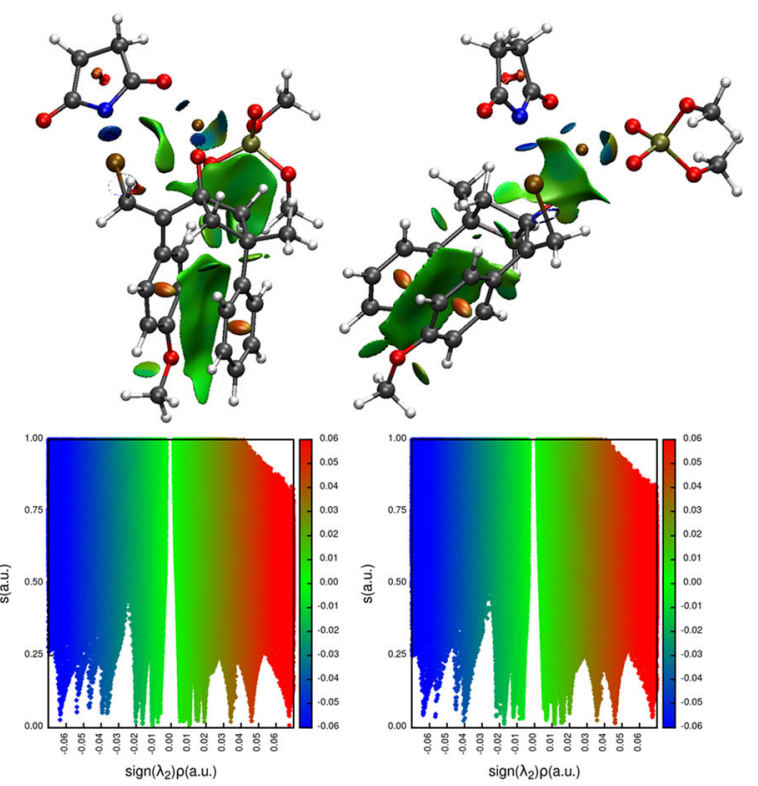

TS3C

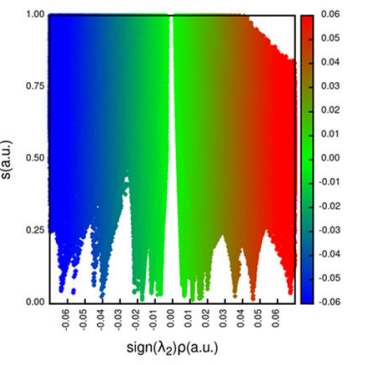

TS3d

Figure S 75. NCI analysis for transition structures TS3a, TS3b, TS3c and TS3d. Top: Isosurfaces. Bottom: $s(\mathbf{r})$ against $\operatorname{sign}\left(\lambda_{2}\right) \rho(\mathbf{r})$ plots (see text). 

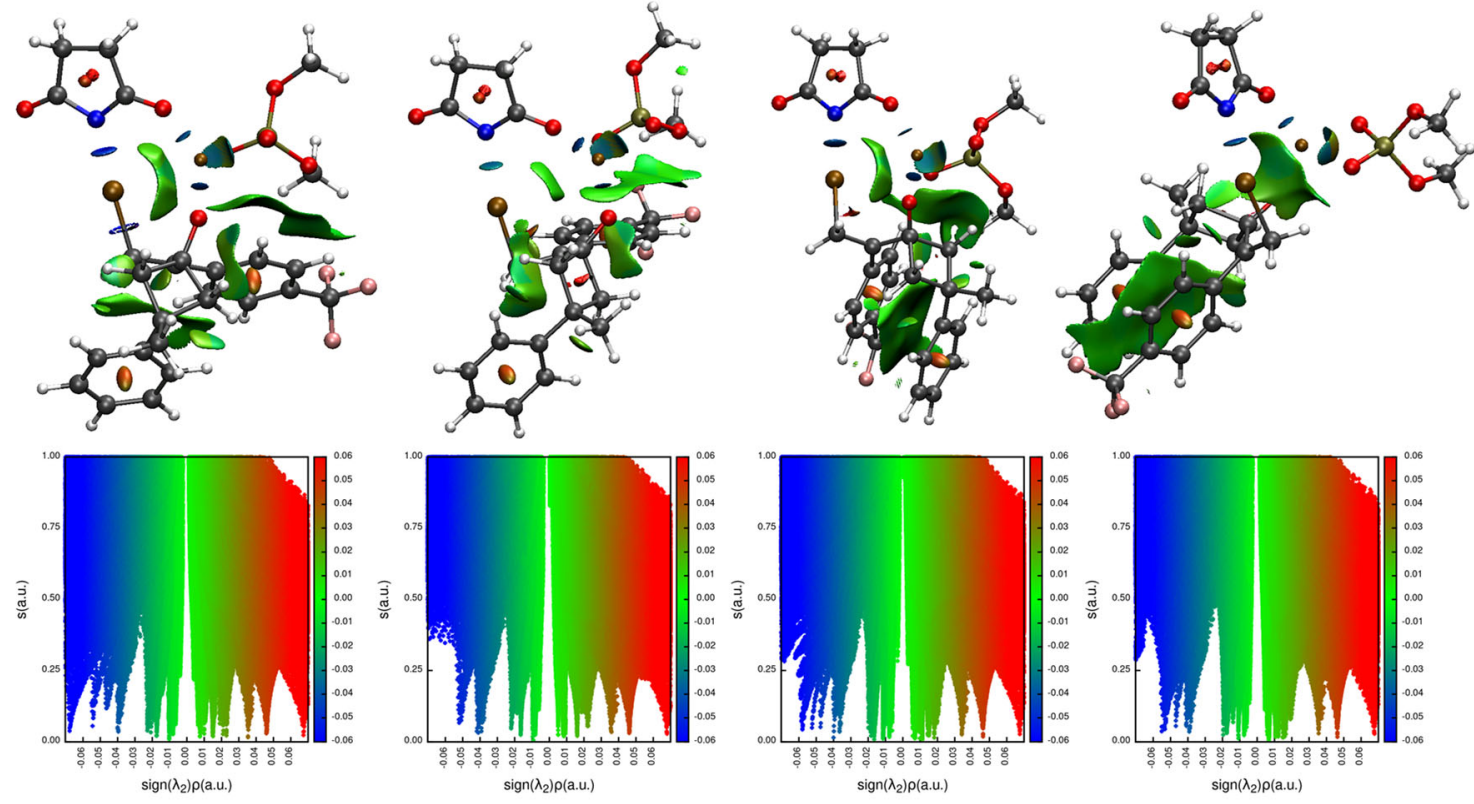

TS4C

TS4d

Figure S 76. NCI analysis for transition structures TS4a, TS4b, TS4c and TS4d. Top: Isosurfaces. Bottom: $\mathrm{s}(\mathbf{r})$ against $\operatorname{sign}\left(\lambda_{2}\right) \rho(\mathbf{r})$ plots $($ see text).

Table S 26 Integration over the volumes of $\operatorname{sign}\left(\lambda_{2}\right) \rho(\mathbf{n})$ for $n=2.5$ according to ref. 37 for TS2a-d

\begin{tabular}{ccccc}
\hline interval & TS2a & TS2b & TS2c & TS2d \\
\hline all & -3.3160424 & -3.30680306 & -3.28056600 & -3.28024501 \\
$\mathbf{- 1 . 0}$ to $\mathbf{- 0 . 0 2}$ & -0.23909918 & -0.23536056 & -0.23501657 & -0.23027198 \\
$\mathbf{- 0 . 0 2}$ to $\mathbf{0 . 0 2}$ & 0.00412829 & 0.00406551 & 0.00424492 & 0.00421830 \\
$\mathbf{0 . 0 2}$ to $\mathbf{1 . 0}$ & 0.30176314 & 0.30120803 & 0.30067717 & 0.29595285 \\
\hline
\end{tabular}

${ }^{\mathrm{a}}$ The interval [-0.1, -0.02] gives information about strong NCI (H-bond, halogen, etc.). The interval [-0.02, $0.02]$ gives information about van der Waals interactions. The interval $[0.02,1.00]$ gives information about steric clashes.

Table S 27 Integration over the volumes of $\operatorname{sign}\left(\lambda_{2}\right) \rho(\mathbf{n})$ for $n=2.5$ according to ref. 37 for TS3a-d

\begin{tabular}{ccccc}
\hline interval & TS3a & TS3b & TS3c & TS3d \\
\hline all & -3.45146392 & -3.46129320 & -3.43458121 & -3.46034759 \\
$\mathbf{- 1 . 0}$ to $\mathbf{- 0 . 0 2}$ & -0.24680436 & -0.24284827 & -0.24965565 & -0.24831046 \\
$\mathbf{- 0 . 0 2}$ to $\mathbf{0 . 0 2}$ & 0.00424362 & 0.00410503 & 0.00446279 & 0.00433981 \\
$\mathbf{0 . 0 2}$ to $\mathbf{1 . 0}$ & 0.31623560 & 0.00410503 & 0.31787636 & 0.31617245 \\
\hline
\end{tabular}

${ }^{a}$ The interval [-0.1, -0.02] gives information about strong NCI (H-bond, halogen, etc.). The interval [-0.02, $0.02]$ gives information about van der Waals interactions. The interval $[0.02,1.00]$ gives information about steric clashes.

Table S 28 Integration over the volumes of $\operatorname{sign}\left(\lambda_{2}\right) \rho(\mathbf{n})$ for $n=2.5$ according to ref. 37 for TS4a-d

\begin{tabular}{ccccc}
\hline interval & TS4a & TS4b & TS4c & TS4d \\
\hline all & -3.48364508 & -3.48392618 & -3.51629465 & -3.48151987 \\
$\mathbf{- 1 . 0}$ to $\mathbf{- 0 . 0 2}$ & -0.23673273 & -0.23556756 & -0.23558497 & -0.23850609 \\
$\mathbf{- 0 . 0 2}$ to $\mathbf{0 . 0 2}$ & 0.00424149 & 0.00417054 & 0.00443372 & 0.00443898 \\
$\mathbf{0 . 0 2}$ to $\mathbf{1 . 0}$ & 0.31364802 & 0.31505788 & 0.31459717 & 0.31451545 \\
\hline
\end{tabular}

${ }^{a}$ The interval [-0.1, -0.02] gives information about strong NCI (H-bond, halogen, etc.). The interval [-0.02, $0.02]$ gives information about van der Waals interactions. The interval $[0.02,1.00]$ gives information about steric clashes. 


\section{ELF Analysis}

The topological analysis of the gradient field of ELF provides basins which are the domains in which the probability of finding an electron pair is maximal. The basins are classified as core and valence basins. The latter are characterised by the synaptic order, i.e. the number of atomic valence shells in which they participate. ${ }^{20}$ Thus, there are monosynaptic, disynaptic, basins and so on. Monosynaptic basins, labelled V(A), correspond to lone pairs or non-bonding regions, while disynaptic basins, labelled $\mathrm{V}(\mathrm{A}, \mathrm{B})$, connect the core of two nuclei $\mathrm{A}$ and $\mathrm{B}$ and, thus, correspond to a bonding region between $\mathrm{A}$ and $\mathrm{B}$. This description recovers the Lewis bonding model, and the representation of the evolution of electron population of basins along the reaction coordinate provides a very intuitive graphical representation of how the molecular system evolves during the reaction. To verify the sequence of events during the course of the reaction we carried out a topological ELF analyses of the IRCs corresponding to TS2a, TS2b, TS2c and TS2d. For the purpose of comparison, we also carried out the analyses of TS3b and TS4b. Monosynaptic basins correspond to lone pairs of individual atoms. Disynaptic basins correspond to bonds. For the exact value of the basins (indicating the electron population) in each point of the IRC see the corresponding table. ${ }^{21}$ Figures illustrate the evolution of the electronic population for the corresponding basins. Graphical representation of the attractors (maxima of electron density) allows a rapid identification of the moment in which a bond is broken or formed. Prior to a bond formation, monosynaptic basins appear in the involved atoms (or in one of them if there is a significant difference in electronegativity). When a bond is broken, monosynaptic basins are visible after breaking. The graphics illustrate how the electron density changes along the reaction resembling a classical description of the mechanism using curved arrows. The punctual changes can be immediately recognized thus identifying the events of the reaction and the order in which they take place.

\footnotetext{
${ }^{20}$ Savin, A. J. Chem. Sci. 2005, 117, 473-475

${ }^{21}$ Only the represntative basins that change during the reaction are collected
} 


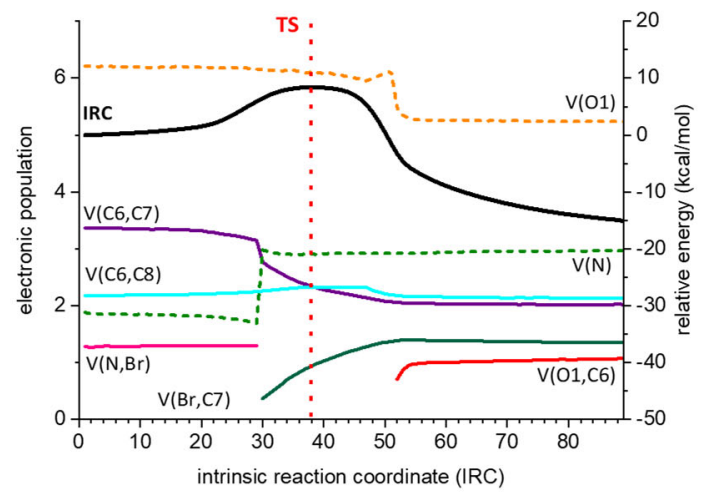

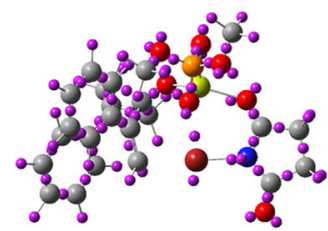

point 29

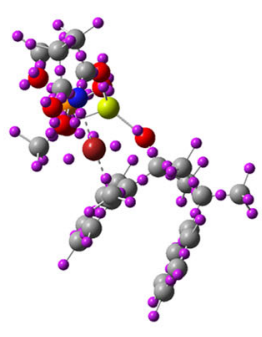

point 37 (TS2a)

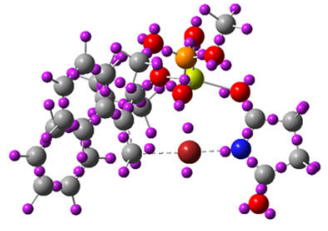

point 30

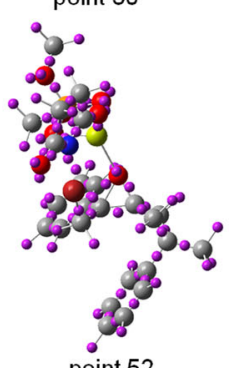

point 52

Figure S 77. Graphical representation of ELF analysis for the IRC corresponding to TS2a. Left: Evolution of the electron density along the IRC. Right. Position of attractors in critical and representative points.
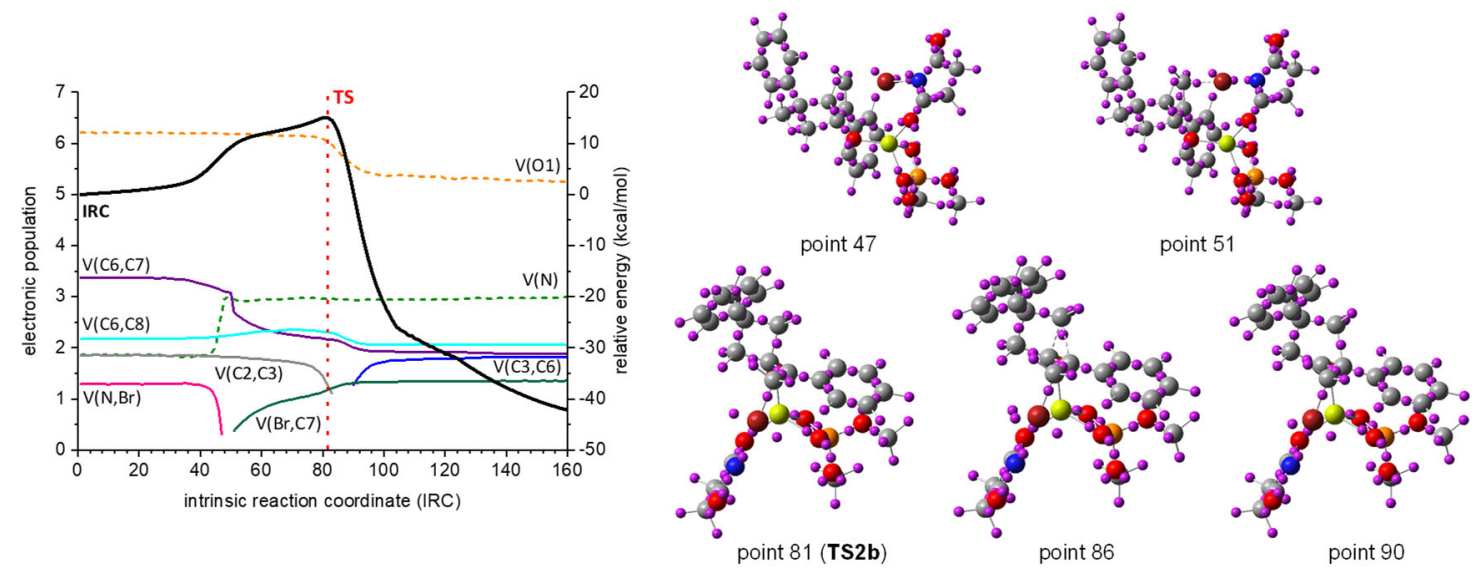

Figure S 78. Graphical representation of ELF analysis for the IRC corresponding to TS2b. Left: Evolution of the electron density along the IRC. Right. Position of attractors in critical and representative point

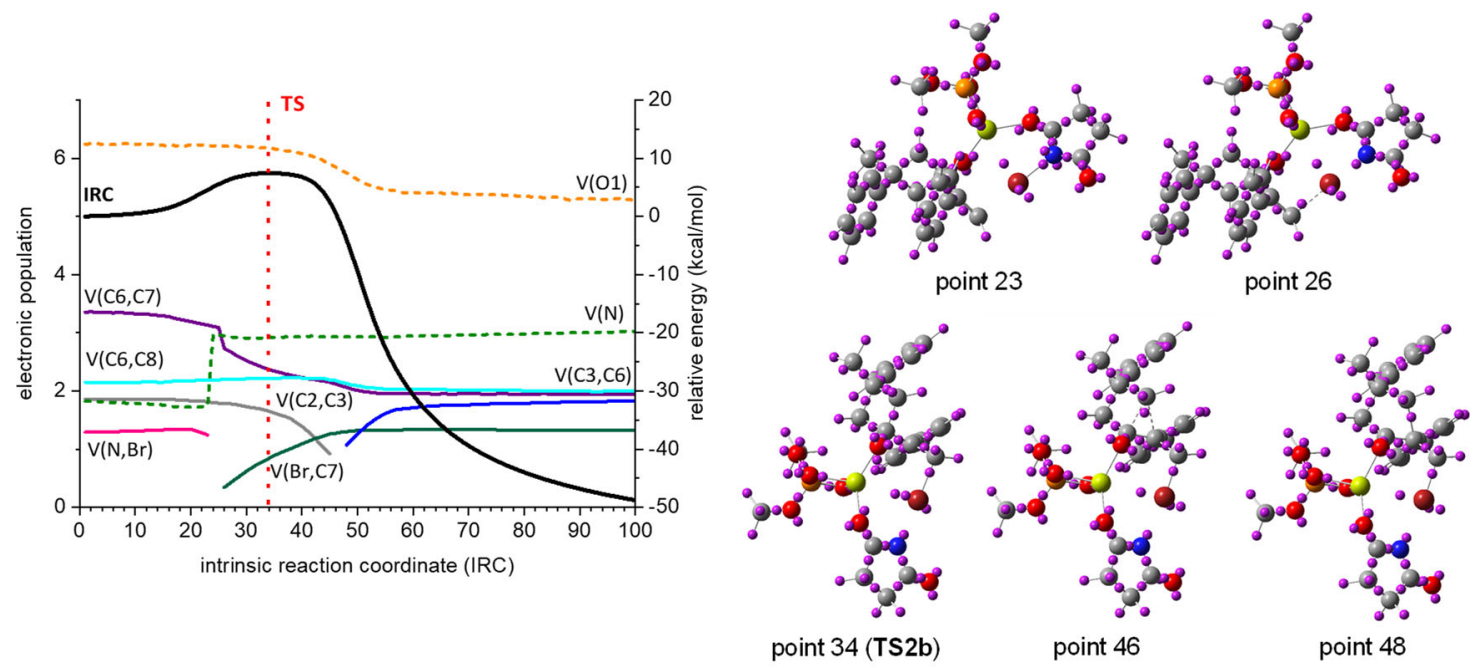

Figure S 79. Graphical representation of ELF analysis for the IRC corresponding to TS2c. Left: Evolution of the electron density along the IRC. Right. Position of attractors in critical and representative point 


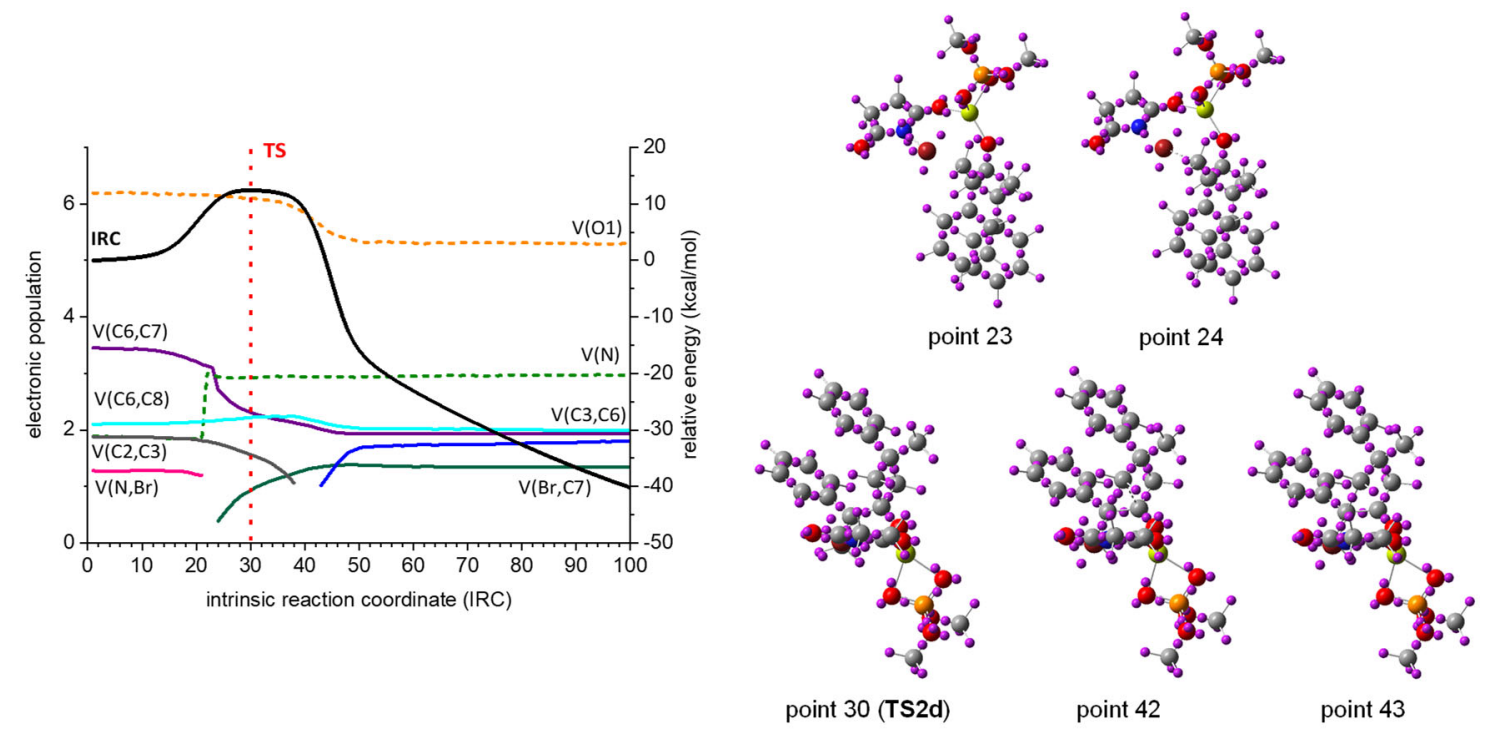

Figure S 80. Graphical representation of ELF analysis for the IRC corresponding to TS2d. Left: Evolution of the electron density along the IRC. Right. Position of attractors in critical and representative point

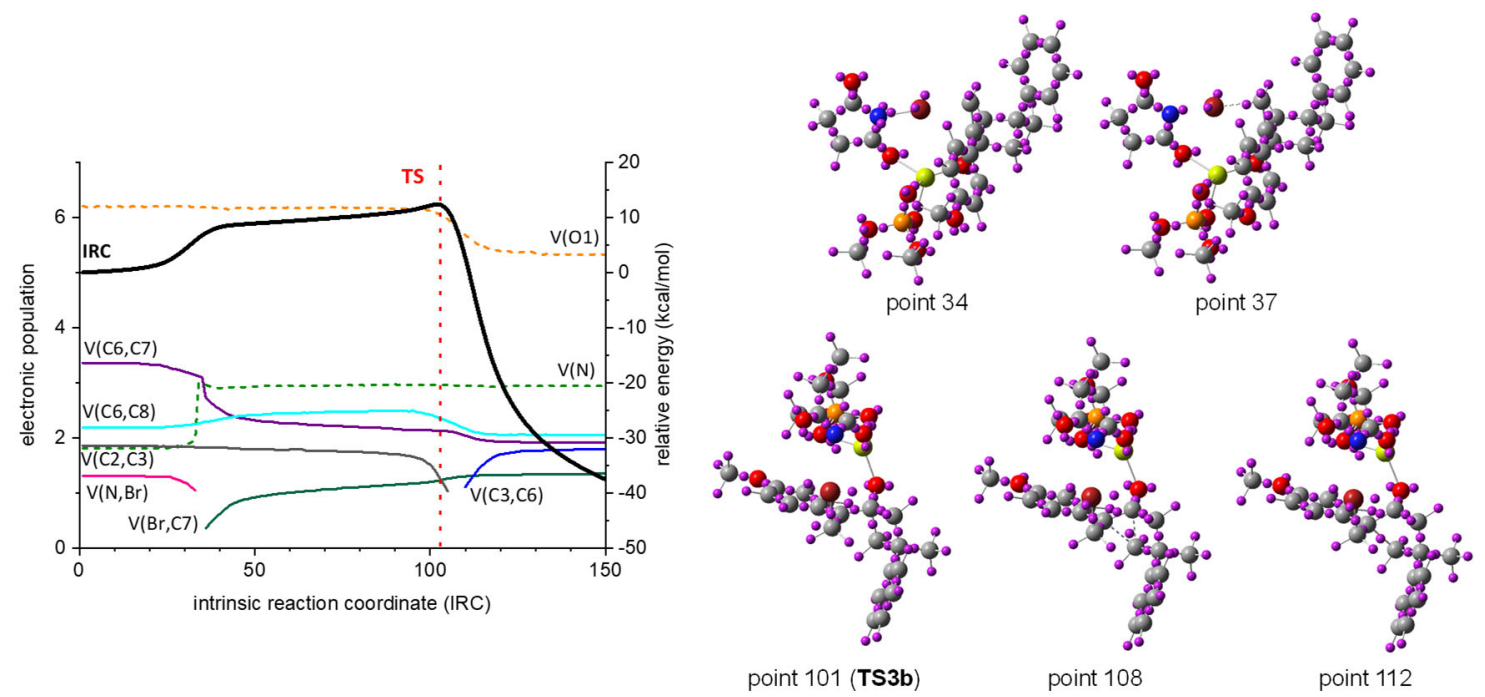

Figure S 81. Graphical representation of ELF analysis for the IRC corresponding to TS3b. Left: Evolution of the electron density along the IRC. Right. Position of attractors in critical and representative point
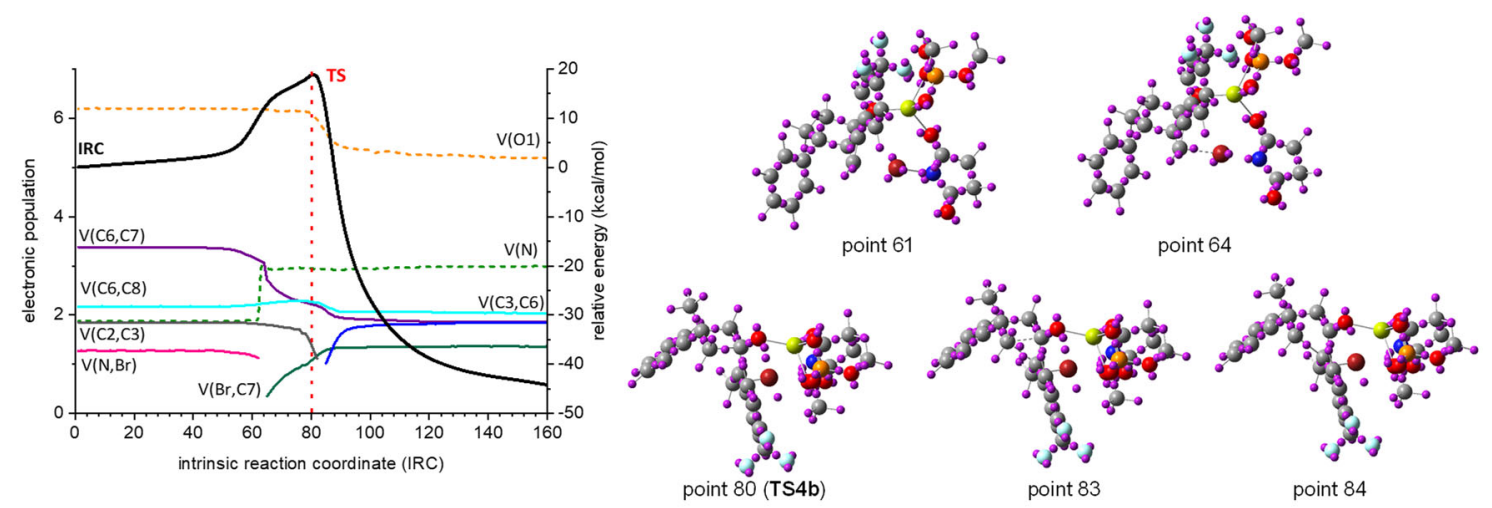

Figure S 82. Graphical representation of ELF analysis for the IRC corresponding to TS4b. Left: Evolution of the electron density along the IRC. Right. Position of attractors in critical and representative point 
Table S 29. ELF basin populations for the IRC corresponding to TS2a.

\begin{tabular}{|c|c|c|c|c|c|c|c|}
\hline & $\frac{0}{0}$ & $\begin{array}{l}\infty \\
\bigcup_{0}^{\infty} \\
\underbrace{0}\end{array}$ & $\stackrel{\hat{0}}{\stackrel{0}{0}}$ & $\stackrel{\widehat{0}}{\overrightarrow{0}}$ & $\begin{array}{l}\hat{U} \\
\text { U } \\
0\end{array}$ & 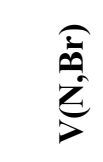 & $\underset{>}{Z}$ \\
\hline 1 & - & 2.18 & - & 6.21 & 3.37 & 1.28 & 1.88 \\
\hline 2 & - & 2.18 & - & 6.21 & 3.37 & 1.27 & 1.86 \\
\hline 3 & - & 2.18 & - & 6.20 & 3.37 & 1.28 & 1.86 \\
\hline 4 & - & 2.18 & - & 6.21 & 3.36 & 1.29 & 1.85 \\
\hline 5 & - & 2.18 & - & 6.21 & 3.36 & 1.29 & 1.86 \\
\hline 6 & - & 2.18 & - & 6.20 & 3.36 & 1.28 & 1.85 \\
\hline 7 & - & 2.18 & - & 6.20 & 3.36 & 1.28 & 1.85 \\
\hline 8 & - & 2.19 & - & 6.20 & 3.35 & 1.28 & 1.85 \\
\hline 9 & - & 2.19 & - & 6.20 & 3.35 & 1.28 & 1.84 \\
\hline 10 & - & 2.19 & - & 6.20 & 3.35 & 1.29 & 1.85 \\
\hline 11 & - & 2.19 & - & 6.19 & 3.35 & 1.29 & 1.85 \\
\hline 12 & - & 2.19 & - & 6.19 & 3.34 & 1.29 & 1.84 \\
\hline 13 & - & 2.19 & - & 6.19 & 3.35 & 1.29 & 1.84 \\
\hline 14 & - & 2.19 & - & 6.20 & 3.35 & 1.29 & 1.84 \\
\hline 15 & - & 2.19 & - & 6.19 & 3.34 & 1.29 & 1.84 \\
\hline 16 & - & 2.19 & - & 6.21 & 3.34 & 1.29 & 1.83 \\
\hline 17 & - & 2.19 & - & 6.20 & 3.34 & 1.29 & 1.82 \\
\hline 18 & - & 2.20 & - & 6.19 & 3.33 & 1.30 & 1.82 \\
\hline 19 & - & 2.20 & - & 6.20 & 3.32 & 1.30 & 1.82 \\
\hline 20 & - & 2.20 & - & 6.19 & 3.32 & 1.30 & 1.82 \\
\hline 21 & - & 2.21 & - & 6.19 & 3.30 & 1.30 & 1.81 \\
\hline 22 & - & 2.21 & - & 6.18 & 3.29 & 1.30 & 1.80 \\
\hline 23 & - & 2.22 & - & 6.18 & 3.27 & 1.30 & 1.80 \\
\hline 24 & - & 2.22 & - & 6.18 & 3.25 & 1.30 & 1.78 \\
\hline 25 & - & 2.22 & - & 6.19 & 3.24 & 1.30 & $\begin{array}{l}1.77 \\
\end{array}$ \\
\hline 26 & - & 2.23 & - & 6.18 & 3.22 & 1.30 & 1.76 \\
\hline 27 & - & 2.24 & - & 6.18 & 3.2 & 1.30 & 1.74 \\
\hline 28 & - & 2.24 & - & 6.17 & 3.17 & 1.30 & 1.72 \\
\hline 29 & - & 2.25 & - & 6.16 & 3.15 & 1.30 & 1.69 \\
\hline 30 & - & 2.26 & 0.37 & 6.15 & 2.77 & - & 2.98 \\
\hline 31 & - & 2.27 & 0.45 & 6.14 & 2.69 & - & 2.95 \\
\hline 32 & - & 2.28 & 0.53 & 6.14 & 2.63 & - & 2.93 \\
\hline 33 & - & 2.29 & 0.61 & 6.13 & 2.57 & - & 2.91 \\
\hline 34 & - & 2.30 & 0.70 & 6.14 & 2.51 & - & 2.91 \\
\hline 35 & - & 2.31 & 0.77 & 6.14 & 2.46 & - & 2.90 \\
\hline 36 & - & 2.32 & 0.83 & 6.12 & 2.41 & - & 2.90 \\
\hline 37 & - & 2.32 & 0.89 & 6.09 & 2.38 & - & 2.91 \\
\hline 38 & - & 2.33 & 0.94 & 6.11 & 2.34 & - & 2.91 \\
\hline 39 & - & 2.33 & 0.99 & 6.10 & 2.31 & - & 2.90 \\
\hline 40 & - & 2.33 & 1.03 & 6.08 & 2.28 & - & 2.92 \\
\hline 41 & - & 2.33 & 1.07 & 6.09 & 2.26 & - & 2.92 \\
\hline 42 & - & 2.33 & 1.11 & 6.06 & 2.24 & - & 2.92 \\
\hline 43 & - & 2.33 & 1.15 & 6.05 & 2.22 & - & 2.92 \\
\hline 44 & - & 2.32 & 1.19 & 6.02 & 2.20 & - & 2.92 \\
\hline 45 & - & 2.32 & 1.22 & 6.00 & 2.18 & - & 2.92 \\
\hline 46 & - & 2.32 & 1.25 & 5.99 & 2.16 & - & 2.93 \\
\hline 47 & - & 2.32 & 1.28 & 5.95 & 2.14 & - & 2.92 \\
\hline 48 & - & 2.27 & 1.31 & 6.00 & 2.12 & - & 2.92 \\
\hline 49 & - & 2.24 & 1.34 & 6.04 & 2.10 & - & 2.92 \\
\hline 50 & - & 2.21 & 1.36 & 6.09 & 2.08 & - & 2.92 \\
\hline 51 & - & 2.19 & 1.37 & 6.12 & 2.07 & - & 2.92 \\
\hline
\end{tabular}




\begin{tabular}{|c|c|c|c|c|c|c|c|}
\hline 52 & 0.71 & 2.18 & 1.39 & 5.47 & 2.06 & - & 2.92 \\
\hline 53 & 0.88 & 2.17 & 1.40 & 5.34 & 2.05 & - & 2.93 \\
\hline 54 & 0.96 & 2.16 & 1.40 & 5.29 & 2.05 & - & 2.92 \\
\hline 55 & 0.98 & 2.16 & 1.40 & 5.27 & 2.04 & - & 2.92 \\
\hline 56 & 0.99 & 2.16 & 1.40 & 5.27 & 2.04 & - & 2.93 \\
\hline 57 & 1.00 & 2.16 & 1.39 & 5.26 & 2.04 & - & 2.93 \\
\hline 58 & 1.00 & 2.16 & 1.39 & 5.26 & 2.04 & - & 2.93 \\
\hline 59 & 1.01 & 2.16 & 1.39 & 5.25 & 2.03 & - & 2.94 \\
\hline 60 & 1.01 & 2.15 & 1.39 & 5.26 & 2.03 & - & 2.94 \\
\hline 61 & 1.01 & 2.15 & 1.39 & 5.26 & 2.03 & - & 2.94 \\
\hline 62 & 1.01 & 2.15 & 1.38 & 5.27 & 2.03 & - & 2.94 \\
\hline 63 & 1.02 & 2.15 & 1.38 & 5.26 & 2.03 & - & 2.95 \\
\hline 64 & 1.02 & 2.15 & 1.38 & 5.26 & 2.03 & - & 2.95 \\
\hline 65 & 1.02 & 2.15 & 1.38 & 5.26 & 2.03 & - & 2.95 \\
\hline 66 & 1.02 & 2.15 & 1.38 & 5.25 & 2.03 & - & 2.95 \\
\hline 67 & 1.02 & 2.15 & 1.37 & 5.25 & 2.03 & - & 2.96 \\
\hline 68 & 1.03 & 2.15 & 1.37 & 5.26 & 2.03 & - & 2.96 \\
\hline 69 & 1.03 & 2.14 & 1.37 & 5.25 & 2.03 & - & 2.96 \\
\hline 70 & 1.03 & 2.14 & 1.37 & 5.25 & 2.03 & - & 2.96 \\
\hline 71 & 1.03 & 2.14 & 1.37 & 5.26 & 2.03 & - & 2.96 \\
\hline 72 & 1.04 & 2.15 & 1.37 & 5.25 & 2.02 & - & 2.96 \\
\hline 73 & 1.04 & 2.14 & 1.37 & 5.24 & 2.03 & - & 2.96 \\
\hline 74 & 1.04 & 2.14 & 1.37 & 5.24 & 2.03 & - & 2.96 \\
\hline 75 & 1.05 & 2.15 & 1.37 & 5.24 & 2.02 & - & 2.96 \\
\hline 76 & 1.05 & 2.15 & 1.36 & 5.24 & 2.02 & - & 2.96 \\
\hline 77 & 1.05 & 2.14 & 1.36 & 5.24 & 2.02 & - & 2.96 \\
\hline 78 & 1.05 & 2.14 & 1.36 & 5.24 & 2.02 & - & 2.96 \\
\hline 79 & 1.06 & 2.14 & 1.36 & 5.24 & 2.02 & - & 2.96 \\
\hline 80 & 1.05 & 2.14 & 1.36 & 5.24 & 2.02 & - & 2.97 \\
\hline 81 & 1.05 & 2.14 & 1.36 & 5.24 & 2.02 & - & 2.96 \\
\hline 82 & 1.06 & 2.14 & 1.36 & 5.24 & 2.02 & - & 2.96 \\
\hline 83 & 1.06 & 2.14 & 1.36 & 5.24 & 2.02 & - & 2.96 \\
\hline 84 & 1.06 & 2.14 & 1.35 & 5.24 & 2.02 & - & 2.97 \\
\hline 85 & 1.06 & 2.13 & 1.36 & 5.24 & 2.02 & - & 2.97 \\
\hline 86 & 1.06 & 2.13 & 1.36 & 5.24 & 2.02 & - & 2.97 \\
\hline 87 & 1.06 & 2.14 & 1.36 & 5.24 & 2.02 & - & 2.97 \\
\hline 88 & 1.07 & 2.14 & 1.35 & 5.24 & 2.03 & - & 2.96 \\
\hline 89 & 1.07 & 2.14 & 1.35 & 5.24 & 2.02 & - & 2.97 \\
\hline
\end{tabular}

Table S 30. ELF basin populations for the IRC corresponding to TS2b.

\begin{tabular}{|c|c|c|c|c|c|c|c|c|}
\hline & $\underset{i}{\tilde{U}}$ & 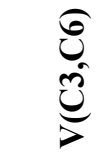 & 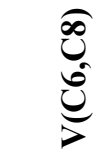 & 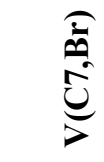 & $\widehat{\overline{0}}$ & 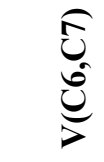 & 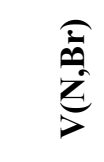 & $\underset{7}{2}$ \\
\hline 1 & 1.85 & - & 2.17 & - & 6.21 & 1.29 & 3.37 & 1.86 \\
\hline 2 & 1.85 & - & 2.17 & - & 6.21 & 1.29 & 3.37 & 1.86 \\
\hline 3 & 1.85 & - & 2.17 & - & 6.20 & 1.29 & 3.37 & 1.86 \\
\hline 4 & 1.85 & - & 2.17 & - & 6.21 & 1.29 & 3.37 & 1.86 \\
\hline 5 & 1.86 & - & 2.17 & - & 6.21 & 1.29 & 3.37 & 1.86 \\
\hline 6 & 1.86 & - & 2.17 & - & 6.2 & 1.29 & 3.37 & 1.86 \\
\hline 7 & 1.86 & - & 2.17 & - & 6.21 & 1.29 & 3.37 & 1.86 \\
\hline 8 & 1.85 & - & 2.18 & - & 6.21 & 1.29 & 3.37 & 1.86 \\
\hline 9 & 1.85 & - & 2.17 & - & 6.21 & 1.29 & 3.37 & 1.86 \\
\hline 10 & 1.85 & - & 2.17 & - & 6.20 & 1.28 & 3.37 & 1.86 \\
\hline 11 & 1.85 & - & 2.17 & - & 6.21 & 1.29 & 3.37 & 1.86 \\
\hline
\end{tabular}




\begin{tabular}{|c|c|c|c|c|c|c|c|c|}
\hline 12 & 1.86 & - & 2.17 & - & 6.21 & 1.29 & 3.37 & 1.86 \\
\hline 13 & 1.85 & - & 2.17 & - & 6.21 & 1.29 & 3.37 & 1.86 \\
\hline 14 & 1.85 & - & 2.17 & - & 6.21 & 1.29 & 3.37 & 1.86 \\
\hline 15 & 1.85 & - & 2.17 & - & 6.21 & 1.29 & 3.37 & 1.86 \\
\hline 16 & 1.85 & - & 2.17 & - & 6.20 & 1.29 & 3.37 & 1.86 \\
\hline 17 & 1.85 & - & 2.17 & - & 6.20 & 1.29 & 3.37 & 1.86 \\
\hline 18 & 1.86 & - & 2.18 & - & 6.20 & 1.29 & 3.37 & 1.86 \\
\hline 19 & 1.86 & - & 2.18 & - & 6.20 & 1.29 & 3.37 & 1.86 \\
\hline 20 & 1.86 & - & 2.17 & - & 6.20 & 1.29 & 3.37 & 1.86 \\
\hline 21 & 1.85 & - & 2.17 & - & 6.21 & 1.28 & 3.37 & 1.86 \\
\hline 22 & 1.85 & - & 2.17 & - & 6.21 & 1.29 & 3.37 & 1.86 \\
\hline 23 & 1.85 & - & 2.17 & - & 6.21 & 1.29 & 3.37 & 1.86 \\
\hline 24 & 1.85 & - & 2.17 & - & 6.20 & 1.29 & 3.37 & 1.86 \\
\hline 25 & 1.85 & - & 2.17 & - & 6.21 & 1.29 & 3.36 & 1.86 \\
\hline 26 & 1.86 & - & 2.17 & - & 6.21 & 1.29 & 3.36 & 1.86 \\
\hline 27 & 1.86 & - & 2.17 & - & 6.21 & 1.29 & 3.36 & 1.85 \\
\hline 28 & 1.85 & - & 2.17 & - & 6.21 & 1.29 & 3.37 & 1.85 \\
\hline 29 & 1.84 & - & 2.18 & - & 6.21 & 1.29 & 3.36 & 1.85 \\
\hline 30 & 1.84 & - & 2.17 & - & 6.20 & 1.29 & 3.35 & 1.83 \\
\hline 31 & 1.84 & - & 2.17 & - & 6.20 & 1.30 & 3.35 & 1.83 \\
\hline 32 & 1.85 & - & 2.17 & - & 6.20 & 1.30 & 3.34 & 1.83 \\
\hline 33 & 1.85 & - & 2.17 & - & 6.20 & 1.30 & 3.34 & 1.82 \\
\hline 34 & 1.85 & - & 2.18 & - & 6.19 & 1.30 & 3.34 & 1.83 \\
\hline 35 & 1.85 & - & 2.18 & - & 6.19 & 1.31 & 3.33 & 1.82 \\
\hline 36 & 1.85 & - & 2.19 & - & 6.20 & 1.30 & 3.32 & 1.82 \\
\hline 37 & 1.85 & - & 2.19 & - & 6.20 & 1.29 & 3.31 & 1.82 \\
\hline 38 & 1.84 & - & 2.19 & - & 6.19 & 1.28 & 3.29 & 1.83 \\
\hline 39 & 1.85 & - & 2.19 & - & 6.19 & 1.27 & 3.27 & 1.84 \\
\hline 40 & 1.84 & - & 2.19 & - & 6.19 & 1.27 & 3.26 & 1.84 \\
\hline 41 & 1.84 & - & 2.20 & - & 6.20 & 1.25 & 3.24 & 1.86 \\
\hline 42 & 1.84 & - & 2.20 & - & 6.20 & 1.23 & 3.23 & 1.86 \\
\hline 43 & 1.83 & - & 2.20 & - & 6.19 & 1.20 & 3.21 & 1.88 \\
\hline 44 & 1.83 & - & 2.21 & - & 6.19 & 1.15 & 3.19 & 1.92 \\
\hline 45 & $\begin{array}{l}1.83 \\
\end{array}$ & - & 2.21 & - & 6.19 & 1.03 & 3.17 & 2.01 \\
\hline 46 & 1.83 & - & 2.22 & - & 6.19 & 0.80 & 3.15 & 2.42 \\
\hline 47 & 1.82 & - & 2.23 & - & 6.19 & 0.31 & 3.12 & 2.68 \\
\hline 48 & 1.82 & - & 2.23 & - & 6.19 & - & 3.10 & 2.99 \\
\hline 49 & 1.82 & - & 2.24 & - & 6.18 & - & 3.09 & 2.97 \\
\hline 50 & 1.81 & - & 2.25 & - & 6.18 & - & 3.07 & 2.96 \\
\hline 51 & 1.81 & - & 2.25 & 0.37 & 6.18 & - & 2.70 & 2.95 \\
\hline 52 & 1.81 & - & 2.26 & 0.44 & 6.18 & - & 2.64 & 2.93 \\
\hline 53 & 1.81 & - & 2.27 & 0.50 & 6.18 & - & 2.60 & 2.92 \\
\hline 54 & 1.8 & - & 2.27 & 0.56 & 6.18 & - & 2.55 & 2.91 \\
\hline 55 & 1.8 & - & 2.28 & 0.61 & 6.18 & - & 2.52 & 2.92 \\
\hline 56 & 1.79 & - & 2.29 & 0.66 & 6.18 & - & 2.48 & 2.92 \\
\hline 57 & 1.79 & - & 2.30 & 0.70 & 6.17 & - & 2.45 & 2.92 \\
\hline 58 & $\begin{array}{l}1.78 \\
\end{array}$ & - & 2.31 & 0.74 & 6.17 & - & 2.43 & 2.93 \\
\hline 59 & 1.78 & - & 2.31 & 0.77 & 6.18 & - & 2.40 & 2.94 \\
\hline 60 & 1.78 & - & 2.32 & 0.80 & 6.17 & - & 2.38 & 2.94 \\
\hline 61 & 1.77 & - & 2.32 & 0.83 & 6.17 & - & 2.36 & 2.95 \\
\hline 62 & 1.77 & - & 2.32 & 0.86 & 6.16 & - & 2.34 & 2.95 \\
\hline 63 & 1.76 & - & 2.33 & 0.88 & 6.17 & - & 2.33 & 2.95 \\
\hline 64 & 1.76 & - & 2.33 & 0.9 & 6.15 & - & 2.31 & 2.95 \\
\hline 65 & 1.75 & - & 2.33 & 0.92 & 6.15 & - & 2.30 & 2.95 \\
\hline 66 & 1.75 & - & 2.34 & 0.94 & 6.14 & - & 2.29 & 2.95 \\
\hline 67 & 1.74 & - & 2.34 & 0.95 & 6.15 & - & 2.28 & 2.95 \\
\hline 68 & 1.74 & - & 2.35 & 0.97 & 6.15 & - & 2.27 & 2.96 \\
\hline 69 & 1.73 & - & 2.35 & 0.98 & 6.15 & - & 2.26 & 2.96 \\
\hline
\end{tabular}




\begin{tabular}{|c|c|c|c|c|c|c|c|c|}
\hline 70 & 1.72 & - & 2.35 & 0.99 & 6.15 & - & 2.25 & 2.96 \\
\hline 71 & 1.71 & - & 2.35 & 1.00 & 6.16 & - & 2.25 & 2.97 \\
\hline 72 & 1.70 & - & 2.35 & 1.02 & 6.15 & - & 2.24 & 2.97 \\
\hline 73 & 1.69 & - & 2.35 & 1.03 & 6.15 & - & 2.23 & 2.97 \\
\hline 74 & 1.68 & - & 2.34 & 1.04 & 6.14 & - & 2.22 & 2.97 \\
\hline 75 & 1.66 & - & 2.35 & 1.05 & 6.14 & - & 2.21 & 2.97 \\
\hline 76 & 1.64 & - & 2.34 & 1.07 & 6.14 & - & 2.20 & 2.97 \\
\hline 77 & 1.62 & - & 2.34 & 1.08 & 6.13 & - & 2.20 & 2.97 \\
\hline 78 & 1.56 & - & 2.33 & 1.10 & 6.12 & - & 2.19 & 2.97 \\
\hline 79 & 1.52 & - & 2.33 & 1.11 & 6.12 & - & 2.19 & 2.96 \\
\hline 80 & 1.44 & - & 2.31 & 1.14 & 6.09 & - & 2.18 & 2.96 \\
\hline 81 & 1.33 & - & 2.31 & 1.16 & 6.05 & - & 2.17 & 2.96 \\
\hline 82 & 1.23 & - & 2.30 & 1.19 & 6.01 & - & 2.16 & 2.95 \\
\hline 83 & 1.11 & - & 2.29 & 1.21 & 5.97 & - & 2.16 & 2.96 \\
\hline 84 & - & - & 2.27 & 1.23 & 5.91 & - & 2.15 & 2.95 \\
\hline 85 & - & - & 2.25 & 1.25 & 5.85 & - & 2.14 & 2.95 \\
\hline 86 & - & - & 2.22 & 1.27 & 5.78 & - & 2.12 & 2.95 \\
\hline 87 & - & - & 2.19 & 1.28 & 5.73 & - & 2.09 & 2.94 \\
\hline 88 & - & - & 2.17 & 1.30 & 5.68 & - & 2.06 & 2.94 \\
\hline 89 & - & - & 2.15 & 1.30 & 5.62 & - & 2.03 & 2.94 \\
\hline 90 & - & 1.27 & 2.13 & 1.31 & 5.58 & - & 2.00 & 2.94 \\
\hline 91 & - & 1.36 & 2.10 & 1.31 & 5.53 & - & 1.99 & 2.93 \\
\hline 92 & - & 1.44 & 2.09 & 1.32 & 5.49 & - & 1.97 & 2.93 \\
\hline 93 & - & 1.51 & 2.08 & 1.32 & 5.47 & - & 1.96 & 2.94 \\
\hline 94 & - & 1.57 & 2.07 & 1.32 & 5.44 & - & 1.95 & 2.94 \\
\hline 95 & - & 1.62 & 2.06 & 1.32 & 5.43 & - & 1.94 & 2.94 \\
\hline 96 & - & 1.66 & 2.06 & 1.32 & 5.41 & - & 1.94 & 2.94 \\
\hline 97 & - & 1.68 & 2.06 & 1.32 & 5.40 & - & 1.93 & 2.94 \\
\hline 98 & - & 1.70 & 2.06 & 1.32 & 5.39 & - & 1.93 & 2.94 \\
\hline 99 & - & 1.71 & 2.06 & 1.32 & 5.38 & - & 1.93 & 2.94 \\
\hline 100 & - & 1.72 & 2.06 & 1.32 & 5.37 & - & 1.93 & 2.94 \\
\hline 101 & - & 1.74 & 2.06 & 1.33 & 5.36 & - & 1.93 & 2.94 \\
\hline 102 & - & 1.75 & 2.06 & 1.33 & 5.37 & - & 1.93 & 2.95 \\
\hline 103 & - & 1.76 & 2.06 & 1.33 & 5.36 & - & 1.93 & 2.94 \\
\hline 104 & - & 1.76 & 2.06 & 1.33 & 5.38 & - & 1.92 & 2.95 \\
\hline 105 & - & 1.76 & 2.06 & 1.34 & 5.37 & - & 1.92 & 2.94 \\
\hline 106 & - & 1.76 & 2.06 & 1.34 & 5.38 & - & 1.92 & 2.94 \\
\hline 107 & - & 1.76 & 2.06 & 1.34 & 5.36 & - & 1.92 & 2.95 \\
\hline 108 & - & 1.77 & 2.06 & 1.34 & 5.37 & - & 1.92 & 2.94 \\
\hline 109 & - & \begin{tabular}{|l|}
1.77 \\
\end{tabular} & 2.06 & 1.34 & 5.37 & - & 1.92 & 2.94 \\
\hline 110 & - & 1.77 & 2.07 & 1.34 & 5.37 & - & 1.92 & 2.94 \\
\hline 111 & - & 1.77 & 2.06 & 1.34 & 5.37 & - & 1.92 & 2.94 \\
\hline 112 & - & 1.77 & 2.07 & 1.34 & 5.35 & - & 1.92 & 2.94 \\
\hline 113 & - & 1.78 & 2.07 & 1.34 & 5.35 & - & 1.92 & 2.94 \\
\hline 114 & - & 1.78 & 2.07 & 1.34 & 5.35 & - & 1.92 & 2.94 \\
\hline 115 & - & 1.78 & 2.06 & 1.34 & 5.34 & - & 1.92 & 2.94 \\
\hline 116 & - & 1.78 & 2.07 & 1.34 & 5.35 & - & 1.92 & 2.94 \\
\hline 117 & - & 1.78 & 2.07 & 1.34 & 5.35 & - & 1.92 & 2.95 \\
\hline 118 & - & 1.78 & 2.07 & 1.34 & 5.35 & - & 1.92 & 2.95 \\
\hline 119 & - & 1.78 & 2.06 & 1.34 & 5.35 & - & 1.92 & 2.95 \\
\hline 120 & - & 1.79 & 2.06 & 1.34 & 5.31 & - & 1.91 & 2.95 \\
\hline 121 & - & 1.79 & 2.06 & 1.34 & 5.32 & - & 1.91 & 2.95 \\
\hline 122 & - & 1.79 & 2.06 & 1.35 & 5.31 & - & 1.91 & 2.96 \\
\hline 123 & - & 1.79 & 2.06 & 1.35 & 5.31 & - & 1.91 & 2.95 \\
\hline 124 & - & 1.79 & 2.06 & 1.35 & 5.3 & - & 1.91 & 2.95 \\
\hline 125 & - & 1.79 & 2.06 & 1.35 & 5.29 & - & 1.91 & 2.95 \\
\hline 126 & - & 1.79 & 2.06 & 1.35 & 5.33 & - & 1.91 & 2.96 \\
\hline 127 & - & 1.80 & 2.06 & 1.35 & 5.33 & - & 1.91 & 2.96 \\
\hline
\end{tabular}




\begin{tabular}{|l|l|l|l|l|l|l|l|l|}
\hline 128 & - & 1.80 & 2.06 & 1.35 & 5.32 & - & 1.90 & 2.96 \\
\hline 129 & - & 1.80 & 2.06 & 1.35 & 5.32 & - & 1.90 & 2.96 \\
\hline 130 & - & 1.80 & 2.06 & 1.35 & 5.31 & - & 1.90 & 2.97 \\
\hline 131 & - & 1.80 & 2.06 & 1.35 & 5.31 & - & 1.90 & 2.97 \\
\hline 132 & - & 1.81 & 2.06 & 1.35 & 5.32 & - & 1.90 & 2.97 \\
\hline 133 & - & 1.81 & 2.06 & 1.35 & 5.31 & - & 1.90 & 2.97 \\
\hline 134 & - & 1.81 & 2.06 & 1.35 & 5.31 & - & 1.90 & 2.96 \\
\hline 135 & - & 1.81 & 2.06 & 1.35 & 5.30 & - & 1.90 & 2.96 \\
\hline 136 & - & 1.81 & 2.06 & 1.35 & 5.30 & - & 1.89 & 2.96 \\
\hline 137 & - & 1.81 & 2.06 & 1.35 & 5.29 & - & 1.89 & 2.97 \\
\hline 138 & - & 1.81 & 2.06 & 1.35 & 5.30 & - & 1.89 & 2.97 \\
\hline 139 & - & 1.81 & 2.06 & 1.36 & 5.30 & - & 1.89 & 2.97 \\
\hline 140 & - & 1.81 & 2.06 & 1.35 & 5.28 & - & 1.89 & 2.97 \\
\hline 141 & - & 1.81 & 2.06 & 1.35 & 5.29 & - & 1.89 & 2.97 \\
\hline 142 & - & 1.81 & 2.06 & 1.35 & 5.28 & - & 1.89 & 2.97 \\
\hline 143 & - & 1.81 & 2.06 & 1.35 & 5.27 & - & 1.89 & 2.97 \\
\hline 144 & - & 1.81 & 2.06 & 1.35 & 5.27 & - & 1.89 & 2.97 \\
\hline 145 & - & 1.81 & 2.06 & 1.35 & 5.27 & - & 1.89 & 2.97 \\
\hline 146 & - & 1.81 & 2.06 & 1.36 & 5.27 & - & 1.88 & 2.97 \\
\hline 147 & - & 1.82 & 2.06 & 1.35 & 5.27 & - & 1.88 & 2.96 \\
\hline 148 & - & 1.82 & 2.06 & 1.35 & 5.27 & - & 1.88 & 2.97 \\
\hline 149 & - & 1.82 & 2.06 & 1.35 & 5.28 & - & 1.88 & 2.97 \\
\hline 150 & - & 1.82 & 2.06 & 1.35 & 5.27 & - & 1.88 & 2.97 \\
\hline 151 & - & 1.82 & 2.06 & 1.35 & 5.28 & - & 1.88 & 2.98 \\
\hline 152 & - & 1.82 & 2.06 & 1.36 & 5.27 & - & 1.88 & 2.98 \\
\hline 153 & - & 1.82 & 2.06 & 1.35 & 5.27 & - & 1.88 & 2.98 \\
\hline 154 & - & 1.82 & 2.06 & 1.35 & 5.26 & - & 1.88 & 2.98 \\
\hline 155 & - & 1.82 & 2.06 & 1.35 & 5.26 & - & 1.88 & 2.98 \\
\hline 156 & - & 1.82 & 2.06 & 1.36 & 5.25 & - & 1.88 & 2.98 \\
\hline 157 & - & 1.82 & 2.06 & 1.35 & 5.25 & - & 1.88 & 2.98 \\
\hline 158 & - & 1.82 & 2.06 & 1.35 & 5.25 & - & 1.88 & 2.98 \\
\hline 159 & - & 1.82 & 2.06 & 1.35 & 5.25 & - & 1.88 & 2.99 \\
\hline 160 & - & 1.82 & 2.05 & 1.36 & 5.24 & - & 1.88 & 2.99 \\
\hline & & & & & & & & \\
\hline
\end{tabular}

Table S 31. ELF basin populations for the IRC corresponding to TS2c.

\begin{tabular}{|c|c|c|c|c|c|c|c|c|}
\hline & 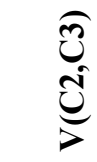 & 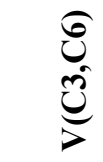 & $\begin{array}{l}\underbrace{\infty}_{0} \\
\stackrel{0}{0}\end{array}$ & 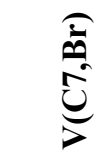 & $\underset{\overline{0}}{\widehat{a}}$ & $\begin{array}{l}\hat{U} \\
0 \\
0 \\
0\end{array}$ & 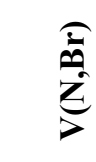 & $Z_{>}^{Z}$ \\
\hline 1 & 1.86 & - & 2.15 & - & 6.24 & 3.35 & 1.29 & 1.82 \\
\hline 2 & 1.86 & - & 2.15 & - & 6.25 & 3.36 & 1.30 & 1.82 \\
\hline 3 & 1.86 & - & 2.15 & - & 6.25 & 3.35 & 1.30 & 1.82 \\
\hline 4 & 1.86 & - & 2.15 & - & 6.24 & 3.35 & 1.30 & 1.81 \\
\hline 5 & 1.86 & - & 2.15 & - & 6.24 & 3.35 & 1.30 & 1.80 \\
\hline 6 & 1.86 & - & 2.15 & - & 6.23 & 3.34 & 1.30 & 1.80 \\
\hline 7 & 1.86 & - & 2.15 & - & 6.24 & 3.35 & 1.30 & 1.80 \\
\hline 8 & 1.86 & - & 2.15 & - & 6.25 & 3.34 & 1.31 & 1.79 \\
\hline 9 & 1.86 & - & 2.15 & - & 6.25 & 3.34 & 1.31 & 1.79 \\
\hline 10 & 1.85 & - & 2.15 & - & 6.24 & 3.32 & 1.31 & $\begin{array}{l}1.79 \\
\end{array}$ \\
\hline 11 & 1.86 & - & 2.15 & - & 6.24 & 3.32 & 1.31 & 1.78 \\
\hline 12 & 1.85 & - & 2.16 & - & 6.24 & 3.32 & 1.32 & 1.78 \\
\hline 13 & 1.85 & - & 2.16 & - & 6.24 & 3.31 & 1.32 & $\begin{array}{l}1.77 \\
\end{array}$ \\
\hline 14 & 1.85 & - & 2.15 & - & 6.23 & 3.3 & 1.33 & 1.77 \\
\hline
\end{tabular}




\begin{tabular}{|c|c|c|c|c|c|c|c|c|}
\hline 15 & 1.85 & - & 2.15 & - & 6.22 & 3.29 & 1.33 & 1.76 \\
\hline 16 & 1.85 & - & 2.16 & - & 6.21 & 3.28 & 1.34 & 1.74 \\
\hline 17 & 1.85 & - & 2.17 & - & 6.22 & 3.25 & 1.34 & 1.73 \\
\hline 18 & 1.84 & - & 2.17 & - & 6.21 & 3.23 & 1.34 & 1.73 \\
\hline 19 & 1.84 & - & 2.17 & - & 6.21 & 3.21 & 1.34 & 1.72 \\
\hline 20 & 1.83 & - & 2.17 & - & 6.23 & 3.19 & 1.34 & 1.72 \\
\hline 21 & 1.83 & - & 2.18 & - & 6.23 & 3.17 & 1.31 & 1.72 \\
\hline 22 & 1.83 & - & 2.18 & - & 6.23 & 3.15 & 1.28 & 1.73 \\
\hline 23 & 1.81 & - & 2.18 & - & 6.22 & 3.13 & 1.24 & 1.75 \\
\hline 24 & 1.80 & - & 2.19 & - & 6.21 & 3.11 & - & 2.98 \\
\hline 25 & 1.79 & - & 2.19 & - & 6.21 & 3.09 & - & 2.96 \\
\hline 26 & 1.79 & - & 2.20 & 0.34 & 6.21 & 2.73 & - & 2.95 \\
\hline 27 & 1.78 & - & 2.20 & 0.41 & 6.20 & 2.68 & - & 2.93 \\
\hline 28 & 1.77 & - & 2.20 & 0.48 & 6.21 & 2.62 & - & 2.92 \\
\hline 29 & 1.75 & - & 2.20 & 0.55 & 6.19 & 2.57 & - & 2.91 \\
\hline 30 & $\begin{array}{l}1.73 \\
\end{array}$ & - & 2.21 & 0.62 & 6.2 & 2.52 & - & 2.91 \\
\hline 31 & 1.71 & - & 2.21 & 0.68 & 6.19 & 2.48 & - & 2.91 \\
\hline 32 & 1.70 & - & 2.22 & 0.74 & 6.17 & 2.44 & - & 2.91 \\
\hline 33 & 1.68 & - & 2.22 & 0.79 & 6.18 & 2.4 & - & 2.91 \\
\hline 34 & 1.65 & - & 2.22 & 0.84 & 6.17 & 2.37 & - & 2.93 \\
\hline 35 & 1.62 & - & 2.22 & 0.89 & 6.16 & 2.34 & - & 2.92 \\
\hline 36 & 1.59 & - & 2.22 & 0.93 & 6.15 & 2.32 & - & 2.92 \\
\hline 37 & 1.56 & - & 2.23 & 0.97 & 6.13 & 2.29 & - & 2.93 \\
\hline 38 & 1.52 & - & 2.23 & 1.01 & 6.12 & 2.27 & - & 2.93 \\
\hline 39 & 1.45 & - & 2.23 & 1.05 & 6.10 & 2.25 & - & 2.93 \\
\hline 40 & 1.39 & - & 2.22 & 1.09 & 6.08 & 2.23 & - & 2.93 \\
\hline 41 & 1.31 & - & 2.22 & 1.13 & 6.06 & 2.21 & - & 2.94 \\
\hline 42 & 1.22 & - & 2.22 & 1.17 & 6.03 & 2.2 & - & 2.94 \\
\hline 43 & 1.11 & - & 2.21 & 1.20 & 6.00 & 2.18 & - & 2.93 \\
\hline 44 & 1.01 & - & 2.21 & 1.23 & 5.95 & 2.17 & - & 2.93 \\
\hline 45 & 0.92 & - & 2.19 & 1.26 & 5.90 & 2.15 & - & 2.93 \\
\hline 46 & - & - & 2.18 & 1.27 & 5.85 & 2.12 & - & 2.93 \\
\hline 47 & - & - & 2.15 & 1.29 & 5.79 & 2.09 & - & 2.93 \\
\hline 48 & - & $\begin{array}{ll}1.07 \\
\end{array}$ & 2.13 & 1.3 & 5.75 & 2.06 & - & 2.93 \\
\hline 49 & - & 1.16 & 2.10 & 1.31 & 5.69 & 2.03 & - & 2.93 \\
\hline 50 & - & 1.26 & 2.09 & 1.32 & 5.63 & 2.01 & - & 2.93 \\
\hline 51 & - & 1.35 & 2.07 & 1.33 & 5.58 & 1.99 & - & 2.93 \\
\hline 52 & - & 1.43 & 2.05 & 1.33 & 5.54 & 1.98 & - & 2.93 \\
\hline 53 & - & 1.50 & 2.05 & 1.33 & 5.51 & 1.97 & - & 2.93 \\
\hline 54 & - & 1.56 & 2.04 & 1.33 & 5.48 & 1.95 & - & 2.92 \\
\hline 55 & - & 1.61 & 2.03 & 1.33 & 5.46 & 1.95 & - & 2.92 \\
\hline 56 & - & 1.65 & 2.03 & 1.33 & 5.45 & 1.95 & - & 2.92 \\
\hline 57 & - & 1.68 & 2.03 & 1.33 & 5.44 & 1.95 & - & 2.93 \\
\hline 58 & - & 1.69 & 2.02 & 1.33 & 5.41 & 1.95 & - & 2.93 \\
\hline 59 & - & 1.70 & 2.02 & 1.34 & 5.40 & 1.95 & - & 2.94 \\
\hline 60 & - & 1.71 & 2.02 & 1.34 & 5.41 & 1.95 & - & 2.94 \\
\hline 61 & - & 1.72 & 2.02 & 1.34 & 5.40 & 1.95 & - & 2.94 \\
\hline 62 & - & 1.73 & 2.02 & 1.34 & 5.40 & 1.95 & - & 2.95 \\
\hline 63 & - & 1.74 & 2.02 & 1.34 & 5.40 & 1.95 & - & 2.95 \\
\hline 64 & - & 1.74 & 2.02 & 1.34 & 5.40 & 1.94 & - & 2.95 \\
\hline 65 & - & 1.75 & 2.02 & 1.34 & 5.40 & 1.95 & - & 2.95 \\
\hline 66 & - & 1.75 & 2.02 & 1.34 & 5.39 & 1.95 & - & 2.95 \\
\hline 67 & - & 1.76 & 2.01 & 1.34 & 5.40 & 1.94 & - & 2.95 \\
\hline 68 & - & 1.76 & 2.01 & 1.34 & 5.38 & 1.95 & - & 2.96 \\
\hline 69 & - & 1.76 & 2.01 & 1.34 & 5.38 & 1.95 & - & 2.96 \\
\hline 70 & - & 1.76 & 2.01 & 1.34 & 5.37 & 1.95 & - & 2.96 \\
\hline 71 & - & 1.77 & 2.01 & 1.34 & 5.38 & 1.95 & - & 2.96 \\
\hline 72 & - & 1.77 & 2.01 & 1.34 & 5.38 & 1.95 & - & 2.96 \\
\hline
\end{tabular}




\begin{tabular}{|l|l|l|l|l|l|l|l|l|}
\hline 73 & - & 1.77 & 2.01 & 1.34 & 5.38 & 1.94 & - & 2.97 \\
\hline 74 & - & 1.78 & 2.01 & 1.34 & 5.37 & 1.94 & - & 2.97 \\
\hline 75 & - & 1.78 & 2.01 & 1.33 & 5.37 & 1.95 & - & 2.97 \\
\hline 76 & - & 1.78 & 2.01 & 1.33 & 5.37 & 1.95 & - & 2.97 \\
\hline 77 & - & 1.79 & 2.01 & 1.33 & 5.36 & 1.95 & - & 2.97 \\
\hline 78 & - & 1.79 & 2.01 & 1.33 & 5.36 & 1.94 & - & 2.97 \\
\hline 79 & - & 1.79 & 2.01 & 1.33 & 5.35 & 1.94 & - & 2.97 \\
\hline 80 & - & 1.79 & 2.00 & 1.33 & 5.35 & 1.94 & - & 2.98 \\
\hline 81 & - & 1.79 & 2.00 & 1.33 & 5.34 & 1.94 & - & 2.98 \\
\hline 82 & - & 1.80 & 2.00 & 1.33 & 5.33 & 1.94 & - & 2.99 \\
\hline 83 & - & 1.80 & 2.00 & 1.33 & 5.33 & 1.95 & - & 2.99 \\
\hline 84 & - & 1.80 & 2.00 & 1.33 & 5.31 & 1.95 & - & 2.99 \\
\hline 85 & - & 1.80 & 2.00 & 1.33 & 5.33 & 1.94 & - & 3.00 \\
\hline 86 & - & 1.80 & 2.00 & 1.33 & 5.33 & 1.94 & - & 2.99 \\
\hline 87 & - & 1.81 & 2.00 & 1.33 & 5.31 & 1.94 & - & 2.99 \\
\hline 88 & - & 1.81 & 2.00 & 1.33 & 5.28 & 1.94 & - & 3.00 \\
\hline 89 & - & 1.81 & 2.00 & 1.33 & 5.29 & 1.94 & - & 3.00 \\
\hline 90 & - & 1.81 & 2.00 & 1.33 & 5.31 & 1.94 & - & 3.00 \\
\hline 91 & - & 1.81 & 2.00 & 1.32 & 5.31 & 1.94 & - & 3.00 \\
\hline 92 & - & 1.81 & 1.99 & 1.32 & 5.30 & 1.94 & - & 3.00 \\
\hline 93 & - & 1.81 & 1.99 & 1.32 & 5.30 & 1.94 & - & 3.00 \\
\hline 94 & - & 1.81 & 1.99 & 1.32 & 5.29 & 1.94 & - & 3.01 \\
\hline 95 & - & 1.82 & 2.00 & 1.33 & 5.29 & 1.94 & - & 3.01 \\
\hline 96 & - & 1.82 & 2.00 & 1.32 & 5.30 & 1.94 & - & 3.01 \\
\hline 97 & - & 1.82 & 2.00 & 1.32 & 5.31 & 1.94 & - & 3.02 \\
\hline 98 & - & 1.82 & 2.00 & 1.32 & 5.30 & 1.94 & - & 3.02 \\
\hline 99 & - & 1.82 & 2.00 & 1.33 & 5.28 & 1.94 & - & 3.03 \\
\hline 100 & - & 1.82 & 2.00 & 1.32 & 5.29 & 1.94 & - & 3.02 \\
\hline & & & & & & & & \\
\hline
\end{tabular}

Table S 32. ELF basin populations for the IRC corresponding to TS2d.

\begin{tabular}{|c|c|c|c|c|c|c|c|c|}
\hline & 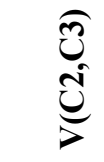 & $\underbrace{0}_{0}$ & $\underbrace{0}_{>}$ & 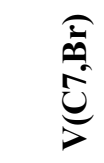 & $\stackrel{\widehat{0}}{2}$ & $\begin{array}{l}\hat{U} \\
0 \\
0 \\
D\end{array}$ & $\begin{array}{l}\widehat{\overline{0}} \\
\stackrel{2}{c}\end{array}$ & $\sum_{>}$ \\
\hline 1 & 1.88 & - & 2.10 & - & 6.19 & 3.45 & 1.28 & 1.89 \\
\hline 2 & 1.88 & - & 2.10 & - & 6.19 & 3.45 & 1.27 & 1.89 \\
\hline 3 & 1.88 & - & 2.11 & - & 6.18 & 3.44 & 1.27 & 1.89 \\
\hline 4 & 1.88 & - & 2.11 & - & 6.18 & 3.44 & 1.27 & 1.88 \\
\hline 5 & 1.87 & - & 2.11 & - & 6.19 & 3.44 & 1.27 & 1.88 \\
\hline 6 & 1.87 & - & 2.11 & - & 6.2 & 3.44 & 1.27 & 1.88 \\
\hline 7 & 1.87 & - & 2.11 & - & 6.2 & 3.43 & 1.27 & 1.89 \\
\hline 8 & 1.87 & - & 2.11 & - & 6.2 & 3.43 & 1.28 & 1.88 \\
\hline 9 & 1.87 & - & 2.11 & - & 6.18 & 3.44 & 1.28 & 1.87 \\
\hline 10 & 1.87 & - & 2.11 & - & 6.18 & 3.42 & 1.28 & 1.88 \\
\hline 11 & 1.87 & - & 2.11 & - & 6.19 & 3.42 & 1.28 & 1.87 \\
\hline 12 & 1.87 & - & 2.11 & - & 6.17 & 3.41 & 1.28 & 1.87 \\
\hline 13 & 1.86 & - & 2.11 & - & 6.16 & 3.4 & 1.28 & 1.86 \\
\hline 14 & 1.86 & - & 2.11 & - & 6.18 & 3.38 & 1.28 & 1.86 \\
\hline 15 & 1.86 & - & 2.12 & - & 6.18 & 3.36 & 1.27 & 1.85 \\
\hline 16 & 1.85 & - & 2.12 & - & 6.17 & 3.34 & 1.27 & 1.85 \\
\hline 17 & 1.85 & - & 2.13 & - & 6.16 & 3.31 & 1.27 & 1.85 \\
\hline 18 & 1.84 & - & 2.13 & - & 6.17 & 3.28 & 1.26 & 1.85 \\
\hline 19 & 1.84 & - & 2.14 & - & 6.16 & 3.24 & 1.24 & 1.85 \\
\hline 20 & 1.83 & - & 2.14 & - & 6.16 & 3.21 & 1.21 & 1.85 \\
\hline 21 & 1.81 & - & 2.15 & - & 6.16 & 3.16 & 1.20 & 1.85 \\
\hline
\end{tabular}




\begin{tabular}{|c|c|c|c|c|c|c|c|c|}
\hline 22 & 1.80 & - & 2.15 & - & 6.16 & 3.13 & - & 3.01 \\
\hline 23 & 1.78 & - & 2.16 & - & 6.15 & 3.10 & - & 2.97 \\
\hline 24 & 1.76 & - & 2.17 & 0.39 & 6.14 & 2.71 & - & 2.94 \\
\hline 25 & 1.73 & - & 2.18 & 0.51 & 6.14 & 2.61 & - & 2.92 \\
\hline 26 & 1.70 & - & 2.18 & 0.63 & 6.13 & 2.52 & - & 2.92 \\
\hline 27 & 1.67 & - & 2.19 & 0.73 & 6.12 & 2.45 & - & 2.92 \\
\hline 28 & 1.63 & - & 2.20 & 0.82 & 6.11 & 2.39 & - & 2.92 \\
\hline 29 & 1.60 & - & 2.21 & 0.88 & 6.10 & 2.35 & - & 2.93 \\
\hline 30 & 1.56 & - & 2.22 & 0.93 & 6.10 & 2.31 & - & 2.93 \\
\hline 31 & 1.53 & - & 2.23 & 0.99 & 6.09 & 2.28 & - & 2.93 \\
\hline 32 & 1.49 & - & 2.23 & 1.03 & 6.08 & 2.25 & - & 2.93 \\
\hline 33 & 1.45 & - & 2.23 & 1.07 & 6.07 & 2.23 & - & 2.94 \\
\hline 34 & 1.40 & - & 2.24 & 1.11 & 6.05 & 2.20 & - & 2.94 \\
\hline 35 & 1.33 & - & 2.24 & 1.14 & 6.04 & 2.18 & - & 2.95 \\
\hline 36 & 1.26 & - & 2.23 & 1.17 & 6.02 & 2.17 & - & 2.94 \\
\hline 37 & 1.17 & - & 2.24 & 1.20 & 5.98 & 2.15 & - & 2.95 \\
\hline 38 & 1.06 & - & 2.24 & 1.23 & 5.94 & 2.13 & - & 2.95 \\
\hline 39 & - & - & 2.23 & 1.26 & 5.89 & 2.11 & - & 2.94 \\
\hline 40 & - & - & 2.2 & 1.29 & 5.84 & 2.09 & - & 2.94 \\
\hline 41 & - & - & 2.18 & 1.32 & 5.75 & 2.07 & - & 2.94 \\
\hline 42 & - & - & 2.16 & 1.33 & 5.68 & 2.04 & - & 2.94 \\
\hline 43 & - & 1.02 & 2.14 & 1.35 & 5.62 & 2.01 & - & 2.94 \\
\hline 44 & - & 1.15 & 2.11 & 1.36 & 5.54 & 1.99 & - & 2.94 \\
\hline 45 & - & 1.28 & 2.09 & 1.37 & 5.47 & 1.97 & - & 2.94 \\
\hline 46 & - & 1.41 & 2.07 & 1.38 & 5.44 & 1.95 & - & 2.94 \\
\hline 47 & - & 1.51 & 2.06 & 1.38 & 5.40 & 1.95 & - & 2.94 \\
\hline 48 & - & 1.60 & 2.05 & 1.39 & 5.39 & 1.93 & - & 2.94 \\
\hline 49 & - & 1.64 & 2.04 & 1.39 & 5.36 & 1.94 & - & 2.93 \\
\hline 50 & - & 1.68 & 2.03 & 1.38 & 5.34 & 1.94 & - & 2.94 \\
\hline 51 & - & 1.70 & 2.03 & 1.38 & 5.32 & 1.94 & - & 2.94 \\
\hline 52 & - & 1.70 & 2.02 & 1.37 & 5.31 & 1.94 & - & 2.94 \\
\hline 53 & - & 1.71 & 2.02 & 1.37 & 5.32 & 1.94 & - & 2.94 \\
\hline 54 & - & 1.71 & 2.02 & 1.37 & 5.33 & 1.94 & - & 2.94 \\
\hline 55 & - & 1.72 & 2.02 & 1.36 & 5.33 & 1.94 & - & 2.94 \\
\hline 56 & - & 1.72 & 2.02 & 1.36 & 5.32 & 1.94 & - & 2.94 \\
\hline 57 & - & 1.73 & 2.02 & 1.36 & 5.32 & 1.94 & - & 2.95 \\
\hline 58 & - & 1.72 & 2.02 & 1.36 & 5.32 & 1.94 & - & 2.95 \\
\hline 59 & - & 1.73 & 2.02 & 1.36 & 5.31 & 1.94 & - & 2.95 \\
\hline 60 & - & 1.73 & 2.02 & 1.35 & 5.31 & 1.94 & - & 2.95 \\
\hline 61 & - & 1.74 & 2.02 & 1.35 & 5.3 & 1.94 & - & 2.95 \\
\hline 62 & - & 1.74 & 2.02 & 1.36 & 5.29 & 1.94 & - & 2.96 \\
\hline 63 & - & 1.74 & 2.02 & 1.36 & 5.31 & 1.94 & - & 2.95 \\
\hline 64 & - & 1.73 & 2.01 & 1.35 & 5.31 & 1.94 & - & 2.95 \\
\hline 65 & - & 1.74 & 2.02 & 1.35 & 5.31 & 1.94 & - & 2.96 \\
\hline 66 & - & 1.74 & 2.02 & 1.35 & 5.31 & 1.94 & - & 2.96 \\
\hline 67 & - & 1.74 & 2.02 & 1.35 & 5.30 & 1.94 & - & 2.97 \\
\hline 68 & - & 1.74 & 2.01 & 1.35 & 5.30 & 1.94 & - & 2.96 \\
\hline 69 & - & 1.75 & 2.02 & 1.35 & 5.32 & 1.94 & - & 2.96 \\
\hline 70 & - & 1.75 & 2.02 & 1.35 & 5.31 & 1.94 & - & 2.97 \\
\hline 71 & - & 1.75 & 2.02 & 1.35 & 5.31 & 1.94 & - & 2.96 \\
\hline 72 & - & 1.75 & 2.01 & 1.35 & 5.31 & 1.94 & - & 2.96 \\
\hline 73 & - & 1.75 & 2.01 & 1.35 & 5.31 & 1.94 & - & 2.95 \\
\hline 74 & - & 1.75 & 2.01 & 1.35 & 5.31 & 1.94 & - & 2.96 \\
\hline 75 & - & 1.75 & 2.01 & 1.35 & 5.31 & 1.94 & - & 2.96 \\
\hline 76 & - & 1.76 & 2.01 & 1.35 & 5.32 & 1.94 & - & 2.96 \\
\hline 77 & - & 1.76 & 2.01 & 1.35 & 5.30 & 1.94 & - & 2.97 \\
\hline 78 & - & 1.76 & 2.01 & 1.35 & 5.30 & 1.94 & - & 2.97 \\
\hline 79 & - & 1.76 & 2.01 & 1.35 & 5.31 & 1.94 & - & 2.97 \\
\hline
\end{tabular}




\begin{tabular}{|l|l|l|l|l|l|l|l|l|}
\hline 80 & - & 1.76 & 2.01 & 1.35 & 5.31 & 1.94 & - & 2.97 \\
\hline 81 & - & 1.77 & 2.01 & 1.35 & 5.31 & 1.94 & - & 2.97 \\
\hline 82 & - & 1.77 & 2.01 & 1.35 & 5.30 & 1.94 & - & 2.97 \\
\hline 83 & - & 1.77 & 2.01 & 1.35 & 5.31 & 1.94 & - & 2.97 \\
\hline 84 & - & 1.77 & 2.00 & 1.35 & 5.30 & 1.94 & - & 2.97 \\
\hline 85 & - & 1.77 & 2.00 & 1.35 & 5.30 & 1.94 & - & 2.97 \\
\hline 86 & - & 1.78 & 2.00 & 1.35 & 5.30 & 1.94 & - & 2.97 \\
\hline 87 & - & 1.78 & 2.00 & 1.35 & 5.31 & 1.94 & - & 2.97 \\
\hline 88 & - & 1.78 & 2.00 & 1.35 & 5.30 & 1.94 & - & 2.97 \\
\hline 89 & - & 1.78 & 2.00 & 1.35 & 5.30 & 1.94 & - & 2.97 \\
\hline 90 & - & 1.78 & 2.00 & 1.35 & 5.30 & 1.94 & - & 2.97 \\
\hline 91 & - & 1.79 & 2.00 & 1.34 & 5.30 & 1.94 & - & 2.97 \\
\hline 92 & - & 1.79 & 2.00 & 1.34 & 5.29 & 1.94 & - & 2.97 \\
\hline 93 & - & 1.79 & 2.00 & 1.34 & 5.30 & 1.94 & - & 2.97 \\
\hline 94 & - & 1.79 & 2.00 & 1.34 & 5.29 & 1.94 & - & 2.98 \\
\hline 95 & - & 1.80 & 2.00 & 1.34 & 5.29 & 1.94 & - & 2.97 \\
\hline 96 & - & 1.79 & 2.00 & 1.34 & 5.29 & 1.94 & - & 2.97 \\
\hline 97 & - & 1.80 & 2.00 & 1.34 & 5.29 & 1.93 & - & 2.97 \\
\hline 98 & - & 1.80 & 2.00 & 1.34 & 5.29 & 1.93 & - & 2.97 \\
\hline 99 & - & 1.80 & 2.00 & 1.34 & 5.30 & 1.93 & - & 2.97 \\
\hline 100 & - & 1.80 & 2.00 & 1.34 & 5.30 & 1.93 & - & 2.97 \\
\hline 101 & - & 1.81 & 2.00 & 1.34 & 5.30 & 1.93 & - & 2.97 \\
\hline 102 & - & 1.81 & 2.00 & 1.34 & 5.30 & 1.93 & - & 2.98 \\
\hline 103 & - & 1.8 & 2.00 & 1.35 & 5.31 & 1.94 & - & 2.98 \\
\hline 104 & - & 1.81 & 2.00 & 1.35 & 5.3 & 1.94 & - & 2.98 \\
\hline 105 & - & 1.81 & 1.99 & 1.34 & 5.29 & 1.93 & - & 2.98 \\
\hline 106 & - & 1.81 & 1.99 & 1.35 & 5.31 & 1.93 & - & 2.98 \\
\hline 107 & - & 1.81 & 1.99 & 1.34 & 5.3 & 1.94 & - & 2.98 \\
\hline 108 & - & 1.81 & 1.99 & 1.34 & 5.29 & 1.93 & - & 2.97 \\
\hline 109 & - & 1.81 & 1.99 & 1.34 & 5.29 & 1.93 & - & 2.98 \\
\hline 110 & - & 1.82 & 1.99 & 1.34 & 5.29 & 1.93 & - & 2.98 \\
\hline & & & & & & & & \\
\hline
\end{tabular}

Table S 33. ELF basin populations for the IRC corresponding to TS3b.

\begin{tabular}{|c|c|c|c|c|c|c|c|c|}
\hline & 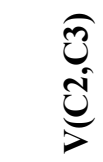 & $\underset{j}{0}$ & 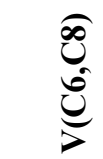 & $\underset{D}{\stackrel{0}{0}}$ & $\stackrel{\widehat{a}}{\partial}$ & $\begin{array}{l}\text { U } \\
\text { Uj } \\
\underset{D}{0}\end{array}$ & 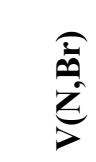 & $\sum_{2}^{2}$ \\
\hline 1 & 1.86 & -- & 2.18 & -- & 6.20 & 3.36 & 1.31 & 1.81 \\
\hline 2 & 1.86 & -- & 2.18 & -- & 6.19 & 3.36 & 1.31 & 1.81 \\
\hline 3 & 1.86 & -- & 2.18 & -- & 6.20 & 3.36 & 1.31 & 1.81 \\
\hline 4 & 1.86 & -- & 2.18 & -- & 6.19 & 3.36 & 1.31 & 1.81 \\
\hline 5 & 1.86 & -- & 2.18 & -- & 6.2 & 3.36 & 1.31 & 1.81 \\
\hline 6 & 1.86 & -- & 2.18 & -- & 6.20 & 3.36 & 1.31 & 1.81 \\
\hline 7 & 1.86 & -- & 2.18 & -- & 6.19 & 3.36 & 1.31 & 1.81 \\
\hline 8 & 1.86 & -- & 2.18 & -- & 6.20 & 3.36 & 1.31 & 1.81 \\
\hline 9 & 1.86 & -- & 2.18 & -- & 6.19 & 3.36 & 1.31 & 1.81 \\
\hline 10 & 1.86 & -- & 2.18 & -- & 6.20 & 3.36 & 1.31 & 1.81 \\
\hline 11 & 1.85 & -- & 2.19 & -- & 6.20 & 3.36 & 1.31 & 1.81 \\
\hline 12 & 1.85 & -- & 2.19 & -- & 6.19 & 3.36 & 1.31 & 1.81 \\
\hline 13 & 1.85 & -- & 2.19 & -- & 6.20 & 3.36 & 1.31 & 1.81 \\
\hline 14 & 1.85 & -- & 2.19 & -- & 6.19 & 3.35 & 1.31 & 1.81 \\
\hline 15 & 1.85 & -- & 2.19 & -- & 6.20 & 3.35 & 1.31 & 1.81 \\
\hline 16 & 1.85 & -- & 2.19 & -- & 6.20 & 3.35 & 1.31 & 1.81 \\
\hline 17 & 1.85 & -- & 2.19 & -- & 6.19 & 3.35 & 1.31 & 1.81 \\
\hline 18 & 1.85 & -- & 2.19 & -- & 6.20 & 3.34 & 1.31 & 1.81 \\
\hline
\end{tabular}




\begin{tabular}{|c|c|c|c|c|c|c|c|c|}
\hline 19 & 1.85 & -- & 2.19 & -- & 6.19 & 3.34 & 1.30 & 1.81 \\
\hline 20 & 1.85 & -- & 2.19 & -- & 6.20 & 3.33 & 1.30 & 1.81 \\
\hline 21 & 1.85 & -- & 2.19 & -- & 6.20 & 3.33 & 1.30 & 1.81 \\
\hline 22 & 1.85 & - & 2.19 & - & 6.20 & 3.33 & 1.30 & 1.81 \\
\hline 23 & 1.84 & - & 2.19 & - & 6.19 & 3.32 & 1.30 & 1.81 \\
\hline 24 & 1.85 & - & 2.19 & - & 6.20 & 3.30 & 1.30 & 1.81 \\
\hline 25 & 1.84 & - & 2.20 & - & 6.19 & 3.29 & 1.29 & 1.81 \\
\hline 26 & 1.84 & - & 2.20 & - & 6.20 & 3.26 & 1.29 & 1.82 \\
\hline 27 & 1.84 & - & 2.21 & - & 6.21 & 3.25 & 1.26 & 1.82 \\
\hline 28 & 1.85 & - & 2.21 & - & 6.20 & 3.24 & 1.24 & 1.83 \\
\hline 29 & 1.84 & - & 2.22 & - & 6.20 & 3.22 & 1.22 & 1.85 \\
\hline 30 & 1.84 & - & 2.23 & - & 6.20 & 3.20 & 1.19 & 1.87 \\
\hline 31 & 1.84 & - & 2.23 & - & 6.20 & 3.18 & 1.16 & 1.89 \\
\hline 32 & 1.84 & - & 2.24 & - & 6.19 & 3.16 & 1.10 & 1.92 \\
\hline 33 & 1.83 & - & 2.25 & - & 6.19 & 3.14 & 1.05 & 1.96 \\
\hline 34 & 1.83 & - & 2.26 & - & 6.19 & 3.12 & - & 2.99 \\
\hline 35 & 1.83 & - & 2.28 & - & 6.18 & 3.1 & - & 2.97 \\
\hline 36 & 1.83 & - & 2.30 & 0.36 & 6.18 & 2.73 & - & 2.96 \\
\hline 37 & 1.83 & - & 2.30 & 0.43 & 6.17 & 2.67 & - & 2.94 \\
\hline 38 & 1.83 & - & 2.31 & 0.5 & 6.18 & 2.61 & - & 2.92 \\
\hline 39 & 1.83 & - & 2.32 & 0.56 & 6.17 & 2.56 & - & 2.91 \\
\hline 40 & 1.82 & - & 2.34 & 0.62 & 6.17 & 2.52 & - & 2.90 \\
\hline 41 & 1.82 & - & 2.35 & 0.68 & 6.16 & 2.48 & - & 2.91 \\
\hline 42 & 1.82 & - & 2.36 & 0.73 & 6.15 & 2.44 & - & 2.91 \\
\hline 43 & 1.81 & - & 2.37 & 0.78 & 6.14 & 2.41 & - & 2.92 \\
\hline 44 & 1.81 & - & 2.38 & 0.81 & 6.16 & 2.39 & - & 2.92 \\
\hline 45 & 1.81 & - & 2.39 & 0.84 & 6.17 & 2.37 & - & 2.92 \\
\hline 46 & 1.80 & - & 2.40 & 0.86 & 6.18 & 2.36 & - & 2.92 \\
\hline 47 & 1.80 & - & 2.40 & 0.88 & 6.17 & 2.35 & - & 2.92 \\
\hline 48 & 1.80 & - & 2.41 & 0.89 & 6.17 & 2.34 & - & 2.92 \\
\hline 49 & 1.80 & - & 2.42 & 0.91 & 6.16 & 2.33 & - & 2.93 \\
\hline 50 & 1.80 & - & 2.42 & 0.92 & 6.16 & 2.32 & - & 2.93 \\
\hline 51 & 1.80 & - & 2.42 & 0.93 & 6.16 & 2.31 & - & 2.93 \\
\hline 52 & 1.80 & - & 2.42 & 0.95 & 6.17 & 2.30 & - & 2.93 \\
\hline 53 & 1.80 & - & 2.43 & 0.95 & 6.17 & 2.30 & - & 2.94 \\
\hline 54 & 1.80 & - & 2.43 & 0.96 & 6.18 & 2.29 & - & 2.94 \\
\hline 55 & 1.80 & - & 2.44 & 0.97 & 6.17 & 2.29 & - & 2.94 \\
\hline 56 & 1.78 & - & 2.44 & 0.98 & 6.17 & 2.28 & - & 2.94 \\
\hline 57 & 1.78 & - & 2.44 & 0.99 & 6.16 & 2.27 & - & 2.94 \\
\hline 58 & 1.78 & - & 2.44 & 0.99 & 6.17 & 2.27 & - & 2.94 \\
\hline 59 & $\begin{array}{l}1.78 \\
\end{array}$ & - & 2.45 & 1.00 & 6.18 & 2.27 & - & 2.95 \\
\hline 60 & 1.79 & - & 2.45 & 1.00 & 6.18 & 2.26 & - & 2.95 \\
\hline 61 & 1.79 & - & 2.45 & 1.01 & 6.17 & 2.26 & - & 2.94 \\
\hline 62 & 1.80 & - & 2.45 & 1.02 & 6.17 & 2.26 & - & 2.94 \\
\hline 63 & 1.80 & - & 2.45 & 1.02 & 6.17 & 2.25 & - & 2.94 \\
\hline 64 & 1.78 & - & 2.46 & 1.03 & 6.17 & 2.25 & - & 2.94 \\
\hline 65 & 1.76 & - & 2.45 & 1.03 & 6.17 & 2.24 & - & 2.94 \\
\hline 66 & 1.76 & - & 2.46 & 1.04 & 6.18 & 2.24 & - & 2.94 \\
\hline 67 & 1.76 & - & 2.46 & 1.04 & 6.18 & 2.23 & - & 2.95 \\
\hline 68 & 1.77 & - & 2.46 & 1.05 & 6.17 & 2.23 & - & 2.95 \\
\hline 69 & 1.77 & - & 2.46 & 1.06 & 6.17 & 2.23 & - & 2.95 \\
\hline 70 & 1.77 & - & 2.47 & 1.06 & 6.18 & 2.22 & - & 2.95 \\
\hline 71 & 1.77 & - & 2.47 & 1.07 & 6.18 & 2.22 & - & 2.95 \\
\hline 72 & 1.77 & - & 2.47 & 1.07 & 6.18 & 2.22 & - & 2.95 \\
\hline 73 & 1.76 & - & 2.47 & 1.07 & 6.18 & 2.21 & - & 2.95 \\
\hline 74 & 1.75 & - & 2.47 & 1.08 & 6.18 & 2.21 & - & 2.95 \\
\hline 75 & 1.75 & - & 2.47 & 1.09 & 6.18 & 2.21 & - & 2.95 \\
\hline 76 & 1.75 & - & 2.48 & 1.09 & 6.18 & 2.20 & - & 2.96 \\
\hline
\end{tabular}




\begin{tabular}{|c|c|c|c|c|c|c|c|c|}
\hline 77 & 1.75 & - & 2.48 & 1.09 & 6.17 & 2.20 & - & 2.96 \\
\hline 78 & 1.75 & - & 2.48 & 1.10 & 6.18 & 2.20 & - & 2.95 \\
\hline 79 & 1.75 & - & 2.48 & 1.10 & 6.17 & 2.20 & - & 2.96 \\
\hline 80 & 1.75 & - & 2.48 & 1.11 & 6.17 & 2.19 & - & 2.96 \\
\hline 81 & 1.74 & - & 2.48 & 1.11 & 6.17 & 2.19 & - & 2.95 \\
\hline 82 & 1.74 & - & 2.48 & 1.11 & 6.17 & 2.19 & - & 2.96 \\
\hline 83 & 1.74 & - & 2.49 & 1.12 & 6.17 & 2.18 & - & 2.96 \\
\hline 84 & 1.73 & - & 2.49 & 1.12 & 6.17 & 2.18 & - & 2.96 \\
\hline 85 & 1.73 & - & 2.49 & 1.13 & 6.17 & 2.18 & - & 2.96 \\
\hline 86 & 1.72 & - & 2.49 & 1.13 & 6.18 & 2.18 & - & 2.96 \\
\hline 87 & 1.72 & - & 2.49 & 1.14 & 6.17 & 2.17 & - & 2.96 \\
\hline 88 & 1.71 & - & 2.49 & 1.14 & 6.17 & 2.17 & - & 2.96 \\
\hline 89 & 1.71 & - & 2.49 & 1.15 & 6.16 & 2.17 & - & 2.96 \\
\hline 90 & 1.71 & - & 2.49 & 1.15 & 6.16 & 2.16 & - & 2.97 \\
\hline 91 & 1.7 & - & 2.49 & 1.16 & 6.16 & 2.16 & - & 2.96 \\
\hline 92 & 1.69 & - & 2.49 & 1.16 & 6.16 & 2.15 & - & 2.96 \\
\hline 93 & 1.68 & - & 2.49 & 1.16 & 6.16 & 2.15 & - & 2.96 \\
\hline 94 & 1.67 & - & 2.48 & 1.17 & 6.15 & 2.15 & - & 2.96 \\
\hline 95 & 1.66 & - & 2.50 & 1.17 & 6.15 & 2.15 & - & 2.96 \\
\hline 96 & 1.64 & - & 2.47 & 1.18 & 6.15 & 2.14 & - & 2.96 \\
\hline 97 & 1.62 & - & 2.46 & 1.18 & 6.15 & 2.14 & - & 2.96 \\
\hline 98 & 1.60 & - & 2.45 & 1.19 & 6.14 & 2.14 & - & 2.96 \\
\hline 99 & 1.55 & - & 2.43 & 1.19 & 6.14 & 2.14 & - & 2.96 \\
\hline 100 & 1.51 & - & 2.42 & 1.20 & 6.12 & 2.14 & - & 2.96 \\
\hline 101 & 1.44 & - & 2.40 & 1.21 & 6.11 & 2.13 & - & 2.96 \\
\hline 102 & 1.34 & - & 2.37 & 1.22 & 6.07 & 2.13 & - & 2.96 \\
\hline 103 & 1.24 & - & 2.35 & 1.23 & 6.05 & 2.13 & - & 2.96 \\
\hline 104 & 1.14 & - & 2.33 & 1.25 & 6.01 & 2.13 & - & 2.96 \\
\hline 105 & 1.03 & - & 2.30 & 1.26 & 5.97 & 2.12 & - & 2.96 \\
\hline 106 & - & - & 2.28 & 1.27 & 5.91 & 2.12 & - & 2.96 \\
\hline 107 & - & - & 2.25 & 1.28 & 5.84 & 2.10 & - & 2.95 \\
\hline 108 & - & - & 2.22 & 1.29 & 5.78 & 2.08 & - & 2.95 \\
\hline 109 & - & - & 2.19 & 1.30 & 5.73 & 2.06 & - & 2.95 \\
\hline 110 & - & 1.11 & 2.17 & 1.30 & $\begin{array}{l}5.68 \\
\end{array}$ & 2.04 & - & 2.95 \\
\hline 111 & - & 1.20 & 2.14 & 1.31 & 5.63 & 2.02 & - & 2.94 \\
\hline 112 & - & 1.29 & 2.12 & 1.31 & 5.57 & 2.00 & - & 2.94 \\
\hline 113 & - & 1.37 & 2.10 & 1.31 & 5.54 & 1.98 & - & 2.94 \\
\hline 114 & - & 1.45 & 2.09 & 1.32 & 5.49 & 1.97 & - & 2.94 \\
\hline 115 & - & 1.51 & 2.08 & 1.32 & 5.49 & 1.96 & - & 2.94 \\
\hline 116 & - & 1.57 & 2.06 & 1.32 & 5.45 & 1.95 & - & 2.93 \\
\hline 117 & - & 1.61 & 2.06 & 1.32 & 5.42 & 1.94 & - & 2.93 \\
\hline 118 & - & 1.65 & 2.06 & 1.32 & 5.42 & 1.94 & - & 2.93 \\
\hline 119 & - & 1.68 & 2.06 & 1.32 & 5.4 & 1.93 & - & 2.93 \\
\hline 120 & - & 1.70 & 2.05 & 1.32 & 5.39 & 1.93 & - & 2.93 \\
\hline 121 & - & 1.71 & 2.05 & 1.32 & 5.39 & 1.93 & - & 2.93 \\
\hline 122 & - & 1.72 & 2.05 & 1.33 & 5.39 & 1.93 & - & 2.94 \\
\hline 123 & - & 1.73 & 2.06 & 1.33 & $\begin{array}{l}5.37 \\
\end{array}$ & 1.93 & - & 2.94 \\
\hline 124 & - & 1.74 & 2.05 & 1.33 & 5.37 & 1.93 & - & 2.94 \\
\hline 125 & - & 1.75 & 2.06 & 1.33 & 5.37 & 1.93 & - & 2.94 \\
\hline 126 & - & 1.76 & 2.06 & 1.33 & $\begin{array}{l}5.37 \\
\end{array}$ & 1.92 & - & 2.94 \\
\hline 127 & - & 1.76 & 2.06 & 1.34 & 5.38 & 1.92 & - & 2.94 \\
\hline 128 & - & 1.76 & 2.05 & 1.34 & 5.38 & 1.92 & - & 2.94 \\
\hline 129 & - & 1.76 & 2.05 & 1.34 & 5.38 & 1.92 & - & 2.95 \\
\hline 130 & - & 1.77 & 2.05 & 1.34 & 5.35 & 1.92 & - & 2.94 \\
\hline 131 & - & 1.77 & 2.05 & 1.34 & 5.34 & 1.92 & - & 2.94 \\
\hline 132 & - & 1.77 & 2.05 & 1.34 & 5.32 & 1.92 & - & 2.94 \\
\hline 133 & - & 1.78 & 2.06 & 1.34 & 5.34 & 1.91 & - & 2.94 \\
\hline 134 & - & 1.78 & 2.05 & 1.34 & 5.33 & 1.91 & - & 2.95 \\
\hline
\end{tabular}




\begin{tabular}{|l|l|l|l|l|l|l|l|l|}
\hline 135 & -- & 1.78 & 2.05 & 1.34 & 5.33 & 1.91 & -- & 2.94 \\
\hline 136 & -- & 1.78 & 2.05 & 1.34 & 5.33 & 1.91 & -- & 2.94 \\
\hline 137 & -- & 1.78 & 2.05 & 1.34 & 5.33 & 1.91 & -- & 2.94 \\
\hline 138 & -- & 1.78 & 2.05 & 1.34 & 5.33 & 1.91 & -- & 2.94 \\
\hline 139 & -- & 1.78 & 2.05 & 1.34 & 5.33 & 1.91 & -- & 2.94 \\
\hline 140 & -- & 1.78 & 2.05 & 1.34 & 5.33 & 1.91 & -- & 2.94 \\
\hline 141 & -- & 1.79 & 2.05 & 1.34 & 5.33 & 1.91 & -- & 2.94 \\
\hline 142 & -- & 1.79 & 2.05 & 1.34 & 5.33 & 1.91 & -- & 2.94 \\
\hline 143 & -- & 1.79 & 2.05 & 1.34 & 5.33 & 1.91 & -- & 2.94 \\
\hline 144 & -- & 1.79 & 2.05 & 1.34 & 5.33 & 1.91 & -- & 2.94 \\
\hline 145 & -- & 1.79 & 2.05 & 1.34 & 5.33 & 1.91 & -- & 2.94 \\
\hline 146 & -- & 1.79 & 2.05 & 1.35 & 5.33 & 1.91 & -- & 2.94 \\
\hline 147 & -- & 1.79 & 2.05 & 1.35 & 5.33 & 1.91 & -- & 2.94 \\
\hline 148 & -- & 1.79 & 2.05 & 1.35 & 5.33 & 1.91 & -- & 2.94 \\
\hline 149 & -- & 1.79 & 2.05 & 1.35 & 5.33 & 1.91 & -- & 2.94 \\
\hline 150 & -- & 1.79 & 2.05 & 1.35 & 5.33 & 1.91 & -- & 2.94 \\
\hline
\end{tabular}

Table S 34. ELF basin populations for the IRC corresponding to TS4b.

\begin{tabular}{|c|c|c|c|c|c|c|c|c|}
\hline & 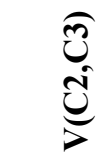 & $\frac{0}{0}$ & 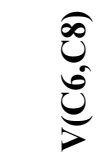 & 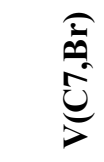 & $\stackrel{\widehat{0}}{\vec{b}}$ & $\begin{array}{l}\hat{U} \\
0 \\
0 \\
0\end{array}$ & $\underset{D}{\stackrel{D}{2}}$ & $\sum_{>}$ \\
\hline 1 & 1.85 & - & 2.17 & - & 6.19 & 3.38 & 1.27 & 1.88 \\
\hline 2 & 1.85 & - & 2.17 & - & 6.20 & 3.38 & 1.28 & $\begin{array}{l}1.89 \\
\end{array}$ \\
\hline 3 & 1.85 & - & 2.17 & - & 6.20 & 3.38 & 1.27 & $\begin{array}{ll}1.88 \\
\end{array}$ \\
\hline 4 & 1.85 & - & 2.17 & - & 6.20 & 3.38 & 1.28 & 1.88 \\
\hline 5 & 1.85 & - & 2.17 & - & 6.19 & 3.38 & 1.28 & 1.88 \\
\hline 6 & 1.85 & - & 2.17 & - & 6.19 & 3.38 & 1.28 & $\begin{array}{l}1.87 \\
\end{array}$ \\
\hline 7 & 1.85 & - & 2.17 & - & 6.21 & 3.38 & 1.28 & 1.87 \\
\hline 8 & 1.85 & - & 2.17 & - & 6.19 & 3.38 & 1.27 & $\begin{array}{l}1.87 \\
\end{array}$ \\
\hline 9 & 1.85 & - & 2.18 & - & 6.19 & 3.38 & 1.27 & $\begin{array}{ll}1.88 \\
\end{array}$ \\
\hline 10 & 1.85 & - & 2.17 & - & 6.19 & 3.38 & 1.27 & 1.88 \\
\hline 11 & 1.85 & - & 2.17 & - & 6.19 & 3.38 & 1.27 & 1.88 \\
\hline 12 & 1.85 & - & 2.17 & - & 6.20 & 3.38 & 1.28 & 1.89 \\
\hline 13 & 1.85 & - & 2.17 & - & 6.20 & 3.38 & 1.27 & 1.88 \\
\hline 14 & 1.85 & - & 2.17 & - & 6.20 & 3.38 & 1.28 & 1.88 \\
\hline 15 & 1.85 & - & 2.17 & - & 6.19 & 3.38 & 1.28 & 1.88 \\
\hline 16 & 1.85 & - & 2.17 & - & 6.19 & 3.38 & 1.28 & 1.87 \\
\hline 17 & 1.85 & - & 2.17 & - & 6.21 & 3.38 & 1.28 & 1.87 \\
\hline 18 & 1.85 & - & 2.17 & - & 6.19 & 3.38 & 1.27 & $\begin{array}{l}1.87 \\
\end{array}$ \\
\hline 19 & 1.85 & - & 2.18 & - & 6.19 & 3.38 & 1.27 & 1.88 \\
\hline 20 & 1.85 & - & 2.17 & - & 6.19 & 3.38 & 1.27 & 1.88 \\
\hline 21 & 1.85 & - & 2.17 & - & 6.19 & 3.38 & 1.27 & $\begin{array}{l}1.88 \\
\end{array}$ \\
\hline 22 & 1.85 & - & 2.17 & - & 6.20 & 3.38 & 1.28 & 1.89 \\
\hline 23 & 1.85 & - & 2.17 & - & 6.20 & 3.38 & 1.27 & 1.88 \\
\hline 24 & 1.85 & - & 2.17 & - & 6.20 & 3.38 & 1.28 & $\begin{array}{l}1.88 \\
\end{array}$ \\
\hline 25 & 1.85 & - & 2.17 & - & 6.19 & 3.38 & 1.28 & 1.88 \\
\hline 26 & 1.85 & - & 2.17 & - & 6.19 & 3.38 & 1.28 & 1.87 \\
\hline 27 & 1.85 & - & 2.17 & - & 6.21 & 3.38 & 1.28 & $\begin{array}{l}1.87 \\
\end{array}$ \\
\hline 28 & 1.85 & - & 2.17 & - & 6.19 & 3.38 & 1.27 & 1.87 \\
\hline 29 & 1.85 & - & 2.18 & - & 6.19 & 3.38 & 1.27 & 1.88 \\
\hline 30 & 1.85 & - & 2.17 & - & 6.19 & 3.38 & 1.27 & 1.88 \\
\hline 31 & 1.85 & - & 2.17 & - & 6.19 & 3.38 & 1.27 & 1.88 \\
\hline 32 & 1.85 & - & 2.17 & - & 6.20 & 3.38 & 1.28 & 1.89 \\
\hline 33 & 1.85 & - & 2.17 & - & 6.20 & 3.38 & 1.27 & 1.88 \\
\hline
\end{tabular}




\begin{tabular}{|c|c|c|c|c|c|c|c|c|}
\hline 34 & 1.85 & - & 2.17 & - & 6.20 & 3.38 & 1.28 & 1.88 \\
\hline 35 & 1.85 & - & 2.17 & - & 6.19 & 3.38 & 1.28 & 1.88 \\
\hline 36 & 1.85 & - & 2.17 & - & 6.19 & 3.38 & 1.28 & 1.87 \\
\hline 37 & 1.85 & - & 2.17 & - & 6.21 & 3.38 & 1.28 & 1.87 \\
\hline 38 & 1.85 & - & 2.17 & - & 6.19 & 3.38 & 1.27 & 1.87 \\
\hline 39 & 1.85 & - & 2.18 & - & 6.19 & 3.38 & 1.27 & 1.88 \\
\hline 40 & 1.85 & - & 2.17 & - & 6.19 & 3.38 & 1.27 & 1.88 \\
\hline 41 & 1.85 & - & 2.17 & - & 6.19 & 3.38 & 1.27 & 1.88 \\
\hline 42 & 1.85 & - & 2.17 & - & 6.20 & 3.37 & 1.28 & 1.89 \\
\hline 43 & 1.85 & - & 2.17 & - & 6.20 & 3.37 & 1.27 & 1.88 \\
\hline 44 & 1.85 & - & 2.17 & - & 6.20 & 3.38 & 1.28 & 1.88 \\
\hline 45 & 1.85 & - & 2.17 & - & 6.19 & 3.36 & 1.28 & 1.88 \\
\hline 46 & 1.85 & - & 2.17 & - & 6.19 & 3.36 & 1.28 & 1.87 \\
\hline 47 & 1.85 & - & 2.17 & - & 6.21 & 3.35 & 1.28 & 1.87 \\
\hline 48 & 1.85 & - & 2.17 & - & 6.19 & 3.36 & 1.27 & 1.87 \\
\hline 49 & 1.85 & - & 2.18 & - & 6.19 & 3.35 & 1.27 & 1.88 \\
\hline 50 & 1.85 & - & 2.17 & - & 6.19 & 3.35 & 1.27 & 1.88 \\
\hline 51 & 1.85 & - & 2.17 & - & 6.20 & 3.34 & 1.25 & 1.88 \\
\hline 52 & 1.85 & - & 2.17 & - & 6.20 & 3.34 & 1.25 & 1.88 \\
\hline 53 & 1.85 & - & 2.18 & - & 6.19 & 3.33 & 1.24 & 1.88 \\
\hline 54 & 1.84 & - & 2.18 & - & 6.20 & 3.32 & 1.24 & 1.88 \\
\hline 55 & 1.84 & - & 2.18 & - & 6.18 & 3.3 & 1.24 & 1.88 \\
\hline 56 & 1.84 & - & 2.19 & - & 6.18 & 3.29 & 1.22 & 1.88 \\
\hline 57 & 1.84 & - & 2.19 & - & 6.18 & 3.26 & 1.22 & 1.89 \\
\hline 58 & 1.84 & - & 2.20 & - & 6.18 & 3.23 & 1.21 & 1.89 \\
\hline 59 & 1.83 & - & 2.20 & - & 6.18 & 3.21 & 1.20 & 1.89 \\
\hline 60 & 1.83 & - & 2.20 & - & 6.19 & 3.18 & 1.17 & 1.89 \\
\hline 61 & 1.83 & - & 2.21 & - & 6.19 & 3.15 & 1.16 & 1.89 \\
\hline 62 & 1.82 & - & 2.22 & - & 6.19 & 3.12 & 1.13 & 1.89 \\
\hline 63 & 1.82 & - & 2.23 & - & 6.18 & 3.09 & - & 3.00 \\
\hline 64 & 1.81 & - & 2.23 & - & 6.18 & 3.07 & - & 2.98 \\
\hline 65 & 1.80 & - & 2.24 & 0.35 & 6.18 & 2.71 & - & 2.95 \\
\hline 66 & 1.80 & - & 2.25 & 0.43 & 6.17 & 2.65 & - & 2.93 \\
\hline 67 & $\begin{array}{l}1.79 \\
\end{array}$ & - & 2.25 & 0.51 & 6.16 & 2.58 & - & 2.92 \\
\hline 68 & 1.78 & - & 2.26 & 0.59 & 6.15 & 2.52 & - & 2.93 \\
\hline 69 & 1.77 & - & 2.27 & 0.65 & 6.14 & 2.47 & - & 2.93 \\
\hline 70 & 1.76 & - & 2.27 & 0.71 & 6.14 & 2.44 & - & 2.94 \\
\hline 71 & 1.75 & - & 2.27 & 0.76 & 6.14 & 2.4 & - & 2.95 \\
\hline 72 & 1.74 & - & 2.28 & 0.8 & 6.15 & 2.37 & - & 2.94 \\
\hline 73 & 1.74 & - & 2.29 & 0.84 & 6.15 & 2.34 & - & 2.95 \\
\hline 74 & 1.72 & - & 2.29 & 0.88 & 6.15 & 2.32 & - & 2.95 \\
\hline 75 & 1.71 & - & 2.29 & 0.91 & 6.15 & 2.30 & - & 2.95 \\
\hline 76 & 1.72 & - & 2.29 & 0.93 & 6.15 & 2.29 & - & 2.96 \\
\hline 77 & 1.62 & - & 2.29 & 0.98 & 6.12 & 2.27 & - & 2.95 \\
\hline 78 & 1.63 & - & 2.29 & 0.98 & 6.14 & 2.26 & - & 2.96 \\
\hline 79 & 1.54 & - & 2.28 & 1.02 & 6.13 & 2.24 & - & 2.95 \\
\hline 80 & 1.40 & - & 2.27 & 1.08 & 6.09 & 2.22 & - & 2.95 \\
\hline 81 & 1.25 & - & 2.26 & 1.12 & 6.03 & 2.21 & - & 2.94 \\
\hline 82 & 1.11 & - & 2.26 & 1.18 & $\begin{array}{l}5.97 \\
\end{array}$ & 2.19 & - & 2.94 \\
\hline 83 & - & - & 2.24 & 1.22 & 5.89 & 2.17 & - & 2.94 \\
\hline 84 & - & - & 2.21 & 1.26 & 5.79 & 2.14 & - & 2.94 \\
\hline 85 & - & 1.02 & 2.18 & 1.29 & 5.71 & 2.09 & - & 2.93 \\
\hline 86 & - & 1.16 & 2.15 & 1.31 & 5.61 & 2.04 & - & 2.93 \\
\hline 87 & - & 1.30 & 2.13 & 1.32 & 5.56 & 2.01 & - & 2.93 \\
\hline 88 & - & 1.43 & 2.10 & 1.33 & 5.50 & 1.97 & - & 2.92 \\
\hline 89 & - & 1.53 & 2.08 & 1.33 & 5.46 & 1.96 & - & 2.92 \\
\hline 90 & - & 1.61 & 2.07 & 1.34 & 5.42 & 1.95 & - & 2.92 \\
\hline 91 & - & 1.66 & 2.06 & 1.33 & 5.41 & 1.94 & - & 2.92 \\
\hline
\end{tabular}




\begin{tabular}{|c|c|c|c|c|c|c|c|c|}
\hline 92 & - & 1.70 & 2.06 & 1.33 & 5.40 & 1.94 & - & 2.93 \\
\hline 93 & - & 1.72 & 2.06 & 1.34 & 5.39 & 1.93 & - & 2.93 \\
\hline 94 & - & 1.74 & 2.06 & 1.34 & 5.38 & 1.93 & - & 2.94 \\
\hline 95 & - & 1.75 & 2.07 & 1.34 & 5.37 & 1.92 & - & 2.94 \\
\hline 96 & - & 1.76 & 2.07 & 1.34 & 5.37 & 1.92 & - & 2.94 \\
\hline 97 & - & 1.76 & 2.07 & 1.34 & 5.36 & 1.92 & - & 2.94 \\
\hline 98 & - & 1.77 & 2.06 & 1.34 & 5.36 & 1.92 & - & 2.95 \\
\hline 99 & - & 1.78 & 2.06 & 1.35 & 5.36 & 1.91 & - & 2.94 \\
\hline 100 & - & 1.78 & 2.06 & 1.35 & 5.35 & 1.91 & - & 2.95 \\
\hline 101 & - & 1.79 & 2.06 & 1.35 & 5.31 & 1.91 & - & 2.94 \\
\hline 102 & - & 1.79 & 2.06 & 1.35 & 5.29 & 1.90 & - & 2.94 \\
\hline 103 & - & 1.80 & 2.07 & 1.35 & 5.31 & 1.90 & - & 2.95 \\
\hline 104 & - & 1.80 & 2.07 & 1.35 & 5.32 & 1.90 & - & 2.96 \\
\hline 105 & - & 1.80 & 2.07 & 1.35 & 5.32 & 1.90 & - & 2.95 \\
\hline 106 & - & 1.80 & 2.06 & 1.36 & 5.33 & 1.90 & - & 2.96 \\
\hline 107 & - & 1.81 & 2.06 & 1.36 & $\begin{array}{l}5.32 \\
\end{array}$ & 1.90 & - & 2.95 \\
\hline 108 & - & 1.81 & 2.06 & 1.36 & 5.31 & 1.89 & - & 2.96 \\
\hline 109 & - & 1.81 & 2.06 & 1.36 & 5.30 & 1.89 & - & 2.96 \\
\hline 110 & - & 1.81 & 2.06 & 1.36 & 5.29 & 1.88 & - & 2.97 \\
\hline 111 & - & 1.81 & 2.06 & 1.36 & 5.29 & 1.88 & - & 2.96 \\
\hline 112 & - & 1.81 & 2.06 & 1.36 & 5.28 & 1.88 & - & 2.97 \\
\hline 113 & - & 1.81 & 2.06 & 1.36 & 5.26 & 1.88 & - & 2.96 \\
\hline 114 & - & 1.82 & 2.06 & 1.35 & 5.25 & 1.88 & - & 2.97 \\
\hline 115 & - & 1.82 & 2.06 & 1.36 & 5.26 & 1.88 & - & 2.96 \\
\hline 116 & - & 1.82 & 2.06 & 1.36 & 5.26 & 1.88 & - & 2.97 \\
\hline 117 & - & 1.82 & 2.06 & 1.36 & 5.25 & 1.87 & - & 2.98 \\
\hline 118 & - & 1.82 & 2.06 & 1.36 & 5.26 & 1.87 & - & 2.98 \\
\hline 119 & - & 1.82 & 2.06 & 1.36 & 5.27 & 1.87 & - & 2.98 \\
\hline 120 & - & 1.83 & 2.06 & 1.36 & 5.26 & 1.87 & - & 2.98 \\
\hline 121 & - & 1.83 & 2.06 & 1.36 & 5.24 & 1.87 & - & 2.97 \\
\hline 122 & - & 1.82 & 2.05 & 1.36 & 5.24 & 1.87 & - & 2.97 \\
\hline 123 & - & 1.83 & 2.05 & 1.36 & 5.25 & 1.87 & - & 2.98 \\
\hline 124 & - & 1.83 & 2.05 & 1.36 & 5.24 & 1.87 & - & 2.98 \\
\hline 125 & - & 1.83 & 2.06 & 1.36 & 5.25 & 1.87 & - & 2.99 \\
\hline 126 & - & 1.83 & 2.05 & 1.36 & 5.25 & 1.87 & - & 2.99 \\
\hline 127 & - & 1.83 & 2.05 & 1.36 & 5.25 & 1.86 & - & 2.99 \\
\hline 128 & - & 1.83 & 2.05 & 1.37 & 5.25 & 1.86 & - & 2.99 \\
\hline 129 & - & 1.84 & 2.05 & 1.36 & 5.24 & 1.87 & - & 2.99 \\
\hline 130 & - & 1.84 & 2.05 & 1.37 & 5.25 & 1.86 & - & 2.98 \\
\hline 131 & - & 1.84 & 2.05 & 1.37 & 5.24 & 1.86 & - & 2.98 \\
\hline 132 & - & 1.84 & 2.05 & 1.37 & 5.24 & 1.86 & - & 2.98 \\
\hline 133 & - & 1.84 & 2.05 & 1.37 & 5.24 & 1.86 & - & 2.99 \\
\hline 134 & - & 1.84 & 2.05 & 1.37 & 5.23 & 1.86 & - & 2.99 \\
\hline 135 & - & 1.84 & 2.05 & 1.37 & 5.23 & 1.86 & - & 2.99 \\
\hline 136 & - & 1.84 & 2.05 & 1.37 & 5.23 & 1.86 & - & 2.99 \\
\hline 137 & - & 1.84 & 2.05 & 1.37 & 5.23 & 1.86 & - & 2.99 \\
\hline 138 & - & 1.84 & 2.05 & 1.37 & 5.21 & 1.86 & - & 2.98 \\
\hline 139 & - & 1.84 & 2.04 & 1.37 & 5.21 & 1.86 & - & 2.99 \\
\hline 140 & - & 1.84 & 2.05 & 1.37 & 5.21 & 1.87 & - & 2.99 \\
\hline 141 & - & 1.84 & 2.05 & 1.36 & 5.20 & 1.86 & - & 2.99 \\
\hline 142 & - & 1.84 & 2.05 & 1.37 & 5.20 & 1.86 & - & 2.99 \\
\hline 143 & - & 1.84 & 2.05 & 1.37 & 5.21 & 1.87 & - & 2.99 \\
\hline 144 & - & 1.84 & 2.05 & 1.37 & 5.20 & 1.87 & - & 2.99 \\
\hline 145 & - & 1.84 & 2.05 & 1.37 & 5.21 & 1.86 & - & 2.99 \\
\hline 146 & - & 1.84 & 2.04 & 1.37 & 5.20 & 1.86 & - & 2.99 \\
\hline 147 & - & 1.84 & 2.04 & 1.37 & 5.19 & 1.86 & - & 2.99 \\
\hline 148 & - & 1.84 & 2.04 & 1.37 & 5.19 & 1.86 & - & 2.99 \\
\hline 149 & - & 1.84 & 2.05 & 1.37 & 5.19 & 1.86 & - & 2.99 \\
\hline
\end{tabular}




\begin{tabular}{|l|l|l|l|l|l|l|l|l|}
\hline 150 & - & 1.85 & 2.04 & 1.37 & 5.19 & 1.86 & - & 2.99 \\
\hline 151 & - & 1.84 & 2.04 & 1.36 & 5.19 & 1.86 & - & 2.99 \\
\hline 152 & - & 1.84 & 2.04 & 1.36 & 5.19 & 1.86 & - & 2.99 \\
\hline 153 & - & 1.84 & 2.04 & 1.37 & 5.20 & 1.87 & - & 2.99 \\
\hline 154 & - & 1.84 & 2.04 & 1.36 & 5.20 & 1.87 & - & 2.99 \\
\hline 155 & - & 1.85 & 2.04 & 1.36 & 5.19 & 1.86 & - & 2.99 \\
\hline 156 & - & 1.85 & 2.04 & 1.36 & 5.20 & 1.87 & - & 3.00 \\
\hline 157 & - & 1.85 & 2.04 & 1.36 & 5.20 & 1.87 & - & 2.99 \\
\hline 158 & - & 1.85 & 2.04 & 1.36 & 5.20 & 1.87 & - & 2.99 \\
\hline 159 & - & 1.84 & 2.04 & 1.36 & 5.20 & 1.87 & - & 2.99 \\
\hline 160 & - & 1.84 & 2.04 & 1.37 & 5.20 & 1.87 & - & 2.99 \\
\hline
\end{tabular}

\section{Stabilization of the intermediate}

A stepwise processed was achieved by placing a dimetylamino group instead the aromatic ring. In this case a stable intermediate iminium ion was located. From this intermediate, paths towards an epoxide and a cyclopentanone were calculated (Scheme S 15).

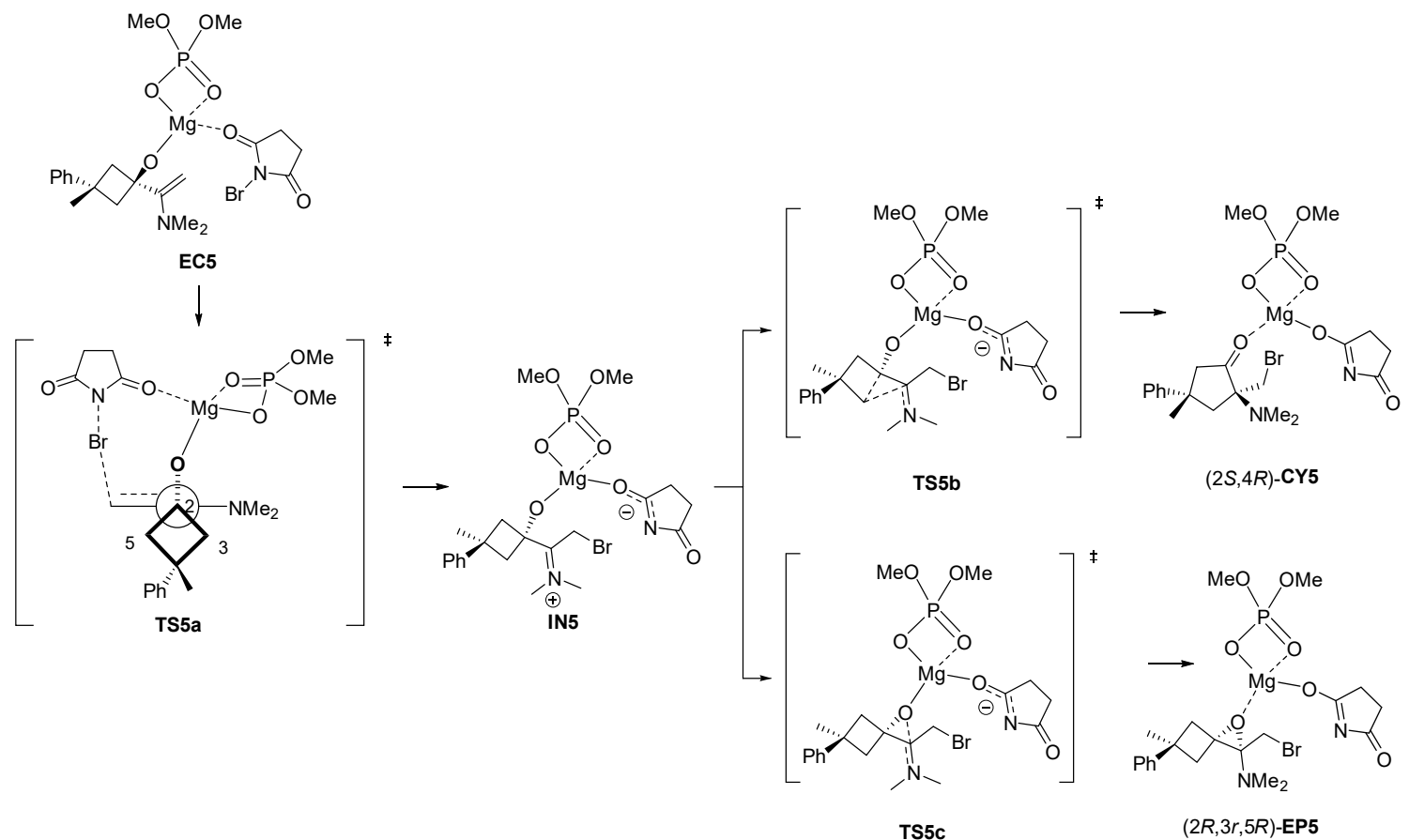

Scheme S 15. Stabilization of intermediate as iminium ion. Only the attack by one face of the iminium ion has been calculated.

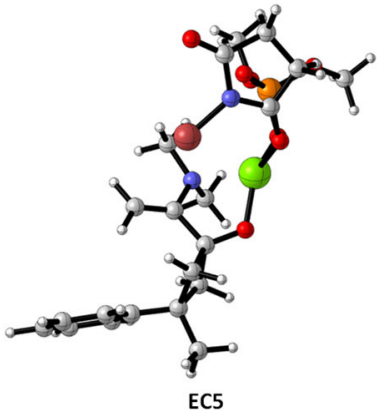

EC5

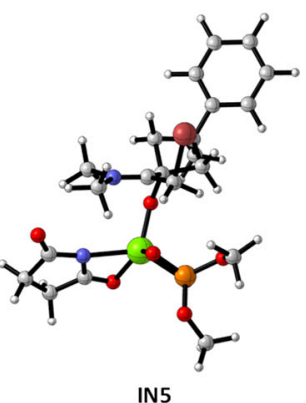

IN5

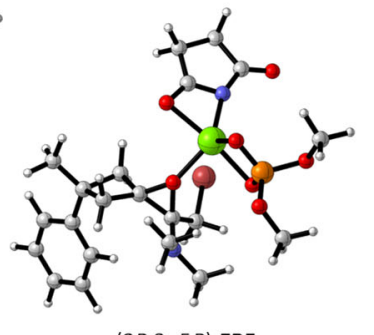

$(2 R, 3 r, 5 R)$-EP5

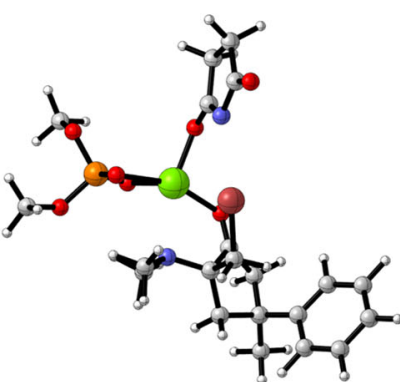

$(2 S, 4 S)-$ CY5 
Figure S 83. Optimized (wb97xd/def2SVP) geometries of encounter complex EC5, intermediate IN5, epoxide $(2 R, 3 r, 5 R)$-EP5 and cyclopentanone $(2 S, 4 R)$-CY5

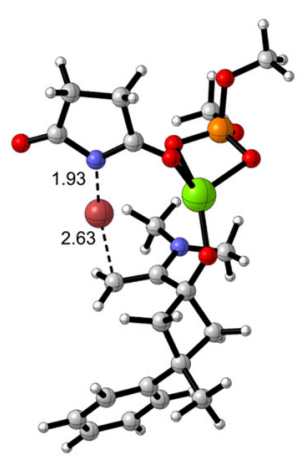

TS5a

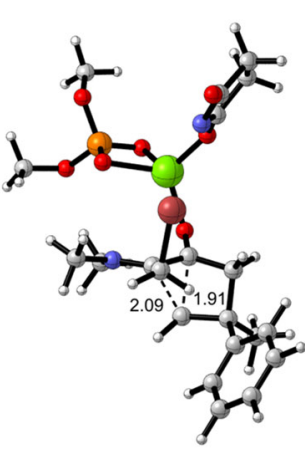

TS5b

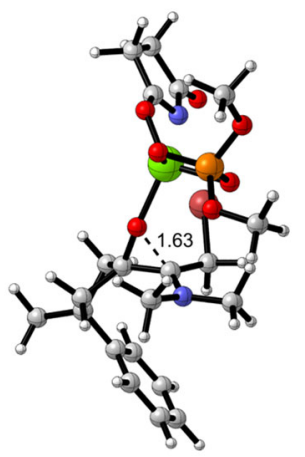

TS5c

Figure S 84. Optimized (wb97xd/def2SVP) geometries of transition structures TS5a, TS5b and TS5c.

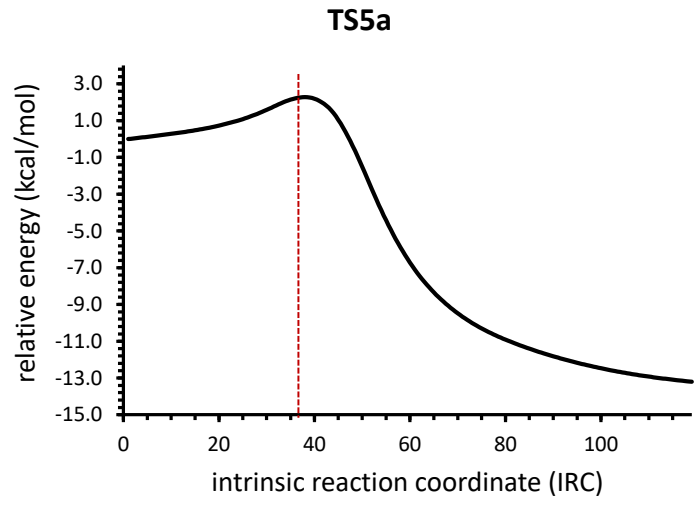

TS5b

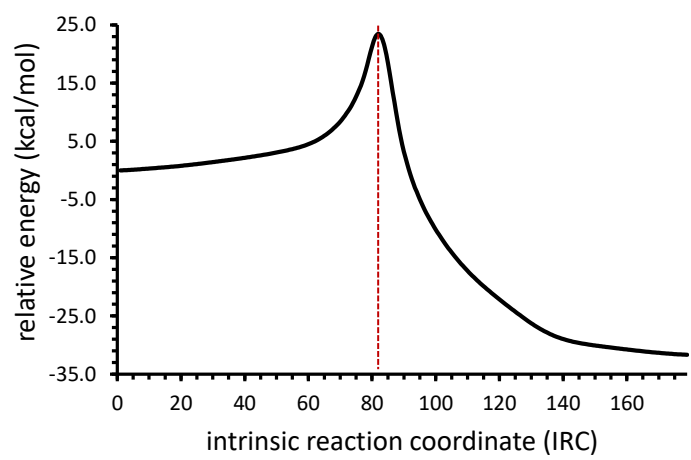

TS5c

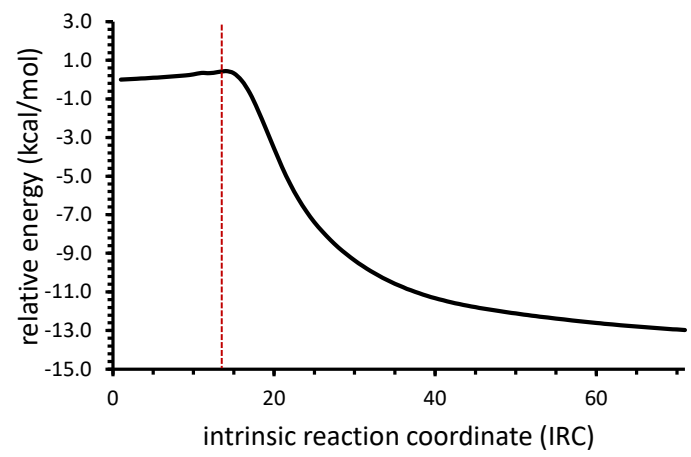

Figure S 85. IRCs (wb97xd/def2svp) corresponding to transition structures TS5a, TS5b and TS5c. 


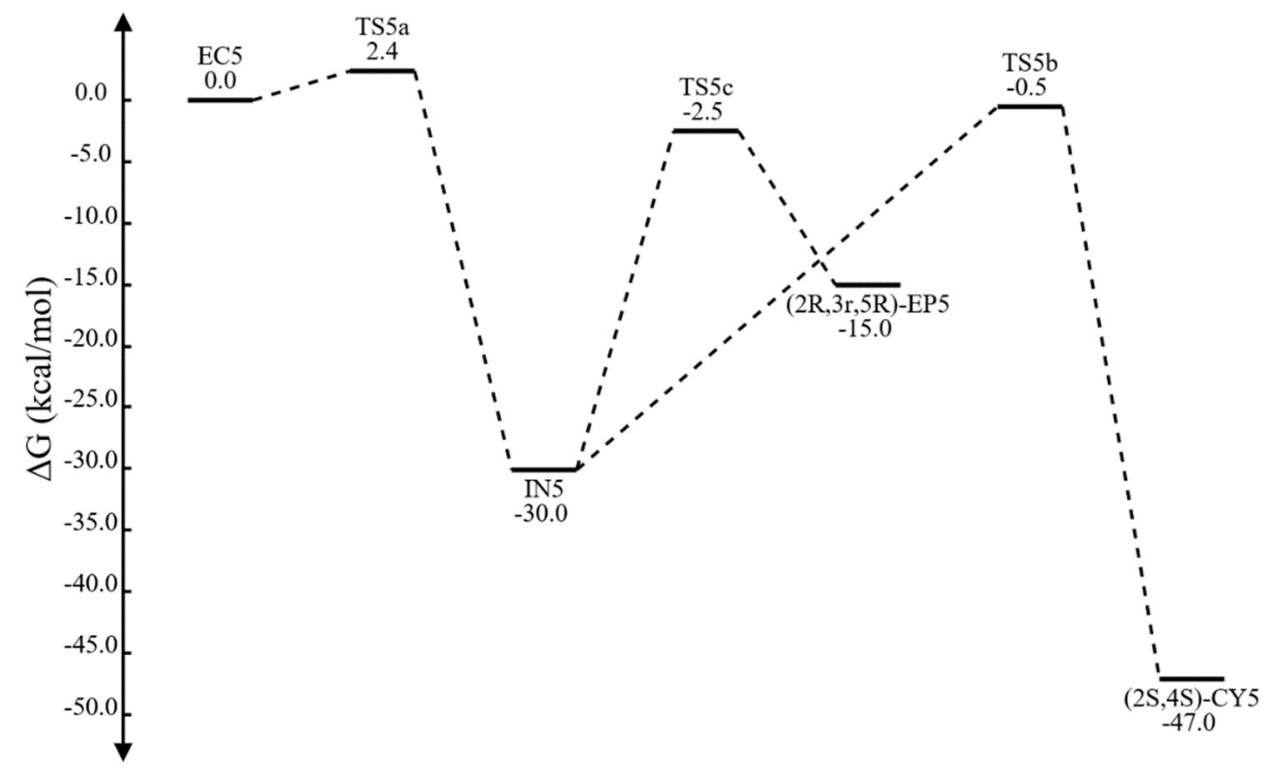

Figure S 86. Energy profiles (wb97xd/def2tzvp/pcm=toluene)//wb97xd/def2svp) for the different pathways from EC5 to EP5 and CY5. Relative free energies are given in $\mathrm{kcal} / \mathrm{mol}$.

Table S 35. Calculated (wb97xd/def2tzvp/pcm=toluene//wb97xd/def2svp) absolute (hartree) and relative (kcal/mol) energies for the transformation of EC5 into EP5 and CY5 .

\begin{tabular}{lccccc}
\hline & $\mathrm{E}_{0}$ & $\Delta \mathrm{E}_{0}$ & $\mathrm{G}$ & $\Delta \mathrm{G}$ & im. freq \\
\hline EC5 & -4569.880067 & 0.0 & -4569.974554 & 0.0 & \\
TS5a & -4569.876584 & 2.2 & -4569.970712 & 2.4 & -118.0 \\
IN5 & -4569.925222 & -28.3 & -4570.022437 & -30.0 & \\
TS5b & -4569.875033 & 3.2 & -4569.974764 & -0.1 & -485.1 \\
TS5c & -4569.884170 & -2.6 & -4569.978612 & -2.5 & -225.6 \\
$(2 S, 4 S)-$ CY5 & -4569.955115 & -47.1 & -4570.049506 & -47.0 & \\
$(2 R, 3 r, 5 R)-$-EP5 & -4569.900108 & -12.6 & -4569.998385 & -15.0 & \\
\hline
\end{tabular}




\section{Real Model with Real Catalyst}

Consideration of the chiral catalyst duplicates the approaches illustrated in Scheme S 14 We located 8 transition structures (TS2a1 and TS2a2 leading to isomeric epoxides and TS2b1, TS2b2, TS2c1, TS2c2, TS2d1 and TS2d2 leading to isomeric cyclopentanones) (Scheme S 16). The optimized structures are given in Figure S 87 and their absolute and relative energies in Table S 36.
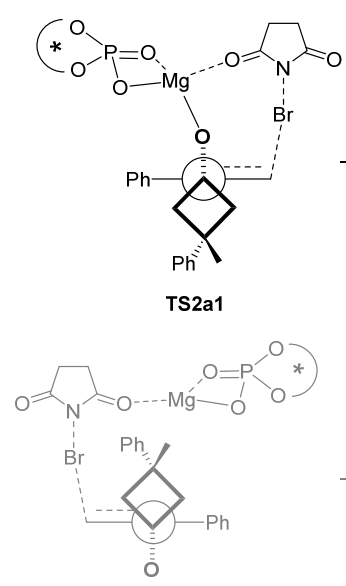

NOT POSSIBLE

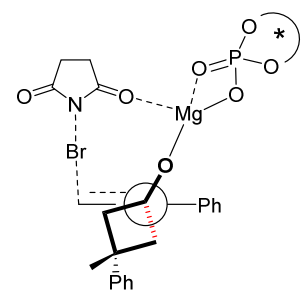

TS2b2

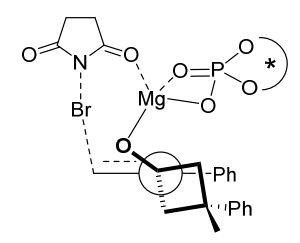

TS2c2

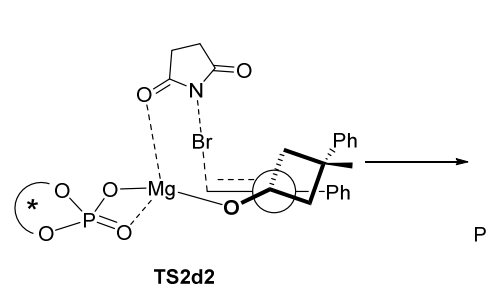

TS2d2

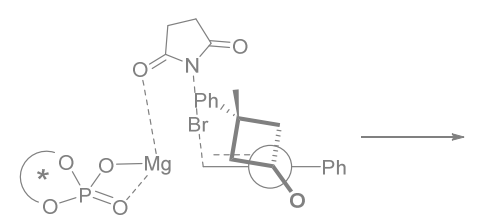

NOT POSSIBLE

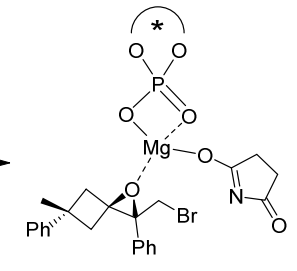

$(2 S, 3 r, 5 S)-E P 2$

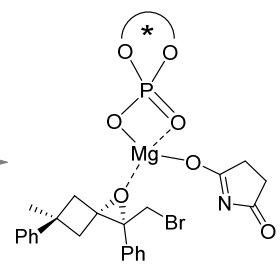

$(2 R, 3 r, 5 R)-\mathrm{EP} 2$

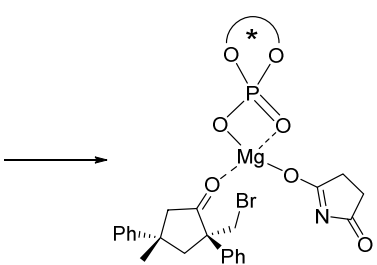

$(2 S, 4 R)-\mathrm{CY} 2$

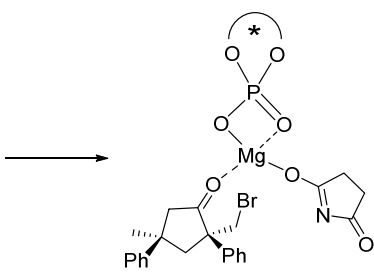

$(2 S, 4 S)-C Y 2$
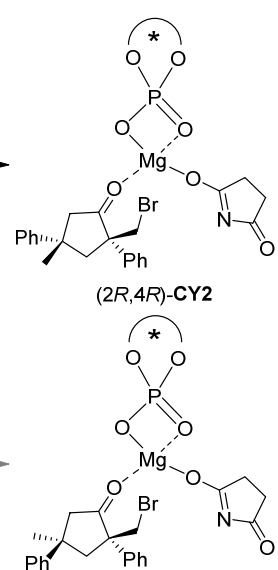

$(2 R, 4 S)-C Y 2$

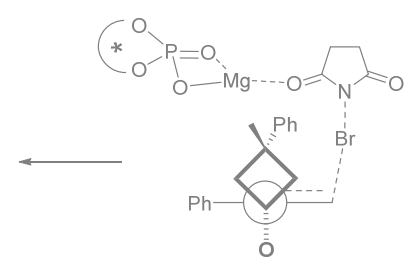

NOT POSSIBLE
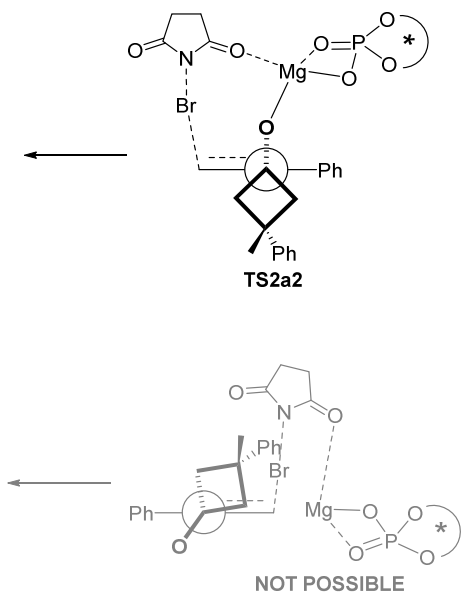

NOT POSSIBLE
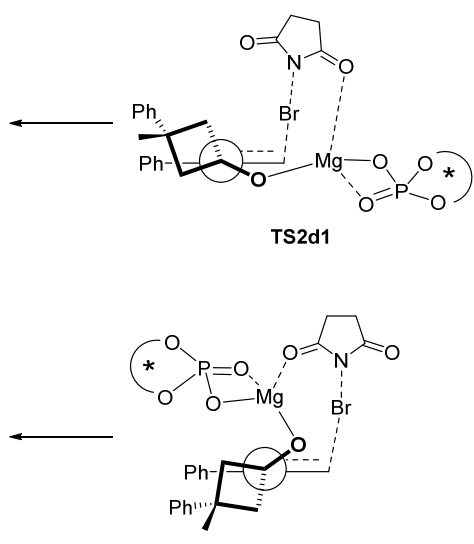

TS2c1

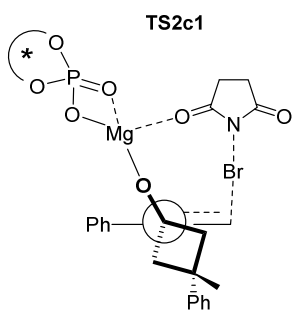

TS2b1

Scheme S 16. Approaches for the real model with the real catalyst. 


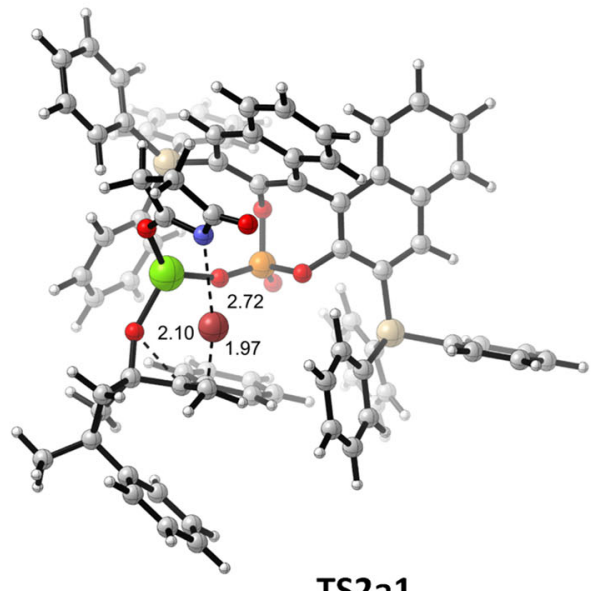

TS2a1

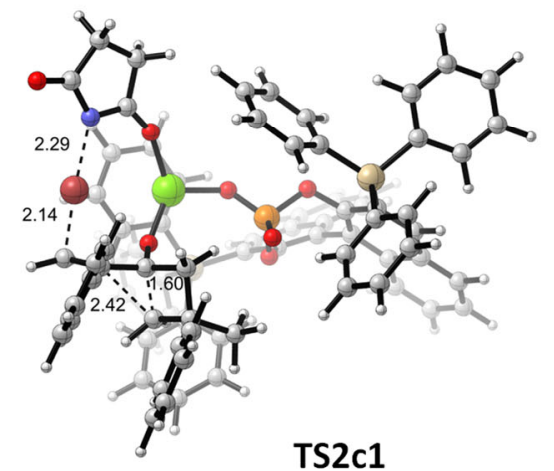

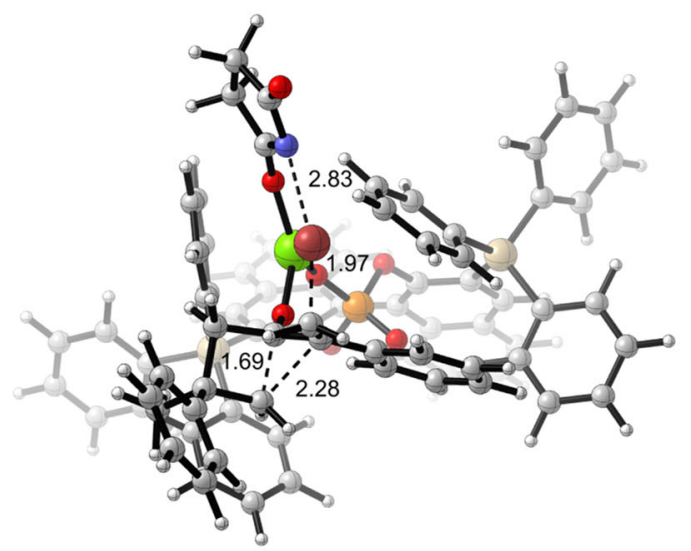

TS2b1

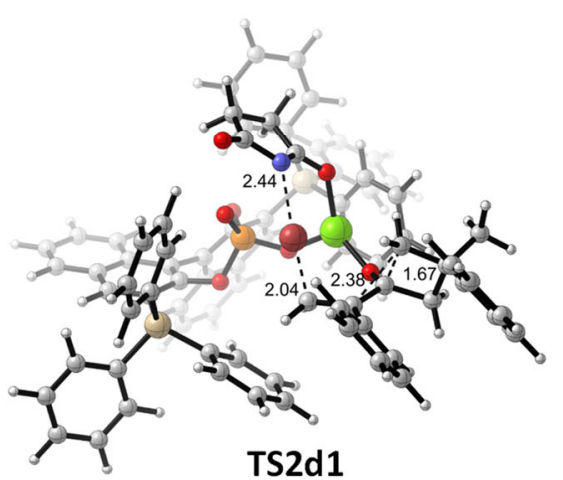

Figure S 87. Optimized (wb97xd/def2SVP:PM6) geometries of transition structures TS2a1, TS2b1, TS2c1 and TS2d1.

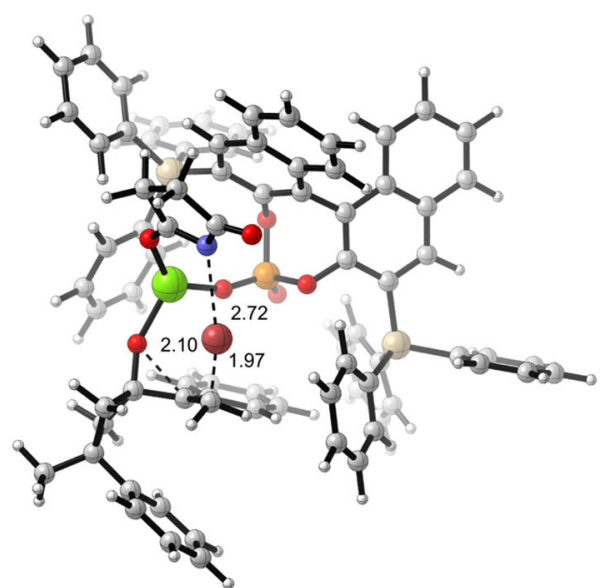

TS2a1

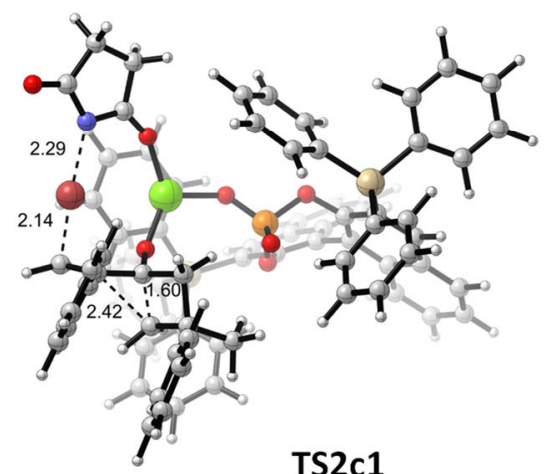

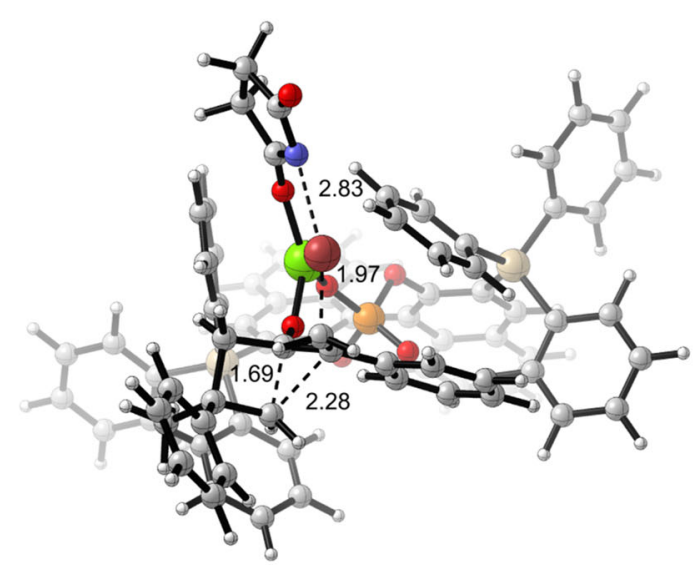

TS2b1

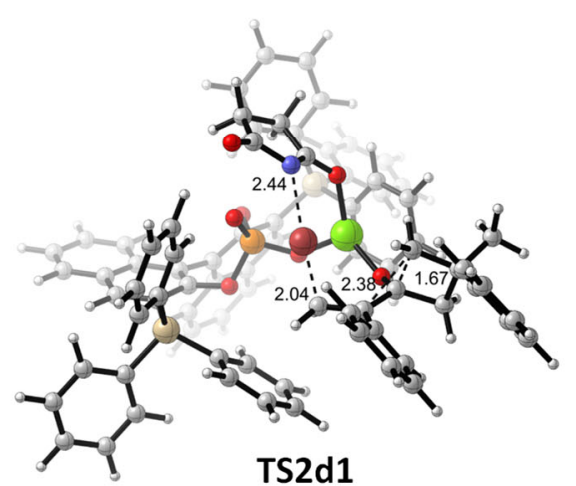

Figure S 88. Optimized (wb97xd/def2SVP:PM6) geometries of transition structures TS2a2, TS2b2, TS2c2 and TS2d2. 
Table S 36. Calculated (wb97xd/def2tzvp/pcm=toluene:PM6//wb97xd/def2svp:PM6) absolute (hartree) and relative $(\mathrm{kcal} / \mathrm{mol})$ energies for the of transition structures illustrated in Scheme S 16.

\begin{tabular}{|c|c|c|c|c|c|c|c|}
\hline & $\mathrm{E}_{0}$ & $\Delta \mathrm{E}_{0}^{\mathrm{a}}$ & $\Delta \Delta \mathrm{E}_{0}^{\mathrm{b}}$ & $\mathrm{G}$ & $\Delta \mathrm{G}^{\mathrm{a}}$ & $\Delta \Delta \mathrm{G}^{\mathrm{b}}$ & im. freq \\
\hline ECr & -5355.664952 & 0.0 & & -5355.843317 & 0.0 & & \\
\hline TS2a1 & -5355.658142 & 4.3 & 0.0 & -5355.831175 & 7.6 & 2.3 & -117.4 \\
\hline TS2b1 & -5355.655543 & 5.9 & 1.6 & -5355.834840 & 5.3 & 0.0 & -194.8 \\
\hline TS2c1 & -5355.650568 & 9.0 & 4.8 & -5355.827010 & 10.2 & 4.9 & -88.9 \\
\hline TS2d1 & -5355.643397 & 13.5 & 9.3 & -5355.821245 & 13.9 & 8.5 & -88.1 \\
\hline TS2a2 & -5355.652947 & 7.5 & 3.3 & -5355.827309 & 10.0 & 4.7 & -106.9 \\
\hline TS2b2 & -5355.653358 & 7.3 & 3.0 & -5355.829366 & 8.8 & 3.4 & -118.3 \\
\hline TS2c2 & -5355.651228 & 8.6 & 4.3 & -5355.827379 & 10.0 & 4.7 & -152.5 \\
\hline TS2d2 & -5355.635444 & 18.5 & 14.2 & -5355.809567 & 21.2 & 15.9 & -119.2 \\
\hline$(2 R, 3 r, 5 R)-\mathrm{EPr}$ & -5355.715864 & -31.9 & & -5355.888529 & -28.4 & & \\
\hline$(2 S, 3 r, 5 S)-\mathrm{EPr}$ & -5355.675336 & -6.5 & & -5355.849791 & -4.1 & & \\
\hline$(2 R, 4 R)-C Y r$ & -5355.769241 & -65.4 & & -5355.942932 & -62.5 & & \\
\hline$(2 R, 4 S)-C Y r$ & -5355.768198 & -64.8 & & -5355.942795 & -62.4 & & \\
\hline$(2 S, 4 R)-C Y r$ & -5355.759003 & -59.0 & & -5355.934484 & -57.2 & & \\
\hline$(2 S, 4 S)-C Y r$ & -5355.753488 & -55.6 & & -5355.930652 & -54.8 & & \\
\hline
\end{tabular}

${ }^{a}$ Referred to ECr. ${ }^{b}$ Referred to the minimum value of the transition structures

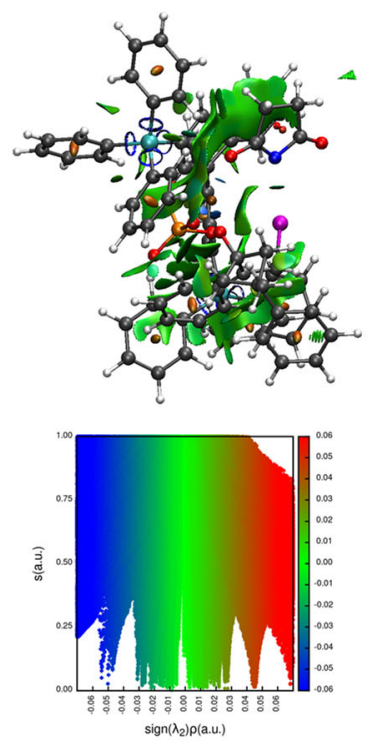

TS2a1
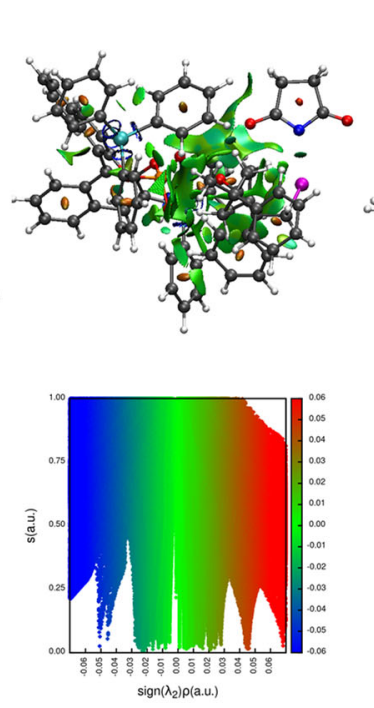

TS2b1
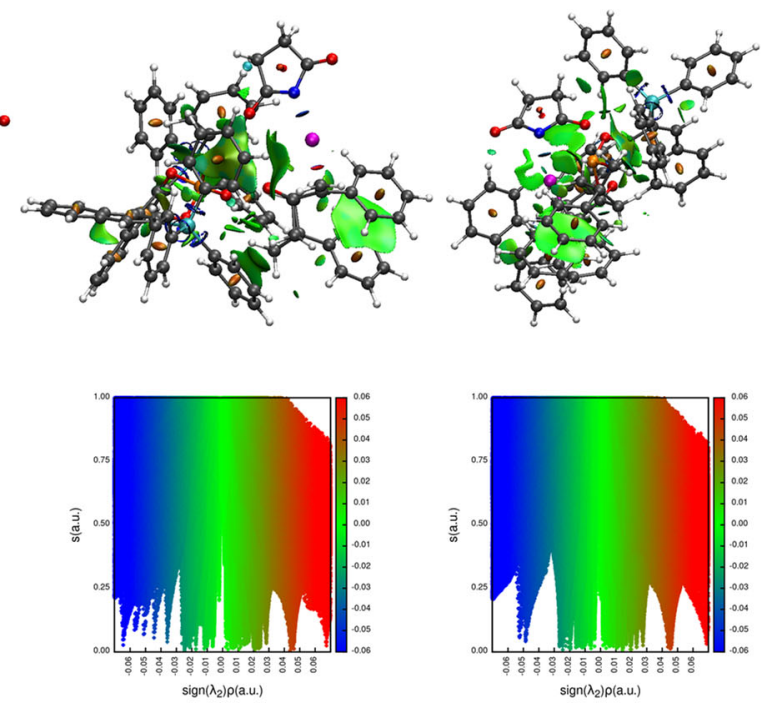

TS2c1

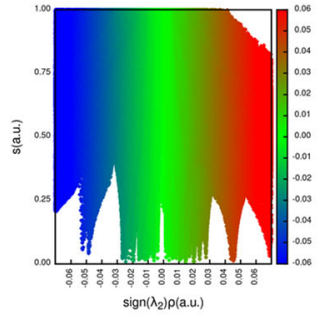

TS2d1

Figure S 89. NCI analysis for transition structures TS2a1, TS2b1, TS2c1 and TS2d1. Top: Isosurfaces. Bottom: $s(\mathbf{r})$ against $\operatorname{sign}\left(\lambda_{2}\right) \rho(\mathbf{r})$ plots (see text). 

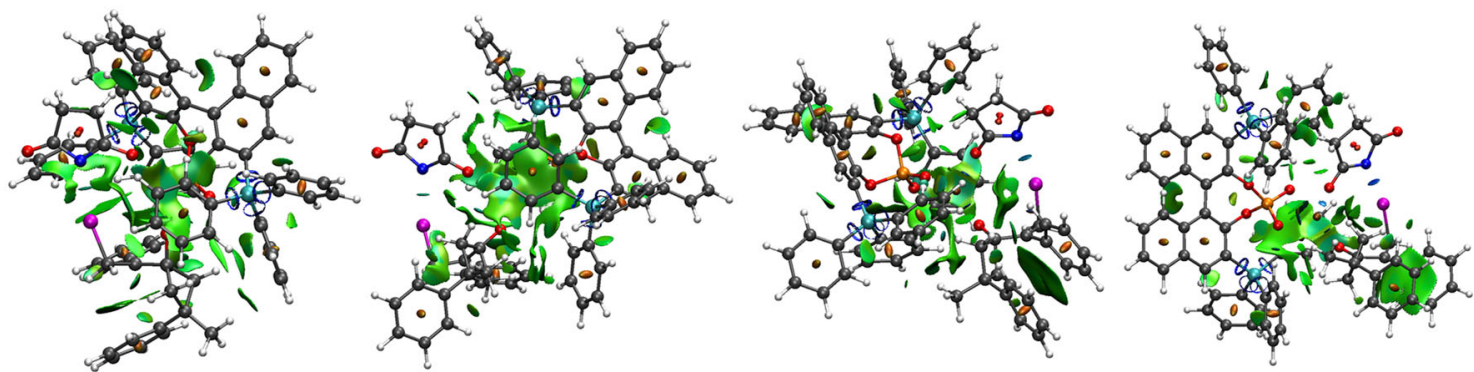

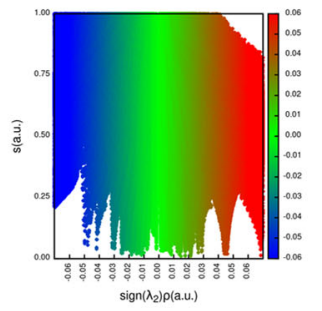

TS2a2

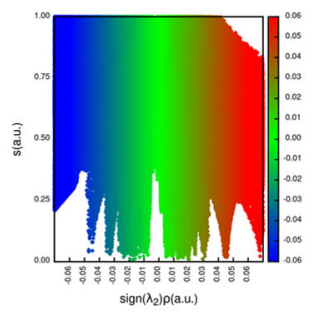

TS2b2

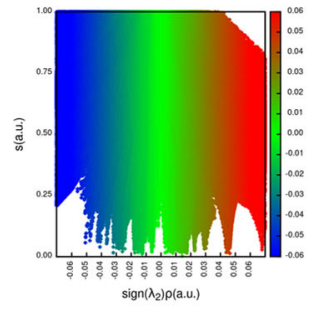

TS2c2

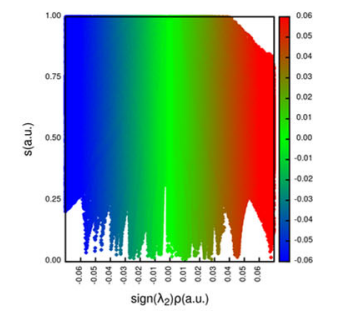

TS2d2

Figure S 90. NCI analysis for transition structures TS2a2, TS2b2, TS2c2 and TS2d2. Top: Isosurfaces. Bottom: $s(\mathbf{r})$ against $\operatorname{sign}\left(\lambda_{2}\right) \rho(\mathbf{r})$ plots (see text).

Table S 37 Integration over the volumes of $\operatorname{sign}\left(\lambda_{2}\right) \rho(\mathbf{n})$ for $\mathrm{n}=2.5$ according to ref. 37 for TS2a1, TS2b1, TS2c1 and TS2d1

\begin{tabular}{ccccc}
\hline interval & TS2a1 & TS2b1 & TS2c1 & TS2d1 \\
\hline all & -8.22861886 & -8.24809882 & -8.23758177 & -8.24182234 \\
$\mathbf{- 1 . 0}$ to $\mathbf{- 0 . 0 2}$ & -0.64825856 & -0.65183371 & -0.65442657 & -0.65456785 \\
$\mathbf{- 0 . 0 2}$ to $\mathbf{0 . 0 2}$ & 0.01078311 & 0.01062379 & 0.01121693 & 0.01112437 \\
$\mathbf{0 . 0 2}$ to $\mathbf{1 . 0}$ & 0.80472338 & 0.80664636 & 0.80362521 & 0.80557497 \\
\hline
\end{tabular}

${ }^{a}$ The interval [-0.1, -0.02] gives information about strong NCI (H-bond, halogen, etc.). The interval [-0.02, $0.02]$ gives information about van der Waals interactions. The interval $[0.02,1.00]$ gives information about steric clashes.

Table S 38 Integration over the volumes of $\operatorname{sign}\left(\lambda_{2}\right) \rho(\mathbf{n})$ for $n=2.5$ according to ref. 37 for TS2a2, TS2b2, TS2c2 and TS2d2.

\begin{tabular}{ccccc}
\hline interval & TS2a2 & TS2b2 & TS2c2 & TS2d2 \\
\hline all & -8.24209417 & -8.23800821 & -8.24454098 & -8.23658102 \\
$\mathbf{- 1 . 0}$ to $\mathbf{- 0 . 0 2}$ & -0.65124256 & -0.65469843 & -0.65292160 & -0.65315603 \\
$\mathbf{- 0 . 0 2}$ to $\mathbf{0 . 0 2}$ & 0.01049493 & 0.01117797 & 0.01144069 & 0.01108816 \\
$\mathbf{0 . 0 2}$ to $\mathbf{1 . 0}$ & 0.80401630 & 0.80813914 & 0.80717058 & 0.80439725 \\
\hline
\end{tabular}

${ }^{a}$ The interval [-0.1, -0.02] gives information about strong NCI (H-bond, halogen, etc.). The interval [-0.02, $0.02]$ gives information about van der Waals interactions. The interval $[0.02,1.00]$ gives information about steric clashes. 


\section{Molecular Dynamic Simulations}

The following file corresponds to the "progdyn.conf" file used in the Progdyn script package. iError! Marcador no definido. It contains information about the level of theory, charge, multiplicity, and all other inputs for a Gaussian electronic structure calculation. Transition structure TS2b, calculated at the $\omega \mathrm{B} 97 \mathrm{XD} / \mathrm{def} 2 \mathrm{svp}$ level of theory was used as the input structure for the molecular dynamics simulations.

\section{--- Start of file}

\#This is the configuration file for PROGDYN. This file is read by progdynstarterHP and \# the awk programs proggenHP, prog1stpoint, prog2ndpoint, and progdynb.

\#The programs won't read anything past the first blank line, \#and this file must end with a blank line.

\#The program has a number of default values but they are unlikely to be what you want. \#Do not delete lines - rather, comment out lines for unwanted options.

\#The values here are read repeatedly and most can be changed in the middle of running jobs \#***The keywords are case sensitive. The following keywords should always be defined:*** \#***method, charge, multiplicity, memory, processors, title \#*** method --The following word is copied exactly to the gaussian input file. method wb97xd/def2svp

\#To do a nonstandard route, make nonstandard 1. For normal calcs, use nonstandard 0 or else leave it out.

\#Then make a file called "nonstandard" containing the nonstandard route with no extra lines. \#nonstandard 0

\# NMRoptions As is NMRtype=1 will add a section for an NMR calc at every NMRevery intervals. If you want to combine the two use nonstandard \#NMRtype 1

\#NMRmethod2 B97D/6-31G*

\#NMRmethod LC-wPBE/6-31G*

\#NMRmethod3 B3LYP/cc-pvtz

\#NMRevery 4

\#NMRrand 1

\#NMRcc 1

\#loadlimit 10.0

\#geometry linear rotationmode 1

$\#^{* * *}$ method2 --The options here are restricted, unrestricted, and read. restricted is the default \#If the method is U..., put unrestricted here and the .com files will have in them guess=mix. \#If you put read here, the .com files will contain guess=tcheck, which sometimes makes things faster, sometimes not.

\#The use of read requires a specifically defined checkpoint file name using the keyword checkpoint.

method 2 restricted

charge 0

multiplicity 1

\#oniomchargemult 11

processors 16

$\#^{* * *}$ memory --The following "word" is copied exactly to the gaussian input file after \%mem=. 
memory 8gb

\#*** killcheck and checkpoint -- You can use a specifically defined checkpoint file name by putting

\#the name after the keyword checkpoint. This is necessary if you use the read option with method2.

\#Defined checkpoint names are an unnecessary modest hastle and if you do not want to bother, use killcheck 1

killcheck 1

\#checkpoint g09.chk

\#*** diagnostics -- 0 prints out nothing extra, 1 (default) prints out extra stuff to a

\#file "diagnostics", 2 adds more stuff, 3 adds velocities to a file "vellist"

\#4 adds the apparent temperature to vellist, but this is meaningless with quasiclassical calculations

diagnostics 4

\#*** title -- the title keyword must be followed by exactly four words (the first after title is loaded by proganal)

title title CB08 TS1b model

$\#^{* * *}$ initialdis -- 0 (default) turns off displacement of the normal modes, so that all trajectories start from the same place

\# and only the energies and signs of the motion in the modes are randomized

\# 1 gives a flat distribution of displacements where all of the possible values are equally likely

\# 2 (recommended) gives a QM-like gaussian distribution of displacements, so that displacements in the middle are more likely that

\# those at the end by $1 / \mathrm{e}$

initialdis 0

$\#^{* * *}$ timestep -- this is the time between points in the trajectory. Typical values would be $1 \mathrm{E}$ 15 or $0.5 \mathrm{E}-15$ or $0.25 \mathrm{E}-15$

timestep $1 \mathrm{E}-15$

\#*** scaling -- this lets you scale the gaussian frequencies by a constant

scaling 1.0

temperature 298.15

$\#^{* * *}$ thermostat 1 puts in a damping factor so as to bring the classical temperature toward the desired temperature.

\#*** use a thermostatmult between 0.95 and 1 , typically 0.995 , so the damping happens slowly

- otherwise there will be

\#*** overadjustment in response to random variation

$\#^{* * *}$ the thermostat is not exact. The second traj point ignores this, so it only applies to later points handled by progdynb.

thermostat 1

thermostatmult 0.999

$\#^{* * *}$ method3, method4, method5, and method6 -- These keywords let you add extra lines to the gaussian input file.

\#method3 and method4 add lines at the top of the input after the lines defining the method, and

\#this is useful to implement things like the iop for mPW1k

\#method5 and method6 add lines after the geometry, after a blank line of course

\#only a single term with no spaces can be added, one per method line. Here are some examples

to uncomment if needed

\#method3 IOp(3/76=0572004280)

\#method3 scrf $=(\mathrm{pcm}$, solvent=ethanol $)$

\#method3 scrf=(pcm,Solvent=dichloromethane) 
\#add the line below with big structures (more than 50 atoms) to get it to put out the distance matrix and the input orientation

method3 iop(2/9=2000)

\#other possibilities

\#method4 emp=gd3bj

method4 int=ultrafine

\#method4 scf=(conver=5)

\#method4 iop(3/124=3)

\#method4 scrf $=(\mathrm{pcm}$, solvent $=\mathrm{dmso}$, read $)$

\#method5 radii=bondi

\#method6

\#*** methodfile -- This keyword lets you add more complicated endings to gaussian input files \#such as a gen basis set. Put after the keyword the number of lines in a file you create called \#methodfile that contains the test you want to add to the end of the gaussian input methodfile 0

$\#^{* * *}$ numimag --This tells the program the number of imaginary frequencies in the starting structure.

$\#$ \#if 0 , treats as ground state and direction of all modes is random

\#if 1 , motion along the reaction coordinate will start out in the direction defined by searchdir

\#if 2, only lowest freq will go direction of searchdir and other imag mode will go in random direction

numimag 1

\#*** searchdir -- This keyword says what direction to follow the mode associated with the imaginary frequency.

\#The choices are "negative" and "positive". Positive moves in the direction defined in the gaussian frequency calculation

\#for the imaginary frequency, while negative moves in the opposite direction. The correct choice can be made either

\#by a careful inspection of the normal modes and standard orientation geometry, or by trial and error.

searchdir negative

\#*** classical -- for quassiclassical dynamics, the default, use 0 . for classical dynamics, use 1

$\#$ \#if there are no normal modes and the velocities are to be generated from scratch, use classical 2

classical 0

\#*** DRP, saddlepoint, and maxAtomMove --to run a DRP use 'DRP 1 ' in the line below, otherwise leave it at 0 or comment it out

\#the treatment of starting saddlepoints is not yet implemented so use saddlepoint no

\#if DRP shows oscillations then decrease maxAtomMove

\#DRP 1

\#saddlepoint no

\#maxAtomMove 0.01

\#*** cannonball -- The program can "fire" a trajectory from a starting position toward a particular target, such as toward

\#a ts. To use this, make a file cannontraj with numAtom lines and three numbers per line that defines the vector

\#for firing the trajectory, relative to the starting geometry's standard orientation. The number following cannonball sets

\#the extra energy being put into the structure in $\mathrm{kcal} / \mathrm{mol}$

\#cannonball 10

\#*** keepevery --This tells the program how often to write the gaussian output file to file dyn, after the first two points. 
\#Use 1 for most dynamics to start with, but use a higher number to save on disk space or molden loading time.

keepevery 1

\#*** highlevel --For ONIOM jobs, the following line states the number of highlevel atoms, \#which must come before the medium level atoms. Use some high value such as 999 if not using ONIOM

highlevel 9999

\#*** fixedatom1, fixedatom2, fixedatom3, and fixedatom 4 - These fix atoms in space.

\#Fixing one atom serves no useful purpose and messes things up, while fixing two atoms

\#fixes one distance and fixing three has the effect of fixing three distances, not just two

\#in current form fixed atoms only are meant to work with no displacements, that is, initialdis=0

\#fixedatom1 16

\#fixedatom2 1

\#fixedatom3 4

\#fixedatom4 20

\#applyforce 1 lets one push atoms together or appart - a positive force pushes them together

\#format is applyforce force - with the units on force the same as in the Gaussian output file

\#applyforce 2 or 3 or 4 applys a polynomical force centered at dist0. 2 is just harmonic, 3 is second order, 4 is third order

\#format is applyforce 4 forcecoefficient dist0 forcecoefficient2 forcecoefficient3

\#then use afatoms to chose the atoms with format afatoms firstatom secondatom [additional atoms]

\#applyforce 20.12 .1

\#afatoms 16123456

\#applyforceB 20.015 .2

\#afatomsB 815

\#applyforceC 20.015 .2

\#afatomsC 815

\#zeroatom pushes the numbered atom toward the origin with a small harmonic potential - good with boxon when you want to keep the reaction in the center

\#zeroatom 16

$\#^{* * *}$ boxon and boxsize - With boxon 1 , a cubic box is set such that atoms that reach the edge \#are reflected back toward the middle. Useful for dynamics with solvent molecules. This is a crude

\#implementation that is ok for a few thousand femtoseconds but will not conserve energy long term.

\#Set the box size so as to fit the entire initial molecule but not have too much extra room.

\#The dimensions of the box are two times the boxsize, e.g. boxsize 7.5 leads to a box that is 15 $\times 15 \times 15$ angstroms

boxon 0

boxsize 8

$\#^{* * *}$ sphereon and spheresize and sphereforce - uses a force to push atoms within a sphere. notice that if the atom is far outside of

\#the sphere then the force is large unless sphereforce is set small

\#sphereon 1

\#spheresize 12.9

\#sphereforce .01

\#setting a value for empiricaldispersion sets its s6 value with the Grimme 2006 algorithm.

Default is 0 , with no empiricaldispersion; 1.0 for correction

empiricaldispersion 0.0

\#*** displacements -- This keyword lets you set the initialdis of particular modes by using a series of lines of the format 
\# displacements NumberOfMode InitialDisForThatMode, as in the example below. You should be able to do as many of these as you like

\# you might consider this for rotations where a straight-line displacement goes wrong at large displacements

\# The choices for InitialDisForThatMode are 0, 1, 2, and 10, where 10 does the same thing as 0 but is maintained for now because

\# a previous version of the program had a bug that made 0 not work.

\#displacements 20

\#displacements 30

\#displacements 40

\#displacements 50

\#displacements 60

\#displacements 70

\#displacements 80

\#displacements 90

\#displacements 100

\#*** etolerance --This sets the allowable difference between the desired energy in a trajectory and the actual

\#energy, known after point 1 from the potential energy + the kinetic energy in the initial velocities.

\#The unit is $\mathrm{kcal} / \mathrm{mol}$ and 1 is a normal value for mid-sized organic systems. For very large and floppy molecules, a larger value

\#may be needed, but the value must stay way below the average thermal energy in the molecule (not counting zpe).

\#If initialdis is not 0 and few trajectories are being rejected, decrease the value.

etolerance 1

$\#^{* * *}$ controlphase --It is sometimes useful to set the phase of particular modes in the initialization of trajectories.

\#The format is controlphase numberOfModeToControl positive or controlphase numberOfModeToControl negative.

\#controlphase 2 positive

\#*** damping -- The damping keyword lets you add or subtract energy from the system at each point, by multiplying the velocities

\#by the damping factor. A damping of 1 has no effect, and since you mostly want to change the energy slowly, normal values range

\#from 0.95 to 1.05 . The use of damping lets one do simulated annealing - you add energy until the structure is moving enough

\#to sample the kinds of possibilities you are interested in, then you take away the energy slowly. damping 1.00

\#at a damping of .9995, the energy is cut in half in 693 points

$\#^{* * *}$ reversetraj --This keyword sets the trajectories so that both directions from a transition state are explored.

reversetraj true

\#updated Aug 9, 2007 to include the possibility of classical dynamics by the keyword classical \#updated Jan 2008 to include fixed atoms, ONIOM jobs, keepevery, and box size

\#update Feb 2008 to include methodfile parameter

\# updated Nov 2008 to allow for start without an initial freq calc using classical = 2

\# update Aug 2010 to include etolerance, damping controlphase and reversetraj

--- End of file 


\section{Cartesian Coordinates}

\section{Catalyst}

CATa-MeOH

01

\begin{tabular}{|c|c|c|c|}
\hline $\mathrm{Mg}$ & -0.1264955464 & 0.7810007121 & 0.2231375775 \\
\hline $\mathrm{O}$ & -1.9720070365 & 0.4044166471 & 0.1335976432 \\
\hline $\mathrm{O}$ & 1.7562687404 & 0.9775358192 & 0.3116246580 \\
\hline $\mathrm{O}$ & -2.7616162815 & -0.3084572562 & -2.2426475454 \\
\hline $\mathrm{O}$ & 2.7993886856 & 0.5170256460 & 2.6434769647 \\
\hline $\mathrm{P}$ & 2.9822303264 & 0.7832895982 & 1.1944727179 \\
\hline $\mathrm{O}$ & 3.8326328148 & -0.3754107527 & 0.3771430088 \\
\hline $\mathrm{O}$ & 3.9333673052 & 2.1085790058 & 1.0161087291 \\
\hline $\mathrm{C}$ & 5.1393146020 & -0.6495026190 & 0.7763753446 \\
\hline $\mathrm{C}$ & 4.7641234238 & 2.3382869339 & -0.0694447135 \\
\hline $\mathrm{C}$ & 6.1081650280 & 0.3307794808 & 0.6095994329 \\
\hline $\mathrm{C}$ & 5.4306905300 & -1.9587331860 & 1.2522142146 \\
\hline $\mathrm{C}$ & 5.8560475959 & 1.5112659659 & 582744 \\
\hline $\mathrm{C}$ & 4.4723798523 & 3.4679638397 & -0.8852767433 \\
\hline $\mathrm{C}$ & 7.3896450435 & 0.1234531209 & 1.2210583231 \\
\hline $\mathrm{C}$ & 6.7086993637 & -2.1762432415 & 1.7378926902 \\
\hline $\mathrm{Si}$ & 4.2742769798 & -3.4525040873 & 31712 \\
\hline $\mathrm{C}$ & 6.7139709662 & 1.7679044844 & 31532 \\
\hline $\mathrm{C}$ & 5.3732748607 & 3.7667949208 & 364907 \\
\hline $\mathrm{Si}$ & 2.7861894120 & 4.31 & 172289 \\
\hline $\mathrm{C}$ & 6772 & 1.14 & 896588 \\
\hline $\mathrm{C}$ & 7.6823354226 & -1.1485614319 & 50916 \\
\hline $\mathrm{H}$ & 6.9896270501 & -3.1648122870 & 272189 \\
\hline $\mathrm{C}$ & 3.4237228832 & -3.5012385853 & 254894 \\
\hline $\mathrm{C}$ & 3.0597279513 & -3.5527927256 & 778158 \\
\hline $\mathrm{C}$ & 2556 & -4.9 & 15662 \\
\hline $\mathrm{C}$ & $7.7596^{\prime}$ & 795 & 637227 \\
\hline $\mathrm{C}$ & 6.4929967353 & 2.9442114287 & 523829 \\
\hline $\mathrm{H}$ & 0180 & 234 & 41715 \\
\hline $\mathrm{C}$ & 1.6495066575 & 742 & 00196 \\
\hline $\mathrm{C}$ & & & 17553 \\
\hline $\mathrm{C}$ & 2.0791336848 & 4.97 & 392992 \\
\hline $\mathrm{C}$ & 9.5897683806 & 28385 & 025593 \\
\hline $\mathrm{H}$ & $8.1541^{\prime}$ & 2.11 & 48830 \\
\hline $\mathrm{C}$ & 8.9503969563 & -1.36629 & 622523 \\
\hline $\mathrm{C}$ & & & \\
\hline $\mathrm{C}$ & 3.7885565282 & 6333 & 78879 \\
\hline $\mathrm{C}$ & 2.8723665305 & 22162 & 39088 \\
\hline $\mathrm{C}$ & 2.2927257229 & 35083 & 577479 \\
\hline $\mathrm{C}$ & 5.9290548375 & -5.5376365382 & 0.0339329561 \\
\hline $\mathrm{C}$ & 5.6522201576 & -5.6057780527 & 2.4258528342 \\
\hline $\mathrm{C}$ & 8.5672769358 & 1.192 & 142030 \\
\hline $\mathrm{H}$ & 7.9170616698 & -0.0287268602 & -1.2394636769 \\
\hline $\mathrm{C}$ & & & \\
\hline $\mathrm{C}$ & 0.2487527510 & 3.1124286825 & -1.6419496276 \\
\hline $\mathrm{C}$ & 2.1948421979 & 1.9344828606 & -2.4337990873 \\
\hline $\mathrm{C}$ & 3.3279592950 & 7.0368723941 & -1.6602520070 \\
\hline $\mathrm{C}$ & 2.5899183232 & 5.6487452121 & -3.4860377219 \\
\hline $\mathrm{C}$ & 2.6080887322 & 4.7117345515 & 1.9341418606 \\
\hline $\mathrm{C}$ & 0.9537612392 & 5.8189448473 & 0.5763905416 \\
\hline $\mathrm{C}$ & 9.8882873065 & -0.3619903775 & 2.4502190657 \\
\hline $\mathrm{H}$ & 10.3251576072 & 1.7026440296 & 1.9559443242 \\
\hline $\mathrm{H}$ & 9.1594302418 & -2.3427207805 & 2.8299775868 \\
\hline $\mathrm{C}$ & 1.7678819408 & -4.5360418914 & -2.0478701917 \\
\hline
\end{tabular}




\begin{tabular}{|c|c|c|c|}
\hline $\mathrm{H}$ & 2.0925201950 & -5.1078525905 & -0.0142332032 \\
\hline $\mathrm{C}$ & 3.1430239510 & -2.7402990931 & -2.8803373636 \\
\hline $\mathrm{H}$ & 4.5721330409 & -1.9241190557 & -1.5088301863 \\
\hline $\mathrm{C}$ & 1.9170528418 & -2.5540317908 & 4.4367363711 \\
\hline $\mathrm{H}$ & 3.4374861563 & -1.5664589240 & 3.3012652411 \\
\hline $\mathrm{C}$ & 1.3385958419 & -4.7911925106 & 3.7391387498 \\
\hline $\mathrm{H}$ & 2.4525300919 & -5.5841887227 & 2.0917773325 \\
\hline $\mathrm{C}$ & 6.7704272798 & -6.6478395493 & 0.0964856294 \\
\hline $\mathrm{H}$ & 5.7045821227 & -5.0918239516 & -0.9311488522 \\
\hline $\mathrm{C}$ & 6.4945581663 & -6.7160304500 & 2.4947223663 \\
\hline $\mathrm{H}$ & 5.2096716884 & -5.2149781627 & 3.3378422212 \\
\hline $\mathrm{C}$ & 8.3780388955 & 2.3820202859 & -3.5991519300 \\
\hline $\mathrm{H}$ & 9.3555992450 & 0.5024031776 & -3.1449954176 \\
\hline $\mathrm{H}$ & 7.1722946401 & 4.1395091830 & -3.8357641190 \\
\hline $\mathrm{C}$ & -0.5682243885 & 2.1006670400 & -2.1615345345 \\
\hline $\mathrm{H}$ & -0.2218572817 & 3.9586213274 & -1.1516401594 \\
\hline $\mathrm{C}$ & 1.3876594973 & 0.9150367387 & -2.9405434547 \\
\hline $\mathrm{H}$ & 3.2717107283 & 1.8416955354 & -2.5252568949 \\
\hline $\mathrm{C}$ & 3.4424949074 & 8.1225134340 & -2.5273875530 \\
\hline $\mathrm{H}$ & 3.5592815534 & 7.1706416915 & -0.6070027281 \\
\hline $\mathrm{C}$ & 2.7010668947 & 6.7322216080 & -4.3581468373 \\
\hline $\mathrm{H}$ & 2.2493901487 & 4.6898866977 & -3.8665342108 \\
\hline $\mathrm{C}$ & 2.0201233217 & 5.2489416484 & 3.0809095547 \\
\hline $\mathrm{H}$ & 3.4697657336 & 4.0643185824 & 2.0352051188 \\
\hline $\mathrm{C}$ & 0.3615615461 & 6.3515108415 & 1.7203279504 \\
\hline $\mathrm{H}$ & 0.5372801855 & 6.0722761396 & -0.3938531965 \\
\hline $\mathrm{H}$ & 10.8531357965 & -0.5347382056 & 2.9164599870 \\
\hline $\mathrm{C}$ & 2.1250633643 & -3.6729225166 & -3.0848042709 \\
\hline $\mathrm{H}$ & 0.9715758463 & -5.2576287494 & -2.2006787316 \\
\hline $\mathrm{H}$ & 3.4398675098 & -2.0729016501 & -3.6848498501 \\
\hline $\mathrm{C}$ & 1.1384166246 & -3.7003704714 & 4.5896968800 \\
\hline $\mathrm{H}$ & 1.7791398541 & -1.7036612483 & 5.0980062826 \\
\hline $\mathrm{H}$ & 0.7395719421 & -5.6869585380 & 3.8684906233 \\
\hline $\mathrm{C}$ & 7.0560878699 & -7.2380105799 & 1.3287655906 \\
\hline $\mathrm{H}$ & 7.2000194472 & -7.0553448930 & -0.8141529926 \\
\hline $\mathrm{H}$ & 6.7085120472 & -7.1760076721 & 3.4552132287 \\
\hline $\mathrm{H}$ & 9.0284738315 & 2.6083746521 & -4.4380699333 \\
\hline $\mathrm{C}$ & 0.0020804686 & 0.9821982115 & -2.7911478069 \\
\hline $\mathrm{H}$ & -1.6450303454 & 2.1860004393 & -2.0968307786 \\
\hline $\mathrm{H}$ & 1.8407487153 & 0.0530713513 & -3.4205649712 \\
\hline $\mathrm{C}$ & 3.1289554146 & 7.9709173270 & -3.8793996093 \\
\hline $\mathrm{H}$ & 3.7713968330 & 9.0863584746 & -2.1499702084 \\
\hline $\mathrm{H}$ & 2.4507990178 & 6.6113775331 & -5.4081560571 \\
\hline $\mathrm{C}$ & 0.8917172646 & 6.0619451154 & 2.9783655268 \\
\hline $\mathrm{H}$ & 2.4415439589 & 5.0226567784 & 4.0556168385 \\
\hline $\mathrm{H}$ & -0.5094695191 & 6.9935918551 & 1.6306867044 \\
\hline $\mathrm{H}$ & 1.6046024766 & -3.7275411668 & -4.0361940734 \\
\hline $\mathrm{H}$ & 0.3733950818 & -3.7539823252 & 5.3574982646 \\
\hline $\mathrm{H}$ & 7.7095828787 & -8.1040840465 & 1.3794550120 \\
\hline $\mathrm{H}$ & -0.6533959654 & 0.1947148171 & -3.1444320262 \\
\hline $\mathrm{H}$ & 3.2142234080 & 8.8163347975 & -4.5558333519 \\
\hline $\mathrm{H}$ & 0.4299779430 & 6.4716132816 & 3.8716070932 \\
\hline $\mathrm{P}$ & -3.0762213114 & -0.0215385240 & -0.8190035423 \\
\hline $\mathrm{O}$ & -3.8305697843 & -1.2417743735 & -0.0281608854 \\
\hline $\mathrm{O}$ & -4.2621487777 & 1.1267547335 & -0.7673645771 \\
\hline $\mathrm{C}$ & -4.8519779253 & -1.7935451560 & -0.7927440083 \\
\hline $\mathrm{C}$ & -5.3409651206 & 1.1038397096 & 0.1013989374 \\
\hline $\mathrm{C}$ & -6.0595543736 & -1.1199215847 & -0.8209257172 \\
\hline $\mathrm{C}$ & -4.5515394529 & -2.9626614341 & -1.5339330686 \\
\hline $\mathrm{C}$ & -6.2527332637 & 0.0557804515 & 0.0759703143 \\
\hline
\end{tabular}




\begin{tabular}{|c|c|c|c|}
\hline $\mathrm{C}$ & -5.4653955217 & 2.2417638240 & 然 \\
\hline & -7.0601552783 & -1.5904599890 & -1.7342414682 \\
\hline $\mathrm{C}$ & -5.5491790632 & -3.4383364745 & -2.3642096207 \\
\hline $\mathrm{si}$ & -2.8662907528 & -3.8210749533 & -1.4680392136 \\
\hline $\mathrm{C}$ & -7.3454790337 & 0.0957001225 & 1.0107546130 \\
\hline $\mathrm{C}$ & -6.5753937802 & 2.3064519959 & 1.76699864 \\
\hline $\mathrm{Si}$ & -4.0803907485 & 3.5088921269 & 1.162336584 \\
\hline $\mathrm{C}$ & -8.2834257521 & -0.9042279697 & -1.957136852 \\
\hline $\mathrm{C}$ & -6.7975305591 & -2.7753728283 & -2.497795896 \\
\hline $\mathrm{H}$ & -5.3756163195 & -4.3207743258 & -2.97575 \\
\hline $\mathrm{C}$ & -1.5491040229 & -2.9553924991 & -0.44056 \\
\hline $\mathrm{C}$ & -2.1921910396 & -4.0038948318 & -3.21102387 \\
\hline $\mathrm{C}$ & -3.0549584252 & -5.5176998868 & -0.66671767 \\
\hline $\mathrm{C}$ & -8.2463196478 & -0.9889168646 & $1.18960^{\circ}$ \\
\hline $\mathrm{C}$ & -7.5257114939 & 1.2564995769 & 1.82842 \\
\hline $\mathrm{H}$ & -6.7154029704 & 3.1617321516 & 2.42 \\
\hline $\mathrm{C}$ & -2.6216620880 & 2.6267825835 & 1.971907119 \\
\hline $\mathrm{C}$ & -4.7069623456 & 4.8103383993 & 2.3707 \\
\hline $\mathrm{C}$ & -3.4713511629 & 961001 & -0.36 \\
\hline $\mathrm{C}$ & -9.2112319745 & -1.3841481197 & -2.8532 \\
\hline $\mathrm{H}$ & -8.4764801700 & 0.0135358015 & -1.41 \\
\hline $\mathrm{C}$ & -7.7821649657 & 3662955 & -3.4 \\
\hline $\mathrm{C}$ & -0.4316360094 & -2.3578887174 & -1.03145 \\
\hline $\mathrm{C}$ & -1.6344200320 & -2.9619894941 & \\
\hline $\mathrm{C}$ & -2.1578589698 & -2.8862920094 & -4.06655 \\
\hline $\mathrm{C}$ & -1.5967435592 & 0918182 & -3.6 \\
\hline $\mathrm{C}$ & -1.8937 & 247728 & -0.2 \\
\hline $\mathrm{C}$ & -4.2920855076 & -6.1472668865 & -0.4642620063 \\
\hline $\mathrm{C}$ & -9.2902946462 & -0.9054020020 & \\
\hline $\mathrm{H}$ & -8.1007794219 & 0232493 & 0.61 \\
\hline $\mathrm{C}$ & -8.6201001653 & 1.3170023764 & 2.73 \\
\hline $\mathrm{C}$ & -1.307 & 774205 & 1.78 \\
\hline $\mathrm{C}$ & -2.8097450458 & 1.4794028946 & 2.7596043870 \\
\hline $\mathrm{C}$ & -5.5221325634 & 374199 & 1.92557 \\
\hline $\mathrm{C}$ & -4.409598 & 773500 & 3.7 \\
\hline $\mathrm{C}$ & -3.6079805312 & 3.9818936294 & -1.68715 \\
\hline $\mathrm{C}$ & -2.7647744440 & 5.6303539632 & -0.14 \\
\hline $\mathrm{C}$ & -8.9676920058 & -2.5741849453 & -3.57778 \\
\hline $\mathrm{H}$ & -10.1372319346 & -0.8397338669 & -3.01071 \\
\hline $\mathrm{H}$ & -7.5714822322 & 4964587 & -3.971004333 \\
\hline $\mathrm{C}$ & 0.5834245668 & -1.7939379149 & -0.2476920892 \\
\hline $\mathrm{H}$ & -0.3314641490 & -2.3437593905 & -2.10 \\
\hline $\mathrm{C}$ & -0.6440948987 & -2.3894230149 & 1.75394666 \\
\hline $\mathrm{H}$ & -2.4953913209 & -3.4069080146 & 1.451833295 \\
\hline $\mathrm{C}$ & -1.5301211319 & -2.9629163596 & -5.309975375 \\
\hline $\mathrm{H}$ & -2.6011899154 & -1.9496727380 & -3.7412832093 \\
\hline $\mathrm{C}$ & -0.9708485277 & -5.2759503241 & -4.8937622013 \\
\hline $\mathrm{H}$ & -1.6192893675 & -6.0764775624 & -3.0097693695 \\
\hline $\mathrm{C}$ & -1.9650921499 & -7.4725805795 & 0.311466408 \\
\hline $\mathrm{H}$ & -0.9230489916 & -5.7269525580 & -0.3754573908 \\
\hline $\mathrm{C}$ & -4.3705943667 & -7.4157904498 & 0.114766465 \\
\hline $\mathrm{H}$ & -5.2063835913 & -5.6376056803 & -0.753262056 \\
\hline $\mathrm{C}$ & -9.4934422123 & 0.2626020699 & 2.853435345 \\
\hline $\mathrm{H}$ & -9.9611513535 & -1.7506747425 & 2.202594675 \\
\hline $\mathrm{H}$ & -8.7423457087 & 2.2126948990 & 3.333058208 \\
\hline $\mathrm{C}$ & -0.2154269195 & 2.4027893202 & 2.334321077 \\
\hline $\mathrm{H}$ & -1.1175240356 & 3.9770368808 & 1.195343527 \\
\hline $\mathrm{C}$ & -1.7281581555 & 0.7866556187 & 3.307154288 \\
\hline $\mathrm{H}$ & -3.8131402596 & 1.0908148961 & 2.90787364 \\
\hline & -6.0271594771 & 6.8121412674 & 2.81624173 \\
\hline
\end{tabular}




$\begin{array}{lrrr}\mathrm{H} & -5.7556530587 & 5.9546495652 & 0.8678295199 \\ \mathrm{C} & -4.9113831268 & 5.6847591094 & 4.6375011898 \\ \mathrm{H} & -3.7745059781 & 3.9398199337 & 4.1106645723 \\ \mathrm{C} & -3.0373002421 & 4.6876127736 & -2.7480961952 \\ \mathrm{H} & -4.1439646988 & 3.0628813846 & -1.8895744497 \\ \mathrm{C} & -2.1889280130 & 6.3347973455 & -1.2050059463 \\ \mathrm{H} & -2.6661236538 & 6.0214693386 & 0.8603278314 \\ \mathrm{H} & -9.7108152242 & -2.9429277139 & -4.2777481544 \\ \mathrm{C} & 0.4770826596 & -1.8084096930 & 1.1490072890 \\ \mathrm{H} & 1.4736922575 & -1.3933703803 & -0.7183431140 \\ \mathrm{H} & -0.7278999408 & -2.4147758103 & 2.8338446664 \\ \mathrm{C} & -0.9328781532 & -4.1553719729 & -5.7249197758 \\ \mathrm{H} & -1.5063247696 & -2.0899427532 & -5.9560369330 \\ \mathrm{H} & -0.5156509598 & -6.2084375274 & -5.2149115477 \\ \mathrm{C} & -3.2074195575 & -8.0830858460 & 0.4975071384 \\ \mathrm{H} & -1.0560568607 & -7.9840779530 & 0.6146748731 \\ \mathrm{H} & -5.3396001405 & -7.8820812017 & 0.2671160046 \\ \mathrm{H} & -10.3248623314 & 0.3156217071 & 3.5491348041 \\ \mathrm{C} & -0.4229493566 & 1.2345156246 & 3.0875649246 \\ \mathrm{H} & 0.7873016951 & 2.7906858037 & 2.2026141338 \\ \mathrm{H} & -1.9013605741 & -0.1174638055 & 3.8819979518 \\ \mathrm{C} & -5.7222266447 & 6.7217658364 & 4.1758235657 \\ \mathrm{H} & -6.6544090016 & 7.6204642341 & 2.4518031420 \\ \mathrm{H} & -4.6673996973 & 5.6135564670 & 5.6934679921 \\ \mathrm{C} & -2.3169983155 & 5.8583161146 & -2.5104952639 \\ \mathrm{H} & -3.1482950165 & 4.3140261513 & -3.7615930031 \\ \mathrm{H} & -1.6459443323 & 7.2555128891 & -1.0114721252 \\ \mathrm{H} & 1.2652168120 & -1.3994345088 & 1.7679353381 \\ \mathrm{H} & -0.4451355156 & -4.2129207415 & -6.6938558204 \\ \mathrm{H} & -3.2674064396 & -9.0706803097 & 0.9450652135 \\ \mathrm{H} & 0.4381421155 & 0.7054651023 & 3.4844131774 \\ \mathrm{H} & -6.1125003215 & 7.4590906701 & 4.8712002494 \\ \mathrm{H} & -1.8622834271 & 6.3984726969 & -3.3353118488 \\ \mathrm{C} & -2.9286146235 & -5.6872461400 & 3.2852119384 \\ \mathrm{H} & -3.4507596341 & -5.4137825591 & 2.3592132050 \\ \mathrm{H} & -2.6185655520 & -6.7368624370 & 3.1994263371 \\ \mathrm{H} & -3.6321225793 & -5.6016887472 & 4.1174394740 \\ \mathrm{O} & -1.8392244960 & -4.8209331361 & 3.5754890712 \\ \mathrm{H} & -1.2402777134 & -4.8231104989 & 2.8172439081 \\ & & & \end{array}$

$\begin{array}{lrrr}\text { CATb-MeOH } & & \\ 01 & & & \\ \mathrm{Mg} & 0.9174894099 & 1.1856007231 & -0.3728477255 \\ \mathrm{O} & 2.6446981626 & 0.6102572757 & 0.0612078671 \\ \mathrm{O} & -5.3894212357 & 2.7298724718 & 0.6982726726 \\ \mathrm{O} & 2.8176336624 & -0.8734140138 & 2.1910325344 \\ \mathrm{O} & -4.7599524838 & 0.3273951627 & 1.6598542680 \\ \mathrm{P} & -4.8616363746 & 1.3582729436 & 0.5659078317 \\ \mathrm{O} & -5.5353918499 & 0.7073144658 & -0.8131302552 \\ \mathrm{O} & -3.2301609751 & 1.3623706740 & -0.0109077862 \\ \mathrm{C} & -5.1103901228 & -0.5495027692 & -1.1563857320 \\ \mathrm{C} & -2.8578338340 & 1.5211512699 & -1.3044506047 \\ \mathrm{C} & -3.9577515519 & -0.6561882146 & -1.9239537858 \\ \mathrm{C} & -5.8693973812 & -1.6554571512 & -0.6921394092 \\ \mathrm{C} & -3.2176799670 & 0.5901232381 & -2.2873371214 \\ \mathrm{C} & -1.9486605194 & 2.6026825559 & -1.5476269173 \\ \mathrm{C} & -3.4764983211 & -1.9789489296 & -2.2406667643 \\ \mathrm{C} & -5.3786823617 & -2.9129048826 & -0.9755712859 \\ \mathrm{Si} & -7.4896783778 & -1.5342668244 & 0.2873964733 \\ \mathrm{C} & -2.8159786663 & 0.8608747284 & -3.6378204151\end{array}$




\begin{tabular}{|c|c|c|c|}
\hline $\mathrm{C}$ & -1.4455743951 & 2.7372081674 & -2.8322376797 \\
\hline $\mathrm{Si}$ & -1.4544760112 & 3.7563828753 & -0.1084493646 \\
\hline $\mathrm{C}$ & -2.3010135790 & -2.2472347357 & -2.9929900302 \\
\hline $\mathrm{C}$ & -4.1941691774 & -3.1109546910 & -1.7286701289 \\
\hline $\mathrm{H}$ & -5.8943436572 & -3.7940558347 & -0.6008128664 \\
\hline $\mathrm{C}$ & -8.0377023103 & 0.1759016975 & 0.8157431813 \\
\hline $\mathrm{C}$ & -7.2657749831 & -2.6666370705 & 1.7719326597 \\
\hline $\mathrm{C}$ & -8.8563636189 & -2.2272503566 & -0.8176965718 \\
\hline $\mathrm{C}$ & -3.3041807030 & 0.1134465151 & -4.7440765165 \\
\hline $\mathrm{C}$ & -1.8847278511 & 1.9172591440 & -3.9020973315 \\
\hline $\mathrm{H}$ & -0.7203296532 & 3.5075301836 & -3.0646838451 \\
\hline $\mathrm{C}$ & -0.6738085179 & 5.3364811264 & -0.8047516807 \\
\hline $\mathrm{C}$ & -0.2124287659 & 2.7977953379 & 0.9981894966 \\
\hline $\mathrm{C}$ & -2.8127362598 & 4.3553111527 & 1.0196212645 \\
\hline $\mathrm{C}$ & -1.8477975816 & -3.5310932709 & -3.1996348187 \\
\hline $\mathrm{H}$ & -1.7457099950 & -1.4274124426 & -3.4175270762 \\
\hline $\mathrm{C}$ & -3.7081095553 & -4.4232103943 & -1.9639934816 \\
\hline $\mathrm{C}$ & -7.9213331495 & 0.6251038039 & 2.1393150841 \\
\hline $\mathrm{C}$ & -8.5836537974 & 1.0539750843 & -0.1363472729 \\
\hline $\mathrm{C}$ & -6.2645795088 & -2.3826492400 & 2.7207753271 \\
\hline $\mathrm{C}$ & -8.0540323694 & -3.8129471830 & 1.9588846712 \\
\hline $\mathrm{C}$ & -10.1933000790 & -2.0768956630 & -0.4043325285 \\
\hline $\mathrm{C}$ & -8.6189339228 & -2.9065909025 & -2.0226228904 \\
\hline $\mathrm{C}$ & -2.8573014170 & 0.3610457087 & -6.0217273362 \\
\hline $\mathrm{H}$ & -4.0434438630 & -0.6572362242 & -4.5641928692 \\
\hline $\mathrm{C}$ & -1.4262950975 & 2.1340104945 & -5.2295864756 \\
\hline $\mathrm{C}$ & -0.9737998489 & 5.8435612583 & -2.0826631380 \\
\hline $\mathrm{C}$ & 0.1191846412 & 6.1485587819 & 0.0310005758 \\
\hline $\mathrm{C}$ & -0.6653800859 & 1.5767986734 & 1.5800741176 \\
\hline $\mathrm{C}$ & 1.0119855604 & 3.3141852571 & 1.4931720389 \\
\hline $\mathrm{C}$ & -2.9761157957 & 3.8957739314 & 2.3340828958 \\
\hline $\mathrm{C}$ & -3.6809976249 & 5.3536536795 & 0.5491620352 \\
\hline $\mathrm{C}$ & -2.5551948935 & -4.6380566800 & -2.6806237882 \\
\hline $\mathrm{H}$ & -0.9341061328 & -3.6909366297 & -3.7639391727 \\
\hline $\mathrm{H}$ & -4.2719617065 & -5.2582234479 & -1.5576834831 \\
\hline $\mathrm{C}$ & -8.3163425104 & 1.9137613148 & 2.4958138703 \\
\hline $\mathrm{H}$ & -7.4992382856 & -0.0273237005 & 2.8946850214 \\
\hline $\mathrm{C}$ & -8.9776637355 & 2.3411236221 & 0.2155515390 \\
\hline $\mathrm{H}$ & -8.6901980475 & 0.7306966217 & -1.1680546528 \\
\hline $\mathrm{C}$ & -6.0701164803 & -3.2164048515 & 3.8212466262 \\
\hline $\mathrm{H}$ & -5.6417266184 & -1.5023162046 & 2.5897548676 \\
\hline $\mathrm{C}$ & -7.8542351576 & -4.6523115292 & 3.0567694752 \\
\hline $\mathrm{H}$ & -8.8322769744 & -4.0521988135 & 1.2398616661 \\
\hline $\mathrm{C}$ & -11.2468919670 & -2.5972908245 & -1.1539732624 \\
\hline $\mathrm{H}$ & -10.4111963917 & -1.5347058600 & 0.5118838533 \\
\hline $\mathrm{C}$ & -9.6697287137 & -3.4246878548 & -2.7815120760 \\
\hline $\mathrm{H}$ & -7.6006864625 & -3.0274084924 & -2.3788116756 \\
\hline $\mathrm{C}$ & -1.8949950908 & 1.3688465708 & -6.2708589314 \\
\hline $\mathrm{H}$ & -3.2520651663 & -0.2192023560 & -6.8498672118 \\
\hline $\mathrm{H}$ & -0.7132019007 & 2.9352974476 & -5.4068394153 \\
\hline $\mathrm{C}$ & -0.4488612437 & 7.0569237623 & -2.5308935361 \\
\hline $\mathrm{H}$ & -1.6453600248 & 5.3004692157 & -2.7371064032 \\
\hline $\mathrm{C}$ & 0.6552708857 & 7.3558159160 & -0.4130807077 \\
\hline $\mathrm{H}$ & 0.2923197125 & 5.8581374901 & 1.0610453499 \\
\hline $\mathrm{C}$ & 0.0632545915 & 0.9155100683 & 2.5722622844 \\
\hline $\mathrm{H}$ & -1.6159272127 & 1.1600582505 & 1.2752912457 \\
\hline $\mathrm{C}$ & 1.7242684622 & 2.6664912569 & 2.5020371705 \\
\hline $\mathrm{H}$ & 1.4184168516 & 4.2273435220 & 1.0831227687 \\
\hline $\mathrm{C}$ & -3.9859266468 & 4.4036699749 & 3.1472388525 \\
\hline $\mathrm{H}$ & -2.3273740372 & 3.1209668773 & 2.7281548621 \\
\hline
\end{tabular}




\begin{tabular}{|c|c|c|c|}
\hline $\mathrm{C}$ & -4.6962324031 & 5.8560395309 & 1.3563548063 \\
\hline $\mathrm{H}$ & -3.5682637331 & 5.7401314297 & -0.4602700797 \\
\hline $\mathrm{H}$ & -2.1954294837 & -5.6474342037 & -2.8521505895 \\
\hline $\mathrm{C}$ & -8.8395279093 & 2.7749295651 & 1.5345382451 \\
\hline $\mathrm{H}$ & -8.2005159665 & 2.2484040065 & 3.5224011472 \\
\hline $\mathrm{H}$ & -9.3814369551 & 3.0099894297 & -0.5389089391 \\
\hline $\mathrm{C}$ & -6.8620120688 & -4.3542065796 & 3.9904178187 \\
\hline $\mathrm{H}$ & -5.2973111546 & -2.9787189649 & 4.5471343225 \\
\hline $\mathrm{H}$ & -8.4732380562 & -5.5362866449 & 3.1831524244 \\
\hline $\mathrm{C}$ & -10.9861049327 & -3.2744212562 & -2.3467264877 \\
\hline $\mathrm{H}$ & -12.2702723395 & -2.4697429211 & -0.8127113323 \\
\hline $\mathrm{H}$ & -9.4604013991 & -3.9427666759 & -3.7132901592 \\
\hline $\mathrm{H}$ & -1.5474483124 & 1.5472052785 & -7.2834399685 \\
\hline $\mathrm{C}$ & 0.3863703051 & 7.8071201845 & -1.7055498300 \\
\hline $\mathrm{H}$ & -0.7007748877 & 7.4171748856 & -3.5238438672 \\
\hline $\mathrm{H}$ & 1.2687271464 & 7.9520023685 & 0.2560126649 \\
\hline $\mathrm{C}$ & 1.2539704105 & 1.4684557249 & 3.0442361065 \\
\hline $\mathrm{H}$ & -0.3251476969 & -0.0236449586 & 2.9498870179 \\
\hline $\mathrm{H}$ & 2.6598595737 & 3.0866362909 & 2.8460154855 \\
\hline $\mathrm{C}$ & -4.8507874826 & 5.3795994763 & 2.6574618688 \\
\hline $\mathrm{H}$ & -4.1067902737 & 4.0237984061 & 4.1570310212 \\
\hline $\mathrm{H}$ & -5.3709675281 & 6.6133944614 & 0.9689359922 \\
\hline $\mathrm{H}$ & -9.1329834179 & 3.7841281522 & 1.8087292378 \\
\hline $\mathrm{H}$ & -6.7069801657 & -5.0045400928 & 4.8469134933 \\
\hline $\mathrm{H}$ & -11.8051746969 & -3.6771115826 & -2.9357433080 \\
\hline $\mathrm{H}$ & 0.8028113079 & 8.7466854360 & -2.0554431567 \\
\hline $\mathrm{H}$ & 1.8346751163 & 0.9475522767 & 3.7946735506 \\
\hline $\mathrm{H}$ & -5.6476268225 & 5.7652368394 & 3.2863928594 \\
\hline $\mathrm{P}$ & 3.4306608167 & -0.3505346354 & 0.9452963137 \\
\hline $\mathrm{O}$ & 3.9301905631 & -1.5232293664 & -0.0925964142 \\
\hline $\mathrm{O}$ & 4.8758775499 & 0.3670042388 & 1.2791198531 \\
\hline $\mathrm{C}$ & 4.5932229777 & -2.5405932941 & 0.5904095474 \\
\hline $\mathrm{C}$ & 6.0465363742 & 0.1642657375 & 0.5611847449 \\
\hline $\mathrm{C}$ & 5.9095757228 & -2.3144004857 & 0.9481216290 \\
\hline $\mathrm{C}$ & 3.8448079807 & -3.6919539519 & 0.9304012971 \\
\hline $\mathrm{C}$ & 6.5997259098 & -1.1022528751 & 0.4248658295 \\
\hline $\mathrm{C}$ & 6.6493028781 & 1.3455118340 & 0.0444771995 \\
\hline $\mathrm{C}$ & 6.5373274987 & -3.2696244475 & 1.8138361056 \\
\hline $\mathrm{C}$ & 4.4838523011 & -4.6339709306 & 1.7149923294 \\
\hline $\mathrm{Si}$ & 2.0566524225 & -3.9792295751 & 0.3911775814 \\
\hline $\mathrm{C}$ & 7.8173786613 & -1.2252665109 & -0.3332893810 \\
\hline $\mathrm{C}$ & 7.8675336270 & 1.2187687281 & -0.5940309446 \\
\hline $\mathrm{Si}$ & 5.7312211576 & 2.9888799297 & -0.0172735569 \\
\hline $\mathrm{C}$ & 7.8317192842 & -3.0756040920 & 2.3646381966 \\
\hline $\mathrm{C}$ & 5.8099593888 & -4.4474924212 & 2.1855914029 \\
\hline $\mathrm{H}$ & 3.9562073286 & -5.5325269846 & 2.0259599139 \\
\hline $\mathrm{C}$ & 1.2491876278 & -2.5194138170 & -0.5124942782 \\
\hline $\mathrm{C}$ & 1.0023984201 & -4.3502446564 & 1.8921820556 \\
\hline $\mathrm{C}$ & 2.0165954418 & -5.3875667171 & -0.8545656794 \\
\hline $\mathrm{C}$ & 8.3873676156 & -2.4816512805 & -0.6751886083 \\
\hline $\mathrm{C}$ & 8.4760695668 & -0.0439924436 & -0.8016560517 \\
\hline $\mathrm{H}$ & 8.3621208344 & 2.0990913331 & -0.9934976241 \\
\hline $\mathrm{C}$ & 4.2717163546 & 2.7368766386 & -1.1951015370 \\
\hline $\mathrm{C}$ & 6.8713537055 & 4.2654619920 & -0.7961049771 \\
\hline $\mathrm{C}$ & 5.1852773319 & 3.6648020660 & 1.6462411129 \\
\hline $\mathrm{C}$ & 8.3899532550 & -4.0115524420 & 3.2052522231 \\
\hline $\mathrm{H}$ & 8.3759616425 & -2.1714375586 & 2.1215881066 \\
\hline $\mathrm{C}$ & 6.4210776968 & -5.3978063873 & 3.0456360788 \\
\hline $\mathrm{C}$ & 0.1665062671 & -1.8365695803 & 0.0491591522 \\
\hline $\mathrm{C}$ & 1.6703611876 & -2.1473159752 & -1.8052880786 \\
\hline
\end{tabular}




\begin{tabular}{|c|c|c|c|}
\hline $\mathrm{C}$ & 3599 & -3.4930401577 & 1784 \\
\hline $\mathrm{C}$ & 0.0692929985 & -5.3996443164 & 1.8971666911 \\
\hline $\mathrm{C}$ & 0.8379198054 & -5.6129030639 & -1.5913867243 \\
\hline $\mathrm{C}$ & 3.1157250537 & -6.2262891258 & -1.0958137023 \\
\hline $\mathrm{C}$ & 9.5636075226 & -2.5583178963 & -1.3865151830 \\
\hline $\mathrm{H}$ & 7.8824542604 & -3.3904150322 & -0.3758662767 \\
\hline $\mathrm{C}$ & 9.6949397069 & -0.1560907973 & -1.5206424897 \\
\hline$C$ & 3.1096092349 & 3.5148321776 & -1.1233255640 \\
\hline $\mathrm{C}$ & 4.3228976648 & 1.7285352132 & -2.1745373235 \\
\hline $\mathrm{C}$ & 7.7233929785 & 5.0470200482 & 0.0031054162 \\
\hline $\mathrm{C}$ & 6.9344455521 & 4.4336202253 & -2.1897775498 \\
\hline $\mathrm{C}$ & 5.3533634411 & 2.9586247429 & 2.8484989496 \\
\hline $\mathrm{C}$ & 4.6227161016 & 4.9538696663 & 1537 \\
\hline $\mathrm{C}$ & 7.6860812540 & -5.1911194902 & 3.5424862271 \\
\hline $\mathrm{H}$ & 9.3786832991 & -3.8396553966 & \\
\hline $\mathrm{H}$ & 5.8599670256 & -6.2887428916 & 3.3129646299 \\
\hline $\mathrm{C}$ & 0.5007866655 & -0.8236793622 & 921905 \\
\hline $\mathrm{H}$ & -0.1975864477 & -2.0921606273 & 1.0347825681 \\
\hline 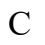 & 1.0683959650 & -1.1001792027 & -2.4879636673 \\
\hline $\mathrm{H}$ & 20956 & 0447 & 522021 \\
\hline $\mathrm{C}$ & 471 & & \\
\hline $\mathrm{H}$ & 241797 & -2.667 & \\
\hline $\mathrm{C}$ & -0.7909115677 & -5.5875598981 & 2.9800052455 \\
\hline $\mathrm{H}$ & 470 & 554 & 5967 \\
\hline $\mathrm{C}$ & 0.7 & 190 & 3147 \\
\hline $\mathrm{H}$ & -0.0270235939 & -4.9728503824 & -1.4416313751 \\
\hline C & 11789 & -7.2590387685 & 7261249 \\
\hline 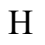 & 4.04 & -6.066 & 5104 \\
\hline $\mathrm{C}$ & 837278 & -1.3 & 22547 \\
\hline $\mathrm{H}$ & 9.9747193404 & -3.5315341325 & -1.6364465918 \\
\hline$U$ & 38702 & 87 & 91641 \\
\hline$C$ & 54347 & 931 & 26689 \\
\hline $\mathrm{H}$ & 3.0385198983 & 4.3181237172 & -0.3997147923 \\
\hline $\mathrm{C}$ & 3.2487691937 & 1.4867018501 & -3.0271476056 \\
\hline 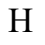 & 113 & 1.0 & 7842 \\
\hline $\mathrm{C}$ & 8.6114683273 & 5.95 & 97331 \\
\hline $\mathrm{H}$ & 7.6852673406 & 4.9476146164 & 1.0842961002 \\
\hline$C$ & 784 & & 42260 \\
\hline 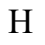 & 6.27 & 3.8 & 16963 \\
\hline $\mathrm{C}$ & 4.9616832452 & 3.5123068917 & 7247202 \\
\hline $\mathrm{H}$ & 952716 & 1.9615917625 & 2.8315937974 \\
\hline $\mathrm{C}$ & 83 & 5. & 308214 \\
\hline 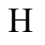 & 6465 & 5.54 & 0.80 \\
\hline $\mathrm{H}$ & 8.1412940913 & -5.9220633906 & 4.2031328873 \\
\hline C & .0260696722 & & -1.9172451034 \\
\hline $\mathrm{H}$ & 03 & -0.4 & 881595 \\
\hline $\mathrm{H}$ & 1.4147619214 & -0.8171395249 & -3.4756055159 \\
\hline $\mathrm{C}$ & -0.7316134779 & -4.7245016919 & 4.0741111677 \\
\hline 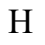 & & & \\
\hline$H$ & -1.51 & -6.40 & 95443 \\
\hline $\mathrm{C}$ & 1.8639122572 & -7.4710332925 & -2.7467127122 \\
\hline $\mathrm{H}$ & -0.1570490660 & -6.8036103207 & -3.0838214032 \\
\hline 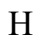 & & -7.8951417705 & -2.2047504916 \\
\hline 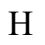 & 11.1675698173 & -1.4644176427 & -2.3564080640 \\
\hline $\mathrm{C}$ & 2.0784740471 & 2.2440080130 & -2.9144035621 \\
\hline $\mathrm{H}$ & 483 & 3.92 & -1.90 \\
\hline$U$ & 3159710201 & 0.6975585901 & -3.7685285930 \\
\hline $\mathrm{C}$ & 8.6617603892 & 6.1060777269 & -1.9551905698 \\
\hline $\mathrm{H}$ & 9.2610606434 & 6.5531492359 & 0.0664256247 \\
\hline $\mathrm{H}$ & 7.8526779074 & 5.4599444566 & -3.8456556358 \\
\hline
\end{tabular}




$\begin{array}{rrrr}\mathrm{C} & 4.3808018847 & 4.7805389187 & 4.1098713805 \\ \mathrm{H} & 5.1016882894 & 2.9482103269 & 4.9858040230 \\ \mathrm{H} & 3.7703509173 & 6.4950773182 & 2.9527330889 \\ \mathrm{H} & -0.5645078098 & 0.3143621514 & -2.4933287101 \\ \mathrm{H} & -1.4080392018 & -4.8628300575 & 4.9123023575 \\ \mathrm{H} & 1.8058456025 & -8.2732439336 & -3.4763807404 \\ \mathrm{H} & 1.2403266260 & 2.0637266250 & -3.5807074062 \\ \mathrm{H} & 9.3508845488 & 6.8166344151 & -2.4018462585 \\ \mathrm{H} & 4.0655872926 & 5.2060283369 & 5.0576608441 \\ \mathrm{O} & -2.4213853577 & -1.0571011398 & 1.8069710158 \\ \mathrm{H} & -3.2465488332 & -0.5128698725 & 1.7869989048 \\ \mathrm{C} & -2.8243242955 & -2.4135587521 & 1.9024528233 \\ \mathrm{H} & -3.5756810349 & -2.6730904269 & 1.1475402776 \\ \mathrm{H} & -1.9484298780 & -3.0497926393 & 1.7501703429 \\ \mathrm{H} & -3.2395440347 & -2.6550274361 & 2.8908222078\end{array}$

\section{Unsubstituted Model}

\begin{tabular}{lrrr} 
CY1 & & & \\
01 & & & \\
$\mathrm{O}$ & 0.0446115596 & 0.5527046608 & 0.9338260313 \\
$\mathrm{C}$ & -0.9578399103 & -0.0410394269 & 0.5895812356 \\
$\mathrm{C}$ & -2.5925680154 & -1.2825521165 & -0.6701385263 \\
$\mathrm{C}$ & -2.0295564588 & -0.5062638119 & 1.5350322730 \\
$\mathrm{C}$ & -2.6360775559 & -1.7052446628 & 0.8048460470 \\
$\mathrm{H}$ & -2.6475813355 & -2.1339380315 & -1.3626896713 \\
$\mathrm{H}$ & -3.4562668899 & -0.6326132676 & -0.8885626406 \\
$\mathrm{H}$ & -1.6191664279 & -0.6937172885 & 2.5353969962 \\
$\mathrm{H}$ & -2.7655107201 & 0.3147414971 & 1.6186345189 \\
$\mathrm{C}$ & -1.272689356 & -0.4852527091 & -0.8425710905 \\
$\mathrm{C}$ & -1.5200599667 & 0.7127507710 & -1.7753545805 \\
$\mathrm{H}$ & -2.3512100226 & 1.3261663231 & -1.4012153553 \\
$\mathrm{H}$ & -1.8009873635 & 0.3286756046 & -2.7659889317 \\
$\mathrm{Mg}$ & 2.0892998865 & 0.6968466717 & 0.7889819695 \\
$\mathrm{O}$ & 3.2244853882 & -0.4846160375 & -0.4925052010 \\
$\mathrm{O}$ & 2.1333971165 & -1.3134427921 & 1.5635188743 \\
$\mathrm{P}$ & 3.0749900445 & -1.6873422063 & 0.4315735196 \\
$\mathrm{O}$ & 2.5046511021 & -2.9907507104 & -0.3302186446 \\
$\mathrm{O}$ & 4.5303004657 & -2.1473973570 & 0.9338927396 \\
$\mathrm{C}$ & 4.6444733685 & -3.0971078046 & 1.9796300917 \\
$\mathrm{H}$ & 4.2529378041 & -4.0787252973 & 1.6662515868 \\
$\mathrm{H}$ & 5.7112713547 & -3.1932295195 & 2.2161779048 \\
$\mathrm{H}$ & 4.0980145107 & -2.7611357710 & 2.8738207485 \\
$\mathrm{C}$ & 3.1249989794 & -3.4144005907 & -1.5328558605 \\
$\mathrm{H}$ & 2.5645788036 & -4.2831276446 & -1.9003993040 \\
$\mathrm{H}$ & 3.1029348899 & -2.6132612934 & -2.2876574261 \\
$\mathrm{H}$ & 4.1720762847 & -3.7070349925 & -1.3545387022 \\
$\mathrm{O}$ & 3.0029330911 & 1.8941355278 & 2.2892940643 \\
$\mathrm{C}$ & 2.9768096562 & 2.8693411214 & 1.5077195865 \\
$\mathrm{C}$ & 3.5206475739 & 4.2579051858 & 1.7538633014 \\
$\mathrm{~N}$ & 2.4233590512 & 2.7247375508 & 0.3010247095 \\
$\mathrm{C}$ & 3.2193525304 & 4.9624351481 & 0.4296637433 \\
$\mathrm{H}$ & 4.5895914505 & 4.1915763976 & 2.0025209712 \\
$\mathrm{H}$ & 3.0144541221 & 4.6971530493 & 2.6259035284 \\
$\mathrm{C}$ & 2.505148147 & 3.8981521147 & -0.4229100829 \\
$\mathrm{Br}$ & -0.0270173622 & 1.9212432585 & -2.0234775954 \\
$\mathrm{H}$ & 2.5625903751 & 5.8382729335 & 0.5296056309 \\
$\mathrm{H}$ & 4.1193661551 & 5.2968542387 & -0.1060406544 \\
$\mathrm{O}$ & 2.0864808870 & 4.0742142215 & -1.5411657709 \\
$\mathrm{C}$ & -0.1333139546 & -1.3793466184 & -1.3479323934 \\
& & & \\
\hline
\end{tabular}




$\begin{array}{lrrr}\mathrm{H} & -0.3878488632 & -1.7632224017 & -2.3472211205 \\ \mathrm{H} & 0.8110988993 & -0.8228821559 & -1.4310345682 \\ \mathrm{H} & 0.0410822949 & -2.2341214495 & -0.6792780685 \\ \mathrm{H} & -2.0077675731 & -2.5945438130 & 0.9695855434 \\ \mathrm{H} & -3.6501205051 & -1.9510065057 & 1.1467601030\end{array}$

EC1

01

O $\quad-0.7402626821$

C $\quad-2.0003602637$

C $\quad-3.1119497957$

C $\quad-2.8156352028$

$-0.4961827432$

$-0.2935271753$

1.4349349416

$-1.2667742546$

9604826101

0.8620447432

1.4890166028

C $\quad-4.0483143193$

$-0.0590564019$

1.6093312018

$\mathrm{H} \quad-2.7680000937$

$-1.6864885641$

1.6836055788

$-3.4456435380$

$-2.0851821714$

4452342019

$-2.3927547140$

1.0455999481

0.8321419272

$-2.8960962695$

1.8257948983

2.6072772695

$-2.0187686956$

$-0.3125649942$

1.0825300381

C $\quad-2.6849513700$

0.5706158209

0.5713598709

$-3.2845121039$

1.3786039551

$-1.3402883771$

$-2.7057818540$

0.4631785036

$-0.9147830557$

$\mathrm{Mg} \quad 1.0105095445$

$-2.4290924598$

O 2.4977019439

0431512861

1.4154034795

O 2.6799518891

.

$-0.1102178323$

$-0.8386196872$

2.2686112126

3.4301817318

$-0.7448060405$

0.9476239152

4.0396610176

$-2.1756344442$

0.5662446032

$0.2203816485 \quad 1.0512112812$

C $\quad 5.6765786787$

$-0.0140540490$

2.0808917426

6.1629045506

$-0.9935778558$

1.9501200905

6.4341717998

0.7765427770

2.0124234426

5.1952927892

0.0181475772

3.0699791110

C $\quad 4.6067882390$

$-2.3868204009$

$-0.7173522473$

4.8808132106

$-3.4468406944$

$-0.7787081835$

3.8811355478

$-2.1469356615$

$-1.5088577221$

5.5089971391

$-1.7694593901$

$-0.8557137231$

1.6016909801

2.0267066303

1.5546052300

$2.6933232201 \quad 0.6338921090$

2.0589877386
3.3976374156

3.3784946658

0.6151925656

2.8933705909

$-0.5623659412$

1.4446514102

4.1002742867

$-0.7315882089$

3.4518223417

2.5719829927

0.7102209282

4.1426613020
3.4934518886

4.0292493171

1.4949041724

3.6742960543

$-1.4722722629$

2.1948912946

2.0356781704

$-0.9738581788$

3.4380631085

5.1961737535

$-0.6407910513$

4.3249052981

3.8353490311

$-1.3428793754$

1.8567744019

3.9292719591

$-2.5865383764$

$-1.2314512847$

$-1.4363759582$

$-1.1869397873$

$-0.1514047035$

$-1.2829776538$

$-1.0372780554$

$-1.4798055839$

$-2.3908843333$

$-0.6983478736$

$-1.4227494196$

$-1.5239456164$

$-2.2653990168$

$-4.6425510157$

$-0.0287941634$

2.6079799285

$-4.7274001383$

0.0809523428

0.8294090260

EP1

01

$\begin{array}{lr}\mathrm{O} & 0.0441686384 \\ \mathrm{C} & -1.1285205353\end{array}$

0.4705090044

0.8052623417

$\begin{array}{ll}-0.3435196859 & 0.8151989752\end{array}$

C $\quad-1.1475243101$

$\begin{array}{ll}-1.8605311640 & 0.8010809982\end{array}$

C $\quad-2.1790524229$

$-0.2864596543$

1.9095535080 


\begin{tabular}{|c|c|c|c|}
\hline $\mathrm{C}$ & 7851 & 10738368 & 1629 \\
\hline $\mathrm{H}$ & -0.3246365767 & -2.2697046287 & 1.4025305481 \\
\hline $\mathrm{H}$ & 1.1518076620 & -2.3526328522 & -0.1811799058 \\
\hline $\mathrm{H}$ & -1.7054051678 & -0.1615367299 & 2.8939260866 \\
\hline $\mathrm{H}$ & -2.9917735730 & 0.4441140306 & 1.7937159872 \\
\hline $\mathrm{C}$ & -0.8940379605 & 0.5884322925 & -0.2978724232 \\
\hline $\mathrm{C}$ & -1.5953357253 & 1.9242397323 & -0.2985409808 \\
\hline $\mathrm{H}$ & -1.0454912220 & 2.6441795992 & -0.9191490611 \\
\hline $\mathrm{H}$ & -1.7111006342 & 2.3069591036 & 0.7214217170 \\
\hline $\mathrm{Mg}$ & 2.0821678295 & 0.3101942372 & 0.6565635154 \\
\hline $\mathrm{O}$ & 2.6901339133 & -1.2409823211 & -0.5810304729 \\
\hline $\mathrm{O}$ & 2.1931966042 & -1.4427129812 & 1.8308218449 \\
\hline $\mathrm{P}$ & 2.6769079950 & -2.2020486861 & 0.6029056989 \\
\hline $\mathrm{O}$ & 1.7160319497 & -3.4714804692 & 0.3589910358 \\
\hline $\mathrm{O}$ & 4.1355510921 & 907019 & 102095 \\
\hline $\mathrm{C}$ & 4.4342061519 & -3.6066131691 & 1.9406412285 \\
\hline $\mathrm{H}$ & 42416 & 616036 & 5465 \\
\hline $\mathrm{H}$ & 1900 & 0612474 & 51525 \\
\hline $\mathrm{H}$ & 4.2417932339 & 23111 & 1817 \\
\hline $\mathrm{C}$ & 1.8696779453 & -4.2549547691 & -0.8129377781 \\
\hline $\mathrm{H}$ & 5881 & -5.0 & 0347 \\
\hline $\mathrm{H}$ & 1.75 & 6242 & 39605 \\
\hline $\mathrm{H}$ & 2.8575878162 & -4.7418167879 & -0.8350695963 \\
\hline $\mathrm{O}$ & 5008 & 168 & 5633 \\
\hline $\mathrm{C}$ & 917 & 645 & 0085 \\
\hline $\mathrm{C}$ & 3.6920 & 69235 & 68307 \\
\hline $\mathrm{N}$ & 2.1433821744 & 2803330 & -0.3201045741 \\
\hline $\mathrm{C}$ & 165 & 4.4 & 2264 \\
\hline $\mathrm{H}$ & 522 & 322 & 7240 \\
\hline $\mathrm{H}$ & 3.5770407401 & 4.5070733348 & 1.2101557433 \\
\hline $\mathrm{C}$ & 413 & & 24518 \\
\hline $\mathrm{Br}$ & 4929 & 360 & 518595 \\
\hline $\mathrm{H}$ & 2.2879648737 & 94065 & 74095354 \\
\hline $\mathrm{H}$ & 3.4301857414 & 4.5923404732 & -1.7999123609 \\
\hline $\mathrm{O}$ & 341 & & 1632 \\
\hline $\mathrm{C}$ & 0913 & 975 & 4780 \\
\hline $\mathrm{H}$ & 0.2219380532 & 7638 & -2.0952000297 \\
\hline $\mathrm{H}$ & 5727 & & 545592 \\
\hline $\mathrm{H}$ & 3686 & -0 . & 92794 \\
\hline $\mathrm{H}$ & 56734 & -2.4 & 2.4403779783 \\
\hline $\mathrm{H}$ & -3.3574272230 & -1.8744169370 & 0.9279913747 \\
\hline \multicolumn{4}{|l|}{ TS1 } \\
\hline \multicolumn{4}{|l|}{01} \\
\hline $\mathrm{O}$ & & & \\
\hline $\mathrm{C}$ & & & \\
\hline $\mathrm{C}$ & 8441 & 7616 & 2.1819100752 \\
\hline $\mathrm{C}$ & 2.7049479128 & -1.0926698175 & 1.6063501713 \\
\hline $\mathrm{C}$ & & & 2.0241376846 \\
\hline $\mathrm{H}$ & 80 & 0.91 & 21624 \\
\hline $\mathrm{H}$ & 2.4077745506 & 2.004 & 588012 \\
\hline $\mathrm{H}$ & 2.3868023716 & -1.6878749765 & 2.4739056770 \\
\hline $\mathrm{H}$ & & & 0.8147007544 \\
\hline $\mathrm{C}$ & 1017 & 0.3283441382 & -0.1152440817 \\
\hline $\mathrm{C}$ & 1.6224053193 & -0.5204683858 & -1.2155255721 \\
\hline $\mathrm{H}$ & 2.5331158903 & -1.1198630970 & -1.1243122995 \\
\hline $\mathrm{H}$ & & -0.0716259687 & -2.2023318265 \\
\hline$M$ & & -0.9026253908 & 11206636542 \\
\hline $\mathrm{O}$ & -2.3904845287 & 0.3903057659 & -0.2701576866 \\
\hline $\mathrm{O}$ & -2.8714038275 & 0.2479332986 & 2.1541441251 \\
\hline
\end{tabular}




$\begin{array}{lr}\mathrm{P} & -3.2149438018 \\ \mathrm{O} & -2.9429168162 \\ \mathrm{O} & -4.7750708682 \\ \mathrm{C} & -5.7480169215 \\ \mathrm{H} & -5.7030300079 \\ \mathrm{H} & -6.7311271630 \\ \mathrm{H} & -5.5996056887 \\ \mathrm{C} & -3.1204212157 \\ \mathrm{H} & -2.8747798125 \\ \mathrm{H} & -2.4554508433 \\ \mathrm{H} & -4.1621553986 \\ \mathrm{O} & -2.2034685589 \\ \mathrm{C} & -2.2074154412 \\ \mathrm{C} & -3.1458098431 \\ \mathrm{~N} & -1.4128176973 \\ \mathrm{C} & -2.7907635766 \\ \mathrm{H} & -4.1816234270 \\ \mathrm{H} & -2.9715979828 \\ \mathrm{C} & -1.6503457216 \\ \mathrm{Br} & 0.1335101228 \\ \mathrm{H} & -2.4410342731 \\ \mathrm{H} & -3.6157747575 \\ \mathrm{O} & -1.0558795184 \\ \mathrm{C} & 0.5880055992 \\ \mathrm{H} & -0.3471601304 \\ \mathrm{H} & 0.3362434652 \\ \mathrm{H} & 1.2121823574 \\ \mathrm{H} & 4.2655755551 \\ \mathrm{H} & 4.2515778317 \\ & \end{array}$

TS1b

01

$\begin{array}{ll}\mathrm{O} & -0.1238087984 \\ \mathrm{C} & 0.9979617128 \\ \mathrm{C} & 1.1803462858 \\ \mathrm{C} & 2.2478864506 \\ \mathrm{C} & 2.5268783422 \\ \mathrm{H} & 0.3498217661 \\ \mathrm{H} & 1.1430306366 \\ \mathrm{H} & 1.9041893924 \\ \mathrm{H} & 3.0360321522 \\ \mathrm{C} & 1.1789561534 \\ \mathrm{C} & 2.4720841413 \\ \mathrm{H} & 3.3598370038 \\ \mathrm{H} & 2.5492734933 \\ \mathrm{Mg} & -0.8034886542 \\ \mathrm{O} & -1.8080464381 \\ \mathrm{O} & -2.8128030293 \\ \mathrm{P} & -3.1129245920 \\ \mathrm{O} & -4.2104065434 \\ \mathrm{O} & -3.7995428013 \\ \mathrm{C} & -4.9154295813 \\ \mathrm{H} & -5.7832181118 \\ \mathrm{H} & -5.1652915610 \\ \mathrm{H} & -4.6731560227 \\ \mathrm{C} & -4.5786563768 \\ \mathrm{H} & -5.3123007324 \\ \mathrm{H} & -3.7043635072 \\ \mathrm{H} & -5.0333933005 \\ \mathrm{O} & 0.2612645096 \\ & \end{array}$

0.9823204358

2.5575745691

0.9027167712

1.1895704713

2.2480650437

0.9821777955

0.5564479580

3.4452954644

4.4531932087

3.1823819180

3.4317432603

$-2.7412665994$

$-3.6264709655$

$-4.8155187682$

$-3.6578414476$

$-5.5841305014$

$-4.4483698136$

$-5.3752298145$

$-4.7767767008$

$-2.0832552145$

$-6.6103689157$

$-5.6464011459$

$-5.0489812851$

1.5966282765

1.3868697193

2.1155816292

2.2452123847

0.4128635178

$-0.0731077596$
0.8676546406

1.0694573527

0.4906000098

1.4815071366

1.7854188601

1.0412101094

2.3692686468

$-0.0184857264$

0.3387603839

$-0.8563076761$

$-0.3775480047$

0.9957540031

0.1153133258

0.1504603956

$-0.9483412883$

$-1.1195729500$

0.1864642632

1.0811719441

$-1.7491321865$

$-1.1651324641$

$-0.9396935301$

$-1.8434672836$

$-2.7550236816$

$-0.3542607378$

$-0.8998133120$

0.5776436193

$-0.9915847866$

1.1972041279

2.9129063984
$-1.9919445122$

$-2.4964451731$

$-4.1841857844$

$-2.5123833991$

$-4.0057195627$

$-4.4866408124$

$-4.7690881390$

$-2.3147928620$

$-1.7864731336$

$-2.4330439044$

$-2.1677384603$

$-2.4611939293$

$-2.4022571006$

$-0.2033428339$

0.0168492960

0.0559189244

0.1696396615

$-0.9348232983$

1.5666958815

2.0333908192

1.3665584971

3.0302021366

2.1000011363

$-1.0961583925$

$-1.9106186389$

$-1.3563875049$

$-0.1757333323$

1.3735011489
$-1.2376164380$

$-0.7615977359$

$-0.7236988090$

$-1.6712615045$

$-1.4252063166$

$-1.3755263621$

0.2071278238

$-2.6937298258$

$-1.4238240884$

0.6798095729

1.2973727250

0.7310689117

2.3638941772

$-1.0356042242$

0.8168409433

$-1.4450372038$

0.0438467457

0.4462571811

0.4396313901

$-0.3006073177$

$-0.1705585664$

0.0830165411

$-1.3720039844$

1.8039664616

1.8482467153

2.4208544004

2.2044641265

$-1.3820516747$ 


$\begin{array}{lrrr}\mathrm{C} & 0.9488717434 & 2.2909703059 & -0.8554180338 \\ \mathrm{C} & 0.9058054317 & 3.7085961550 & -1.4017244929 \\ \mathrm{~N} & 1.7592228303 & 2.1534150959 & 0.1735113810 \\ \mathrm{C} & 1.8479578916 & 4.4591819468 & -0.4696240902 \\ \mathrm{H} & -0.1340606718 & 4.0667863801 & -1.3882033530 \\ \mathrm{H} & 1.2258416189 & 3.6950987792 & -2.4544345440 \\ \mathrm{C} & 2.3690430865 & 3.3674965815 & 0.4776450385 \\ \mathrm{Br} & 2.2434740234 & -0.1701213424 & 1.0108244242 \\ \mathrm{H} & 2.7010804468 & 4.9346450389 & -0.9738974778 \\ \mathrm{H} & 1.3523299117 & 5.2373273378 & 0.1287701565 \\ \mathrm{O} & 3.1823918391 & 3.5353126540 & 1.3492813700 \\ \mathrm{C} & -0.0388314659 & -2.5183631891 & 1.5264396741 \\ \mathrm{H} & -0.5192021978 & -1.5236092924 & 1.5864155304 \\ \mathrm{H} & -0.7875278561 & -3.1607798478 & 1.0444420082 \\ \mathrm{H} & 0.1927202160 & -2.8662257362 & 2.5414879131 \\ \mathrm{H} & 3.3817533158 & -4.1968442450 & -0.7606702036 \\ \mathrm{H} & 2.6623748468 & -4.6184093498 & -2.3266059643\end{array}$

TS1c

01

$\mathrm{O}$

C $\quad-1.9214903129$

C $\quad-3.0274852503$

C $\quad-3.1314455328$

C $\quad-4.0960833332$

$\mathrm{H} \quad-3.2107860822$

$\mathrm{H} \quad-2.8167025573$

$\mathrm{H} \quad-3.2454294555$

$\mathrm{H} \quad-3.0227949218$

C $\quad-1.1405490651$

C $\quad-0.0282667438$

$\mathrm{H} \quad-0.0491968549$

$\mathrm{H} \quad 0.3169472951$

$\mathrm{Mg} \quad-0.1777075717$

O $\quad-0.6595201185$

O $\quad-1.2443930234$

$\mathrm{P} \quad-1.3279072285$

O $\quad-2.8771497694$

O $\quad-0.6441972825$

C $\quad-0.9481700429$

$\mathrm{H} \quad-2.0071743123$

$\mathrm{H} \quad-0.3148712842$

$\mathrm{H} \quad-0.7383795817$

C $\quad-3.2196236095$

$\mathrm{H} \quad-4.3138253225$

$\mathrm{H} \quad-2.8642259069$

$\mathrm{H} \quad-2.7849223916$

O $\quad 1.7035366972$

C $\quad 2.7455749365$

C $\quad 3.9017238871$

N $\quad 2.9739583310$

C $\quad 4.8992012458$

$\mathrm{H} \quad 3.5306467882$

$\mathrm{H} \quad 4.2721136714$

C $\quad 4.2303213950$

$\mathrm{Br} \quad 1.3839998987$

$\mathrm{H} \quad 5.8789754933$

H $\quad 5.0834311619$

O $\quad 4.7103977587$

C $\quad-1.4806400840$
1.2063246278

1.8039551615

1.0040828572

2.8308403485

2.0761379747

0.0684337226

0.7563135685

2.6066144335

3.9177181129

2.7791033833

3.4061759113

3.3550165610

4.3530933551

$-0.3557400461$

$-1.0985671125$

$-2.1357154510$

$-2.2927539256$

$-2.4537273221$

$-3.6486373478$

$-4.8777091145$

$-5.1472818542$

$-5.6473647162$

$-4.8210630108$

$-2.5147196967$

$-2.5680150485$

$-1.6199578606$

$-3.4074444225$

$-0.8610472858$

$-0.7443316690$

$-1.7191757225$

0.2082029738

$-1.1980636497$

$-2.7355450235$

$-1.7066099946$

0.0634280418

1.9554154247

$-0.9265134903$

$-1.8968500249$

0.8081739319

3.1088269935
1.4524209019

0.4610498993

$-0.2928958692$

0.9104807528

$-0.0106111457$

0.2482842048

$-1.3421881623$

1.9784460400

0.7762320511

$-0.3266690259$

0.3163788276

1.4085360160

$-0.1097376261$

1.1259609366

$-0.8000527680$

1.3768849543

$-0.1337721239$

$-0.5566858090$

$-0.6641296291$

$-0.0269023214$

$-0.1713144983$

$-0.4853872406$

1.0519258143

$-1.9283429639$

$-1.9907743252$

$-2.4634389253$

$-2.4063760878$

1.4405925686

0.7506434126

0.8698652871

$-0.1401671577$

$-0.1591506790$

0.6737886981

1.9057367984

$-0.7235169367$

$-0.1529733643$

0.2582435286

$-0.9878839934$

$-1.5362391632$

$-1.7336637386$ 


$\begin{array}{ll}\mathrm{H} & -1.2097391456 \\ \mathrm{H} & -2.5634101074 \\ \mathrm{H} & -0.9471027752 \\ \mathrm{H} & -4.4035456752 \\ \mathrm{H} & -5.0095805863\end{array}$

2.2382809880

3.2488193029

3.9935379199

2.6538415848

1.7143006103

TS1d

01

C

C

C

$\mathrm{H}$

C

C

$\mathrm{H}$

$\mathrm{H}$

$\mathrm{Mg}$

$\mathrm{O}$

$\mathrm{O}$

$\mathrm{P}$

$\mathrm{O}$

$\mathrm{O}$$$
\text { C }
$$$$
\mathrm{H}
$$

$\mathrm{H}$

$\mathrm{H}$

C

$\mathrm{H}$

H $\quad 5.0663399820$

$\mathrm{H} \quad 5.7835878070$

O $\quad-0.8883176150$

C $\quad-1.9714413778$

C $\quad-2.7818291519$

$\mathrm{N} \quad-2.5257432000$

C $\quad-3.9705498977$

$\mathrm{H} \quad-3.0477521510$

$\mathrm{H} \quad-2.1525006611$

C $\quad-3.7236225059$

$\mathrm{Br} \quad-1.2383390935$

$\mathrm{H} \quad-4.0316559175$

$\mathrm{H} \quad-4.9458651802$

O $\quad-4.4563321174$

C $\quad-0.7328151661$

$\mathrm{H} \quad-1.5311630715$

$\mathrm{H} \quad-1.1131542454$

$\mathrm{H} \quad 0.0598608747$

$\mathrm{H} \quad-1.3525642918$

$\mathrm{H} \quad-1.6722636255$

O $\quad 0.6965084699$

C $\quad-1.5185728120$

$\mathrm{H} \quad-1.6206598826$

$\mathrm{H} \quad-2.3353693830$
2.7834202524

4.0702992178

4.2037517358

4.9268026905

3.7624604459

3.0041362949

1.9450254284

1.4003817392

2.2296276107

$-0.2135340401$

$-1.0539335015$

$-0.6933004191$

$-1.1956411199$

$-0.3786086524$

$-2.7276743693$

$-3.1445745436$

$-2.6394806224$

$-4.2265087690$

$-2.9312455024$

$-0.5342043116$

0.1604963938

$-0.2974114505$

$-1.5638683807$

$-1.3044856921$

$-1.7199007487$

$-2.8036219562$

$-2.9866439867$

$-2.4620095882$

$-3.6974889180$

$-1.9578737319$

0.5582364210

$-3.9875497871$

$-2.7832491143$

$-1.7585366916$

4.2485808368

4.0019274964

4.9458518808

4.7550863219

4.1790643883

5.0929075392

1.6939656294

2.9090271817

2.0201147861

2.9306409324
$-1.2923803263$
$-2.3551431639$

$-1.8570233963$

$-2.1016058417$

$-0.8947947892$

0.4802960533

0.5573933060

1.4267258087

1.8411485951

0.9023383714

2.2422972220

$-0.8756299384$

$-1.8084385381$

$-1.5577395115$

$-2.8635580433$

0.7875137553

1.5313773308

$-0.8658194620$

0.1155346732

$-0.1217269950$

$-0.1420109728$

$-1.4463615570$

$-1.7817934492$

$-1.4050372867$

$-2.1642257977$

0.7940945972

0.4904823234

1.8187969441

0.7726044509

1.2759526429

0.7847412909

1.4758559575

$-0.3343899909$

0.5405679483

2.4873334928

1.5971252102

$-0.5747508581$

$-1.4034639190$ 0.0894427707

1.0051527161

$-1.5088498506$

$-1.4268179267$

$-2.1441553611$

$-0.6735332343$

$-2.0051746780$

2.9231253773

1.4369448446

0.9265156896

1.1061022999

1.7392636938

0.3740350580

\section{Real model with achiral catalyst}

(2S,4R)-CY2

01
$\begin{array}{lr}\text { O } & 0.7332191310 \\ \text { C } & -0.4278912248\end{array}$
$-0.5871603570$
$-0.4627994537$
$-0.8216872406$ 


\begin{tabular}{|c|c|c|c|}
\hline $\mathrm{C}$ & -1.0803804729 & 6505598126 & -1.4 \\
\hline $\mathrm{C}$ & -2.3162503686 & -1.3523025886 & -1.9337187817 \\
\hline $\mathrm{C}$ & -2.4856330309 & 0.1943756525 & -1.922190126 \\
\hline $\mathrm{H}$ & -0.4477028370 & 0.9029806257 & -2.3388443613 \\
\hline $\mathrm{H}$ & -1.0212081463 & 1.5347085442 & -0.8200373939 \\
\hline $\mathrm{H}$ & -1.8038870873 & -1.6450993650 & -2.866495194 \\
\hline $\mathrm{H}$ & -3.2733468691 & -1.8877682336 & -1.917537014 \\
\hline $\mathrm{C}$ & -2.7911705828 & 0.7319215421 & $-3.327618591^{\prime}$ \\
\hline $\mathrm{H}$ & -3.7345599240 & 0.3281201278 & -3.7214945467 \\
\hline $\mathrm{H}$ & -2.8775991784 & 1.8284090493 & -3.308732462 \\
\hline $\mathrm{H}$ & -1.9898539500 & 0.4609468909 & -4.0326747265 \\
\hline $\mathrm{C}$ & -3.6237839846 & 0.5818629848 & 56796359 \\
\hline $\mathrm{C}$ & -3.4305013907 & 1.3062395734 & 0212223181 \\
\hline $\mathrm{C}$ & -4.9251735639 & 0.1495161804 & -1.2596033589 \\
\hline $\mathrm{C}$ & -4.4926384889 & 1.5632552672 & 1.0795712144 \\
\hline $\mathrm{H}$ & -2.4386187587 & 1.6628800590 & 3965 \\
\hline $\mathrm{C}$ & -5.9891677269 & 0.4110719128 & -0.402 \\
\hline $\mathrm{H}$ & -5.1178030803 & 65530 & -2.1715968793 \\
\hline $\mathrm{C}$ & -5.7755043261 & 1.1164070727 & 0.7807688348 \\
\hline $\mathrm{H}$ & -4.3075551726 & 3721 & \\
\hline $\mathrm{H}$ & 2668 & 7830 & -0.6 \\
\hline $\mathrm{C}$ & -1.3929737374 & -1.7073609231 & 58511 \\
\hline $\mathrm{C}$ & 4161 & 9517 & 39315 \\
\hline $\mathrm{H}$ & 523 & -3.0 & -1 . \\
\hline $\mathrm{H}$ & 7601 & 3065 & 54600 \\
\hline $\mathrm{C}$ & -2.0998080592 & -1.6455782139 & 0.6215286003 \\
\hline $\mathrm{C}$ & 168 & 3975 & 1937 \\
\hline $\mathrm{C}$ & 4466 & 7408 & 0.7 \\
\hline $\mathrm{C}$ & -2.1235673394 & -1.0607589116 & 2.9742054622 \\
\hline $\mathrm{H}$ & 9466 & 8330 & 490 \\
\hline$\sigma_{0}$ & 130341 & 514891 & 25869 \\
\hline 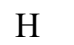 & 6161 & 11481 & 18055 \\
\hline $\mathrm{C}$ & -3.4107176439 & -1.5727151481 & 3.1051257221 \\
\hline $\mathrm{H}$ & 936 & -0.6 & 276 \\
\hline $\mathrm{H}$ & 144 & -2.5 & 642 \\
\hline $\mathrm{Mg}$ & 87273 & 676796 & -0.0371358558 \\
\hline $\mathrm{O}$ & & & 2154 \\
\hline $\mathrm{O}$ & 52 & 2.5 & 5220 \\
\hline $\mathrm{D}+2>$ & 41964 & 2.45 & 3495 \\
\hline $\mathrm{O}$ & -0.6050574738 & 2.74 & 89936 \\
\hline $\mathrm{O}$ & & & \\
\hline $\mathrm{C}$ & 748 & 4.9 & 7998 \\
\hline $\mathrm{H}$ & 343042 & 5.3321668987 & 1.9411423114 \\
\hline $\mathrm{H}$ & & & 2.6537167340 \\
\hline $\mathrm{H}$ & & & 5397158 \\
\hline $\mathrm{C}$ & 3202 & 804 & 169 \\
\hline $\mathrm{H}$ & -2.1759800976 & 3.3360289203 & 2.6114735192 \\
\hline $\mathrm{H}$ & & & 2.9594102967 \\
\hline $\mathrm{H}$ & 08 & 3.12 & 250 \\
\hline $\mathrm{O}$ & 4688980 & 0.3146276339 & -1.9660696374 \\
\hline $\mathrm{C}$ & 4.1945530633 & -0.0433167760 & -1.3638729554 \\
\hline $\mathrm{C}$ & & & -1.9721841687 \\
\hline $\mathrm{N}$ & & & -0.026509460 \\
\hline $\mathrm{C}$ & 4273745 & -0.8337505107 & -0.7388231443 \\
\hline $\mathrm{H}$ & 5.8845416039 & 0.1867100560 & -2.6597013136 \\
\hline $\mathrm{H}$ & & -1.4615195292 & -2.572469446 \\
\hline $\mathrm{C}$ & 566901367 & -0.4245866897 & 0.4563307170 \\
\hline $\mathrm{Br}$ & 0.4143913113 & -3.7378658771 & 0.4229083611 \\
\hline $\mathrm{H}$ & 6.6252233062 & -1.8882695593 & -0.619042981 \\
\hline $\mathrm{H}$ & 7.2663527842 & -0.2449759484 & -0.71133954 \\
\hline
\end{tabular}




$\begin{array}{lrrr}\mathrm{O} & 5.7861679121 & -0.4823274029 & 1.6109543239 \\ \mathrm{H} & -6.6039801779 & 1.3152147581 & 1.4637101570 \\ \mathrm{H} & -3.9227165221 & -1.5399613892 & 4.0690975442\end{array}$

\begin{tabular}{|c|c|c|c|}
\hline \multicolumn{4}{|c|}{$(2 \mathrm{~S}, 4 \mathrm{R})-\mathrm{CY} 3$} \\
\hline $\mathrm{O}$ & 0.8853328265 & -0.6398495459 & -0.4908719827 \\
\hline $\mathrm{C}$ & -0.2860559548 & -0.6100249873 & -0.8200283165 \\
\hline $\mathrm{C}$ & -0.9924106303 & 0.5882074880 & -1.3864669778 \\
\hline $\mathrm{C}$ & -2.1810898664 & -1.4239609386 & -1.9219242056 \\
\hline $\mathrm{C}$ & -2.3964647435 & 0.1141565223 & -1.8218825586 \\
\hline $\mathrm{H}$ & -0.3929805287 & 0.9034844442 & -2.2578355486 \\
\hline $\mathrm{H}$ & -0.9388759136 & 1.4402434160 & -0.6941781637 \\
\hline $\mathrm{H}$ & -1.6893704539 & -1.6489939026 & -2.8841638766 \\
\hline $\mathrm{H}$ & -3.1206715843 & -1.9899331419 & -1.9084316450 \\
\hline $\mathrm{C}$ & -2.7574250330 & 0.7148274803 & -3.1883532809 \\
\hline $\mathrm{H}$ & -3.6982939192 & 0.3022566040 & -3.5792220098 \\
\hline $\mathrm{H}$ & -2.8766196712 & 1.8055205947 & -3.1089366393 \\
\hline $\mathrm{H}$ & -1.9678359609 & 0.5071242263 & -3.9272554048 \\
\hline $\mathrm{C}$ & -3.5175498196 & 0.4214448915 & -0.8161802298 \\
\hline $\mathrm{C}$ & -3.3095608968 & 1.0896174367 & 0.3924577809 \\
\hline $\mathrm{C}$ & -4.8160338480 & -0.0276942929 & -1.0975569285 \\
\hline $\mathrm{C}$ & -4.3534858364 & 1.2767237836 & 1.2989615227 \\
\hline $\mathrm{H}$ & -2.3194100593 & 1.4559348710 & 0.6600369127 \\
\hline $\mathrm{C}$ & -5.8626530048 & 0.1647687248 & -0.2012370850 \\
\hline $\mathrm{H}$ & -5.0200203033 & -0.5573860844 & -2.0318011307 \\
\hline $\mathrm{C}$ & -5.6339392761 & 0.8154707696 & 1.0102315673 \\
\hline $\mathrm{H}$ & -4.1553057286 & 1.7825992689 & 2.2463358510 \\
\hline $\mathrm{H}$ & -6.8618744688 & -0.2000353117 & -0.4497226393 \\
\hline $\mathrm{C}$ & -1.2115813466 & -1.8140738794 & -0.7793388733 \\
\hline $\mathrm{C}$ & -0.5178806695 & -3.1403551200 & -1.1168702872 \\
\hline $\mathrm{H}$ & 0.0752898908 & -3.0434313937 & -2.0359558853 \\
\hline $\mathrm{H}$ & -1.2835402177 & -3.9116609225 & -1.2705075331 \\
\hline $\mathrm{C}$ & -1.8806904563 & -1.8278361286 & 0.6036907433 \\
\hline $\mathrm{C}$ & -1.2347705599 & -1.3168443501 & 1.7399043153 \\
\hline $\mathrm{C}$ & -3.1589871644 & -2.3602808824 & 0.7657257377 \\
\hline $\mathrm{C}$ & -1.8658663801 & -1.3107253876 & 2.9752624153 \\
\hline $\mathrm{H}$ & -0.2270515544 & -0.8996656010 & 1.6829251854 \\
\hline $\mathrm{C}$ & -3.8076898894 & -2.3528753870 & 1.9974069942 \\
\hline $\mathrm{H}$ & -3.6953863907 & -2.7781908219 & -0.0877685080 \\
\hline $\mathrm{C}$ & -3.1662271830 & -1.8081361946 & 3.1146428776 \\
\hline $\mathrm{H}$ & -1.3586843026 & -0.9196584059 & 3.8588560203 \\
\hline $\mathrm{H}$ & -4.8157381408 & -2.7605687471 & 2.0652664354 \\
\hline $\mathrm{Mg}$ & 2.3663193100 & 0.7107627247 & -0.0854699097 \\
\hline $\mathrm{O}$ & 1.5773594886 & 0.9881447374 & 1.8847635084 \\
\hline $\mathrm{O}$ & 1.4228024421 & 2.5609083215 & -0.0150654471 \\
\hline $\mathrm{P}$ & 1.0851731001 & 2.3589132426 & 1.4596178901 \\
\hline $\mathrm{O}$ & -0.5095265943 & 2.5578767856 & 1.6324681302 \\
\hline $\mathrm{O}$ & 1.7131597453 & 3.4874279368 & 2.4107829846 \\
\hline $\mathrm{C}$ & 1.5726172355 & 4.8583908455 & 2.0746738470 \\
\hline $\mathrm{H}$ & 0.5214476957 & 5.1792138862 & 2.1567553708 \\
\hline $\mathrm{H}$ & 2.1817154249 & 5.4295368343 & 2.7856567389 \\
\hline $\mathrm{H}$ & 1.9265231223 & 5.0474482647 & 1.0501521173 \\
\hline $\mathrm{C}$ & -1.1126724796 & 2.4466902550 & 2.9134592384 \\
\hline $\mathrm{H}$ & -2.0391808908 & 3.0354371732 & 2.8911881353 \\
\hline $\mathrm{H}$ & -1.3523780218 & 1.3962954859 & 3.1334878933 \\
\hline $\mathrm{H}$ & -0.4527071115 & 2.8449787111 & 3.6994306118 \\
\hline $\mathrm{O}$ & 3.1433365526 & 0.5098207008 & -2.0996632822 \\
\hline $\mathrm{C}$ & 4.2264506860 & 0.1571882974 & -1.5926967212 \\
\hline $\mathrm{C}$ & 5.4856357201 & -0.2580337986 & -2.3187283083 \\
\hline
\end{tabular}




$\begin{array}{ll}\mathrm{N} & 4.3505055545 \\ \mathrm{C} & 6.4406761282 \\ \mathrm{H} & 5.8145204987 \\ \mathrm{H} & 5.2622901422 \\ \mathrm{C} & 5.6226561341 \\ \mathrm{Br} & 0.6804488699 \\ \mathrm{H} & 6.7835934189 \\ \mathrm{H} & 7.3399271199 \\ \mathrm{O} & 6.0302385285 \\ \mathrm{H} & -6.4490325509 \\ \mathrm{O} & -3.7150997738 \\ \mathrm{C} & -5.0277381774 \\ \mathrm{H} & -5.7443956418 \\ \mathrm{H} & -5.1231770107 \\ \mathrm{H} & -5.2739994156\end{array}$

0.1192376994

$-0.5709770877$

0.5634768233

$-1.1158037390$

$-0.2973338637$

$-3.8460988491$

$-1.6152958506$

0.0611923839

$-0.4259567206$

0.9629181556

$-1.7234443626$

$-2.1763490604$

$-1.6271919216$

$-3.2567406364$

$-1.9947740992$
$-0.2591032998$

$-1.1666003589$

$-2.9716747982$

$-2.9697079793$

0.1097740682

0.2323005455

$-1.1472007118$

$-1.1518613540$

1.2334076602

1.7220702467

4.3426297565

4.5328081942

3.8972081474

4.3270237477

5.5859712887

\section{(2S,4R)-CY4}

01

O $\quad 0.8622306163$

C $\quad-0.1216616186$

C $\quad-0.3965905547$

$-0.8010098850$

$-0.9753506877$

0.0709588775

$-0.2753830080$

$-0.6293125837$

C $\quad-1.9543716073$

C $\quad-1.8364495033$

$\mathrm{H} \quad 0.3252204908$

$-2.0497562046$

$-1.9236382123$

$-0.6414541811-2.3140233112$

$-0.1396119444$

$-0.7128651670$

$-2.6384919108$

0.7919538597

$-1.8688935718$

$-1.4134301891$

$-2.7675984670$

$-2.3107701948$

$-2.9888206169$

$-2.4074089937$

$-1.6076994715$

$-0.6981821573$

$-3.8372930860$

$-2.9848451035$

$-1.0692138963$

$-4.1328462505$

$-1.8658605306$

0.3035807620

$-4.2730078411$

$-1.2390772270$

$-1.3655458240$

$-4.2826714931$

0.2963512499

$-1.6959443092$

$-2.8829285310$

1.3303035354

$-0.8174815987$

$\begin{array}{ll}0.0647510267 & -1.9478238165\end{array}$

$-4.2429154706$

2.0786250115

$-0.1793556868$

1.5662981100

$-0.5843158859$

$-1.5109261697$

0.8172007159

$-1.3233129009$

$-5.2328105794$

$-0.7366609263$

$-2.6277998941$

1.8241293538

2.8608076888

$-0.4229354841$

$-4.8835063329$

$-3.2360625940$

0.6092884630

0.5194474521

$-6.2844983366$

$-1.9799915264$

$-1.5333706306$

$-0.3015109600$

$-3.3839282192$

0.0388619114

$-3.7242929053$

$-0.7399845433$

$-0.0005430042$

$-4.0779434449$

0.0771843981

$-1.5450525916$

$-1.3984183388$

0.8371028112

1.8841092706

$-0.6741311201$

$-1.5847474940$

0.8542530093

$-0.1079596624$

2.8791011004

$\begin{array}{ll}-0.5270747514 & 1.9263581450\end{array}$

$\begin{array}{ll}-0.9976395975 & 1.8333677372\end{array}$

$\begin{array}{ll}-2.1616538935 & 0.0733832109\end{array}$

$\begin{array}{ll}-0.2367942485 & 2.8380141141\end{array}$

$-3.6773055491$

0.4510241560

3.6864283896

$-1.1206295955$

0.6998224560

1.8016695929

$-0.0568500288$

$\mathrm{Mg} \quad 2.2711604181$

$\mathrm{O} \quad 1.2774418243$

1.7064041481

2.2910895229

2.6961034020

1.7191419369

$-0.5399987913$

0.9330670406

$2.7391636695 \quad 1.4008566801$ 


$\begin{array}{lr}\mathrm{O} & 1.4623055136 \\ \mathrm{C} & 0.9520774971 \\ \mathrm{H} & -0.1161495424 \\ \mathrm{H} & 1.5208202520 \\ \mathrm{H} & 1.0764267035 \\ \mathrm{C} & -0.9324320182 \\ \mathrm{H} & -0.7300826319 \\ \mathrm{H} & -2.0024492911 \\ \mathrm{H} & -0.3513174154 \\ \mathrm{O} & 2.8054781233 \\ \mathrm{C} & 3.9376100056 \\ \mathrm{C} & 5.1047601621 \\ \mathrm{~N} & 4.2116704086 \\ \mathrm{C} & 6.1859197710 \\ \mathrm{H} & 5.3573374436 \\ \mathrm{H} & 4.8077030252 \\ \mathrm{C} & 5.5167180779 \\ \mathrm{Br} & 0.2405526248 \\ \mathrm{H} & 6.5366766505 \\ \mathrm{H} & 7.0764484681 \\ \mathrm{O} & 6.0432070650 \\ \mathrm{H} & -5.6564195823 \\ \mathrm{C} & -4.5140186276 \\ \mathrm{~F} & -5.7767154326 \\ \mathrm{~F} & -4.5537759279 \\ \mathrm{~F} & -4.0189790493\end{array}$

4.1877547204

5.2605360270

5.4287351150

6.1571225963

5.0585297079

3.0582646059

4.1200070002

2.8558406416

2.4379436276

$-0.0529950874$

$-0.2947367901$

$-0.9342188721$

0.0224265658

$-0.9511152869$

$-0.3354217886$

$-1.9302677533$

$-0.3249292276$

$-3.5970670175$

$-1.9596313238$

$-0.3596188304$

$-0.1673358547$

2.4039509283

0.5100222577

0.0801364796

1.8226283997

0.4113693633
1.1871019164

0.4109110660

0.6231265652

0.6850859237

$-0.6636230040$

2.7451403450

2.9562729830

2.8763527013

3.4449645364

$-2.0563096355$

$-1.5921343493$

$-2.3091393567$

$-0.3203723204$

$-1.2281236013$

$-3.1962543337$

$-2.6687809013$

0.0092339472

1.7205770857

$-0.9669567510$

$-1.4843460289$

1.0776543879

0.0858077302

3.8404183323

3.8733436863

3.5460904510

5.0776074895

\section{$(2 \mathrm{~S}, 4 \mathrm{~S})-\mathrm{CY} 2$}

01

O $\quad 0.9660724224$

C $\quad-0.1157630791$

C $\quad-0.5185325257$

$-0.7346463463$

$-0.7662475797$

$-0.4679436671$

0.1153065609

$-1.0249604238$

$-2.1646722310$

C $\quad-2.2009473562$

$-1.5607546202$

$-1.8591937855$

C $\quad-2.0291996214$

$-0.1037764308$

$-0.2569135707$

$-2.3578359359$

0.0547510880

1.1512468731

$-3.0344306526$

$-0.1901703762$

$-2.2478198760$

$-1.9961169359$

$-1.9234766976$

$-1.7902424002$

$-2.6758426810$

$-3.2373277127$

0.8803692952

$-1.5812047050$

$-2.8288027681$

1.9132381412

$-1.4841342056$

$-2.6373904268$

$-3.9066881888$

0.6801793155

$-1.8088266694$

$-2.5535236136$

0.8023795083

$-1.5755507224$

0.0780252816

$-0.4231944057$

$-2.4695844661$

$-0.6537126094$

$-3.8051689053$

$-4.3350499197$

$\begin{array}{ll}1.0358058171 & -4.6220670736\end{array}$

$-1.8558625968$

$-0.4460350285$

$-5.6432216828$

$-1.3990731571$

$-3.7215656573$

$\begin{array}{ll}1.2459319044 & -5.9303535944\end{array}$

$-2.2874592980$

$-1.0280249206$

1.6342168488

$-4.2345778912$

$-3.3473974139$

0.5037452354

$-6.4477779216$

$-4.8071443449$

$-1.0326009090$

$-6.0353884591$

$-1.7893534312$

1.9955666714

$-6.5491019537$

$-1.2190162361$

$-1.7610830804$

$-0.6737297397$

$-0.6923124667$

$-3.2097378771$

$-0.7047385359$

$-0.1339741670$

$-3.3979072389$

$-1.6315959915$

$-1.5461566319$

$-3.8985061319$

$-0.6675821682$

$-1.8462397603$

$-1.4361423892$

0.6911832076

1.6263491070

$-0.5915832865$

1.0340325203

$-3.0566684034$

$-2.0517140610$

$-0.3600273019$

2.8636622000 


$\begin{array}{lrrr}\mathrm{H} & -0.2916931116 & -0.0924373669 & 1.4171805421 \\ \mathrm{C} & -3.6541274821 & -1.8225608967 & 2.2698012011 \\ \mathrm{H} & -3.5455150186 & -2.7324905565 & 0.3326402735 \\ \mathrm{C} & -3.0487334057 & -0.9690583869 & 3.1911749785 \\ \mathrm{H} & -1.3434198988 & 0.2997049470 & 3.5779142764 \\ \mathrm{H} & -4.5964723728 & -2.3172579273 & 2.5144602609 \\ \mathrm{Mg} & 2.5085930904 & 0.6103615208 & -0.2722556875 \\ \mathrm{O} & 1.4020403627 & 1.6150615531 & 1.2623619643 \\ \mathrm{O} & 1.7042485281 & 2.3567219985 & -1.0753744168 \\ \mathrm{P} & 1.0460376407 & 2.6979280709 & 0.2596753524 \\ \mathrm{O} & -0.5428197039 & 2.8215138048 & -0.0026715210 \\ \mathrm{O} & 1.4634299119 & 4.1378340689 & 0.8248242633 \\ \mathrm{C} & 1.4296679618 & 5.2721707206 & -0.0270329735 \\ \mathrm{H} & 0.3950311744 & 5.5174704735 & -0.3165533876 \\ \mathrm{H} & 1.8566867199 & 6.1112245306 & 0.5353150320 \\ \mathrm{H} & 2.0246978400 & 5.0956648169 & -0.9355387420 \\ \mathrm{C} & -1.4255021985 & 3.1076351023 & 1.0702146056 \\ \mathrm{H} & -2.4291737097 & 3.2221598818 & 0.6423419341 \\ \mathrm{H} & -1.4352825730 & 2.2839342778 & 1.7993896723 \\ \mathrm{H} & -1.1374436019 & 4.0402292941 & 1.5804822287 \\ \mathrm{O} & 3.6400456790 & -0.3143333476 & -1.8579791620 \\ \mathrm{C} & 4.5344294034 & -0.5494925057 & -1.0209836375 \\ \mathrm{C} & 5.8327393285 & -1.2882625286 & -1.2494932561 \\ \mathrm{~N} & 4.3821171055 & -0.1284074280 & 0.2416822939 \\ \mathrm{C} & 6.4777629173 & -1.2532925773 & 0.1363543642 \\ \mathrm{H} & 6.4090530720 & -0.7745586789 & -2.0326600511 \\ \mathrm{H} & 5.6129084447 & -2.2988682080 & -1.6232625756 \\ \mathrm{C} & 5.4735437894 & -0.4899891550 & 1.0205006094 \\ \mathrm{Br} & 0.4728565149 & -3.7218509750 & 0.7542184751 \\ \mathrm{H} & 6.6439744016 & -2.2487974526 & 0.5721208903 \\ \mathrm{H} & 7.4427693818 & -0.7273902869 & 0.1632250665 \\ \mathrm{O} & 5.6215983758 & -0.2524655052 & 2.1896081478 \\ \mathrm{H} & -3.6848170340 & 0.6656468990 & -7.4736143064 \\ \mathrm{H} & -3.5137894763 & -0.7876153256 & 4.1623325846 \\ & & \end{array}$

\section{$(2 \mathrm{~S}, 4 \mathrm{~S})-\mathrm{CY} 3$}

01

$\begin{array}{lrrr}\mathrm{O} & 1.2024152176 & -0.6889794397 & -0.3519494433 \\ \mathrm{C} & 0.2543374366 & -0.7167935967 & -1.1163903187 \\ \mathrm{C} & 0.1265643314 & 0.1160232636 & -2.3522952462 \\ \mathrm{C} & -1.6551927829 & -1.4802861092 & -2.3160382486 \\ \mathrm{C} & -1.3258050345 & -0.0557091487 & -2.8282649374 \\ \mathrm{H} & 0.8419657649 & -0.3243816053 & -3.0722327383 \\ \mathrm{H} & 0.4666995283 & 1.1457483090 & -2.1666340979 \\ \mathrm{H} & -1.2513483170 & -2.2135853980 & -3.0338422057 \\ \mathrm{H} & -2.7347893149 & -1.6612451467 & -2.2431476990 \\ \mathrm{C} & -2.2304356835 & 0.9987721114 & -2.1640941763 \\ \mathrm{H} & -1.9444617060 & 2.0062744716 & -2.4983589771 \\ \mathrm{H} & -3.2818784624 & 0.8261890902 & -2.4383043553 \\ \mathrm{H} & -2.1481805004 & 0.9734932966 & -1.0688461248 \\ \mathrm{C} & -1.4709639106 & 0.0730927618 & -4.3393857656 \\ \mathrm{C} & -2.4564530541 & -0.6363041417 & -5.0359397302 \\ \mathrm{C} & -0.6623384539 & 0.9602572676 & -5.0607577102 \\ \mathrm{C} & -2.6214369774 & -0.4755430116 & -6.4107630082 \\ \mathrm{H} & -3.1134403236 & -1.3268277797 & -4.5025907676 \\ \mathrm{C} & -0.8242680142 & 1.1234145621 & -6.4352387167 \\ \mathrm{H} & 0.1071897384 & 1.5394136343 & -4.5451218628 \\ \mathrm{C} & -1.8038311861 & 0.4040561760 & -7.1172068663 \\ \mathrm{H} & -3.3948671799 & -1.0435845524 & -6.9326108284 \\ \mathrm{H} & -0.1778404270 & 1.8181207473 & -6.9760413554\end{array}$




\begin{tabular}{|c|c|c|c|}
\hline $\mathrm{C}$ & 67455253 & -1.6574366527 & -0.9515511041 \\
\hline $\mathrm{C}$ & -0.4746127367 & -3.1233078021 & -0.8255377260 \\
\hline $\mathrm{H}$ & 0.2438810848 & -3.3735420958 & -1.6177473593 \\
\hline $\mathrm{H}$ & -1.3491981278 & -3.7786641673 & -0.9290071013 \\
\hline $\mathrm{C}$ & -1.8030874488 & -1.2474616174 & 0.2484041831 \\
\hline $\mathrm{C}$ & -1.3375057350 & -0.4289409935 & 1.2884318706 \\
\hline $\mathrm{C}$ & -3.0983167528 & -1.7562700410 & 0.3544551003 \\
\hline $\mathrm{C}$ & -2.1492985504 & -0.1222212582 & 2.3708969776 \\
\hline $\mathrm{H}$ & -0.3304769013 & -0.0100123402 & 1.2749541112 \\
\hline $\mathrm{C}$ & -3.9233567390 & -1.4599640531 & 1.4384191808 \\
\hline $\mathrm{H}$ & -3.4951305583 & -2.4168663515 & -0.4203180002 \\
\hline $\mathrm{C}$ & -3.4505637931 & -0.6297925214 & 2.4600447055 \\
\hline $\mathrm{H}$ & -1.7808076646 & 0.5096535385 & 06909215 \\
\hline $\mathrm{H}$ & -4.9258562857 & -1.8861959131 & 1.4725938411 \\
\hline $\mathrm{Mg}$ & 2.7552614564 & 0.6284472506 & -0.0377285514 \\
\hline $\mathrm{O}$ & 1.5758859982 & 1.6804508620 & 1170121 \\
\hline $\mathrm{O}$ & 2.0078056335 & 584972 & 316732 \\
\hline $\mathrm{P}$ & 5546 & 2739 & 6385 \\
\hline $\mathrm{O}$ & -0.3215427452 & 2.7640325794 & -0.0331010302 \\
\hline $\mathrm{O}$ & 7837 & 083 & 8171 \\
\hline $\mathrm{C}$ & 1860 & 759 & 36422 \\
\hline $\mathrm{H}$ & 6783 & 539 & 813489 \\
\hline $\mathrm{H}$ & 8677 & 70174 & 53312 \\
\hline $\mathrm{H}$ & 671 & 7494 & 98542 \\
\hline $\mathrm{C}$ & -1.26 & 910 & 5317 \\
\hline $\mathrm{H}$ & -2.2429886878 & 2.7143175061 & 0.6430609606 \\
\hline $\mathrm{H}$ & -1.0 & 006 & 1035 \\
\hline $\mathrm{H}$ & 3017 & 7535 & 5049 \\
\hline $\mathrm{O}$ & 4557 & 63124 & 91649 \\
\hline $\mathrm{C}$ & 4.7954577378 & -0.5660264165 & -0.7010938393 \\
\hline $\mathrm{C}$ & 491 & 250 & 30531 \\
\hline $\mathrm{N}$ & 902 & 2180 & 59491 \\
\hline $\mathrm{C}$ & 6.6791603508 & -1.2864684905 & 5355678 \\
\hline $\mathrm{H}$ & & & 23395 \\
\hline $\mathrm{H}$ & 49 & 345 & 83487 \\
\hline $\mathrm{C}$ & 5.6454 & 208758 & 1.3798440994 \\
\hline $\mathrm{Br}$ & 0.3634948348 & -3.6110777831 & 0.8503254398 \\
\hline $\mathrm{H}$ & 87 & -2 & 664 \\
\hline $\mathrm{H}$ & 5493 & -0.7701581696 & 49082 \\
\hline $\mathrm{O}$ & 5.7449442735 & -0.2697894388 & 2.5534686577 \\
\hline $\mathrm{H}$ & -1.929 & 80651 & 674894 \\
\hline $\mathrm{O}$ & 00 & -0 . & 046 \\
\hline $\mathrm{C}$ & -5.4610661419 & 5956126 & 3.7069640476 \\
\hline $\mathrm{H}$ & -6.1286604956 & -0.4566215036 & 2.8876485310 \\
\hline $\mathrm{H}$ & & & \\
\hline $\mathrm{H}$ & -5.8415232544 & -0.3707783512 & 4.6520119338 \\
\hline \multicolumn{4}{|l|}{$(2 \mathrm{~S}}$, \\
\hline \multicolumn{4}{|l|}{01} \\
\hline $\mathrm{O}$ & -1.61 & & \\
\hline $\mathrm{C}$ & -0.8228105379 & -1.1789880455 & 0.8947678362 \\
\hline $\mathrm{C}$ & & & \\
\hline $\mathrm{C}$ & & 238819 & $00=80<01200$ \\
\hline $\mathrm{C}$ & 41822 & -1.2431327953 & 65579 \\
\hline $\mathrm{H}$ & -1.7033586325 & -1.2963757928 & 2.7766444476 \\
\hline $\mathrm{H}$ & & & \\
\hline $\mathrm{H}$ & & -3.3388354607 & 2.4452451378 \\
\hline $\mathrm{H}$ & 1.8495800505 & -2.7774425406 & 2.0890957734 \\
\hline $\mathrm{C}$ & 1.5707150496 & -0.1597348711 & 2.7476695873 \\
\hline $\mathrm{H}$ & 1.3195019348 & 0.7254987441 & 3.348990237 \\
\hline
\end{tabular}




\begin{tabular}{|c|c|c|c|}
\hline $\mathrm{H}$ & 197 & 295 & 43 \\
\hline 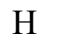 & 1.6540874118 & 0.1670510152 & 1.7018314710 \\
\hline & .3893834885 & -1.5794325194 & 098956321 \\
\hline $\mathrm{C}$ & . 1875138549 & -2.5757544049 & 4.9841330043 \\
\hline $\mathrm{C}$ & .4610073614 & -0.8490346580 & 5.2493080590 \\
\hline $\mathrm{C}$ & .1293946818 & -2.8439421751 & 6.3508812709 \\
\hline 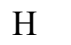 & 8725824789 & -3.1559725789 & 3621110071 \\
\hline $\mathrm{C}$ & -0.5217342695 & -1.1144997310 & 6.6159389130 \\
\hline$U$ & 1.0868573215 & -0.0550196333 & 03544 \\
\hline C & 0.2721803085 & -2.1154184119 & 9134 \\
\hline $\mathrm{H}$ & 1.7592202404 & -3.6291526707 & 6.7749373227 \\
\hline $\mathrm{H}$ & -1.1963111687 & -0.5339172093 & 7.2489529907 \\
\hline $\mathrm{C}$ & 0.30888 & & \\
\hline $\mathrm{C}$ & ).2202475242 & -3.4677764404 & 7027 \\
\hline $\mathrm{H}$ & -1.0743992187 & 7294 & 0322 \\
\hline$H$ & 502598 & 287924 & 4240 \\
\hline $\mathrm{C}$ & 149 & -1.5 & \\
\hline $\mathrm{C}$ & 512 & 943 & 7746 \\
\hline $\mathrm{C}$ & 2.6744108713 & -2.0897092777 & 587523 \\
\hline $\mathrm{C}$ & 2.13 & & \\
\hline $\mathrm{H}$ & & & \\
\hline $\mathrm{C}$ & 3.68 & -1.5 & 3565 \\
\hline $\mathrm{H}$ & & & \\
\hline C & 292 & -0 & 544 \\
\hline $\mathrm{H}$ & 18 & & \\
\hline $\mathrm{H}$ & 4.67889 & -2.0 & 7878 \\
\hline $\mathrm{Mg}$ & 75 & & \\
\hline 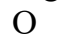 & -1.44 & & 3434 \\
\hline 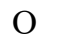 & -2.0 & & \\
\hline $\mathrm{D}$ & -1.1356044687 & & 342620 \\
\hline $\mathrm{O}$ & & & \\
\hline $\mathrm{O}$ & & & \\
\hline $\mathrm{C}$ & -1.19 & & \\
\hline & & & \\
\hline & 47 & & 620 \\
\hline & 1642 & 4.7 & 403 \\
\hline C & & & 19127 \\
\hline & & & \\
\hline & & & 207 \\
\hline 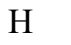 & 1.2714939132 & 78556 & 7663 \\
\hline P & & & \\
\hline 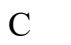 & & & \\
\hline $\mathrm{C}$ & 358 & -0.7 & 4842 \\
\hline $\mathrm{N}$ & -4.7691250923 & & -0.9580880218 \\
\hline $\mathrm{C}$ & & & \\
\hline $\mathrm{H}$ & & & 24 \\
\hline 11 & -6.5515861982 & -1.8551232125 & 8484 \\
\hline $\mathrm{C}$ & 3629 & & -1. \\
\hline $\mathrm{Br}$ & & & \\
\hline - & & -1 & 3597 \\
\hline 11 & -7.8494979692 & 0.3929568974 & -1.4382619122 \\
\hline $\mathrm{O}$ & & & -3.0089258039 \\
\hline 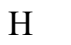 & & & \\
\hline $\mathrm{C}$ & 085459385 & 0.1795198612 & -2.7109365011 \\
\hline $\mathrm{F}$ & 5.5767725033 & -0.6115687594 & -2.8381001024 \\
\hline $\mathrm{F}$ & & & \\
\hline & & & \\
\hline
\end{tabular}

(2S,4S)-CY5

01 


\begin{tabular}{|c|c|c|c|}
\hline $\mathrm{O}$ & 638183 & 05639 & 701 \\
\hline C & -1.3335896929 & -0.7839061942 & 1.3673401025 \\
\hline & -2.5609539145 & -2.3942328240 & 0.0816548469 \\
\hline & -2.4684981124 & -1.2776909139 & 9810470 \\
\hline C & -3.4873166130 & -1.9127944385 & 1.2436967705 \\
\hline & -2.1271530811 & -3.3616298748 & 0.369819662 \\
\hline $\mathrm{H}$ & -3.1098418315 & -2.5541564692 & -0.8559461376 \\
\hline $\mathrm{H}$ & -2.0123514257 & -2.0744338275 & 2.8260183392 \\
\hline $\mathrm{H}$ & -2.8395838273 & -0.5299176280 & 2.9203662481 \\
\hline $\mathrm{C}$ & -4.1991403029 & -3.0987271908 & 1.9105388046 \\
\hline $\mathrm{H}$ & -4.8189440702 & -2.7538131615 & 2.7514428970 \\
\hline $\mathrm{H}$ & -4.8582637216 & -3.6207183956 & 1.2024059398 \\
\hline $\mathrm{H}$ & -3.4698296912 & -3.8293430459 & 3061 \\
\hline $\mathrm{C}$ & -4.5459382160 & -0.9197202108 & 0.7405445959 \\
\hline $\mathrm{C}$ & -5.4409793752 & -1.3338165694 & -0.2577564547 \\
\hline $\mathrm{C}$ & -4.6728838862 & 0.3870060008 & 1.2234278324 \\
\hline $\mathrm{C}$ & -6.4136105257 & -0.4766204831 & 21972 \\
\hline $\mathrm{H}$ & -5.3768093950 & -2.3480436898 & -0.6602160497 \\
\hline $\mathrm{C}$ & -5.6456125763 & 1.2525383370 & 0.7187863928 \\
\hline $\mathrm{H}$ & -3.99 & 0.7 & 00725 \\
\hline $\mathrm{C}$ & -6.51 & & 72008 \\
\hline $\mathrm{H}$ & -7.0922813991 & -0.827 & 21279 \\
\hline $\mathrm{H}$ & -5.7102529041 & 2.2707367566 & 116278 \\
\hline $\mathrm{C}$ & -1.44 & -1.3 & 8490 \\
\hline $\mathrm{C}$ & -1.9 & -0.2 & 5308 \\
\hline $\mathrm{H}$ & -2.9103263247 & 0.1530207952 & -0.6789914090 \\
\hline $\mathrm{H}$ & 21538 & -0.65 & 1994 \\
\hline $\mathrm{Mg}$ & 1.29 & & 0992 \\
\hline $\mathrm{O}$ & 2.779 & -0.46 & 37972 \\
\hline $\mathrm{O}$ & 2.6885975881 & -1.1975371977 & 1.3243097224 \\
\hline$P$ & 3.51 & -1.2 & 5682 \\
\hline $\mathrm{O}$ & 3.77 & 342 & 6416 \\
\hline $\mathrm{O}$ & 4.9939096084 & 8217 & 0.2302463234 \\
\hline $\mathrm{C}$ & 5.7592740253 & -0.9781245971 & 42558 \\
\hline $\mathrm{H}$ & 18 & -2. & 572 \\
\hline $\mathrm{H}$ & 309 & 324 & 1.33 \\
\hline $\mathrm{H}$ & 5.2132107612 & -0.7409645636 & 2.2951193904 \\
\hline $\mathrm{C}$ & & & 3421 \\
\hline $\mathrm{H}$ & 4.48 & 9211 & 30241 \\
\hline $\mathrm{H}$ & 3.9855177632 & 2130215 & -2.4244263020 \\
\hline $\mathrm{H}$ & & & -1.4984360585 \\
\hline $\mathrm{O}$ & & & 64607 \\
\hline $\mathrm{C}$ & 1.89 & 691 & 5332 \\
\hline $\mathrm{C}$ & 2.7405152508 & 4.1312222622 & 0.8027898149 \\
\hline $\mathrm{N}$ & & & 958306 \\
\hline $\mathrm{C}$ & & & 855 \\
\hline $\mathrm{H}$ & 3.7933512730 & 3.8158376357 & 2105700 \\
\hline $\mathrm{H}$ & & & .8275043709 \\
\hline $\mathrm{C}$ & & & \\
\hline $\mathrm{Br}$ & 94 & 1.302 & 537698 \\
\hline $\mathrm{H}$ & 2.0055366234 & 6.1233024795 & 0.1696978926 \\
\hline $\mathrm{H}$ & 3472 & 5.4949904834 & 857714 \\
\hline $\mathrm{O}$ & & & \\
\hline $\mathrm{H}$ & -7.276 & 73087 & 265190 \\
\hline $\mathrm{N}$ & -0.0807372121 & -1.7817734017 & -0.4247873461 \\
\hline $\mathrm{C}$ & & & 0.3460767536 \\
\hline $\mathrm{H}$ & 307733820 & -2.7842272870 & 1424680282 \\
\hline $\mathrm{H}$ & -0.1879783955 & -3.8687775733 & 0.0552345975 \\
\hline $\mathrm{H}$ & 1.4248298475 & -3.1202664629 & 0.175481586 \\
\hline $\mathrm{C}$ & 0.1372118752 & -2.0358346427 & -1.850527279 \\
\hline
\end{tabular}




$\begin{array}{lrrr}\mathrm{H} & 0.0235929785 & -1.1185846637 & -2.4399029772 \\ \mathrm{H} & 1.1774778754 & -2.3578999937 & -1.9763935703 \\ \mathrm{H} & -0.5372258210 & -2.8215538775 & -2.2410212890\end{array}$

EC2

01

O 1.1551415629

C $\quad 1.9685576686$

C $\quad 2.7650276986$

$-0.4600184195$

$-0.6175353191$

$-0.9709461449 \quad 0.3509686476$

C $\quad 3.3386080162$

$-2.2626838849$

$-0.0650307196$

C $\quad 4.1064021037$

$-0.2415792249$

0.4642129439

$\mathrm{H} \quad 2.6428275164$

$-1.5797780435$

0.3045486529

2.5415250497

$-2.4138292794$

$-1.1464722211$

H 3.4371344494

$-3.1988677642$

0.4701446126

3.5603015201

3990838318

$-0.4210249251$

0.3588849034

1.3594743983

C $\quad 4.6939354920$

$-2.1085538321$

1.6178782177

5.0919654962

$-3.1258222202$

1.4778415374

H $\quad 5.5212366018$

$-1.4667185887$

1.9601224811

3.9323572200

$-2.1480710180$

2.4102718500

$-1.6031777882-0.7553239292$

C $\quad 5.1892573437$

$-0.5058456580$

$-0.9388187593$

C $\quad 5.4106843445$

$-2.7469742712$

$-1.5306158564$

C $\quad 7.0723748044$

$-0.5457011871$

$-1.8724080398$

0.3975225594

$-0.3424293182$

C $\quad 6.4428844428$

$-2.7921618171$

$-2.4661978164$

$\mathrm{H} \quad 4.7610789460$

C $\quad 7.2784042534$

$-3.6169240636$

$-1.4015068307$

$-1.6899182423-2.6417648119$

7.7203556490

0.3245420665

$-2.0017050588$

$\mathrm{H} \quad 6.5939658613$

$-3.6940786563$

$-3.0641775440$

1.1871415332

$-1.1656312600$

1.6594234757

$-0.7516828852$

2.8621911062

$-0.2548680280$

2.9825148227

2.5884233062

$-0.9477048011$

3.7789341515

1.0641813544
-0.1522544188

$-1.8224262834$

1.5268673489

$\begin{array}{ll}-2.6885181715 & 0.4554282500\end{array}$

$-0.4427596212$

$-1.5716668773$

2.4516936921

$-1.6933785771$

$-3.2889611370$

0.3322147499

$-2.8914246406 \quad-0.2990524143$

$\mathrm{H} \quad 0.3160688821$

C $\quad-2.4237661445$

$-2.1805133867$

2.3366272717

$-1.0121420177$

$-0.8653697541$

3.2668704307

$-2.6875933192$

$-3.0437098546$

1.2764922603

$\mathrm{H} \quad-1.8921476849$

$-3.9462547631$

$-0.5162611616$

$-3.1993708845$

$-1.9606020483$

3.0737645818

$-3.5154327701 \quad 1.1772813739$

$-3.6674953831$

0.1266241093

$-0.9304153022$

$\begin{array}{ll}\mathrm{Mg} & -0.5618462741 \\ \mathrm{O} & -2.5210623056\end{array}$

0.1266241093

$-0.0525369095$

$-0.4842394949-2.3088585808$

O $\quad-1.9800904290$

$-0.0870004200$

$-1.3704310176$

$-3.1107437787$

$-0.0870004200$

$-1.2299385458$

O $\quad-4.1554363330$

$-1.2941421612$

$\begin{array}{rr}1.1226149149 & -1.9775785055 \\ 1.0025257640 & -3.2951172917\end{array}$

$-4.5195502508$

$-3.3618648191$

$-5.2370220681$

$-5.0352044105$

1.9416538636

$-3.5315619603$

$-3.7068619707$

0.8342877545

$-4.0176661990$

$-5.2054492232$

$-1.2011245091$

$-0.2813942330$

$-5.7432526489$

$-2.1569714365$

$-0.2991522317$

$-4.8029541982$

$-1.0233570886$

0.7273127937

$-5.9017105907$

$-0.3881146775$

$-0.5436783339$

$-0.6570582331$

2.2063117640

$-1.1748797636$

$-1.2628542393$

2.9621512494

$-0.4323728435$ 


$\begin{array}{lr}\mathrm{C} & -2.3551355668 \\ \mathrm{~N} & -1.0830535505 \\ \mathrm{C} & -2.7423803732 \\ \mathrm{H} & -3.1607722329 \\ \mathrm{H} & -1.9992381558 \\ \mathrm{C} & -1.9453056872 \\ \mathrm{Br} & 0.0316204245 \\ \mathrm{H} & -2.4817870200 \\ \mathrm{H} & -3.8099311565 \\ \mathrm{O} & -1.9882015383 \\ \mathrm{H} & 8.0857187643\end{array}$

3.9166639670

3.0449402513

4.6386546356

3.2855298586

4.5708192140

3.9628948953

1.8842570431

5.7070914818

4.5643597020

4.1389875294

$-1.7218250506$
$-0.8278163359$

0.9195751425

0.4630848637

$-1.2366315372$

$-1.6352381683$

1.5647865165

1.8177294314

0.4616796909

0.7098977706

2.7427644838

$-3.3768292459$

EC3

01

O $\quad 1.1150658508$

C $\quad 1.9220155910$

C 2.6645869831

$-1.0244704948$

$-0.7587422367$

$-1.4737967281$

0.2443870991

$-2.8319347250$

$-0.0231338426$

3.3196894747

$-0.8006993474$

$-2.1858146096$

0.2931778050

4.0232163110

$-3.0570535076$

0.3555549131

$\mathrm{H} \quad 2.6092692267$

2.3344712720

$-3.7116148999$

$-1.0976439844$

3.4826413531

3.5308356998

$-0.3153361102$

0.5473798400

$-0.0698617746$

$-0.6779445695$

$-2.3903864532$

1.0876722344

5.1335065747

6.0091396658

$-1.7636097803$

$-0.6783155261$

5.4661384336

$-3.4404116448$

$-0.4463623716$

4.7787512663

4.5681896790

$-2.1266481494$

$-0.6918043252$

$-2.5450986770$

$-1.6865998095$

1.7310578977

$-3.7967666656$

2.3172325934

4.3588008291
5.3699313964

$-1.6257532323$

2.4217180065

$-4.1136264895$

$-4.5425396070$

3.5587087894

1.8018333342

3.7507260486

5.9228550716

$-1.9349900927$

3.6618515430

$\begin{array}{ll}-0.6440909313 & 1.9800628279\end{array}$

$-3.1827586653$

4.2402464340

$\begin{array}{ll}-5.0971363181 & 3.9967527095\end{array}$

4.7246343444

$-1.1963972253$

3.9967527095
4.1808781500

6.5384772130

$-1.5336480644$

1.5874876910

1.6983053954

$-1.1267677862$

2.7578997118

$-0.7370004089$

2.8312284777

2.7140545629

$-1.2524946602$

3.7007175106

1.1607428339

$-2.0776454099$

1.5256998687

$-0.6166705525$

$-2.9653776876$

0.5136561791

$-1.7030622715$

2.4616720289

$-1.1993593023$

$-3.4575297669$

0.4303303257

$-1.9182846699$

$-3.2761927490$

$-0.2462340971$

$-2.4924078589$

$-2.1943433698$

2.4014766909

$-0.9811004159$

3.2428310089

$-0.9544540716$

$-3.0728088338$

1.3780891633

$-2.8696242665$

$-4.1258905136$

$-0.3906351128$

$-3.2451622362$

$-1.8862722207$

3.1290716600

$-0.3037636134 \quad-0.9332022445$

$\begin{array}{lll}0.3010973015 & 0.0378449509\end{array}$

$-2.4258192495$

$-0.8709176566$

$-2.1347369105$

$-0.2148466457$

$-1.1809297525$

$-1.2279343238$

$-0.8387763224$

1.0436447557

$-1.8683274783$

$0.8670904515 \quad-3.1310731403$

$\begin{array}{ll}0.1596347428 & -3.0585650742\end{array}$

$1.8462646065 \quad-3.4486611927$ 


$\begin{array}{cc}\mathrm{H} & -3.8297861602 \\ \mathrm{C} & -5.3160438537 \\ \mathrm{H} & -6.0444075755 \\ \mathrm{H} & -4.8226916657 \\ \mathrm{H} & -5.8319730727 \\ \mathrm{O} & -0.4643033066 \\ \mathrm{C} & -0.9001613464 \\ \mathrm{C} & -1.8736391348 \\ \mathrm{~N} & -0.6127922663 \\ \mathrm{C} & -2.0547864931 \\ \mathrm{H} & -2.7888671348 \\ \mathrm{H} & -1.4927725658 \\ \mathrm{C} & -1.2855229097 \\ \mathrm{Br} & 0.3814507630 \\ \mathrm{H} & -1.6404256855 \\ \mathrm{H} & -3.1002958392 \\ \mathrm{O} & -1.2185465269 \\ \mathrm{H} & 6.1181107838 \\ \mathrm{O} & -4.1552872422 \\ \mathrm{C} & -4.5983763352 \\ \mathrm{H} & -5.6616763418 \\ \mathrm{H} & -4.4849887094 \\ \mathrm{H} & -4.0598146820\end{array}$

0.4921795507

$-0.8581022373$

$-1.6737729483$

$-0.7256178345$

0.0734069583

1.7466422870

2.6351397112

3.7072188455

2.7899424760

4.5749838043

3.1708949285

4.2377427543

3.8700747087

1.5375679969

5.5878520359

4.6836053772

4.1382849687

$-3.4278184246$

$-3.4824009829$

$-4.3042186901$

$-4.5018977847$

$-3.8006970431$

$-5.2674194490$
$-3.8729863234$

0.1542777514

0.2257341052

1.1279303849

$-0.1287020607$

$-1.3800254394$

$-0.6657145952$

$-1.0718384557$

0.6611485986

0.1736541541

$-1.3703784316$

$-1.9549525267$

1.2774248828

1.5861109455

0.0643963128

0.4916418933

2.4370842066

5.2150399748

1.3766603156

0.3278071226

0.5133848068

$-0.6470284318$

0.3085545454

EC4

01

O $\quad-1.1905436972$

C $\quad-2.0693949327$

$-0.3311755444$

0.5427259828

$-0.2040920775$

$-0.4919184498$

$-3.2773048384$

$-1.2113940051$

$-0.4596037481$

C $\quad-3.1034197311$

0.9428189795

$-0.3015297678$

$-4.2860257629$

$-0.0362646568$

$-0.5238674299$

$-3.2758230256$

$-1.7253112022$

0.5115084320

$-3.3515702354$

$-1.9505606471$

$-1.2725633519$

$-3.0322023442$

1.2742772650

0.7419451514

$-3.0564509241$

1.8273161097

$-0.9547941346$

$0.1003637628-1.9014908064$

$-4.9439388684$

$-0.7126519749$

$-2.0620004142$

$-5.6691302909$

-0.7126519749
1.0561380799

$-1.9807371802$

$-4.1985224750$

0.0538473722

$-2.7088413466$

$-0.0204157581$

0.5358958272

$-5.3693045103$

1.1847250092

1.0963983364

-5.8068230774
-6.0047073538

$-1.2028177006$

0.9312815789

2.0292188514

0.7999544051

$-5.3263498632$

2.1210653346

1.8642099177

$-7.0401445044$

$-1.1845040131$

$-2.1556392857$

0.5034875711

$-7.4628639007$

0.0228903112

2.4184501633

$2.1612532949 \quad 2.4571145183$

$-7.1648803282$

$-2.120622701$

2.1621740879

$-7.5183169587$

$-1.3226992657$

$-0.2353484131$

$-1.8328218057$

0.6445235678

$-2.8291589401$

$-2.2655075107$

1.4450963292

$-2.7433798072$

$-1.0171051629$

0.5605268324

$-3.7900541098$

$-0.2964519336$

$-1.3146001250$

$-1.9862458990$

$-2.5165106151 \quad-1.2599793599$

$-0.3804682562$

$-1.1561658125$

$-2.8498874776$

0.7972633817

$-3.5283432633$

$-1.4288184902$

0.5576476270
-1.1888626532

$-2.6647424534$

$-0.5461151087$

$-2.1672717731-3.0317882831$

1.7330430218

$-0.2149245118$

$-3.3827513993$ 


\begin{tabular}{ll}
$\mathrm{C}$ & 1.6092063523 \\
$\mathrm{H}$ & 0.4696795588 \\
$\mathrm{H}$ & 2.5690734801 \\
$\mathrm{Mg}$ & 0.5778008150 \\
$\mathrm{O}$ & 2.5225252389 \\
$\mathrm{O}$ & 1.6416848934 \\
$\mathrm{P}$ & 2.8751502343 \\
$\mathrm{O}$ & 3.4488750310 \\
$\mathrm{O}$ & 4.0992413613 \\
$\mathrm{C}$ & 4.5060841370 \\
$\mathrm{H}$ & 4.9106887271 \\
$\mathrm{H}$ & 5.2917495375 \\
$\mathrm{H}$ & 3.6605909454 \\
$\mathrm{C}$ & 4.5421572675 \\
$\mathrm{H}$ & 4.7563565716 \\
$\mathrm{H}$ & 4.2804062938 \\
$\mathrm{H}$ & 5.4307536655 \\
$\mathrm{O}$ & 1.3256609622 \\
$\mathrm{C}$ & 2.1910763236 \\
$\mathrm{C}$ & 3.5135583971 \\
$\mathrm{~N}$ & 2.1264642859 \\
$\mathrm{C}$ & 4.1881424725 \\
$\mathrm{H}$ & 4.0413944238 \\
$\mathrm{H}$ & 3.3516661285 \\
$\mathrm{C}$ & 3.2794780742 \\
$\mathrm{Br}$ & 0.7471852190 \\
$\mathrm{H}$ & 4.2882118404 \\
$\mathrm{H}$ & 5.1863219176 \\
$\mathrm{O}$ & 3.4441170116 \\
$\mathrm{H}$ & -8.2715691227 \\
$\mathrm{C}$ & 2.5542285883 \\
$\mathrm{~F}$ & 2.7892189805 \\
$\mathrm{~F}$ & 3.7419174340 \\
$\mathrm{~F}$ & 2.0640994518 \\
& \\
\hline
\end{tabular}

$-3.3643897894$

$-4.4503577356$

$-2.0172974272$

$-0.3342484064$

$-0.5406736199$

$-2.9105434977$

$-0.8462451490$

$-1.4628674077$

$-2.4699596509$

$-0.8349043583$

$-1.5409783618$

$-2.9506605665$

$-4.0058254370$

$-2.4495573914$

$-2.4665392456$

1.4561128799

2.1307477977

2.5259351499

2.6152391082

3.3920263095

1.5773457638

3.0210576471

3.3287730765

2.1610854748

4.4469464515

3.0394753136

3.7779285797

0.0390580658

$-4.5045264436$

$-5.2313223233$

$-4.0811011081$

$-5.3434989719$
$-1.7098879627$

$-1.5000442926$

$-2.3302113230$

$-0.8522929646$

$-3.7165158256$

1.0362540487

0.1471959794

2.1439388518

1.2751038584

0.7909242963

2.1138773655

3.3270724074

3.1392495696

3.7653454863

4.0269864010

$-0.1156439250$

$-0.3150164706$

$-1.0588521300$

0.3200770455

1.8029550585

1.2657390311

1.8608582051

$-0.0086263107$

0.7964086782

2.0520328846

2.8278267675

$-0.4181560206$

$-1.1435444601$

1.0908690396

0.5042587142

$-1.5093367357$

3.1524334725

$-2.5950728844$

$-1.4957998178$

$-3.0522551358$

$-3.5152394506$

EC5

01

O $\quad-0.8878564081$

C $\quad-2.0094976799$

C $\quad-3.1255272467$

$-0.6009694701$

$-0.5485706783$

$-1.6021223533$

0.5567758901

$-0.4755481938$

$-2.0690556682$

$-2.3872265567$

0.9121568623

1.4260152716

$-0.4867111278$

0.4300058218

$-1.3464573019$

$-0.5522718676$

$-0.3707702965$

$-1.4988759275$

0.8786561067

$-1.3839893842$

$-2.4898272721$

1.0007615776

1.7763582356

$-0.1328272936$

$-2.2816708180$

1.9881849689
1.9509394368

1.5043114982

1.5740684017

1.6390517504

2.4717928885

0.7589590252

2.5739093645

0.9104231684

2.9633360796

3.0778589888

3.0099852638

3.8142086119

0.4981664224

$-0.1694529351$

0.1367587003

$-1.1775868696$

0.0991585852

$-0.8702432120$

0.6537520407

$-1.5376012308$

$-1.6864340025$

$-1.1354788115$
1.1721795554 


\begin{tabular}{lr}
$\mathrm{C}$ & -1.6411139625 \\
$\mathrm{C}$ & -2.2962656925 \\
$\mathrm{H}$ & -3.1779158344 \\
$\mathrm{H}$ & -2.0140035310 \\
$\mathrm{Mg}$ & 0.8095354793 \\
$\mathrm{O}$ & 3.2465610391 \\
$\mathrm{O}$ & 2.3447935372 \\
$\mathrm{P}$ & 3.4190638524 \\
$\mathrm{O}$ & 3.5224533466 \\
$\mathrm{O}$ & 4.8573485720 \\
$\mathrm{C}$ & 5.2259321855 \\
$\mathrm{H}$ & 5.2302567036 \\
$\mathrm{H}$ & 6.2386340556 \\
$\mathrm{H}$ & 4.5336400857 \\
$\mathrm{C}$ & 4.3619524702 \\
$\mathrm{H}$ & 4.1931401161 \\
$\mathrm{H}$ & 4.1237735682 \\
$\mathrm{H}$ & 5.4231657328 \\
$\mathrm{O}$ & 1.9362944361 \\
$\mathrm{C}$ & 2.5800494095 \\
$\mathrm{C}$ & 4.0404941502 \\
$\mathrm{~N}$ & 2.0489664109 \\
$\mathrm{C}$ & 4.3631252323 \\
$\mathrm{H}$ & 4.6019661559 \\
$\mathrm{H}$ & 4.1716737296 \\
$\mathrm{C}$ & 3.0249433626 \\
$\mathrm{Br}$ & 0.2946926003 \\
$\mathrm{H}$ & 4.9876397461 \\
$\mathrm{H}$ & 4.8395570629 \\
$\mathrm{O}$ & 2.7752735287 \\
$\mathrm{H}$ & -7.8713912397 \\
$\mathrm{~N}$ & -0.4432635641 \\
$\mathrm{C}$ & -0.5716676497 \\
$\mathrm{H}$ & -0.8923511604 \\
$\mathrm{H}$ & -1.3053082720 \\
$\mathrm{H}$ & 0.4098782694 \\
$\mathrm{H}$ & 0.1059957231 \\
& 0.3065100339 \\
$\mathrm{H}$ & 1.0564708688 \\
\hline
\end{tabular}

EP2

01

$\begin{array}{ll}\mathrm{O} & -0.4468264800 \\ \mathrm{C} & 0.8161418656 \\ \mathrm{C} & 1.7481340124 \\ \mathrm{C} & 1.9411516581 \\ \mathrm{C} & 2.9330572315 \\ \mathrm{H} & 1.6880930351 \\ \mathrm{H} & 1.6519288516 \\ \mathrm{H} & 1.9596338189 \\ \mathrm{H} & 1.9881800757 \\ \mathrm{C} & 3.4431919312 \\ \mathrm{H} & 3.9778840380 \\ \mathrm{H} & 4.1391092324 \\ \mathrm{H} & 2.6108459950 \\ \mathrm{C} & 4.0869709218 \\ \mathrm{C} & 4.7997674535 \\ \mathrm{C} & 4.5021445826 \\ \mathrm{C} & 5.8952575185\end{array}$

$\begin{array}{ll}-0.5594157471 & -0.3288788732\end{array}$

$0.1005135119-1.2935984801$

$0.6958220482-1.0602055503$

$0.0370466170 \quad-2.3449817262$

$\begin{array}{ll}-0.5953518361 & 1.1483609643\end{array}$

$-0.0244312646 \quad-0.8868114351$

$\begin{array}{ll}-1.7558797636 & 0.8246815915\end{array}$

$-1.3352715103-0.1832029118$

$-2.5817804574 \quad-1.2127105023$

$\begin{array}{ll}-1.3210340233 & 0.5656085372\end{array}$

$\begin{array}{ll}-2.3995793702 & 1.4055482132\end{array}$

$-3.3502315885 \quad 0.8482502154$

$-2.1955182124 \quad 1.7760318285$

$-2.4917114754 \quad 2.2561564572$

$-2.4648498463 \quad-2.3467463738$

$-3.3500122338 \quad-2.9729796450$

$-1.5552368645-2.9193186876$

$-2.4308833701-2.0501222337$

$\begin{array}{ll}1.0673045461 & 1.6066597177\end{array}$

$\begin{array}{ll}1.8179157829 & 0.8871136721\end{array}$

$\begin{array}{ll}2.1539661411 & 0.9928622532\end{array}$

$2.5396551227 \quad-0.1424982393$

$2.8223211240 \quad-0.3438720131$

$\begin{array}{ll}1.2301027190 & 1.1804711559\end{array}$

$2.8268442044 \quad 1.8551472164$

$3.1547496648 \quad-0.9698221422$

$2.3317925809-0.6356124856$

$3.7222957939-0.2821880078$

$2.1012927137 \quad-1.0242839584$

$3.7735114924-1.9568761917$

$-0.0409696451-2.3306620215$

$-1.3139329490 \quad-0.5964290842$

$-2.7388705812 \quad-0.2316526796$

$\begin{array}{ll}-2.8385304835 & 0.8121724438\end{array}$

$-3.2433988596-0.8853026043$

$-3.2192403178-0.3437276448$

$-1.2045202716-1.9449675287$

$-0.1556745836 \quad-2.1950869317$

$-1.7526368428-1.9795727453$

$-1.6349724901-2.7020292894$
0.3314510940

0.5711350691

$-0.5257045336$

1.3124840991

0.4280710868

$-1.3895751465$

$-0.8616502776$

1.0765201317

2.4014015612

1.1652435688

0.4713789390

1.9679933996

1.6096580600

$-0.1774248478$

0.5861883162

$-1.4903087477$

0.0511879406
$-0.5627767075$

0.0308177516

0.5014833523

$-0.6608533190$

0.1591141647

$-0.1757870592$

1.5432919435

$-1.7345742871$

$-0.5170636755$

1.4003404790

2.0663807521

1.1131467915

1.9682480583

$-0.6079048229$

$-1.5387431251$

$-0.3645649454$

$-2.2134369619$ 


\begin{tabular}{|c|c|c|c|}
\hline $\mathrm{H}$ & 4.4919500088 & 55597 & -1.7413 \\
\hline$f$ & 5.5981894255 & -2.0292139918 & \\
\hline 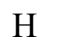 & .9579621717 & -2.1040479465 & ת \\
\hline $\mathrm{C}$ & .2982779648 & -1.2601626077 & -1.9643063225 \\
\hline $\mathrm{H}$ & 4360123419 & 0.6614182286 & \\
\hline $\mathrm{H}$ & 9046145892 & -3.0583842604 & -0.836242987 \\
\hline $\mathrm{C}$ & .4155502738 & 1.1069310056 & 7822 \\
\hline $\mathrm{C}$ & .9921012668 & 0.3538484762 & 8030 \\
\hline $\mathrm{H}$ & 9457483226 & -0.7257867849 & \\
\hline $\mathrm{H}$ & .0352019189 & 0.6375347188 & 2.0359850463 \\
\hline $\mathrm{C}$ & -0.8247800408 & 2.5367462106 & 0.4365738821 \\
\hline $\mathrm{C}$ & 58934 & 3.06 & \\
\hline $\mathrm{C}$ & 0189 & 3.3 & \\
\hline $\mathrm{C}$ & 3676 & 4.38 & -1.091 \\
\hline $\mathrm{H}$ & -0.5159271592 & 82039 & 12752 \\
\hline $\mathrm{C}$ & 66 & & \\
\hline $\mathrm{H}$ & & & \\
\hline $\mathrm{C}$ & 153 & & \\
\hline $\mathrm{H}$ & 42 & & 5683 \\
\hline $\mathrm{H}$ & 73 & 5.2 & 229 \\
\hline $\mathrm{H}$ & & & \\
\hline $\mathrm{Mg}$ & & & 68216 \\
\hline $\mathrm{O}$ & & & \\
\hline $\mathrm{O}$ & & & \\
\hline $\mathrm{P}$ & -0.9 & -3.2 & -0.7 \\
\hline $\mathrm{O}$ & & & \\
\hline $\mathrm{O}$ & 66 & -4.7 & 019 \\
\hline 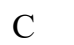 & 493 & -5.5 & 746 \\
\hline $\mathrm{H}$ & 5533 & 268 & 00283 \\
\hline$H$ & & & \\
\hline $\mathrm{H}$ & 39 & -5.0 & 1544 \\
\hline $\mathrm{C}$ & 0.77 & -4.1 & 850 \\
\hline $\mathrm{H}$ & & & \\
\hline $\mathrm{H}$ & & & \\
\hline $\mathrm{H}$ & 36 & & 057 \\
\hline $\mathrm{O}$ & 3168 & 796 & 681808 \\
\hline $\mathrm{C}$ & & & \\
\hline 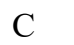 & 35 & & \\
\hline $\mathrm{N}$ & 3381 & 19977 & 22392 \\
\hline $\mathrm{C}$ & & 07886 & -0.5452988093 \\
\hline $\mathrm{H}$ & & & \\
\hline $\mathrm{H}$ & & & -2.52 \\
\hline $\mathrm{C}$ & -3.9777 & 031514 & 44252 \\
\hline $\mathrm{Br}$ & 90356 & 0.67 & 5108 \\
\hline $\mathrm{H}$ & & & \\
\hline $\mathrm{H}$ & & & \\
\hline $\mathrm{O}$ & -4.0282640601 & 1.4135327511 & 1.5175679438 \\
\hline & 7.1543455708 & -1.6825170717 & -2.49470211 \\
\hline
\end{tabular}

EP3

01

$\begin{array}{lrrr}\mathrm{O} & -2.0049647340 & -0.9651705384 & -2.7461223143 \\ \mathrm{C} & -1.4991322749 & -0.2082587416 & -1.6521221536 \\ \mathrm{C} & -1.6832604647 & 1.2911596544 & -1.5742968415 \\ \mathrm{C} & -1.8690407907 & -0.3908706016 & -0.1943060186 \\ \mathrm{C} & -1.7362568479 & 1.1568651464 & -0.0200551026 \\ \mathrm{H} & -2.6611293530 & 1.5698350036 & -1.9907365593 \\ \mathrm{H} & -0.8961846110 & 1.9135658280 & -2.0188970813 \\ \mathrm{H} & -2.9031961357 & -0.7468314475 & -0.0904014338 \\ \mathrm{H} & -1.1937616171 & -1.0248743685 & 0.3947087282\end{array}$




\begin{tabular}{|c|c|c|c|}
\hline$C$ & 3046567 & 565 & 420 \\
\hline & -3.0087642585 & 1.5604897671 & 1.6969345718 \\
\hline & -2.8713125185 & 2.9200961713 & 0.5582116785 \\
\hline & -3.8752982335 & 1.4956161640 & 357010948 \\
\hline & -0.4600590137 & 1.5871741200 & 0.6890593554 \\
\hline & 0.2745832358 & 2.6981351662 & 0.2615142299 \\
\hline & -0.0352780261 & 0.9246718362 & 1.8471823123 \\
\hline & 1.4074957754 & 3.1221683473 & 0.9542222374 \\
\hline $\mathrm{H}$ & -0.0364160159 & 3.2481790469 & -0.6291732262 \\
\hline & 1.0955798478 & 1.3443512156 & 2.5441941914 \\
\hline & -0.5985632292 & 0.0641822357 & 2.2170593001 \\
\hline 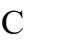 & 1.8258850694 & 2.4440951242 & 2.0968746493 \\
\hline & 1.9679185080 & 3.9879737651 & 094550 \\
\hline & 1.4088284823 & 0.8063645407 & 3.4417824410 \\
\hline $\mathrm{c}$ & -0.5798255380 & -0.9944403924 & -2.5030171562 \\
\hline & 0.1768167205 & -0.2547340649 & -3.5799071786 \\
\hline $\mathrm{H}$ & -0.4571648848 & 570 & 2130 \\
\hline & 6651868 & 134 & 9204 \\
\hline & -0.0951288521 & 0413 & 3053 \\
\hline & -0.9324497318 & -3.1995809828 & 674997 \\
\hline & 1.1215789108 & 3435 & 1250 \\
\hline c & 26344 & -4.51 & 1813 \\
\hline $\mathrm{H}$ & -1.8999743714 & -2.8498548428 & -0.9612279444 \\
\hline C & 3566 & 5775 & 2283 \\
\hline & 4589 & 306 & 7687 \\
\hline $\mathrm{C}$ & 0.625 & -5.0 & -1.5 \\
\hline $\mathrm{H}$ & -1.2568173202 & -5.1778629252 & 408154 \\
\hline $\mathrm{H}$ & 8662 & -4.5 & 2773 \\
\hline $\mathrm{Mg}$ & -3.33 & -2.4 & 1047 \\
\hline $\mathrm{O}$ & -4.9514809799 & -1.30 & -3.732 \\
\hline & -4.38 & -2.0 & 51950 \\
\hline & -5.31 & 655 & 60926 \\
\hline $\mathrm{O}$ & -5.15 & 033 & 33134 \\
\hline $\mathrm{O}$ & -6.8697021763 & -1.4856086891 & -2.0518289838 \\
\hline & & -1.6 & 1056 \\
\hline & 998 & 506 & 8239 \\
\hline $\mathrm{H}$ & -8.4440292419 & 829664 & -0.8426780205 \\
\hline $\mathrm{H}$ & -6.84 & & \\
\hline C & -5.7 & & 131 \\
\hline $\mathrm{H}$ & $-5.4^{\top}-x+2$ & 192 & 4680 \\
\hline $\mathrm{H}$ & -5.437 & 5792 & -3.4998770604 \\
\hline $\mathrm{H}$ & -6.86 & & 561088 \\
\hline $\mathrm{O}$ & -3.35 & -4.66 & 07680 \\
\hline $\mathrm{C}$ & -2.3496686846 & 000342 & -4.0454828717 \\
\hline $\mathrm{C}$ & -1.5042186069 & & -4.4542722844 \\
\hline & & & \\
\hline $\mathrm{C}$ & 7222 & 85820 & -5.2884397219 \\
\hline $\mathrm{H}$ & -2.1212083622 & -6.5044089921 & -4.9981445112 \\
\hline $\mathrm{H}$ & & & 31370 \\
\hline $\mathrm{C}$ & -0.71 & -3.6 & 6903 \\
\hline $\mathrm{Br}$ & 5731 & 00086 & -2.8590410703 \\
\hline $\mathrm{H}$ & 0.6070871247 & -5.2835525613 & -4.9024348755 \\
\hline $\mathrm{H}$ & & & -6.3456502405 \\
\hline $\mathrm{O}$ & -0.006 & -2.73 & -5.6525971370 \\
\hline $\mathrm{H}$ & 2.7163951510 & 2.7715951938 & 204259 \\
\hline 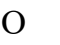 & 0.8557727078 & -6.3371349537 & -1.3276335108 \\
\hline $\mathrm{C}$ & & & -1.7937211932 \\
\hline $\mathrm{H}$ & & -7.9685747517 & -1.4930018881 \\
\hline $\mathrm{H}$ & 2.1243283845 & -6.8585941304 & -2.8936596056 \\
\hline 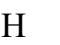 & 2.9347900201 & -6.4350131770 & -1.3482103819 \\
\hline
\end{tabular}


EP4

01

O $\quad-0.2694501292$

0.3200916031

$-0.4736867824$

$-0.7452519372$

$-0.9144224632$

0.0317907556

C $\quad-2.1434565354$

$-1.1218903760$

$-0.8069342348$

$-2.1878424681$

0.5736986006

C $\quad-2.0438485919$

$-2.5963254842$

$-0.7855450915$

$-2.8639988804$

$-0.5238638856$

0.0760278662

$-2.3002162254$

$-0.9587196963$

$-0.0021013359$

$-1.0782133362$

$-1.9677028452$

1.6489951786

0.0734826448

$-2.8458908751$

$-1.8280387481$

$-1.6474516732$

$-3.5496535363$

$-0.7598405140$

$-2.4804660669$

$-3.6718446599$

1.2066218923

$-1.3935721067$

$-4.5416712185$

1.9151087327

$-0.7790984215$

$-3.1656026838$

0.8034869913

$-3.2382759919$

$-3.1364761783$

1.7647194146

$-3.0647823616$

$-4.0608373905$

$-0.6778516803$

C $\quad-4.5396870962$

$-2.7699269452$

$-1.7137514817$

$-0.3215201638$

$-4.6011791735$

$-2.3807329510$

$-2.0547673925$

$-4.3621617819$

$-2.0056005614$

-2.0547673925
-5.6405619397

$-3.3089796012$

$-0.9853953923$

$-4.6971296527$

$-2.0481256213$

0.4841891177

$-4.2261284260$

$-2.0183200085$

$-4.0066021163$

$-5.3178089807$

$-3.1902282655$

$-6.6495965627$

$-3.0070525311$

$-0.6959379187$

$-0.2535524234$

0.6543686508

0.5292006454

1.9329209902

0.2098894905

1.1119934194

1.8589653304

$-0.7164165163$

1.2113646828

2.1363183358

1.8237902452

$-0.6776328636$

0.2981565302

$-0.8277525982-1.0491008921$

C $\quad 2.1780876540$

$-0.8581042936$

1.2781826210

$\begin{array}{ll}\text { C } & 2.8025520995 \\ \text { C } & 3.4829564715\end{array}$

-0.8581042936
-1.1400737069

$-1.4089310998$

$\mathrm{H} \quad 1.4312308132$

$-0.6619464359$

$-1.8265021512$

$\begin{array}{ll}-1.1703839380 & 0.9209529207\end{array}$

C 4.1110713485

H 2.5593344306

$-0.7345305919$

2.3331449329

4.4557020958

$-1.3038119513$

$-0.4218015157$

$\mathrm{H} \quad 3.7480788060$

$-1.2463531165$

$-2.4622917790$

$-1.2935454646 \quad 1.6956014192$

$\mathrm{Mg} \quad-0.4566979329$

2.3251089254

$-0.8956719260$

$\mathrm{Mg} \quad-0.4566979329$

2.9356159808

0.6483073719

$2.3147407790 \quad-1.6446114137$

$2.7484191133 \quad-0.2843150086$

$-2.9295064382$

1.6352035874

0.2535155928

O $\quad-3.9640977271$

O $\quad-3.7922683971$

4.0970443361

4.2310327500

$-0.3338380373$

C $\quad-4.8424383685$

$-5.6515440159$

3.5123820169

$-1.2802502166$

$-5.2317037181$

5.2520012189

$-1.0740122782$

$-4.4685277582$

4.0688624962

1.6964366526

$-1.1878210681$

$-4.4331731482$

$-5.1037044552$

0.8406778857

- 3022626961

1.5906480979

$-3.5967643851$

1.6363836598

1.7382280281

$-4.9904323791$

2.6292112217

2.3040427937

0.9208471667

2.5909106793

1.7713658032

$-2.4599228398$

$2.5949458295 \quad-1.6413006587$

1.8672272316

2.5937290987

$-1.9508612026$

$\begin{array}{ll}2.5658681691 & -0.3320752407\end{array}$

1.5973731938

$-0.5576926280$

C $\quad 3.9678251859$

$\mathrm{H} \quad 3.6016035247$

3.5015994

$-2.5154266514$ 


$\begin{array}{lr}\mathrm{H} & 3.5761838756 \\ \mathrm{C} & 2.7692634718 \\ \mathrm{Br} & 0.0162740365 \\ \mathrm{H} & 4.5597338527 \\ \mathrm{H} & 4.6161889518 \\ \mathrm{O} & 2.8430165629 \\ \mathrm{H} & -6.3167822995 \\ \mathrm{C} & 5.8905544924 \\ \mathrm{~F} & 6.5875251239 \\ \mathrm{~F} & 6.5069889029 \\ \mathrm{~F} & 5.9888959838\end{array}$

1.7344798543

2.4900504246

$-0.6373997322$

1.5990966464

3.3631957927

2.3851071991

$-4.6458138088$

$-1.5297900466$

$-2.1476198422$

$-0.3623888213$

$-2.2613520669$
$-2.5968767123$

0.3990953559

3.4839738942

$-0.3993404809$

$-0.3062675793$

1.6013741164

$-2.5421756761$

$-0.8204155425$

0.1344707949

$-1.0660513017$

$-1.9339486380$

EP5

01

O $\quad-0.2105845477$

C $\quad-1.5658562225$

C $\quad-2.4842498271$

$-0.6185287972$

$-0.1528154423$

$-0.4192217147$

1.2987898677

0.9116296137

$-0.3419951935$

$-1.3464463057$

1.9337541770

1.7285763031

1.8352624815

2.8615583844

1.4773991229

1.8883428526

0.6956117306

$-0.4174810861$

1.6309652847

$-0.5792514751$

$-1.1885152963$

1.4694897480

2.5089070990

0.3641379123

$-1.4579625005$

2.2172595919

$-1.1050801483$

$-0.6558801546$

$-0.5890534413$

$-1.3570429311$

0.0467164969

$-1.5522091060$

$-1.4407828138$

$-2.2543226006$

$-3.7551122857$

$-2.4738387989$

$-2.8061243181$

$-3.8064405223$

$-2.8041890179$

$-2.0676431469$

$-4.7239246833$

$-5.5971882747$

$-4.3403373147$

$-5.0259485922$

1.6831747599

2.3951643846

3.8470486960

1.8746133493

4.1384356098
0.6266098791

0.6921164271

1.8696388400

0.6271686198

1.5329514152

2.8170013972

1.8600871919

1.1556085703

$-0.3548950741$

2.7245507994

2.4063761316

3.3682620958

3.3287458287

0.6810335478

$-0.1685693236$

0.6707023780

$-0.9953040207$

$-0.1906611138$

$-0.1547503570$

1.3161060293

$-0.9949866247$

$-1.6414278039$

$-0.1362698293$

$-0.3517461110$

$-1.7857112707$

$-2.3374833455$

$-2.2702992977$

0.4740236054

$-0.9649242022$

1.3472289314

0.0603651001

0.3884293520

$-0.5491625651$

0.3061223445

0.7486484103

$-0.3068872296$

1.1153527098

$-0.6433884813$

$-0.2643815423$

$-1.5432989742$

$-0.9184380342$

1.7850266935

1.1988281820

0.2060463156

0.4587590053
1.4752370346 


$\begin{array}{lrrr}\mathrm{H} & 2.6308951631 & 3.9603628936 & 2.5256455873 \\ \mathrm{H} & 1.4152785523 & 4.4486050400 & 1.3383156283 \\ \mathrm{C} & 3.6077070990 & 2.8186806827 & -0.3162428709 \\ \mathrm{Br} & 0.0049105214 & 1.0515241505 & -1.9722217815 \\ \mathrm{H} & 3.1811034873 & 4.9379873307 & -0.2532845024 \\ \mathrm{H} & 4.3920587848 & 4.4079499541 & 0.9181245990 \\ \mathrm{O} & 4.3782327346 & 2.6475037061 & -1.2236889295 \\ \mathrm{H} & -7.5972127683 & 0.2357126724 & -1.6408585598 \\ \mathrm{~N} & -1.5167814623 & -2.4763506655 & -0.2365778989 \\ \mathrm{C} & -1.0738242184 & -3.1705231173 & 0.9671402587 \\ \mathrm{H} & -1.1601151218 & -2.5388462008 & 1.8556921322 \\ \mathrm{H} & -1.7088891203 & -4.0557513553 & 1.1188302362 \\ \mathrm{H} & -0.0191103669 & -3.4911082111 & 0.8943728464 \\ \mathrm{C} & -1.4151491088 & -3.3313531883 & -1.4038594494 \\ \mathrm{H} & -0.3708417270 & -3.5520124486 & -1.6993233724 \\ \mathrm{H} & -1.9062699210 & -4.2863916720 & -1.1696600842 \\ \mathrm{H} & -1.9464299950 & -2.9090880752 & -2.2669264743\end{array}$

IN5

0

$-0.9862786520$

$-0.9660013293$

$-0.8864770761$

$-0.6867166568$

0.2784255371

$-0.2384493168$

$-0.9505406125$

$-2.0122889416$

$-1.7686747322$

$-0.2699387051$

$\begin{array}{ll}\text { C } & -2.0122889416 \\ \text { C } & -2.7431380023\end{array}$

$-0.9420424638$

$-1.3591964580$

$-1.4157915184$

0.8730080927

$-1.7529607371$

$-2.4324386607$

$-1.6710020058$

0.9340618375

$-0.2659636512$

$-2.7742209152$

$-0.5543338903$

$-2.5860840818$

$-1.8174756309$

0.6690755669

$-2.4135071679$

$-1.4397877816$

$-2.7699146596$

$-2.8967698858$

$-2.4114140020$

$-2.9574503055$

$-2.7810159480$

$-0.7257147703$

$-3.5229525865$

$-1.3264812060$

$-1.5519706298$

$-2.8878523065$

$-4.2393731582$

$-0.7846735441$

$-1.1985468213$

$-4.8726538899$

0.4189761521

$-1.5270349727$

$-1.8564888825 \quad-0.7722065229$

C $\quad-5.0312426853$

0.5520482240

$-1.4244231742$

$\begin{array}{ll}C & -5.0312426853126729 \\ \mathrm{H} & -4.2727153320\end{array}$

1.2667645904

$-1.8690435494$

C $\quad-6.4153498323$

$-1.7285257926$

$-0.6676483274$

$\mathrm{H} \quad-4.5567497247$

$-2.8083787153$

$-0.5179402159$

$-7.0333991884$

$-0.5217378321$

$-0.9921281473$

$\mathrm{H} \quad-6.7306534231$

1.5016506332

$-1.6829477078$

$-7.0150020032$

$-2.5769344968$

$-0.3296246676$

$-0.2528646870$

1.1440217505

$-0.3824710735$

1.1700598485

1.5690990200

$\begin{array}{ll}\mathrm{C} & -0.5538879287 \\ \mathrm{H} & -0.6735760138\end{array}$

1.8194778148

0.6974949752

1.5397569705

2.1912379606

$\mathrm{Mg} \quad 2.0361882813$

$-0.3891016754$

$-1.0009748754$

$\begin{array}{ll}1.2147607587 & 0.5857009972\end{array}$

O 2.0765346972

1.4910763030

$-1.8790527365$

2.2184220362

$-0.5478503259$

P $\quad 2.2120393732$

O $\quad 1.0029990010$

3.2965156082

$-0.5533588850$

3.1218714094

$-0.3105057222$

$\begin{array}{ll}\mathrm{O} & 3.5216279085 \\ \mathrm{C} & 4.0518930714\end{array}$

3.8847988389

$-1.3827852493$

$\mathrm{H} \quad 3.3681812385$

4.7025665174

$-1.6645551085$

5.0013039000

4.3127241812

$-1.0379204527$

$\mathrm{H} \quad 4.2283538839$

3.2502489872

$-2.2638149938$

0.9153225611

4.2786268589

0.4606267727

$\mathrm{H} \quad-0.0526431657$

4.7828639532

0.3454786564

$\mathrm{H} \quad 0.9698863107$

3.8306634013

1.4665775566 


$\begin{array}{lrrr}\mathrm{H} & 1.7229718505 & 5.0214193129 & 0.3678087016 \\ \mathrm{O} & 3.4811118758 & -1.5167885856 & -2.0877648452 \\ \mathrm{C} & 3.8676644516 & -2.0795938387 & -1.0469713137 \\ \mathrm{C} & 5.0064221319 & -3.0666575055 & -0.9173817614 \\ \mathrm{~N} & 3.2551664943 & -1.8207311379 & 0.1179711658 \\ \mathrm{C} & 5.0391553860 & -3.3249884926 & 0.5885133531 \\ \mathrm{H} & 5.9244532105 & -2.6260609405 & -1.3310093759 \\ \mathrm{H} & 4.7784003639 & -3.9587054627 & -1.5192590905 \\ \mathrm{C} & 3.8393661889 & -2.5352337981 & 1.1340126688 \\ \mathrm{Br} & -2.2010572279 & 1.2709516916 & 2.6147126546 \\ \mathrm{H} & 4.9349544411 & -4.3820778028 & 0.8702712704 \\ \mathrm{H} & 5.9524370351 & -2.9526997720 & 1.0757324500 \\ \mathrm{O} & 3.4685676791 & -2.5670325677 & 2.2905345232 \\ \mathrm{H} & -8.1174483461 & -0.4183795564 & -0.9083499577 \\ \mathrm{~N} & 0.2618347188 & -1.0781664811 & 1.9073751611 \\ \mathrm{C} & 0.3579348289 & -2.5092217478 & 1.5947857431 \\ \mathrm{H} & 0.3571130977 & -2.6396804388 & 0.5088073909 \\ \mathrm{H} & 1.3037560685 & -2.8787273176 & 2.0039261302 \\ \mathrm{H} & -0.4936612017 & -3.0295562669 & 2.0572557857 \\ \mathrm{C} & 0.9666762101 & -0.6280683884 & 3.1142937477 \\ \mathrm{H} & 1.7056205833 & 0.1303093662 & 2.8208657129 \\ \mathrm{H} & 0.2514659883 & -0.2214050603 & 3.8430413855 \\ \mathrm{H} & 1.5087182327 & -1.4774009033 & 3.5374542575\end{array}$

TS2a

01

$\begin{array}{lrrr}\mathrm{O} & 0.4954553344 & -1.0559903412 & 1.6688631060 \\ \mathrm{C} & 0.8631483624 & -2.3348521328 & 1.4025669843 \\ \mathrm{C} & 2.0053744991 & -3.0033600649 & 2.2108052070 \\ \mathrm{C} & -0.0329783483 & -3.5074830766 & 1.8611634482 \\ \mathrm{C} & 1.2500334973 & -4.3519952353 & 2.1099102150 \\ \mathrm{H} & 1.9510201940 & -2.6123550963 & 3.2371681725 \\ \mathrm{H} & 3.0425764041 & -2.9210439717 & 1.8599968029 \\ \mathrm{H} & -0.4947197010 & -3.2095570924 & 2.8132285762 \\ \mathrm{H} & -0.8195603050 & -3.8946460931 & 1.1985135403 \\ \mathrm{C} & 1.1320579985 & -2.2217293287 & -0.0901376169 \\ \mathrm{C} & 0.1778743940 & -2.7025982793 & -1.0426459267 \\ \mathrm{H} & -0.2434389320 & -3.6823655533 & -0.7989971417 \\ \mathrm{H} & 0.4535015937 & -2.6002043303 & -2.0942306437 \\ \mathrm{Mg} & -0.3759346331 & 0.5291424591 & 1.0818056673 \\ \mathrm{O} & 0.4474959208 & 1.5121834787 & -0.5780800212 \\ \mathrm{O} & 0.7202618803 & 2.1811741311 & 1.7904889088 \\ \mathrm{P} & 1.0376931935 & 2.5615342562 & 0.3524991555 \\ \mathrm{O} & 2.6330764139 & 2.7249906202 & 0.1753511708 \\ \mathrm{O} & 0.4649522090 & 4.0099496218 & -0.0532496118 \\ \mathrm{C} & 0.7118565128 & 5.1188823578 & 0.7929993774 \\ \mathrm{H} & 1.7864602044 & 5.3629921372 & 0.8222462244 \\ \mathrm{H} & 0.1597812356 & 5.9728222884 & 0.3809783698 \\ \mathrm{H} & 0.3641656292 & 4.9148557036 & 1.8171730598 \\ \mathrm{C} & 3.1524973933 & 2.9257337211 & -1.1274263487 \\ \mathrm{H} & 4.2436300778 & 3.0027838674 & -1.0363872025 \\ \mathrm{H} & 2.8986505192 & 2.0789470695 & -1.7839326872 \\ \mathrm{H} & 2.7596985001 & 3.8551674532 & -1.5707387493 \\ \mathrm{O} & -2.2978452795 & 0.9655065981 & 1.2381713840 \\ \mathrm{C} & -3.2922849367 & 0.7568686579 & 0.5078327255 \\ \mathrm{C} & -4.5985630882 & 1.5075277487 & 0.6808267535 \\ \mathrm{~N} & -3.3473790764 & -0.1209062648 & -0.4836413727 \\ \mathrm{C} & -5.4796315745 & 0.9333584700 & -0.4243777495 \\ \mathrm{H} & -4.4023401118 & 2.5860053768 & 0.5941145238 \\ \mathrm{H} & -4.9764953541 & 1.3310184122 & 1.6986837031\end{array}$




$\begin{array}{lrrr}\mathrm{C} & -4.5963452390 & -0.1230047457 & -1.1014929378 \\ \mathrm{Br} & -1.5232121760 & -1.4733390701 & -0.8473747169 \\ \mathrm{H} & -6.3976448778 & 0.4497217671 & -0.0619422261 \\ \mathrm{H} & -5.7810253751 & 1.6735754502 & -1.1793463519 \\ \mathrm{O} & -4.9221467335 & -0.8365740849 & -2.0113829198 \\ \mathrm{C} & 2.3383398349 & -1.5208323548 & -0.5237808834 \\ \mathrm{C} & 2.8948915115 & -1.8198179671 & -1.7842067433 \\ \mathrm{C} & 3.0334608421 & -0.6287883177 & 0.3212553907 \\ \mathrm{C} & 4.1053429418 & -1.2624221811 & -2.1782273205 \\ \mathrm{H} & 2.4055261379 & -2.5365232820 & -2.4439199921 \\ \mathrm{C} & 4.2346115374 & -0.0662162782 & -0.0828058871 \\ \mathrm{H} & 2.5908381780 & -0.3536848012 & 1.2786432612 \\ \mathrm{C} & 4.7762998770 & -0.3827267083 & -1.3295905357 \\ \mathrm{H} & 4.5284366924 & -1.5201749834 & -3.1508380069 \\ \mathrm{H} & 4.7383711891 & 0.6429615560 & 0.5755630058 \\ \mathrm{H} & 5.7245087712 & 0.0604341264 & -1.6425745294 \\ \mathrm{C} & 1.2721343357 & -5.2355216195 & 3.3535888574 \\ \mathrm{H} & 0.5723127573 & -6.0798272336 & 3.2579739435 \\ \mathrm{H} & 2.2783414573 & -5.6514686825 & 3.5171381656 \\ \mathrm{H} & 0.9884509438 & -4.6566990726 & 4.2457690089 \\ \mathrm{C} & 1.6024076325 & -5.1639527274 & 0.8659133266 \\ \mathrm{C} & 2.8070828485 & -5.0194946211 & 0.1713522208 \\ \mathrm{C} & 0.6702743935 & -6.0902874030 & 0.3732933597 \\ \mathrm{C} & 3.0636718837 & -5.7538053339 & -0.9888983649 \\ \mathrm{H} & 3.5637611752 & -4.3175505342 & 0.5242697075 \\ \mathrm{C} & 0.9211902631 & -6.8265126551 & -0.7801237793 \\ \mathrm{H} & -0.2795820470 & -6.2265408317 & 0.8976835470 \\ \mathrm{C} & 2.1210788940 & -6.6551178582 & -1.4733819309 \\ \mathrm{H} & 4.0106310780 & -5.6137930442 & -1.5153598344 \\ \mathrm{H} & 0.1741809750 & -7.5350745736 & -1.1448731705 \\ \mathrm{H} & 2.3182718807 & -7.2258603229 & -2.3832762281 \\ & & & \\ & & & \end{array}$

TS2b

01

$\begin{array}{lrrr}\mathrm{O} & -0.1706470726 & -0.8195503489 & 1.6358279802 \\ \mathrm{C} & -1.2934151926 & -0.6299161314 & 0.9782612371 \\ \mathrm{C} & -2.5287945645 & -1.8195930281 & 1.1001577140 \\ \mathrm{C} & -2.3252677616 & 0.3217953304 & 1.6133013188 \\ \mathrm{C} & -3.4647204362 & -0.7312971705 & 1.6591153418 \\ \mathrm{H} & -2.0984956870 & -2.4954753351 & 1.8504377610 \\ \mathrm{H} & -2.8653299796 & -2.4020557483 & 0.2329087858 \\ \mathrm{H} & -1.9574324012 & 0.5850589628 & 2.6115460969 \\ \mathrm{H} & -2.5110489536 & 1.2518105125 & 1.0602661859 \\ \mathrm{C} & -1.2893710471 & -0.8174479381 & -0.4662537547 \\ \mathrm{C} & -2.0787964419 & 0.0861335688 & -1.3224536745 \\ \mathrm{H} & -3.0060153383 & 0.4553478617 & -0.8779896954 \\ \mathrm{H} & -2.2559344960 & -0.2632465968 & -2.3430835065 \\ \mathrm{Mg} & 1.5149298052 & 0.0465615997 & 1.2372130781 \\ \mathrm{O} & 2.6400061419 & -0.6445109538 & -0.4034806663 \\ \mathrm{O} & 3.1364018404 & -1.0135934981 & 1.9931582631 \\ \mathrm{P} & 3.6434064569 & -1.2360572663 & 0.5739242318 \\ \mathrm{O} & 3.9050009497 & -2.8098432586 & 0.3378210767 \\ \mathrm{O} & 5.0878572418 & -0.5728762528 & 0.3214651283 \\ \mathrm{C} & 6.1265533847 & -0.7842352613 & 1.2615567349 \\ \mathrm{H} & 6.4274451924 & -1.8446373430 & 1.2895897335 \\ \mathrm{H} & 6.9823806884 & -0.1757969118 & 0.9437380452 \\ \mathrm{H} & 5.8100963824 & -0.4779227764 & 2.2701034654 \\ \mathrm{C} & 4.4081044406 & -3.2323366482 & -0.9165000769 \\ \mathrm{H} & 4.3182509452 & -4.3259040935 & -0.9547344999 \\ \mathrm{H} & 3.8290153589 & -2.7894919027 & -1.7420730536\end{array}$




\begin{tabular}{lr}
$\mathrm{H}$ & 5.4676732719 \\
$\mathrm{O}$ & 1.6607137306 \\
$\mathrm{C}$ & 1.7431274182 \\
$\mathrm{C}$ & 2.6248098569 \\
$\mathrm{~N}$ & 1.1102026808 \\
$\mathrm{C}$ & 2.4381054697 \\
$\mathrm{H}$ & 3.6562606091 \\
$\mathrm{H}$ & 2.2872799550 \\
$\mathrm{C}$ & 1.4265117205 \\
$\mathrm{Br}$ & -0.7409634926 \\
$\mathrm{H}$ & 2.0311718791 \\
$\mathrm{H}$ & 3.3571111981 \\
$\mathrm{O}$ & 0.9756621777 \\
$\mathrm{C}$ & -3.9342272443 \\
$\mathrm{H}$ & -4.4685560233 \\
$\mathrm{H}$ & -4.6206710635 \\
$\mathrm{H}$ & -3.0770472352 \\
$\mathrm{C}$ & -4.6775628443 \\
$\mathrm{C}$ & -5.3647417729 \\
$\mathrm{C}$ & -5.1775791637 \\
$\mathrm{C}$ & -6.5009715525 \\
$\mathrm{H}$ & -5.0160762625 \\
$\mathrm{C}$ & -6.3124103723 \\
$\mathrm{H}$ & -4.6727504438 \\
$\mathrm{C}$ & -6.9760077044 \\
$\mathrm{H}$ & -7.0175533823 \\
$\mathrm{H}$ & -6.6786147909 \\
$\mathrm{H}$ & -7.8620580230 \\
$\mathrm{C}$ & -0.4020779157 \\
$\mathrm{C}$ & -0.0947213701 \\
$\mathrm{C}$ & 0.1530392872 \\
$\mathrm{C}$ & 0.7216550045 \\
$\mathrm{H}$ & -0.4536840061 \\
$\mathrm{C}$ & 0.9460605026 \\
$\mathrm{H}$ & -0.0292014523 \\
$\mathrm{C}$ & 1.2303613860 \\
$\mathrm{H}$ & 0.9634833156 \\
$\mathrm{H}$ & 1.3632686573 \\
$\mathrm{H}$ & 1.8635045384 \\
& \\
\hline
\end{tabular}

TS2c

01

$\begin{array}{lr}\mathrm{O} & 0.7089150540 \\ \mathrm{C} & -0.5044228991 \\ \mathrm{C} & -1.1940474632 \\ \mathrm{C} & -1.7427150990 \\ \mathrm{C} & -2.4642465205 \\ \mathrm{H} & -0.6191464043 \\ \mathrm{H} & -1.3198869682 \\ \mathrm{H} & -1.3131704044 \\ \mathrm{H} & -2.2821050825 \\ \mathrm{C} & -2.8158093730 \\ \mathrm{H} & -3.6157907081 \\ \mathrm{H} & -3.1677044966 \\ \mathrm{H} & -1.9349413374 \\ \mathrm{C} & -3.7108801767 \\ \mathrm{C} & -3.9899120191 \\ \mathrm{C} & -4.6543942268 \\ \mathrm{C} & -5.1577044336 \\ \mathrm{H} & -3.2795593443\end{array}$

$-2.9531125096$

1.9685792036

3.0233752508

4.1763417961

3.2567067599

5.1993937471

3.8134367815

4.5160705366

4.5261384825

1.5690279267

6.1641979301

5.4151896234

5.0297133828

$-1.0441523405$

$-0.1798585863$

$-1.9046512690$

$-1.2736842748$

$-0.4200624311$

$-1.4307837184$

0.8858370869

$-1.1437773305$

$-2.4648571156$

1.1775017934

1.6931921551

0.1634318586

$-1.9489219262$

2.2051274840

0.3910840490

$-1.8129378864$

$-1.7410099968$

$-2.8658405527$

$-2.6925076445$

$-0.9091531401$

$-3.8290237865$

$-2.9179315655$

$-3.7476813435$

$-2.6063278182$

$-4.6362329414$

$-4.5016590427$
$-1.0322664668$

1.3222760284

0.6304617348

1.0858252770

$-0.4982719842$

$-0.0259693362$

1.2063148915

2.0766040364

$-0.9690270224$

$-1.2612694939$

0.3087336822

$-0.5900918637$

$-1.9668895922$

3.0843185783

3.5070692920

3.0903620875

3.7353746004

0.7930952135

0.1119878864

0.7168676545

$-0.6434406115$

0.1715906378

$-0.0375308027$

1.2531979750

$-0.7263320622$

$-1.1705623255$

$-0.0878409437$

$-1.3223723880$

$-1.0828703403$

$-2.4544801018$

$-0.3274421608$

$-3.0509257143$

$-3.0605379115$

$-0.9335450407$

0.7440245113

$-2.2960290573$

$-4.1115400382$

$-0.3297317126$

$-2.7691303674$
0.5244115239

0.3197755535

1.5219117644

0.3456109152

1.4058328663

2.4273541198

1.4631596815

0.7422828665

$-0.5876768286$

2.6904226991

2.5107148346

3.4629698266

3.0822721571

0.8888503317

1.2150644413

0.1273475422

0.7731428162

1.8162602441
$-2.3122787702$

$-1.7764546949$

$-1.0671216425$

$-2.8114410145$

$-1.9461496195$

$-1.2963867696$

0.0169348152

$-3.7409050615$

$-3.0307343947$

$-2.7008571614$

$-3.4358838724$

$-1.9998249958$

$-3.2316481466$

$-1.2401562051$

0.0903354636

$-1.9408689606$

0.7096671328

0.6616500185 


\begin{tabular}{|c|c|c|c|}
\hline$C$ & 247069229 & -0.3152636216 & $J$ \\
\hline$U_{2}$ & -4.4744431531 & -0.1250108269 & -2.9894158139 \\
\hline 急 & -6.0789828399 & 0.0015944071 & 049988625 \\
\hline $\mathrm{H}$ & 5.3451819217 & .0314272109 & 1.7543683875 \\
\hline $\mathrm{H}$ & -6.5429068197 & -0.9103138721 & 07916 \\
\hline $\mathrm{C}$ & -0.6989772755 & -1.0504413665 & -1.192829792 \\
\hline $\mathrm{C}$ & -0.0646070113 & -2.1369654234 & -1.8581944899 \\
\hline $\mathrm{H}$ & 0.2880869350 & -1.9305579727 & -2.8723100761 \\
\hline $\mathrm{H}$ & .4695705293 & -3.1366377841 & -1.6730899749 \\
\hline $\mathrm{C}$ & -1.5558486488 & -1.3133897144 & -0.0313590765 \\
\hline $\mathrm{C}$ & -1.2462220264 & -0.7252497519 & 1.2060355892 \\
\hline $\mathrm{C}$ & -2.6620203008 & -2.1667832792 & 501294 \\
\hline $\mathrm{C}$ & 47160899 & -0.9988698050 & \\
\hline $\mathrm{H}$ & -0.3574133854 & -0.0916243795 & 1.294029894 \\
\hline $\mathrm{C}$ & -3.4830476849 & -2.3913039764 & 0.9537345007 \\
\hline $\mathrm{H}$ & 2851 & 2130 & 77233 \\
\hline $\mathrm{C}$ & 545 & 6263 & \\
\hline $\mathrm{H}$ & -1.7873749624 & -0.5678933757 & 3.28 \\
\hline $\mathrm{H}$ & -4.3676765085 & 583704 & 0.85 \\
\hline $\mathrm{Mg}$ & 7090 & 45076 & 9413 \\
\hline $\mathrm{O}$ & 4743 & 183 & \\
\hline $\mathrm{O}$ & 2.3086888778 & 2.9525705806 & -0.6175778469 \\
\hline $\mathrm{P}$ & 0084 & 4963 & 66618 \\
\hline $\mathrm{O}$ & 4982 & 486 & 89686 \\
\hline $\mathrm{O}$ & 659956 & 02756 & 1.96 \\
\hline $\mathrm{C}$ & 3.3253772075 & 2144275 & 1.9841506027 \\
\hline $\mathrm{H}$ & 75 & & 053 \\
\hline $\mathrm{H}$ & 450 & 801 & 07905 \\
\hline $\mathrm{H}$ & 3.8870691342 & 48583 & 1.0545147138 \\
\hline $\mathrm{C}$ & 6664 & & \\
\hline $\mathrm{H}$ & 832 & 4.16 & 1000 \\
\hline$H$ & 56976 & 3723 & 49409 \\
\hline $\mathrm{H}$ & 0.2116748903 & 3.4699982133 & 3.0625741544 \\
\hline $\mathrm{O}$ & & & -0.9534787081 \\
\hline $\mathrm{C}$ & 26 & 982 & -0.3 \\
\hline $\mathrm{C}$ & 5.9875830133 & -0.7255706975 & 0.0946677172 \\
\hline $\mathrm{N}$ & 3.8826621939 & -1.7987745629 & 0.0637497361 \\
\hline $\mathrm{C}$ & & & 09495522187 \\
\hline $\mathrm{H}$ & 57 & 63 & 0.698 \\
\hline $\mathrm{H}$ & 6.6126737177 & -0.6576709253 & -0.8080903466 \\
\hline $\mathrm{C}$ & 1866 & -2.6807816105 & 0.73 \\
\hline$D_{0}$ & & -2.0413443500 & -0.7461013835 \\
\hline $\mathrm{H}$ & 6.8597843294 & -2.7359520921 & 0.4245019009 \\
\hline $\mathrm{H}$ & 6.3623400561 & -1.9220904139 & 1.9134747884 \\
\hline $\mathrm{O}$ & 4084308819 & -3.7598675273 & \\
\hline $\mathrm{H}$ & & -0.3475078841 & \\
\hline $\mathrm{H}$ & -3.8068778276 & -2.0038368856 & 3.05002162 \\
\hline
\end{tabular}

TS2d

01

$\begin{array}{lrrr}\mathrm{C} & 0.9003732528 & -1.0497363942 & 0.4645477108 \\ \mathrm{C} & 1.9118560997 & -1.8536625131 & 1.3516646645 \\ \mathrm{C} & 2.5930918332 & -0.5793272376 & 1.9111075220 \\ \mathrm{H} & 2.5584135290 & -2.5849801315 & 0.8566190064 \\ \mathrm{H} & 1.2983786243 & -2.3723510412 & 2.0972656588 \\ \mathrm{C} & 1.1929505957 & -0.8526312436 & -0.9815405085 \\ \mathrm{C} & 0.0777469223 & -0.6750229182 & -1.8691189010 \\ \mathrm{H} & -0.7863329698 & -1.3090973496 & -1.6236823476 \\ \mathrm{H} & 0.3269486124 & -0.6323073373 & -2.9328316569 \\ \mathrm{Mg} & -2.0884183874 & -0.4233791159 & 0.7850393857\end{array}$




\begin{tabular}{|c|c|c|c|}
\hline $\mathrm{O}$ & 987 & -1 & 35261 \\
\hline 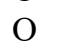 & .2094786968 & -1.1590324256 & 391525905 \\
\hline $\mathrm{P}$ & -4.0615208910 & -1.9483847225 & 1466285001 \\
\hline $\mathrm{O}$ & -3.9972443239 & -3.5102332124 & -0.2365487075 \\
\hline $\mathrm{O}$ & -5.6264827543 & -1.5896678866 & 0.0472794350 \\
\hline $\mathrm{C}$ & -6.2682388438 & -1.6110988265 & -1.2153054523 \\
\hline $\mathrm{H}$ & -6.2708014306 & -2.6278179086 & -1.6410869812 \\
\hline $\mathrm{H}$ & -7.3035118335 & -1.2825952648 & -1.0602530469 \\
\hline $\mathrm{H}$ & -5.7681350088 & -0.9300781563 & -1.9208914509 \\
\hline $\mathrm{C}$ & -4.5535801895 & -4.4664716135 & 0.648312522 \\
\hline $\mathrm{H}$ & -4.3363311504 & -5.4591166198 & 0.2344159106 \\
\hline $\mathrm{H}$ & -4.1063356637 & -4.3793365401 & 1.6502263556 \\
\hline $\mathrm{H}$ & -5.6453773875 & -4.3401131864 & 968280 \\
\hline $\mathrm{O}$ & -2.4326606756 & 1.4297612080 & 1.3924376885 \\
\hline $\mathrm{C}$ & -2.3939491314 & 2.5937368722 & 0.9266624865 \\
\hline $\mathrm{C}$ & -3.0376381216 & 781 & 6927 \\
\hline $\mathrm{N}$ & 395 & & \\
\hline $\mathrm{C}$ & -2.7507412344 & & 6990 \\
\hline $\mathrm{H}$ & -2.5897904404 & 9134 & 90581 \\
\hline $\mathrm{H}$ & 2078 & 3.5 & 1.7 \\
\hline $\mathrm{C}$ & -1.93 & & -0.4 \\
\hline $\mathrm{Br}$ & -0.6820495891 & 6710 & 653681 \\
\hline $\mathrm{H}$ & 220 & & 103 \\
\hline $\mathrm{H}$ & 900 & & \\
\hline $\mathrm{O}$ & 4172 & 4.92 & \\
\hline $\mathrm{O}$ & -0.3738140577 & 027923 & 310010 \\
\hline $\mathrm{C}$ & 323 & & \\
\hline $\mathrm{H}$ & 134 & 551 & 165 \\
\hline $\mathrm{H}$ & 1.9714987996 & 1610 & 971233 \\
\hline $\mathrm{C}$ & 2661 & & 37653 \\
\hline $\mathrm{H}$ & 075 & -1.1 & 9027 \\
\hline $\mathrm{H}$ & 2.809 & 7757 & 0589 \\
\hline $\mathrm{H}$ & 1.4168047813 & -0.5540639682 & 3.7437434695 \\
\hline $\mathrm{C}$ & & & \\
\hline $\mathrm{C}$ & 755 & & 333 \\
\hline $\mathrm{C}$ & 09769 & 0.77 & 67479 \\
\hline $\mathrm{C}$ & 6748 & & \\
\hline $\mathrm{H}$ & & -2 & 718 \\
\hline $\mathrm{C}$ & 5.9270377884 & 33303 & 07965 \\
\hline $\mathrm{H}$ & 3.9219 & & 573468 \\
\hline$C$ & & & \\
\hline $\mathrm{H}$ & 81 & -2.2 & 415 \\
\hline $\mathrm{H}$ & 6.3070328561 & 1.8286341582 & 0.2884420674 \\
\hline $\mathrm{H}$ & 7.8456474294 & -0.10 & 6166114578 \\
\hline$C$ & & & \\
\hline $\mathrm{C}$ & & & 360 \\
\hline $\mathrm{C}$ & 2.8206453511 & 315792 & -2.2249697971 \\
\hline$C$ & & & \\
\hline$U$ & & & -0.9269363952 \\
\hline $\mathrm{C}$ & 141726 & 0.7839266680 & -2.7412821935 \\
\hline $\mathrm{H}$ & 2.0438260118 & 1.3105991667 & -2.3229008087 \\
\hline $\mathrm{C}$ & 74171746 & & \\
\hline- & & & \\
\hline $\mathrm{H}$ & 4.2968010351 & 1.7276000791 & -3.2490949727 \\
\hline 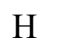 & 6.0832571228 & 0.0031294617 & -3.02161008 \\
\hline
\end{tabular}

TS3a

01

$\begin{array}{llll}\text { O } & 0.4144706507 & -0.1747326069 & 1.8827967482\end{array}$

$\begin{array}{llll}\text { C } & 1.6660818539 & -0.0599354598 & 1.3790579178\end{array}$ 


\begin{tabular}{|c|c|c|c|}
\hline $\mathrm{C}$ & 177 & 35893258 & 2.12 \\
\hline $\mathrm{C}$ & .6589568844 & -1.2094197666 & 85549656 \\
\hline $\mathrm{C}$ & 3.7920025658 & -0.1789187709 & 8619775 \\
\hline $\mathrm{H}$ & 2.3394478434 & 0.9427033168 & .1647868242 \\
\hline $\mathrm{H}$ & 2.8465909552 & 1.9026700606 & 4721 \\
\hline $\mathrm{H}$ & 2.3136564510 & -1.6583332942 & 2.6395814981 \\
\hline $\mathrm{H}$ & 2.8239303206 & -2.0185630603 & 0.9729251968 \\
\hline $\mathrm{C}$ & 4.6488245431 & -0.4211434448 & 3.2076553889 \\
\hline $\mathrm{H}$ & 5.2919555738 & -1.3059909474 & 3.0834432922 \\
\hline $\mathrm{H}$ & 5.3024918348 & 0.4429097549 & 3.4044970398 \\
\hline $\mathrm{H}$ & 4.0133363091 & -0.5826302303 & 4.0917421601 \\
\hline $\mathrm{C}$ & 4.6883697798 & 0.0081284841 & 0.7463060164 \\
\hline $\mathrm{C}$ & 4.8843101048 & 1.2416821926 & 01193016461 \\
\hline $\mathrm{C}$ & 5.3577081422 & -1.1071796125 & 0.2198539054 \\
\hline $\mathrm{C}$ & 5.7030807939 & 1.3565745595 & -1.0063110580 \\
\hline $\mathrm{H}$ & 4.3906583495 & 9057 & \\
\hline $\mathrm{C}$ & 6.1748432449 & -0.9991275199 & -0.9010415198 \\
\hline $\mathrm{H}$ & 5.2243935573 & -2.0845524136 & 56977 \\
\hline $\mathrm{C}$ & 6.3473228063 & 0.2376192680 & -1.5250294562 \\
\hline $\mathrm{H}$ & 5.8340134403 & 2.33 & $-1.4^{\top}>x+2$ \\
\hline $\mathrm{H}$ & 6.6777264152 & 86741 & \\
\hline $\mathrm{C}$ & 1.5442826791 & 0.3401058946 & -0.0997268656 \\
\hline $\mathrm{C}$ & 2.0015 & 627 & 0606 \\
\hline $\mathrm{H}$ & 072 & -1.1 & 5602 \\
\hline $\mathrm{H}$ & 2.0545890248 & -0.1729 & -2.1 \\
\hline $\mathrm{C}$ & 0.8291943313 & 1.5584619649 & -0.4141606341 \\
\hline $\mathrm{C}$ & 146 & 2.4 & \\
\hline $\mathrm{C}$ & 210 & 1.9 & -1. \\
\hline $\mathrm{C}$ & -0.3179204514 & 3.5862010518 & 0.3087363403 \\
\hline $\mathrm{H}$ & 1441 & 862 & 323 \\
\hline$\sigma_{0}$ & 7272 & 61032 & -2.0 \\
\hline $\mathrm{II}$ & 669657 & 1.33 & -2.5 \\
\hline $\mathrm{C}$ & .5092069586 & 3.9692121603 & -1.0305105955 \\
\hline $\mathrm{H}$ & 241 & & 712 \\
\hline $\mathrm{H}$ & 2348 & 216 & -3.1 \\
\hline $\mathrm{Mg}$ & -1.3231503623 & -0.66 & 1.3288125119 \\
\hline $\mathrm{O}$ & -2.3449 & & -0.1 \\
\hline $\mathrm{O}$ & 2790 & 0.4 & 563 \\
\hline $\mathrm{P}$ & -3.2887997510 & 2365 & 56063 \\
\hline $\mathrm{O}$ & -3.3764706213 & 327 & 0.9026104316 \\
\hline $\mathrm{O}$ & 87 & 0.4 & \\
\hline $\mathrm{C}$ & 088965 & 3086 & 2742 \\
\hline $\mathrm{H}$ & -5.9897206299 & 1.7647814471 & 1.6826043357 \\
\hline $\mathrm{H}$ & & 0.2480069794 & 1.0868847753 \\
\hline $\mathrm{H}$ & & 10447 & \\
\hline $\mathrm{C}$ & -3.71 & 140 & -0.2 \\
\hline $\mathrm{H}$ & -3.4633074102 & 4.2905197784 & -0.1700216098 \\
\hline $\mathrm{H}$ & & & \\
\hline $\mathrm{H}$ & -4.78 & 3.12 & -0.5 \\
\hline $\mathrm{O}$ & -2.0498694332 & -2.5017252443 & 1.1388950033 \\
\hline $\mathrm{C}$ & -2.1660026565 & -3.3061773479 & 0.1907579340 \\
\hline $\mathrm{C}$ & & & \\
\hline $\mathrm{N}$ & & -3.3354127026 & -0.8968272962 \\
\hline $\mathrm{C}$ & -2.9888658333 & -5.0886762903 & -1.1657996267 \\
\hline $\mathrm{H}$ & -4.2156954201 & -3.9143567926 & 0.2629725191 \\
\hline $\mathrm{H}$ & .0958710695 & -5.0345533991 & \\
\hline $\mathrm{C}$ & .7848149071 & -4.3531411838 & -1.767724399 \\
\hline $\mathrm{Br}$ & 0.3371904815 & -1.8764833371 & -1.0614560669 \\
\hline $\mathrm{H}$ & -2.7436040783 & -6.1563319002 & -1.0757966191 \\
\hline $\mathrm{H}$ & -3.8332857240 & -5.0089869757 & -1.865272523 \\
\hline
\end{tabular}




$\begin{array}{lrrr}\mathrm{O} & -1.2528725261 & -4.6084346329 & -2.8146906930 \\ \mathrm{O} & -1.1504993432 & 5.1244535817 & -1.2278635966 \\ \mathrm{C} & -1.4406970844 & 5.5471701730 & -2.5387504897 \\ \mathrm{H} & -2.0812872911 & 4.8184855887 & -3.0629606035 \\ \mathrm{H} & -1.9782617903 & 6.4977076264 & -2.4463903227 \\ \mathrm{H} & -0.5207777393 & 5.7101598077 & -3.1238643684 \\ \mathrm{H} & 6.9832969380 & 0.3257148128 & -2.4084130924\end{array}$

TS3b

01

O $\quad-0.4515803986$

C $\quad-1.5572580918$

$-0.5738030990$

1.8910380125

$-0.5552752112$

1.1728129996

$\begin{array}{ll}\text { C } & -2.6201170956 \\ \text { C } & -2.7241583650\end{array}$

$-1.8861916145$

1.3435976283

C $\quad-3.6941507077$

0.2976387370

1.7072561096

$-2.1172522586$

$-0.9046241547$

1.8573782140

$-2.8791953376$

$-2.4834916480$

2.1145577246

$-2.4181087731$

$-2.5265137588$

0.4900613893

$-3.0369272513$

0.7218067453

2.6700555232

$1.1213687614 \quad 1.0531548446$

$-1.4661577301$

$-0.8201478657$

$-0.2582157970$

C $\quad-2.4136869235$

$-0.1678153891$

$-1.1968844432$

$-3.4112645200$

$-0.0036628697$

$-0.7794505898$

H $\quad-2.4968014759$

$-0.6624196891$

$-2.1694223471$

$\mathrm{Mg} \quad 1.1059167425$

0.5026059945

1.5295882083

$-0.1597750398 \quad 0.1369163654$

$\mathrm{O} \quad 2.5704917678$

0.1119223482

2.5841052983

$-0.2359006038$

1.2872789303

3.5608825334

$-1.6742568818$

1.4515977654

$0.7602583749 \quad 0.9817905454$

O $\quad 4.7911917618$

0.7602583749
1.0678737221

2.0116977017

5.7159751552

0.1761120932

2.3023595982

1.8294989929

1.6172380596

6.3998533067

1.4611803225

2.8983024081

5.1963926752
4.9993691448

$-2.2375150818$

0.3819191023

5.5526540894

$-3.0951075906$

0.7855067763

4.3318221969

$-2.5852409331$

$-0.4204220983$

5.7153870224

$-1.5097823066$

$-0.0328020595$

0.7465083992

2.3147956785

1.0248110021

$3.1528838815 \quad 0.1373029899$

3.7833342001

0.1629112591

C $\quad 2.4784070167$

3.5448716476

$-0.8620362760$

0.3390843479
2.4425580429

4.6997670918

$-1.0510967070$

$2.9817486083 \quad 0.1065676406$

$4.2995745093 \quad 1.1234391474$

$4.4462386506-1.6503760543$

2.6265406082

1.0459930976

1.5786165501

$-1.3685723627$

$\mathrm{Br} \quad-1.5130081115$

5.7684383434

$-0.8088650981$

$4.4718069298-1.8106711405$

3.2039458888

4.9566169411

$-2.6638853038$

$\mathrm{O} \quad 0.6374947867$

C $\quad-4.0709324542$

$-1.1807208413$

3.3171014942

$-0.3758238682 \quad 3.7006227260$

$-4.7161328913$

$-4.6198635056$

$-2.1306145574$

3.4079186379

$-3.1698224075$

$-1.2327586242$

3.9467979601

$-4.9601831889$

$-0.8408387469$

1.0158646972

$-2.0011777014$

0.4756143906

$-5.5266336420$

0.3739026012

0.8215970001

C $\quad-5.6283476864$

$-1.9482322081$

$-0.2563238011$

$\mathrm{H} \quad-5.0406823057$

$-2.9681535315$

0.6305463534

$\begin{array}{ll}0.4315110389 & 0.0895977577\end{array}$

$\mathrm{H} \quad-5.2183282026$

1.2938833635

1.2452764594 


$\begin{array}{lrrr}\mathrm{C} & -7.3571884864 & -0.7294033885 & -0.4569780669 \\ \mathrm{H} & -7.1326716603 & -2.8662643147 & -0.6722274612 \\ \mathrm{H} & -7.3125288920 & 1.3919326086 & -0.0551366573 \\ \mathrm{H} & -8.2825212623 & -0.6847601327 & -1.0349137331 \\ \mathrm{C} & -0.3789726027 & -1.6292134520 & -0.7786819851 \\ \mathrm{C} & 0.0227090480 & -1.4993289933 & -2.1227486757 \\ \mathrm{C} & 0.3521773966 & -2.5266649082 & 0.0401256964 \\ \mathrm{C} & 1.1237176636 & -2.1750853623 & -2.6217172838 \\ \mathrm{H} & -0.4849262269 & -0.7978413626 & -2.7852562591 \\ \mathrm{C} & 1.4192874460 & -3.2367128603 & -0.4590373546 \\ \mathrm{H} & 0.0765021578 & -2.6506566268 & 1.0851287136 \\ \mathrm{C} & 1.8385726102 & -3.0441242064 & -1.7863638636 \\ \mathrm{H} & 1.4290741243 & -2.0000134841 & -3.6521955841 \\ \mathrm{H} & 1.9815130267 & -3.9245757411 & 0.1732457467 \\ \mathrm{O} & 2.9311263227 & -3.7177462769 & -2.1623239269 \\ \mathrm{C} & 3.4441488974 & -3.5448888885 & -3.4628518837 \\ \mathrm{H} & 4.3377277553 & -4.1758630397 & -3.5294369669 \\ \mathrm{H} & 2.7188305322 & -3.8681110154 & -4.2273912393 \\ \mathrm{H} & 3.7263614557 & -2.4952948667 & -3.6463848579\end{array}$

TS3e

01

$\begin{array}{lrrr}\mathrm{O} & -0.7232621025 & 0.5335623343 & 2.2923468531 \\ \mathrm{C} & 0.4931036775 & 0.3172438240 & 1.7644149868 \\ \mathrm{C} & 1.1942921686 & 1.5173667060 & 1.0582824919 \\ \mathrm{C} & 1.7219793463 & 0.3371902117 & 2.8028019025 \\ \mathrm{C} & 2.4637311694 & 1.3819953936 & 1.9354035416 \\ \mathrm{H} & 0.6285851809 & 2.4257014804 & 1.2995277141 \\ \mathrm{H} & 1.3197524596 & 1.4743555169 & -0.0262071511 \\ \mathrm{H} & 1.3007704529 & 0.7493801908 & 3.7294234712 \\ \mathrm{H} & 2.2464162507 & -0.6027576423 & 3.0304781671 \\ \mathrm{C} & 2.8393702367 & 2.6623653182 & 2.6866415894 \\ \mathrm{H} & 3.6368474161 & 2.4703212509 & 3.4213970521 \\ \mathrm{H} & 3.2038564087 & 3.4278281578 & 1.9841514167 \\ \mathrm{H} & 1.9660251114 & 3.0701951415 & 3.2177517723 \\ \mathrm{C} & 3.7047796164 & 0.8502384117 & 1.2283626619 \\ \mathrm{C} & 4.0013141224 & 1.1994395051 & -0.0928031999 \\ \mathrm{C} & 4.6346392131 & 0.0646980716 & 1.9218102954 \\ \mathrm{C} & 5.1746889796 & 0.7654706111 & -0.7084944992 \\ \mathrm{H} & 3.3010068280 & 1.8165337510 & -0.6594784047 \\ \mathrm{C} & 5.8105551687 & -0.3699578283 & 1.3143888268 \\ \mathrm{H} & 4.4409400095 & -0.2087358801 & 2.9624531572 \\ \mathrm{C} & 6.0850375856 & -0.0226594283 & -0.0077760704 \\ \mathrm{H} & 5.3728549125 & 1.0416222647 & -1.7467448667 \\ \mathrm{H} & 6.5191194759 & -0.9797488252 & 1.8798736376 \\ \mathrm{C} & 0.6734302543 & -1.0586312833 & 1.1717713699 \\ \mathrm{C} & 0.0478683100 & -2.1406433000 & 1.8547929732 \\ \mathrm{H} & -0.2811738203 & -1.9333641306 & 2.8766643649 \\ \mathrm{H} & 0.4434581652 & -3.1431814170 & 1.6638486694 \\ \mathrm{C} & 1.5188948261 & -1.3122278856 & 0.0145819068 \\ \mathrm{C} & 1.2796697171 & -0.6542248803 & -1.2102789297 \\ \mathrm{C} & 2.6015343722 & -2.1945551745 & 0.1128676446 \\ \mathrm{C} & 2.1259934637 & -0.8583652624 & -2.2806880874 \\ \mathrm{H} & 0.3956277809 & -0.0159531510 & -1.3106888980 \\ \mathrm{C} & 3.4904185718 & -2.3568967707 & -0.9422353134 \\ \mathrm{H} & 2.7951247991 & -2.7143505830 & 1.0539036642 \\ \mathrm{C} & 3.2639452879 & -1.6729835733 & -2.1432758306 \\ \mathrm{H} & 1.9467854129 & -0.3775631304 & -3.2434910776 \\ \mathrm{H} & 4.3613446191 & -2.9970044177 & -0.8106041530 \\ \mathrm{Mg} & -2.1899142927 & 0.9321240207 & 1.1225215593\end{array}$




$\begin{array}{ll}\mathrm{O} & -1.3874460117 \\ \mathrm{O} & -2.3152492886 \\ \mathrm{P} & -1.7166304423 \\ \mathrm{O} & -0.4258871724 \\ \mathrm{O} & -2.6931371315 \\ \mathrm{C} & -3.3134989796 \\ \mathrm{H} & -2.5663003792 \\ \mathrm{H} & -3.9962912836 \\ \mathrm{H} & -3.8813070206 \\ \mathrm{C} & 0.3860172307 \\ \mathrm{H} & 1.1418605373 \\ \mathrm{H} & 0.8917082772 \\ \mathrm{H} & -0.2066355402 \\ \mathrm{O} & -4.0395786581 \\ \mathrm{C} & -4.5471448844 \\ \mathrm{C} & -6.0079801051 \\ \mathrm{~N} & -3.9059206588 \\ \mathrm{C} & -6.1453170197 \\ \mathrm{H} & -6.2190003325 \\ \mathrm{H} & -6.6281755862 \\ \mathrm{C} & -4.7546623633 \\ \mathrm{Br} & -1.7593867280 \\ \mathrm{H} & -6.8881492095 \\ \mathrm{H} & -6.3932614764 \\ \mathrm{O} & -4.4432803126 \\ \mathrm{H} & 7.0086573776 \\ \mathrm{O} & 4.0774801698 \\ \mathrm{C} & 5.2539244238 \\ \mathrm{H} & 5.9116377264 \\ \mathrm{H} & 5.0336704424 \\ \mathrm{H} & 5.7683002439 \\ & \end{array}$

1.1587196205

2.9541036627

2.6441754804

3.5814499657

3.0253652528

4.2998444252

5.1010510567

4.3115160842

4.4768620259

3.3856781759

4.1811272686

2.4066496535

3.4429097231

0.2610200879

$-0.7151304596$

$-0.7432433178$

$-1.8213845208$

$-2.0863470617$

0.1319686205

$-0.6444639985$

$-2.7227755201$

$-2.0513329129$

$-2.7603885525$

$-1.9974705366$

$-3.8168704386$

$-0.3577734065$

$-1.7320960487$

$-2.4971134720$

$-2.1436965293$

$-3.5673334815$

$-2.3727434445$
$-0.8530150642$

0.5845266966

$-0.7775437757$

$-1.0154693840$

$-1.9972370864$

$-2.0233490669$

$-2.1439011859$

$-2.8819386442$

$-1.0974561720$

$-2.1563784655$

$-2.1605086778$

$-2.1201028314$

$-3.0836864197$

0.9296073776

0.3299857465

$-0.0761724215$

$-0.0192450842$

$-0.7857394094$

$-0.7074111722$

0.8271225367

$-0.6597523935$

0.7738064595

$-0.3366914461$

$-1.8532861428$

$-1.0464266915$

$-0.4858460933$

$-3.2068739582$

$-3.1321962633$

$-2.3202616431$

$-2.9806122648$

$-4.0922492679$

TS3d

01

C $\quad 0.5605027946$

C $\quad 1.4974962128$

$-1.0457066069$

$-1.7968894951$

$-0.4761779602$

$-2.5291900044$

$-2.3065615049$

$-0.9327470554$

$-0.8740835793$

$-1.4958123540$

$-0.8888071693$

$-0.4823762346$

$-1.6225763456$

$-1.2634328733$

$-1.9596346044$

$-3.5411377617$

$-1.5349925298$

$-1.6011044740$

$-2.6392207135$

$-1.2218076924$

$-0.9826992631$

$-4.4219298252$

$-5.4444028291$

$-4.2994825743$

$-4.2436377114$

1.4229207985

2.5302535366

3.7541808101
0.6668857392

1.6765520045

2.2530615620

1.2871439288

2.3770318205

$-0.7758249492$

$-1.7541122655$

$-1.5178062863$

$-2.7992915224$

0.7313826906

1.5550014757

$-0.8608202254$

0.1518363038

$-0.1471871256$

0.0095280777

$-1.2591165689$

$-1.6275736983$

$-1.1384617091$

$-1.9945082148$

0.7820328457

0.4253736672

1.7826970504

0.8466867985

1.1575578355

0.5782407261

1.1133206645 


\begin{tabular}{ll}
$\mathrm{N}$ & -2.1425210083 \\
$\mathrm{C}$ & -3.1951249565 \\
$\mathrm{H}$ & -3.1552226709 \\
$\mathrm{H}$ & -4.5957402276 \\
$\mathrm{C}$ & -2.2779649987 \\
$\mathrm{Br}$ & -0.9254686911 \\
$\mathrm{H}$ & -4.0749085391 \\
$\mathrm{H}$ & -2.6662052349 \\
$\mathrm{O}$ & -1.7582441264 \\
$\mathrm{O}$ & -0.7365128966 \\
$\mathrm{C}$ & 1.1085656340 \\
$\mathrm{H}$ & 0.2619214104 \\
$\mathrm{H}$ & 1.5441065698 \\
$\mathrm{C}$ & 1.7858617122 \\
$\mathrm{H}$ & 2.3662299267 \\
$\mathrm{H}$ & 2.0577728192 \\
$\mathrm{H}$ & 0.7172893405 \\
$\mathrm{C}$ & 3.5605177290 \\
$\mathrm{C}$ & 4.4612514789 \\
$\mathrm{C}$ & 4.0816511033 \\
$\mathrm{C}$ & 5.8334750330 \\
$\mathrm{H}$ & 4.0795332387 \\
$\mathrm{C}$ & 5.4552244414 \\
$\mathrm{H}$ & 3.4120931386 \\
$\mathrm{C}$ & 6.3373531738 \\
$\mathrm{H}$ & 6.5141294726 \\
$\mathrm{H}$ & 5.8358567637 \\
$\mathrm{H}$ & 7.4142329714 \\
$\mathrm{C}$ & 2.3579266994 \\
$\mathrm{C}$ & 3.4285329771 \\
$\mathrm{C}$ & 2.6346682365 \\
$\mathrm{C}$ & 4.7261002462 \\
$\mathrm{H}$ & 3.2541705394 \\
$\mathrm{C}$ & 3.9281265913 \\
$\mathrm{H}$ & 1.8234628007 \\
$\mathrm{C}$ & 4.9925679713 \\
$\mathrm{H}$ & 5.5233675103 \\
$\mathrm{H}$ & 4.1494912402 \\
$\mathrm{O}$ & 6.2150499308 \\
$\mathrm{C}$ & 7.3272724053 \\
$\mathrm{H}$ & 8.2103218996 \\
$\mathrm{H}$ & 7.4193145921 \\
$\mathrm{H}$ & 7.2722069825 \\
& \\
\hline
\end{tabular}

2.7932012864

4.8320728914

3.9679354911

3.5230410332

4.1262104536

1.0202757652

5.2151103631

5.7021405875

4.6344032665

$-1.2884363115$

0.3185807278

0.7668378482

1.0688915037

$-0.2814306948$

$-0.9921047185$

0.7371620079

$-0.4388676690$

$-0.2680157131$

$-1.2825848777$

0.9227112815

$-1.1210189065$

$-2.2199889382$

1.0892014167

1.7388091709

0.0689870635

$-1.9265956637$

2.0255413790

0.2043094052

$-0.6735804534$

$-1.5119594892$

0.4597419098

$-1.2375543313$

$-2.4244023064$

0.7702946099

1.1340416778

$-0.0707938331$

$-1.9225695969$

1.6707389913

0.3185675342

$-0.4703702884$

0.0523389159

$-0.5822094922$

$-1.4699202811$
$-0.5424498924$

0.0853715694

2.1271424226

1.2062509813

$-0.9223890721$

$-1.3555148310$

$-0.4514280256$

0.4991574729

$-1.8792662506$

0.8478420878

1.3451527925

1.8801572815

0.6757384484

3.7446504538

4.3533463643

4.0624091752

3.9555985201

2.0040767217

2.3561449714

1.4904684512

2.1873247286

2.7708120000

1.3131807515

1.2127872584

1.6610875742

2.4728707193

0.8991594566

1.5350376341

$-1.2026868470$

$-0.8660645085$

$-1.9997612189$

$-1.2796576549$

$-0.3004984028$

$-2.3695984366$

$-2.2820861087$

$-2.0035150404$

$-0.9954906605$

$-2.9440242789$

$-2.3886493501$

$-2.0466595619$

$-2.4322136029$

$-0.9537762586$

$-2.5104034599$

TS4a

01

O $\quad-0.4918203398$

C $\quad 0.3473209458$

$-0.5483818971$

1.6469778763

$\begin{array}{ll}-1.5969135236 & 1.4306006901 \\ -1.8791151918 & 2.3727405906\end{array}$

$\begin{array}{ll}-1.8791151918 & 2.3727405906 \\ -3.0450813451 & 1.6962410020\end{array}$

$-0.1086160128$

1.3588038932

$-3.3913236047$

$-1.6471537546$

2.0830910507

3.3987774578

2.1892731091

2.5062750967

$-1.3916271812$

$-3.0311916401$

$-3.6166516643$

$-1.2246602300$

2.5858404397

0.9055641109

0.0084464749

$-1.0980812399$

$-1.8782835335$

$-2.9667998536$

$-1.5484619443$

$-0.9952085765$

$-2.0889795814$

$0.7125777734 \quad 1.0182739280$ 


\begin{tabular}{|c|c|c|c|}
\hline & 343844 & 589 & 518 \\
\hline c & -1.3940164844 & 2.5632501642 & 9535070 \\
\hline $\mathrm{P}$ & -1.0720600528 & 3.1508105145 & 5630522748 \\
\hline $\mathrm{O}$ & 0.3674786966 & 3.8734336960 & 0.6139236957 \\
\hline $\mathrm{O}$ & 2.0744954103 & 4.3340080647 & 0.1369689630 \\
\hline $\mathrm{c}$ & -2.3264940516 & 5.3956638170 & 1.0414462882 \\
\hline & -1.4167818254 & 5.9940358354 & 1.2124561469 \\
\hline $\mathrm{H}$ & -3.0969073668 & 6.0325284101 & 0.5893888469 \\
\hline $\mathrm{H}$ & 72831416 & 5.009599 & 2.006922660 \\
\hline $\mathrm{C}$ & 0.9312572552 & 4.3760485595 & -0.5867590109 \\
\hline $\mathrm{H}$ & 1.9051150823 & 4.8123878560 & -0.3327162830 \\
\hline $\mathrm{H}$ & 1.0730648816 & 3.5680625333 & 32698 \\
\hline & 0.2886360841 & 5.1541222482 & 662513 \\
\hline $\mathrm{c}$ & -3.7460845368 & 0.4681592122 & 0.9721376202 \\
\hline C & -4.5300322153 & -0.0376081408 & 0.1394297172 \\
\hline C & 806325 & 76361 & 6832 \\
\hline 1 & -4.1777245115 & 242 & -0.866 \\
\hline $\mathrm{C}$ & -6.5386953050 & 4995 & -1.0189166983 \\
\hline $\mathrm{H}$ & 300961 & 33592 & 24859 \\
\hline & 569 & -0.1 & 1512 \\
\hline C & 571 & -1.2 & -1.6 \\
\hline 1 & -1.9928450888 & -1.3953880493 & 10646 \\
\hline & 714 & -1. & 449 \\
\hline $\mathrm{H}$ & -7.0 & & 80046 \\
\hline $\mathrm{O}$ & -5.24 & $-1.97-x=$ & 01787 \\
\hline C & & 51463 & 826417 \\
\hline C & 79 & -0. & 7418 \\
\hline $\mathrm{C}$ & 387 & 492 & 0.7 \\
\hline C & 3.3283020181 & 55055 & -1.594 \\
\hline & & -0.8 & 5038 \\
\hline & 979 & 690 & 9195 \\
\hline $\mathrm{H}$ & 1.23 & 922 & 5267 \\
\hline c & & & -0.6223758730 \\
\hline & & & 6647 \\
\hline & & & 9562 \\
\hline $\mathrm{C}$ & 716829 & -4.3393772677 & 3.2597674628 \\
\hline & & $-5.3 \mathrm{c}$ & \\
\hline & 78 & -4.3 & 3.5 \\
\hline $\mathrm{H}$ & 327 & -4.0068253024 & 4.1 \\
\hline$C_{1}+$ & & 1439 & 97547 \\
\hline 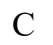 & & & \\
\hline $\mathrm{C}$ & 1.66 & -5.0 & 285 \\
\hline$C$ & 3.9208500870 & -3.6884371436 & -0.7809815155 \\
\hline $\mathrm{H}$ & 329995 & -2.3595159436 & 242536 \\
\hline $\mathrm{C}$ & & & \\
\hline $\mathrm{H}$ & & 92316 & 13073 \\
\hline$C$ & 3.4384162457 & -4.7990095919 & -1.4663558549 \\
\hline 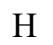 & & & \\
\hline $\mathrm{H}$ & & & \\
\hline $\mathrm{H}$ & 3.9402834295 & -5.1522374763 & -2.3695875826 \\
\hline $\mathrm{C}$ & 4.4984052983 & 3.0348760861 & -0.8966083946 \\
\hline $\mathrm{F}$ & & 3.7699033025 & \\
\hline & & 3.8628237206 & 0141650027 \\
\hline $\mathrm{F}$ & 7110878412 & 2.5566430451 & 187774 \\
\hline
\end{tabular}

TS4b

01

$\begin{array}{lrrr}\mathrm{O} & -0.5281150000 & 0.0597230000 & 1.9778500000 \\ \mathrm{C} & -1.5838540000 & -0.2876470000 & 1.2672950000 \\ \mathrm{C} & -2.3886300000 & -1.7339900000 & 1.6462330000\end{array}$ 


\begin{tabular}{|c|c|c|c|}
\hline $\mathrm{C}$ & -2.9190910000 & 0.4045550000 & 000 \\
\hline $\mathrm{C}$ & -3.6845730000 & -0.9238740000 & 1.8503160000 \\
\hline$H$ & -1.8896620000 & -2.0420230000 & 2.5747850000 \\
\hline $\mathrm{H}$ & -2.4084130000 & -2.5805420000 & 0.9475790000 \\
\hline $\mathrm{H}$ & -2.7674130000 & 0.9754670000 & 2.526755000 \\
\hline $\mathrm{H}$ & -3.3079400000 & 1.0883340000 & 0.8370160000 \\
\hline $\mathrm{C}$ & -1.3581390000 & -0.7626230000 & -0.0947070000 \\
\hline & -2.2649730000 & -0.3848700000 & -1.1761400000 \\
\hline$H$ & -3.2992510000 & -0.1857350000 & -0.889309000 \\
\hline $\mathrm{H}$ & -2.2112150000 & -0.9837290000 & -2.0890070000 \\
\hline $\mathrm{Mg}$ & 0.8779720000 & 1.2708240000 & 1.4475200000 \\
\hline $\mathrm{O}$ & 2.3313270000 & 0.5649640000 & 0.093 \\
\hline $\mathrm{O}$ & 2.6343530000 & 1.0003080000 & 70000 \\
\hline$P$ & 3.3476400000 & 0.5941010000 & 1.2258880000 \\
\hline O & 4.0899260000 & -0.8168760000 & 1.4484320000 \\
\hline 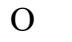 & 4.5427830000 & 1.5995870000 & 90000 \\
\hline $\mathrm{C}$ & 5.4838390000 & 1.9780680000 & 1.8292000000 \\
\hline $\mathrm{H}$ & 380000 & 1.1123640000 & 80000 \\
\hline $\mathrm{H}$ & 6.1525580000 & 2.7201350000 & 70000 \\
\hline $\mathrm{H}$ & 4.9782830000 & 2.4201180000 & 2.701 \\
\hline $\mathrm{C}$ & 40000 & -1.3476 & \\
\hline $\mathrm{H}$ & 5.1096140000 & -2.3880090000 & 0.6496500000 \\
\hline $\mathrm{H}$ & 4.3277730000 & -1.3222900000 & -0.559 \\
\hline $\mathrm{H}$ & 80000 & -0.7822420000 & 10000 \\
\hline $\mathrm{O}$ & 0.5092090000 & 3.1138150000 & 80000 \\
\hline $\mathrm{C}$ & 60000 & 20000 & 70000 \\
\hline $\mathrm{C}$ & 690000 & 5.3200850000 & 70000 \\
\hline $\mathrm{N}$ & 6900000 & 3.6335740000 & -1.1 \\
\hline $\mathrm{C}$ & 80000 & 000 & -1.2 \\
\hline $\mathrm{H}$ & 150000 & 5.2731590000 & 30000 \\
\hline $\mathrm{H}$ & 0.4695950000 & 5.8452130000 & 50000 \\
\hline $\mathrm{C}$ & 700000 & 4.7403840000 & 50000 \\
\hline $\mathrm{Br}$ & -1.3542800000 & 1.4170930000 & 10000 \\
\hline $\mathrm{H}$ & 40000 & 20000 & 80000 \\
\hline $\mathrm{H}$ & 110000 & 6.1532780000 & 700000 \\
\hline $\mathrm{O}$ & 6530000 & 07870000 & 00000 \\
\hline $\mathrm{C}$ & 600000 & 90000 & 50000 \\
\hline $\mathrm{H}$ & 3690000 & -0.3348610000 & 10000 \\
\hline $\mathrm{H}$ & 9060000 & -2.0608650000 & 100000 \\
\hline $\mathrm{H}$ & -3.4540180000 & -0.8330210000 & 710000 \\
\hline $\mathrm{C}$ & -4.7984780000 & -1.2275620000 & 50000 \\
\hline $\mathrm{C}$ & 40000 & -2.5276030000 & 80000 \\
\hline $\mathrm{C}$ & -5.6648490000 & -0.2111930000 & 0.4361320000 \\
\hline $\mathrm{C}$ & -6.08229900 & -2.80127800 & 150000 \\
\hline $\mathrm{H}$ & -4.3915020000 & -3.3486450000 & 0.7280410000 \\
\hline $\mathrm{C}$ & -6.7147620000 & -0.4797540000 & 20000 \\
\hline $\mathrm{H}$ & -5.5182480000 & 0.8107840000 & 0.7948310000 \\
\hline $\mathrm{C}$ & -6.9241060000 & -1.7767780000 & -0.9061400000 \\
\hline $\mathrm{H}$ & -6.2419650000 & -3.8235280000 & -0.8285660000 \\
\hline $\mathrm{H}$ & -7.3724410000 & 0.3310170000 & -0.7604140000 \\
\hline $\mathrm{H}$ & -7.7428720000 & -1.9883170000 & -1.5968010000 \\
\hline $\mathrm{C}$ & -0.1424870000 & -1.5419710000 & -0.4053090000 \\
\hline $\mathrm{C}$ & 0.3077280000 & -1.6644020000 & -1.7318480000 \\
\hline $\mathrm{C}$ & 0.5814020000 & -2.1935230000 & 0.607 \\
\hline $\mathrm{C}$ & 1.4199110000 & -2.4323180000 & -2.0368830000 \\
\hline $\mathrm{H}$ & -0.1843180000 & -1.1212060000 & -2.5384090000 \\
\hline $\mathrm{C}$ & 1.6800120000 & -2.9846220000 & 0.300447000 \\
\hline $\mathrm{H}$ & 0.2904330000 & -2.0712300000 & 1.648963000 \\
\hline $\mathrm{C}$ & 2.0942720000 & -3.1129220000 & -1.022284000 \\
\hline & 1.7635530000 & -2.5027090000 & -3.07004600 \\
\hline
\end{tabular}




$\begin{array}{lrrr}\mathrm{H} & 2.2241770000 & -3.4878880000 & 1.0993080000 \\ \mathrm{C} & 3.2523290000 & -4.0066120000 & -1.3942390000 \\ \mathrm{~F} & 3.9071550000 & -4.4603880000 & -0.3221790000 \\ \mathrm{~F} & 4.1380480000 & -3.3660070000 & -2.1654510000 \\ \mathrm{~F} & 2.8317840000 & -5.0706390000 & -2.0844950000\end{array}$

TS4c

01

$$
0.2099257810
$$




$\begin{array}{lrrr}\mathrm{C} & 4.3435846322 & -0.4771295764 & -0.5464722509 \\ \mathrm{C} & 5.8332455531 & -0.2905648545 & -0.3320192629 \\ \mathrm{~N} & 3.8669546704 & -1.5293100096 & 0.1003387713 \\ \mathrm{C} & 6.1914728673 & -1.4306534787 & 0.6156112199 \\ \mathrm{H} & 6.0116989500 & 0.7156605373 & 0.0738480476 \\ \mathrm{H} & 6.3403342622 & -0.3376294594 & -1.3072308978 \\ \mathrm{C} & 4.8731348584 & -2.1945303428 & 0.7995907101 \\ \mathrm{Br} & 1.6543434458 & -2.1158050339 & -0.3944787468 \\ \mathrm{H} & 6.9523740698 & -2.1205987520 & 0.2247645222 \\ \mathrm{H} & 6.5393958498 & -1.0912773468 & 1.6019102359 \\ \mathrm{O} & 4.7262082215 & -3.2011129119 & 1.4388701832 \\ \mathrm{H} & -7.0173112407 & -0.7958396416 & 1.6852787060 \\ \mathrm{C} & -3.8863196928 & -1.7320692308 & 4.1847178741 \\ \mathrm{~F} & -4.1785968254 & -0.4901935637 & 4.5947921562 \\ \mathrm{~F} & -3.2166720003 & -2.3216943698 & 5.1785573802 \\ \mathrm{~F} & -5.0411092730 & -2.3843141075 & 4.0352366296\end{array}$

TS4d

01

C $\quad 0.8434333826$

$-1.0713207222$

0.3752853772

$\begin{array}{lll}1.8592044127 & -1.9471574286 & 1.1819654839\end{array}$

$\begin{array}{lllll}\text { C } & 2.5848880085 & -0.7350056289 & 1.8164647665\end{array}$

$\mathrm{H} \quad 2.4776622076 \quad-2.6509692570 \quad 0.6162750505$

$\begin{array}{llll}\mathrm{H} & 1.2538844348 & -2.5082406439 & 1.9029340708\end{array}$

$\begin{array}{lllll}\text { C } & 1.1210949015 & -0.7347930753 & -1.0378812556\end{array}$

$\begin{array}{lllll}\text { C } & 0.0072399416 & -0.4144952471 & -1.8913704485\end{array}$

$\mathrm{H} \quad-0.8746923067 \quad-1.0504492125 \quad-1.7207601620$

$\mathrm{H} \quad \begin{array}{llll}0.2594792441 & -0.2645599230 & -2.9449361074\end{array}$

$\begin{array}{llll}\mathrm{Mg} & -2.1369072550 & -0.4189113606 & 0.7690433661\end{array}$

$\begin{array}{llll}\mathrm{O} & -3.6258594582 & -1.7432656347 & 1.3599810740\end{array}$

$\begin{array}{llll}\text { O } & -3.1898593119 & -1.0024355037 & -0.9628010660\end{array}$

$\begin{array}{lllll}\mathrm{P} & -4.0595696067 & -1.9001092217 & -0.0908468250\end{array}$

$\begin{array}{llll}\mathrm{O} & -3.9439869168 & -3.4150032106 & -0.6207866761\end{array}$

$\begin{array}{llll}\mathrm{O} & -5.6268127527 & -1.5633703652 & -0.2134691811\end{array}$

$\begin{array}{lllll}\text { C } & -6.2253295152 & -1.4777731794 & -1.4948379073\end{array}$

$\mathrm{H} \quad-6.1915195837 \quad-2.4499190358 \quad-2.0131562950$

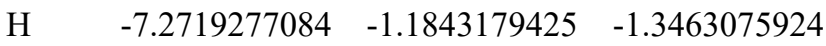

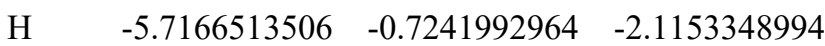

$\begin{array}{lllll}\text { C } & -4.5131508695 & -4.4645552278 & 0.1424069082\end{array}$

$\mathrm{H} \quad-4.2562618444 \quad-5.4070896130 \quad-0.3569580212$

$\begin{array}{llll}\mathrm{H} & -4.1085395498 & -4.4678246335 & 1.1659590458\end{array}$

$\begin{array}{llll}\mathrm{H} & -5.6099983277 & -4.3680185312 & 0.1918697452\end{array}$

$\begin{array}{llll}\text { O } & -2.4658030791 & 1.3529246128 & 1.5757390671\end{array}$

$\begin{array}{llll}\text { C } & -2.3972160949 & 2.5652611587 & 1.2564087291\end{array}$

$\begin{array}{llll}\text { C } & -3.0276430804 & 3.6522464722 & 2.1083922641\end{array}$

$\begin{array}{llll}\mathrm{N} & -1.7888857073 & 3.0491513080 & 0.1850785924\end{array}$

$\begin{array}{llll}\mathrm{C} & -2.7052099449 & 4.9265964517 & 1.3358110266\end{array}$

$\begin{array}{llll}\mathrm{H} & -2.5918881463 & 3.6117045472 & 3.1177199042\end{array}$

$\begin{array}{llll}\mathrm{H} & -4.1016414339 & 3.4418456081 & 2.2165333181\end{array}$

$\begin{array}{llll}\text { C } & -1.8840117886 & 4.4374866926 & 0.1339922463\end{array}$

$\begin{array}{llll}\mathrm{Br} & -0.7021841013 & 1.3766328390 & -1.1248754348\end{array}$

$\begin{array}{llll}\mathrm{H} & -3.5939965758 & 5.4547603051 & 0.9615349854\end{array}$

$\begin{array}{llll}\mathrm{H} & -2.1104391409 & 5.6581216278 & 1.9008229819\end{array}$

$\begin{array}{llll}\mathrm{O} & -1.3972376221 & 5.1376551006 & -0.7137819279\end{array}$

$\begin{array}{lllll}\text { O } & -0.4233306608 & -1.2518262884 & 0.6959145433\end{array}$

$\begin{array}{llll}\text { C } & 1.5818171317 & 0.2156663896 & 1.1334620419\end{array}$

$\begin{array}{llll}\mathrm{H} & 0.8033113535 & 0.6090954721 & 1.7976065207\end{array}$

$\begin{array}{llll}\mathrm{H} & 1.9756702315 & 1.0277262879 & 0.5139595852\end{array}$

$\begin{array}{llll}\text { C } & 2.4900198629 & -0.6938002154 & 3.3443326235\end{array}$

H $\quad 3.0910044603 \quad-1.5000582973 \quad 3.7922518039$ 


$\begin{array}{ll}\mathrm{H} & 2.8688830771 \\ \mathrm{H} & 1.4467017753 \\ \mathrm{C} & 4.0426578548 \\ \mathrm{C} & 4.8951442437 \\ \mathrm{C} & 4.5891207606 \\ \mathrm{C} & 6.2392386183 \\ \mathrm{H} & 4.4987362419 \\ \mathrm{C} & 5.9350684200 \\ \mathrm{H} & 3.9602634759 \\ \mathrm{C} & 6.7641725785 \\ \mathrm{H} & 6.8811493657 \\ \mathrm{H} & 6.3339925547 \\ \mathrm{H} & 7.8132336297 \\ \mathrm{C} & 2.4788868996 \\ \mathrm{C} & 3.4297362859 \\ \mathrm{C} & 2.8200578455 \\ \mathrm{C} & 4.7101681803 \\ \mathrm{H} & 3.1743045537 \\ \mathrm{C} & 4.1046769788 \\ \mathrm{H} & 2.0830978638 \\ \mathrm{C} & 5.0559783977 \\ \mathrm{H} & 5.4492598714 \\ \mathrm{H} & 4.3727308963 \\ \mathrm{C} & 6.4547703968 \\ \mathrm{~F} & 7.3043934108 \\ \mathrm{~F} & 6.9329551278 \\ \mathrm{~F} & 6.4835601861\end{array}$

TS5a

01

$\begin{array}{lll}\mathrm{O} & -0.4964995793 \\ \mathrm{C} & -1.6989367148 & -0 .\end{array}$

C $\quad-2.8276876109$

C $\quad-2.6245757786$

C $\quad-3.8373761907$

$\mathrm{H}-2.5547248289$

$\mathrm{H} \quad-3.0799766872$

$\mathrm{H} \quad-2.2771870515$

$\mathrm{H} \quad-2.7110060643$

C $\quad-4.6343970764$

$\mathrm{H} \quad-5.1926762413$

$\mathrm{H} \quad-5.3631565981$

$\mathrm{H} \quad-3.9634429514$

C $\quad-4.8040426539$

C $\quad-5.2999114470$

C $\quad-5.2804165688$

C $\quad-6.2201126621$

$\mathrm{H} \quad-4.9663723800$

C $\quad-6.1978568900$

$\mathrm{H} \quad-4.9218628664$

C $\quad-6.6691915027$

$\mathrm{H} \quad-6.5868864172$

$\mathrm{H} \quad-6.5463964663$

C $\quad-1.4795719318$

C $\quad-1.9469682239$

$\mathrm{H} \quad-2.6694090279$

$\mathrm{H} \quad-1.8579976546$

$\mathrm{Mg} \quad 1.2290905772$

O 2.5182510991

O $\quad 2.5299796272$
0.2648919092

$-0.8160092576$

$-0.5848866855$

$-1.6948878372$

0.6388258152

$-1.5944947886$

$-2.6605789645$

0.7448176038

1.5305587826

$-0.3726207165$

$-2.4767307517$

1.7075204634

$-0.2920205363$

$-0.5305228854$

$-1.5551633595$

0.7283765968

$-1.3201145693$

$-2.5516963925$

0.9691434893

1.5343793941

$-0.0534965634$

$-2.1209544284$

1.9617101950

0.2378113747

$-0.7476985068$

1.3599505679

0.4104625934

3.7306868006

3.6726084927

1.4018491810

1.4714226842

1.0074588444

1.1233061309

1.7979229745

0.6554876170

0.9608255548

0.7025279581

1.1777980233

0.3301070290

0.4115378824

$-1.5821109429$

$-1.6020585689$

$-2.1089428444$

$-2.0926774927$

$-1.2453862182$

$-2.5707190665$

$-2.1110355353$

$-2.5489067252$

$-2.0867803743$

$-2.9368816940$

$-3.0338453940$

$-2.7388586475$

$-2.4836219633$

$-4.3588175476$

$-0.6752142194$

$-0.5689698650$

$-1.5754442326$

0.5601216200

$-0.4084887930$

$-1.9975840596$

$-2.3977359729$

0.7941054168

1.5014879324

$-0.4026623786$

0.5395123667

$-1.2283646097$

$-0.5150971699$

$-0.2347218799$

$-1.3190058496$

1.0431180875

$-1.1341775047$

$-2.3313717959$

1.2350887372

1.9083282842

0.1442440570

$-1.9991236430$

2.2435716180

$-0.5867983799$

0.3953596168

1.1176027236

0.3163214906

$-0.5142023852$

$-0.5902841693$

$-2.1161284376$
1.7456686448

1.1157137117

1.5378268646

1.6411491840

1.6838133872

2.5153703473

0.8539771196

2.6561735173

1.0808644740

2.9900146545

3.1070388006

3.0070632224

3.8555787123

0.5192796227

$-0.2106291075$

0.1958653538

$-1.2429010132$

0.0256283468

$-0.8337524498$

0.7600865793

$-1.5640275076$

$-1.8007342211$

$-1.0686442864$

$-0.4122454571$

$-1.2495135114$

$-0.8766313886$

$-2.3333570301$

1.0102604900

$-0.7547374916$

1.2009750876 


$\begin{array}{lrrr}\mathrm{P} & 3.1872001336 & -1.8038283785 & -0.1359598248 \\ \mathrm{O} & 3.0902158520 & -3.1138506243 & -1.0757746165 \\ \mathrm{O} & 4.7693086316 & -1.5358881729 & -0.0057634154 \\ \mathrm{C} & 5.5711720135 & -2.4478157703 & 0.7275681838 \\ \mathrm{H} & 5.5938386710 & -3.4355794851 & 0.2393360487 \\ \mathrm{H} & 6.5880447659 & -2.0371112190 & 0.7566655561 \\ \mathrm{H} & 5.1902577587 & -2.5655075593 & 1.7532187855 \\ \mathrm{C} & 3.5737543761 & -3.0493147789 & -2.4062214907 \\ \mathrm{H} & 3.3400714780 & -4.0079942301 & -2.8857171975 \\ \mathrm{H} & 3.0886615912 & -2.2313518971 & -2.9620418332 \\ \mathrm{H} & 4.6640869819 & -2.8914915178 & -2.4231931730 \\ \mathrm{O} & 2.2817986531 & 1.1720108359 & 1.5603052284 \\ \mathrm{C} & 2.5725374509 & 2.1567086442 & 0.8894684691 \\ \mathrm{C} & 3.8375184189 & 2.9668231735 & 1.0364286020 \\ \mathrm{~N} & 1.8180560897 & 2.6817966274 & -0.1063129499 \\ \mathrm{C} & 3.7071466303 & 4.0802226995 & -0.0031765697 \\ \mathrm{H} & 4.6835062507 & 2.2875662737 & 0.8554732317 \\ \mathrm{H} & 3.9191350938 & 3.3192710236 & 2.0741783733 \\ \mathrm{C} & 2.3940756080 & 3.8057510733 & -0.7248859228 \\ \mathrm{Br} & 0.1732199551 & 1.8373261280 & -0.6487097972 \\ \mathrm{H} & 3.6558909147 & 5.0872803046 & 0.4346079681 \\ \mathrm{H} & 4.5171597847 & 4.0906950879 & -0.7453347879 \\ \mathrm{O} & 1.9113298222 & 4.4082146978 & -1.6364198593 \\ \mathrm{H} & -7.3852245715 & 0.2908984552 & -2.3756011061 \\ \mathrm{~N} & -0.6111266955 & -1.5614787880 & -0.8531698267 \\ \mathrm{C} & -0.5545883683 & -2.8787950959 & -0.2201131566 \\ \mathrm{H} & -0.6529222849 & -2.7901737201 & 0.8657572232 \\ \mathrm{H} & -1.3450784479 & -3.5428223828 & -0.6139202825 \\ \mathrm{H} & 0.4235786935 & -3.3325730019 & -0.4321789659 \\ \mathrm{C} & -0.2076786362 & -1.5619262545 & -2.2448536455 \\ \mathrm{H} & 0.1834820845 & -0.5756568700 & -2.5288162424 \\ \mathrm{H} & 0.6036665408 & -2.2890557463 & -2.3706392383 \\ \mathrm{H} & -1.0390594777 & -1.8324571367 & -2.9224274241 \\ & & & \\ & & & \\ \mathrm{H} & & & \end{array}$

TS5b

01

$\begin{array}{lrrr}\mathrm{O} & 0.0182896017 & -1.0374450131 & 1.4931805717 \\ \mathrm{C} & -1.0783888211 & -0.8039737271 & 0.8682835491 \\ \mathrm{C} & -2.5169674894 & -2.0636595714 & 0.7703726606 \\ \mathrm{C} & -2.1547504347 & 0.0399104587 & 1.5471769044 \\ \mathrm{C} & -3.3346019690 & -0.9615540291 & 1.4651082014 \\ \mathrm{H} & -2.0936611987 & -2.8117583534 & 1.4514175740 \\ \mathrm{H} & -2.9384154358 & -2.5593749440 & -0.1125984072 \\ \mathrm{H} & -1.8264239879 & 0.2171176719 & 2.5781161683 \\ \mathrm{H} & -2.2899690849 & 1.0189582387 & 1.0665665772 \\ \mathrm{C} & -3.8052392806 & -1.4081848197 & 2.8568704032 \\ \mathrm{H} & -4.2826200590 & -0.5695087381 & 3.3859557987 \\ \mathrm{H} & -4.5400026413 & -2.2237651744 & 2.7770903801 \\ \mathrm{H} & -2.9550339755 & -1.7604398959 & 3.4610094156 \\ \mathrm{C} & -4.5486061359 & -0.4784732673 & 0.6787437309 \\ \mathrm{C} & -5.3469970653 & -1.3771007973 & -0.0386521972 \\ \mathrm{C} & -4.9415516931 & 0.8646421056 & 0.7195446919 \\ \mathrm{C} & -6.4848518134 & -0.9447132592 & -0.7175230555 \\ \mathrm{H} & -5.0849969478 & -2.4377708696 & -0.0670250334 \\ \mathrm{C} & -6.0781037505 & 1.3018072288 & 0.0412139918 \\ \mathrm{H} & -4.3523568132 & 1.5886850670 & 1.2870935335 \\ \mathrm{C} & -6.8517833944 & 0.3993233458 & -0.6857507818 \\ \mathrm{H} & -7.0885783867 & -1.6644706264 & -1.2747126535 \\ \mathrm{H} & -6.3579557715 & 2.3565999879 & 0.0810770066 \\ \mathrm{C} & -1.1941995939 & -1.1058394891 & -0.5369658063\end{array}$




\begin{tabular}{|c|c|c|c|}
\hline $\mathrm{C}$ & -1.9369014341 & -0.1284808010 & -1.40007 \\
\hline $\mathrm{H}$ & -2.8760386285 & 0.1979617919 & -0.946792263 \\
\hline $\mathrm{H}$ & -2.1487979256 & -0.4983933474 & -2.40732337 \\
\hline $\mathrm{Mg}$ & 1.7220101010 & -0.1761588026 & 0.98895307 \\
\hline $\mathrm{O}$ & 2.6531903759 & -0.9785671368 & -0.72078001 \\
\hline $\mathrm{O}$ & 3.3696891270 & -1.2773214108 & $1.6259246^{\circ}$ \\
\hline $\mathrm{P}$ & 3.7293133815 & -1.5756983302 & 0.1772531 \\
\hline $\mathrm{O}$ & 3.8761419405 & -3.1707552664 & -0.0184506 \\
\hline $\mathrm{O}$ & 5.1671723405 & -1.0021874338 & -0.24671211 \\
\hline $\mathrm{C}$ & 6.2813231054 & -1.1851902394 & 0.6124982 \\
\hline $\mathrm{H}$ & 6.5649191381 & -2.2487276312 & 0.6711909 \\
\hline $\mathrm{H}$ & 7.1146529451 & -0.6121175561 & 0.1881279 \\
\hline $\mathrm{H}$ & 6.0572822892 & -0.8179684709 & 1.6250622 \\
\hline $\mathrm{C}$ & 4.2546932737 & -3.6901568338 & -1.28035036 \\
\hline $\mathrm{H}$ & 4.2635936293 & -4.7838425398 & -1.19 \\
\hline $\mathrm{H}$ & 3.5387660777 & -3.3903092210 & -2.06 \\
\hline $\mathrm{H}$ & 5.2577274242 & -3.3393736675 & -1.57021333 \\
\hline $\mathrm{O}$ & 1.8366425866 & 1.7048968800 & 1.30109272 \\
\hline $\mathrm{C}$ & 1.8185775954 & 2.8501080238 & 0.7519024 \\
\hline $\mathrm{C}$ & 2.4296181434 & 4.0441205942 & 1.470 \\
\hline $\mathrm{N}$ & 1.3001127724 & 3.1291946923 & -0.41746591 \\
\hline $\mathrm{C}$ & 2.2187715631 & 5.1703000471 & 0.46959652 \\
\hline $\mathrm{H}$ & 3.4823720869 & 3.8275656254 & 1.70565806 \\
\hline $\mathrm{H}$ & 1.9122076993 & 4.1890980276 & 2.43 \\
\hline $\mathrm{C}$ & 1.4512510311 & 4.4868613299 & -0.67792713 \\
\hline $\mathrm{Br}$ & -0.7263124728 & 1.4084514141 & -1.45997357 \\
\hline $\mathrm{H}$ & 1.6246583011 & 6.0132189329 & 0.85040047 \\
\hline $\mathrm{H}$ & 3.1535374718 & 5.5896785748 & 0.06943849 \\
\hline $\mathrm{O}$ & 1.0406383891 & 5.0578810432 & -1.65746006 \\
\hline $\mathrm{H}$ & -7.7390933682 & 0.7413972922 & -1.22219766 \\
\hline $\mathrm{N}$ & -0.3687858482 & -1.9911 & \\
\hline $\mathrm{C}$ & 0.2431373258 & -3.1129329553 & -0.43670505 \\
\hline $\mathrm{H}$ & 0.0963467262 & -3.0421247638 & 0.64240176 \\
\hline $\mathrm{H}$ & -0.1982824213 & -4.0527201023 & -0.80993830 \\
\hline $\mathrm{H}$ & 1.3249095648 & -3.1215489231 & -0.62242406 \\
\hline $\mathrm{C}$ & -0.2053507261 & -2.0429893540 & -2.57821326 \\
\hline $\mathrm{H}$ & -0.1610956708 & -1.0368900391 & -3.01216980 \\
\hline $\mathrm{H}$ & 0.7636023183 & -2.5140344733 & -2.78437049 \\
\hline $\mathrm{H}$ & -0.9961150869 & -2.6347352773 & -3.07251857 \\
\hline
\end{tabular}

TS5c

01

$\begin{array}{lrrr}\mathrm{O} & -0.1445715782 & -0.3905741650 & 1.0725377774 \\ \mathrm{C} & -1.5210616503 & -0.0942567145 & 1.1105103210 \\ \mathrm{C} & -2.5115344629 & -0.4984317494 & 2.2016887680 \\ \mathrm{C} & -2.1071059778 & 1.3142336488 & 1.1360309816 \\ \mathrm{C} & -3.4024928910 & 0.6759314634 & 1.7166950365 \\ \mathrm{H} & -2.0849660328 & -0.2704290533 & 3.1898119582 \\ \mathrm{H} & -2.9240154289 & -1.5119931711 & 2.2189003638 \\ \mathrm{H} & -1.5768551843 & 1.9127292822 & 1.8926298227 \\ \mathrm{H} & -2.1729776620 & 1.8917719292 & 0.2068404793 \\ \mathrm{C} & -4.1457615230 & 1.4535889717 & 2.7967158628 \\ \mathrm{H} & -4.6087915743 & 2.3625997240 & 2.3845895663 \\ \mathrm{H} & -4.9466084865 & 0.8389210709 & 3.2353635376 \\ \mathrm{H} & -3.4593290261 & 1.7556595476 & 3.6023426735 \\ \mathrm{C} & -4.3347434982 & 0.2922376293 & 0.5676048132 \\ \mathrm{C} & -4.7492172187 & -1.0185087041 & 0.3145841668 \\ \mathrm{C} & -4.7990942150 & 1.3029093930 & -0.2880066157 \\ \mathrm{C} & -5.5915901615 & -1.3128558337 & -0.7594211472 \\ \mathrm{H} & -4.4141318785 & -1.8363343379 & 0.9532609303\end{array}$




$\begin{array}{lrrr}\mathrm{C} & -5.6400110823 & 1.0155539941 & -1.3587768234 \\ \mathrm{H} & -4.4907080135 & 2.3379180344 & -0.1168279245 \\ \mathrm{C} & -6.0386433956 & -0.2995349410 & -1.6016875591 \\ \mathrm{H} & -5.8976824720 & -2.3465272804 & -0.9356445827 \\ \mathrm{H} & -5.9832635357 & 1.8225032132 & -2.0098252356 \\ \mathrm{C} & -1.1856997682 & -0.8594718474 & -0.0914332335 \\ \mathrm{C} & -1.0586008137 & -0.1009356876 & -1.4062225331 \\ \mathrm{H} & -2.0606798230 & 0.2518687154 & -1.6922219008 \\ \mathrm{H} & -0.6570463405 & -0.7332077555 & -2.1985676578 \\ \mathrm{Mg} & 1.7496978830 & 0.0079705573 & 0.5744434978 \\ \mathrm{O} & 2.0176117034 & -1.4447359885 & -0.9503900383 \\ \mathrm{O} & 2.6832688312 & -1.6744225076 & 1.4187407012 \\ \mathrm{P} & 2.7155430688 & -2.3499465386 & 0.0590385310 \\ \mathrm{O} & 2.0303666860 & -3.8105441976 & 0.1613911239 \\ \mathrm{O} & 4.2040810947 & -2.6694700206 & -0.4532593553 \\ \mathrm{C} & 5.1656461253 & -3.1910621534 & 0.4506633961 \\ \mathrm{H} & 4.9072360895 & -4.2186953922 & 0.7552388753 \\ \mathrm{H} & 6.1292758032 & -3.2022652124 & -0.0729921999 \\ \mathrm{H} & 5.2386496284 & -2.5604033501 & 1.3489375064 \\ \mathrm{C} & 2.1379073136 & -4.7259501802 & -0.9141421680 \\ \mathrm{H} & 1.4464626201 & -5.5530578932 & -0.7078065490 \\ \mathrm{H} & 1.8647191823 & -4.2579149946 & -1.8732755102 \\ \mathrm{H} & 3.1615619082 & -5.1227901535 & -0.9953882014 \\ \mathrm{O} & 2.7076565366 & 1.6006106189 & 1.0731352451 \\ \mathrm{C} & 2.8012594507 & 2.7714296261 & 0.5864087023 \\ \mathrm{C} & 3.7265248740 & 3.7955305571 & 1.2223638309 \\ \mathrm{~N} & 2.1477020630 & 3.2069278499 & -0.4617489131 \\ \mathrm{C} & 3.5343586121 & 5.0087412268 & 0.3229833087 \\ \mathrm{H} & 4.7512300871 & 3.3956330945 & 1.2413723977 \\ \mathrm{H} & 3.4268387650 & 3.9528815463 & 2.2695001496 \\ \mathrm{C} & 2.4877074041 & 4.5342312902 & -0.7032939988 \\ \mathrm{Br} & 0.1386156221 & 1.4278667511 & -1.3368120016 \\ \mathrm{H} & 3.1521060740 & 5.8999953273 & 0.8408670373 \\ \mathrm{H} & 4.4441100154 & 5.3142686457 & -0.2140478583 \\ \mathrm{O} & 2.0342626218 & 5.2221249343 & -1.5828196665 \\ \mathrm{H} & -6.6948211060 & -0.5302766824 & -2.4434845539 \\ \mathrm{~N} & -1.3325665309 & -2.2196714219 & -0.1893538712 \\ \mathrm{C} & -1.2060494437 & -3.0702538754 & 0.9878818134 \\ \mathrm{H} & -1.0375456429 & -2.4784191820 & 1.8898599168 \\ \mathrm{H} & -2.0996241314 & -3.7014653347 & 1.1231752166 \\ \mathrm{H} & -0.3182032697 & -3.7119941213 & 0.8788958555 \\ \mathrm{C} & -1.0290079387 & -2.9109500805 & -1.4353489066 \\ \mathrm{H} & 0.0273926406 & -2.7922498940 & -1.7280576964 \\ \mathrm{H} & -1.2341647375 & -3.9781469513 & -1.2839611388 \\ \mathrm{H} & -1.6817402092 & -2.5786412756 & -2.2544873239\end{array}$

\section{Real Model with Real Catalyst}

$\begin{array}{lrrr}\text { (2R,4R)-CYr } & & & \\ 01 & & & \\ \text { O } & -2.3331166078 & -1.7724600244 & 0.2418225517 \\ \text { C } & -3.2453540315 & -1.0327399451 & -0.0883920137 \\ \text { C } & -5.2470441729 & -0.1929619605 & -1.1029416603 \\ \text { C } & -3.3285233808 & 0.4396268306 & 0.1445231470 \\ \text { C } & -4.8219556901 & 0.7947334979 & 0.0120743560 \\ \text { H } & -4.9283433348 & 0.2236748345 & -2.0699161225 \\ \text { H } & -6.3314652331 & -0.3521004334 & -1.1698298658 \\ \text { H } & -2.7725692734 & 0.8565647298 & -0.7226376242 \\ \text { H } & -2.7820122360 & 0.7945310375 & 1.0290062958 \\ \text { C } & -4.4728057947 & -1.5201570907 & -0.8700503676\end{array}$




\begin{tabular}{|c|c|c|c|}
\hline & 36 & 369 & 60 \\
\hline $\mathrm{H}$ & 5.5183041504 & -1.9465561961 & 208178 \\
\hline $\mathrm{H}$ & -6.2792136947 & -2.6455845958 & -0.4370319892 \\
\hline $\mathrm{O}$ & 0.4815640634 & -2.1791079690 & 2.4736988557 \\
\hline $\mathrm{C}$ & 0.0565638321 & -1.1680594157 & 3.0655668632 \\
\hline $\mathrm{C}$ & 0.1835495618 & -0.8549518668 & 4.5381905823 \\
\hline I & -0.6104564345 & -0.2281554081 & 24890 \\
\hline $\mathrm{C}$ & -0.6051124735 & 0.4472449820 & 4.6610698607 \\
\hline $\mathrm{H}$ & -0.2147025938 & -1.6923953851 & 51278704778 \\
\hline $\mathrm{H}$ & 1.2487496721 & -0.7521838340 & 4.7894501857 \\
\hline $\mathrm{C}$ & -1.0880827636 & 0.7341483911 & 4786 \\
\hline $\mathrm{Br}$ & -4.5339236796 & -4.1596677568 & 26530 \\
\hline $\mathrm{H}$ & 0.0017094816 & & \\
\hline $\mathrm{H}$ & -1.4779087102 & 0.3798840651 & 6135 \\
\hline $\mathrm{O}$ & -1.8187890251 & 1.6538649597 & 2.9246114696 \\
\hline $\mathrm{C}$ & 123 & 168 & 8453 \\
\hline $\mathrm{H}$ & & & \\
\hline $\mathrm{H}$ & 1749 & 050 & 8268 \\
\hline $\mathrm{H}$ & -4.4217483767 & & -1.3127775413 \\
\hline$C$ & 684 & & \\
\hline $\mathrm{C}$ & 123 & & \\
\hline $\mathrm{C}$ & 2147 & & 456 \\
\hline $\mathrm{C}_{\mathrm{S}}$ & 556 & & \\
\hline$H_{2}$ & 926 & & \\
\hline $\mathrm{C}$ & 1478 & & \\
\hline $\mathrm{H}$ & -3.9000161976 & & 54194 \\
\hline$C_{3}$ & 745 & & \\
\hline $\mathrm{H}$ & 644 & & \\
\hline $\mathrm{H}$ & -5.2118 & & \\
\hline $\mathrm{H}$ & & & \\
\hline$\theta^{2}$ & 411 & 92 & 400 \\
\hline $\mathrm{C}$ & 367 & & 026 \\
\hline 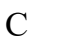 & -2.9710377179 & 693 & -2.9060286954 \\
\hline $\mathrm{C}$ & & & \\
\hline 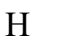 & & & \\
\hline $\mathrm{C}_{\mathrm{S}}$ & 2536 & 775 & 272 \\
\hline $\mathrm{H}$ & & & \\
\hline C & & & \\
\hline-3 & 472 & 305 & 803 \\
\hline $\mathrm{H}$ & -1.7989876637 & 6661 & 8326 \\
\hline $\mathrm{H}$ & & & \\
\hline $\mathrm{Mg}$ & -0.3 & & \\
\hline $\mathrm{O}$ & 612 & 286 & 431 \\
\hline $\mathrm{P}$ & & & \\
\hline 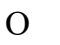 & & & \\
\hline 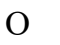 & & & 007 \\
\hline $\mathrm{O}$ & 5560 & 159589 & 27176 \\
\hline $\mathrm{C}$ & & & \\
\hline $\mathrm{C}$ & & & 02 \\
\hline $\mathrm{C}$ & 2.38 & & 057 \\
\hline 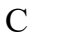 & 0.6492089478 & 084671 & 0.4039870063 \\
\hline$a_{2}$ & & & \\
\hline $\mathrm{C}$ & & & -0.06 \\
\hline $\mathrm{C}$ & & & 475 \\
\hline 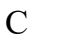 & 0.5838251322 & 3.5824110367 & 1.6666812097 \\
\hline $\mathrm{Si}$ & & & \\
\hline $\mathrm{C}$ & & & \\
\hline$C$ & 5.3697377648 & -1.4749463497 & 0.2201926077 \\
\hline & & 3451751027 & \\
\hline & & & \\
\hline
\end{tabular}




\begin{tabular}{|c|c|c|c|}
\hline $\mathrm{C}$ & 1.3385684579 & 3.0591310345 & 2.7499441635 \\
\hline $\mathrm{H}$ & -0.0675274830 & 4.4377477806 & 1.8833803506 \\
\hline $\mathrm{C}$ & -0.8438411656 & 2.8551782570 & -2.3906437097 \\
\hline $\mathrm{C}$ & -1.8683936578 & 4.6930315734 & -0.1616614665 \\
\hline $\mathrm{C}$ & 0.7347700087 & 5.3215382709 & -1.5965129518 \\
\hline $\mathrm{C}$ & 5.2973518699 & 2.0127583108 & 1.5821468297 \\
\hline $\mathrm{C}$ & 5.7961118198 & -0.2447523271 & 0.7911209232 \\
\hline $\mathrm{H}$ & 6.1385921751 & -2.2210654108 & -0.0153945802 \\
\hline $\mathrm{C}$ & 4.4904064685 & -3.3860250642 & -2.5592302782 \\
\hline $\mathrm{C}$ & 4.1890110743 & -4.7762816145 & 0.1654659136 \\
\hline $\mathrm{C}$ & 1.7625767290 & -3.5196359893 & -1.0700187606 \\
\hline $\mathrm{C}$ & 2.7886206825 & 1.9625507127 & 4.8855364502 \\
\hline $\mathrm{H}$ & 3.5828046180 & 0.5672493886 & 3.4737002828 \\
\hline $\mathrm{C}$ & 1.2222722254 & 3.5996326140 & 4.0592302244 \\
\hline $\mathrm{C}$ & -2.1741460704 & 2.4879908835 & -2.6398976092 \\
\hline $\mathrm{C}$ & 0.1572696168 & 2.4267721763 & -3.2814645217 \\
\hline $\mathrm{C}$ & -2.3871156841 & 5.8825825029 & -0.7023356906 \\
\hline $\mathrm{C}$ & -2.4896603622 & 4.1390195135 & 0.9691657831 \\
\hline $\mathrm{C}$ & 1.7332817293 & 5.9090879072 & -0.8037272835 \\
\hline $\mathrm{C}$ & 0.5261860580 & 5.8156968137 & -2.8961724598 \\
\hline $\mathrm{C}$ & 6.6354667949 & 2.2357976414 & 1.7954382425 \\
\hline $\mathrm{H}$ & 4.5798674381 & 2.8096705393 & 1.7771256592 \\
\hline $\mathrm{C}$ & 7.1732894383 & 0.0133370633 & 1.0333998975 \\
\hline $\mathrm{C}$ & 5.4523103615 & -4.3565602787 & -2.8833627344 \\
\hline $\mathrm{C}$ & 4.1943552637 & -2.3824331895 & -3.4988328679 \\
\hline $\mathrm{C}$ & 3.9771202217 & -6.0822990958 & -0.3138709586 \\
\hline $\mathrm{C}$ & 4.7762026262 & -4.6082258788 & 1.4278017940 \\
\hline $\mathrm{C}$ & 1.0091188699 & -3.6537059635 & 0.1155552248 \\
\hline $\mathrm{C}$ & 1.1019543899 & -3.5045246599 & -2.3028798928 \\
\hline $\mathrm{C}$ & 1.9394288828 & 3.0739835483 & 5.1055670367 \\
\hline $\mathrm{H}$ & 3.3377123385 & 1.5269887489 & 5.7230871102 \\
\hline $\mathrm{H}$ & 0.5390458773 & 4.4371648396 & 4.2189811740 \\
\hline $\mathrm{C}$ & -2.4995084934 & 1.6960101811 & -3.7472944216 \\
\hline $\mathrm{H}$ & -2.9817220540 & 2.8197728969 & -1.9729228011 \\
\hline $\mathrm{C}$ & -0.1646402475 & 1.6216033972 & -4.3787082835 \\
\hline $\mathrm{H}$ & 1.2065700234 & 2.7077538667 & -3.1192873503 \\
\hline $\mathrm{C}$ & -3.4902005363 & 6.5125543834 & -0.1154637795 \\
\hline $\mathrm{H}$ & -1.9318594759 & 6.3388185070 & -1.5857160204 \\
\hline $\mathrm{C}$ & -3.5815332283 & 4.7790783376 & 1.5677146292 \\
\hline $\mathrm{H}$ & -2.1301970794 & 3.1972411651 & 1.4113208993 \\
\hline $\mathrm{C}$ & 2.5042917888 & 6.9689706016 & -1.2963286024 \\
\hline $\mathrm{H}$ & 1.9284189151 & 5.5458292406 & 0.2077595474 \\
\hline $\mathrm{C}$ & 1.2957439067 & 6.8746530800 & -3.3890426856 \\
\hline $\mathrm{H}$ & -0.2362134029 & 5.3741503698 & -3.5436313954 \\
\hline $\mathrm{C}$ & 7.5873608751 & 1.2227653776 & 1.5314189978 \\
\hline $\mathrm{H}$ & 6.9684763321 & 3.2078536310 & 2.1648926581 \\
\hline $\mathrm{H}$ & 7.8997001837 & -0.7699770260 & 0.8027645201 \\
\hline $\mathrm{C}$ & 6.0985116738 & -4.3330317054 & -4.1244773517 \\
\hline $\mathrm{H}$ & 5.7144246024 & -5.1425923516 & -2.1718243042 \\
\hline $\mathrm{C}$ & 4.8411398970 & -2.3599832321 & -4.7393687351 \\
\hline $\mathrm{H}$ & 3.4513967814 & -1.5965140110 & -3.2775537176 \\
\hline $\mathrm{C}$ & 4.3594990281 & -7.1916005956 & 0.4467042329 \\
\hline $\mathrm{H}$ & 3.5052249670 & -6.2479740394 & -1.2868965811 \\
\hline $\mathrm{C}$ & 5.1595789892 & -5.7187977202 & 2.1904043229 \\
\hline $\mathrm{H}$ & 4.9372518629 & -3.6097257558 & 1.8398712005 \\
\hline $\mathrm{C}$ & -0.3903534296 & -3.7647524872 & 0.0599986820 \\
\hline $\mathrm{H}$ & 1.5095012992 & -3.7090413056 & 1.0957210544 \\
\hline $\mathrm{C}$ & -0.2956653612 & -3.6130068430 & -2.3600996420 \\
\hline $\mathrm{H}$ & 1.6607335660 & -3.3910989799 & -3.2379787050 \\
\hline $\mathrm{H}$ & 1.8441466163 & 3.4968683337 & 6.1074642142 \\
\hline
\end{tabular}




$\begin{array}{lrrr}\mathrm{C} & -1.4942671872 & 1.2588613639 & -4.6147199951 \\ \mathrm{H} & -3.5341041640 & 1.4204391421 & -3.9359650571 \\ \mathrm{H} & 0.6271243864 & 1.2646011590 & -5.0371621952 \\ \mathrm{C} & -4.0835505428 & 5.9656288773 & 1.0262788068 \\ \mathrm{H} & -3.8805296270 & 7.4329918615 & -0.5435099454 \\ \mathrm{H} & -4.0350072829 & 4.3482704036 & 2.4594090276 \\ \mathrm{C} & 2.2859132415 & 7.4532472729 & -2.5887614092 \\ \mathrm{H} & 3.2763610293 & 7.4134366357 & -0.6722144949 \\ \mathrm{H} & 1.1262935708 & 7.2459895780 & -4.3972614468 \\ \mathrm{H} & 8.6476587838 & 1.4119568410 & 1.7093323014 \\ \mathrm{C} & 5.7921602738 & -3.3348373580 & -5.0542316267 \\ \mathrm{H} & 6.8402316202 & -5.0906649576 & -4.3647375983 \\ \mathrm{H} & 4.6018038956 & -1.5773494958 & -5.4568240108 \\ \mathrm{C} & 4.9541788273 & -7.0106125655 & 1.6999142408 \\ \mathrm{H} & 4.1916526957 & -8.1964044181 & 0.0651117735 \\ \mathrm{H} & 5.6154651856 & -5.5738724391 & 3.1673214003 \\ \mathrm{C} & -1.0394761813 & -3.7325510074 & -1.1888611293 \\ \mathrm{H} & -0.9710499085 & -3.9737656043 & 0.9684973152 \\ \mathrm{H} & -0.7976416283 & -3.6014217358 & -3.3264893031 \\ \mathrm{H} & -1.7456177914 & 0.6389474487 & -5.4727823363 \\ \mathrm{H} & -4.9315353562 & 6.4630796710 & 1.4922777492 \\ \mathrm{H} & 2.8858260727 & 8.2757933169 & -2.9724704388 \\ \mathrm{H} & 6.2944064197 & -3.3157315377 & -6.0191659061 \\ \mathrm{H} & 5.2523484329 & -7.8737258720 & 2.2914844174 \\ \mathrm{H} & -2.1290833165 & -3.8108442955 & -1.2317149835\end{array}$

$\begin{array}{lrrr}\text { (2R,4S)-CYr } & & & \\ 01 & & & \\ \mathrm{C} & -3.4538414764 & -1.2797112173 & -0.4637782937 \\ \mathrm{C} & -2.9509993807 & -2.1282843069 & 0.6558475463 \\ \mathrm{C} & -4.1873956866 & -2.5973726496 & 1.4390883527 \\ \mathrm{H} & -2.1340630702 & -1.6490233726 & 1.2157901931 \\ \mathrm{H} & -2.4658964031 & -2.9796813549 & 0.1424070389 \\ \mathrm{C} & -4.9782644023 & -1.3631263950 & -0.5594253258 \\ \mathrm{C} & -5.4316221814 & -1.4717377190 & -2.0197926693 \\ \mathrm{H} & -5.0964571755 & -0.5957493596 & -2.5862697200 \\ \mathrm{H} & -6.5241828960 & -1.5471724811 & -2.0758070318 \\ \mathrm{Mg} & -0.7150969929 & -0.6374431613 & -1.7793407749 \\ \mathrm{O} & -0.2772897818 & 0.6786696486 & -0.0713290851 \\ \mathrm{O} & 1.3320614154 & -0.2608158208 & -1.7033141360 \\ \mathrm{O} & -0.7159481546 & -2.7752768773 & -1.3432748941 \\ \mathrm{C} & -0.8872274076 & -3.0063179571 & -2.5588662211 \\ \mathrm{C} & -0.9993665041 & -4.3650539117 & -3.2122207853 \\ \mathrm{~N} & -0.9952474987 & -1.9951182164 & -3.4263282524 \\ \mathrm{C} & -1.2684321760 & -4.0042182379 & -4.6729488824 \\ \mathrm{H} & -1.7963847323 & -4.9449337412 & -2.7259049363 \\ \mathrm{H} & -0.0538615249 & -4.9084114249 & -3.0529918365 \\ \mathrm{C} & -1.1984917966 & -2.4687714449 & -4.7065731013 \\ \mathrm{Br} & -4.7232299607 & -3.0208519816 & -2.9750314391 \\ \mathrm{H} & -0.5334140810 & -4.4153125328 & -5.3781569033 \\ \mathrm{H} & -2.2636116245 & -4.3134630632 & -5.0239695707 \\ \mathrm{O} & -1.3110707311 & -1.7871583154 & -5.6973524919 \\ \mathrm{O} & -2.7715113681 & -0.6548101111 & -1.2586659940 \\ \mathrm{C} & -5.2585699866 & -2.6023594256 & 0.3133365753 \\ \mathrm{H} & -5.1083458333 & -3.5008980121 & -0.3044296129 \\ \mathrm{H} & -6.2822271346 & -2.6507201363 & 0.7021352646 \\ \mathrm{C} & -3.9720245340 & -4.0048148842 & 2.0087068969 \\ \mathrm{H} & -3.1968431757 & -3.9922963348 & 2.7892309524 \\ \mathrm{H} & -4.8918367641 & -4.4085644875 & 2.4545508094 \\ \mathrm{H} & -3.6548315556 & -4.6977381860 & 1.2143244584\end{array}$




\begin{tabular}{|c|c|c|c|}
\hline $\mathrm{C}$ & -4.6075197346 & -1.6282287106 & 2.5518867748 \\
\hline $\mathrm{C}$ & -3.9655155323 & -0.4097523364 & 2.7870647184 \\
\hline $\mathrm{C}$ & -5.7294382151 & -1.9350744643 & 3.3358434394 \\
\hline $\mathrm{C}$ & -4.4384762593 & 0.4786080546 & 3.7533687547 \\
\hline $\mathrm{H}$ & -3.0942122274 & -0.1132506142 & 2.2007896495 \\
\hline $\mathrm{C}$ & -6.2004272334 & -1.0560070349 & 4.3053605884 \\
\hline $\mathrm{H}$ & -6.2645893080 & -2.8746276633 & 3.1764626282 \\
\hline $\mathrm{C}$ & -5.5575944200 & 0.1637919337 & 4.5158759614 \\
\hline $\mathrm{H}$ & -3.9222325862 & 1.4354592953 & 3.8911093932 \\
\hline $\mathrm{H}$ & -7.0794751520 & -1.3225527715 & 4.8964765314 \\
\hline $\mathrm{H}$ & -5.9291230520 & 0.8622596242 & 5.2683718711 \\
\hline $\mathrm{C}$ & -5.5935014874 & -0.0524050400 & -0.0179794112 \\
\hline $\mathrm{C}$ & -4.9614467316 & 1.1808818474 & -0.2182943377 \\
\hline $\mathrm{C}$ & -6.8235553136 & -0.0656691049 & 0.6485408901 \\
\hline $\mathrm{C}$ & -5.5240811486 & 2.3588672840 & 0.2695076863 \\
\hline $\mathrm{H}$ & -4.0060972530 & 1.2354166108 & -0.7431784905 \\
\hline $\mathrm{C}$ & -7.3868363038 & 1.1104849258 & 1.1345769322 \\
\hline $\mathrm{H}$ & -7.3524323872 & -1.0037169081 & 0.8158248666 \\
\hline $\mathrm{C}$ & -6.7348205247 & 2.3285288689 & 0.9552737781 \\
\hline $\mathrm{H}$ & -4.9966267054 & 3.3059569910 & 0.1234432653 \\
\hline $\mathrm{H}$ & -8.3396198418 & 1.0694843199 & 1.6658463168 \\
\hline $\mathrm{H}$ & -7.1692430596 & 3.2499719330 & 1.3477606154 \\
\hline $\mathrm{P}$ & 1.1804042629 & 0.6323903140 & -0.4884261095 \\
\hline $\mathrm{O}$ & 2.0892373117 & 0.2216786642 & 0.7858872988 \\
\hline $\mathrm{O}$ & 1.8526199770 & 2.0623996747 & -0.8655593749 \\
\hline $\mathrm{C}$ & 3.4629396371 & 0.3043872713 & 0.6322180098 \\
\hline $\mathrm{C}$ & 2.1016834671 & 2.9685855128 & 0.1422486270 \\
\hline $\mathrm{C}$ & 4.0505727190 & 1.5552406603 & 0.7019191530 \\
\hline $\mathrm{C}$ & 4.1901407453 & -0.8889154505 & 0.3862211360 \\
\hline $\mathrm{C}$ & 3.1916583245 & 2.7435757945 & 0.9641083101 \\
\hline $\mathrm{C}$ & 1.2226019213 & 4.0695615772 & 0.2953946442 \\
\hline $\mathrm{C}$ & 5.4571273762 & 1.6628254392 & 0.4447527166 \\
\hline $\mathrm{C}$ & 5.5496959000 & -0.7852432123 & 0.2060012908 \\
\hline $\mathrm{Si}$ & 3.3819147401 & -2.6001993101 & 0.3615413190 \\
\hline $\mathrm{C}$ & 3.4084234624 & 3.6334582211 & 2.0663581602 \\
\hline $\mathrm{C}$ & 1.4687840848 & 4.9536684318 & 1.3202769271 \\
\hline $\mathrm{Si}$ & -0.3103841366 & 4.3299606926 & -0.7816476436 \\
\hline $\mathrm{C}$ & 6.1310465649 & 2.9140146613 & 0.3674021597 \\
\hline $\mathrm{C}$ & 6.2098855247 & 0.4752179193 & 0.2157310211 \\
\hline $\mathrm{H}$ & 6.1640471487 & -1.6771411823 & 0.0331704074 \\
\hline $\mathrm{C}$ & 1.8411462203 & -2.6568732896 & 1.4214615178 \\
\hline $\mathrm{C}$ & 3.0477393665 & -3.2363570626 & -1.3663008563 \\
\hline $\mathrm{C}$ & 4.6504145964 & -3.7515509203 & 1.1488131887 \\
\hline $\mathrm{C}$ & 4.4264431966 & 3.4185464718 & 3.0378475032 \\
\hline $\mathrm{C}$ & 2.5491363280 & 4.7598085227 & 2.2256876144 \\
\hline $\mathrm{H}$ & 0.8136099109 & 5.8178319354 & 1.4856973053 \\
\hline $\mathrm{C}$ & -1.7454167131 & 4.4041981144 & 0.4180084089 \\
\hline $\mathrm{C}$ & -0.1461731743 & 5.9608381154 & -1.6951767287 \\
\hline $\mathrm{C}$ & -0.5648434325 & 3.0016104682 & -2.0694671228 \\
\hline $\mathrm{C}$ & 7.4806433677 & 2.9754459400 & 0.1201760947 \\
\hline $\mathrm{H}$ & 5.5622651253 & 3.8346077720 & 0.4998978900 \\
\hline $\mathrm{C}$ & 7.6076450441 & 0.5724643497 & -0.0260513974 \\
\hline $\mathrm{C}$ & 0.7753568820 & -3.5111683130 & 1.0987246401 \\
\hline $\mathrm{C}$ & 1.7747489867 & -1.9130480822 & 2.6134915946 \\
\hline $\mathrm{C}$ & 2.9114948129 & -2.3962682754 & -2.4794935713 \\
\hline $\mathrm{C}$ & 2.9029209356 & -4.6266843893 & -1.5452030637 \\
\hline $\mathrm{C}$ & 4.6474455705 & -3.9918469567 & 2.5317614772 \\
\hline $\mathrm{C}$ & 5.6393702563 & -4.3647044559 & 0.3599881961 \\
\hline $\mathrm{C}$ & 4.6035134642 & 4.2936447694 & 4.0811695807 \\
\hline $\mathrm{H}$ & 5.0678684925 & 2.5409981571 & 2.9509354129 \\
\hline
\end{tabular}




\begin{tabular}{|c|c|c|c|}
\hline $\mathrm{C}$ & 517 & 06410 & 772 \\
\hline $\mathrm{C}$ & -2.5339531363 & 5.5518346974 & 0.5873305503 \\
\hline $\mathrm{C}$ & -1.9798057095 & 3.2840199110 & 2362691474 \\
\hline $\mathrm{C}$ & -1.1882724710 & 6.3389845134 & -2.5617967083 \\
\hline $\mathrm{C}$ & 0.9689440705 & 6.8023243398 & -1.5714987891 \\
\hline $\mathrm{C}$ & 0.4370040008 & 2.8504986174 & -3.0504614386 \\
\hline $\mathrm{C}$ & -1.6711235680 & 2.1421708310 & -2.0934843229 \\
\hline$C$ & 8.2341513419 & 1.7926453332 & -0.0673081406 \\
\hline $\mathrm{H}$ & 7.9756915292 & 3.9470114654 & 0.0623936903 \\
\hline $\mathrm{H}$ & 8.1747061220 & -0.3479824887 & -0.1874202570 \\
\hline $\mathrm{C}$ & -0.3024804245 & -3.6675148143 & 1.9794684255 \\
\hline $\mathrm{H}$ & 0.7558789522 & -4.0469245787 & 0.1437734240 \\
\hline $\mathrm{C}$ & 0.6877075158 & -2.0527180322 & 5753 \\
\hline $\mathrm{H}$ & 2.5624790818 & -1.1973810853 & 2.8657016287 \\
\hline $\mathrm{C}$ & 2.6351559802 & -2.9312723528 & -3.7465780350 \\
\hline $\mathrm{H}$ & 2.9984681352 & -1.3055056277 & -2.3806823381 \\
\hline . & 2.6357771652 & 3297456 & -2.8083841186 \\
\hline П & 3.0042459911 & -5.3097962990 & -0.6969955227 \\
\hline 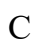 & 5.6119737572 & -4.8230127123 & 3.1145882347 \\
\hline $\mathrm{H}$ & 4464 & 3692 & 7199 \\
\hline $\mathrm{C}$ & 6.6034 & -5.1 & \\
\hline $\mathrm{H}$ & 5.6642806748 & -4.2069279214 & -0.721 \\
\hline $\mathrm{C}$ & 3.7726529032 & 71088 & 2453 \\
\hline 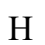 & 5.3891 & 4.1 & 049 \\
\hline 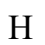 & 2.104 & 855 & 3.4 \\
\hline $\mathrm{C}$ & -3.5359485212 & 5.5891690199 & 1.5647431751 \\
\hline $\mathrm{H}$ & 39069 & 7742 & 27635 \\
\hline $\mathrm{C}$ & -2.97 & 2154 & 7408 \\
\hline $\mathrm{H}$ & -1.3884449788 & 16215 & 1.11 \\
\hline $\mathrm{C}$ & -1.1192398441 & 7.5381732466 & -3.2776409998 \\
\hline $\mathrm{H}$ & 9425 & 5.6 & 4381 \\
\hline 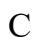 & 3323 & 2828 & 26853 \\
\hline $\mathrm{H}$ & 1.8025847469 & 6.5317368396 & -0.9197145727 \\
\hline $\mathrm{C}$ & 24235 & & 1616822 \\
\hline- & 483 & 599 & 8408 \\
\hline 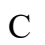 & -1.7904870683 & 1.1563613062 & -3.0913671146 \\
\hline $\mathrm{H}$ & -2.4636826872 & 2.2237756118 & -1.3412973076 \\
\hline 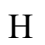 & 9.3 & & 4348 \\
\hline $\mathrm{C}$ & -0.3459386722 & 75202 & 213 \\
\hline $\mathrm{H}$ & -1.1051201265 & -4.3557838763 & 1.7285527542 \\
\hline 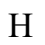 & 0.6452630825 & & \\
\hline $\mathrm{C}$ & 2.5 & -4.3 & -3.9 \\
\hline 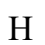 & 2.5178756338 & -2.2611583359 & -4.5967649471 \\
\hline $\mathrm{H}$ & 2.5405906080 & -6.2353495571 & -2.9357952463 \\
\hline $\mathrm{C}$ & 6.591 & & \\
\hline 1 & 59 & -5.0 & 154 \\
\hline $\mathrm{H}$ & 7.3619017615 & -5.6647919625 & 0.3185258391 \\
\hline $\mathrm{H}$ & & & 5.0493754860 \\
\hline 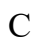 & .7490302730 & & \\
\hline $\mathrm{H}$ & -4.141 & 6.4 & 44959 \\
\hline $\mathrm{H}$ & -3.1396120026 & 2.4651425436 & 2.8677941587 \\
\hline $\mathrm{C}$ & & & -3.1402041784 \\
\hline 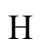 & .9300877513 & 12778 & -3.9459188503 \\
\hline $\mathrm{H}$ & 1.9097406292 & 559444 & -2.1843623059 \\
\hline $\mathrm{C}$ & -0.7742317447 & 1.0024013268 & -4.0465990527 \\
\hline $\mathrm{H}$ & & 1.7199098609 & -4.7530039958 \\
\hline 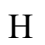 & & 0.5625544971 & -3.1730376568 \\
\hline $\mathrm{H}$ & -1.1826380992 & -3.0615562252 & 3.8575131860 \\
\hline $\mathrm{H}$ & 2.2996610220 & -4.7244963286 & -4.8982492788 \\
\hline $\mathrm{H}$ & 7.3409148004 & -6.0705954393 & 2.7724703416 \\
\hline
\end{tabular}




$\begin{array}{lrrr}\mathrm{H} & -4.5175876184 & 4.5112705872 & 3.1571839196 \\ \mathrm{H} & 0.0490546971 & 9.3056764666 & -3.6982586279 \\ \mathrm{H} & -0.8688498223 & 0.2396962607 & -4.8343517794\end{array}$

\begin{tabular}{|c|c|c|c|}
\hline \multicolumn{4}{|c|}{$(2 \mathrm{~S}, 4 \mathrm{R})-\mathrm{CYr}$} \\
\hline $\mathrm{O}$ & -1.0565345481 & -3.3012194389 & -1.1380848672 \\
\hline $\mathrm{C}$ & -2.2327304779 & -2.9562588158 & -1.1790701662 \\
\hline $\mathrm{C}$ & -2.7727536428 & -1.6344376405 & -0.7508744044 \\
\hline $\mathrm{C}$ & -4.0900131328 & -2.6504966083 & -2.5105370470 \\
\hline $\mathrm{C}$ & -4.1114158774 & -1.4477442890 & -1.5151693843 \\
\hline $\mathrm{H}$ & -2.0317052697 & -0.8484538469 & -0.9692301226 \\
\hline $\mathrm{H}$ & -2.8456490223 & -1.6584995558 & 0.3525357952 \\
\hline $\mathrm{H}$ & -3.5109934242 & -2.3660851842 & -3.4060594010 \\
\hline $\mathrm{H}$ & -5.0892155408 & -2.9458536526 & -2.8517726893 \\
\hline $\mathrm{C}$ & -4.0868154037 & -0.1140597579 & -2.2729769989 \\
\hline $\mathrm{H}$ & -4.9670643652 & 0.0078199072 & -2.9191344557 \\
\hline $\mathrm{H}$ & -4.0686646981 & 0.7296337904 & -1.5649025065 \\
\hline $\mathrm{H}$ & -3.1884045340 & -0.0326224598 & -2.9064228037 \\
\hline $\mathrm{C}$ & -5.3801148088 & -1.5142729970 & -0.6470809736 \\
\hline $\mathrm{C}$ & -5.3697871716 & -1.6350702929 & 0.7444102816 \\
\hline $\mathrm{C}$ & -6.6270645118 & -1.4688962878 & -1.2900207338 \\
\hline $\mathrm{C}$ & -6.5635861471 & -1.7347867469 & 1.4614517913 \\
\hline $\mathrm{H}$ & -4.4363441662 & -1.6787408656 & 1.3069633636 \\
\hline $\mathrm{C}$ & -7.8180827044 & -1.5637947282 & -0.5779463822 \\
\hline $\mathrm{H}$ & -6.6774647123 & -1.3669415414 & -2.3776334785 \\
\hline $\mathrm{C}$ & -7.7913384251 & -1.7046847024 & 0.8090245613 \\
\hline $\mathrm{H}$ & -6.5191828665 & -1.8459998250 & 2.5465730878 \\
\hline $\mathrm{H}$ & -8.7712556030 & -1.5300987363 & -1.1102063585 \\
\hline $\mathrm{C}$ & -3.3420744596 & -3.7959159167 & -1.7847531799 \\
\hline $\mathrm{C}$ & -2.8607427967 & -4.8468408301 & -2.7851034301 \\
\hline $\mathrm{H}$ & -2.1424543982 & -4.4134928932 & -3.4925498177 \\
\hline $\mathrm{H}$ & -3.7200122637 & -5.2369351914 & -3.3444635737 \\
\hline $\mathrm{C}$ & -4.1801539852 & -4.3998524872 & -0.6517900799 \\
\hline $\mathrm{C}$ & -3.6619698342 & -4.5415361036 & 0.6373486638 \\
\hline $\mathrm{C}$ & -5.4942647130 & -4.8088237872 & -0.8995951155 \\
\hline $\mathrm{C}$ & -4.4528376236 & -5.0490393075 & 1.6663705873 \\
\hline $\mathrm{H}$ & -2.6444611980 & -4.2217971355 & 0.8743519845 \\
\hline $\mathrm{C}$ & -6.2813722961 & -5.3235134799 & 0.1241560010 \\
\hline $\mathrm{H}$ & -5.9250379979 & -4.7041488095 & -1.8977292881 \\
\hline $\mathrm{C}$ & -5.7640060269 & -5.4385541429 & 1.4145258714 \\
\hline $\mathrm{H}$ & -4.0374890743 & -5.1201372469 & 2.6729424062 \\
\hline $\mathrm{H}$ & -7.3104601090 & -5.6222234295 & -0.0838036434 \\
\hline $\mathrm{Mg}$ & 0.5586202698 & -2.5590476036 & -0.0824621102 \\
\hline $\mathrm{O}$ & 0.3067348791 & 0.6320085834 & 1.5516027175 \\
\hline $\mathrm{O}$ & 0.8287489590 & -0.7151058251 & -0.6307099202 \\
\hline $\mathrm{O}$ & 1.7213632973 & -2.7535538601 & 1.6813168317 \\
\hline $\mathrm{C}$ & 0.7261763366 & -2.4130700784 & 2.3587220347 \\
\hline $\mathrm{C}$ & 0.7365582893 & -1.8295141986 & 3.7456426870 \\
\hline $\mathrm{N}$ & -0.4950693464 & -2.4971800347 & 1.8147117601 \\
\hline $\mathrm{C}$ & -0.7303002996 & -1.4561596902 & 3.9440570563 \\
\hline $\mathrm{H}$ & 1.4246899568 & -0.9738495156 & 3.7465283563 \\
\hline $\mathrm{H}$ & 1.1199847599 & -2.5744096556 & 4.4578687757 \\
\hline $\mathrm{C}$ & -1.4268874811 & -1.9876694249 & 2.6904626180 \\
\hline $\mathrm{Br}$ & -1.9965320559 & -6.4030694268 & -2.0040755122 \\
\hline $\mathrm{H}$ & -1.1993019405 & -1.8751890919 & 4.8437639253 \\
\hline $\mathrm{H}$ & -0.8839245849 & -0.3653193481 & 3.9464966457 \\
\hline $\mathrm{O}$ & -2.6247698038 & -1.9591211915 & 2.4983552360 \\
\hline $\mathrm{H}$ & -8.7218623369 & -1.7886713253 & 1.3741454979 \\
\hline $\mathrm{H}$ & -6.3878228521 & -5.8258394657 & 2.2224324545 \\
\hline
\end{tabular}




\begin{tabular}{|c|c|c|c|}
\hline$?$ & 0.9771370486 & 0.5404632645 & 0.2347085771 \\
\hline $\mathrm{O}$ & 2.5771395519 & 0.8249274606 & 0.4764430196 \\
\hline & 0.6097300197 & 1.7416146196 & -0.8254373878 \\
\hline & 3.4213285874 & 1.1484776935 & -0.5522159532 \\
\hline $\mathrm{C}$ & 0.9658700284 & 3.0386840231 & -0.5323282596 \\
\hline$C$ & 3.2986677867 & 2.4002565669 & -1.1277151616 \\
\hline $\mathrm{C}$ & 4.4118713022 & 0.2120066892 & -0.9613501888 \\
\hline $\mathrm{C}$ & 2.3091011209 & 3.3734763749 & -0.5891208056 \\
\hline $\mathrm{C}$ & -0.0448958497 & 3.9937652750 & -0.2202881493 \\
\hline $\mathrm{C}$ & 4.1194436689 & 2.7268158748 & -2.2583192537 \\
\hline $\mathrm{C}$ & 5.2492189561 & 0.5610953192 & -1.9966617895 \\
\hline $\mathrm{Si}$ & 4.6014808091 & -1.5067341576 & -0.2048754133 \\
\hline $\mathrm{C}$ & 2.7217730662 & 4.6677620512 & -0.1287815289 \\
\hline C & 0.3561514355 & 5.2583320994 & 0.1471087218 \\
\hline $\mathrm{Si}$ & -1.9044712455 & 3.7091279139 & -0.4243502497 \\
\hline $\mathrm{C}$ & 3.9630486297 & 3.9302339995 & -3.0031017478 \\
\hline $\mathrm{C}$ & 5.1169271061 & 1.8017405689 & -2.6784320445 \\
\hline $\mathrm{H}$ & 6.0355500056 & -0.1214781866 & -2.3447528360 \\
\hline $\mathrm{C}$ & 4.7002940020 & -1.5568797741 & 1.6610836340 \\
\hline $\mathrm{C}$ & 3.2189065484 & -2.5920489055 & 6200993 \\
\hline $\mathrm{C}$ & 6.2155036251 & 3361182 & 6490363 \\
\hline $\mathrm{C}$ & 4.0900266957 & 5.0358384305 & 0.0055262799 \\
\hline $\mathrm{C}$ & & & 058191 \\
\hline $\mathrm{H}$ & -0.3776508162 & 5672 & 3721382 \\
\hline $\mathrm{C}$ & -2.6329504080 & 2.5828275300 & 1130822 \\
\hline $\mathrm{C}$ & -2.7293088307 & 980311 & 6138560 \\
\hline $\mathrm{C}$ & -2.3337108639 & 3.1078763199 & -2.1540937210 \\
\hline $\mathrm{C}$ & 4.7739504827 & 73893 & 1484839 \\
\hline $\mathrm{H}$ & 3.18 & 7906 & 963457 \\
\hline $\mathrm{C}$ & 5.9489354855 & 2.1242424411 & -3.7855829804 \\
\hline $\mathrm{C}$ & 5.2415069732 & -2.7118445080 & 2.2599007133 \\
\hline $\mathrm{C}$ & 4.2528768241 & -0.5178617484 & 2.4898282850 \\
\hline $\mathrm{C}$ & 2.4721078345 & -2.1898382826 & -1.9973709829 \\
\hline $\mathrm{C}$ & 2.946 & 1922800 & 2889141 \\
\hline $\mathrm{C}$ & 7.4281476448 & -1.8036812067 & 7944061 \\
\hline $\mathrm{C}$ & & & \\
\hline $\mathrm{C}$ & 4.4441668751 & 65846 & 353735 \\
\hline $\mathrm{H}$ & 4.8643565957 & 15110 & 1845778 \\
\hline $\mathrm{C}$ & 2.1267879913 & 6.9024444598 & 0911686 \\
\hline $\mathrm{C}$ & -3.9732847859 & 2.1717714776 & 0.7293994523 \\
\hline $\mathrm{C}$ & -1.9122337361 & 2.1841719172 & 8604967 \\
\hline $\mathrm{C}$ & -2.65 & 52185 & 4497111 \\
\hline $\mathrm{C}$ & -3.4098274911 & 5.7357374994 & 0.9488443479 \\
\hline $\mathrm{C}$ & & & 046188 \\
\hline $\mathrm{C}$ & -3.5974521554 & 3.4355052851 & -2.6811813915 \\
\hline $\mathrm{C}$ & 5.7882797869 & 3.3030552469 & -4.4692326476 \\
\hline $\mathrm{H}$ & 4.6308974463 & 5.1361130809 & 15752257 \\
\hline $\mathrm{H}$ & 6.7177271904 & 1.4077447810 & -4.0875184563 \\
\hline $\mathrm{C}$ & 5.3240115320 & -2.8238370067 & 3.6507631443 \\
\hline $\mathrm{H}$ & 5.6130556595 & -3.5370164264 & 1.6464097911 \\
\hline $\mathrm{C}$ & 4.3347661079 & -0.6299235999 & 3.8842118063 \\
\hline $\mathrm{H}$ & 3.8287467292 & 0.4002595214 & 2.0643037725 \\
\hline $\mathrm{C}$ & 1.4812604195 & -3.0293682020 & -2.5321783670 \\
\hline $\mathrm{H}$ & 2.6432860465 & -1.2137860520 & -2.4665280337 \\
\hline $\mathrm{C}$ & 1.9469972666 & -4.6785284224 & -0.8127542327 \\
\hline $\mathrm{H}$ & 3.4963837420 & -4.1716646361 & 0.6026483177 \\
\hline $\mathrm{C}$ & 8.6481384308 & -2.3008829816 & -0.7516627875 \\
\hline $\mathrm{H}$ & 7.4326271211 & -1.0845990710 & 0.5427615604 \\
\hline . & 7.4711046212 & -3.6511364918 & -2.3777142742 \\
\hline $\mathrm{H}$ & 5.3256338215 & -3.5002241380 & -2.373885236 \\
\hline
\end{tabular}




\begin{tabular}{|c|c|c|c|}
\hline$C$ & 44953979 & 7.2348611892 & 0.8009576891 \\
\hline $\mathrm{H}$ & 5.5005130816 & 6.5411280793 & 0.5607694295 \\
\hline $\mathrm{H}$ & 1.3509570266 & 7.6229440815 & .9712409586 \\
\hline $\mathrm{C}$ & -4.5750193848 & 1.3732165984 & 1.7042172035 \\
\hline $\mathrm{H}$ & -4.5631570405 & 2.4717684236 & -0.1399707556 \\
\hline $\mathrm{C}$ & -2.5172770794 & 1.3797321534 & 2.9751718756 \\
\hline $\mathrm{H}$ & -0.8623062177 & 2.4688213141 & 2.1366006400 \\
\hline $\mathrm{C}$ & -3.2530645240 & 7.5963011156 & -1.1308040810 \\
\hline $\mathrm{H}$ & -2.1357904235 & 6.0999234589 & -2.1992534980 \\
\hline $\mathrm{C}$ & -4.0070633502 & 6.9949849131 & 1.0876442461 \\
\hline $\mathrm{H}$ & -3.4861599910 & 5.0220452789 & 1.7744291452 \\
\hline $\mathrm{C}$ & -1.8235454944 & 1.9405527993 & -4.2319460119 \\
\hline $\mathrm{H}$ & -0.4663258254 & 2.0648725881 & -2.5674047843 \\
\hline $\mathrm{C}$ & -3.9677268449 & 3.0232817482 & -3.9651526989 \\
\hline $\mathrm{H}$ & -4.3086393387 & 4.0273200553 & -2.0973412032 \\
\hline $\mathrm{H}$ & 6.4306277622 & 77812 & 93183 \\
\hline $\mathrm{C}$ & 4.8683634198 & -1.7816 & 4.4657310717 \\
\hline $\mathrm{H}$ & 5.7430312065 & -3.7208796724 & 36705 \\
\hline $\mathrm{H}$ & 3.9831794314 & 0.1868152998 & 4.5112791127 \\
\hline $\mathrm{C}$ & 1.2259986333 & -4.27 & 31000 \\
\hline $\mathrm{H}$ & 0.9209193188 & -2.713 & -3.4107890370 \\
\hline $\mathrm{H}$ & 1.7523788586 & -5.6490084177 & -0.3562964188 \\
\hline $\mathrm{C}$ & 3225 & 1450 & 05887 \\
\hline $\mathrm{H}$ & 8458 & 9652 & 79555 \\
\hline $\mathrm{H}$ & 7.4850806802 & -4.3718051072 & -3.1924601319 \\
\hline $\mathrm{H}$ & 3.7494672422 & 8.2248112816 & 1.1546463254 \\
\hline $\mathrm{C}$ & 19990 & 0.9 & 50601 \\
\hline $\mathrm{H}$ & 55838 & 362 & 3393 \\
\hline $\mathrm{H}$ & -1.9309866884 & 979580 & 3.8336083223 \\
\hline $\mathrm{C}$ & 6070 & 606 & 05747 \\
\hline $\mathrm{H}$ & 4651 & 6787 & 26177 \\
\hline $\mathrm{H}$ & 1490 & 043 & 58845 \\
\hline $\mathrm{C}$ & -3.0779975488 & 2.2775206819 & -4.7453441394 \\
\hline $\mathrm{H}$ & -1.1 & 99 & 5790 \\
\hline $\mathrm{H}$ & -4.94 & 806 & 14193 \\
\hline $\mathrm{H}$ & 4.9331578211 & 0115 & 2139623 \\
\hline $\mathrm{H}$ & 5407 & -4.9 & 84632 \\
\hline $\mathrm{H}$ & 8420 & -3.6 & 03534 \\
\hline $\mathrm{H}$ & -4.30 & 3121 & 24972 \\
\hline $\mathrm{H}$ & -4.3935858080 & 8.9038 & 0.1557142062 \\
\hline $\mathrm{H}$ & -3.3610918692 & 1.9660740754 & -5.7480889187 \\
\hline \multicolumn{4}{|c|}{$-\mathrm{CYr}$} \\
\hline \multicolumn{4}{|c|}{01} \\
\hline $\mathrm{C}$ & -3.681 & & \\
\hline $\mathrm{C}$ & -4.52 & 776 & 205526 \\
\hline $\mathrm{C}$ & -5.7719954413 & -1.1260294796 & 1.6271351729 \\
\hline $\mathrm{H}$ & & & 1.1791018887 \\
\hline $\mathrm{H}$ & -3.8 & -1.9 & 25225 \\
\hline $\mathrm{C}$ & -4.2310224956 & 0.1265094102 & 0.1394366963 \\
\hline $\mathrm{C}$ & -3.1302881747 & 1.1841174744 & 0.0169613552 \\
\hline $\mathrm{H}$ & & & -0.8461717701 \\
\hline $\mathrm{H}$ & -3.580 & 2.1748532024 & -0.1229706028 \\
\hline $\mathrm{O}$ & -0.8312552268 & -2.5834153907 & 2.4508685600 \\
\hline $\mathrm{C}$ & -1.2588785204 & -1.9463031360 & 3.4673079104 \\
\hline $\mathrm{C}$ & -0.3159053452 & -1.4353149728 & 4.5332644698 \\
\hline $\mathrm{N}$ & -2.5201486797 & -1.6397839486 & 3.6597554356 \\
\hline $\mathrm{C}$ & -1.2549979546 & -0.6638474620 & 5.4515973156 \\
\hline $\mathrm{H}$ & 0.1997047659 & -2.2834312373 & 5.0075276032 \\
\hline $\mathrm{H}$ & 0.4420765205 & -0.8157476665 & 4.0298289356 \\
\hline
\end{tabular}




\begin{tabular}{|c|c|c|c|}
\hline $\mathrm{C}$ & -2.6323761286 & -0.8654683310 & 4 \\
\hline $\mathrm{Br}$ & -1.9295126970 & 1.3310702140 & 1.540433904 \\
\hline $\mathrm{H}$ & -1.0374009968 & 0.4184404762 & \\
\hline $\mathrm{H}$ & -1.2832109823 & -1.0210599383 & 6.4902022 \\
\hline $\mathrm{O}$ & -3.6684598290 & -0.4009005974 & 5.22350892 \\
\hline $\mathrm{O}$ & -2.6108101847 & -1.6834327733 & -0.0522730 \\
\hline $\mathrm{C}$ & -5.1780144004 & 0.2835939157 & \\
\hline $\mathrm{H}$ & -4.5725655191 & 0.5755707802 & \\
\hline $\mathrm{H}$ & -5.9411858998 & 1.0588096836 & 1.21291820 \\
\hline $\mathrm{C}$ & -6.2335917578 & -1.2600544760 & \\
\hline $\mathrm{H}$ & -6.6023197595 & -2.2790925059 & 3.274942 \\
\hline $\mathrm{H}$ & -7.0512635549 & -0.5612640177 & \\
\hline $\mathrm{H}$ & -5.4068052547 & -1.0457277369 & \\
\hline $\mathrm{C}$ & -6.9303170497 & -1.4242679948 & 0.6 \\
\hline $\mathrm{C}$ & -6.8437972319 & -2.3523376227 & \\
\hline $\mathrm{C}$ & -8.1239134051 & -0.6981912009 & \\
\hline $\mathrm{C}$ & -7.8932961681 & -2.5317464403 & -1.2 \\
\hline $\mathrm{H}$ & 133831 & -2.9345604877 & \\
\hline $\mathrm{C}$ & 850326 & -0.8749405666 & \\
\hline $\mathrm{H}$ & 347570 & 0.0389126023 & 1. \\
\hline $\mathrm{C}$ & -9.0 & -1.792 & \\
\hline $\mathrm{H}$ & -7.78 & -3.2522110194 & -2 \\
\hline $\mathrm{H}$ & -10 & -0.2887718958 & \\
\hline $\mathrm{H}$ & 0774763 & -1.9282584860 & \\
\hline $\mathrm{C}$ & 261671 & 0.1101315763 & -1.2 \\
\hline $\mathrm{C}$ & -4.35 & -0.5894306851 & \\
\hline $\mathrm{C}$ & 982270 & 0.8383260466 & -1 \\
\hline $\mathrm{C}$ & 48093 & -0.5992264374 & \\
\hline $\mathrm{H}$ & -3.4 & -1.1 & -2 \\
\hline $\mathrm{C}$ & 118452 & 0.8291999645 & -2. \\
\hline $\mathrm{H}$ & 525476 & 1.4056108289 & \\
\hline $\mathrm{C}$ & 696637 & 058907 & \\
\hline $\mathrm{H}$ & -4.5884821436 & -1.1616331767 & -4 \\
\hline $\mathrm{H}$ & -7.6 & 570 & -2 \\
\hline $\mathrm{H}$ & -6.72 & 984001 & -4 \\
\hline $\mathrm{Mg}$ & -0.7 & 187687 & \\
\hline $\mathrm{O}$ & 0.6 & -1.0 & \\
\hline $\mathrm{P}$ & 1.56 & 765823 & 2327 \\
\hline $\mathrm{O}$ & 1.17 & 0.32 & 2.2 \\
\hline $\mathrm{O}$ & 486262 & -0.6926212130 & \\
\hline $\mathrm{O}$ & 115881 & 1.1928355051 & \\
\hline $\mathrm{C}$ & 07989 & 552239 & -0 . \\
\hline $\mathrm{C}$ & 209847 & 2.0725776706 & 0.0 \\
\hline $\mathrm{C}$ & & & \\
\hline $\mathrm{C}$ & 852364 & -2.1308677172 & -0.7 \\
\hline $\mathrm{C}$ & 4.13 & 1.6353070319 & -0.1 \\
\hline $\mathrm{C}$ & 243746 & 3.38 & 0.5 \\
\hline $\mathrm{C}$ & 5.0881742183 & 0.1899779734 & -2.00591151 \\
\hline $\mathrm{C}$ & 335156 & 417710 & -1.8 \\
\hline $\mathrm{Si}$ & 3.2895845022 & -3.6798288406 & 51370 \\
\hline $\mathrm{C}$ & 5.2238822421 & 2.4695694867 & 0.28167289 \\
\hline $\mathrm{C}$ & 3.59 & & \\
\hline $\mathrm{Si}$ & 0.8142525331 & 4.1781085654 & 0.62448905 \\
\hline $\mathrm{C}$ & 5.5682326718 & 1.3169798998 & -2.73 \\
\hline $\mathrm{C}$ & 5.3171059950 & -1.1074841808 & -2.54877548 \\
\hline $\mathrm{H}$ & 4.9871672340 & -3.2298860681 & -2.33639551 \\
\hline $\mathrm{C}$ & 3.6716404074 & -3.9194941131 & 1.85425583 \\
\hline $\mathrm{C}$ & 1.4259548698 & -3.7397591791 & -0.24349411 \\
\hline $\mathrm{C}$ & 4.0041083922 & -5.1526876645 & -0.88593141 \\
\hline $\mathrm{C}$ & 6.5804646093 & 2.0425118510 & 0.2318219 \\
\hline
\end{tabular}




\begin{tabular}{|c|c|c|c|}
\hline 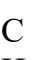 & 4.9421319117 & 3.7660333961 & 0.7953457698 \\
\hline & 3.4241742329 & 5.2283948293 & 1.2411153213 \\
\hline & -0.0753319343 & 3.9561529961 & 2.2553511114 \\
\hline & 1.1161421946 & 6.0234586242 & 0.3676947605 \\
\hline & -0.3375313572 & 3.6595893582 & -0.7685809397 \\
\hline c & 6.2648650291 & 1.1584452168 & -3.9044716055 \\
\hline & 5.3721616093 & 2.3191461056 & -2.3489065328 \\
\hline C & 6.0518821998 & -1.2381739121 & -3.7594343590 \\
\hline C & 3.6154926342 & -5.2298382135 & 2.3706189107 \\
\hline & 3.9939784866 & -2.8676161638 & 2.7231569637 \\
\hline C & 0.8677660457 & -3.1463680811 & -1.3938907142 \\
\hline $\mathrm{C}$ & 0.5891098092 & -4.4702058754 & 0.6172257455 \\
\hline $\mathrm{C}$ & 5.3238614322 & -5.5472116674 & -0.6011004788 \\
\hline C & 3.2818465516 & -5.8600358114 & -1.8583752030 \\
\hline C & 7.5962600609 & 2.8705194823 & 0.6435116785 \\
\hline $\mathrm{H}$ & 6.8105308424 & 1.0396030836 & -0.1291262891 \\
\hline $\mathrm{C}$ & 6.0160628228 & 4.6032533185 & 298941 \\
\hline C & -1.1270693397 & 7079 & 948117 \\
\hline $\mathrm{C}$ & 08508 & 2.88524 & 81986 \\
\hline C & 1.2184315103 & 6.5376183467 & -0.9352360680 \\
\hline C & 1.2669263300 & 6.9001866089 & 6604 \\
\hline C & -0.0225740659 & 2.7273149768 & 98171 \\
\hline $\mathrm{C}$ & -1.5968931679 & 4.2901642512 & -0.8151520437 \\
\hline $\mathrm{C}$ & 49445 & 4833 & 4067 \\
\hline $\mathrm{H}$ & 3438 & 4797 & 3141 \\
\hline $\mathrm{H}$ & 6.2294942902 & -2.241 & -4.1570531431 \\
\hline $\mathrm{C}$ & 3.8695493616 & -5.4758747808 & 3.7221838641 \\
\hline $\mathrm{H}$ & 3.380 & 521 & 78343 \\
\hline $\mathrm{C}$ & 4.248 & -3.11 & 9682 \\
\hline $\mathrm{H}$ & 4.0496490136 & -1.8325606177 & 2.3619414516 \\
\hline $\mathrm{C}$ & -0.491 & -3.31 & 49352 \\
\hline $\mathrm{H}$ & 2285 & 2037 & 464660 \\
\hline $\mathrm{C}$ & -0.7735013986 & -4.6517349122 & 486476 \\
\hline $\mathrm{H}$ & 0.9829607661 & -4.9139173303 & 1.5400394368 \\
\hline $\mathrm{C}$ & 880 & -6.6 & 3367 \\
\hline $\mathrm{H}$ & 5.911 & -5.01 & 0.15 \\
\hline $\mathrm{C}$ & 3.8641381872 & -6.9395105697 & -2.5342105874 \\
\hline $\mathrm{H}$ & 2.2541213601 & 29648 & -2.1016536079 \\
\hline $\mathrm{C}$ & 7.31 & 4.1 & 9919 \\
\hline $\mathrm{H}$ & 8.629 & 997 & 35306 \\
\hline $\mathrm{H}$ & 5.7846119888 & 5.6005 & 1.5860648142 \\
\hline $\mathrm{C}$ & -1.915 & 677027 & 3.7088400860 \\
\hline $\mathrm{H}$ & -1.34 & 5.69 & 78765 \\
\hline $\mathrm{C}$ & -0.6179935666 & 2.6652766956 & 4.2461340337 \\
\hline $\mathrm{H}$ & 1.0236956307 & 2.1942301070 & 2.9346199818 \\
\hline $\mathrm{C}$ & & 7.89 & \\
\hline $\mathrm{H}$ & 1.104 & 5.8814469749 & -1.8025193030 \\
\hline $\mathrm{C}$ & 1.5112373989 & 8.2616451525 & 1.2440259824 \\
\hline $\mathrm{H}$ & 1.1921 & 6.5304914182 & 2.4807962321 \\
\hline $\mathrm{C}$ & -0.9417 & 2.421 & -2.7790288201 \\
\hline $\mathrm{H}$ & 0.9452310440 & 2.2140875290 & -1.7708951990 \\
\hline $\mathrm{C}$ & -2.5122533506 & 3.9922992394 & -1.8288789562 \\
\hline $\mathrm{H}$ & & 5.0262592679 & -0.0570172714 \\
\hline $\mathrm{H}$ & 7.0830931568 & -0.2451604676 & -5.3530637242 \\
\hline $\mathrm{C}$ & 4.1857934800 & -4.4157143313 & 4.5798643513 \\
\hline $\mathrm{H}$ & 3.8233749159 & -6.4913055735 & 4.1089847474 \\
\hline $\mathrm{H}$ & 1.4926638216 & -2.2845446254 & 4.740302322 \\
\hline $\mathrm{C}$ & -1.3131720580 & -4.0900286375 & -0.856324744 \\
\hline $\mathrm{H}$ & -0.9073822784 & -2.8700498057 & -2.6024663891 \\
\hline $\mathrm{H}$ & -1.4020400703 & -5.2467887301 & 0.9793530079 \\
\hline
\end{tabular}




$\begin{array}{lr}\mathrm{C} & 5.1762570893 \\ \mathrm{H} & 6.9275945928 \\ \mathrm{H} & 3.2929623214 \\ \mathrm{H} & 8.1334395006 \\ \mathrm{C} & -1.6706780642 \\ \mathrm{H} & -2.7252935783 \\ \mathrm{H} & -0.4241493999 \\ \mathrm{C} & 1.6099448735 \\ \mathrm{H} & 1.5394468090 \\ \mathrm{H} & 1.6228722870 \\ \mathrm{C} & -2.1858112765 \\ \mathrm{H} & -0.6786371282 \\ \mathrm{H} & -3.4757823180 \\ \mathrm{H} & 4.3823538171 \\ \mathrm{H} & -2.3575390430 \\ \mathrm{H} & 5.6291883227 \\ \mathrm{H} & -2.3010503815 \\ \mathrm{H} & 1.7992539308 \\ \mathrm{H} & -2.8979451325\end{array}$

(2S,5R)-EPr

01

O $\quad-0.6950646953$

C $\quad-0.5794381907$

C $\quad-1.2088459163$

C $\quad-0.8880200013$

C $\quad-0.9461618383$

$\mathrm{H} \quad-2.2843063116$

$\mathrm{H} \quad-0.7439977441$

$\mathrm{H} \quad-1.9028553206$

$\mathrm{H} \quad-0.1900047642$

C $\quad 0.6227715855$

C $\quad 1.5806446961$

$\mathrm{H} \quad 1.0627377868$

H 2.2006316296

$\mathrm{Mg} \quad-1.5411046645$

O $\quad-0.3041534786$

O $\quad 1.1100471959$

O $\quad-3.2724900036$

C $\quad-2.5599368499$

C $\quad-2.9618473817$

$\mathrm{N} \quad-1.2967397792$

C $\quad-1.6918008501$

$\mathrm{H} \quad-3.8380976771$

$\mathrm{H} \quad-3.2604327910$

C $\quad-0.6633339124$

$\mathrm{Br} \quad 2.7989484514$

$\mathrm{H} \quad-1.3093075934$

$\mathrm{H} \quad-1.8105396816$

O $\quad 0.5206543923$

C $\quad 1.1923614657$

C $\quad 1.9940073480$

C $\quad 0.9666115843$

C $\quad 2.5471848798$

$\mathrm{H} \quad 2.1845664270$

C $\quad 1.5227311496$

$\mathrm{H} \quad 0.3740821306$

C 2.3089856003

$\mathrm{H} \quad 3.1686110026$

$\mathrm{H} \quad 1.3509754897$
$-7.3222691804-2.2441614363$

$-6.9218879091-1.0465142383$

$-7.4814108846 \quad-3.2850382442$

$4.8207142305 \quad 1.4461100034$

$3.5343700948 \quad 4.5411275643$

$5.3186516011 \quad 3.9410614082$

$\begin{array}{ll}1.8017623362 & 4.8898201643\end{array}$

$8.7628257790 \quad-0.0575680781$

$8.2855011339-2.1610677581$

$8.9309958869 \quad 2.0941721324$

$3.0535098306-2.8137476850$

$1.6915215400 \quad-3.5411299108$

$4.4952021495 \quad-1.8564756973$

$\begin{array}{ll}-4.6071935382 & 5.6331093289\end{array}$

$-4.2646412269-1.1200795293$

$-8.1616848832 \quad-2.7684484220$

$3.3562759326 \quad 5.4106265582$

$9.8215003894 \quad-0.2216866587$

$2.8204960363 \quad-3.6024643081$

2.7709592945

3.9543378577

0.9855101077

0.2060079531

0.5381270137

$\begin{array}{rr}5.2867437645 & 0.5381270137 \\ 4.1385319485 & -1.2648762364\end{array}$

$5.6664318949-0.9595528924$

$\begin{array}{lll}5.1658938748 & 0.7330772264\end{array}$

$5.8874619343 \quad 1.3297513783$

$3.7818440440 \quad-1.4839657113$

$3.7359452466-2.0087177465$

$3.3605271911 \quad 0.8147772266$

$2.6078156637-0.0748077483$

$\begin{array}{ll}1.9818953220 & -0.8119216468\end{array}$

$3.3424558640 \quad-0.6046213638$

$\begin{array}{ll}0.9024054481 & 0.6363858286\end{array}$

$-0.1807413082 \quad 1.6242343762$

$\begin{array}{ll}-2.1283708731 & 2.6794208047\end{array}$

$1.5309241845 \quad-0.6148212418$

$1.3241718348 \quad-1.6238990683$

$1.5244975938 \quad-3.0674804777$

$0.9268341346-1.4774664994$

$1.1250398070-3.8153279925$

$0.9026185144 \quad-3.2953739479$

$2.5729920658 \quad-3.2175194113$

$\begin{array}{ll}0.8768932745 & -2.7055439235\end{array}$

$1.4563209507 \quad 0.8883544414$

$1.8877898271-4.5065232585$

$0.1922418469-4.3849496673$

$0.7098884172 \quad-2.8741485149$

$\begin{array}{ll}4.0045408549 & 2.0411244665\end{array}$

$\begin{array}{ll}5.1407164592 & 1.8963659409\end{array}$

$3.4684440651 \quad 3.3078221996$

$5.7492516146 \quad 3.0201536631$

$\begin{array}{ll}5.5540829930 & 0.9026554819\end{array}$

$4.0792580219 \quad 4.4301726765$

$2.5558948978 \quad 3.4043573748$

$\begin{array}{ll}5.2217826911 & 4.2890411597\end{array}$

$\begin{array}{ll}6.6389460126 & 2.9006606365\end{array}$

$3.6519274022 \quad 5.4192890849$ 


\begin{tabular}{|c|c|c|c|}
\hline $\mathrm{H}$ & 2.7451552620 & 5.6976144483 & 5.1695292818 \\
\hline $\mathrm{C}$ & -2.0503438546 & 6.4381548339 & -1.6757829721 \\
\hline $\mathrm{H}$ & -1.8330225916 & 6.5085945696 & -2.7523524052 \\
\hline $\mathrm{H}$ & -2.1363782380 & 7.4620959906 & -1.2833189331 \\
\hline $\mathrm{H}$ & -3.0228492859 & 5.9385018817 & -1.5489691230 \\
\hline $\mathrm{C}$ & 0.3964986958 & 6.3768638062 & -1.0960233446 \\
\hline $\mathrm{C}$ & 0.6090077616 & 7.5637997570 & -0.3803269685 \\
\hline $\mathrm{C}$ & 1.4365541036 & 5.8998039788 & -1.8987493185 \\
\hline $\mathrm{C}$ & 1.8252274783 & 8.2361180362 & -0.4420727886 \\
\hline $\mathrm{H}$ & -0.1872164275 & 7.9602400251 & 0.2549953122 \\
\hline $\mathrm{C}$ & 2.6621779153 & 6.5668136241 & -1.9591953774 \\
\hline $\mathrm{H}$ & 1.3087721095 & 4.9887953093 & -2.4855205601 \\
\hline $\mathrm{C}$ & 2.8638579806 & 7.7330647422 & -1.2268282888 \\
\hline $\mathrm{H}$ & 1.9675470581 & 9.1529233976 & 0.1337170260 \\
\hline $\mathrm{H}$ & 3.4636258187 & 6.1643850228 & -2.5823359680 \\
\hline $\mathrm{H}$ & 3.8243443085 & 8.2502920961 & -1.2682491655 \\
\hline $\mathrm{P}$ & 0.3582665886 & -1.5579164747 & 1.5510236298 \\
\hline $\mathrm{O}$ & 1.1534493120 & -1.5236800015 & 0.1073167968 \\
\hline $\mathrm{O}$ & -0.8671330763 & -2.6101232941 & 1.1619991637 \\
\hline $\mathrm{C}$ & 1.4488981686 & -2.7025882212 & -0.5263111559 \\
\hline $\mathrm{C}$ & -1.5537649082 & -2.4032512880 & 0.0038935532 \\
\hline $\mathrm{C}$ & 0.4092461428 & -3.3832998275 & -1.1432111208 \\
\hline $\mathrm{C}$ & 2.7930222818 & -3.1793425130 & -0.5540353387 \\
\hline $\mathrm{C}$ & -0.9438378547 & -2.7656925237 & -1.1861703496 \\
\hline $\mathrm{C}$ & -2.8586069173 & -1.8291738436 & 0.0517646808 \\
\hline $\mathrm{C}$ & 0.6512181766 & -4.6829842433 & -1.6963420073 \\
\hline $\mathrm{C}$ & 3.0267434184 & -4.3987939550 & -1.1482051706 \\
\hline $\mathrm{Si}$ & 4.3170045114 & -2.1557694733 & -0.0887790139 \\
\hline $\mathrm{C}$ & -1.6320693379 & -2.5255927270 & -2.4168118770 \\
\hline $\mathrm{C}$ & -3.5559585289 & -1.6988527381 & -1.1322454223 \\
\hline $\mathrm{Si}$ & -3.7016325557 & -1.2796857341 & 1.6533670069 \\
\hline $\mathrm{C}$ & -0.3856107051 & -5.5117470051 & -2.2107746161 \\
\hline $\mathrm{C}$ & 1.9795232553 & -5.1905161349 & -1.6954767273 \\
\hline $\mathrm{H}$ & 4.0412862849 & -4.8122550331 & -1.2146438561 \\
\hline $\mathrm{C}$ & 4.5387359035 & -0.8552566034 & -1.4207929046 \\
\hline $\mathrm{C}$ & 4.3215326127 & -1.3080860494 & 1.5767102766 \\
\hline $\mathrm{C}$ & 5.7957908099 & -3.3268140175 & -0.1022893762 \\
\hline $\mathrm{C}$ & -1.0312128096 & -2.7461015715 & -3.6894432858 \\
\hline $\mathrm{C}$ & -2.9711155077 & -2.0399692684 & -2.3795347190 \\
\hline $\mathrm{H}$ & -4.5758318373 & -1.2964052893 & -1.1519006927 \\
\hline $\mathrm{C}$ & -5.5147999370 & -0.9981490165 & 1.2171674873 \\
\hline $\mathrm{C}$ & -3.6394639788 & -2.5802030062 & 2.9993602473 \\
\hline $\mathrm{C}$ & -3.0155755020 & 0.3036775849 & 2.4285685391 \\
\hline $\mathrm{C}$ & -0.1076215707 & -6.7552752729 & -2.7243143960 \\
\hline $\mathrm{H}$ & -1.4165236571 & -5.1575091705 & -2.1844764337 \\
\hline $\mathrm{C}$ & 2.2370600335 & -6.4767452511 & -2.2423520382 \\
\hline $\mathrm{C}$ & 5.8169977039 & -0.4691268333 & -1.8609531494 \\
\hline $\mathrm{C}$ & 3.4139176915 & -0.2128594149 & -1.9625944169 \\
\hline $\mathrm{C}$ & 3.7150097571 & -1.8656977182 & 2.7102200027 \\
\hline $\mathrm{C}$ & 5.0094206994 & -0.0840750570 & 1.7043094897 \\
\hline $\mathrm{C}$ & 6.4177725432 & -3.6891943560 & -1.3100930986 \\
\hline $\mathrm{C}$ & 6.2918259951 & -3.8602822766 & 1.0973762160 \\
\hline $\mathrm{C}$ & -1.7465236728 & -2.5680825386 & -4.8474739363 \\
\hline $\mathrm{H}$ & 0.0124961893 & -3.0574257241 & -3.7349181627 \\
\hline $\mathrm{C}$ & -3.6935832237 & -1.8871852839 & -3.5968660930 \\
\hline $\mathrm{C}$ & -5.9520915992 & 0.1442217069 & 0.5253276094 \\
\hline $\mathrm{C}$ & -6.4617503747 & -1.9812780814 & 1.5495673468 \\
\hline $\mathrm{C}$ & -4.3908529837 & -2.3352226892 & 4.1671561040 \\
\hline $\mathrm{C}$ & -2.9020604585 & -3.7679302275 & 2.9095637329 \\
\hline $\mathrm{C}$ & -3.5079202867 & 1.6005597635 & 2.1521871922 \\
\hline
\end{tabular}




\begin{tabular}{|c|c|c|c|}
\hline $\mathrm{C}$ & 0328 & 887 & 23 \\
\hline $\mathrm{C}$ & 1.2195791956 & -7.2442995727 & -2.7521490617 \\
\hline & -0.9207122678 & -7.3750674682 & -3.1084705254 \\
\hline $\mathrm{H}$ & 3.2655903015 & -6.8475809102 & -2.2424468587 \\
\hline $\mathrm{C}$ & .9671910401 & 0.5204486803 & -2.837387783 \\
\hline $\mathrm{H}$ & 6.7128398403 & -0.9350609488 & -1.444097879 \\
\hline $\mathrm{fl}$ & 3.5652170033 & 0.7743246994 & -2.9453887269 \\
\hline $\mathrm{H}$ & 2.3951197931 & -0.4591736365 & -1.6269717664 \\
\hline $\mathrm{C}$ & 3.7469384978 & -1.1887173724 & 20921 \\
\hline $\mathrm{H}$ & 3.1820968785 & -2.8238029973 & 2.6564280115 \\
\hline$C_{3}$ & 5.0330589407 & 0.5927868593 & 2.9281240294 \\
\hline $\mathrm{H}$ & 5.5452639517 & 0.3475327043 & 0.8522741764 \\
\hline $\mathrm{C}$ & 41618 & -4.56 & \\
\hline $\mathrm{H}$ & 6.0547929978 & -3.2913808043 & -2.261 \\
\hline $\mathrm{C}$ & 7.3850932394 & 95760 & 85869 \\
\hline $\mathrm{H}$ & 4340679 & -3.5977332905 & 12322 \\
\hline $\mathrm{C}$ & .1017384246 & 07890 & -4.80 \\
\hline $\mathrm{H}$ & -1.2650509956 & -2.74 & 9603 \\
\hline $\mathrm{H}$ & -4.7326363483 & -1.5491553966 & -3.5492361737 \\
\hline $\mathrm{C}$ & 6430 & 0.3 & \\
\hline $\mathrm{H}$ & $-5.2 ?$ & & 195 \\
\hline $\mathrm{C}$ & -7.8110160845 & -1.8 & 5627 \\
\hline $\mathrm{H}$ & -6.16 & 899 & 143 \\
\hline $\mathrm{C}_{\mathrm{S}}$ & 3917 & -3.2 & 681 \\
\hline 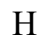 & -4.9 & 342 & \\
\hline $\mathrm{C}$ & -2.9152759261 & 87424 & 94565 \\
\hline$U_{1}$ & 299 & 131 & 808 \\
\hline $\mathrm{fl}$ & -3.1 & & 7542 \\
\hline $\mathrm{H}$ & 5273 & 1.75 & 1.3 \\
\hline $\mathrm{C}$ & -1.7362191272 & 52913 & 4.2864008032 \\
\hline$U_{-1}$ & -1.7 & -0.8 & 291 \\
\hline $\mathrm{H}$ & 1.42 & 176 & -3.1 \\
\hline $\mathrm{C}$ & 337 & 1.1 & -3.38 \\
\hline $\mathrm{H}$ & 6.9 & & \\
\hline $\mathrm{H}$ & 2.6 & & -3 \\
\hline $\mathrm{C}$ & 4.39 & 0.04 & 89152 \\
\hline $\mathrm{H}$ & 3.2363347366 & -1.6224338841 & 4.7965564433 \\
\hline $\mathrm{H}$ & & & \\
\hline 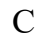 & 7.9 & $-5 .($ & 98344 \\
\hline $\mathrm{H}$ & 19534 & 79796 & -2.2580751106 \\
\hline$U$ & 448 & & 84688 \\
\hline $\mathrm{H}$ & 50 & -2.0 & -5.7 \\
\hline C & 60883 & 6047 & 54563 \\
\hline $\mathrm{H}$ & -7.6201986330 & 315594 & -0.3433092218 \\
\hline $\mathrm{H}$ & & & \\
\hline $\mathrm{C}$ & & -4.4 & 5.1 \\
\hline $\mathrm{H}$ & -4.9885349978 & -3.0620348517 & 26437 \\
\hline$H^{2}$ & 10331 & 4851 & 7123244 \\
\hline $\mathrm{C}$ & & & \\
\hline $\mathrm{H}$ & 70 & 3.68 & $260+2+3$ \\
\hline $\mathrm{H}$ & -1.0433700760 & 1.1183345648 & 5.1161524262 \\
\hline 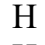 & & 07442 & -4.1508138641 \\
\hline $\mathrm{H}$ & & & \\
\hline $\mathrm{H}$ & 8.8461782089 & -5.7650091577 & -0.1198181198 \\
\hline $\mathrm{H}$ & -9.2805474122 & -0.5469921899 & 0.2706616625 \\
\hline $\mathrm{H}$ & & 51651202460 & \\
\hline & & & 4.61012 \\
\hline
\end{tabular}

$(2 \mathrm{~S}, 5 \mathrm{~S})-\mathrm{EPr}$

01 


\begin{tabular}{|c|c|c|c|}
\hline & 1.8125134809 & 218 & 0.4427158059 \\
\hline & -2.8747020360 & 2.3977099147 & -0.0870006982 \\
\hline & -3.2097275356 & 2.6005154346 & -1.5464841624 \\
\hline & -4.3469956523 & 2.3691038076 & 0.2650701813 \\
\hline & -4.5519986745 & 3.2041232917 & -1.0415975642 \\
\hline & -3.3778150358 & 1.6258917363 & -2.0312657761 \\
\hline & -2.5360660755 & 3.1935854424 & -2.171029251 \\
\hline$\Pi$ & -4.7532133565 & 1.3544164215 & 0.1304030693 \\
\hline & 38657 & & \\
\hline & -1.7795128521 & 3.0520676864 & 0.659040928 \\
\hline & -1.9821639866 & 3.3605805201 & \\
\hline 11 & -2.8790305122 & 2.8636831225 & 2.5071 \\
\hline $\mathrm{H}$ & -2.0500327859 & 4.4333806303 & 2.32 \\
\hline $\mathrm{Mg}$ & -0.3488068125 & 0.2621981689 & 8007 \\
\hline $\mathrm{O}$ & 0.8399213795 & 0.6571784719 & -0.2332282244 \\
\hline $\mathrm{O}$ & -0.4511350857 & 22727 & 2330 \\
\hline & 3280 & -0.7 & \\
\hline & 0097 & 870 & 361 \\
\hline 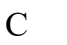 & 2.3027759833 & -1.4234226699 & 4.3008503734 \\
\hline $\mathrm{N}$ & 137 & & \\
\hline $\mathrm{C}$ & 507 & -0.5 & \\
\hline $\mathrm{H}$ & 918076 & -2.0 & 009 \\
\hline $\mathrm{H}$ & 2351 & -2.1 & \\
\hline $\mathrm{C}$ & & & \\
\hline $\mathrm{Br}$ & -0.46 & & \\
\hline $\mathrm{H}$ & 3.0807696742 & -0.7768850674 & 428873 \\
\hline $\mathrm{H}$ & 28 & -0.5 & \\
\hline $\mathrm{O}$ & & & \\
\hline $\mathrm{C}$ & -0.7411 & 3.8 & 93955 \\
\hline$f_{1}>$ & & & \\
\hline $\mathrm{C}$ & 222 & 3.3 & 651 \\
\hline $\mathrm{C}$ & 430 & $5.8 \mathrm{~s}$ & 7054 \\
\hline $\mathrm{H}$ & -0.8097632381 & 5.5549810143 & 1.2338422254 \\
\hline $\mathrm{C}$ & & & \\
\hline $\mathrm{H}$ & 20 & & \\
\hline $\mathrm{C}$ & 5592 & 9332 & 864 \\
\hline H & & & \\
\hline $\mathrm{H}$ & & & \\
\hline $\mathrm{H}$ & 030 & & 546 \\
\hline $\mathrm{C}$ & 7894 & 598 & 6139 \\
\hline $\mathrm{H}$ & & & 20714 \\
\hline $\mathrm{H}$ & -5.7 & & 362 \\
\hline $\mathrm{H}$ & -5.850 & 172812 & 2493 \\
\hline $\mathrm{C}$ & & & -0.7243130583 \\
\hline $\mathrm{C}_{\mathrm{S}}$ & & & \\
\hline $\mathrm{C}$ & 29 & & 648 \\
\hline $\mathrm{C}$ & 240397 & 6.9007857881 & -0.9500756412 \\
\hline $\mathrm{H}$ & & & \\
\hline $\mathrm{C}$ & -5.3 & & 01 \\
\hline $\mathrm{H}$ & -6.16 & 4.61 & 6007 \\
\hline $\mathrm{C}$ & 106172 & 7.4362414501 & -0.0598845704 \\
\hline $\mathrm{H}$ & & & \\
\hline $\mathrm{H}$ & & & \\
\hline $\mathrm{H}$ & 7493593 & 8.4958791897 & 0.2009059890 \\
\hline 1 & 0.3991974877 & -0.3303998393 & -1.303664498 \\
\hline $\mathrm{O}$ & & & \\
\hline 0 & 0802 & -1.3831381903 & $-0.21296564 t$ \\
\hline $\mathrm{C}$ & 2.4211749604 & -1.8040410356 & -0.7532127954 \\
\hline & 1601060 & 171700252 & \\
\hline & & & \\
\hline
\end{tabular}




\begin{tabular}{|c|c|c|c|}
\hline $\mathrm{C}$ & 3607 & 33759 & 23808 \\
\hline $\mathrm{C}$ & 0.6414498548 & -3.5011811582 & -0.4297326747 \\
\hline $\mathrm{C}$ & -1.8085024987 & -3.3355378693 & -0.5823555996 \\
\hline $\mathrm{C}$ & 2.7313628499 & -3.4018219413 & 1.0355680107 \\
\hline$C$ & 4.4445985809 & -1.7145595766 & 0.5131179368 \\
\hline $\mathrm{Si}$ & 4.3210171445 & 0.3732335475 & -1.3470476123 \\
\hline . & 0.5627459928 & -4.8815255017 & -0.8153187715 \\
\hline$C$ & -1.8805237612 & -4.6798321363 & -0.8695097265 \\
\hline $\mathrm{Si}$ & -3.4386644149 & -2.4555387214 & -0.2327911994 \\
\hline $\mathrm{C}$ & 2.2796316049 & -4.4425383440 & 1.8936063505 \\
\hline $\mathrm{C}$ & 4.0081601308 & -2.8246505916 & 1.2899131865 \\
\hline $\mathrm{H}$ & 5.4238120471 & -1.2828923893 & 0.7544813281 \\
\hline $\mathrm{C}$ & 3.4651786028 & & 542770 \\
\hline $\mathrm{C}$ & 4.2591690404 & 1.7974712784 & -0.1395628516 \\
\hline $\mathrm{C}$ & 6.1142432214 & -0.0186475503 & -1.7667341296 \\
\hline $\mathrm{C}$ & 1.7206052973 & -5.6845177771 & -1.0174840172 \\
\hline $\mathrm{C}$ & -0.7125542716 & -5.4715520060 & 3695697 \\
\hline $\mathrm{H}$ & -2.8485228403 & -5.1843254703 & -0.9842959944 \\
\hline $\mathrm{C}$ & -4.1088126872 & -1.5026353772 & -1.6926968626 \\
\hline $\mathrm{C}$ & -4.6918045897 & 9437 & 276342 \\
\hline $\mathrm{C}$ & -3.28 & & \\
\hline $\mathrm{C}$ & 3.0731718745 & -4.913 & \\
\hline $\mathrm{H}$ & 1.2867414230 & 03732 & 73186 \\
\hline 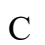 & 4.8114966864 & -3.344 & 849 \\
\hline $\mathrm{C}$ & 3.4051 & & 63452 \\
\hline $\mathrm{C}$ & 2.9596266250 & -0.1487355735 & -3.8259598429 \\
\hline $\mathrm{C}$ & 3.306 & 6682 & 53888 \\
\hline 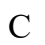 & 8418 & & 05251 \\
\hline $\mathrm{C}$ & 75376 & -0.5989124285 & -3.0032887478 \\
\hline $\mathrm{C}$ & 7.1458501001 & 0.2304035096 & -0.8456036362 \\
\hline 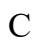 & 1.609 & -7. & 7080 \\
\hline $\mathrm{H}$ & 2.707 & 1925 & 16376 \\
\hline $\mathrm{C}$ & -0.7957479577 & -6.8408561161 & -1.4096410381 \\
\hline $\mathrm{C}$ & 5684 & 1531 & 884355 \\
\hline $\mathrm{C}$ & 9437 & 8745 & 31152 \\
\hline $\mathrm{C}$ & -4.8389270887 & -4.1791024957 & 1.5713789276 \\
\hline $\mathrm{C}$ & -5.4717357853 & -4.4119516189 & -0.7507009969 \\
\hline $\mathrm{C}$ & -2.1 & & 3892 \\
\hline $\mathrm{C}$ & -4.3860114431 & -0.5 & 250 \\
\hline $\mathrm{C}$ & 4.3610305946 & -4.3720127891 & 3.1324969899 \\
\hline 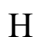 & 0649 & & 16430 \\
\hline 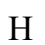 & 5.79 & -2 & 006 \\
\hline 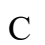 & 2.8617468408 & 609515 & 446394 \\
\hline $\mathrm{H}$ & 3.7785141175 & 2.9610189372 & -2.6672340335 \\
\hline$C$ & 18004 & & \\
\hline $\mathrm{H}$ & 14 & -1.20 & 508 \\
\hline $\mathrm{C}$ & 3.2839725710 & 2.9009822331 & 1.7997132140 \\
\hline $\mathrm{H}$ & 2.554 & & 1619365 \\
\hline 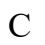 & 52 & 47 & 540615 \\
\hline $\mathrm{H}$ & 5.921 & 07224 & -1.0549246627 \\
\hline $\mathrm{C}$ & 7.7665541991 & -0.9248028094 & -3.3116112642 \\
\hline $\mathrm{H}$ & & & -3.7461489221 \\
\hline 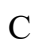 & 8.4715465070 & -0.0938473745 & -1.1528770057 \\
\hline $\mathrm{H}$ & 6.9283328461 & 6041567 & 8882249 \\
\hline $\mathrm{C}$ & 0.3384947036 & -7.5938855504 & -1.5801347567 \\
\hline $\mathrm{H}$ & & -7.5961119110 & -1.5470810195 \\
\hline 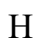 & .7839163223 & -7.2817738305 & -1.5648663673 \\
\hline $\mathrm{C}$ & -6.0269901334 & -0.6065055028 & -2.9031281498 \\
\hline $\mathrm{H}$ & -6.1777932380 & -1.6232559533 & -1.0187118921 \\
\hline$C$ & -3.8035635035 & -0.3605665075 & -3.8216301100 \\
\hline
\end{tabular}




$\begin{array}{lrrr}\mathrm{H} & -2.1802988687 & -1.1855233010 & -2.6788137890 \\ \mathrm{C} & -5.7496913497 & -5.1835962497 & 1.9199790590 \\ \mathrm{H} & -4.2453964096 & -3.7116449536 & 2.3615357028 \\ \mathrm{C} & -6.3820222338 & -5.4154460317 & -0.4032222939 \\ \mathrm{H} & -5.3792469355 & -4.1260622931 & -1.8023464856 \\ \mathrm{C} & -2.0934678998 & -0.4708563226 & 3.2262318804 \\ \mathrm{H} & -1.3210036369 & -2.0483025366 & 1.9549342916 \\ \mathrm{C} & -4.3431841326 & 0.2827147316 & 2.7629234927 \\ \mathrm{H} & -5.3075630671 & -0.5881721384 & 1.0464483196 \\ \mathrm{H} & 4.9843907066 & -4.7598159243 & 3.9402855926 \\ \mathrm{C} & 2.3559716929 & 1.5653191113 & -5.4293078625 \\ \mathrm{H} & 2.8348138815 & 3.5901750478 & -4.8602727537 \\ \mathrm{H} & 1.9924214524 & -0.5437848582 & -5.7138003026 \\ \mathrm{C} & 4.2037540655 & 3.9437137018 & 1.6797975827 \\ \mathrm{H} & 2.5480460775 & 2.9058078744 & 2.6079470422 \\ \mathrm{H} & 5.8599451378 & 4.7410179450 & 0.5477492561 \\ \mathrm{C} & 8.7835158217 & -0.6730726404 & -2.3867863663 \\ \mathrm{H} & 8.0048072551 & -1.3730775278 & -4.2736064923 \\ \mathrm{H} & 9.2607792430 & 0.1070014591 & -0.4319348090 \\ \mathrm{H} & 0.2649297045 & -8.6436797907 & -1.8707022688 \\ \mathrm{C} & -5.1788986037 & -0.1493734720 & -3.9201272394 \\ \mathrm{H} & -7.1004478544 & -0.4510972318 & -2.9816499558 \\ \mathrm{H} & -3.1310469418 & -0.0038334081 & -4.6019380615 \\ \mathrm{C} & -6.5224233784 & -5.8020924122 & 0.9334461206 \\ \mathrm{H} & -5.8548599261 & -5.4831305159 & 2.9608454879 \\ \mathrm{H} & -6.9819303800 & -5.8955735811 & -1.1737479718 \\ \mathrm{C} & -3.1924140469 & 0.3420122531 & 3.5445053779 \\ \mathrm{H} & -1.2292129701 & -0.4916072614 & 3.9036586230 \\ \mathrm{H} & -5.2170507779 & 0.8749644004 & 3.0291314667 \\ \mathrm{H} & 1.9247904147 & 1.8506341207 & -6.3864609551 \\ \mathrm{H} & 4.1884923559 & 4.7672647759 & 2.3910939235 \\ \mathrm{H} & 9.8142628145 & -0.9252196754 & -2.6262210926 \\ \mathrm{H} & -5.5936874693 & 0.3652956176 & -4.7848496585 \\ \mathrm{H} & -7.2310120375 & -6.5827549637 & 1.2040787272 \\ \mathrm{H} & -3.1440807603 & 0.9941246738 & 4.4165107114 \\ & & & \end{array}$

ECr

01

$\begin{array}{lrrr}\mathrm{O} & -2.1867530339 & 1.3223010795 & 0.9396434406 \\ \mathrm{C} & -2.6656458197 & 2.4715672201 & 0.3933827916 \\ \mathrm{C} & -2.6012878950 & 2.6120500501 & -1.1560934505 \\ \mathrm{C} & -4.2123130701 & 2.6079422720 & 0.2441995632 \\ \mathrm{C} & -4.0133342330 & 3.2529826883 & -1.1504431019 \\ \mathrm{H} & -2.6276081011 & 1.6020422481 & -1.5923964829 \\ \mathrm{H} & -1.7738421271 & 3.1648917248 & -1.6166321665 \\ \mathrm{H} & -4.6290068604 & 1.5972898270 & 0.1267256675 \\ \mathrm{H} & -4.7815033286 & 3.1431109719 & 1.0162995798 \\ \mathrm{C} & -2.0867896104 & 3.6921170290 & 1.1576196449 \\ \mathrm{C} & -2.5269965076 & 3.9769543080 & 2.3988470315 \\ \mathrm{H} & -3.3463435214 & 3.4094374457 & 2.8475065116 \\ \mathrm{H} & -2.1200472908 & 4.8161068985 & 2.9710854686 \\ \mathrm{Mg} & -0.8709123384 & 0.0310020889 & 1.0457971093 \\ \mathrm{O} & 0.5045649284 & 0.4845004197 & -0.4415076564 \\ \mathrm{O} & -0.5406106811 & -0.5486608114 & -2.6658502296 \\ \mathrm{O} & 0.6021695709 & -0.3756037352 & 2.4674575977 \\ \mathrm{C} & 1.4329031079 & 0.1692794212 & 3.1839508634 \\ \mathrm{C} & 2.7862923382 & -0.3702395873 & 3.5477349047 \\ \mathrm{~N} & 1.2706009089 & 1.3872536726 & 3.7698968932 \\ \mathrm{C} & 3.3802108688 & 0.6609105899 & 4.5076548797 \\ \mathrm{H} & 3.3460394173 & -0.4543965370 & 2.6020796682\end{array}$




\begin{tabular}{|c|c|c|c|}
\hline & 2.6944753381 & -1.3902477380 & 3.94 \\
\hline C & 2.3760144205 & 1.7986019147 & 20474 \\
\hline & -0.1948408965 & 7481811 & 516869 \\
\hline & 3.5266227333 & 0.2805522131 & 5.527794943 \\
\hline & 4.3428229792 & 1.0703544091 & 4.167324725 \\
\hline & 2.4452584584 & 2.8413415714 & 5.12957260 \\
\hline & -0.9990437598 & 4.5054691458 & 0.5566117025 \\
\hline & -1.0548668827 & 5.9041490241 & 0.60 \\
\hline & 0.0997282070 & 3.8927667887 & -0.064320589 \\
\hline & -0.0326997492 & 6.6754844034 & 0.05421727 \\
\hline $\mathrm{H}$ & -1.9347853039 & 6.3883154090 & 0320030976 \\
\hline $\mathrm{C}$ & 1.12436 & 4.66663 & \\
\hline $\mathrm{H}$ & 0.1518135669 & 2.8029430337 & -0.139693482 \\
\hline $\mathrm{C}$ & 1.0642599886 & 6.0591856719 & -0.5451974482 \\
\hline $\mathrm{H}$ & -0.1027989059 & 7.7650626734 & 120328 \\
\hline $\mathrm{H}$ & 1.9779312209 & 4.16700 & \\
\hline $\mathrm{H}$ & 1.8669222319 & 6.6622628337 & -0.9759477343 \\
\hline $\mathrm{C}$ & -4.9733495621 & 2.7846072082 & -2.2433120798 \\
\hline $\mathrm{H}$ & -5.992 & 3.15 & 2623 \\
\hline & $-4.6547 \mathrm{c}$ & 3.1500982103 & -3.2 \\
\hline $\mathrm{H}$ & $-5.0064 \mathrm{C}$ & 1.6829514837 & -2.2828621924 \\
\hline C & -3.959 & 4.77 & 00985 \\
\hline $\mathrm{C}$ & -3.1848 & 5.44 & 1466 \\
\hline C & -4.69 & 5.55 & 5123 \\
\hline C & -3.1328933598 & 6.8357942495 & -2.1570197469 \\
\hline $\mathrm{H}$ & -2.59 & 4.86 & 00755 \\
\hline $\mathrm{C}$ & -4.64 & 6.95 & -0.2 \\
\hline $\mathrm{H}$ & -5.31 & 847 & \\
\hline $\mathrm{C}$ & -3.8619840423 & 7.5973753533 & 314044 \\
\hline $\mathrm{H}$ & -2.50 & 7.3 & 2431 \\
\hline $\mathrm{H}$ & -5.22 & 924 & 0203 \\
\hline $\mathrm{H}$ & -3.8179683316 & 8.68 & -1.27 \\
\hline $\mathrm{P}$ & & -0.6 & 99124 \\
\hline $\mathrm{O}$ & 837 & -1.5 & 5104 \\
\hline $\mathrm{O}$ & 266 & 404 & -0.2 \\
\hline $\mathrm{C}$ & & & -0.6788743915 \\
\hline C & 74 & & \\
\hline C & 1.91 & 585 & 026 \\
\hline $\mathrm{C}$ & 53358 & -1.3203681277 & -0.3666132811 \\
\hline $\mathrm{C}$ & & & -0.413 \\
\hline $\mathrm{C}$ & -1.7 & & -0.6 \\
\hline $\mathrm{C}$ & 2.656 & 241 & 3134 \\
\hline $\mathrm{C}$ & 4.3406499065 & -1.8156747990 & 33474 \\
\hline G. & & & \\
\hline C & 18 & -5.1 & -0 \\
\hline$C$ & 3951 & 888 & -0.9 \\
\hline $\mathrm{Si}$ & -3.4 & & 976736 \\
\hline$C$ & & & \\
\hline$c$ & 57 & -2.9 & 110 \\
\hline $\mathrm{H}$ & 5.2959636570 & -1.3448748976 & 0.9304134732 \\
\hline $\mathrm{C}$ & & & \\
\hline S & & & \\
\hline$C$ & & 64666 & -1.94 \\
\hline $\mathrm{C}$ & 1.8240361841 & -5.9313860481 & -0.8859388422 \\
\hline $\mathrm{C}$ & 5541 & -5.7880649420 & \\
\hline $\mathrm{H}$ & -2.7591838507 & -5.5643610972 & -1.090473236 \\
\hline $\mathrm{C}$ & 4.1267894905 & -1.8665288006 & -1.80333835 \\
\hline $\mathrm{C}$ & -4.6633791462 & -4.1276578537 & 0.1459074623 \\
\hline & 2075822917 & -1.6848680317 & \\
\hline & & 931004 & \\
\hline
\end{tabular}




\begin{tabular}{|c|c|c|c|}
\hline $\mathrm{H}$ & 1.2244100149 & -5.0310261511 & 1.8247526499 \\
\hline C & 4.6482439552 & -3.3550653207 & 2.5815945441 \\
\hline C & 2.4157459172 & 2.0547990613 & -2.6471618727 \\
\hline $\mathrm{C}$ & 2.8877025903 & -0.0779494650 & -3.6900965666 \\
\hline $\mathrm{C}$ & 3.1035374065 & 2.0727214580 & 0.6648366453 \\
\hline $\mathrm{C}$ & 5.5204097869 & 1.9517740835 & 0.6718118225 \\
\hline $\mathrm{C}$ & 6.5890920023 & 1.0233977577 & -2.4779942077 \\
\hline $\mathrm{C}$ & 6.4627653944 & -1.3440104103 & -1.9984791932 \\
\hline $\mathrm{C}$ & 1.7695140039 & -7.2596956587 & -1.2312112441 \\
\hline $\mathrm{H}$ & 2.7897405985 & -5.4554259499 & -0.7122537471 \\
\hline $\mathrm{C}$ & -0.6349387108 & -7.1668676090 & -1.3840463388 \\
\hline $\mathrm{C}$ & -5.4971129519 & -1.5352257508 & -1.8092515815 \\
\hline $\mathrm{C}$ & -3.3342620424 & -1.4820274588 & -2.8932822923 \\
\hline $\mathrm{C}$ & -4.7951866733 & -4.5244695567 & 1.4861808048 \\
\hline $\mathrm{C}$ & -5.4507492675 & -4.7636271380 & -0.8290200004 \\
\hline $\mathrm{C}$ & -2.1156590983 & -1.826 & 782161 \\
\hline $\mathrm{C}$ & -4.1994006435 & -0.7394058647 & 0587 \\
\hline $\mathrm{C}$ & 4.1736432081 & -4.3513511308 & 34312 \\
\hline $\mathrm{H}$ & 2.5380407272 & -5.7321260108 & 497044 \\
\hline $\mathrm{H}$ & 5.6071910008 & -2.87 & 94024 \\
\hline $\mathrm{C}$ & 1.5608328119 & 2.38 & 8818206 \\
\hline $\mathrm{H}$ & 2.5436162683 & 2.7840775198 & -1.8373509696 \\
\hline $\mathrm{C}$ & 2.0369 & 1774 & 42829 \\
\hline$H$ & 1095282 & -1.0 & 94011 \\
\hline$C$ & 3.1532529279 & 2057 & 38717 \\
\hline $\mathrm{H}$ & 2.1194205283 & 515989 & 0.2928252870 \\
\hline$C$ & 5.56 & 06 & 229 \\
\hline $\mathrm{H}$ & 6.45 & 1.5 & 0.2 \\
\hline $\mathrm{C}$ & 7.8533639535 & 0.8419552199 & -3.0476027808 \\
\hline 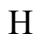 & 6.15 & 751 & 3347 \\
\hline $\mathrm{C}$ & 1439019 & -1.5262603413 & 48699 \\
\hline 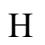 & 61368 & 2187 & 7169091 \\
\hline $\mathrm{C}$ & 0.5272242189 & -7.8911415056 & -1.4752937975 \\
\hline $\mathrm{H}$ & 2.694 & -7.8 & 10765 \\
\hline $\mathrm{H}$ & -1.600 & -7.6 & 1729 \\
\hline $\mathrm{C}$ & -6.0558048398 & -0.8337330294 & -2.8793264942 \\
\hline $\mathrm{H}$ & 35276 & -1.8 & 967201 \\
\hline 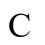 & 2727 & 2941 & 86737 \\
\hline $\mathrm{H}$ & -2.2594853615 & -1.7129019851 & -2.9323070414 \\
\hline $\mathrm{C}$ & -5.6951649179 & -5.5348834556 & 1.8452849071 \\
\hline 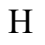 & -4.19 & -4.0 & 637559 \\
\hline $\mathrm{C}$ & -6.3499123419 & -5.7737698947 & 491644 \\
\hline $\mathrm{H}$ & -5.3751215867 & -4.4729196353 & -1.8808772062 \\
\hline $\mathrm{C}$ & -2.0004880399 & -0.9897014448 & 3.1695845427 \\
\hline 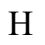 & -1.3790829762 & & \\
\hline$C$ & -4.09 & 701 & 45815 \\
\hline $\mathrm{H}$ & -5.0612420671 & -0.6062978218 & 0.7977692941 \\
\hline $\mathrm{H}$ & & & 4.2624580775 \\
\hline $\mathrm{C}$ & 3 & 23 & -4.7 \\
\hline$\Pi$ & 1.035 & 8241 & -3.7031317697 \\
\hline $\mathrm{H}$ & 1.8774069947 & -0.4583084698 & -5.5585840905 \\
\hline$C$ & & & 2.1719814953 \\
\hline $\mathrm{H}$ & 2.229 & 3.5227062970 & 2.0061609915 \\
\hline 11 & 6.5263781022 & 3.2381684963 & 2.0847965442 \\
\hline $\mathrm{C}$ & 8.4252789969 & -0.4338159611 & -3.0921690262 \\
\hline t & & 1.6929684907 & -3.4612694927 \\
\hline $\mathrm{H}$ & & -2.5209203457 & -2.6096083044 \\
\hline $\mathrm{H}$ & 0.4977767822 & -8.9485079347 & -1.7446053653 \\
\hline$c$ & -5.2522386038 & -0.4496418050 & -3.9609707824 \\
\hline $\mathrm{H}$ & -7.1140286673 & -0.5846035819 & -2.8753531628 \\
\hline
\end{tabular}




$\begin{array}{lrrr}\mathrm{H} & -3.2574482136 & -0.4689717425 & -4.7946241927 \\ \mathrm{C} & -6.4729996463 & -6.1605177900 & 0.8670668545 \\ \mathrm{H} & -5.7898267178 & -5.8321323287 & 2.8874270543 \\ \mathrm{H} & -6.9560305566 & -6.2569539206 & -1.2346742578 \\ \mathrm{C} & -2.9936239054 & -0.0387256493 & 3.4440218423 \\ \mathrm{H} & -1.1642431511 & -1.1167940852 & 3.8589088242 \\ \mathrm{H} & -4.8713053738 & 0.8102829336 & 2.8060401229 \\ \mathrm{H} & 0.6830882196 & 1.7224689426 & -5.5652822969 \\ \mathrm{H} & 4.4163107813 & 4.2463282922 & 2.9425186968 \\ \mathrm{H} & 9.4083259086 & -0.5750537894 & -3.5370152201 \\ \mathrm{H} & -5.6873316751 & 0.1005555136 & -4.7929763911 \\ \mathrm{H} & -7.1735087814 & -6.9452582394 & 1.1457959995 \\ \mathrm{H} & -2.8954882447 & 0.6153126495 & 4.3088901948\end{array}$

TS2a1

01

O

C $\quad-1.2201518751$

C $\quad-1.2663473256$

C $\quad-1.7491071778$

C $\quad-1.1787966838$

$\mathrm{H} \quad-2.2715840645$

$\mathrm{H} \quad-0.5228474681$

$\mathrm{H} \quad-2.8461442090$

$\mathrm{H} \quad-1.4493169639$

C $\quad 0.1146488073$

C $\quad 0.5876554875$

$\mathrm{H} \quad 0.4155420979$

$\mathrm{H}$
$\mathrm{H}$

$\mathrm{Mg} \quad-2.0979322268$

O $\quad-0.6285192972$

O $\quad 1.0353381601$

O $\quad-3.4791157815$

C $\quad-3.3720590671$

C $\quad-4.5995834056$

$\mathrm{N} \quad-2.2396388841$

C $\quad-4.0053615861$

$\mathrm{H} \quad-5.0888146484$

$\mathrm{H} \quad-5.3236544057$

C $\quad-2.4887123050$

$\mathrm{Br} \quad-0.4078179767$

$\mathrm{H} \quad-4.2898317793$

$\mathrm{H} \quad-4.2413652049$

$\mathrm{O} \quad-1.6486511177$

C $\quad 0.8613925929$

C $\quad 2.1596510523$

C $\quad 0.3032235824$

C $\quad 2.8563523390$

$\mathrm{H} \quad 2.6454738702$

C $\quad 0.9955379813$

$\mathrm{H} \quad-0.7201038690$

C 2.2720663244

$\mathrm{H} \quad 3.8545711742$

$\mathrm{H} \quad 0.5293022326$

$\mathrm{H} \quad 2.8067069926$

C $\quad-2.0077434153$

$\mathrm{H} \quad-2.0264866303$

$\mathrm{H} \quad-1.5874411938$

H $\quad-3.0462403858$

C $\quad 0.2526918242$
3.2172095359

4.4031346661

5.5693601575

5.4090466289

6.5586371394

5.5965945603

5.6236478220

5.3816936423

5.3257411023

3.7508255806

3.6058881095

4.5275011592

3.3350395591

1.3154470156

0.3749310618

$-0.7587944374$

0.8161270585

0.4450784059

0.0955676463

0.3315678507

$-0.1618853332$

$-0.7850545438$

0.9213620190

$-0.0579749696$

2.1708101032

0.5960358368

$-1.1448752762$

$-0.2584289341$

3.1766924550

2.6489036508

3.0813603391

2.0665875942

2.6904172910

2.4779178466

3.4112425147

1.9581162420

1.6591118822

2.3698889342

1.4441514808

7.8337811480

8.3886897903

8.5000294854

7.5973650863

6.8689791194
0.4699399625

0.2847676746

1.3068992138

$-0.7579765600$

0.1184739165

1.7504031158

2.1116216700

$-0.7046208052$

$-1.8113594058$

0.0465067023

$-1.3565592133$

$-1.9206840155$

$-1.4206476874$

0.1093027510

0.8898728263

2.5683564106

$-1.2112958558$

$-2.4224318389$

$-3.2482367521$

$-3.0746965105$

$-4.6255304599$

$-2.8032790328$

$-3.2030606148$

$-4.3871868161$

$-2.2608181769$

$-5.3703731798$

$-5.0524848389$

$-5.2274370897$

1.1331606271

0.9348562547

2.4343246738

1.9792401659

$-0.0404044542$

3.4646914257

2.5968761686

3.2379044629

1.8021410427

4.4430629308

4.0388732052

0.2352860667

$-0.7146465794$

1.0039645827

0.5116014897

$-0.3162756256$ 


\begin{tabular}{|c|c|c|c|}
\hline $\mathrm{C}$ & 1.3697280014 & 6.6669904453 & 0.5002283955 \\
\hline $\mathrm{C}$ & 0.4718666166 & 7.3439477067 & -1.6191349591 \\
\hline & 2.6621498571 & 6.9091008280 & 0.027706936 \\
\hline 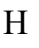 & 1.2483507409 & 6.3024544219 & 1.5209755290 \\
\hline 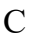 & 1.7559561302 & 7.5913246070 & -2.0930469073 \\
\hline $\mathrm{H}$ & -0.3820101373 & 7.5081107559 & -2.2820025126 \\
\hline $\mathrm{C}$ & 2.8617122270 & 7.3675727993 & -1.27015943 \\
\hline $\mathrm{H}$ & 3.5163584521 & 6.7308807906 & 0.684710963 \\
\hline 1 & 1.8968526050 & 7.9542136707 & -3.113298400 \\
\hline $\mathrm{H}$ & 3.8716168113 & 7.5514167814 & -1.641843292 \\
\hline 1 & 0.2721626837 & -0.7830289528 & 1.3033618559 \\
\hline $\mathrm{O}$ & 1.2101973700 & -1.0332624526 & -0.0305134944 \\
\hline $\mathrm{O}$ & -0.6610662314 & -2.1415117900 & 1.279769881 \\
\hline $\mathrm{C}$ & 1.7331805313 & -2.2761904509 & -0.2882 \\
\hline $\mathrm{C}$ & -1.3166205819 & -2.4519582469 & 0.11410 \\
\hline $\mathrm{C}$ & 0.8683248162 & -3.2724884355 & -0.7129554026 \\
\hline $\mathrm{C}$ & 3.1349537623 & -2.5028859753 & -0.150105958 \\
\hline $\mathrm{C}$ & -0.5705570066 & -2.9655296697 & -0.9 \\
\hline C & -2.7234146762 & -2.2603979743 & 0.0270 \\
\hline $\mathrm{C}$ & 1.3644456950 & -4.6066047530 & -0.8 \\
\hline $\mathrm{C}$ & 3.6135305887 & -3.7728653756 & -0.383108112 \\
\hline $\mathrm{Si}$ & 4.4483595229 & -1.1944568488 & 0.20 \\
\hline $\mathrm{C}$ & -1.2106739153 & -3.2008 & -2.1 \\
\hline $\mathrm{C}$ & -3.3522014524 & -2.611 & -1.1478 \\
\hline $\mathrm{Si}$ & -3.7827789501 & -1.616 & \\
\hline $\mathrm{C}$ & 0.5184782924 & -5.712 & -1.1 \\
\hline $\mathrm{C}$ & 2.7556954346 & -4.8518698099 & -0.7278 \\
\hline $\mathrm{H}$ & 078445 & -3.99 & -0.3 \\
\hline $\mathrm{C}$ & 4.1895507677 & 0.3698233230 & -0.8065 \\
\hline $\mathrm{C}$ & & & \\
\hline $\mathrm{C}$ & 82958 & -1.89 & -0.4 \\
\hline $\mathrm{C}$ & -0.4901255295 & 59408 & -3.36 \\
\hline $\mathrm{C}$ & -2.6234443030 & -3.059 & -2.2800 \\
\hline $\mathrm{H}$ & -4.4409059551 & -2.5267509714 & -1.25755 \\
\hline $\mathrm{C}$ & -5.5714080927 & -1.7184248522 & 0.8532 \\
\hline $\mathrm{C}$ & 733461 & -2.678 & 2.98 \\
\hline $\mathrm{C}$ & -3.4191631141 & 0.1515714319 & 2.0009 \\
\hline $\mathrm{C}$ & & -6.97 & \\
\hline $\mathrm{H}$ & 868736 & -5.55 & -1.2 \\
\hline $\mathrm{C}$ & 3.2626076999 & $-6.16^{\prime}$ & -0.90 \\
\hline $\mathrm{C}$ & 5.1306679605 & 1.410 & -0.69900 \\
\hline $\mathrm{C}$ & 3.1701357763 & 0.4814672165 & -1.7635415114 \\
\hline $\mathrm{C}$ & 275464 & -1.29 & \\
\hline $\mathrm{C}$ & 915733 & $-0.06^{\prime}$ & 2.43 \\
\hline $\mathrm{C}$ & 6.4190967733 & -1.8023862286 & -1.77 \\
\hline $\mathrm{C}$ & 6.9890840189 & -2.526 & 0.461 \\
\hline $\mathrm{C}$ & -1.1432198993 & -3.7761750856 & -4.550517711 \\
\hline $\mathrm{H}$ & 0.5973856052 & -3.6179579852 & -3.319 \\
\hline $\mathrm{C}$ & -3.2745530092 & -3.3397901103 & -3.5124727607 \\
\hline $\mathrm{C}$ & -6.0932580774 & -0.7766300654 & -0.0500228396 \\
\hline $\mathrm{C}$ & -6.3907257458 & -2.786 & \\
\hline $\mathrm{C}$ & -4.5566926666 & -2.5302375924 & 4.0130094204 \\
\hline $\mathrm{C}$ & -2.5599135320 & -3.5927506055 & 3.1568102824 \\
\hline $\mathrm{C}$ & -4.0833879257 & 1.2883019317 & 1.4881426670 \\
\hline $\mathrm{C}$ & -2.5893129183 & 0.3156210009 & 3.131231066 \\
\hline $\mathrm{C}$ & 2.4246742443 & -7.2095262158 & -1.2170490463 \\
\hline $\mathrm{H}$ & 0.3651627887 & -7.8079590787 & -1.5722272687 \\
\hline $\mathrm{H}$ & 4.3369379434 & -6.3345302881 & -0.790733022 \\
\hline $\mathrm{C}$ & 5.0600607443 & 2.5288675376 & -1.535560221 \\
\hline $\mathrm{H}$ & 5.9401995292 & 1.3548220204 & 0.0339313 \\
\hline
\end{tabular}




\begin{tabular}{|c|c|c|c|}
\hline & 198 & 86 & 68 \\
\hline & .3917311524 & -0.2874749240 & -1.8533157711 \\
\hline & 3.9785269468 & -1.0135537992 & 534867449 \\
\hline 11 & 2.8797409313 & -1.8805803846 & 2.7183057164 \\
\hline $\mathrm{C}$ & 5.9832218415 & 0.2159131042 & 3.7908509385 \\
\hline $\mathrm{H}$ & 6.4982657058 & 0.3031432041 & 1.7074292799 \\
\hline $\mathrm{C}$ & 7.6228774965 & -2.3315056339 & -2.2523864181 \\
\hline $\mathrm{H}$ & 5.7399055042 & -1.3152829772 & -2.4791236316 \\
\hline $\mathrm{C}$ & 761 & 6922604 & \\
\hline $\mathrm{H}$ & .7614804454 & -2.6142122953 & 1.5274612471 \\
\hline C & -2.5534566344 & -3.6885799600 & -4.6256525013 \\
\hline$\Pi$ & -0.5695891431 & -4.0156981373 & -5.4477610213 \\
\hline Ut & 9386162 & 11988 & -3.5 \\
\hline $\mathrm{C}$ & -7.4016057305 & -0.8875075525 & 84550 \\
\hline $\mathrm{H}$ & -5.4704501491 & 5492426 & 58926 \\
\hline$C$ & 7123 & 37391 & 4662 \\
\hline $\mathrm{H}$ & -6.0177894125 & -3.5493353420 & 3052 \\
\hline C & 3146 & 80433 & 5.18 \\
\hline $\mathrm{H}$ & -5.3772370439 & 857275 & 45433 \\
\hline O & 290 & 33 & \\
\hline $\mathrm{H}$ & & 276 & \\
\hline$c$ & -3.9013000408 & 2.5510973718 & 96322 \\
\hline $\mathrm{H}$ & -4.7 & 482 & \\
\hline C & 0204 & 7651 & 3.7 \\
\hline $\mathrm{H}$ & -2.0 & 7737 & 3.5 \\
\hline 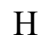 & 2.82 & 44565 & -1.3521196704 \\
\hline $\mathrm{C}$ & 4.05 & 541 & 3076 \\
\hline $\mathrm{H}$ & 2461 & 9450 & 00480 \\
\hline $\mathrm{H}$ & 2.33 & 575 & -3.3 \\
\hline$C$ & & 6722 & \\
\hline $\mathrm{H}$ & 3.26 & 165 & \\
\hline $\mathrm{H}$ & 6.84 & 013 & 997 \\
\hline C & 8.51 & 60875 & 733106 \\
\hline $\mathrm{H}$ & & -2.2 & \\
\hline H & 47 & 647 & 0.6 \\
\hline $\mathrm{H}$ & 497 & 9597 & -5.5 \\
\hline$C$ & & & 063609 \\
\hline & & & \\
\hline $\mathrm{H}$ & 254 & 7077 & 1.0 \\
\hline 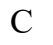 & -3.4210023766 & -4.2106846326 & 5.3378252579 \\
\hline $\mathrm{H}$ & & & \\
\hline $\mathrm{H}$ & & & \\
\hline $\mathrm{C}$ & 6638 & 6969 & 0615 \\
\hline $\mathrm{H}$ & -4.3873728737 & 3.4241669579 & 1.6460421741 \\
\hline $\mathrm{H}$ & & & \\
\hline & & 913630 & \\
\hline $\mathrm{H}$ & 337 & -0.0427051962 & 5.8053592147 \\
\hline $\mathrm{H}$ & 9.4476945101 & -3.3694125206 & -1.7460551523 \\
\hline & 51879 & 82301082 & -0.48 \\
\hline & 04796043 & -4.8049624771 & \\
\hline & 0089054511 & 3.6517987 & 3.7068396 \\
\hline
\end{tabular}

TS2a2

01

$\begin{array}{lllr}\mathrm{O} & -3.3349282661 & -1.3765051542 & -0.9012585557 \\ \mathrm{C} & -4.1355904664 & -1.9118116307 & 0.0690505073 \\ \mathrm{C} & -5.2143627800 & -1.1114617961 & 0.8509257607 \\ \mathrm{C} & -5.2764650682 & -2.8645317149 & -0.3411536630 \\ \mathrm{C} & -6.0621990043 & -2.4039121065 & 0.9148954431 \\ \mathrm{H} & -5.6722746071 & -0.3827523690 & 0.1644858359\end{array}$




\begin{tabular}{|c|c|c|c|}
\hline $\mathrm{H}$ & -4.9568575542 & -0.5958412413 & 1.7827776381 \\
\hline & -5.7255376289 & -2.4491920254 & -1.2540876317 \\
\hline $\mathrm{H}$ & 5.0846914081 & -3.9333738371 & -0.5031498889 \\
\hline $\mathrm{C}$ & -2.9735139041 & -2.4667249769 & 0.8525116776 \\
\hline $\mathrm{C}$ & -2.6275342025 & -3.8962458037 & 0.6893068968 \\
\hline-1 & -3.4924544383 & -4.5030605786 & 0.4171876736 \\
\hline $\mathrm{H}$ & -2.1726707440 & -4.3048966417 & 1.5962617677 \\
\hline 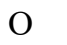 & -0.6762190142 & -2.0039444939 & -3.2965772052 \\
\hline 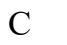 & 0.3198863181 & -2.7886326130 & -3.3660736656 \\
\hline $\mathrm{f}$ & 1.5462712134 & -2.4436812510 & -4.1823155297 \\
\hline $\mathrm{N}$ & 0.4097498970 & -3.9307778300 & -2.7207662194 \\
\hline $\mathrm{C}$ & 2.3958811324 & -3.6996003688 & -4.0392626632 \\
\hline 11 & 1.9924891650 & 494048704 & 057001 \\
\hline 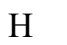 & 1.2656046428 & -2.1791656453 & -5.2103809304 \\
\hline$e^{2}$ & 1.6260609341 & -4.5414476464 & -3.0123331156 \\
\hline $\mathrm{Br}$ & -1.2884068165 & -4.0833972892 & -0.7675653150 \\
\hline $\mathrm{H}$ & 845667 & & 1003 \\
\hline$U^{\prime}$ & 0984 & & 3783 \\
\hline $\mathrm{O}$ & 75358 & -5.5 & 02870 \\
\hline 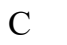 & -2.1101971676 & & 97417 \\
\hline C & -0.96 & 493 & 2697 \\
\hline $\mathrm{C}$ & -2.346 & -0.2 & 1.71 \\
\hline $\mathrm{C}$ & -0.1259025655 & -1.2929060617 & 623491 \\
\hline-1 & 542 & & 363 \\
\hline $\mathrm{C}$ & & & \\
\hline-1 & 891603 & 0.2 & \\
\hline $\mathrm{C}_{\mathrm{S}} \mathrm{x}$ & -0.4089912348 & 0.0673429943 & 3.1154662991 \\
\hline 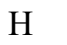 & 25 & & 653 \\
\hline $\mathrm{H}$ & -1.7 & & 016 \\
\hline $\mathrm{H}$ & 0.2551941264 & 0.7 & $3.6^{\circ}$ \\
\hline 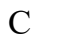 & 41732 & & \\
\hline & 51092 & -3.2 & 4216 \\
\hline $\mathrm{H}$ & 4834 & -1.7 & 1.6 \\
\hline $\mathrm{H}$ & -7.8195815041 & -1.6159856577 & -0.0890095498 \\
\hline$e^{-1}$ & 43 & & 5805 \\
\hline 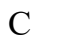 & -5.0 & 179 & 451 \\
\hline $\mathrm{C}$ & -5.9989312813 & -4.6747505713 & 50417 \\
\hline$C$ & -4.71 & & 9318 \\
\hline-3 & -4.8 & -1. & 3.3 \\
\hline $\mathrm{C}$ & -5.6558535681 & -5.5519408230 & 3.0 \\
\hline $\mathrm{H}$ & -6.4965028594 & -5.0648884444 & 1.1225472288 \\
\hline$C$ & 3753 & & \\
\hline $\mathrm{H}$ & -4.2 & -3.3 & 756 \\
\hline $\mathrm{H}$ & -5.8916976895 & -6.6140522553 & 2.9437508864 \\
\hline $\mathrm{H}$ & -4.7260203539 & -5.7647419095 & 4.9797299500 \\
\hline $\mathrm{Mg}$ & & & \\
\hline 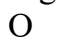 & 008 & 150 & -2.0 \\
\hline $\mathrm{O}$ & -0.4296957490 & -0.1618980244 & -0.7163049447 \\
\hline $\mathrm{D}$ & & & -0.8857872503 \\
\hline $\mathrm{O}$ & & 2.17 & -0.88 \\
\hline r & 1.61 & 536696 & 88505 \\
\hline $\mathrm{C}$ & 0.1086528955 & 2.8049884677 & 0.2341444788 \\
\hline$C_{1}>$ & & & 0.8591888960 \\
\hline 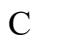 & & 2.9714166027 & 157393 \\
\hline$C$ & -1.2191035294 & 3.3244190229 & 0.2396963297 \\
\hline $\mathrm{C}$ & 2.3799417494 & 2.5392527667 & 1.2071083221 \\
\hline 列 & & & \\
\hline $\mathrm{C}$ & 0.4556012015 & 3.5661095909 & 510100702 \\
\hline $\mathrm{C}$ & -1.6496742327 & 4.0211283448 & 1.3473427069 \\
\hline $\mathrm{Si}$ & -2.4256919773 & 3.2611140339 & -1.2080323282 \\
\hline
\end{tabular}




\begin{tabular}{|c|c|c|c|}
\hline C & 39442024 & 3.4720789160 & 46849 \\
\hline 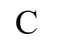 & 5.0336116907 & 1.6322726659 & 1.0099603730 \\
\hline $\mathrm{Si}$ & 4.5151642865 & -1.0210823539 & 0387653 \\
\hline $\mathrm{C}$ & 1.2036317961 & 3.5993039761 & 7302867142 \\
\hline $\mathrm{C}$ & -0.8526752959 & 4.1277982384 & 73608 \\
\hline $\mathrm{H}$ & -2.6407582595 & 4.4932075093 & 1.3682065172 \\
\hline $\mathrm{C}$ & -2.1485853830 & 1.8756489486 & -2.4345985032 \\
\hline $\mathrm{C}$ & -4.1962546278 & 3.0488390229 & -0.5900785462 \\
\hline $\mathrm{C}$ & -2.2200300773 & 4.9218355954 & -2.0532962689 \\
\hline $\mathrm{C}$ & 3.2444668184 & 4.8625647828 & 1.6172579532 \\
\hline $\mathrm{C}$ & 4.7938702215 & 3.0006830841 & 1.3118096926 \\
\hline $\mathrm{H}$ & 6.0817265297 & 1.3088709728 & 0.9599038746 \\
\hline $\mathrm{C}$ & 4.8351271510 & -1.2393798066 & -1.4924653802 \\
\hline $\mathrm{C}$ & 6.1605520837 & -1.2660902081 & 1.2371394549 \\
\hline $\mathrm{C}$ & 3.3242099064 & -2.3309675382 & 0.9487228065 \\
\hline $\mathrm{C}$ & 0.6883416584 & 4.1808124399 & 5627 \\
\hline $\mathrm{H}$ & 2.1974772209 & 3.1497959524 & 3.752 \\
\hline $\mathrm{C}$ & -1.3490746738 & 4.7430118979 & 3.7017659871 \\
\hline $\mathrm{C}$ & -3.0819597625 & 547918 & -2.569 \\
\hline $\mathrm{C}$ & -1.0346289973 & 1.9049130249 & -3.29 \\
\hline $\mathrm{C}$ & 8530485 & 2.61091 & 77572 \\
\hline $\mathrm{C}$ & -5.2352051219 & 3.2789798360 & -1.5117079283 \\
\hline $\mathrm{C}$ & 3753 & 36233 & 96595 \\
\hline $\mathrm{C}$ & -3.2141771576 & 5.9114197067 & -2.0706568735 \\
\hline $\mathrm{C}$ & 19786 & 5.71 & 1.7920828533 \\
\hline $\mathrm{H}$ & 5894323 & 81735 & 95460 \\
\hline $\mathrm{C}$ & 2766 & 3.9 & 308 \\
\hline $\mathrm{C}$ & 9523 & -2.42 & -1.9 \\
\hline $\mathrm{C}$ & 4.4944336743 & -0.2724956741 & -2.4476338046 \\
\hline $\mathrm{C}$ & 8357 & -1.4044225956 & 44029 \\
\hline $\mathrm{C}$ & 7.3773003458 & 80178 & 7613 \\
\hline $\mathrm{C}$ & 4316 & 5570 & 93771 \\
\hline $\mathrm{C}$ & 2.6992110819 & -3.1864080110 & 0.0277735568 \\
\hline $\mathrm{C}$ & 5984 & 4.7 & 4.8 \\
\hline $\mathrm{H}$ & 2037 & 961 & 5.7 \\
\hline $\mathrm{H}$ & -2.3498297455 & 64898 & 3371909 \\
\hline $\mathrm{C}$ & -2.925 & -0.1 & 47118 \\
\hline $\mathrm{H}$ & 1391 & 0.7 & 27017 \\
\hline $\mathrm{C}$ & -0.8540278797 & 447372 & -4.2663201146 \\
\hline $\mathrm{H}$ & -0.2759322345 & 2.6946976924 & -3.1906945704 \\
\hline $\mathrm{C}$ & 8062 & 2.4 & 29517 \\
\hline $\mathrm{H}$ & -3.7530364708 & 7935 & 9699 \\
\hline $\mathrm{C}$ & -6.5714081193 & 96901 & 234372 \\
\hline $\mathrm{H}$ & -5.0115868101 & 1868 & -2.5331757385 \\
\hline $\mathrm{C}$ & -0.73 & & \\
\hline $\mathrm{H}$ & -0.17 & 4.4 & 94415 \\
\hline $\mathrm{C}$ & -2.9789356735 & 7.1485160550 & -2.6833215330 \\
\hline $\mathrm{H}$ & -4.18 & & -1.6021951850 \\
\hline$C$ & 77 & 5.23 & 586190 \\
\hline $\mathrm{H}$ & 4.1177556028 & 6.781 & 1.9577645725 \\
\hline $\mathrm{H}$ & 6.8922393193 & 3.5261383960 & 1.4538445884 \\
\hline $\mathrm{C}$ & & -2.6177671997 & -3.2664879245 \\
\hline$U$ & 5.7292843697 & -3.2057006126 & -1.1994741053 \\
\hline $\mathrm{C}$ & 4.8008887294 & -0.4726336750 & -3.8020569868 \\
\hline $\mathrm{H}$ & 3.9604124357 & 0.6421534951 & -2.1597094341 \\
\hline $\mathrm{C}$ & 7.4094066715 & -1.5529621220 & 3.3134828205 \\
\hline $\mathrm{H}$ & & -1.4021335723 & 3.2155674127 \\
\hline $\mathrm{C}$ & 8.5943967372 & -1.4184505146 & 1.2115515071 \\
\hline $\mathrm{H}$ & 7.3957024203 & -1.1556641735 & -0.5516523230 \\
\hline $\mathrm{C}$ & 2.3619788571 & -3.6459431881 & 2.7629429595 \\
\hline
\end{tabular}




\begin{tabular}{lrrr}
$\mathrm{H}$ & 3.5911457612 & -1.9049613096 & 3.0638640052 \\
$\mathrm{C}$ & 1.9441148147 & -4.2818865762 & 0.4674149098 \\
$\mathrm{H}$ & 2.7971948267 & -3.0178561688 & -1.0492550992 \\
$\mathrm{H}$ & -0.9910031482 & 5.2406054770 & 5.7541855178 \\
$\mathrm{C}$ & -1.7994745407 & -0.0985421150 & -4.4201554656 \\
$\mathrm{H}$ & -3.7014878785 & -0.9010124372 & -3.7324603737 \\
$\mathrm{H}$ & 0.0359979631 & 0.9368812634 & -4.8950324139 \\
$\mathrm{C}$ & -6.8907181215 & 2.6636110981 & 0.1478728333 \\
$\mathrm{H}$ & -6.1120033096 & 2.0884893525 & 2.0751745889 \\
$\mathrm{H}$ & -7.3643054121 & 3.2785451838 & -1.8669532161 \\
$\mathrm{C}$ & -1.7432661884 & 7.4120130013 & -3.2798831492 \\
$\mathrm{H}$ & 0.2268155657 & 6.6420509776 & -3.7155120575 \\
$\mathrm{H}$ & -3.7594772144 & 7.9060693154 & -2.6924562622 \\
$\mathrm{H}$ & 6.4680944822 & 5.9288298013 & 1.8977478922 \\
$\mathrm{C}$ & 5.4501080466 & -1.6380314148 & -4.2105175893 \\
$\mathrm{H}$ & 6.2832699572 & -3.5299974327 & -3.5825669299 \\
$\mathrm{H}$ & 4.5235701167 & 0.2851725190 & -4.5312910553 \\
$\mathrm{C}$ & 8.6126479057 & -1.5617904708 & 2.6012909935 \\
$\mathrm{H}$ & 7.4176860246 & -1.6638086627 & 4.3952468016 \\
$\mathrm{H}$ & 9.5271157882 & -1.4245934621 & 0.6516885253 \\
$\mathrm{C}$ & 1.7758536778 & -4.5133935395 & 1.8333893452 \\
$\mathrm{H}$ & 2.2396296726 & -3.8289727382 & 3.8269458007 \\
$\mathrm{H}$ & 1.5080829025 & -4.9651550927 & -0.2700354061 \\
$\mathrm{H}$ & -1.6661546862 & -0.8625242008 & -5.1890093980 \\
$\mathrm{H}$ & -7.9309376681 & 2.5218420630 & 0.4345231572 \\
$\mathrm{H}$ & -1.5603124277 & 8.3729236391 & -3.7567929900 \\
$\mathrm{H}$ & 5.6950477174 & -1.7893634479 & -5.2595747667 \\
$\mathrm{H}$ & 9.5576559016 & -1.6805283287 & 3.1266315978 \\
$\mathrm{H}$ & 1.2047644052 & -5.3741369154 & 2.1726685729 \\
& & & \\
\hline & & &
\end{tabular}

TS2b1

01

$\begin{array}{lrrr}\mathrm{O} & -2.5965405453 & 0.0725331975 & -0.1240065408 \\ \mathrm{C} & -3.7883840327 & -0.1976658965 & -0.6092394322 \\ \mathrm{C} & -4.2001925942 & 0.4808338668 & -2.1007989921 \\ \mathrm{C} & -4.9655417215 & 0.6359506754 & -0.0405821334 \\ \mathrm{C} & -5.3695667885 & 1.2187249688 & -1.4206697378 \\ \mathrm{H} & -3.3174213703 & 1.0929995743 & -2.3338111367 \\ \mathrm{H} & -4.4310872481 & -0.1585293777 & -2.9623446074 \\ \mathrm{H} & -4.5434781604 & 1.3945055720 & 0.6326256399 \\ \mathrm{H} & -5.7275520701 & 0.0805821486 & 0.5192609185 \\ \mathrm{C} & -4.0570575689 & -1.5564690229 & -1.0980901240 \\ \mathrm{C} & -5.3947778750 & -2.1435888507 & -0.8304302560 \\ \mathrm{H} & -6.2142044353 & -1.4206561910 & -0.8354468300 \\ \mathrm{H} & -5.6687973454 & -3.0111173292 & -1.4326752085 \\ \mathrm{O} & -2.3214279748 & -0.6489110000 & 3.1230966161 \\ \mathrm{C} & -3.1286895847 & -1.2197647759 & 3.9222854798 \\ \mathrm{C} & -3.1966794723 & -0.7967962936 & 5.3861809094 \\ \mathrm{~N} & -3.9517036865 & -2.1919117334 & 3.6097780674 \\ \mathrm{C} & -4.2582112122 & -1.7284942542 & 5.9518416557 \\ \mathrm{H} & -2.2021965209 & -0.9101735548 & 5.8421357614 \\ \mathrm{H} & -3.4553976606 & 0.2717624705 & 5.4440227616 \\ \mathrm{C} & -4.6950435283 & -2.5517827628 & 4.7275869700 \\ \mathrm{Br} & -5.0303198295 & -2.6472135924 & 1.0388030743 \\ \mathrm{H} & -5.1344827031 & -1.2151207193 & 6.3719772138 \\ \mathrm{H} & -3.8835406813 & -2.4133891987 & 6.7259416007 \\ \mathrm{O} & -5.5656751709 & -3.3863541405 & 4.7389898782 \\ \mathrm{C} & -5.2289558662 & 2.7427796119 & -1.4983851901 \\ \mathrm{H} & -5.9813633316 & 3.2300026441 & -0.8599902281 \\ \mathrm{H} & -5.3772131786 & 3.0948090830 & -2.5305222477\end{array}$




\begin{tabular}{|c|c|c|c|}
\hline $\mathrm{H}$ & 070 & 851 & 106 \\
\hline & -6.7539586311 & 0.8188967982 & -1.9130799664 \\
\hline $\mathrm{C}$ & -6.9959862115 & 0.5588851417 & -3.2666678941 \\
\hline $\mathrm{C}$ & -7.8376997657 & 0.7697555597 & -1.0272805258 \\
\hline 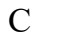 & -8.2745898328 & 0.2358478024 & -3.7190564394 \\
\hline $\mathrm{H}$ & -6.1765507296 & 0.6135525455 & -3.9878493984 \\
\hline $\mathrm{C}$ & -9.1173941171 & 0.4462857296 & -1.4747694683 \\
\hline $\mathrm{H}$ & -7.6833478212 & 0.9860439011 & 0.0328289668 \\
\hline$c$ & -9.3399775650 & 0.1723186434 & -2.8232328435 \\
\hline 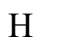 & -8.4376738947 & 0.0326480892 & -4.7796409691 \\
\hline $\mathrm{H}$ & -9.9450616108 & 0.4074063085 & -0.7634607889 \\
\hline $\mathrm{H}$ & -10.3407810088 & -0.0861729804 & -3.1746916263 \\
\hline $\mathrm{C}$ & -3.0430030839 & -2.3423242807 & 864997773 \\
\hline $\mathrm{C}$ & -3.3172979189 & -3.6679496528 & -2.2061714965 \\
\hline $\mathrm{C}$ & -1.7730837501 & -1.8001971442 & -2.092 \\
\hline $\mathrm{C}$ & -2.3729643896 & -4.4040962453 & -2.9008286310 \\
\hline $\mathrm{H}$ & -4.2648202499 & 119539 & 3150902 \\
\hline $\mathrm{C}$ & 790916 & 252 & 98790 \\
\hline $\mathrm{H}$ & -1.5035203623 & -0.801 & -1.7516931044 \\
\hline$C$ & 8668310 & 86447268 & 7734513 \\
\hline $\mathrm{H}$ & & & \\
\hline $\mathrm{H}$ & 0.14 & 57607 & -2 \\
\hline $\mathrm{H}$ & -0.3802719378 & -4.4242384762 & -3.7462615073 \\
\hline $\mathrm{Mg}$ & 1742 & 523 & 195 \\
\hline $\mathrm{O}$ & & & \\
\hline $\mathrm{P}$ & 247512 & 0.14 & 03872 \\
\hline $\mathrm{O}$ & & 4649 & 2688 \\
\hline 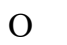 & & & 2500 \\
\hline $\mathrm{O}$ & 2.69 & -0.2 & \\
\hline$C$ & 2.7902206865 & 2.4187461341 & -0.1315564095 \\
\hline 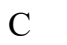 & & 135 & 2111 \\
\hline 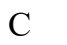 & & & 3966 \\
\hline$C$ & 2.2524547126 & 3.693 & 17473 \\
\hline$C$ & 4.6142309012 & 99752 & -0.2371326448 \\
\hline $\mathrm{C}$ & & -1 . & 5046 \\
\hline $\mathrm{C}$ & & 547 & 2059 \\
\hline $\mathrm{C}$ & 3.0436695000 & 4.5949583820 & 0.8621211123 \\
\hline . & & & $-0 .($ \\
\hline 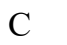 & & 978 & 8646 \\
\hline $\mathrm{C}$ & 132093 & -1.8444028713 & -0.9787392015 \\
\hline $\mathrm{Si}$ & & -3.2725149814 & -0.0318977411 \\
\hline$C$ & & & 90599 \\
\hline 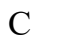 & 4.35 & 699 & 6228 \\
\hline $\mathrm{H}$ & 2.6680452633 & 018822 & 1.1259513242 \\
\hline $\mathrm{C}$ & & & 641710 \\
\hline 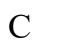 & & & \\
\hline $\mathrm{C}$ & 0.1931141751 & 5.9186446324 & 0.1598378337 \\
\hline $\mathrm{C}$ & 635554 & 49422 & -1.5037595875 \\
\hline$C$ & & & \\
\hline- & & -2.8 & -1.2860879495 \\
\hline$C$ & 3.0967066846 & -4.0395534520 & -1.7360145544 \\
\hline $\mathrm{C}$ & 4.2344371270 & -4.4380104101 & 1.0873801835 \\
\hline 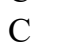 & & & \\
\hline $\mathrm{C}$ & 6.8 & 3.5 & 6394 \\
\hline $\mathrm{H}$ & 6.5474693231 & 1.6120754840 & 1.3220427141 \\
\hline 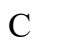 & & & \\
\hline O & & & -2.0546980614 \\
\hline 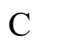 & 0.3763287646 & 2.9331012462 & -2.7601924790 \\
\hline $\mathrm{C}$ & 0.0395551780 & 3.1793884841 & 2.5630827559 \\
\hline$r$ & -1.7278552246 & 2.5242314655 & 1.0423568133 \\
\hline
\end{tabular}




\begin{tabular}{|c|c|c|c|}
\hline $\mathrm{C}$ & 0.7120737136 & 6.7926181811 & -0.8121226023 \\
\hline $\mathrm{C}$ & -0.4723915696 & 6.4586845344 & 1.2699920222 \\
\hline $\mathrm{C}$ & 7.6903991874 & 1.5430848619 & -2.2424843708 \\
\hline $\mathrm{H}$ & 6.1848553421 & 2.7209619479 & -1.2924562850 \\
\hline $\mathrm{C}$ & 7.4673628481 & -0.8528309663 & -2.1128874534 \\
\hline $\mathrm{C}$ & 3.3959630414 & -5.3799798953 & -2.0170352627 \\
\hline $\mathrm{C}$ & 2.6134766592 & -3.2150940921 & -2.7683289583 \\
\hline $\mathrm{C}$ & 3.6727499226 & -4.9383324750 & 2.2724579282 \\
\hline $\mathrm{C}$ & 5.5371342873 & -4.8371602601 & 0.7384577251 \\
\hline $\mathrm{C}$ & 1.3085888820 & -2.3084886923 & 1.8543444545 \\
\hline $\mathrm{C}$ & 0.4699372235 & -3.6249326996 & 0.0080575643 \\
\hline $\mathrm{C}$ & 6.3894473876 & 4.8093328643 & 2.4989949111 \\
\hline $\mathrm{H}$ & 7.8624455828 & 3.2156176796 & 2.6339563191 \\
\hline $\mathrm{H}$ & 4.7419746400 & 6.1588209845 & 2.2443481821 \\
\hline $\mathrm{C}$ & -2.1956578823 & 3.8988156948 & -3.2806050937 \\
\hline $\mathrm{H}$ & -2.1164258409 & 4.7716620540 & -1.3182885499 \\
\hline $\mathrm{C}$ & -0.2409790419 & 2.6638371827 & -3.9896039426 \\
\hline $\mathrm{H}$ & 1.3867977945 & 2.5426761365 & -2.5832178036 \\
\hline $\mathrm{C}$ & -0.6483681614 & 2.5860436033 & 3.6290111055 \\
\hline $\mathrm{H}$ & 1.0107011791 & 3.6435924850 & 2.7612487361 \\
\hline $\mathrm{C}$ & -2.4452859086 & 1.9735684131 & 2.1196134376 \\
\hline $\mathrm{H}$ & -2.1522805716 & 2.4516933019 & 0.0313739925 \\
\hline $\mathrm{C}$ & 0.5705706176 & 8.1769428993 & -0.6721290140 \\
\hline $\mathrm{H}$ & 1.2302756715 & 6.4006077492 & -1.6921697213 \\
\hline $\mathrm{C}$ & -0.6151759772 & 7.8447102744 & 1.4103219224 \\
\hline $\mathrm{H}$ & -0.8902294857 & 5.8062585322 & 2.0401849268 \\
\hline $\mathrm{C}$ & 8.1691094135 & 0.2455498929 & -2.5416876134 \\
\hline $\mathrm{H}$ & 8.2330510007 & 2.4176575597 & -2.6077896229 \\
\hline $\mathrm{H}$ & 7.8121762179 & -1.8608832360 & -2.3576866597 \\
\hline $\mathrm{C}$ & 3.2211206832 & -5.8909345236 & -3.3094324135 \\
\hline $\mathrm{H}$ & 3.7682589168 & -6.0426040225 & -1.2320509238 \\
\hline $\mathrm{C}$ & 2.4410017831 & -3.7256605068 & -4.0588048549 \\
\hline $\mathrm{H}$ & 2.3572356684 & -2.1600570564 & -2.5663424052 \\
\hline $\mathrm{C}$ & 4.3957486466 & -5.8147576381 & 3.0915749647 \\
\hline $\mathrm{H}$ & 2.6614593438 & -4.6540244527 & 2.5750977169 \\
\hline $\mathrm{C}$ & 6.2600468251 & -5.7123020363 & 1.5548414603 \\
\hline $\mathrm{H}$ & 6.0049685523 & -4.4661952898 & -0.1774516806 \\
\hline $\mathrm{C}$ & -0.0042677209 & -2.1408894486 & 2.3325085853 \\
\hline $\mathrm{H}$ & 2.1332433687 & -1.8400286119 & 2.4052785943 \\
\hline $\mathrm{C}$ & -0.8315866525 & -3.4719778558 & 0.4849132406 \\
\hline $\mathrm{H}$ & 0.6281757319 & -4.2061005087 & -0.9054357546 \\
\hline $\mathrm{H}$ & 6.9861222952 & 5.5163709881 & 3.0788455161 \\
\hline $\mathrm{C}$ & -1.5258051426 & 3.1433627858 & -4.2500630354 \\
\hline $\mathrm{H}$ & -3.1908973422 & 4.2849808405 & -3.4858423467 \\
\hline $\mathrm{H}$ & 0.2868892147 & 2.0769648974 & -4.7386684139 \\
\hline $\mathrm{C}$ & -1.8935930359 & 1.9951930208 & 3.4110228196 \\
\hline $\mathrm{H}$ & -0.2099398580 & 2.5857873627 & 4.6245072650 \\
\hline $\mathrm{H}$ & -3.4572891487 & 1.5734422236 & 1.9572170793 \\
\hline $\mathrm{C}$ & -0.0933755699 & 8.7044997232 & 0.4403333447 \\
\hline $\mathrm{H}$ & 0.9755223848 & 8.8440716781 & -1.4301273460 \\
\hline $\mathrm{H}$ & -1.1337042663 & 8.2516653537 & 2.2756062196 \\
\hline $\mathrm{H}$ & 9.0841585550 & 0.1228463467 & -3.1245842091 \\
\hline $\mathrm{C}$ & 2.7464373781 & -5.0635855607 & -4.3306469020 \\
\hline $\mathrm{H}$ & 3.4586520171 & -6.9313866267 & -3.5177115717 \\
\hline $\mathrm{H}$ & 2.0806095388 & -3.0769227237 & -4.8539195237 \\
\hline $\mathrm{C}$ & 5.6893694729 & -6.2022538289 & 2.7343221427 \\
\hline $\mathrm{H}$ & 3.9474741840 & -6.1933187240 & 4.0077965125 \\
\hline $\mathrm{H}$ & 7.2675583449 & -6.0110521648 & 1.2744489510 \\
\hline $\mathrm{C}$ & -1.0762547836 & -2.7296983317 & 1.6477773871 \\
\hline $\mathrm{H}$ & -0.1685573318 & -1.6148750222 & 3.2818240995 \\
\hline
\end{tabular}




$\begin{array}{lrrr}\text { H } & -1.6636016794 & -3.9447789351 & -0.0343708813 \\ H & -2.0032647959 & 2.9369905621 & -5.2052821998 \\ \text { H } & -2.4446299711 & 1.5512230192 & 4.2410056315 \\ \text { H } & -0.2039384811 & 9.7815363470 & 0.5487274960 \\ \text { H } & 2.6195547178 & -5.4579306873 & -5.3365013850 \\ \text { H } & 6.2518943992 & -6.8820907193 & 3.3708847572 \\ \text { H } & -2.1000400974 & -2.7013454123 & 2.0524236572\end{array}$

TS2b2

01

O $\quad-2.7139192827$

0.1724743427

0.3560995530

C $\quad-4.0248449090$

0.0122096137

0.3943398918

C $\quad-4.9313646817$

1.3226180293

0.8827731641

C $\quad-4.7956509747$

0.1218482297

$-0.9488383341$

C $\quad-5.6808996266$

1.3007527413

$-0.4653328781$

$\mathrm{H} \quad-4.1904035158$

2.1276676777

0.9918391987

$-5.5328818454$

1.2606841253

1.7997680448

$\mathrm{H} \quad-4.0801442665$

0.4189991257

$-5.3032522168$

$-0.7865292377$

$-1.7290159582$

C $\quad-4.5613441986$

$-0.9124655656$

$-1.2949029632$

1.4160466693

C $\quad-5.7262757686$

$-1.7543012351$

1.1091401443

$\mathrm{H} \quad-6.4275602077$

$-1.3308830434$

0.3856857842

$\mathrm{H} \quad-6.2612707794$

$-2.1732546196$

1.9643447273

$\mathrm{Mg} \quad-1.0706980106$

$-0.6740832412$

$-0.3430766696$

O $\quad 0.2068811748$

0.6585933509

0.7866790131

O $\quad 1.0165981425$

O $\quad-1.8151933464$

$-0.7631977188$

$-1.0739850794$

$-2.1230448193 \quad-1.5053312287$

C $\quad-2.0626070397$

$-3.2419927756$

$-2.0317706307$

C $\quad-1.4212155160$

$-3.6569447603$

$-3.3497323678$

$\mathrm{N} \quad-2.8706669825$

$-4.1552808558$

$-1.5284833554$

C $\quad-1.9662198928$

$-5.0628399966$

$-3.5586109763$

$-0.3258441576$

$-3.5972490099$

$-3.2544157500$

$-1.7148282268$

$-2.9418874117$

$-4.1323260597$

C $\quad-2.9234695143$

$-5.2590900038$

$-2.3703286626$

$\mathrm{Br} \quad-4.6326431506$

$-3.1209281980$

0.1539982751

$-5.2041210946-4.4971125533$

$-2.5203395112$

$-5.8446358072$

$-3.5202647388$

$-1.1929625436$

$-6.2317361027$

$-2.2007065957$

$2.5835289244-1.2779487125$

$-5.4799285256$

2.4644480368

$-2.2927202260$

$-5.9931451646$

3.4331423082

$-0.8013423827$

$-4.4095047270$

2.8273928165

$-1.3619339682$

0.9974361334

$-0.3703456341$

$\begin{array}{ll}1.5162344500 & 0.6669061848\end{array}$

$-7.9527443881$

0.2432983112

$-1.3639726089$

$\begin{array}{rr}1.2706924389 & 0.7247069291\end{array}$

$-9.3240225563$

1.4441064126

$-0.0059620486-1.3098433367$

$-9.1768822001$

$-0.159005649$

$-2.1967765912$

$-7.2245016620$

0.5027542469

$-0.2608682449$

$-9.9133832867$

1.6835662666

1.5464415375

$-9.6492975117$

$-0.6024630261$

$-2.0932456442$

$-11.0137887187$

0.3047433378

$-0.2143396126$

$-3.9466307940$

$-0.9907930389$

2.7417154270

3.6506790748

$-0.0128271958 \quad 3.1642761208$

$-3.0197169588$

3.1642761208
4.9313043902

C $\quad-3.7774039066$

$-2.0396062624$

3.3465860329

2.8092446178

4.4595083405

C $\quad-2.5161968763$

$-0.0324699667$

2.4537970560 


\begin{tabular}{|c|c|c|c|}
\hline $\mathrm{C}$ & -2.8904108037 & -1.0433581877 & 5.3437288622 \\
\hline $\mathrm{H}$ & -4.0553397674 & -2.8454798819 & 5.6128831838 \\
\hline $\mathrm{H}$ & -1.8066293740 & 0.7361938631 & 4.7709054356 \\
\hline $\mathrm{H}$ & -2.4797810859 & -1.0646388319 & 6.3556496865 \\
\hline $\mathrm{P}$ & 1.4114330201 & 0.2755688024 & -0.0475184439 \\
\hline $\mathrm{O}$ & 2.1395275262 & 1.5068330184 & -0.8230856643 \\
\hline $\mathrm{O}$ & 2.6186622542 & -0.1352121970 & 0.9594010578 \\
\hline $\mathrm{C}$ & 2.6936459377 & 2.4963334335 & -0.0393102837 \\
\hline $\mathrm{C}$ & 3.8955730509 & -0.2206262750 & 0.4442585462 \\
\hline $\mathrm{C}$ & 3.9219893851 & 2.2498855714 & 0.5497075479 \\
\hline $\mathrm{C}$ & 1.9601345031 & 3.6949465066 & 0.1503930274 \\
\hline $\mathrm{C}$ & 4.5983017496 & 0.9564521401 & 0.2487139670 \\
\hline $\mathrm{C}$ & 4.4070647276 & -1.4994572126 & 0.1054466865 \\
\hline $\mathrm{C}$ & 4.4480975601 & 3.2274544612 & 1.4549653167 \\
\hline $\mathrm{C}$ & 2.4963834215 & 4.6538657503 & 0.9776573911 \\
\hline $\mathrm{Si}$ & 0.2816939084 & 3.9953436400 & -0.6717514398 \\
\hline $\mathrm{C}$ & 5.9020754839 & 0.8824332502 & -0.3427982130 \\
\hline $\mathrm{C}$ & 5.6773492258 & -1.5682918780 & -0.4171661773 \\
\hline $\mathrm{Si}$ & 3.3921984457 & -3.0911044729 & 0.2317814925 \\
\hline $\mathrm{C}$ & 5.6399851793 & 3.0155394869 & 2.2036099064 \\
\hline $\mathrm{C}$ & 3.7325317512 & 4.4452250239 & 1.6517595801 \\
\hline $\mathrm{H}$ & 1.9677020999 & 5.5969882818 & 1.1609286834 \\
\hline $\mathrm{C}$ & 0.4307711506 & 5.5083096552 & -1.7777106311 \\
\hline $\mathrm{C}$ & -0.2432705767 & 2.5806988688 & -1.7763453823 \\
\hline $\mathrm{C}$ & -0.9644105296 & 4.3333823536 & 0.6841025808 \\
\hline $\mathrm{C}$ & 6.6597642818 & 2.0383864534 & -0.6846625578 \\
\hline $\mathrm{C}$ & 6.4515279889 & -0.3973600919 & -0.6479750244 \\
\hline $\mathrm{H}$ & 6.1144625551 & -2.5326537946 & -0.7033867062 \\
\hline $\mathrm{C}$ & 3.2447040141 & -3.7923939480 & -1.4969899271 \\
\hline $\mathrm{C}$ & 4.3075438212 & -4.3038136263 & 1.3377575407 \\
\hline $\mathrm{C}$ & 1.7244870547 & -2.8127195770 & 1.0284682398 \\
\hline $\mathrm{C}$ & 6.1122783220 & 3.9737000695 & 3.0666131117 \\
\hline $\mathrm{H}$ & 6.1768836169 & 2.0730574853 & 2.0902422643 \\
\hline $\mathrm{C}$ & 4.2545407282 & 5.4204860755 & 2.5454958948 \\
\hline $\mathrm{C}$ & -0.7188341389 & 5.9330966998 & -2.4691309078 \\
\hline $\mathrm{C}$ & 1.6308147995 & 6.2077043516 & -1.9703321952 \\
\hline $\mathrm{C}$ & -1.3859885121 & 1.7911799746 & -1.5612917639 \\
\hline $\mathrm{C}$ & 0.6034165063 & 2.2710312944 & -2.8561195842 \\
\hline $\mathrm{C}$ & -1.5332949093 & 5.6038290368 & 0.8672210593 \\
\hline $\mathrm{C}$ & -1.3143003055 & 3.2969489655 & 1.5673402072 \\
\hline $\mathrm{C}$ & 7.9063901075 & 1.9258395983 & -1.2497482960 \\
\hline $\mathrm{H}$ & 6.2372603514 & 3.0261675983 & -0.5001454126 \\
\hline $\mathrm{C}$ & 7.7487426821 & -0.4792365392 & -1.2248535663 \\
\hline $\mathrm{C}$ & 2.5203172727 & -3.0744610877 & -2.4647636353 \\
\hline $\mathrm{C}$ & 3.8589262658 & -4.9994423318 & -1.8680291434 \\
\hline $\mathrm{C}$ & 5.4989667203 & -3.9916355275 & 2.0081746961 \\
\hline $\mathrm{C}$ & 3.7394716449 & -5.5777519062 & 1.5236383670 \\
\hline $\mathrm{C}$ & 1.7290611781 & -2.3187006057 & 2.3454503003 \\
\hline $\mathrm{C}$ & 0.4944621286 & -3.0308155349 & 0.3851231827 \\
\hline $\mathrm{C}$ & 5.4200153023 & 5.1966612888 & 3.2344717506 \\
\hline $\mathrm{H}$ & 7.0271375993 & 3.7877770672 & 3.6330117842 \\
\hline $\mathrm{H}$ & 3.7009365180 & 6.3531054981 & 2.6817586407 \\
\hline $\mathrm{C}$ & -0.6691415049 & 7.0394558738 & -3.3227248423 \\
\hline $\mathrm{H}$ & -1.6662351195 & 5.3995730609 & -2.3518525748 \\
\hline $\mathrm{C}$ & 1.6816977638 & 7.3159050638 & -2.8254935911 \\
\hline $\mathrm{H}$ & 2.5459011581 & 5.8963383567 & -1.4617812453 \\
\hline $\mathrm{C}$ & -1.6620801956 & 0.7017712577 & -2.4025151526 \\
\hline $\mathrm{H}$ & -2.0818428094 & 2.0180494969 & -0.7382544430 \\
\hline $\mathrm{C}$ & 0.3422442157 & 1.1668684210 & -3.6782244176 \\
\hline $\mathrm{H}$ & 1.4980326572 & 2.8711738951 & -3.0536931277 \\
\hline
\end{tabular}




\begin{tabular}{|c|c|c|c|}
\hline $\mathrm{C}$ & -2.4397280303 & 5.8371751454 & 1.9082892293 \\
\hline $\mathrm{H}$ & -1.2765547291 & 6.4315520835 & 0.2011860384 \\
\hline $\mathrm{C}$ & -2.2160178981 & 3.5335879094 & 2.6109053388 \\
\hline $\mathrm{H}$ & -0.8831427365 & 2.2852460531 & 1.4489208644 \\
\hline $\mathrm{C}$ & 8.4669267509 & 0.6536488930 & -1.5135611656 \\
\hline $\mathrm{H}$ & 8.4672530498 & 2.8274015960 & -1.5046088430 \\
\hline $\mathrm{H}$ & 8.1599073155 & -1.4675399499 & -1.4458664771 \\
\hline $\mathrm{C}$ & 2.4115009708 & -3.5576886539 & -3.7731678336 \\
\hline $\mathrm{H}$ & 2.0259327470 & -2.1212270301 & -2.2088107264 \\
\hline $\mathrm{C}$ & 3.7464917214 & -5.4844294702 & -3.1763443188 \\
\hline $\mathrm{H}$ & 4.4347280355 & -5.5791030392 & -1.1425876947 \\
\hline $\mathrm{C}$ & 6.1196789222 & -4.9348568794 & 2.8369388913 \\
\hline $\mathrm{H}$ & 5.9585576044 & -3.0070144355 & 1.8995152112 \\
\hline $\mathrm{C}$ & 4.3586983403 & -6.5200818470 & 2.3506251431 \\
\hline $\mathrm{H}$ & 2.8021728447 & -5.8468538059 & 1.0270187853 \\
\hline $\mathrm{C}$ & 0.5271810510 & -2.0371141897 & 3.0048857592 \\
\hline $\mathrm{H}$ & 2.6731805498 & -2.1283047313 & 2.8667258417 \\
\hline $\mathrm{C}$ & -0.7135401506 & -2.7730853616 & 1.0539070151 \\
\hline $\mathrm{H}$ & 0.4613659688 & -3.4146552439 & -0.6406192644 \\
\hline $\mathrm{H}$ & 5.8081611570 & 5.9519617343 & 3.9205195118 \\
\hline $\mathrm{C}$ & 0.5322721802 & 7.7338293314 & -3.5004844397 \\
\hline $\mathrm{H}$ & -1.5641241094 & 7.3586581704 & -3.8522074523 \\
\hline $\mathrm{H}$ & 2.6193221599 & 7.8494523771 & -2.9656540535 \\
\hline $\mathrm{C}$ & -0.7813083924 & 0.3736898565 & -3.4426534807 \\
\hline $\mathrm{H}$ & -2.6022032017 & 0.1336889792 & -2.2873805289 \\
\hline $\mathrm{H}$ & 1.0276554137 & 0.9194534843 & -4.4857117271 \\
\hline $\mathrm{C}$ & -2.7803173956 & 4.8015120261 & 2.7821985671 \\
\hline $\mathrm{H}$ & -2.8745533552 & 6.8251664507 & 2.0382236123 \\
\hline $\mathrm{H}$ & -2.4703095174 & 2.7319467937 & 3.2990430940 \\
\hline $\mathrm{H}$ & 9.4604098922 & 0.5778776689 & -1.9595965570 \\
\hline $\mathrm{C}$ & 3.0228006105 & -4.7634223459 & -4.1302605706 \\
\hline $\mathrm{H}$ & 1.8567647104 & -2.9878944380 & -4.5152727510 \\
\hline $\mathrm{H}$ & 4.2236314549 & -6.4223836936 & -3.4501653724 \\
\hline $\mathrm{C}$ & 5.5517975555 & -6.1998922165 & 3.0070488725 \\
\hline $\mathrm{H}$ & 7.0443641314 & -4.6802037470 & 3.3499843017 \\
\hline $\mathrm{H}$ & 3.9105484660 & -7.5022225785 & 2.4858115747 \\
\hline $\mathrm{C}$ & -0.6891335029 & -2.2711850241 & 2.3607936123 \\
\hline $\mathrm{H}$ & 0.5434765726 & -1.6340861042 & 4.0140257403 \\
\hline $\mathrm{H}$ & -1.6727546872 & -3.0679125916 & 0.5988132279 \\
\hline $\mathrm{H}$ & 0.5712530342 & 8.5944189677 & -4.1651299409 \\
\hline $\mathrm{H}$ & -0.9795147766 & -0.4982892254 & -4.0637332888 \\
\hline $\mathrm{H}$ & -3.4790727552 & 4.9829808095 & 3.5957946780 \\
\hline $\mathrm{H}$ & 2.9373763204 & -5.1385075983 & -5.1479945727 \\
\hline $\mathrm{H}$ & 6.0338418917 & -6.9334034091 & 3.6499791412 \\
\hline $\mathrm{H}$ & -1.6244795181 & -2.0949221518 & 2.8867751580 \\
\hline \multicolumn{4}{|c|}{$\mathrm{TS} 2 \mathrm{c} 1$} \\
\hline \multicolumn{4}{|c|}{01} \\
\hline $\mathrm{O}$ & -2.6042633426 & 0.6803082257 & 0.0488968659 \\
\hline $\mathrm{C}$ & -3.1910993631 & -0.1933617252 & -0.7853914975 \\
\hline $\mathrm{C}$ & -2.4309881064 & -1.5131631655 & -1.1165724768 \\
\hline $\mathrm{C}$ & -3.0870374679 & 0.1658788311 & -2.3411701363 \\
\hline $\mathrm{C}$ & -2.4575215726 & -1.2157107587 & -2.6414769973 \\
\hline $\mathrm{H}$ & -1.4099922403 & -1.4578144493 & -0.7160645813 \\
\hline $\mathrm{H}$ & -2.8978368276 & -2.4467442450 & -0.7838653561 \\
\hline $\mathrm{H}$ & -2.3794687951 & 1.0050137482 & -2.4117543154 \\
\hline $\mathrm{H}$ & -4.0075795695 & 0.4293452853 & -2.8818975685 \\
\hline $\mathrm{C}$ & -1.0606931435 & -1.1294182915 & -3.2666590251 \\
\hline $\mathrm{H}$ & -1.1230607602 & -0.7226476238 & -4.2883991749 \\
\hline $\mathrm{H}$ & -0.6054800698 & -2.1292137820 & -3.3180443078 \\
\hline
\end{tabular}




\begin{tabular}{|c|c|c|c|}
\hline $\mathrm{H}$ & 190 & 44127 & 654 \\
\hline & -3.3187308580 & -2.1245261315 & -3.5058693145 \\
\hline & -3.4505679884 & -3.4896511853 & -3.2370592543 \\
\hline $\mathrm{C}$ & -3.9272464726 & -1.6225319737 & -4.6637415337 \\
\hline $\mathrm{C}$ & -4.1838935619 & -4.3219386029 & -4.0820231722 \\
\hline & -2.9743766941 & -3.9147487009 & 506002918 \\
\hline $\mathrm{C}$ & -4.6594232590 & -2.4485912349 & -5.5126976006 \\
\hline 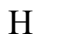 & -3.8223342154 & -0.5621599002 & -4.9099653463 \\
\hline $\mathrm{C}$ & -4.7953860791 & -3.8054544686 & -5.2219163347 \\
\hline-7 & -4.2780848239 & -5.3839941532 & -3.8438079889 \\
\hline $\mathrm{H}$ & -5.1247455198 & -2.0305590186 & -6.4083942545 \\
\hline $\mathrm{C}$ & -4.6819516184 & -0.3067571798 & -0.5853359978 \\
\hline $\mathrm{C}$ & 11595549 & 0.8023945266 & 603946 \\
\hline $\mathrm{H}$ & -4.8184310273 & 1.7427845022 & .0051255313 \\
\hline $\mathrm{H}$ & -6.4562199094 & 503 & 89400 \\
\hline $\mathrm{C}$ & -5.4723165020 & -1.4661057097 & -1.0595251126 \\
\hline G & -5.3950749577 & 1943 & 24184 \\
\hline $\mathrm{C}$ & 2537 & & 0758 \\
\hline 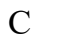 & 007605 & & 69945 \\
\hline $\mathrm{H}$ & 107022 & 5099 & 5110 \\
\hline $\mathrm{C}$ & & & \\
\hline $\mathrm{H}$ & 6497 & -0. & \\
\hline 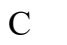 & -6.9593277163 & -3.6260384563 & -2.0225568520 \\
\hline $\mathrm{H}$ & 9355 & & 1069 \\
\hline $\mathrm{H}$ & 610 & -2 & 6925 \\
\hline $\mathrm{O}$ & -2.1277703940 & 0.0411040931 & 9003044 \\
\hline$C$ & 14430 & & 033 \\
\hline $\mathrm{C}$ & 0418 & -0. & 569 \\
\hline T & 1618 & -0.6 & 768 \\
\hline 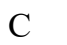 & -3.8545798443 & -1.4672182299 & 6.1633271191 \\
\hline $\mathrm{H}$ & 2704 & 386 & 091 \\
\hline $\mathrm{H}$ & 5584 & 357 & 035 \\
\hline $\mathrm{C}$ & -4.8917099970 & -1.1975997874 & 5.0 \\
\hline $\mathrm{Br}$ & -4.9762384243 & -0.0398006527 & 743374 \\
\hline 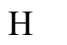 & 728 & & 444 \\
\hline $\mathrm{H}$ & 4900 & 920 & 0886 \\
\hline $\mathrm{O}$ & -6.0642446941 & -1.4512344766 & 5.1254246798 \\
\hline $\mathrm{H}$ & 3978 & & 91918 \\
\hline $\mathrm{H}$ & 388 & 479 & 33833 \\
\hline $\mathrm{Mg}$ & -1.5736329094 & 81785 & 1.6318098563 \\
\hline $\mathrm{O}$ & & & \\
\hline $\mathrm{P}$ & & & 18852 \\
\hline 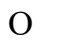 & 523 & 2057 & 022671 \\
\hline $\mathrm{O}$ & 3662876 & 1.2687067114 & -0.7889762996 \\
\hline ? & & 15752 & \\
\hline 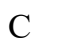 & & & -0.4 \\
\hline $\mathrm{C}$ & 3.7302879263 & -0.3661142254 & 0.1254888161 \\
\hline $\mathrm{C}$ & 19441 & & -0.2212628931 \\
\hline . & & & -0.2464018306 \\
\hline $\mathrm{C}$ & & 0.72 & 80190 \\
\hline$C$ & 4.1639594685 & -1.6975562370 & -0.1106612418 \\
\hline $\mathrm{C}$ & 1068899 & 365490 & 0.2630512850 \\
\hline 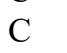 & & & \\
\hline $\mathrm{Si}$ & -0.02 & 301 & 025 \\
\hline$C$ & 5.3261615939 & 0.5165458880 & -1.4776072426 \\
\hline $\mathrm{C}$ & .1949105883 & -1.8899423172 & -1.0010519089 \\
\hline r. & & & \\
\hline 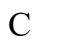 & 5.9356521878 & 2.9607868886 & 0.6203712373 \\
\hline$C$ & 4.0040358091 & 4.4455860334 & 0.4267601558 \\
\hline $\mathrm{H}$ & 2.2259804191 & 5.6581994292 & 0.2746504552 \\
\hline
\end{tabular}




\begin{tabular}{|c|c|c|c|}
\hline $\mathrm{C}$ & 8727954 & 09914 & 56290 \\
\hline $\mathrm{C}$ & -0.9918563709 & 3.3029004465 & 0.9755985830 \\
\hline $\mathrm{C}$ & -0.2429557300 & 5.8142736561 & -0.4777784527 \\
\hline $\mathrm{C}$ & 5.8800710108 & 1.5684679125 & -2.2612043544 \\
\hline $\mathrm{C}$ & 5.7945769886 & -0.8090141953 & -1.7036350712 \\
\hline $\mathrm{H}$ & 5.5690007975 & -2.9011056077 & -1.2157519007 \\
\hline $\mathrm{C}$ & 2.7700478137 & -4.3206506804 & -0.7555350544 \\
\hline$C$ & 4.7782669904 & -4.1688643409 & 1.5426601241 \\
\hline $\mathrm{C}$ & 2.0326733493 & -2.9530002407 & 1.8900004553 \\
\hline $\mathrm{C}$ & 6.6977672538 & 4.0079437330 & 1.0764869068 \\
\hline $\mathrm{H}$ & 6.3742712699 & 1.9657076769 & 0.5353786853 \\
\hline $\mathrm{C}$ & 4.8228937959 & 5.5123941650 & 0.8897342896 \\
\hline $\mathrm{C}$ & -1.9232214230 & 3.9206380957 & 4826 \\
\hline $\mathrm{C}$ & -0.0380208857 & 2.4551968554 & -2.9748025852 \\
\hline $\mathrm{C}$ & -0.3277071997 & 3.0450674364 & 2.1947805029 \\
\hline $\mathrm{C}$ & -2.3984619583 & 3.2350831964 & 0.9373095227 \\
\hline $\mathrm{C}$ & 0.0933661406 & 6.5515625041 & -1.6268852442 \\
\hline $\mathrm{C}$ & -0.7061556313 & 6.5015036326 & 0.6543997485 \\
\hline $\mathrm{C}$ & 6.8695635246 & 1.3198202102 & -3.1806487795 \\
\hline $\mathrm{H}$ & 638693 & 38934 & 977461 \\
\hline $\mathrm{C}$ & 6.82 & 844606 & 7853 \\
\hline $\mathrm{C}$ & 3.1843343649 & -5.6496118714 & 54059 \\
\hline $\mathrm{C}$ & 1.8293687825 & 8414532 & 252188 \\
\hline $\mathrm{C}$ & 4.6725 & 57174 & 26016 \\
\hline $\mathrm{C}$ & 5.93 & -4.5 & 7549 \\
\hline $\mathrm{C}$ & 2.2042080555 & -2.0207778364 & 2.9284433739 \\
\hline $\mathrm{C}$ & 8371 & 9647 & 1.8 \\
\hline 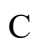 & 6.1421361279 & 6090 & 36727 \\
\hline $\mathrm{H}$ & 7.741 & 3.83 & 0162 \\
\hline $\mathrm{H}$ & 4.3764995089 & 6.5046415964 & 0.9986615818 \\
\hline $\mathrm{C}$ & -2.44 & 3.5 & 2908 \\
\hline $\mathrm{H}$ & -2.467 & 4.6 & 39741 \\
\hline $\mathrm{C}$ & -0.5572034638 & 336675 & -4.2330648164 \\
\hline . & 0.900 & & 51431 \\
\hline 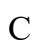 & 4442 & 2.7 & 0641 \\
\hline $\mathrm{H}$ & 0.7684652917 & 3.0 & 194011 \\
\hline $\mathrm{C}$ & -3.1311481744 & 2.9638810915 & 2.1047130907 \\
\hline 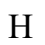 & -2.9 & & 5603 \\
\hline $\mathrm{C}$ & -0.0320819202 & 185 & -1.6416833640 \\
\hline $\mathrm{H}$ & 0.4548400532 & 6.0444626849 & -2.5264782281 \\
\hline $\mathrm{C}$ & -0.833 & & 350036 \\
\hline 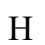 & -0.97 & 5.9 & 701 \\
\hline 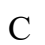 & 7.3606005819 & 0.0070163030 & -3.3754938856 \\
\hline $\mathrm{H}$ & 7.2769919485 & 2.1429859539 & -3.7716236426 \\
\hline 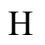 & 7.18 & -2.05 & -2.8113537752 \\
\hline$C$ & 55 & -6.4 & -1.99 \\
\hline $\mathrm{H}$ & 3.9042600101 & -6.1072678440 & -0.2553232394 \\
\hline $\mathrm{C}$ & 1.3237822587 & -4.5389306126 & -2.7012704682 \\
\hline-1 & & & -1.5170957703 \\
\hline$c$ & 2608 & 250719 & 505346 \\
\hline $\mathrm{H}$ & 3.7879539964 & -4.2044717941 & 3.4795484170 \\
\hline $\mathrm{C}$ & & & 1.4972053998 \\
\hline 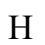 & 6.0509490434 & -4.3311803016 & -0.2142738327 \\
\hline$C$ & 1.2214824892 & -1.8646853398 & 3.9108765561 \\
\hline $\mathrm{H}$ & 3.0981581869 & -1.3884827789 & 2.9649766404 \\
\hline 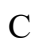 & -0.1302642821 & & 2.8289938052 \\
\hline 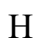 & 0.6765298655 & -4.4183904196 & 1.0271015823 \\
\hline $\mathrm{H}$ & 6.7612511121 & 6.1293032760 & 1.5612787527 \\
\hline $\mathrm{C}$ & -1.7599992003 & 2.6788322271 & -4.6684049563 \\
\hline $\mathrm{H}$ & -3.3831811966 & 4.0157038827 & -4.1805611843 \\
\hline
\end{tabular}




$\begin{array}{lrrr}\mathrm{H} & -0.0170055274 & 1.4202428571 & -4.8685333260 \\ \mathrm{C} & -2.4643477392 & 2.7407403900 & 3.3161485480 \\ \mathrm{H} & -0.5396835819 & 2.5666201810 & 4.2941384496 \\ \mathrm{H} & -4.2202223597 & 2.9383749648 & 2.0744318220 \\ \mathrm{C} & -0.4960298600 & 8.6184743313 & -0.5073801769 \\ \mathrm{H} & 0.2306453391 & 8.5041371847 & -2.5370633558 \\ \mathrm{H} & -1.1949694840 & 8.4166339995 & 1.5240746735 \\ \mathrm{H} & 8.1508059623 & -0.1764883090 & -4.1063191801 \\ \mathrm{C} & 1.7489411637 & -5.8588207229 & -2.8785874643 \\ \mathrm{H} & 3.0072486698 & -7.4424369656 & -2.1266517609 \\ \mathrm{H} & 0.5973863583 & -4.1037844755 & -3.3845561361 \\ \mathrm{C} & 6.8403725604 & -5.5764504310 & 2.8556238772 \\ \mathrm{H} & 5.5989539448 & -5.4299861537 & 4.6138304273 \\ \mathrm{H} & 7.8472907425 & -5.5628330022 & 0.9466139934 \\ \mathrm{C} & 0.0606104019 & -2.6425565734 & 3.8655631955 \\ \mathrm{H} & 1.3569226875 & -1.1297113963 & 4.6999915525 \\ \mathrm{H} & -1.0395225049 & -4.1516066277 & 2.7837428359 \\ \mathrm{H} & -2.1611338307 & 2.4198721900 & -5.6459511104 \\ \mathrm{H} & -3.0359670099 & 2.5491669515 & 4.2241433079 \\ \mathrm{H} & -0.5944562624 & 9.7021465276 & -0.5191546011 \\ \mathrm{H} & 1.3568264751 & -6.4521455429 & -3.7015735000 \\ \mathrm{H} & 7.6361725824 & -6.1180920915 & 3.3619994621 \\ \mathrm{H} & -0.7031091143 & -2.5246202961 & 4.6383926227\end{array}$

$\begin{array}{lrrr}\text { TS2c2 } & & & \\ \text { O 1 } & & & \\ \text { O } & -0.9487964326 & -3.4097662783 & -1.0164605411 \\ \text { C } & -2.2023096366 & -3.0306463661 & -1.2034189397 \\ \text { C } & -2.4695039059 & -1.6710843753 & -1.8975951342 \\ \text { C } & -2.9494450662 & -3.7308597205 & -2.5313056267 \\ \text { C } & -3.2183696367 & -2.3158634608 & -3.0924375825 \\ \text { H } & -1.5088235796 & -1.2237062805 & -2.1761424831 \\ \mathrm{H} & -3.0237205496 & -0.9338404763 & -1.3076693549 \\ \mathrm{H} & -2.1212576067 & -4.2759229689 & -2.9973600618 \\ \mathrm{H} & -3.8142329737 & -4.3976519786 & -2.4223842164 \\ \mathrm{C} & -2.5184744305 & -2.0685299779 & -4.4336243693 \\ \mathrm{H} & -2.9683815240 & -2.6773740311 & -5.2325543132 \\ \mathrm{H} & -2.6153969621 & -1.0095319586 & -4.7161281501 \\ \mathrm{H} & -1.4484087518 & -2.3127460864 & -4.3640612145 \\ \mathrm{C} & -4.7011522065 & -1.9961750863 & -3.2340563766 \\ \mathrm{C} & -5.2505997822 & -0.7904734304 & -2.7953684692 \\ \mathrm{C} & -5.5445865248 & -2.9075893388 & -3.8835816180 \\ \mathrm{C} & -6.6044052558 & -0.5100157779 & -2.9794661195 \\ \mathrm{H} & -4.6203724803 & -0.0505408512 & -2.2933132286 \\ \mathrm{C} & -6.8963026189 & -2.6330574380 & -4.0706231927 \\ \mathrm{H} & -5.1386095164 & -3.8537331119 & -4.2525433704 \\ \mathrm{C} & -7.4344290841 & -1.4304598258 & -3.6134285415 \\ \mathrm{H} & -7.0085820769 & 0.4381242328 & -2.6177078636 \\ \mathrm{H} & -7.5329297976 & -3.3617546763 & -4.5769310600 \\ \mathrm{C} & -3.2127784326 & -3.5770689313 & -0.2946850140 \\ \mathrm{C} & -2.8899892218 & -4.7965133358 & 0.4122074459 \\ \mathrm{H} & -2.1105928686 & -5.3997187215 & -0.0600029167 \\ \mathrm{H} & -3.7434002757 & -5.3594685638 & 0.8015439717 \\ \mathrm{C} & -4.5370155435 & -2.9614910640 & -0.0829942953 \\ \mathrm{C} & -4.6370223236 & -1.6939518094 & 0.5060164698 \\ \mathrm{C} & -5.7018201161 & -3.6552188798 & -0.4363363609 \\ \mathrm{C} & -5.8897146920 & -1.1270297927 & 0.7175926394 \\ \mathrm{H} & -3.7347216071 & -1.1660611510 & 0.8287476629 \\ \mathrm{C} & -6.9479369344 & -3.0648483637 & -0.2624834305 \\ \mathrm{H} & -5.6298039195 & -4.6486575687 & -0.8847305501\end{array}$




\begin{tabular}{|c|c|c|c|}
\hline & -7.0417670509 & -1.8001758851 & 0.31 \\
\hline $\mathrm{H}$ & -5.9635379336 & -0.1450732392 & 1888784877 \\
\hline 11 & -7.8480089708 & -3.5937611859 & -0.5787044163 \\
\hline $\mathrm{Mg}$ & 0.5253970492 & -2.6695871051 & -0.0123181843 \\
\hline $\mathrm{O}$ & 0.3494976826 & 0.3912842571 & 1.8573886287 \\
\hline $\mathrm{O}$ & 0.7549034139 & -0.7910886153 & -0.444427356 \\
\hline $\mathrm{O}$ & 1.1863826711 & -2.8904732237 & 1.8157439790 \\
\hline $\mathrm{C}$ & 0.8141578302 & -2.5319226749 & \\
\hline C & 1.6924470182 & -1.6969242470 & 3.8758010059 \\
\hline $\mathrm{N}$ & -0.3519776490 & -2.8351125277 & 3.5105556700 \\
\hline $\mathrm{C}$ & 0.7689839095 & -1.3941499243 & 5.0480172977 \\
\hline $\mathrm{H}$ & 2.0137788474 & -0.8103514387 & 3.31 \\
\hline $\mathrm{H}$ & 2.5928017238 & -2.2743956058 & 4.1415557045 \\
\hline $\mathrm{C}$ & -0.4852630883 & -2.2265036244 & 4.7529856747 \\
\hline $\mathrm{Br}$ & -1.9293922696 & -3.8794386736 & 71490 \\
\hline$U_{2}$ & 9813 & 36930 & 6.0 \\
\hline $\mathrm{H}$ & 0.4603342034 & 29540 & 26513 \\
\hline $\mathrm{O}$ & -1.4481575546 & -2.3180395542 & 5.4729488063 \\
\hline $\mathrm{H}$ & -8.49 & 2831 & 3249 \\
\hline $\mathrm{H}$ & 009 & & \\
\hline $\mathrm{P}$ & 5478 & 792 & \\
\hline $\mathrm{O}$ & 32543 & 9608 & \\
\hline $\mathrm{O}$ & 219 & 330 & 7337 \\
\hline C & $3.1 \mathrm{C}$ & & \\
\hline $\mathrm{C}$ & 0.7234710125 & 36893 & 5624 \\
\hline $\mathrm{C}$ & 2.95 & & \\
\hline $\mathrm{C}$ & 4.14 & 400 & 2384 \\
\hline$C$ & 3059 & 3.3 & -0.4 \\
\hline $\mathrm{C}_{\mathrm{S}}$ & -0.2404064130 & 2879809 & \\
\hline$C_{1}$ & 05 & & \\
\hline $\mathrm{C}$ & 52 & & 035 \\
\hline $\mathrm{Si}$ & 4.4925313943 & 198877 & 099743 \\
\hline $\mathrm{C}_{\mathrm{S}}$ & & & \\
\hline $\mathrm{C}$ & 0.16 & 325 & \\
\hline $\mathrm{Si}$ & -2.0 & 693 & \\
\hline$C$ & & & 871954 \\
\hline $\mathrm{C}$ & & & \\
\hline $\mathrm{H}$ & 5.58 & 656 & \\
\hline $\mathrm{C}$ & 4982 & 2963 & 02263 \\
\hline $\mathrm{C}$ & & & \\
\hline$C_{1}$ & 54 & & \\
\hline $\mathrm{C}$ & 111 & 136 & 397397 \\
\hline $\mathrm{C}$ & 1849 & 2168 & 0.5754926992 \\
\hline $\mathrm{H}$ & & & \\
\hline$C_{1}$ & 94 & & $2 .($ \\
\hline $\mathrm{C}$ & -2.9 & 954701 & 500 \\
\hline $\mathrm{C}$ & -2.886 & & -0.943 \\
\hline $\mathrm{C}$ & & & \\
\hline $\mathrm{H}$ & 55 & $4.5 \mathrm{~S}$ & -2.67 \\
\hline $\mathrm{C}$ & 5.2288171580 & 2.1451827096 & -4.0871030663 \\
\hline $\mathrm{C}$ & & & \\
\hline$C_{1}$ & & & \\
\hline $\mathrm{C}$ & 10922 & 1764852 & -1.935 \\
\hline $\mathrm{C}$ & 2.8847762025 & -3.8126242812 & 0.0190991163 \\
\hline $\mathrm{C}_{\mathrm{S}}$ & & & \\
\hline$f^{2}+2$ & & & \\
\hline $\mathrm{C}$ & 1862555594 & 6.3249120538 & 0.2996487547 \\
\hline $\mathrm{H}$ & 4.5583703480 & 4.3892709348 & -0.5215613920 \\
\hline & & 60?24444200 & \\
\hline & & $1.2+37+2$ & \\
\hline
\end{tabular}




\begin{tabular}{|c|c|c|c|}
\hline $\mathrm{C}$ & -1.3725107476 & 1.8784944310 & 2.9849181878 \\
\hline $\mathrm{C}$ & -3.1591946166 & 6.0155494963 & 0.3178480782 \\
\hline & -3.3146873528 & 5.1549709793 & 2.5718677043 \\
\hline & -2.1918413711 & 2.2575696625 & -1.9794660933 \\
\hline 乙 & -4.2455656056 & 3.2096206860 & -1.1372099216 \\
\hline$y$ & 4.9422259688 & 3.3092662840 & -4.7547313205 \\
\hline $\mathrm{H}$ & 3.7230536305 & 5.1094933183 & -4.7792600164 \\
\hline 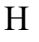 & 5.9701883656 & 1.4463167584 & -4.4839596925 \\
\hline $\mathrm{C}$ & 5.6144971861 & -2.5578182816 & 3.5641257572 \\
\hline $\mathrm{H}$ & 5.5757538034 & -3.4233734354 & 1.5992470684 \\
\hline$c$ & 4.8164437397 & -0.2836738671 & 3.7576202259 \\
\hline $\mathrm{H}$ & 4.1071129663 & 0.6474395982 & 1.9512493892 \\
\hline $\mathrm{C}$ & 1.5195026542 & -3.4139244905 & -2.3974764981 \\
\hline $\mathrm{H}$ & 2.5666015416 & -1.5322205638 & -2.5196365251 \\
\hline $\mathrm{C}$ & 1.9651038896 & 7111025 & -0.4369414074 \\
\hline $\mathrm{H}$ & 3.3911553766 & -3.9825503544 & 0.9760215198 \\
\hline $\mathrm{C}$ & 8.5064791984 & -2.0079551226 & -1.1751277413 \\
\hline $\mathrm{H}$ & 7.3398023996 & -0.7915783903 & 90568 \\
\hline $\mathrm{C}$ & 7.2759782316 & -3.5353561875 & -2.5911466778 \\
\hline $\mathrm{H}$ & 5.1361414084 & 345612 & -2.3771611968 \\
\hline $\mathrm{C}$ & 3.2357801527 & 7.2199321937 & 0.8451185268 \\
\hline $\mathrm{H}$ & 5.2304017776 & 6.6326817716 & 50289 \\
\hline $\mathrm{H}$ & 1.1868 & 7.49 & 4049 \\
\hline $\mathrm{C}$ & -3.8114952240 & 0.51 & 503121 \\
\hline $\mathrm{H}$ & & & 7633 \\
\hline $\mathrm{C}$ & -1.6175 & 0.95 & 817 \\
\hline $\mathrm{H}$ & -0.4018904711 & 9374 & 5218 \\
\hline $\mathrm{C}$ & -3.7813 & 7.19 & 1066 \\
\hline $\mathrm{H}$ & -2.8643957498 & 5.905947 & -0.729 \\
\hline $\mathrm{C}$ & & & 8608 \\
\hline $\mathrm{H}$ & -3.1449 & 4.36 & 7944 \\
\hline $\mathrm{C}$ & -2.8295551542 & 1.9566 & 71345 \\
\hline $\mathrm{H}$ & -1.1361093745 & 1.98 & -1.86 \\
\hline $\mathrm{C}$ & -4.8843528418 & 2.9085988583 & 84152 \\
\hline $\mathrm{H}$ & -4.8212792319 & 3.7030346405 & 020436 \\
\hline $\mathrm{H}$ & 5.45 & 3.55 & -5.68 \\
\hline $\mathrm{C}$ & 5.3520753875 & -1.4291800208 & 62500 \\
\hline $\mathrm{H}$ & & & \\
\hline $\mathrm{H}$ & 4.6112 & 0.59 & 3401 \\
\hline $\mathrm{C}$ & 1.2939 & -4.58 & -1.65 \\
\hline $\mathrm{H}$ & 0.9774 & -3.2519677627 & -3.3283659063 \\
\hline $\mathrm{H}$ & 1.7889901495 & -5.6768232819 & 0.1510195063 \\
\hline $\mathrm{C}$ & 8.49262 & -2.9958904325 & -2.1 \\
\hline $\mathrm{H}$ & 9.4523613070 & -1.58 & -0.8 \\
\hline $\mathrm{H}$ & 7.2619104169 & -4.30 & -3.35 \\
\hline $\mathrm{H}$ & 3.54600 & 8.21 & 3703 \\
\hline $\mathrm{C}$ & -2.8285454030 & 0.2681953719 & 4.0675501683 \\
\hline $\mathrm{H}$ & -4.7591125661 & -0.0148689080 & 3.1565352377 \\
\hline $\mathrm{H}$ & -0.8382817609 & 0.7571317998 & 4.7513312900 \\
\hline $\mathrm{C}$ & -4.1716085161 & 7.3637535894 & 2.0581998449 \\
\hline $\mathrm{H}$ & -3.9632101697 & 7544607 & 03563 \\
\hline $\mathrm{H}$ & -4.2408205409 & 6.4635513119 & 4.0182147232 \\
\hline $\mathrm{C}$ & -4.1732470427 & 2.2890291383 & -3.3772935476 \\
\hline $\mathrm{H}$ & -2.2716901903 & 1.4694466539 & -3.9863434582 \\
\hline $\mathrm{H}$ & -5.9312653111 & 3.1660053513 & -2.4838704542 \\
\hline $\mathrm{H}$ & 5.5684236247 & -1.4446273932 & 5.4154685017 \\
\hline $\mathrm{H}$ & 0.5850664652 & -5.3295868916 & -2.0149098961 \\
\hline 7. & 9.4270148350 & -3.3441456022 & -2.6007234701 \\
\hline $\mathrm{H}$ & -2.9989541300 & -0.4744894757 & 4.8510981669 \\
\hline U & -4.6560299906 & 8.2849586374 & 2.374844417 \\
\hline
\end{tabular}




\begin{tabular}{|c|c|c|c|}
\hline $\mathrm{H}$ & -4.6661359775 & 2.0646121532 & -4.3211196944 \\
\hline \multicolumn{4}{|c|}{$\mathrm{TS} 2 \mathrm{~d} 1$} \\
\hline 01 & & & \\
\hline $\mathrm{C}$ & 3.9743791950 & 1.3730508631 & -1.1950737590 \\
\hline $\mathrm{C}$ & 4.9092448584 & 2.2189133728 & -2.1142403448 \\
\hline $\mathrm{C}$ & 5.7667653723 & 2.7574345809 & -0.9415658170 \\
\hline $\mathrm{H}$ & 5.4439382030 & 1.6903201553 & -2.9085991011 \\
\hline $\mathrm{H}$ & 4.2779626542 & 2.9885011097 & -2.5705024771 \\
\hline $\mathrm{C}$ & 4.2636022232 & -0.0527124087 & -0.9359129099 \\
\hline $\mathrm{C}$ & 3.1698796103 & -0.8787270724 & -0.4740913301 \\
\hline $\mathrm{H}$ & 2.2484757369 & -0.6811548290 & -1.0424992544 \\
\hline $\mathrm{H}$ & 3.4014813258 & -1.9470580761 & -0.4055214840 \\
\hline $\mathrm{O}$ & 1.0332128519 & 2.5829179615 & 1.7305551168 \\
\hline $\mathrm{C}$ & 0.8614637854 & 1.9440030699 & 2.8107933184 \\
\hline $\mathrm{C}$ & -0.3282306404 & 2.1997726766 & 3.7091093951 \\
\hline $\mathrm{N}$ & 1.6587331414 & 0.9926514197 & 3.2578095448 \\
\hline $\mathrm{C}$ & -0.2255553297 & 1.0711941166 & 4.7253561249 \\
\hline $\mathrm{H}$ & -0.2509563749 & 3.2087436014 & 4.1431990881 \\
\hline $\mathrm{H}$ & -1.2411333198 & 2.1750738382 & 3.0952760171 \\
\hline $\mathrm{C}$ & 1.1189841365 & 0.4149178627 & 4.4040049360 \\
\hline $\mathrm{Br}$ & 2.6745404320 & -0.1944255350 & 1.3880788261 \\
\hline $\mathrm{H}$ & -1.0000139491 & 0.3087846311 & 4.5650608430 \\
\hline $\mathrm{H}$ & -0.2565896151 & 1.3826597970 & 5.7777911308 \\
\hline $\mathrm{O}$ & 1.6264764372 & -0.4812657434 & 5.0305797685 \\
\hline $\mathrm{O}$ & 2.6944657661 & 1.7202879667 & -1.2168235170 \\
\hline $\mathrm{C}$ & 4.8572508813 & 2.0249875843 & 0.0631398015 \\
\hline $\mathrm{H}$ & 4.1404501075 & 2.6635348713 & 0.5933068976 \\
\hline $\mathrm{H}$ & 5.3220874557 & 1.3440349443 & 0.7820212811 \\
\hline $\mathrm{C}$ & 5.7068265390 & 4.2804916882 & -0.7846725284 \\
\hline $\mathrm{H}$ & 6.2284993614 & 4.7772986675 & -1.6168046520 \\
\hline $\mathrm{H}$ & 6.1920108395 & 4.5908473617 & 0.1532465589 \\
\hline $\mathrm{H}$ & 4.6636395893 & 4.6300251107 & -0.7685373141 \\
\hline $\mathrm{C}$ & 7.2242806411 & 2.3174159901 & -0.9755947077 \\
\hline $\mathrm{C}$ & 7.9598203768 & 2.4777641615 & -2.1572760042 \\
\hline $\mathrm{C}$ & 7.8838687539 & 1.8123427164 & 0.1473211084 \\
\hline $\mathrm{C}$ & 9.3033057495 & 2.1199829300 & -2.2240114516 \\
\hline $\mathrm{H}$ & 7.4714777066 & 2.8866820034 & -3.0462918570 \\
\hline $\mathrm{C}$ & 9.2292385513 & 1.4490979533 & 0.0850235153 \\
\hline $\mathrm{H}$ & 7.3473107669 & 1.6870673447 & 1.0901075078 \\
\hline $\mathrm{C}$ & 9.9435726402 & 1.5963009050 & -1.1010516705 \\
\hline $\mathrm{H}$ & 9.8539049061 & 2.2506672182 & -3.1582575418 \\
\hline $\mathrm{H}$ & 9.7192312750 & 1.0455489202 & 0.9737653138 \\
\hline $\mathrm{H}$ & 10.9960663627 & 1.3096032514 & -1.1499505714 \\
\hline $\mathrm{C}$ & 5.6078493641 & -0.6539685142 & -0.9436558076 \\
\hline $\mathrm{C}$ & 6.4487604937 & -0.6081649244 & -2.0641675230 \\
\hline $\mathrm{C}$ & 6.0452736856 & -1.3322134861 & 0.2100198733 \\
\hline $\mathrm{C}$ & 7.7044955142 & -1.2056685022 & -2.0254002628 \\
\hline $\mathrm{H}$ & 6.1131580182 & -0.1355612383 & -2.9853416368 \\
\hline $\mathrm{C}$ & 7.3137194456 & -1.8956974029 & 0.2541044909 \\
\hline $\mathrm{H}$ & 5.3963747207 & -1.3815391562 & 1.0878873931 \\
\hline $\mathrm{C}$ & 8.1466462419 & -1.8318239621 & -0.8633164151 \\
\hline $\mathrm{H}$ & 8.3460703585 & -1.1660414108 & -2.9066474935 \\
\hline $\mathrm{H}$ & 7.6515405630 & -2.3915128375 & 1.1656736189 \\
\hline $\mathrm{H}$ & 9.1425876202 & -2.2770881102 & -0.8288515875 \\
\hline $\mathrm{Mg}$ & 1.1224542170 & 1.9760033087 & -0.1342023617 \\
\hline $\mathrm{O}$ & -0.4446524512 & 0.8968160000 & -0.4799201797 \\
\hline $\mathrm{P}$ & -1.2718725785 & -0.0107904292 & 0.4300282775 \\
\hline $\mathrm{O}$ & -0.7508082886 & -0.4595184290 & 1.7414120891 \\
\hline $\mathrm{O}$ & -2.7362108199 & 0.6880839554 & 0.6942150255 \\
\hline
\end{tabular}




\begin{tabular}{|c|c|c|c|}
\hline & 017301493 & -1.2218389075 & 917 \\
\hline & -3.6460243171 & 0.8417024727 & -0.3187065648 \\
\hline $\mathrm{C}$ & -2.6924575856 & -2.0846237017 & -0.19163398 \\
\hline $\mathrm{C}$ & -4.2886911546 & -0.2837564516 & -0.8037898374 \\
\hline $\mathrm{C}$ & -3.9150703769 & 2.1504740066 & -0.8035767218 \\
\hline & -3.9971590807 & -1.6187886329 & -0.2093177604 \\
\hline $\mathrm{C}$ & -2.3510647035 & -3.4069070491 & 0.2084169084 \\
\hline $\mathrm{C}$ & -5.1933408278 & -0.1356844153 & -1.9071858192 \\
\hline $\mathrm{C}$ & -4.8373612418 & 2.2961220890 & -1.8142215677 \\
\hline $\mathrm{Si}$ & -3.0376083354 & 3.6678636977 & -0.1049235627 \\
\hline $\mathrm{C}$ & -5.0302725417 & -2.4356162562 & 0.3556585198 \\
\hline C & -3.3616079178 & -4.2076634801 & 0.6907228068 \\
\hline G. & -0.6639032866 & -4.2411427808 & 0.0036459667 \\
\hline $\mathrm{C}$ & -5.7995322237 & -1.2469835389 & -2.5592890280 \\
\hline $\mathrm{C}$ & -5.4815999120 & 1.1706193543 & -2.3979982770 \\
\hline $\mathrm{H}$ & -5.0894527920 & 3.2868252204 & 0593 \\
\hline$C$ & -3.2234693644 & 3.8469041009 & 174015700 \\
\hline 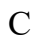 & -1.2383605455 & 3.6849875761 & -0.6376126549 \\
\hline $\mathrm{C}$ & -3.8423987880 & 5.1800408770 & 6595691 \\
\hline $\mathrm{C}$ & -6.3694573386 & -1.9815329398 & 00820 \\
\hline C & -4.7009411739 & -3.7428288767 & 0.81 \\
\hline $\mathrm{H}$ & -3.1630790812 & -5.2398801622 & 1.0070421694 \\
\hline $\mathrm{C}$ & 0.3019371472 & 5992 & 285306 \\
\hline $\mathrm{C}$ & 2521033 & 5396 & 24910 \\
\hline $\mathrm{C}$ & 0.4720475476 & 19863 & 16429 \\
\hline $\mathrm{C}$ & -6.6666946963 & 228537 & 5580785 \\
\hline $\mathrm{H}$ & -5.5632846987 & 344002 & 03059 \\
\hline $\mathrm{C}$ & -6.3925512689 & 3602 & 42284 \\
\hline $\mathrm{C}$ & -2.9414302887 & 5.0973491419 & 2.3322395660 \\
\hline $\mathrm{C}$ & -3.65605 & 3755 & 02701 \\
\hline te & -0.8750894163 & 3.0608899929 & -1.8471033239 \\
\hline C & -0.2625980900 & 2496 & 303 \\
\hline $\mathrm{C}$ & -5.0846085820 & 5.6179860976 & -0.3913870098 \\
\hline $\mathrm{C}$ & 408 & 3333 & 73688 \\
\hline $\mathrm{C}$ & -7.32 & 09914 & 24138 \\
\hline $\mathrm{H}$ & -6.6306833605 & -0.9710973219 & 0.2004210113 \\
\hline $\mathrm{C}$ & 6880 & -4.56 & 392658 \\
\hline te & 1.084 & 6310 & 384922 \\
\hline $\mathrm{C}$ & 0.3370121224 & -3.2674989967 & 2.4871700533 \\
\hline $\mathrm{C}$ & -1.1558773712 & -6.2173507217 & -1.9988311416 \\
\hline$C$ & -1.3530618398 & 7309136 & 0.2668506177 \\
\hline $\mathrm{C}$ & 0.08 & 4244745 & 978371 \\
\hline $\mathrm{C}$ & 1.7859757881 & -4.0220216846 & -1.3496732219 \\
\hline $\mathrm{C}$ & -6.9790364673 & 0.2354690944 & -4.0706741747 \\
\hline $\mathrm{H}$ & -7.116 & -1.93 & -4.0941354911 \\
\hline $\mathrm{H}$ & -6.6 & 002614 & -3.835 \\
\hline $\mathrm{C}$ & -3.0809810332 & 5.2898839432 & 3.7094872098 \\
\hline $\mathrm{H}$ & -2.6 & & 1.7144897898 \\
\hline $\mathrm{C}$ & -3.801 & 2.99 & 39560408213 \\
\hline $\mathrm{H}$ & -3.8768292848 & 1.8087753375 & 2.1599275470 \\
\hline $\mathrm{C}$ & 0.4225931273 & 3.1997465657 & -2.3610870081 \\
\hline $\mathrm{H}$ & -1.5962007926 & & -2.3974529235 \\
\hline $\mathrm{C}$ & 1.0399145473 & 4.5411706788 & -0.432055361 \\
\hline $\mathrm{H}$ & -0.4954773861 & 4.8482333895 & 1.0476145070 \\
\hline $\mathrm{C}$ & -5.7243839383 & 6.7255420686 & -0.9572719773 \\
\hline $\mathrm{H}$ & -5.5652368260 & 5.1000478745 & \\
\hline $\mathrm{C}$ & .8947675270 & 6.9838921486 & -2.51863957 \\
\hline $\mathrm{H}$ & -2.2893092893 & 5.5619865676 & -2.3556550354 \\
\hline $\mathrm{C}$ & -7.0049463079 & -4.1027441875 & 1.5003478 \\
\hline $\mathrm{H}$ & -8.3478663036 & -2.4212237961 & $1.19259562 \mathrm{C}$ \\
\hline
\end{tabular}




\begin{tabular}{lr}
$\mathrm{H}$ & -5.4516590481 \\
$\mathrm{C}$ & 1.8731407487 \\
$\mathrm{H}$ & 1.0893608407 \\
$\mathrm{C}$ & 1.1385337457 \\
$\mathrm{H}$ & -0.2500145729 \\
$\mathrm{C}$ & -1.4921535595 \\
$\mathrm{H}$ & -0.9511466966 \\
$\mathrm{C}$ & -1.6876337904 \\
$\mathrm{H}$ & -1.3044449915 \\
$\mathrm{C}$ & 0.9823663703 \\
$\mathrm{H}$ & -0.9306271135 \\
$\mathrm{C}$ & 2.6802590012 \\
$\mathrm{H}$ & 2.1270939799 \\
$\mathrm{H}$ & -7.6754536908 \\
$\mathrm{C}$ & -3.5129265521 \\
$\mathrm{H}$ & -2.8593330653 \\
$\mathrm{H}$ & -4.1374873151 \\
$\mathrm{C}$ & 1.3782716838 \\
$\mathrm{H}$ & 0.6937222457 \\
$\mathrm{H}$ & 1.7778050611 \\
$\mathrm{C}$ & -5.1296100839 \\
$\mathrm{H}$ & -6.6848103545 \\
$\mathrm{H}$ & -3.4285642090 \\
$\mathrm{H}$ & -7.7770656305 \\
$\mathrm{C}$ & 1.9018945178 \\
$\mathrm{H}$ & 2.4659672602 \\
$\mathrm{H}$ & 1.1748006028 \\
$\mathrm{C}$ & -1.7576453617 \\
$\mathrm{H}$ & -1.5465204546 \\
$\mathrm{H}$ & -1.8931770373 \\
$\mathrm{C}$ & 2.2785023083 \\
$\mathrm{H}$ & 0.6635419307 \\
$\mathrm{H}$ & 3.6838448765 \\
$\mathrm{H}$ & -3.6251890834 \\
$\mathrm{H}$ & 2.3788918948 \\
$\mathrm{H}$ & -5.6269400811 \\
$\mathrm{H}$ & 2.5201696407 \\
$\mathrm{H}$ & -2.0181090115 \\
$\mathrm{H}$ & 2.9694895487 \\
& \\
\hline
\end{tabular}

$-5.5675206255$

$-5.5423743518$

$-6.3447925398$

$-3.3189854186$

$-2.3527534029$

$-7.4865956427$

$-5.4180427840$

$-8.3018188411$

$-6.8791783081$

$-2.1703331943$

$-2.1701327887$

$-3.6174262586$

$-4.7448894410$

0.3663741851

4.2368614691

6.2598219691

2.1709551674

3.9631529489

2.7163863082

5.1177394884

7.4098568558

7.0561106091

7.5149548932

$-4.7394247054$

$-4.4535080735$

$-6.4275500896$

$-2.4564700092$

$-8.5305959561$

$-7.6590234767$

$-9.1116901455$

$-2.6883767187$

$-1.4417367355$

$-4.0337617042$

4.3875928166

4.0929941616

8.2721880378

$-4.4896964349$

$-9.5171071375$

$-2.3781271508$
1.7107748651

3.0310943063

1.2033160127

3.6358772882

2.3024469214

$-2.4849042557$

$-2.7163600786$

$-0.2170990548$

1.3481932902

$-3.2757109558$

$-2.2896724637$

$-2.3459728355$

$-0.6018820699$

$-4.9015053079$

4.5239019457

4.1486982875

4.5838509504

$-1.6671219273$

$-3.2997552734$

0.1251841002

$-2.0222712947$

$-0.5672802178$

$-3.3455934163$

1.9370180984

3.9113116610

3.2437950513

4.3102046295

$-1.5948777890$

$-3.5574518922$

0.4791822226

$-3.3118535898$

$-4.0180254760$

$-2.3759464716$

5.5958367568

$-2.0838289624$

$-2.4617877173$

4.8058927121

$-1.9717370377$

$-4.0913977601$

TS2d2

01

C $\quad-4.2652531333$

$-0.7343638590$

$-0.2046975305$

$-0.0450557223$

1.0947550420

$-1.2847523734$

1.9898071325

C $\quad-4.5395358131$

0.3549335068

1.0781022188

0.7782119976

1.3013304376

$\mathrm{H} \quad-4.0888441907$

$-1.3886044028$

$-1.1322371302$

$-5.2086080039$

$-1.5651653367$

$-2.4942536962$

C $\quad-4.7721401116$

$-0.6654144609$

$-2.8504950100$

$-4.2456977119$

$-1.9548913882$

$-3.1782989055$

$-0.4033263884$

$-0.9540504266$

$\mathrm{Mg} \quad-1.2745580064$

0.9294592583

0.2862624623

$-0.2366523818$

$-0.0531303745$

0.2862624623
-1.7653414704

$-2.2439273771$

$-0.4074985958$

$-0.7696439756$

$-3.4002556606$

$-0.8287463062$

$-0.5327795343$

$-4.2683597381$

$-0.2310634179$

C $\quad 0.5525930091$

$-4.0101510615$

$-1.8127891069$

$-1.1843457446$

$-5.5642656613$

$-1.0250176426$

C $\quad 0.4420427563$

H $\quad 0.3898852234$

$-4.3692473584$

0.8529071043 


\begin{tabular}{|c|c|c|c|}
\hline $\mathrm{H}$ & 5195 & -3.7469458329 & 2513 \\
\hline C & -0.7092698870 & -5.3037507791 & -2.0026364280 \\
\hline & -3.1794387142 & -2.8920165831 & -2.3895170513 \\
\hline & 1.3486739208 & -5.8006247760 & -1.60274479 \\
\hline $\mathrm{H}$ & 0.2033363961 & -6.4471406510 & -0.4162348110 \\
\hline 0 & -1.1414563094 & -6.0930301002 & \\
\hline $\mathrm{O}$ & -3.1989755552 & -0.1724273492 & -0.7613668111 \\
\hline $\mathrm{C}$ & -3.9462333102 & -2.0419932382 & 0.7866661654 \\
\hline $\mathrm{H}$ & -2.8533570127 & -2.1319392532 & 0.7860739592 \\
\hline $\mathrm{H}$ & -4.3821494820 & -3.0099940236 & 0.5214565816 \\
\hline $\mathrm{C}$ & -3.5032732784 & -1.0474162973 & 3.0934427997 \\
\hline $\mathrm{H}$ & -3.9028812021 & -0.3676330970 & 3.8613315385 \\
\hline 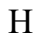 & -3.2401725750 & -1.9970288146 & 4830 \\
\hline $\mathrm{H}$ & -2.5853800786 & -0.6055555411 & 2.6769705274 \\
\hline $\mathrm{C}$ & -5.8019019926 & -1.8769565331 & 2.6000479252 \\
\hline$C$ & -6.7053060673 & -1.0339613536 & 3.2605996202 \\
\hline $\mathrm{C}$ & -6.0758555964 & -3.2466 & \\
\hline $\mathrm{C}$ & -7.8582295165 & -1.5377622529 & 4462 \\
\hline $\mathrm{H}$ & -6.5056734848 & 0.0404406538 & 3.3058367900 \\
\hline $\mathrm{C}$ & -7.2309716480 & 00628 & 62320 \\
\hline $\mathrm{H}$ & -5.3869684302 & 150514 & 0575 \\
\hline , & -8.1300121908 & -2.9049597839 & 3.807 \\
\hline $\mathrm{H}$ & -8.5489810596 & -0.8589170573 & 22625 \\
\hline $\mathrm{H}$ & -7.4277382392 & -4.83 & 2826 \\
\hline 11 & -9.0 & -3.30 & \\
\hline $\mathrm{C}$ & -6.4761160093 & -2.0269944479 & -0.7335874914 \\
\hline C & 15483 & 21578 & 23471 \\
\hline C & 6017295 & 0502 & 12223 \\
\hline $\mathrm{C}$ & 5699 & -1.97 & \\
\hline $\mathrm{H}$ & -7.3938632972 & -0.2677275066 & 0.1343038530 \\
\hline $\mathrm{C}$ & 5146 & -4.0 & 63426 \\
\hline $\mathrm{H}$ & -5.8 & -3.93 & 95050 \\
\hline $\mathrm{C}$ & -8.8442021073 & -3.3335256021 & -0.0065414028 \\
\hline $\mathrm{H}$ & -9.4711403926 & 441668 & 0.7756220481 \\
\hline & -7.9 & 566 & 08740 \\
\hline $\mathrm{H}$ & -9.7613651994 & -3.84 & 95576 \\
\hline $\mathrm{P}$ & 0.9990570683 & 0.8728275564 & -0.5974752354 \\
\hline $\mathrm{O}$ & 2.2 & & 65190 \\
\hline $\mathrm{O}$ & 1.48 & 2.31 & 239440 \\
\hline $\mathrm{C}$ & 3.5713812778 & 0.8549206540 & 0.0885974827 \\
\hline $\mathrm{C}$ & & 3.2781770541 & -0.1903389047 \\
\hline $\mathrm{C}$ & 3.8 & 377 & 73593 \\
\hline 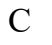 & 4.5463290659 & -0.1618092774 & 96201 \\
\hline $\mathrm{C}$ & 2.8849218308 & 3.2216189189 & 0.5006870622 \\
\hline$C$ & 0.67 & & 0.0756493911 \\
\hline $\mathrm{C}$ & 5.21 & 2.60 & -0.2 \\
\hline$C$ & 5.8271151184 & 0.2370192311 & -0.4496898011 \\
\hline $\mathrm{Si}$ & 4.3202229745 & -2.0313634817 & 0.0596148546 \\
\hline$C$ & & & \\
\hline $\mathrm{C}$ & 0.890 & 5.13 & 1.0869987882 \\
\hline $\mathrm{Si}$ & -0.9823236163 & 4.2581834383 & -0.8372858258 \\
\hline $\mathrm{C}$ & 5.5878843823 & 3.9695867004 & -0.4575114059 \\
\hline$C_{1}$ & & 1.6063638080 & -0.5686731300 \\
\hline $\mathrm{H}$ & 6.6232029151 & -0.4978300566 & -0.6283640817 \\
\hline $\mathrm{C}$ & 3.2439031395 & -2.5504753865 & 1.5052143507 \\
\hline $\mathrm{C}$ & 3.6700685127 & -2.8551099738 & -1.4896789412 \\
\hline$C$ & 6.0397349158 & -2.7188375064 & 04262067771 \\
\hline $\mathrm{C}$ & 4.2234657838 & 4.0744763716 & 2.443060612 \\
\hline $\mathrm{C}$ & 2.0794094885 & 5.1026239355 & 1.8698627847 \\
\hline $\mathrm{H}$ & 0.1327168146 & 5.8834168031 & 1.33978077 \\
\hline
\end{tabular}




\begin{tabular}{|c|c|c|c|}
\hline & 081746007 & 4.1355238089 & 0.4774288606 \\
\hline $\mathrm{C}$ & -1.1379380368 & 5.8913273016 & -1.7516655432 \\
\hline $\mathrm{C}$ & -1.1258746222 & 2.9047029639 & 182564317 \\
\hline $\mathrm{C}$ & 6.8595461540 & 4.3126427066 & -0.8474620547 \\
\hline 11 & 4.8471616611 & 4.7500975464 & -0.2831957491 \\
\hline $\mathrm{C}$ & 7.4995571084 & 1.9919483378 & -0.9591370867 \\
\hline 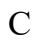 & 3.2197020742 & -3.9277452163 & 1.8066666867 \\
\hline $\mathrm{C}$ & 2.4860548657 & -1.6790790991 & 2.2973493855 \\
\hline $\mathrm{C}$ & 2.8184587107 & -2.1933631778 & -2.3853429106 \\
\hline $\mathrm{C}$ & 3.9926676575 & -4.2040891702 & -1.7383939770 \\
\hline $\mathrm{C}$ & 6.4908115808 & -2.7859406352 & 1.7545126196 \\
\hline $\mathrm{C}$ & 6.8989755380 & -3.1442244314 & -0.6007933052 \\
\hline $\mathrm{C}$ & 4.3730261990 & 4.9603905483 & 3.4820090414 \\
\hline $\mathrm{H}$ & 4.9831597752 & 3.3123555800 & 2.2662149515 \\
\hline $\mathrm{C}$ & 2.2697107015 & 6.0109629010 & 2.9470451734 \\
\hline $\mathrm{C}$ & -3.2824265640 & 7837839 & 234668 \\
\hline $\mathrm{C}$ & -2.2800766528 & 3.0329210091 & 1.3499655107 \\
\hline $\mathrm{C}$ & -2.2816566665 & 6.0932859311 & -2.5462413248 \\
\hline $\mathrm{C}$ & -0.1670693704 & 6.9017381713 & -1.6969387929 \\
\hline$C$ & -0.1824727047 & 7786294 & 53008 \\
\hline $\mathrm{C}$ & -2.0997908593 & 891 & -2.0594873069 \\
\hline $\mathrm{C}$ & 7.8337685877 & 3.3162807132 & -1.0923789295 \\
\hline $\mathrm{H}$ & 9025 & 3231 & 4181 \\
\hline $\mathrm{H}$ & 8.23 & 1.20 & 6807 \\
\hline $\mathrm{C}$ & 2.4488253018 & 124535 & 063802 \\
\hline $\mathrm{H}$ & 3.8077507485 & -4.6377073257 & 1.2180837653 \\
\hline $\mathrm{C}$ & 8506 & -2.1 & 998 \\
\hline $\mathrm{H}$ & 5927 & -0.60 & 358 \\
\hline $\mathrm{C}$ & 2.2893494928 & -2.8649000019 & -3.4965496893 \\
\hline 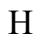 & 554128 & 0959 & 29161 \\
\hline $\mathrm{C}$ & 3.4760822791 & 5393 & 07832 \\
\hline $\mathrm{H}$ & 4.6553542757 & -4.7526163327 & -1.0642272269 \\
\hline $\mathrm{C}$ & 7.7697497349 & -3.2725332430 & 2.0501557198 \\
\hline 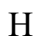 & 307 & -2 & 345 \\
\hline $\mathrm{C}$ & 8.176 & 728 & 9800 \\
\hline $\mathrm{H}$ & 6.5798679442 & 089970 & -1.6456987038 \\
\hline $\mathrm{C}$ & 1581 & & 67607 \\
\hline 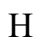 & 6233 & 4.8 & 0284 \\
\hline $\mathrm{H}$ & 1.4955879464 & 9355 & 73150 \\
\hline $\mathrm{C}$ & -4.2085836061 & 5.0264841799 & 1.7123592488 \\
\hline 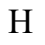 & 7198 & 35775 & 0.006 \\
\hline $\mathrm{C}$ & -3.19 & 2.93 & 0241 \\
\hline $\mathrm{H}$ & -1.5355717483 & 2.2275951597 & 1.2186302581 \\
\hline $\mathrm{C}$ & -2.4531839220 & 7.2842013184 & -3.2586081599 \\
\hline 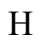 & -3.04 & 5.31 & \\
\hline $\mathrm{C}$ & -0.33 & 8.09 & 23297 \\
\hline $\mathrm{H}$ & 0.7398961709 & 6.7724176646 & -1.1024294969 \\
\hline $\mathrm{C}$ & -0.1704200667 & 1.835 & -4.0930086040 \\
\hline . & 0.57 & 3.66 & 12001 \\
\hline $\mathrm{C}$ & -2.0963737081 & 0.8522835957 & -3.0049195702 \\
\hline $\mathrm{H}$ & -2.8905101529 & 1.9128687511 & -1.2899983984 \\
\hline $\mathrm{H}$ & & & -1.3960028384 \\
\hline 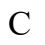 & 1.6884110523 & -3.5334958545 & 3.6418386901 \\
\hline $\mathrm{H}$ & 2.4422652798 & -5.4795151983 & 3.0881589076 \\
\hline $\mathrm{H}$ & 1.1188803603 & -1.4748418815 & 3.9542588935 \\
\hline$C$ & 2.6167863226 & -4.2005642303 & -3.7326082839 \\
\hline $\mathrm{H}$ & 1.6119551384 & -2.3374728889 & -4.1672479377 \\
\hline $\mathrm{H}$ & 3.7412739950 & -5.9078539586 & -3.0392719221 \\
\hline $\mathrm{C}$ & 8.6134985297 & -3.6972213237 & 1.0202348497 \\
\hline $\mathrm{H}$ & 8.1059165506 & -3.3204553338 & 3.0837633827 \\
\hline
\end{tabular}




$\begin{array}{lrrr}\mathrm{H} & 8.8303630694 & -3.9623295572 & -1.1108808113 \\ \mathrm{H} & 3.5251580949 & 6.6491321873 & 4.5608524046 \\ \mathrm{C} & -4.1627619956 & 3.9347756690 & 2.5838904293 \\ \mathrm{H} & -4.9588015417 & 5.8012623541 & 1.8492355940 \\ \mathrm{H} & -3.1521683590 & 2.0923450450 & 3.0839443128 \\ \mathrm{C} & -1.4810550950 & 8.2878855206 & -3.1899974246 \\ \mathrm{H} & -3.3411622808 & 7.4293428190 & -3.8698777346 \\ \mathrm{H} & 0.4231783844 & 8.8704628102 & -2.3595352647 \\ \mathrm{C} & -1.1124425564 & 0.8110847227 & -4.0049452468 \\ \mathrm{H} & 0.5903482947 & 1.8134897760 & -4.8707098583 \\ \mathrm{H} & -2.9145561940 & 0.1134465723 & -3.0114933926 \\ \mathrm{H} & 1.0868953819 & -3.9126650829 & 4.4650546496 \\ \mathrm{H} & 2.2030584913 & -4.7223178362 & -4.5942314111 \\ \mathrm{H} & 9.6068175076 & -4.0774190367 & 1.2495786142 \\ \mathrm{H} & -4.8732130840 & 3.8624559812 & 3.4043560286 \\ \mathrm{H} & -1.6141416089 & 9.2144221529 & -3.7447488847 \\ \mathrm{H} & -1.0915001598 & -0.0182465171 & -4.7114905746\end{array}$

